

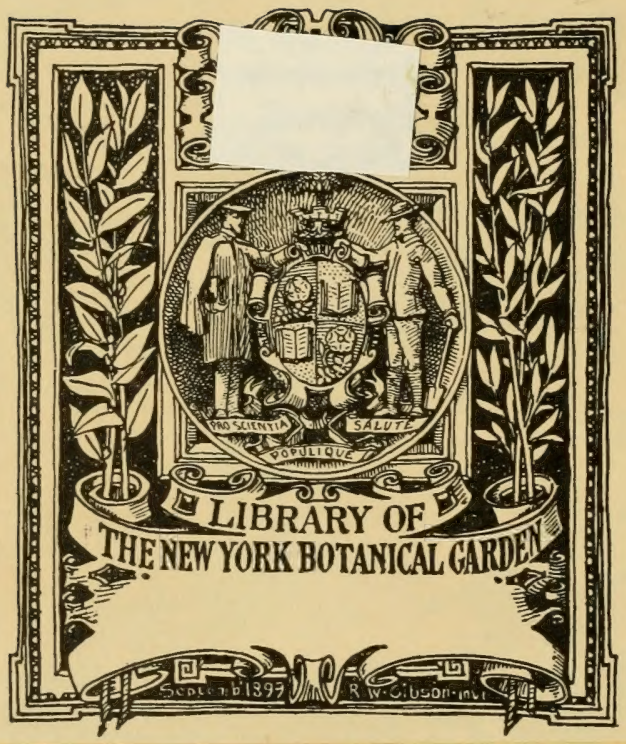








\section{THE FLORA OF THE DUTCH WEST INDIAN ISLANDS ST. EUSTATIUS, SABA AND ST. MARTIN.}

PROEFSCHRIFT TER VERKRIJGING VAN DEN GRAAD VAN DOCTOR IN DE PLANT- EN DIERKUNDE AAN DE RIJKS-UNIVERSITEIT TE UTRECHT, OP GEZAG VAN DEN RECTOR MAGNIFICUS DR. JAN DE VRIES, HOOGLEERAAR IN DE FACULTEIT DER WIS- EN NATUURKUNDE, VOLGENS BESLUIT VAN DEN SENAAT DER UNIVERSITEIT TEGEN DE BEDENKINGEN VAN DE FACULTEIT DER WIS- EN NATUURKUNDE TE VERDEDIGEN OP DONDERDAG 1 JULI 1909, DES NAMIDDAGS TE 4 UREN DOOR ISAÄC BOLDINGH, GEBOREN TE PURMEREND :-: :-:

BOEKHANDEL EN DRUKKERIJ

voorheen

E. J, BRILL: - LEIDEN

1909. 



\section{THE FLORA OF THE DUTCH}

WEST INDIAN ISLANDS $\mathrm{S}^{\mathrm{T}}$. EUSTATIUS, SABA AND $S^{T}$. MARTIN. 



\section{The Flora of the}

Dutch West Indian Islands St. Eustatius,

Saba and St. Martin. 



\title{
THE FLORA OF THE DUTCH WEST INDIAN ISLANDS ST. EUSTATIUS, SABA AND ST. MARTIN.
}

PROEFSCIIRIFT TER I'ERKRIJGING IAN DEN GR.I.II) WAN IOOCTOR IN IIE PLANT- EN IMERKUNIME A.IN IOF RIJKS-LNIVERSITEIT TE UTRECHT, OP GEZAG TAN IDEN RECTOR MAGNIFICLS DR. JAN LE I'RIES, HOOGLELRA.IR IN DE FACLITEIT LER WIS- EN N.ITUURKLNDE, VOI. GENS BESLLTT VAN DEN SENAAT DER UNIVERSITEIT TEGEN DE BEDENKINGEN VAN DE FACLLTEIT DER WIS. EN NATLURLLNDE TE IERDEDIGEN OP LONIERDAG 1 JULI 1909, DES NAMIDI)AGS TE 4 UREN DOOR ISA IC BOLDINGH, GEBOREN TE PURIIERENI) : : :-:

\author{
LIBRARY \\ NEW YORK \\ BOTANICAL \\ OAROEN.
}

Boekhandel en Drukkerij

voorheen

E. J. BRILL. - LEIDEN

1909. 
.86 
Aan mijne Moeder

en aan de nagedachtenis van mijn Vader. 

Gaarne de gewoonte volgend om ook op papier eon woord te richten tot hen, die de leiders zijn geweest bij de studie in dat levenstijdperk, dat ligt tusschen de eerste inschrijving als Student ain etur. Hoogetichoul en de: Promotie plechtigherd, is het mịj een groot voorrecht, hier dank te brengen aan $U$, Hooggeleerde Heeren Professoren in de Faculteit dor Wis- en Natuurkunde aan de Utrechtsche Hoogeschool.

Geldt mijn dank in 't algemeen $U$ allen, ik hecht er veel wiarde aan in 't bijzonder $U$ te noemen, Hooggeleerde Hubrech en Wichmann.

Maar bovenal Hooggeachte Promotor, Hooggeleerde Wenr, mijn dank aan $U$, bij wien ik het voorrecht heb gehad de laatste jaren van mijn studietijd assistent te zijn. Ik acht mij gelukkig, dat het einde van mijn student-zijn niet als dadelijk gevolg meesleept het einde van mijn assistentschap.

De herinnering aan den grooten steun, dien ik van U mocht ondervinden zal mij steeds bij blijven, tegelijk met die aan de alangename wijze, waarop (iij mij invoerde in de studie der Botanie.

Met grooten dank voor hunnen steun bij mijne studiereis noem ik hier: de Matitschappij tot bevoriering van het Natumkmolig Onderzoek der Nederlandsche Koloniën, de Koninklijke Akademie van Wetenschappen te Amsterdam, de Commissie van het F. W. van Eedenfonds en de Directie van het Provinciaal Utrechtsch Genootschap van Kunsten en Wetenschappen. 



\section{CONTENTS.}

Preface.

Additions and Corrections.

\section{FIRST PART.}

\section{SYSTEMATICAL.}

Pteridophyta 1, Gymnospermae 8, Monocotyledoneae 8, Dicotyledoneae 44.

\section{SECOND PART.}

\section{HISTORICAL.}

CHAPTER I. HISTORICAL SKETCH.

St. Eustatius 211, Saba 214, St. Martin 214.

CHAPTER II. NOTES ON THE LARGER COLLECTIONS.

Collection W. F. J. Suringar 216, Collections Mrs. J. vau Grol-Meyers 217, Collection A. C. W. Lionarons 225, Collection I. Boldingh 229.

\section{THIRD PART.}

\section{PHYTOGEOGRAPHICAL.}

SECTION A. OROLOGICAL, GEOLOGICAL AND METEOROLOGICAL.

CHAPTER I. GEOLOGICAL AND OROLOGICAL NOTES.

Introduction 235, St. Eustatius 237, Saba 239, St. Martin 241, General notes 243.

CHAPTER II. METEOROLOGICAL NOTES.

SECTION B. DISTRIBUTION OF THE WILD PLANTS ENUMERATED IN THE FIRST PART.

CHAPTER I. DISTRIBUTION OF ALL THE WILD PLANTS ENUMERATED IN THE FIRST PART. 
CHAPTER II. DISTRIBUTION OF THE PLANTS KNOWN ON THE ANTILLES ONLY, ENUMERATED IN THE FIRST PART.

SECTION C. THE VEGETATION OF ST. EUSTATIUS, $S A B A$ AND ST. MARTIN.

CHAPTER I. NOTES ON THE ASPECT OF THE VEGETATION OF THE THREE DUTCH WEST INDIAN ISLANDS.

Introduction 265, St. Eustatius 266, Saba 271, St. Martin 276.

CHAPTER II. DESCRIPTION OF THE DIFFERENT TYPES OF THE VEGETATION.

Introduction 28:, Eriodendronvegetation $25:$, Crotonvegetation 286, Littoralvegetation 288, Vegetation of the Cultivated Region 291.

Literature.

Vernacular Names.

Maps of the Dutch West Indian Islands. 


\section{STELLINGEN.}

I.

Bij de bestudeering van de Flora der West-Indische Eilanden moet meer dan tot nu toe geschied is, gelet worden op de verspreiding der planten op elli eiland in 't bijzonder.

II.

De indeeling der Antillen volgens E. Suess is ook om floristische redenen te verkiezen boven die van P. T. Cleve.

\section{III.}

Conocarpus erectus Linn. is geen Mangroveplant. IV.

Batis maritima Linn. behoort niet tot de Orde der Centrospermae. 
De nieuwere onderzoekingen over de embryozak fer Planerogamen verminderen de warde der phylogenetische beschouwingen, zooals die door O. Porsch zijn gegeven.

VI.

Op phylogenetisehe gronden is het gebruik van het begrip "Gesammiart" niet te verledigen; ook hreft het geene andere dan verwarende nitwerking. (Ascherson und (iräbner: Syuopsis der Mitteleuropäischen F'lora).

VII.

Onze kennis van de Bacteriaceae en Cyanophyceac is te onvolledig om daaruit conclusies te trekken omtrent de cytologie van de oercel.

\section{VIII.}

De Appendicularia zijn geen neotenische Ascidienlarven.

IX.

De Cetacea vormen eene monophyletische groep. $\mathrm{X}$.

Hyolithidae zijn verwant met Cephalopoda. 
XI.

De White Wall op St. Eustatius is ontstaan door plaatselijke opheffing.

\section{XII.}

In een herbarium is alphabetische rangschikking van de Species eener zelfele Plantenfanilie de beste. 





\section{THE FLORA OF THE DUTCH WEST INDIAN ISLANDS.}

I. The Flora of St. Eustatius, Saba and St. Martin. 


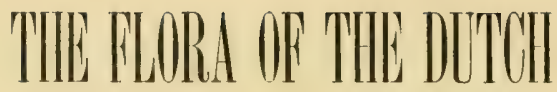 WLST" INDIII ISLANINS.}

\section{FIRST VOLUME}

St. Eustatius, Saba and St. Martin

\section{BOLDINGH.}

Boekifandei。 EN DRUKKeriJ

voorheen

E. J. BRILL - LEIDEN

1909. 


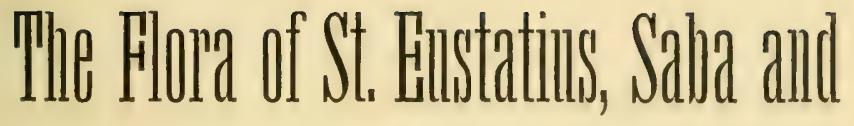 St. Mlaptill.}

\section{BOLDINGH.}

LIBRARY

NEV YURK

BOTANICAL

UARLEN.

Boekhandel ex Drukkeit.

voorheren

E. J. BRILL - LEIDEN

1909. 



\section{PREFACE.}

Mrs. J. van Grol-Meyers, wife to Mr. G. J. van Grol, administrator of the Dutch West Indian Island St. Eustatius, sent to the "Koloniaal Museum in Haarlem" a nice collection of dried plants collected in St. Eustatius in the years 1904-1906.

She also gave such a collection to the Herbarium of the Botanical Laboratory of the University of Utrecht.

The latter collection of about 350 numbers gave rise to a renewel investigation to the Florit of the Dutch West Indian Islands. I am glad to say that the collection made by Mrs. J. van GrolMeyers is a very good one. The name of Mr. van Grol himself is well known to the student of the Melocacti of the Dutch West Indian Islands, and the work Mrs, van Grol did now, proved to be a great help for the stuily of the Flora of the Dutch West Indies Prof. Dr, F. A. F. C. Went, Director of the Botanical Laboratory and Garden of the University of Utrecht kindly intrusted to me the determination of the collection of the above mentioned West Indian Plants, and in 1305 he proposed to me to make a voyage to the three Dutch West Indian Islands St. Eustatius, Saba and St. Martin in order to get acquainted with the vegetation there and to get a collection of the plants from the three Islands.

I wish to express my thinks to „De Maatschappij ter Bevordering van het Natuurkundig ()nderzoek ler Nederlandsche Koloniën ("Society for promoting seientific Investigation in the Dutch Colonies"), De Koninklijke Akalemie van Wetenschanpen (Korthalsfonds) te Amsterdam, the "Commissie van het F. W. van Eedenfonds in Haarlem” and the „Directie van het Provinciaal Utrechtsch Genootschap van Kunsten en Wetenschappen" (Provincial Utrecht Society of Art and Science), who subsided and so made it jmssible for me to undertake the above mentioned investigations.

Most of all I am indebted to the kindness of Prof. F. A. F. C. Went who, after having taken the initiative for my study of the 
Flora of the Dutch West Indies, has helperd me so energetically by his personal advice as well as by his influence with the above mentioned Societies.

I am glad to have an opportunity now to express my thanks to all those who have helpenl me in any way in promoting my studies. As to the Dutch Antilles I thankfully mention the Governor of Curaçao Jhr. J. O. de Jong van Beek en Donk and the admini. strators of St. Eustatius, G. J. van Grol, and 'T. G. Groebe, the ad. ministrator of Sala H. J. Beilujon, the administrator of st. Martin A. J. C. Brouwer and the maire of the French Part of St. Martin D. Beauperthuy.

Moreover: in St. Enstatius: Mrr. J. van Grol-Meyers, Mr. J. Morgan Griffith, Rev. P. Delgueur, Mr. J. G. C. Every, Messs: Hill and Mr. Hassel; in Saba: Dr. A. C. W. Lionarons, Rev. Mulder and Mr. T. Holms; in St. Martin: Mr. J. A. Gravestein, Mr. L. A. van Romond, Mr. A. A. van Romondt, Mr. D. J. van Romondt, Mr. W. E. van Romondt, Mr. W. Netherwood and Mr. F. Morales.

During my royage to the West Indies I experienced much friendly help from Sir Daniel Morris, Mr. J. R. Bovell, R. P. Duss, Mr. Stephens and Mr. Shepherd.

I also highly appreciate the assistance I got in Holland where I was informed with the greatest loyality by Mr. L. A. Bakhuis, Dr. J. Boeke, Dr. Mr. Greshoff, Dr, G. A. F. Molengraaff;, Dr. J. Valckenier Suringar and Dr. C. E. A. Wichmann.

According to the wishes of the "Commissie van het $\mathrm{F}$. W. van Eedenfonds" I paid much attention to the cultivated plants too; in a former publication: Lijst van Planten die door de bewoners van de drie Nederlandsche Antillen, St. Eustatius, Saba en St. Martin als geneeskrachtig worden beschouwd (Bull. Kolon. Mus. Haarlem n. 38 (2907) p. 93-112), I wrote on the use made of certain plants and in this Flora now I have enumerated all the cultivated plants I met with and which I was alyle to determine. I also feel very much indebted to the following Directors of "'s Rijks Herbarium" at Leiden viz: Dr. J. M. Janse, Dr. J. P. Lotsy and Dr. J. W. C. Goethart, who greatly facilitaled by their kind assistance my working there on the collection of Dr. F. W. R. Suringar.

But most of all I kindly thank Prof. I Urban for his assistance when I was working in the magnificent Herbarium Krug et Urban in the Künigl. Bot. Garten und Museum steglitz-Dahlem (Berlin.).

Although the time I could be there was only very short, my visit 
however has been of great use to me owing to the kind assistaner: of l'rol. Urban in loking after doubtiul determinations and un. determinated flantis and in describing sone new species. He hatis enablent me hy his great knowledge to bring vit the names of some plants that were collected without flowers or fruits.

I feel inclined to express my thanks to Mr. O. E. Schulz and Dr. F. Niedenzu for the determination of some plants and to Dr. $\mathrm{H}$. Hallier, Dr. A. A. Pulle and Dr. S. H. Koorders for their advices. My best thanks also to Mr. S. Perrin who has helped me in looking after the English text of this Flora.

This Flora is dividen in Three Parts; whereas the different Parts, Sections and Chaprers need no further explanation a short account of the methods I foilowed in nomenclature, synonymy and distri. bution of plants is given here.

The enumeration of Families and Genera is very nearly given after the order of De Dalle Torre et Harms: Gencra siphonogamarum ad systema Englerianum Conscripta (1908).

In a few cases $I$ thougt it better to follow:

Urb.in: Symbolae Antillanae vol. I, II, III, IV (fasc, 1 and 2), ? VI (fasc. 1) and Urban: Additamenta ad Cognitionem Florae Indiae Occidentalis $1892-97$.

The names of the species used are mostly drawn from the two latter papers, Ensler und l'rantl.: Die natürlichen Ptlanzenfamilie up to 1909, Engler: Regnum Vegetabile up to 1909, or from other monographs.

Whenever I could not find any name in the ahove mentioned pullications I trok the name I thought hest after the Internatio. nal rules for Botanical Nomenclature chiefly of Vilscular Plants. Wien 1905. (Verh. Int. Bot. Congr. 1805 Wien.).

Besides the names thus given I also mention the synonyms that are used in: Grisebach: Flora of the British West Indian Islands, Duss: Flore phanérogamiıue des Antilles françaises, Mrillspaugh: Flora of the Island of St. Croix, and Jackson: Index Kervensis up to 1909 and in a very few cases still another one.

I tried to get aim at the wreatest completeness possihle in giving the data about the names thus used, and I also mentioned the pages where the plants are given up in: Grisebach: Flora of the 
British West Indian Islands, Duss: Flure phanérogamique des Antilles françaises, Millspaugh: Flore of the Island of St. Croix and in a few cases still some other work.

To every plant is added the name of the Dutch West Indian Leeward Island where it is found in this order: St. Eustatius, Sabia, St. Martin; whenever I saw or collected it also on Anguilla this Island too is mentioned ${ }^{1}$ ) and when the plant is also known from St. Croix this Island is named after Anguilla.

I have mentionel as much as I could find about the distribution of the plants in all parts of the world, and these data are taken from the following publications in this order:

Urban: Symbolae Antillanae Vol. I, II, III, IV (fasc. 1 and 2), V, VI (fasc. 1) and Additamenta ad Cognitionem Florae Indiae Occi. dentalis (1892-97), Engler: Regnum vegetabile up to April 1909 , Engler und Prantl: Die Natürlichen Pflanzenfamilien up to 1909 , newer Monographs and Jackson: Index Kewensis up to 1909.

For the abbreviated names see under Literature.

Prof. Urban was so kind as to allow me the use of the not yet published data from the Herbar Krug et Urhan Berlin (Steglitz. Dahlem). A few data are from "'s Rijksherlarium" at Leiden. Whenever I could not find that a plant was known in other parts of the world besides the Antilles, then the names of all the Islands where it is found are enumerated.

The Systematic Part consists of the plants belonging to the collections of Suringar, van Grol-Meyers, Lionarons and Boldiugh. The numbers with B. alded are from the collection Boldingh, marked G. are from the collection van Grol-Meyers when they belong to the collection in the Herbarium at Utrecht, marked $H$ when they belong to the collection in the Koloniaal Museum at Haarlem; the numbers markod L. are from the collection Lionarons and the numbers where no initials are added are from the collection Suringar.

The numbers of the collections Suringar were not given by Suringan when he collected the plants (as Suringar dit not jut any num. ber on the labels) but they are only the last ciphors of the long number that is fixed to the paper on which the plants are moun. ted in "s Rijks Herbarium" at Leiden.

As I am sure that when giving the name under which the plant

1) See Literature: Boldingh. 
is mounted and these ciphers the plant is sufficiently indicated, I thought it hetter not to give the long numbers that are used in "s Rijks Herbarium".

The plants collected in the differcut Islamls are thus given: collection Boldingh, collection Suringar, both in the order of the day of collecting, collections Van (irol-Meyers and collection Lionarons. Not all tho vernacular names people told me are given here, but the names I have given in this flora are used without any donbt. The heights where the plants were collected are given with the greatest accuracy possible.

Whenever a plant struck my attention although 1 did not think it necessary to collect it, I made some notes ahout it; these notes are also to be found in this Flora, printed with small type.

As to the flowering and fruiting time of the plants I did not think it necessary to mention with every plant whether I collected flowers on fruits as the greater part of the plants are collecterl with flowers, erther ly Suringar, Van Grol-Aleyers or by myself:

I wish to draw the attention to the fact that I have tried to rencer this Flora of the Dutch West Indian Islands as complete as possible, loth hy what my own investigations have taught me and what I could find elsewhere.

Only the genus Melocactus has not been studied with the sane care, as I was sure I could not add anything to the work done hy Suringar on this head by the data I could bring together with out giving too much time to it: So I refer to the studies of F. W. R. Suringar on Melocactus and those of J. Valckenier Suringar.

Botanical Laboratory of the University in Utrecht.

June 1909. 


\section{Additions and Corrections.}

Paige

13 Line 19 from top. For 1859 read 1889.

17 Line from top. After Millspauch 437 insert, Panicum lanatum Rottb. in Act. litt. univ. Hafn. I (1778) 269.

47 Line 16 from bottom. After 81 insert 60 a.

79 Line 4 from top. For macrantha read, macracantha.

100 Line 15 a from top. Insert, St. Martin: Tintamarre: n. 3376 B. 106 Line 16 from bottom. After (Symb.). insert, Baham. (Hitchc.). 119 Line 1 from top and line 8 from bottom. For 1885 read 1883. 164 Line 15 from top. For Antill., Cuba to French Islands. (Gris. Fl.). read: Jamaica, Hispaniola, Portorico, St. Thomas, Guadeloupe, Dominica, Martinique, St. Lucia, St. Vincent, Barbados. (Herb. Krug et Urban).

178 Line 11 from top. For Antill. (Gris. Fl.). read: St. Thomas, St. Jan, Guadeloupe, Desirade, Les Saintes, MarieGalante, Dominica, Martinique, St. Vincent, Barbados. (Herb. Krug et Urban).

235 Line 9a from bottom. Insert, J. W. W. Spencer. Reconstruction of the Antillean Continent. Bull. Geol. Soc. Amer. 6. 1894 p. 103-146.

Map of St. Eustatius: Bengalen is to lie put to the N. E. of the Quill. Map of 'The Dutch W. I. Islinds: For Barbala reeul Barbuda. Map of Saba: For Marizot read Marigot. 


\section{CONTENTS.}

Preface.

Pages.

Additions and Corrections.

\section{FIRST PART.}

\section{SYSTEMATICAL.}

Pteridophyta 1, Gymmospermae 8, Monocotyleroneat 8, Dicotyledoneae 44.

\section{SECOND PART.}

HISTORICAL.

CHAPTER I. HISTORICAL SKETCH.

St. Eustatius 211, Saba 214, St. Martin 214.

CHAPTER II. NOTES ON THE LARGER COLLECTIONS.

Collection W. F. J. Suringar 216, Collections Mrs. J. van Grol-Meyers 217, Collection A. C. W. Lionarons 225, Collection I. Boldingh 229.

\section{THIRD PART.}

PHYTOGEOGRAPHICAL.

233

SECTION A. OROLOGICAL, GEOLOGICAL AND METEOROLOGICAL.

CHAPTER I. GEOLOGICAL AND OROLOGICAL NOTES.

Introduction 235, St. Eustatius 237, Sala 239, St. Martin 241 , General notes 243.

CHAPTER II. METEOROLOGICAL NOTES.

SECTION B. DISTRIBUTION OF THE WILD PLANTS ENUMERATED IN THE FIRST PART.

CHAPTER I. DISTRIBUTION OF ALL THE WILD PLANTS ENUMERATED IN THE FIRST PART. 
CHAPTER II. DISTRIBUTION OF THE PLANTS KNOWN ON THE ANTILLES ONLY, ENUMERATED IN THE FIRST PART.

SEUTION C. THE VEGETATION OF ST. EUSTATIUS, $S A B A$ AND ST. MLARTIN.

CHAPTER I. NOTES ON THE ASPECT OF THE VEGETATION OF THE THREE DUTCH WEST INDIAN ISLANDS.

Introduction 265, St. Eustatius 266, Saha 271, St. Martin 276.

CHAPTER II. DESCRIPTION OF THE DIFFERENT TYPES OF THE VEGETATION.

Introduction 282, Eriodendronvegetation 282, Crotonvegetation 286, Littoralvegetation 288, Vegetation of the Cultivated Region 291.

- Literature.

Vernacular Names.

Index to the First Part.

Maps of the Dutch West Indian Islands. 


\section{FIRST PART}

SYSTEMATICAL 



\section{Pteridophyta.}

\section{Hymenophyllaceae.}

\section{Trichomanes Linn.}

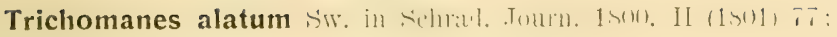
Giris. F!. 60t.

Saba: Mountain S00 M.: 1. 2204 B., 2206 B., 1, 6.

Antill., Amer. austral. (Symb.).

\section{Hymenophyllum Smith.}

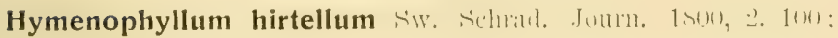
Gris. Fl. 659.

Saba: Jountain 800 M.: n. $181+$ B., 1815 B., 1816 B., 2201 B., 7. Antill., Amer. centr., Moxico. (Christensen 1906.)

\section{Cyatheaceae.}

Cyathea J. H. Sm.

Cyathea arborea J. L. Sm. in Mém. Acad. Scienc. 'l'urin V (1790) 417 ; Gris. Fl. 704.

Saba: Mountain 600 M.: 11.17 .55 B.

Antill., Bras. (Symb.).

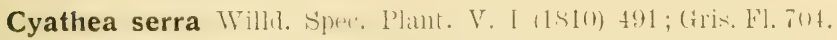

St. Eustatius: Top of the Quill: n. 163, 16t, 171, 172, 173, 174,175 .

I only saw two specimens of a Ferntree in the Quill, in a very deep gut and it was impossible for me to get a part of it; most probably it was Cyatheal serra.

Saba: Mountain near. Windwardsicte 600 M.: n. 2170 B.; Rendez-rous Mountain: n. 46.

Antill., Bras., Mexico. (Symb.). 
Hemitelia R, Bl:

Hemitelia horrida $R$. Br. Prodr. I (1s10) 158; Gris. Fl. T0ti. Saba: Mountain 800 M.: n. 2221 B., 2225 B., 165, 188 L., 189 L. Antill., Amer, austr. sept. (Symb.).

\section{Alsophila R. Br.}

Alsophila phalerata Mirt. Ie. Cr. Bras (1834) 67 t. 30 f. I. t. 42 .

Saba: Mountain 800 M.: n. 2220 B., 39, 40, 41, 42, 43, 44, 45,170 .

Amer. trop. (Christensen 1906.).

\section{Polypodiaceae.}

Dryopteris Adans.

Dryopteris opposita Lrh, in Symbolie Antillanae IV (190:3) 14. Aspidium conterminum Willd. Spec. Plant. V. I. (1810) 249; Gris. Fl. 691.

Nephrodium oppositum Diels in Engl.-Prantl. Nat. Pflanzenfam. I. 4 (1889) 172 (non Hook). (Symb.).

Saba: Gut near Bottom: n. 1434 B.; Top of the Mountain: n. 1798 B., 1808 B., 2151 B., 2195 B.; Mountain near Hellsgate:

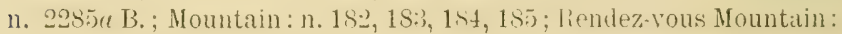
n. 186 ; Crespeen: 11. $253 \mathrm{~A}$; locality unknown: 11. $1660 a$ B., $220 \mathrm{~L}$. Antill., Amer. cont. trop. (Symb.).

Dryopteris parasitica 1). Kitze. Rev. (ien. Pl. 2 (1891) \$11.

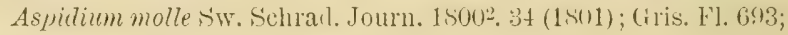
Millspaugh 468.

St. Eustatius: lope of the Quill :ino M.: n. 407 B.; inner slope of the Quill 350 M.: n. 463 B.; Quill: n. 116, 122; locality unknown: n. $258 \mathrm{H} ., 258 a \mathrm{G} ., 276 \mathrm{G} . ;$ Bengalen: 17. 16.

Saba: Gut near the Bottom: n. $1423 a$ B., 1481a B.; Booloyhill: n. 1592a B.; llellsuate to Sulphumines: n. 1673 B.; Mountain: n. $1750 a$ B., 1761 a B.; Mountaintop: n. 1832 B.; Sulphurmi. nes to Marypoint: n. 2063 a B.; Hountain near Hellsgate: n. 2.28:a B.; Mountain: n. 224, 280, 234: Rend(\%-vous. 4 -600 M.: n. 16!): Jenkeygut: $n .15$.

St. Martin: Milrigothill -.Milrigot 1)-200 M.: n. 2644 B.; in an 


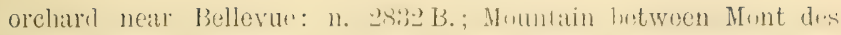
Accords and Centryhill 200-300 M.: n. 2895 B.; hill on the northern side of Cul de sac: n. $3007 a$ B.; Milldrumhill $300-400$ M.: n. 3182 B., $318: 3$ B.; Mount Paradis $300-400$ M.: n. 3239 B.; gut from Bethlehem to Cul de sac: 11. 3413 B.; Centryhill: 11. 76.

St. Croix ex Millspaugh.

Baham., Bermud., Antill., Amer, trop., Africa, Asia austroorient., Australia, Nova Zeal. (Symb.).

Dryopteris reticulata (Th), Fymliolite Antillanar IV 11 (n):i) 2:).

Menisrium reticulatum Sw. in Schurad. Journ, 1800. II (1801) 10 ; Gris. Fl. 687.

Saba: Mountain 800 M.: n. 1822 B., 2215 B.

Amer. trop. (Christensen 1906).

\section{Aspidium Sw:}

Aspidium Plumierii Prest. liul. Ma(mk. I (18:30)

Aspidium trifotiatum forma Gris. Flor. W. I. (1864) 695.

St. Eustatius: Quill: n. 123; Bengalen: n. 82; inner slope of the Quill: 11. 437 B.

Saba: Mountain 800 M. : n. 2199 B., 1, 2; locality unknown: n. $212 \mathrm{I}$.

Portorico, Guadeloupe, Dominica, Martinique, St. Vincent, Grenada, 'L'obago, 'I'rinidad. (Symb.).

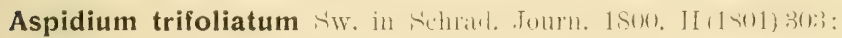
Gris. Fl. 695 (p.p.). (Syml).).

Saba: Mountain S00 M.: n. 1823 B.; Mountain neal Hellsgate 600 M.: 11. 2286 B.; Crespeen-Rendez-vous 400 M.: 11. 3, 4; Gainliygut: n. $22 \overline{\text {. }}$

Antill., Amer, centr. and austr. (Symb.).

\section{Polybotrya $H$. et $B$.}

Polybotrya cervina Kilfs. Enum. (1824) 55.

Olfersia cervina Kze. in Regensl). Floril VII. I (1824) 312: Gris. El. 678 .

Saba: Mountain 800 M. : n. 1819 B., 1824 B., 1826 B., $2118 \alpha$ B., $2202 \mathrm{~B} .$, Mountain 6-800 M.: n. 70, 71, 72, 168, 202 I., $204 \mathrm{~L}$.

Antill., Amer. cont, trop. (Symb.).

\section{Nephrolepis Schott.}

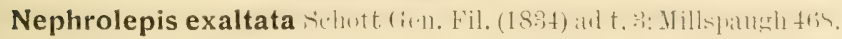
Aspidium exaltatum Sw. in Schrad. Journ. 1800. II (1S01) 32; Gris. Fl. 6SS. 
St. Eustatius: Quill : 11. 137; Top of the Quill : n. 13s, 141, 142, 143, 144; Gilboohill: 1. 1125 B.

Saba: Mountain 800 M.: n. 1802 B., 2194 B., 2197 B.; Mountain near Hellsgate to00 M.: n. 2285 B.; Mountain: n. 62, 63, 64, $65,66,67 ;$ locality unknown: n. $172 \mathrm{~L}$.

St. Croix ex Millspaugh.

Bermud., Antill., Amer. cont. trop, Afr. trop., A sia austro orient., Polynes., Australia sept. (Syml).).

\section{Diplazium Sw.}

Diplazium semihastatum ('. ('hristensen Inlex F'ilicum (1906) 239. Asplenium bipartitum Spr. Syst. IV (1827) 85.

St. Eustatius: T'op of the Quill: n. 100.

Cuba, Jamaica, Portorico. (Christensen 1906).

Diplazium striatum Pres]. T'ent. jterid. (1\$36) 114.

Asplenium striatum Linn. Spec. (1753) 1082; Gris. Fl. 686.

Saba: Mountain 800 M.: n. 1833 B., 2203 B., 181, 238, 239.

Antill., Amer, cont, trop. (Symb.).

\section{Asplenium Linn.}

Asplenium auritum Sw. in Sehranl. Journ. 1500 ll (1.501) 52: Giris. F1. 682.

W. I. : Suringar: n. 240.

Antill., Amer, cont. trop., Ins. afric, orient., India orient. (Symb.). Asplenium cristatum Lam. Enc. 2. (1786) 310.

Asplenium rhizophorum Linn. y cicutarium Sw.; Gris. Fl. 684.

St. Eustatius: 'Inp of the Quill 400 M.: 11 . :3.2.2 B., 3333 R.: inner slope of the (quill :350) M.: n. 41 !) B.; Bottom of the: Quill 300 M.: n. 436 B., 451 B.; slope of the lower part of the Quill near Bengalen: n. 730 B.; Bengalen: n. 96, 97, 98; top of the Quill: n. 94; Quill: n. 106, 111, 112, 113, 114; locality unknown: 3. 95.

Saba: Springlaygut: n. 2111 B.; Mount Paradis 300-400 M.: n. 3230 B.; Gainbygut: n. 6; Hellsgategut near Devilshand: n. 7; Mountain: n. 2, 3, 4, 8, 10 .

Antill, Amer. cont. trop., Afr. trop. (Symb.).

Asplenium monanthes Linn. Mant. (1767) 130.

Saba: Marypointgut: n, 12.

Amer, trop., Ins. Hawaiï, Africa and Antlant. Ins. (Christen. sen 1906).

Asplenium obtusifolium Linn. spec. (175) 10.50; (iris. Fl. tis1.

St. Eustatius: Bottom of the Quill Bo(k M.: n. 141 B.. 4tit B. Antill., Amer. cont. trop. (Symb.) 


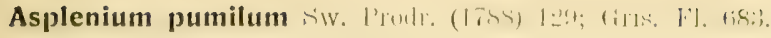

Saba: Hollsgate to Marypoint: 11.2041 B.

St. Martin: Milldrumliell $300-400$ M.: H. 3178 B., 3181 B.: Mount Paradis 300-400 M.: 11. 3:13a B.

Antill., from Mexico to Columbia. (Symb.).

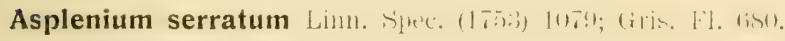

St. Eustatius: slope of the Quill 350 M.: 1]. 387 B.; Bottom of the Quill 300 M.: 11. $44 \pm$ B., 450 B.; Quill: n. 179; Top of the Quill : n. 177, 178; locality unknown: 17. 150.

Saba: locality unknown: n. $211 \mathrm{~L}$.

Antill., Amer. centr. and austr., Ins. Societ. (Symb.).

\section{Blechnum Linı.}

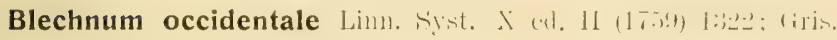
Fl. p. 673 ; Millspaugh 469.

St. Eustatius: slope of the quill $350 \mathrm{M} .:$ 11. 385 B.; innor slope of the Quill 350 M.: N. 1225 B.;Top of the Quill: 11. 135; slope of the Quill: 11. 78, 79, 136.

Saba: Bottom 200 M.: 11. 1449 B.; Hellsgate: 1]. 1689 B.; Springbaygut: 11. 2113 B.; $211 \pm$ B. ; Mountain near Windward side 600 H.: n. 2168 B., 2172 B.; Mountain: n. 35; Mountain 6-800 M.: 12. 233, 233; Peperpot: 11. 25, 26; Crespeen-Rendezvous 400 M.: n. 34; Gainbygut: n. 24.

St. Martin: Marigotroad near Marigot: 1. 2803 B.; Hount Patradis $300-400$.I.: n. $3342 \mathrm{~B}$.

St. Croix ox Millspaugh.

Antill., Amer. cont. trop. (Symb.).

\section{Stenochlaena J. Sn.}

Stenochlaena sorbifolia J. Sm. in Hook. Journ. of Bot. IV (1842) 149 ; Gris. Fl. 674.

St. Eustatius: Buttom of tlu! (quill : 3111 M.: 11,460 B., 45:3 B., Top of the Quill: n. 242a B.; Quill: n. 130, 131, 132, 132; Boven: n. 80 ; Bengalen: n. 81.

Saba: T'op of the Mnuntain: 11. 22. 1; lucality unknown: 11. $181 \mathrm{~L}$. Antill., Amer. cont. trop., Afr. trop., Asia austro-orient., Ins. Monsun., Polynes. (Symb.).

Hemionitis Linn.

Hemionitis palmata Limn. Spec. (1753) 1075; (iris. Hl. f67 Millspaugh 469. 
St. Martin: Naliuboyhill 50-200 M.: n. 2716 B.; Mountain

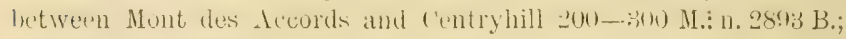
Alillitumhill 200-300 M.: n. 3118 B.

St. Croiz ex Millspaugh.

Antill., from Mexico to Peru. (Symb.).

\section{Neurogramme Lk.}

Neurogramme calomelanos Diels in E'Hgl. L'riutl Nit. P'llanzenfium. I. 4 (1899) 264.

Gymogramme calomelanos Kaulf. Enum. Fil. (1824) 76; Gris. H.1. 679; Millspaugh 469.

St. Eustatius: in a garden in Oranjestad: 13. 68 B.; top of the

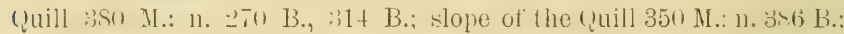
slope of the Quill near Bengalen: n. 744 B.; in a garden near Glassbottle: 1. 1208 B.; top of Pangahill: n. 128; seashore: n. 125; Boven: n. 126, 127, 129; Greatgut: n. 124; locality unknown: 1). 140 G., $189 \mathrm{H}$.

Saba: along roaks between Botton and Windwardsile 2(x) M.: 11. 1302 B.; 1367 B.; along tho seashore near Forthbaylinding: n. 1649 B.; Saddde: 11. 1659 B.; Mountain S00 M.: n. 1795 B.; gut

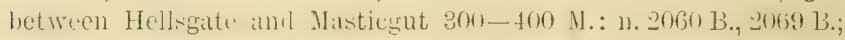
Springloaycut: n. $2089 \mathrm{~B}$; seashore near Forthbaylanding: $n$.

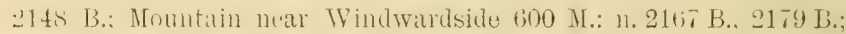
Ladderlanding: n. 232, 240; Greathill 200-400 M.: 11. 18; Moun. tain: n. 167; Peperpot: n. 10; Crespeen-Rendez-vous: n. 17; locality unknown: n. 147 今 B., 199 L.

I saw Neurogramme calomelanos also: near the Sulphurmines: Crespeenhill.

St. Martin: Milldrumhill :300-400 M.: n. 3177 B.

St. Croix ex Millspaugh.

Antill., Amel. cont. trop., Afr. trop., Samoa. (Symb.).

\section{Chleilanthes Sw.}

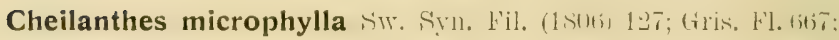
Millspaugh 469 .

St. Eustatius: alung rouds in Grinjestad: 11. 94:3, 13; Cultumvlakte; n. 118; Quill: 11. 121; Oranjestad: n. 115.

Saba: betwcen Bottom and Marypoint 100-200 M.: 11. 1513 B.; seashore near Forthlaylanding: n. 1651 B.; Laddorgut: 11. 1996 B.; Bottuin: 11. 228.

St. Croix ex Millspaugh.

Antill., Amer. cont. trop. (Symb.). 
Adiantum Limn.

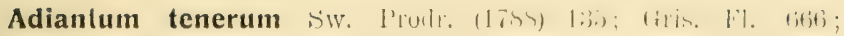
Millspaugh $46 \%$. M a i d e n ha il:

St. Dustatius: Mountain (dedit MIr, Falk): 11. 10t, 105, 106; Bengalen: 1]. 110; locality unknown: n. 109.

Saba: Paris 400 M.: n. 1373 B.; lower part of the Bottom Mountain 250 M.: n. 1412 B.; along roads between Bottom and

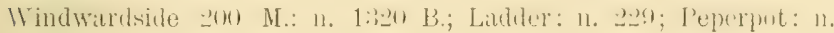
13; Greathill: n. 14, 15, 16; Gainbygut: $11 . \diamond$.

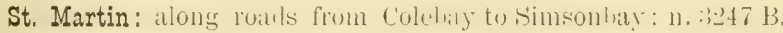

St. Croix ex Millspaugh.

Antill., Amer. cont. trop. (Symb.).

\section{Pteris Linn.}

Pteris biaurita Linn. Spec. (1753) 1076; Gris. Li. (669.

Saba: Gut between Hellsgate and Masticgut 300-400 M.: n.

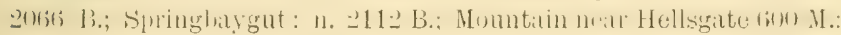
n. 2283 B.; Peperpot: n. 30, 36, 37, 38; (Aainlygut: n. 27, 28, 29. Antill., Amer., Afr., Asia trop. (\$ymb.).

\section{Lonchitis Linn.}

Lonchitis hirsuta Linn. Spec. (17533) 1078.

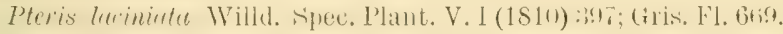
Saba: Top of the Mountain 800 M.: 1. 2218 B. Antill., Americ. cont. trop. (Symb.).

\section{Vittaria Siv.}

Vittaria lineata siv. in schrul. Jourı. 1so0. II (1S01) 7.:; (ilis. Fil. 671.

St. Eustatius: 'l'ol, of the (y)uill $4(0)$ 11.: 11 . :3:35) 13.; slope of the Quill 350 M.: 1. 405 B.; top of the Quill: n. 157. 158.

Baham., Antill., Amer. cont. trop. untill Florida, Afr. trop. untill Cap, Asia austro orient. untill Japon. (Symb.).

\section{Heteropteris Fee.}

cf. Paltoním Presl. (Christensen 1906).

Heteropteris lanceolata Fee ex Moore Ind. Fil. (15.7) 34:3: Diels in Engl.Prantl. Nat. Pflanzenfum. I. 4. (1899) 305 fig. 161 E.

Paltonium lanceolatum Pr. Epim. 156 (1849) 156. (Christensen 1906).

Taenitis lanceolata R. Br. Prodr. (1810) 154; Gris. Fl. 671; Millspaugh 469. 
St. Eustatius: T'op of the Quill 400 M. : 11. 243 B., $316 a$ B.; slope of the Quill near Bengalen 250 M.: 11. 4993 B.

St. Martin: Mount P'aralis 300-100 M.: 11. 3231 B.

St. Croix ex Millspaugh.

Balıam., Antill., Amer. centr. (Symb.).

\section{Polypodium Linn.}

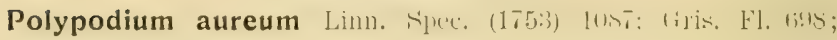
Milispaugh 469.

St. Eustatius: along roads in Oranjestad: 11. $531 \mathrm{~B}$; slope of the Quill near Bengalen 250 M. : n. 698 B.

Saba: Windwardside-Hellsgate: n. $1661 \mathrm{~B}$; lower part of the Bottom Mountain 300 M.: n. $1 \pm 41$ B.; Windwardsicle: n. $19 \pm 5$ B.; Mountain near Hellsgate 600 M. : n. 2287 B.; Quarter: n. 236.

St. Martin: Mountain between Mont des Accords and centry-

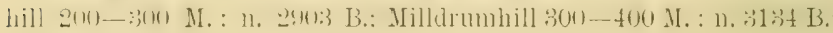
Nount Paradis 300-400 M. : 17. 3223 B., 3233 B., 3235 B.

St. Croix ex Millspaugh.

Baham., Antill., Amer. cont. trop. (Symb.).

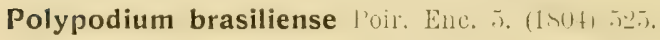

Polypodium neriïfolinm Schkuhr Kr. Gew. 1. 14. t. 15. (1806); Gris. Fl. 698 .

St. Eustatius: 'Iop of the Quill 400 M. : n. 291 B., 300 B.: S21 B.; 'T'op of the Quill 500 M. : 13. 485 B., 83, 140; Quill :n. 134, 139.

Saba: along roads between Bottom and TVindwardside $200 \mathrm{M}$.: 11. 129士 B; Parishill 200-400 M.: 11. 225 , 226.

St. Martin: Milldumhill $300-400$ M.: n. $314 \pm$ B.

Amer: trop. (Christensen 1906.).

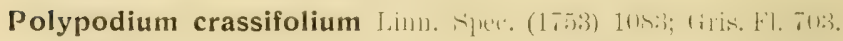

Saba: Mountain so0 M.: 11. 2193 B.; 48, 49, 51; locality unknown: n. $61 \mathrm{~L}$.

Antill., Amer. cont. trop. (Syml).).

Polypodium loriceum Lill1. Sike. (17):i) 1086; (iris. Fl. 698.

Saba: Mountain 800 M.: 11. 2191 B., 232. 237.

Antill., Amer: cont. tiop. (Symb.).

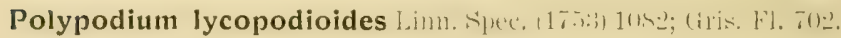
Bastard catclaw.

St. Eustatius: T'op of the Quill 380 M. : n. 259 B., 266 B.,

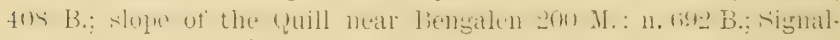
hill 200 M.: 1). 105] J3.; Slope of the Quill: n. 151, 153; Quill:11.

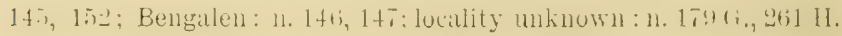


Saba: Paris 400 M.: n. 137t B., 5t: between Bollom and Marypoint 100-200 M. : 11. 1499 B.; Mountain lio0 M. : 11. 17+3 B., 57; Windwardside: 17. $19 \pm 1$ B.; Springluaygut: 11. 6094 B.; Moun. tain near Hellsgate: n. 2282 B.; Peperpot: n. 55; Crespeen-liendezvous 400 M.: 11. 60; locality unknown: 11. 56, 49 l.

St. Martin: Nakedboylill 200-260 M. : 11. 272.2 B., 272t B.; Mountain letween Mont des Accords and Centryhill $200-300$ M. : 11. 2902 B.: Mount Paradis $300-400$ M.: n. 3227 B., 3229 B.; Mount Paradis 200-300 M.: 11. 3318 B.; Centryhill : 11. 75.

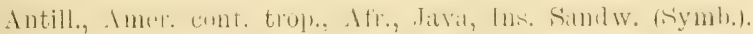

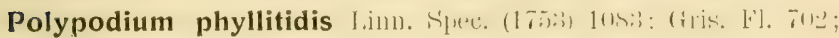
Millspaugh 470 .

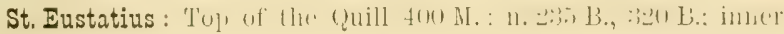
slope of the Quill 350 M. : 11. 462 B.; Top of the Quill 500 M. :

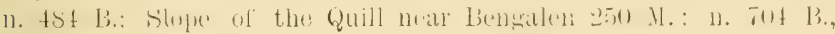
705 B.; Slope of the Quill: n. 159; Quill: n. 101, 160, 161; Ben. ralen: 13. 16:- locality unkmown: 11. $15: 3$ G., $257 \mathrm{H}$.

Saba: Windwardside-Hellsgate: n. 1667 B.: Springbaygut:

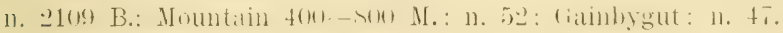

St. Martin: Millarumhill $800--400$ M. : n. 3138 13.; French frontier : 11. 7 t.

St. Croix ex Millspaugh.

Baham., Antill., from liloricia until Bras. (Symb.).

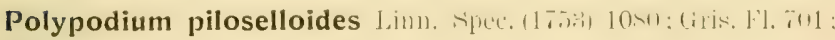
Millspauch 470.

Lepicystis piloselloides Diels in Engl.-1'runtl. Nat. Ptlanzenfan. 1. 4 (1899) 323.

Saba: Boobyhill 300 M.: n. 1581 B.; Mountain 800 M.: 1. $180 \pm$ B.; St. Giles: 1. 1866 B.; Mountain near Windwardside 600 M.: n. 2171 B.; Crispeen-Rendez-vous t00 M.: n. ti1, 235; Hellsgategut near Devilshand: 11. 53.

St. Martin: Mount Paradis $300-400$ M. : 1. 3218 B.

St. Croix ex Millspaugh.

Antill., Amer. cont. trop. (Symb.).

\section{Polypodium plumula 11. R. Will. Nis. I (1,411, 17.}

St. Eustatius: Top of tho Quill 400 M.: 1 . 222 B., 237 B., 311 B.; slope of the Quill 350 M. : 11. 406 B.; slope of the Quill

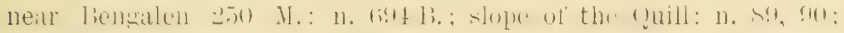

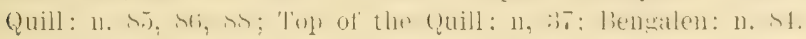

Saba: Crispeen: 11. 68.

Florida, Aner. trop. (Christensen 1906). 
Polypodium serrulatum Mett. Fil, hort. Lifis. (1S5) 50 .

Tiphopteris serrulate Kaulf. Enum. (1824) 85; Gitis. Fl. 703. Saba: Mountain 800 M.: n. 1834 B.

Antill., Amer. cont. trop., Afr., Ins. Sandw. (Symb.).

Polypodium squamatum Limu. spec. (1753) 10 s6.

Lepicystis squamate J. Sm. Brit. and For. Ferns (1866) 80.

St. Eustatius: slope of the Quill near Bengalen (n-2(n) Mr.: n. $643 \mathrm{~B}, 663 \mathrm{~B}$.

Saba: along roals between l3ottom and Windwardside 200 M.: n. 1306 B.; Mountain 600 M.: n. 1771 B.; Laddergut 0-200 MI. : n. 2021 B.; Paris: n. 32 ; Peperpot: n. 22 ; Mountain: n. 9; Gainbygut: n. 21; Liddergut: n. 31 ; Hellsgategut: n. 33.

St. Martin: Mountiin between Mont des Accords and Centryhill 2010-300 M.: 11. 245) B.: Mount Paradis 300-400 M. : 11. 32:32 B.; French frontier: $\mathrm{n}, 77$.

Antill., Amer. cont. from. Mexico to P'eru. (Symb.).

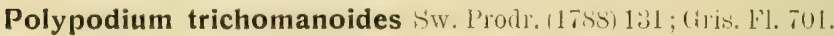

Saba: Mountain 800 M.: 11. 1817 B., 1835 B., 1837 B.

Antill., Amer, austr., Ascencion, Asia austro-orient. (Symb.).

\section{Elaphoglossum Schott.}

Elaphoglossum conforme Schott. (ichl. itl. t. 14 (linit).

Acrostichum conforme Sw. Syn. 10192 t. 1 f. 1 (1806); Gris. ['1. 677.

St. Eustatius: Top of the Quill 400 M.: 11. 302 B, 327 B., $363 \mathrm{~B}, 384 \mathrm{~B}, 180$; locality unknown: n. $1147 \alpha \mathrm{B}$.

Saba: Mountain 800 M.: n. 1820 B., 0 .

Amer. trop. (Christensen 1906).

Elaphoglossum latifolium J. Sim. in llouk. Lom! Jorrlı. bot. I (1842) 197.

Acrostichum latifolium Sw. Prodr. (1788) 128; Gris. F1. 677.

Saba: Mountain 800 M.: n. 2208 B.

Antill., Amer. cont. trop. (Symb).

Elaphoglossum petiolatum Urls, in symlolite Antillanite $V$ (1903) 61.

Acrostichum viscosum Vent. ap. Sw. Syn. Fil. (1806) 193; (tris. Fl. 676.

St. Eustatius: Top of the Quill: n. $192 a$ B., $248 a$ B.

Antill., Amer, cont. trop. until Argentinia, Africa trop., Asia austro-orient., Ins. Monsun. (Symb.). 


\section{Gleicheniaceae.}

Gleichenia J. L. Smith.

Gleichenia bifida Spreng. Syst. IV. I (1827) 27.

Mertensia furcata Gris. F'l. W. I. (1861)651 (non Spreng.). (Symb.).

Saba: Mountain 800 M. : n. 2227 B., 69.

Antill., Amer. cont. trop. (Symb.).

\section{Ophioglossaceae.}

Ophioglossum Linn.

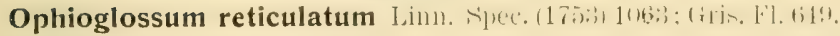

Saba: Mountain 6-800 M.: 11. 73.

Antill., Amer. cont. trop., Africa. (Symb.).

\section{Lycopodiaceae.}

\section{Lycopodium Limn.}

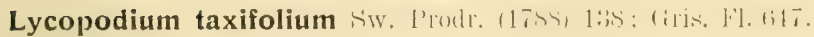

St. Eustatius: 'I'op of the Quill: 11, $479 \Leftrightarrow \mathrm{B}$.

Saba: 'T'op of the Mountain: 11. $2200 a$ B., 27.

Antill., Amer. cont. from Mexico to Brasil. austr. (Symb.).

Lycopodium verticillatum Linn. 1il. vipll. (1781) t4h; (iris. Fl.1it.

Saba: Mountain 650 M. : n. 21, 26.

Antill., Amer: cont. trop., Afr. austr. et orient., l'olynosia. (Symb.).

\section{Psilotaceae.}

Psilotum Sw.

Psilotum nudum Gris. Veg. Kar. (1857) 130.

Psilotum triquetrum Siv. in Schrarl. Journ. 1800 II (1S01) 109; Gris. Fl. 648.

Saba: Crespeen-Rendez-vous: n. 1761a B., 30.; Paris: 11. 20; Greathill: 11, 15; Mountain: n, 25.

Baham., Antill., Trop, countries of both hemispis. (Symb.).

\section{Selaginellaceae.}

Selaginella Spring.

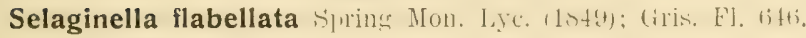

Saba: Mountain: n. 1778a B., 22, 24, 29, 214 L.

Antill., Amer: merid. sept. (Pulle.) 
Selaginella substipitata spring. Mon. Lye. (1st!n: Gris. Fl. fit5. Saba: Mountain 6- 800 M.: 11. $1813 a$ B., $2206 a$ B., $2321 a$ B., $2.227 a$ B., $23,28$.

Antill., Panama to Brasil. and Peru. (Gris. Fl.).

\section{Gymnospermae.}

\section{Pinaceae.}

45. Juniperus Linn.

Juniperus bermudiana Linn. Suec. (17.):3) 10:30.

Cultivated [Bermudas, Barluados.] (Ind. Kew.).

Juniperus virginiana Linn. Spec. (1753) 1039.

Cultivated [Amer, septentr.] (Ind. Kew.).

\section{Monocotyledoneae.}

\section{Potamogetonaceae.}

\section{Cymodocea C. H. Königg.}

Cymodocea manatorum Arihers. in Sitzungsher. (ies. naturforsch. Freunde, Berlin (1868) 19; Duss 473; Millspaugh 470.

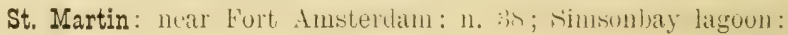
n. $3260 \mathrm{~B}$.

Suringar saw the Cymodocea manatorum also in Simsonbay lagoon.

St. Croix ex Millspaugh.

Bermud., Key West, Antill. (Symb.).

\section{Gramineae.}

\section{Zea Linn.}

Zea Mays Linn. Spec, (1753) 971.

Cultivated. [Amer, austr. Peru. ?] (Symb.).

\section{Saccharum Linn.}

Saccharum officinarum Limn. Sipec. (1753) it.

Cultivated. [Asia austro-orient.?] (Symb.). 


\section{Andropogon Linn.}

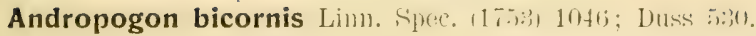

Anatherum bicorne Beauv. agrost. (1812) 15; Gris. F'. 559.

Saba: Greathili: n. 214; between Bottom and Marypoint $300 \mathrm{M}_{.}:$n. 1480 o B.

Antill., Amer. cont. trop., 'J'eneriffit. (Syml).).

Andropogon contortus Lim. Sip. (1753) 1045; I) 11, 5.2S.

Androprogon secundus Willd. ex Nees. Agrost. Bras (1829) 364. Gris, Fl, 558.

St. Eustatius: Near Boren 200 M.: n. 1238 B., 1239 B.

Domingo, lläti, Antigua, Guadeloupe. (Ierb. Krug et Urban.).

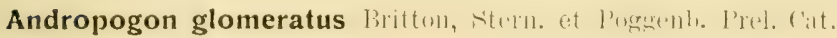
(18S8) 67 .

Andropogon macrow Michx. Hlor: bor: amer. I (1803) 56.

Anatherem macroum Gris. Flor. W. I. (1864) 559.

Saba: Mountain near Windwardside 600 M.: M. 2169 B.

Baham., Antilk, Amer. centr. and sept. (Symb.).

Andropogon Schoenanthus Limn. Spece, 117) $10+1$.

Culticated. [Ind. orient., Afr, trop., Asia.] (Hackel 1859). Lomongrass.

Andropogon sorghum Brot. Flor: lusit. I (1) (1) 4$)$ SS.

Cultivated. Creole corm, Guinea corn.

Andropogon zizanioides $(H$, syml). Ant. IV. (1!1):3) 7(?.

Andropogon squarrosus Linm. fil. Suppl. (1781) 438; Duss 529.

Vetiveria an undinace Griseb. Flor: IV. I. (1864) 559.

St. Eustatius: locality unknown: n. 287 G.

Antill., Bras., from Ind. orient.? (Symb.).

\section{Anthephora Schrel.}

Anthephora hermaphroditica (). Kt\%e. Fier. If (1S41) 759 !

Anthephora eleyans Schreb. Beschr. Griis. III (1810) 105 t. 14: Gris. Fl. 556; Duss 527; Millspaugh 471.

St. Eustatius: Along roads in Oranjestad: n. 559 B., 1094 B., $1107 \mathrm{~B}$.

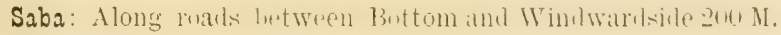
n. 1842 B.; Ladder: n. :376.

St. Martin: Guanabay: n, $2+27$ B.

St. Croix ex Millspaugh.

Antill., Curacao, Aruba, Amer. cont. trop. (Symb.). 


\section{Tragus Hall.}

Tragus racemosus Haller. HList. Stirp. Hels. II (17tis) 203.

Lappago aliena Spreng. Neue Lntd. III (1822) 15; Gris. Fl. 557.

Nozia alienc Millsp. in Millspaugh 471.

St. Eustatius: Betwen Oranjestal aml lort le llinel wh the top of the Cliff: n. 707 B.; Hill near Concordia: n. 946 B.; near White Wall: n. 1259 B.

Saba: St. Giles: n. 1870 B.

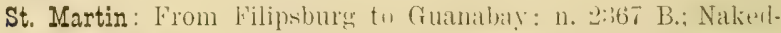

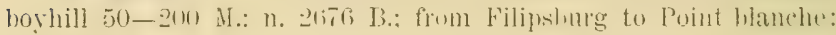
n. $340+$ B.

Anguilla Boldingh.

St. Croix ex Millspaugh.

Antill., Curaça, Aruba, trop. and subtrop. countries of both hemisph. (Symb.).

\section{Paspalum Linn.}

Paspalum conjugatum Bergius in $\Lambda$ ct. helv. VII (1772) $129 \mathrm{t}$. S; Gris. Fl. 541; Duss 591; Millspaugh 471. Crabgrass.

Saba: Butween Bottom and Marypoint 1(x) - gun M.: n. 1470 R.: Mountain 600 M.: n. 1776 B.; Gainbygut: n. 101.

St. Martin Mountain between Mont dex Aleorels and rentry-

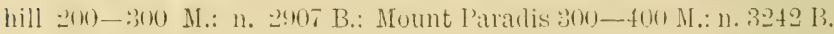

St. Croix ex Millspaugh.

Antill., trop. countries of both hemisph. (Syml.).

Paspalum fimbriatum H. B. K. Nov. (ien. I (1S1) 4.3 t. 24; Gris. Fl. 542; Duss 511; Millspaugh 472.

St. Eustatius: Alon: roads in () Janjestiul: 11, 1109 B.; along roads near Glassbottle: n. 1213 B.; Slope of the Quill: n. 963.

Saba: Mlong roalds between Bottom and Windwardside zon M.:

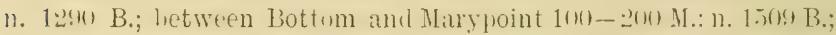

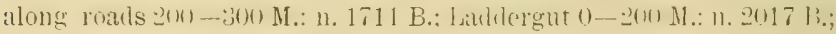
Peperpot: n. 1018; Crespeen-Rendez-vous: 11. (s)8.

Anguilla Boldingh.

St. Croix ex Millspaugh.

Baham., Antill., Amer. austr. (Symb.).

Paspalum hemisphaericum Poir. in Lam. Sinc. I (1s) f) s1.

Paspalum panniculatum Willd. Spec. plant. I (1798) 381 ; (non Linn.) Gris. Fl. 5 43 ; Duss 512. (Symb.). 


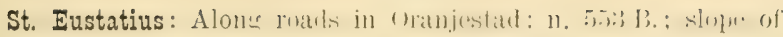
the Quill 200 M.: n. 879 B.; slope of the Quill on the side of White WVall 200 M.: n. 1167 B.

Saba: Between Bottom and Marypoint 100_-200 M.: n. 1525 B.;

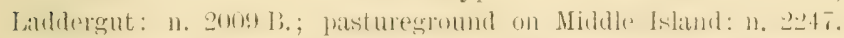

St. Martin: near Mulletpond: 11. 2763 B., $2782 \mathrm{~B}$; ; in pasture ground near Bellevue: n. 282 ; B.; between Telvedere and Oyster. pond : $17.3068 \mathrm{~B}$; Lowlands: n. $3281 \mathrm{~B}$.

Anguilla Boldingh.

Antill., warmer parts of Amer: (Syml.).

\section{Isachne $\mathrm{Pr}$. Br.}

Isachne arundinaceae (iris, Fl, IV. I. (1 isit) 5...): I) uss 52:3.

Saba: 'l'op of the Mountain: 11. 2196 B.

Jamaica, Portorico, St. Kitts, Guadeloupe, Dominica, Martinique, St. Vincent, Grenada. (Symb.).

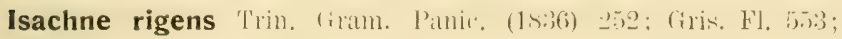
Duss 524.

Saba: Top of the Mountain 800 M.: n. 1386.

Antill., Venezuela. (Gris. Fl.).

\section{Panicum Linn.}

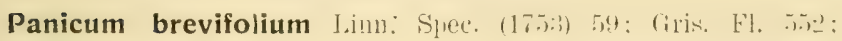
Duss 522.

Saba: Lower part of the Bottom MLountain 300 M.: 11. 1433 B.;

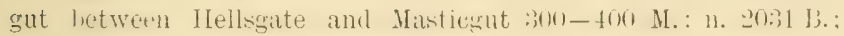
Springbaygut: n. 2100 B., 2122 B.

Antill., Cuba and Mexico to Bras. (Gris. Fl.).

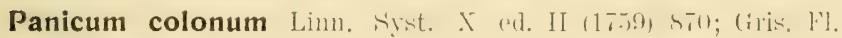
545 ; Duss 515; Millspaugh 472.

Saba: Middle Island: n. 1613 B., $1613 a$ B.

St. Martin: Cul de Sac: 11, 2614 B.; in pasture ground near Bellevtue: n. 2841 B.; Centry hill: n. 606; Colebayhill: n. 207.

St. Croix ex Millspaugh.

Antill., Curaça, trop. and warmer countries of both hemisph. (Symlo.).

Panicum diffusum Sw. Prodr. (1788) 23: Duss 518.

St. Eustatius: Gut near Fort de Wind 50 M.: n. 839 B.

St. Martin : from Filipsburg to fuanabay: n. 2:3roB.: Mrarigotroad:

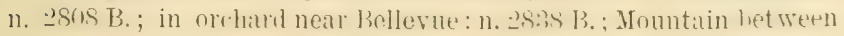


Iront des Acrords and Centry hill: n. 2sist B.; along roads from Colebayhill to Simsonsbay: n. $3015 \mathrm{~B}$; ; betwoen Belveclere and Oysterpond: n. 3077 B.; Tintamarre: n. 3394 B.

Anguilla Boldingh.

St. Croix (Symb).

Cuba, Jamaica, Hispaniola, St. Thomas, St. Jan. St. Barthélemy, Antigua, Guadeloupe, Martini(jue. (Symb.).

Panicum divaricatum Linn. Syst. $X$ ed. II (17!n) SiL; Gris. Fl. 5็1; Duss 521; Millspaugh 472. Ca n e grass, wi l d c a ne.

St. Eustatius: Top of the ouill $400 \mathrm{MI}$ : n. Osis B.; inner slope of the Quill $350 \mathrm{M}$.: n. 417 B.; slope of the Quill near Bengalen 150 M.: n. 676 B.; slope of the Quill near Bengalen 100 M.: n. 750 B.; seashore near White Wall: n. 850 B.; from Little Mountain to Yenusbay: n. 990 13.; slope of the Quill: n. 58t; Sugarloaf: n. $56 \pm$ B.; Quill: n. 194 (

Saba: between Bottom and Marypoint 400 M.: n. 148t B; gut between Hellsgate and Masticgut: n. 2070 B.; Peperpot: n. 561, 589; Rendez-vous 400 M.: n. 593 ; locality unknown: n. 303 L.

St. Martin: Marigothill - Marigot 0-200 M.: n. 2645 B.; Mountain between Mont des Aecords and rentrybill $20(1)-.390)$ M.: n. 2896 B.; Milldrumhill: n. 3050 B.; near Bellevue: n. $3356 a$ B.; Centryhill: n. 559; near the French frontier: n. $5638,586$.

I saw Panienm divarieatum in St. Martin atso on Mount Pruradis 300-100 M, hills on the northern side of Cul de Sac and Nakedboyhill $100-200 \mathrm{M}$.

St. Croix ex Millspaugh.

Key Ins., Baham., Antill., Amer. cont. trop. (Symb.).

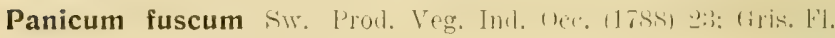
546 ; Duss 516; Millspaugh 472.

St. Eustatius: in deserted plantations at the seashore near Bengalen: 13. $598 \mathrm{~B}$; slope of the lower part of the Quill near Bengalen: n. $724 \mathrm{~B}$; ; along roads in Oranjestad: 11. $1095 \mathrm{~B}$; Cultumrlakte: n. 610 .

Saba: between Bottom and Marypoint 100-200 M. : 17. 1543 $\mathrm{B}$, 1562 B.; Boobyhill: 1583 B.; locality unkncwn : 11.147 bis L.

St. Martin: Nakedboyhill: n. 2699 B. ; along roads near Bethlehem: n. $2945 \mathrm{~B}$.

St. Croix ex Millspaugh.

Jamaica. (Ind. Kew.)

Panicum geminatum Forsk. F'lor, aleg. arah. (1765) 18.

Panicum paspaloides Pers. Syn. I (1805) 81; Gris. El. 515; Duss 514; Millspiangh 473. 
St. Martin: between Belvedere and Oysterpond: n. 3069 B. Anguilla Boldingh.

St. Croix ex Millspaugh 473.

Flumila, Bermud., Tathan, Antill. Arthat, trop conntries of hoth hemisp. (excl. Australia.) (Symb.).

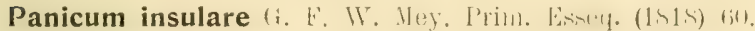

Tricholaend insularis Gris. Hlor. W. I. (186t) 557.

Panicum leucophaeum H. B. K. Nov. Gen. I (1815) 97; Duss 52:.

Syntherisma insularis Millsp. in Millspaugh 437.

L 0 ng g ras.

St. Eustatius: in a garden in Oranjestad: n. $67 \mathrm{~B}, 1068$ a B.; along roals in Oranjestad: 11. $575 \mathrm{~B} ., 1070$ B., $1096 \mathrm{~B} . ;$ slope of the lower part of the Quill near Bengalen: n. $723 \mathrm{~B}$; slope of the Quill 300 M.: n. 881 B.; along roals near English Quarter: n. 1000 B., 770; near Boren: 11. 1251 B.: Culturvlakte: n. 771 ; Nountain near Bengalen: n. 772.

Saba: Boobyhill 300 M.: n. $15 \% 9$ B.; Laddelgut 200 M. : n. 2003 B.; Ladder: n. 1195; Greathill: n. 1206; locality unknown: 11. $294 \mathrm{~L}$.

I saw Panicum insulare in great quantities also: Gut between Hells. gate ind Masticgut; Crespeenhill; Flat point.

St. Martin : from Filipsburg to Guanabay: n. $2397 \mathrm{~B}$; lowel part of the Milldrumhill: n. 3175 B.; Mount Paradis $300-400 \mathrm{M}$. ก. $33: 31 \mathrm{~B}$.

I also saw Panicum insulare: Nakedhoyhill; Marigothill-Marigot.

Anguilla Boldingh.

St. Croix ex Millspaugh.

Florida austr., Baham., Amer. cont. from 'l'exas to Patagonia. (Symb.).

Panicum maximum Jiley. Ic. 1)]. rill. I 117s1) 2, t. 13: (iris. Fl. 549; Duss 519; Millspaugh 47. G u i neag a s s.

St. Eustatius: in a garden in Oranjestad: n. 1063 B.; slope of the Quill: n. 1404; Cultuurvlakte: n. 1402.

Saba: Boobyhill 300 M.: n. 1580 B.; Bottom: n. 1411; locality unknown: n. $240 \mathrm{~L}$.

St. Martin: Top of the hills on the northern side of Cul we Sac : n. 2988 B.

I also saw Panicum maximum, and in great quantities: from Bethle. hem to Cul de Sic, Top of Mount Paradis 100 M. 
St. Croix ex Millspaugh.

Baham., Antill.; Amer. cont. trop., Afr. trop. and subtrop. (Symb.).

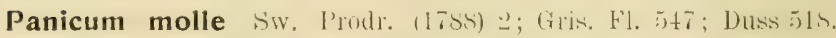
Punicum barbinode T'rin. in Millspaugh 472 . P a r a g r a s s,

St. Eustatius: slope of the Quill near Glasshottle: 11. $1201 \mathrm{~B}$.

I did not collect this plant in Saba, but from my notes it appears that I saw the plant cultivated in Windwardside.

I do not remember that I saw this plant in St. Martin, but I believe that, as it is cultivated in St. Eustatius and Saba, I may not hold myself entitlerl to say that P'anicum molle might not be found in St. Martin as well.

Anguilla Boldingh.

St. Croix ex Millspaugh,

Baham., Antill., Curaçao, Amer, cont. trop. (Symb.).

Panicum prostratum lam. III. I (1791) 171: (iris. Fl. 546: Duss 515; Millspaugh 473. R u n n ing g rass.

St. Martin: Nakedboyhill in_-200 M.: n. 20:18 B.; between Belvedere and Gysterpond: 11. 3071 B.; Momnt Paralis 150-400 M.: n. 3190 B., 200-300 M.: n. 3315 B.; lowerpart of Milldrum. hill: n. 3165 M.: n. 3165 B.; Tintamarre: n. 3377 B., 3383 B.

Anguilla Boldingh.

St. Croix ex Millspaugh.

Antill., Curaçao, trop. countries of both hemisph. (Symb.).

Panicum sanguinale Linn. Apece. (1753i) 5i: Duss 52.3.

Digitaria marginata Lk. Enum. I (1821) 102 et Digitaria setigera Roth. ap. R. et Sch. Syst. II (1817) 474?: Gris. Fl. 544.

Syntherisma sumfuimule Dulac. l'lor. Hautes-P'yrén. (1867) 77: Millspaugh 473. Haygrass.

St. Eustatius: along roads in Oranjestad: n. .3:0 B., $1097 \mathrm{~B}$; Bengalen : n. 614 B., its B.: slope of the Quill near Bengaleu no M.: n. 662 B.; slope of the Quill: n. 1860; locality unknown : n. 2600.

Saba: along rouls between Bottom and Windwardside $200 \mathrm{M}$.: n. 1326 B.; lowel part of the Bottom Mountain 250 M. : n. 1430 B.: between Bottom and Marypoint 100-200 M.: n. 1560 B.; top of the Cliff near the seaside of Boobybill: n. 1604 B.; Laddergut 0-200 M.: n. 2013 B.; gut between Hellsgate and Masticgut 300-400 M.: n. 2068 B.; locality unknown: n. 2603, 273 L.

St. Martin: from Filijsbures to (iuauabily: n. 2:36:3 B.; along the saltpond near Filipshurg: n. "H.to B.: from Filipsburg to Bel-

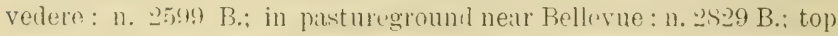
of the hills on the northern side of Cul de Sac 200-300 M.: n. 


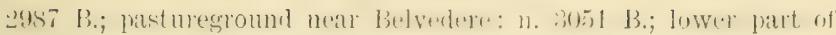

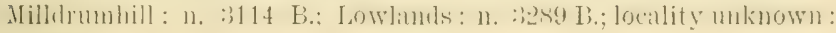
ก. 2601 .

1 also saw Pauicum sanguinale in 'l'intamarre.

Anguilla Boldingh.

St. Croix ex Millspaugh.

Antill., trop, countries of both hemispl. (Symb.).

\section{Ichnanthus Beanv.}

Ichnanthus pallens Mruntw ex Isenth. El. Hongk. (ISti1) 414.

Ianicum pallens Sw. Prod. Veg. Ind. Oce. (1788) 23; Gris. F'l. 550: Duss 520.

Saba: Top of the Nountain : 11. 1794 B., 2213 B.; locality un. known: 11. $206 \mathrm{~L}$.

St. Martin: locality unknown: n. $3430 a$ B.

Antill., Guiana Gallica. (Herb). Krug et Urban.).

\section{Oplismenus Beauv.}

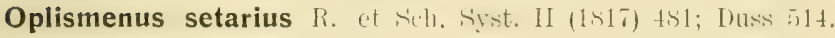

Orthopogon setarius Spreng. Syst. I (1825) 306; Gris. Fl. 545. comp. Millspaugh 473. Kunning mountaingrass.

St. Eustatius : inner slope of the Quill $350 \mathrm{M} .:$ n. $41 \pm$ B.; Quill: 11. 260 H., $27 t$ G.

Saba: Lower part of the Bottom Mountain 250 M. : n. 1408 B.; Bottom 200 M.: n. 1453 B.; Boolyyhill 350 MI.: n. 1600 B.; Mountain near Windwardside 600 M.: 11. 2182 B.; locality unknown: 11. $53 \mathrm{I}$.

St. Martin: Marigothill-Marigot 0-200 M. : 11. 2643 B.

I also satw Oplismenus setarius near Bellevue under old Mangifera trees amd on the hill between Mont des Aceords and Centryhill.

Anguilla Boldingh.

St. Croix ox Millspaugh.

Bermud., Antill., Amer. cont. trop, Asia tron. (Symb.).

\section{Setaria Beaur.}

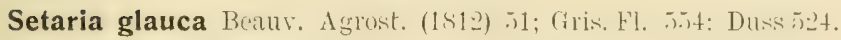
Chactochloa glauca (Linn.) Scribn. in U. St. Dep. of Agr. Div. of Agrost. Bull. n. 4 (1897) 39; Millspaugh 473.

St. Eustatius: in a garden in Oranjestad: n. 523 B.; along roads in Oranjestad: n. 1108 B.; slope of the Quill: n, 435 . 
Saba: along roads between Bottom and Windwardside 200 M.: n. 1289 B., 1328 B.; Mountain 600 M. : n. 1772 B.; locnlity un. known: 11. $133 \mathrm{~L}$.

St. Croix ex Millspaugh.

Bermud., Baham., Antill., trop. and temp. countries of both hemispl.. (Symb.).

Setaria setosa Beauv. Atrost. (1812) 51: (iris FJ. 55; Duss 525.

Chaetochloa setosa (Sw.) Scribn. in U.S. Dep. Agr. Div. Agrost. Bull. 4 (1897) 37; Millspaugh 473.

St. Eustatius: Slope of Signathill: n. 10.it B.; along roals in Oranjestat: 11. $1130 \mathrm{~B}$.; slope of Signalhill : 11. 451; seashore: 11. 3̈́t, 349: along roals in Oranjestal: 11. 452: locality unknown: 11. 298 6.

Saba: 'Tuy of' the Cliff near the seaside of Booly-hill : n, 160s B.; Mountain S00 M.: n. 1812 B.; Bottom: n. 1857 B.; gut between Hellsgate and Masticgut 300-400 M. : n. 2064 B.

St. Martin: Nakedhoyhill 50-200 MI.: 11, 26889 B., 2694 B.: between Belvedere and Oysterpond: n. 3070 B.

St. Croix ex Millspaugh.

Antill., Amer. cont. trop. (Symb.).

Setaria viridis Beaux. Agrost. (1S12) j1: (iris. Fl. 5ist.

St. Martin : from Filipsburg to Guanabay : 13. 2404 B.

Jamaica, Europa and Asia sept. (Gris. Fl.).

\section{7t. Cenchrus Linn.}

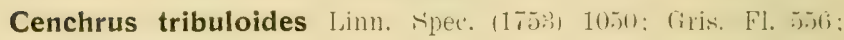
Duss 526. Burrgrass.

St. Eustatius: Slope of the luwer part of signalhill : 17, 109 B.: slope of the Quill near Bengalen 100 M. : 11. fit: B.: Fort de Wind: n. So-3 13.; alung roads near (Blassbottle: n. S6:3 B.: lower part of the hills near Concorlia: n. 9st B.: along roals near Bengilen: n. 1191 B; locality unknown : n, 249 G., $265 \mathrm{H}$.

Saba: along roals between Buttom and Windwardside 200 M. : n. 1283 B.; letween Bottom and Marypoint 100-200 M.: n. 1539 B., 1563 B.; along roads: n. 1710 B.; Ladder: 17. 1372. Masticgut

I also saw Cenchrus tribuloiles in the gut between Hellsgate and

St. Martin: from Filipstury to Guanabay : 11. 2fole 13.: Colehay (coll. George) : n. 2518 B.; neal Mulletpond: n. 2758 B., 2775 B.; in pasture ground netr Bellerwe: n. 2s30 B.: allong roads near Bethlehem: n. 2932 B.: Tintamarre: n. 3385 B. boyhill.

I also saw Cenchrus tribuloides: Top of Mount Paradis 400 M.; Naked. 
Anguilla Boldingh.

Bermud., Baham., Antill., Aruba, Bonaire, Amer. cont, trop. (Symı.).

\section{Pennisetum L. Cl. Rich.}

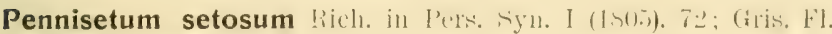
555; Duss 525.

St. Eustatius: Slope of the Quill : n. 241.

'Irop. countries of both hemisph. (Ind. Kew.).

\section{Pharus Linn.}

Pharus glaber 11. B. K. Nov, (ien, I (1sia) 196: (iris. Fl. 536; Duss 505; Millspaugh 474 .

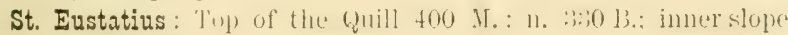
of the Quill 350 M. : 17. 413 B., 415 B., 1221 B.; Bottom of the Quill 300 M.: n. 908 B.; slope of the Quill: n. 1380; Quill : n. 1377, 1385, 1379; Bengalen : 1. 1382; locality unknown: n. 1381; Quill: n. $225 \mathrm{G}$.

Saba: Boobyhill 300 M.: n. 1573 B.; 350 M.: n. 1613 B.; locality unknown: $17.34 \mathrm{~L}$.

St. Martin: Mountain letween Mont des Acends and contryhill $200-300$ M.: n. 2858 B.

St. Croix ex Millspaugh.

Antill., Amer: austr. (Symb).

\section{Aristida Limn.}

Aristida americana Limm, syst. I el. II (17is!) sig; Cris. Fl. 534; Duss 503.

Bouteloua juncifolia Lag. Gen. et Spec. nov. (1816). 5.

Bouteloun litigusa Lag. (ien, et Spee. nuv. (1S16). 5. Millspaugh 475. Mr u legrass.

St. Eustatius: between onanjestal and furt he Wind on the

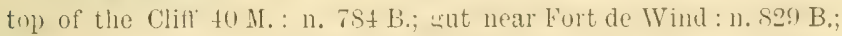
slope of the Quill : n. 104:3, 1055. 1079; neal Sugarloaf: n. 10s4; locality unknown: 17. $215 \mathrm{H}$.

Saba: Paris to0 M. : 11. 1372 B.; along the forthgut 150-200 M.: n. 1631 B.; along the seashore near Forthbaylanding: n, 1648 B., 2147 B.

St. Martin : Lowlands: n. 3275 B.

Anguilla Boldingh.

St. Croik ex Millspaugh.

Baham., Antill., Bonaire, Curaçao, Aruba, Amer, cont, trop. (Symb.). 
230. Sporobolus R. Br.

Sporobolus indicus R. Br. Prodr. I 1s111) 171); (iris. Fl. 533; Duss 503; Millspaugh 47t. L i z a r d 1 as s.

Saba: Paris 400 M.: 1. 1376 B.; between Bottom and Marypoint 100-200 M.: n. 1505 B.; Boobyhill 300 M.: n. 1618 B.; St. Johns 250 M.: n. 1730 B.; Windwardside: n. 1916 B.; Rendezvous 400 M.: 11.725.

St. Martin: Along ruitus trom Marigut to (irande ('alse: 11. 3356 B.

St. Croix ex Millspaugh.

Bermucl, Baham., Antill., Amer. sept., media, austro-occid, Afr., Australia. (Symb.).

Sporobolus virginicus kth. liciv. (irinl. I (1423) ti7; (iris. lil. 533; Duss 503; Millspaugh 474.

St. Eustatius: Seashore near Oranjestad: n. 12 B., 18 B.; seashore near English Quarter: n. 1008 B; near Boven 200 M.: n. 1236 B.; near White Wall: n. 1261 B.; Concordiabay: 11. 563; Thutheray: n. a6t; T'astureground on the slope of the (euill: 11. 305; White Wall: n. 302, 315.

Saba: Ladderlanding: n. 562; Paris: n. 1376 B;; Laddergut อ0 M.: n. 1975 B.

St. Martin: Alowg the Siltpond near filipshurg: 11. 2039 B.,

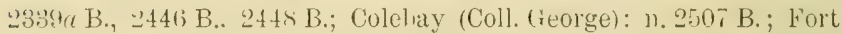
Willem: n. 2800 B.; Mountain between Mont des Accords and Centryhill $200-300$ 11.: n. 290.5 13.; in pasturegromm near Colel ray: n. 3024 B.; between Belvedere and Oysterpond: 11. 3067 B.; Lowlands: n. 3276 B.: Fort Amsterdam: n. 300.

Anguilla Boldingh.

St. Croix ex Millspaugh.

Bermud., Baham., Antill., Aruba, Amer, cont., Afr. trop., Australia, Ins. Pacif. (Symb).

\section{Cynodon Pers.}

Cynodon dactylon Pel's. Syll. I (1S0.) Si) (iris. Fl. itt); Duss 509 ; Millspaugh 474. B a ha $\mathrm{m}$ a $\mathrm{gras}$ s.

St. Eustatius: Along roads in Oranjestad: n. 1061 B.; slope of the Quill near Glassbottle: n. 1200 B.

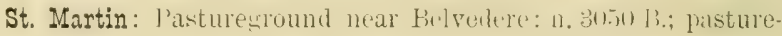

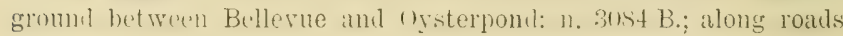
from Grande Case to Narigot: n. 3093 B.

St. Croix ex Millspaugh.

This grass ats I was told was introduced by Mr. Every from St. Martin 
into St. Eustatius; in St. Eustatius it is cultivated on a very small scale, but in St. Martin I saw many pieces of pastureground that consisted cliefly of Cynodon ditetylon.

Bermud., Baham., Antill., Curaça, trop. and warmer countries of both liemisph. (Symb.).

\section{Chloris Sw.}

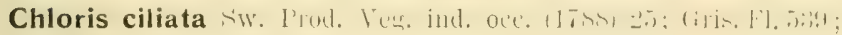
Duss 508; Millspaugh 474.

St. Martin: Mountain between Mont des Accolds and Centry. hill 200-300 M.: n. 2877 B.

St. Croix ex Millspaugh.

Antill., Mexico. (Gris Hi.).

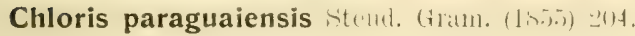

Chloris barbata Siv. Flor: I (1797) 200; Gris. Fl. 549; Duss 508.

Saba: along roads between Bottom and Windwardside 200 MI.: 17. $1299 \mathrm{~B}$; along the seashore near Forthbalanding: n. $1644 \mathrm{~B}$; along roads $200--300$ M.: n. 1712 B.

St. Martin: Cul de Sac: n. 2617 B; along roarls in Prince. quarter: 11. 2913 B.

St. Croix (Symb.).

Baham., Antill., trol). countries of both hemisp. (Symb.).

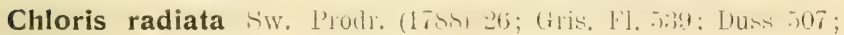
Millspaugh 475 .

Saba: Mountain 600 M.: n. 1777 B.

St. Croix ex Millspaugh.

Baham, Antill., Amer. cont. trop. (symb.).

\section{0t. Eleusine Gärtn.}

Eleusine indica (rilitu. Fuct. I (17tis) 8; (fris. Fl. 5t0; Duss 508; Millspaugh 475. D u t ch gl as s.

St. Eustatius: along luals in (Manjestad: n. .j) 4 B.; in a garden near Glassbottle: 11. 1211 B.; in a garden in Oraujestad: n. 1278 B; Cultuurvlakte: 1). 7S6; locality unknown: 11. 264 G., $237 \mathrm{H}$.

Saba: lower part of the Bottom Mountain 250 MI.: n. 1390 B.;

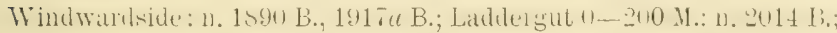
Bottom: n. 755; locality unknown: n. $23 \mathrm{~L}$.

St. Martin: Colebay (Coll. George): n. 2521 B.; MarigothillMarigot 0-200 M.: n. 2655 B; in pastureground near Bellevue: 11. 24-23 B: in pastureground between Belvedere and usterpond: n. $3086 \mathrm{~B}$.

$I$ also saw Eleusine indica in Tintamarre. 
Anguilla Boldingh.

St. Croix ex Millspangh.

Baham., Antill., Bonaire, Curaça, trop). and warmer countries of both hemisph. (Symb.).

\section{Dactyloctenium Willd.}

Dactyloctenium aegyptium Willi. Enum. Hort. Berol. (1809) 1029; Gris. Fl. 5 40 ; Duss 50s; Millspaugh 475.

Eleusine aegyptiaca Desf. F'lor. Atl. I (1798) 85.

St. Eustatius: seashore near Oranjestad: 11. 39 B.; White Wall: 11. $820 \mathrm{~B}, 1260 \mathrm{~B}$.

Saba: between Bottom and Marypoint 100)-200 M.: 11. 1514 B.; along the seashore near Forthbaylanding: 1). $1647 \mathrm{~B}$.

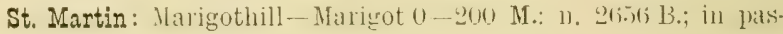
tureground near Colebay: n. 3023 B;; Centryhill: 11. 654.

St. Croix ex Millspaugh.

Baham., Antill., Bonaire, Curaçao, Aruba, trop. and warmer countries of both hemisph. (Syml).).

\section{Leptochloa Beauv.}

Leptochloa filiformis Beauv. Agrost. (1\$12) 71; Dus.s. 507.

Leptochloa mucronatı Kth. Rév. Gram. I (1829) 91; Gris. F'l. 537 ; Millspaugh 475.

St. Martin: Boobyhill 300 M.: n. 1616 B.

St. Croix ex Millspaugh.

Bermud., Antill., Curaçao, Amer. sopt., Nova Granata, Asia and Afr. trop. (Symb.).

Leptochloa virgata Btaluv. Igrost. (1412) 71; (iris. Fl. 5as'; Duss 507; Millspaugh 475.

St. Eustatius: Lower fart of the slope of the hills near (oncordia: n. 940 B.: near Boven: n. 1250 B.

Saba: hetween Buttom and Narypoint 100 - 2100 M.: 11. 12:30 B,; Mountain 175l li; gut between H(dlsgate and Manticgut :3(u)100 M.: n. 2055 B.; Peperpot: n. 5 ว.

St. Croix ex Millspaugh.

Antill., Amer. trop. cont. (Syml).).

310. Pappophorum Schreb.

Pappophorum alopecuroideum Vahl symh. hot. III (1764) 10) t. 51; Gris. Fl. 537. 
Pa)pophorum leguroideum Schrad, in Schult, Mant. II (1824) 342; Duss 506. Crabgras

St. Eustatius: Fetween Granjentall and loort de Wiml on the top of the Cliff 40 M.: n. 772 B.; seashore near White Wall: 1). 856 B.; near Boven 200 M.: n. 1233 B.; near Whito Wall on the

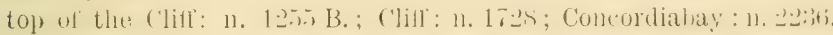

Saba: Laddergut 50 M. : 11. $197+$ B.; Ladder: 11. 2231; at the seashore: n. $2137 \mathrm{~B}$.

St. Martin: T'intamarre: n. 3386 J.

Trinidad, La 'linta, Bras. to Lrugnaly (trir. lis).

\section{Eragrostis Host.}

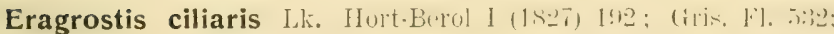
Duss 502; Millspaugh 475 .

St. Eustatius: in a gimben in Mrinjestad: 11. 1i!n Li, Bengalen: n. $615 \mathrm{~B}$; between Oranjestad and Fort de Wind on the top of the Cliff 40. M.: n. 794 B.; along roads in Oranjestad: n. 1073 B.; Cliff: 1. 2144; slope of the Quill: 11. 2131; Cultuurvlakte: ก. 2145 .

Saba : along roads between Bottom and Windwardside 29(x) N.: 11. 13192 B.: hetween Bottom and Marypoint 100-20n A. : 11. 1561 B.; top of the Cliff near the seaside of Boobyhill : n. 1602 B.; Mountain 600 M. : n. 1746 B.; Ladder: 11. 2080.

St. Martin: Colebay (Coll. Ceorge): 17. 2511 B.; Nakedboyhill 50-200 M. : n. 2686 B.; in an orchard near Bellevue: n. 2847 B. 'T'op of the hills on the northern side of Cul de Sac 200-300 M.: n. 2986 B.; in a plantation of cotton near Belvedere: n. 3409 B.;

St. Croix ex Millspaugh.

Baham., Antill., Amer. trop., Afr. trop., Ind. orient. (Symb.).

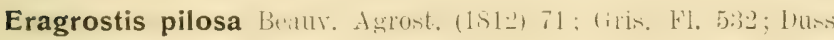
503 ; Millspaugh 475.

Saba: along roads between Bottom and Windwardside $200 \mathrm{M}$. n. 1308 B.; Crispeen 400 M. : n. 1767 B.; Windwardside in 1891 a B.;

St. Martin: Nakedboyhill 50-200 M.: 10. 2678 B; between Mount Vernon and Orientbay: n. 2942 B.; from Belvedere to Oysterpond : n. 30.53 B.; lower part of the Milldrumhill : n. 3157 B.

St, Croix ex Millspaugh.

Antill., warmer countries of both hemisph. (Symb.).

Eragrostis tephrosanthos sihult, iyst. Mint. II (1S:) :316.

Eragrostis poatoides Gris. Fl. W. I. (1 1964$) 532$; (p. p.). (Symb.). 
St. Martin: Fort Willem 0-50 M.: 11. 2550 B.; near Oysterpond: $n, 3053 \mathrm{~B}$.

Antill., Amer. austr. (Symb.).

\section{Festuca Linn.}

Festuca Myurus Linı, Spec. (1753) 74.

St. Eustatius: from Signalhill to T'umblakowndicklay: 11. $129 a$ B.; Signalhill: n. $982 a$ B., 1040 B.; near Boven: n. $1237 a$ B. Europa, Amer. and Asia boreal. (Ind. Kew.).

\section{Cyperaceae.}

Juncellus C. B. Clarke.

\section{c f. 459 C y perus Lin n. sect. 2.}

Juncellus laevigatus (C. B. Clarke in Ifuek, f. Fl. Brit. Ind. II (1893) 596.

Cyperus mucronatus Rottb. Descr, et Ic. (1773) 1) 19 t. 8 fig. 4; Gris. Fl. 562.

St. Martin: near the saltponds of Filipsburg: n. 2444 B.

Antill, in almost all warmer countries of both hemisph. (Symb.).

\section{Cyperus Limn.}

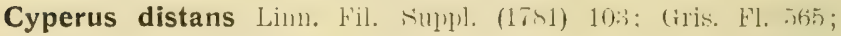
Duss 540.

St. Eustatius: Slope of the Quill 200 M.: n. 23, 29.

Saba: Top of the Mountain: 11. 1745a B.; Rendez vous Mountain: n. 209 .

Antill, warmer countries of both hemisph. (Symb.).

Cyperus elegans Linn. Spec. (1762) 68.

Cyperus viscosus Stvartz Prod. (1788) 20; Gris. Irl. 563; Duss 537.

Cyperus confertus Gris. Fl. W. I. (1864) 563 pro magna parte, non Swartz. Millspaugh 476. (Symb).

St. Eustatius: locality unknown: n. $266 \mathrm{H}$.

St. Martin: along the saltpond near Filipsburg: 11. $2447 \mathrm{~B}$;

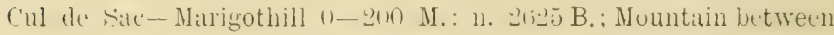
Mont des Accords and Centryhill 200-300 N.: n. 2906 B.

St. Croix ex Millspaugh.

Florida, Antill., Bonaire, Curaça, Amer. centr., Argentinia. (Symb.). 


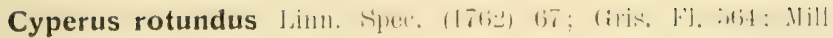
spaugh 477. Nutgrass.

Cypenes purpureo-variegatus Boeck. Cyp. Novae. Ifoft 2 (1890) 37; Duss 539.

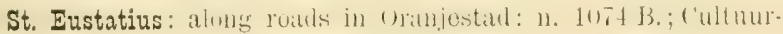
vlakte: n. 35 ; locality unknown: n. 159 G.

Saba: Top of the Cliff near the seashore of Boobyhill: 11. 1603 B.; Mountain 800 M.: n. 2186 B.

St. Martin: in pastureground near Bellevue: 11. 2828 B.; along

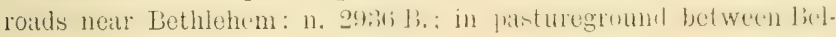
vedere and Oysterpond: 11. 3085 B..

Anguilla Boldingh.

St. Croix ex Millspaugh.

Balam., Antill., warmer countries of both hemisph. (Symb.).

Mariscus Gïrtn.

of. 459. Cyperus Linn. sect. 6.

Mariscus brizaeus C. B. Clarke in symbolate Antillalle: II (1900) 52.

Cyperus bruneus Swartz Fll. Ind. Oce. I (1797) 116 partim. (Symb.); Duss 539. Bullgrass.

St. Eustatius: slope of the Quill near Bengalen 100 M.: 11. $671 \mathrm{~B}, 699 \mathrm{~B}$; ; locality unknown: n. $115 \mathrm{G} ., 262 \mathrm{H}$.

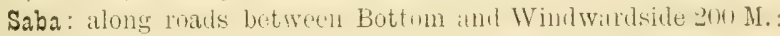
11. 1343 B.; neal Flat Point: n. 2129 B.; Greathill: n. 46.

1 also saw Mariscus brizaeus: Green Island.

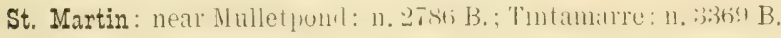

St. Croix ex Millspaugh.

Baham., Antill., Florida. (Symb.).

Mariscus capillaris Vahl Enum. II (1806) 372.

C!npens capillaris (Sw.) Millspaugh 476.

Saba: along the Forthgut 150-200 M.: n. 1639 B.; Mountain 600 M.: n. 1738 B.; Springhaygut: 11. 2097 B., 2101 B.

St. Martin: from Filipsburg to Guanabay: n. 2856 B.; along roads from Grande Case to Marigot: n. 3103 B.

Anguilla Boldingh.

St. Croix (Symb.).

Cuba, Jamaica, Hispaniola. (Symb.).

Mariscus flavus Vahl Enum. II (1806) 374.

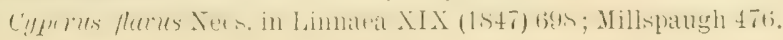


Cyperus flevomariscus Gris. FJ. W. I. (1864) 567 partim; Duss 542. (Symb.).

Saba: Nountain 600 M.: N. 1749 B.

St. Croix ox Millspaugh.

Antill., from Mexico and 'lexas to Patagonia. (Symb.).

\section{Torulinium Desv.}

\section{cf. 459. Cyperus sect. 5.}

Torulinium confertum Desv. in Hiln. Iroll. (15:5) 15.

-Cyperus forax L. C. Rich. in Acta Soc. Hist. Nat. Paris I (1792) 106 ; Duss 541.

St. Eustatius: slope of the Quill: 11. 43.

Saba: MLountain 600 M.: 11. 1773 B.

Bermud., Baham., Antill., Aruba, in warmer countries of both homisph. (Symb.).

\section{Kyllinga Rottb.}

Kyllinga brevifolia liottl,. Deser. et Ic. (1763) p, 1; t. 1 figr. $3 ;$; Gris. Fl. 568; Duss 5t3; Millspaugh 475.

Kyllinga monocephala Thunb. Fl. Japon. (1784) 35; Gris. H. 568 .

St. Martin: Hill on the morthem side of ('al de size: 11. 2077 B.: Mount Paradis 200- 400 M.: n. 3206 B.; Retreat: n. 42.

St. Croix ex Millspaugh.

Bermud., Antill., warmer countries of both hemisph. (Sirmh.).

\section{Heleocharis $\mathrm{R} . \mathrm{Bl}$.}

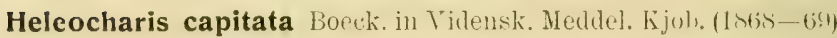
159; Duss 545; Millspaugh 477.

Scirpus capitatus Linn. Spec. (1753) 48; Gris. L1. 570 (partim.).

St. Martin: along the saltpond near l'ilipshur: 11, 24.51 B.

St. Croix ox Millspaugh.

Bernut, Baham., wallmex countrites of both hemisph. (Symb.).

\section{Fimbristylis Vahi.}

Fimbristylis spathacea liotl. Nov. Pl. Sip. (1S21) '2t.

Scirpus obtusifolius Gris. Fl. W. I. (1864) 571.

St. Martin: Lowlands: n. $3298 a$ B.

Antill, in trop. contries of both hemisph. (Symb.).

473. Dichromena Vah].

Dichromena radicans Schlecht et. Cham. in Limnaea VI (1831) 38. 
Rhynchospora Persoonianc Gris. Fl. W. I. (1864) 577.

Rhynchospora Humboldtiana Gris. H. W. I. (1864) 577 syn. excl. (Symb.).

Saba: between Bottom and Marypoint 300 M.: n. 1582 B.; Crespeen 400 M.: n. 1765 B.; Mountain 600 M.: n. 1775 B., 37.

Antill., Amer: centr. (Symb.).

\section{Cladium Crantz.}

Cladium restioides Ienth. in Benth. et Hook, fi. (ien. I'l. III (1883) 1066 .

Machaenina restioides Vahl. Enum. II (1806) 238; Gris, Fl. 573.

Saba: Mountain S00 M.: n. 2210 B.. +2, 208 L.

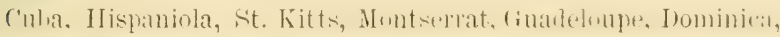
Martinique, St. Vincent. (Symb.).

\section{Rhynchospora Vahl.}

Rhynchospora polyphylla lialil. Enum. II (1Soti) 2:30: liris. Fl. 575; Duss 551.

Saba: Mountain 600-\$00 M.: n. 1790 B:, 49.

Antill., Amer. centr., Nova Granata, Venezuela. (Symb.).

\section{1ち. Scleria Berg.}

Scleria lithosperma Sw. Prod. 117.s, 1S; Duss .55: Millskungh 477. Nountaingrass.

Scleria filiformis Sw. Prod. (1788) 19; Gris. Fl. 579.

St. Eustatius: 'Top of the Quill 400 M.: n, $277 a$ B., $269 \mathrm{H}$.

St. Croix ex Millspaugh.

Biham., Antill., warmer countries of both hemisph. (Symb.).

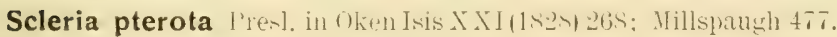

Scleria pratensis Nees in Mart. Fl. Bras. II pars I (1843) 179 t. 23; Gris. Fl. 577; Duss 553.

Saba: lower part of the Bottom Mountain son M.: 11. 14:3: 13; Mountain 600 M.: n. 1747 B., 1750 B.; locality unknown: 13. 51, 132 L., $18 t$ L.

St. Croix ex Millspaugh.

Antill., Amer, cont. trop. (Symb.).

\section{Palmae.}

528. Phoenix Linn.

Phoenix dactylifera Linn. Spec. (1753) 1188.

Cultisated [Afr. sept.] (Symb).). 


\section{Thrinax Linn. f.}

Thrinax spec.

St. Eustatius: Gilboolill: n. 1120 a B.

Saba: Windwardside-Bottom: 11. $298 \mathrm{~L}$.

\section{Oreodoxa Willd.}

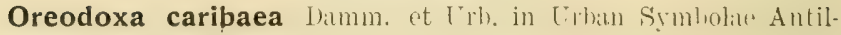
lanae. IV. (1903) 129.

Cultivated. [Portorico.] (Symlı.).

\section{Euterpe Mart.}

Euterpe spec. (prob. Oenocarpus Mart.).

Saba: Mountain: n, 135, 136, 137.

\section{Cocos Jinn.}

Cocos nucifera Linn. Spec. (1753) 1188.

Cultivated. [Amer: austr. sept. occid.?] (Symb.).

\section{Araceae.}

\section{Anthurium Sehott.}

Anthurium cordatum (i. I)on. in sweet. Ilort. brit, ed. 111 (1839) 683. Monkeytail.

St. Eustatius: Top of the Quill 400 M.: n. 182 B., 232 B., 234 B.; slope of the Quill 350 M.: n. 409 B.; slope of the Quill near Bengalen 250 M.: n. 697 B., 736 B.; slope of the Quill: n. 31, 33, 34, 35; locality unknown: $195 \mathrm{G}$.

Saba: Mountain 600 M.: n. $17+1$ B.; Paris: n. 82; locality mnknown: 1. $287 \mathrm{~L}$.

St. Martin: Nakielhoyhill :200-265 M.: 16, 27:20) B.: Mountain letween Nont les Accords and ('entryhill 200 - 301 N1.: 11. 25is B.; gut from Bethlehem to Cul de Sac 100-200 M.: n. 3415 B.; locality unknown: $\mathrm{n} .36$.

St. Croix (Engler 1898).

Jamaica, St. Kitts, Martinique. (Engler 18:18).

\section{Philodendron Schott.}

Philodendron dispar s'ehott. Syn. Al. (155ti) it: (iris. I'. 510; Duss 479. 
St. Iustatius: Top of the Quill 400 M.: n. 298 B.; slope of the Quill 350 M.: n. 389 B.; Quill: 11.913 B.; Jottom of the Quill 300 M.: 11, 432 B., 468 B., 14, 29, 30; locality unknown: n. 220 G.

Saba: Windwardside-Hellsgate: 11. 167t B.; sut between Hellsgate and Masticgut $300-400$ M.: n. 2034 B.

Guadeloupe, Martinicque, St. Vincent. (Symb.).

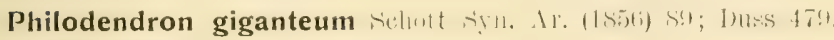

St. Eustatius: I saw Philodendron giganteum on the top of the (huill. Saba: Mountain: n. 2275 a B.

Portorico. St. Kitts, Guadeloupe, Martinique, St. Vincent. (Symb.).

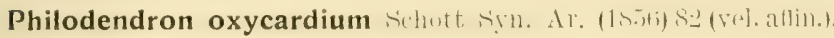

St. Eustatius: 'I'op of the Quill: n. $298 \mathrm{~S}$., 394 B., $389 \mathrm{~B}$; inner slope of the Quill: n. $457 \mathrm{~B}$.

Saba: Mountain: n. $2076 a$ B.; gut near Devilshand: n. 2086 13. Antill., Guatemala. (symb.).

\section{Colocasia Schott.}

Colocasia esculenta Schot.t Melet. I (1832) 18.

Cultivated. [India orient.] (Symb.).

\section{Xanthosoma Schott.}

Xanthosoma spec.

Cultivated.

\section{Lemnaceae \\ 79.. Lemna Linn.}

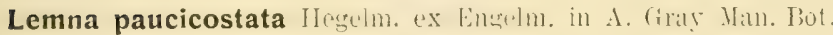
$\mathrm{V}$ ed. (Jan. 1868) 681; Duss 484.

Lemna minor Griseb. Fl. WV. I. (186t) 512 (p. p.). (Symb.): Millspaugh 478.

St. Martin: Lowlands: n. 3285 B. ; near Vineyard: n. 3350 a B.

St. Croix ex Millspaugh.

Antill, Anner, cont., Afr., Asia austro-orient., Jajon., Australia. (Symb.).

\section{Bromeliaceae.}

S40. Bromelia Limu.

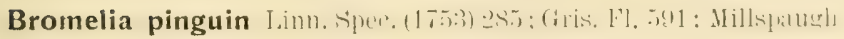
478. Pingwing. 
St. Eustatius: aloug roads in Oranjestad: n. $103 \mathrm{G}$.

I saw Bromelia pinguin in gardens in Oranjestad.

Saba: Bottom: n. 2292 B.; Peperpot: n. 79, 80.

I also saw 3romelia pinguin: Crespeenhill.

St. Martin: alone roals from (o)lebay to fimsonshay: n. 325n B.

St. Croix ex Millspaugh.

Antill., Amer. cont., Venozuela. (Syml.); also cultivated.

\section{Ananas Adans.}

Ananas sativus Sehult. f. in li. ot Sch. ,ist. TII (18:30) 125:3. Cultivaterl. [Brisilia.] (Symb.).

\section{Wiltmackia Mez.}

Wittmackia lingulata Mez. in Mart. F'. Bras, III. :?, (18:1) 27t: Duss 570.

Aechmea lingulata Baker in Journ. Bot. XVII (1879) 164.

Saba: Greathill: n. $1377 a$ B., Paris 300 M.: 11. 82; Bottom: n. 85 ; locality unknown: n. $293 \mathrm{~L}$.

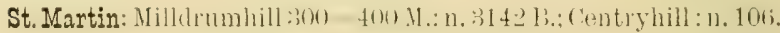

St. Thomas, Virgin. Isl., Vierpuez, Crablen Isl., St: Lucia, Martinipur, Guadeloupe, st. Vincent, Becounia, Antigui, Tobagn, Trinidad. (Mez 1896).

\section{Pitcairnia l'Hérit.}

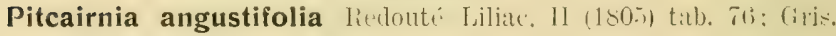
Fl. 59t; Millspaugh 478. Klapper.

St. Eustatius: Top of the Quill 400 M. : n. 246 B., 304 B., 366 B., 383 B.; along roads in Oranjestad: n. 529 B.; near Maryglory 150. M.: n. 1134 B.; Quill: 11. 89, 97; Bengalen: n. 113; Greatgut: n. 88 .

Saba: along roads hetween Buttum and IVindwardside zoo M.: n. 136i B: top of the Cliff near the seaside of Buohyhill : n. 1600 ]3.; Laddergut 0-200 M.: n. 1973 B. ; Paris: 11. 115; Wellgut: n. 101.

I also saw Pitcairnia angustifolia: near the Sulphumines: Hellsgate to Marypoint.

St. Croix ex Millspaugh.

Portorico, Gromala, St. 'Thmmas, Antigna, Dominicas, Sit. Vincent, Martinique, Guadeloupe, St. Croix. (Mez 1896). 


\section{Tillandsia Linn.}

Tillandsia excelsa Gris. Flor. W. I. (1864), 597.

Saba: T'op of the Mountain: 11. 2222 B.

Cuba, Jamaica. (Nez 1896).

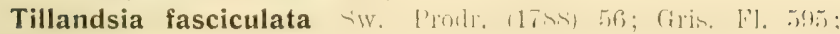
D) uss 572 .

Saba: St. John's 250 ML: n. 1728 B.; Paris: n. 116; locality unknown: n. $21 \mathrm{~L}$.

St. Martin: Milldrumbill $300-400$ M.: n. 3145 B, Centryhill : n. 105 .

l'lorida austral., Baham., Antill., Mexico, Amer. centr, and austro-sept. (Symb.).

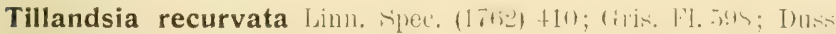
574; Millspaugh 478. Old man's beard.

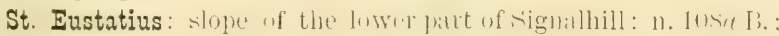
along roads in Oranjestad: 581 B.; Signalhill: n. 91, 96; locality unknown: 1). $217 \mathrm{H}$.

Saba: along the forthgut 150-200 M. : n. 1634 B.; Bottom : n.93.

St. Martin: Princequarter (Coll. George.): n. 2486 B.: Cul de Sac: 11. 2620 B.; Nakedboyhill 280 M.: n. 2736 B.; lower part of the hills letween Nont Chambord and Mount Vernon: n. $2937 \_$B.; hills on the eastern side al Cul de Sac n. 3441 B.

I also saw Tillandsia resurvata: gut from Bethlehem to Cul de Sac.

Anguilla Boldingh.

St. Croix ex Millspaugh.

Baham., Antill., warmer countries of Amer: (Symb.).

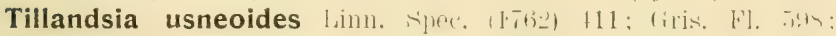
Duss 574; Millspaugh 478. Old man's beard.

St. Eustatius: locality unknown: n. 295 (i.

I also saw Tillandsia usneoides near Boven.

Saba: along the Forthgut 150-200 M.: n. 1638 B.; Peperpot: n. 92,94 ; Paris: 11.95.

St. Martin: Gut from Bethlehem to Cul de Sac 100-200 N. : n. 3416 B.; top of Centryhill: n. 107.

St. Croix ex Millspaugh.

Antill., warmer countries of Amer. (Symb).).

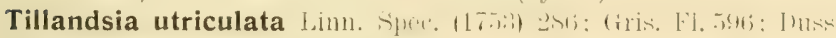
573; Millspaugh 478. Wild pine.

St. Eustatius: Top of Signalhill 22:5 M.: 11. 1031 B.; T'op of Pongo: n. 90; 'Top of the Quill: 11. 99. 
Saba: Slope of the Mountain near St. John's: n. 1735 B.; Paris 200 M.: n. 83, 84; Jenkygut: n. 114; locality unknown: n. 81 .

St. Martin: Nakedboyhill 260 M.: n. 2729 B.

St. Croix ex Millspaugh.

Florida austral., Baham., Antill., Curaçao, Mexico, Venezueli, Guyana. (Symb.).

\section{Vriesea Lin 11.}

Vriesea guadalupensis Mez in (" he camblle Monogl: vol. IX (1896) 596. (vel affin.).

St. Eustatius: Top of the Quill: 11. 245 B., 315 B.

Saba: Hountain near Windwardside: n. 2181 B.; St. John's: n. 86 .

Guadeloupe. (Mez 1896).

\section{Catopsis Griseb.}

Catopsis nutans (iricel). Fl. Wr. I. (1Stit) 5991; Duks 57.

St. Eustatius: on the slope of the Cliff near Oranjestad: $n$. 5 . B.; rocky slope of the hills near Tumbledownlicklity: n. 152 B.; top of the Quill: n. $303 \mathrm{~B} ., 100,101$.

Saba: along roads between Bottom and Windwardside 200 M.: n. 1358 B.; Boobyhill 300 M.: n. 1622 B.; slope of the Mountain near St. John's.: n. 1734 B.

St. Martin: Nakedboyhill 50-2110 M. : n. grte9 B.; Mountain between Mont des Accorts imil Contryhill $200-300$ M. : 13. 245t B.: Mount Paradis 300-400 M.: n. 3237 B.

Antill., Amer. centr. (Symb.).

\section{Commelinaceae.}

\section{Commelina Linn.}

Commelina virginica Limn, Spece (17(i2) 4i2: Millspangh 47.

Commelina elegans H. B. K. Nov. Gen. I (1815) 259; Gris. Fl. 525; Duss 498. Watergrass.

St. Eustatius: seashore neal Tumbleduwndickhay: n. 1, is B.: along roats in Oranjestad: 11 . 56.5 B.; slope of the Quill nuar Bengalen 50-200 M.: 17. 640 B., 653 B., 654 B.; Signalhill 150 M.: n. $10 \overline{3}$ B.; Mountain near Bengalen: n. 262; Cultuurvlakte: n. 281 ; locality unknown: n. $2 \mathrm{H}, 2 \mathrm{G}$.

I also saw Commelina virginica at the seashore near Oranjestad. 
Saba: between Bottom and Marypoint 100-200 M.: n. 1471 B.. 1531 13.; Mountain 400 M.: n. 1770 B.; Laddergut 0 -200 M.: n. 2015 B.; Windwardside: n. 2318 B.; Peperpot: n. 284 ; Jenkeygut: 11. 285; Bottom: n. 280 ; locality unknown: n. $15 \mathrm{~L}$.

St. Martin: From Filipsburg to Guanabay: n. 2381 B.; Moun. tain between Mont des Accords and Centryhill 0-200 Mr: $\mathrm{n}$. $2853 \mathrm{~B}$.; along roads from Colebay to Simsonsbay: n. $3249 \mathrm{~B}$; Mount Paradis 200-300 M.: 11. 3307 B.; roakls near Colebayhill : 11. 264; John Ednyhill: n. 263.

I also saw Commelina virginica near Saline; near I3ellevue; Nakedboythill.

St. Croix ex Millspaugh.

Antill., Curaçao, warmer countries of Amer. cont. (Symb).).

\section{Tradescantia Iinn.}

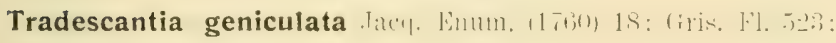
Duss 495.

St. Martin: Milldrumhill 300-400 M.: n. 3141 B.: Nount Paradis $300-400$ M.: n. 3222 B., 3345 B.: locality unknown: n. $3622 \mathrm{~B}$.

Antill., Amer. cont. trop., from Nexico to Paraguay, Ins. Sindw. (Symb.).

\section{Callisia Jinn.}

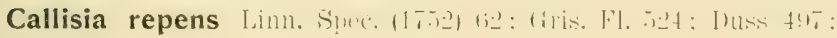
Millspaugh 479 .

St. Eustatius: cultuurvlakte: n. 261.

Saba: between Bottom and Marypoint 100-200 M. : n. 1459a B.; Springbaygut: n. 2099 B.; Bottom: n. 27:3 locality unknown: n. $311 \mathrm{~L}$.

St. Martin: from Filipsburg to Guanabay: n. 2380 B.; from Filipsburg to Belvedere: n. $2591 \mathrm{~B}$; hill on the northern side of Cul de Sac 0-100 M.: n. 3000 B.

I also saw Callisia repens: on the hill between Mont tes Accords aml Centryhill; top of Mount Paradis $400 \mathrm{ML}$.

St. Croix ex Millspaugh.

Antill., Mexico, Amer, centr., Venezuela, Peru, Bras. (Symb.).

\section{Rhoeo Hance.}

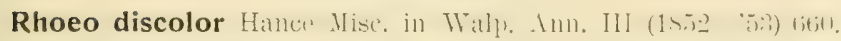
Cultivated. [Mexico, Antill.] (Symb.). 


\section{Zebrina Schnizl.}

Zebrina pendula sehnizl. in Bot. Zeitw. VII (1849) 870; Millspaugh 479.

Saba: Lowol part of the Bottom Mountain 250 M. : n. 1416 B.; along roads between Bottom and Windwardside 200 M. : n. 1335 B. : Windwardside n. 1936 B., 2314 B.; Crespeen-Ronde\%-vous: n. 28:3 Gainbygut: n. 283; locality unknown: n. $42 \mathrm{~L}$.

St. Croiz ex Millspaugh.

Antill., Mexico, Amer. centr. (Symb.).

\section{Liliaceae,}

\section{Aloe Limn.}

Aloe vera Linn. Spec. (1753) 320.

Aloe vulgaris Lam. Enc. I (1783) 86; Gris. Fl. 582; Duss 566; Millspaugh 479. Al 0 e.

St. Eustatius: locality unknown: n. 3589 B., $102 \mathrm{G}$.

I also saw Aloe vera on Signalhill.

st. Martin: I saw Aloe vera: along roads from Filipsburg to Naked. boyhill; along roads near Bethlehem; along roads near Belvedere.

Bermud., Antill., Indig. in mediterr. countries and Ins. Canar. (Symb.).

\section{Allium Linn.}

Allium ascalonicum Linn. Amoen. Acad. IV (1754) 454.

Cultivated. [Everywhere cultivated.] (Ind. Kew.) Charlot ten.

\section{Yucca Linn.}

Yucca aloifolia Linn. Spece (175.3) 314: Gris. Fl. 559: Dnss 557. Adam's needle. Spanish dagger.

St. Martin: Near Orientbay: n. $2922 a$ B., 3358 B.

Cuba, Haïti, Jamaica, 'lobago. (Herb. Krug et Urban.).

\section{Sanseviera Thunb.}

Sanseviera guineensis Willt. Sine. Plint. II (1799) 159 R ham m i. Cultivated. [Afr. trop.] (Symb.). 


\section{Smilax Iinn.}

Smilax coriacea Spr. Syst. Vog. II (1825) 103.

Smilax havanensis Gris. Flor: W. I. (1864) 586 (pro parte) non Jacq. (Symb.). White withe.

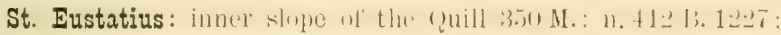
slope of the Quill near Bengalen $150 \mathrm{H},: \mathrm{n}, 678 \mathrm{~B}, 735 \mathrm{~B}$; loca. lity unknown: n. $188 \mathrm{G} ., 263 \mathrm{II}$.

St. Croix. (Symb.)

Haiti, Domingo, Portorico, (Symb.).

Smilax guianensis Vitman summi l'lint. Y $(1-191)$ t2u.

Smilax macropplylla IVilld. Spec. Plant. IV 2 (1806) 786; Gris. Fl. 586; Duss 560. Basket hoop.

St. Eustatius: 'l'op of the Quill: n. 189 B.; Quill: n. 2022; Bengalen 150-200 M.: n. 2019.

Saba: Lower pirt of the Buttom Mumtain 2501 M.: 11. 142:) L.: Hellggate: n. 2023.

Antill, Guiana. (Symb.).

Smilax ilicifolia Kth, Enum. V (1850) 171.

Smilax havanensis Griseb. Flor. W. I. (1864) 586 (p. p. non L.) (Symb.); Millspaugh 479.

St. Martin: Top of the hill on the northern side of Cul do Sac 200-300 M.: 11. 3013 B.; Lowlands: n. 3262 B., 3277 B.

St. Croix ex Millspaugh.

Cuba. (Symb.).

\section{Amaryllidaceae.}

\section{Zephyranthes Herb.}

Zephyranthes carinata IIerb. in I Hot. Miag. LII (1-2.) t. 2594. Amaryllis carinata Spreng. Syst. Cur. post (1827) 132; Gris. Fl. 584; Duss 562. White snowdrop.

St. Eustatius: in a garden in Oranjestad: n. $42 \mathrm{H}$.

I also saw Zephyranthes carinata: slope of the Quill near Glassbottle.

Saba: Bottom: n. 3, 4, 5 .

St. Martin: slope of Centryhill: n, 6 . Antill., Mexico. (Symb.). Also cultivated.

\section{Crinum Linn.}

Crinum americanum Linn. Spec. (1753) 292. Cultivated. [Amer, austr.] (Ind. Kew.). 


\section{Hymenocallis Salisb.}

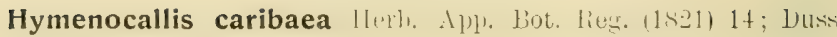
55.); Millspaugh 480 .

Pancratium caribaen Lim. Spec. (1753) 291; Gris. F'l. 583. White lily.

St. Eustatius: Glassgut near Oranjestad: n. $51 \mathrm{~B}$; slope of the Quill near Bengalen 100 M.: n. 674 B.; near Maryglory I50 M.: 11. 1148 B.; Oranjestad: n. 25; locality unknown: n. $298 \mathrm{H} ., 298 a \mathrm{G}$.

Saba: Boolyhil! : n. 1572 B.; along the Forthgut 150-200 Al.: n. 1635 B.; Bottom: n. 26.

St. Martin: Guanabay: 11. 2429 B.; Mountain between Mont des Accords and Centryhill 0-200 M.: n. 2851 B.

St. Croix ex Millspaugh.

F'lorida, Baham., Antill. (Syml).). Also cultivated.

\section{Eucharis Planch.}

Eucharis grandiflora ['linch, et Linden in Fl. Hes serres. Sír. I (LX) (1853-1854) 255.

Cultivated. [New Granada.] (Ind, Kew.)

Eucharis lily.

\section{Hippeastrum Herb.}

Hippeastrum puniceum Urh, in syml. Antill. IV. (19(13) 151. Amaryllis equestris Ait. Hort. Kew. I (1789) 417; Gris. Fl. 584.

Hippeastrum equestre Herb. App. Bot. Reg. (1821) 31; Duss 561 ; Millspaugh 480. 11. 23.

St. Eustatius: Little Mountain: n. 15; locality unknown:

Saba: l'eperpot: n. 14; Bottom: n. 24.

St. Croix ex Millspaugh.

Antill., Amer. trop. (Symb.). Also cultivited.

\section{Fourcroya Vent.}

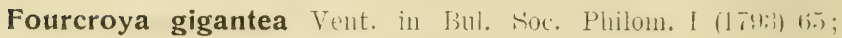
Gris. Fl. 582: Duss 558, Karita, l'ita.

St. Eustatius: along loads in Oranjestad: n. 101 (i.

I also saw Fourcroya giganten: on the top of the Cliff near (iallowshay; Bergje; slope of Signalhill. 
St. Martin: along roads near Belvelere: 11. 3359 B.

Antigual, French Islands to Bras. (Gris. Fi.).

\section{Hypoxis Limm.}

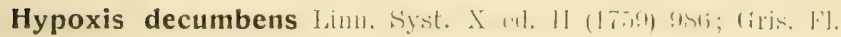
585 ; Duss 562. (procumbens errore).

Saba: Mountain 600-800 M.: 1]. 1785 B., 1796 B., 2191 B.; Mountain near Windwardside 600 M.: n. 2161 B.; Jénckygut: n. 2. Antill., Amer. cont, trop. (Symb.).

\section{Dioscoreaceae.}

\section{Dioscorea Linn.}

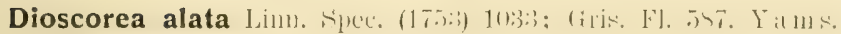
St. Eustatius: locility unknown: n. $245 \mathrm{H}$.

Saba: Mountain neal Windwardside: n. $2157 \mathrm{~B}$.

Antill. Patria veris. Asia austro-orient. (Symb.). Also cultivated.

Dioscorea trifida Linu. f. suluh. (17.81) t27; (iris. Fl. 55\%.

Saba: 'Top of the Mountain 800 M.: n. 1789 B.; Mountain near Windwardsit\}e: n. 2155 B.

Antill., Patria Amer.alustral. (Symb.).

\section{Iridaceae.}

\section{Belamcanda Adans.}

Belamcanda punctata Hoench. Mreth. Suppl. (1791) 214.

Cultivated. [China.] (Ind. Kerv.).

\section{Eleutherine Herl).}

Eleutherine plicata lierh. in Bot. Iiex. (1843) t. 57. Red root. Cultivated. [India occid.] (Ind. Kew.)

\section{Musaceae.}

1318. Musa Linn.

Musa paradisiaca Limn. Spec. (1753) 1043.

Musa sapientium Linn. Syst. X €d. II (1759) 1303.

Cultivated. [India orient.] (Symb.). 


\section{Heliconia limm.}

Heliconia Bihai Linn. Mant. II (1771) $211 ;$; tris. Hl. kt10; Duss 579. Wild banana.

St. Eustatius: Bottom of the Quill 300 M.: n. 449 B.; Quill: 11. $1077 \mathrm{~B} ., 295,223 \mathrm{G}$.

Saba: Mountain 500 M.: 11. 2223 B.

Antill., Amer. cont. trop. (Symb.).

\section{Zingiberaceae.}

\section{Costus Linn.}

Costus speciosus Suf. in l'rans. Linn. Soc. I (1791) 249. Shel1. plant.

Cultivated. [Ins. Malay.] (Schumann 1904.).

\section{Cannaceae.}

1363. Canna Limn.

Canna indica Linn. Spec. (1753) 1. Ca n n o n, I n d i an s hot. Cultivated. [Cosmop. trop.] (Ind. Kew.).

\section{Marantaceae.}

1370. Maranta Linn.

Maranta arundinacea Linn, Spec. (175:3). ‥ \1 1. 11 w root. Cultivated. [Baham., Antill., Amer, cont. trop.] (Symb.).

\section{Orchidaceae.}

\section{Spiranthes L. Cl. Rich.}

Spiranthes elata L. ('I. Rich. Grch. Eur. Allu. (1s)18) :it; (ilis. Fl. $6+1$.

St. Martin: Centryhill: 11. 72.

Antill., Amer. austr. (Symb.).

\section{Stenorrhynchus L. Cl. Rich.}

Stenorrhynchus orchioides L. Cl. Rich. (hreh. Eur. 11817) 37; Gris. Fl. 642.

S'lenorrlynchus lancrolatus L. Cl. Kich. 1. c.; Gris. H'l. 642.

St. Eustatius: 'T'op of the Quill 400 MI. : n. $229 a$ B.

Baham., Antill., Amer. cont. trop. (Symb.). 


\section{Physurus L. Cl. Rich.}

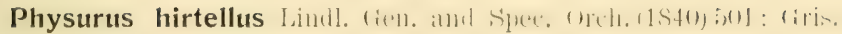
F1. 642; Duss 606.

St. Eustatius: Top of the Quill: n. 65,66 .

Cula, Jamaica, Portorico, Guadeloupe, Dominica, Martini(pue, St. Vincent, Grenada, 'T'rinidad. (Symb.).

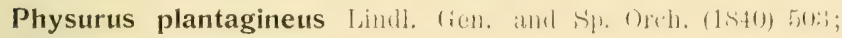
Gris. Fl. 648; Duss 605.

St. Eustatius: I'op of the Quill 400 M.: n. 229 B.

Saba: Mountain near Hellsgate: 11. $2284 a$ B.; Mountain 600-800 M.: n. 1, 2, 3, 4, 67, 68, 69, $176 \mathrm{~L}$.

Cuba, Jamaica, Portorico, Guadeloupe, Dominica, Martinique, St. Vincent, Grenada, I'rinidad. (Symb.).

\section{Prescottia Lindl.}

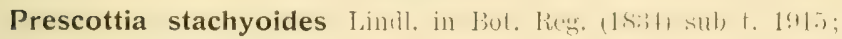
Ciris. Fl. 639: Duss 603.

St. Eustatius: Top of the Quill: i1. 71.

Antill., Amer, centr., Bras. (Symb.).

\section{Microstylis Nutt.}

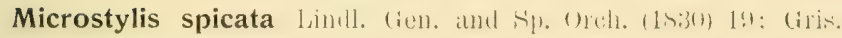
F'l. 612; Duss 590.

Saba: 'l'op of the Mountain 6-S00 M.: 11. 1828 B., 2224 B., $22: 8 \mathrm{~B}$.

Cuba, Jamaica, Hispaniola, Martinique. (Symb.).

\section{Polystachya Hook.}

Polystachya luteola Ilook. Exot. Flor. (lase) tal, 103; (iris. Fl. 628; Duss 597.

St. Eustatius: Quill: 11.70.

Baham., Antill., Amel: trop. (Symb.).

\section{Epidendrum Linn.}

Epidendrum anceps Jacq. Sel. (1763) 224 t. $13 \mathrm{~S}$.

Epidendrum fuscatum Sm. Spic. bot. (1791) 21 t. 23; Gris. FI. 617; Duss 591.

St. Eustatius: Top of the Quill 400 M. : 11. 250 B., 381 B.; top of the Quill: 11. 92, 93, 95; Quill: 11.96 ; locality unknown:n.94.

Saba: Windwardside-Hellsgate: 11. 1666 B.; Crispeen 400 M.: 
11. 1763 B.; Mountain 800 M.: 11. 1818 B.; Springbaygut: n. 2090 B., 2108 B.; Mountain 600 M.: n. 117 ; Mountain 800 M.: 13. 97; Masticgut: n. 119; Wellridge: n. 118 ,

St. Martin: Milldrumhill $300-400$ M.: n. 3149 B.

Antill., Amer. cont. trop. (Symb.).

Epidendrum ciliare Linn. Syst. $\mathrm{X}$ ed. (17.90) 1246; (iris. H.615; Duss 590 ; Millspaugh 481.

St. Eustatius: Slope of the Quill neill Bengalen: 11, 715 B; ; top) Panga hill: n. 101; top of the Quill: n. 102; Boven: 11. 103; locality unknown: n. $281 \mathrm{G} ., 268 \mathrm{H} ., 308 \mathrm{G}$.

Saba: between Bottom and Marypoint 250 M.: n. 1554 B.; Boobyhill 300 M.; n. 1597 B.; Crispeen 400 M.: n. 1727 B.; Paris 2-400 M.: n. 80, 99; Greathill 2-400 M.: n. 81; Rendez-vous 400 M.: ก. 100; Hellsgate: n. 98; locality unknown: n. 165 L.

St. Martin: Nakedhoyhill $200-260$ M. : 11. $27: 7$ B.: Mountain

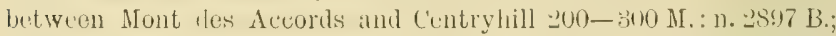
Milldrumhill $300-400$ H.: n. 3154 B.; gut from Bethlehem te Cul de Sac 100-200 M.: n. 3421 B.; Centryhill: n. 104, 105; French frontier: n. 106.

St. Croix ex Millspaugh.

Antill., Amer, austr-sept. and centr. (Symb.).

Epidendrum difforme Jac1, Enum. (1760) :2!); Duss 5!1.2.

Epidendrum umbellatum Sw. Prodr. (1788) 121; Gris. Fl. 618.

St. Eustatius: Top of the Quill 400 MI. : 11. 241 B., 107, 10S. Antill., Amer. trop. (Symb.).

Epidendrum globosum Jacy. Enum. (1760) 2!); (tris. Fl. (i1!); Duss 594.

St. Eustatius: Top of the Quill: n. 83.

Saba: Mountain 800 M.: 11. 1827 B.; Mountain near TVindwardside 600 M.: n. 2177 B.; Mountain: n. 82; Rendez-vous 400 M.: 11.84 .

Antill., Amer. cont. trop. (Symb.).

Epidendrum papilionaceum Tahl in West st. (roix (1793) 303.

St. Eustatius: Signalhill: n, 110 B.; Boven: n. 112; locality unknown: n. 280a G.

Saba: Forthbay 30 M.: n. 5.

St. Martin: neal Mulletpond: n. 2784 B.: Lowlands: n. 325t B., 3278 B.

Balıam., Antill. (Symb.). 


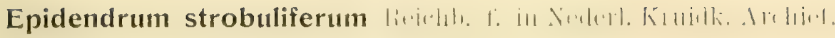
IV. (1859) 333; Gris. 11. 618; Duss 592.

Saba: Rendez-vous 400 M.: n. 109.

St. Croix ex Millspaugh.

Jamaica, 'Trinidad, (tuiana. (Gris. Fl..).

1619. Brassavola R. I3r.

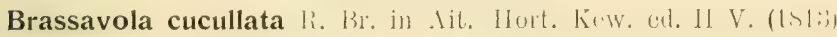
216; Gris. Fl. 620; Duss 594.

St. Eustatius: Lower part of the slope of the hills near Concordia: n. 941 B.; Boven: n. 90.

Saba: Greathill 2-400 M.: 11. 91; Histicent: n. 110.

St. Martin: Milldrumhill $300-400$ N.: D. 3139 B.; gut from Bethlehem to Cul de Sac 100-200 M.: 1. 3419 B.; Centryliill: 11. 111. Antill., Mexico to Venezuela. (Gris. F'l.).

Brassavola rigida (coml), nov.).

Tetramicra rigida Lindl. Gen, et sp. Oreh. (1831) 119; (tris. F'. 6022 .

St. Eustatius: Gut near Fort de Wind 50 M. : n. 825 B. ; Littlo Mountain n. 75; Greatgut: n. 73, 7t, 78; locality unknown: n. $76,77,51 \mathrm{H}$.

Ind. occid. (Ind. Kew.).

1733. Ornithidium Salisb.

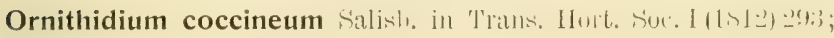
Gris. Fl. 626: Duss 596.

Saba: Mountain 800 M.: n. 1801 B.

Antill., Venezuela. (Symb.).

1779. Oncidium SW.

Oncidium spec. veris: ${ }^{1}$ )

St. Eustatius: Signalhill 200 M.: n, $134^{* * *}$ B., $1049 \mathrm{~B}^{* * *}$; neal White Wall: 11. S49*** B.; Signalhill-Red Cliff: $n .56^{*}, 58^{*}, 60^{*}$; slope of Signalhill: n. $61^{* * *}$; Signalhill: n. $57^{*}$; Red Clift': n. $4 \varepsilon^{*}$;

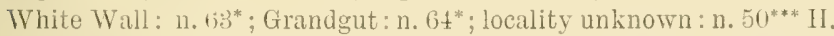

Saba : between Bottom and Marypoint 100__ 200 M.: n. 1469*** B. $2039^{* * *}$ B.; Laddergut: n. 53***; Peperpot: n. 54**; Paris: n. $55^{* *}$.

St. Martin: Nakedboyhill 280 M.: n. $2733^{* * * *}$ B. Centryhill: n. 52**; Princequarter: 11. 51**, 59; roal near Oysterpond: n. 50**. Antill. (Symb.).

1) *) Uncidum variegatum Sir. in Act. Holm. XXI (1500) 240.

₹.*) Oncidium sylvestre Lindl. in Anm. and Mlog. Nat. Hist. IlI Ser. I (1s5S) 33르..

***) Uncidium tetrapetalum IV. (L.) Gris. Fl. 6\%1. 


\section{Dicotyledoneae.}

51. Casuarinaceae.

1855. Casuarina Linn.

Casuarina equisetifolia Linn. Amoen. IV (175!) $14 \%$. ('a s u it rine. Cultivated, |Afr, occid., Australia, Ins. P’acif, ind Malay. |. (Symh.).

\section{Piperaceae.}

\section{Piper Linn.}

Piper dilatatum L. ('l. Rich. in Act. Soc. Hist. nat. I'ar. (17 172 ) 105. Artanthe Bredemeyeri Griseb. Flor. W. Ind. (1859) 170.

Piper Bredemeyeri Jacq. Eclog. (1811-44) 125 f. 84; Millspaugh 481.

St. Eustatius: Inner slope of the Quill $350 \mathrm{M}$.: n. $416 \mathrm{~B}$, 424 B. top of the Quill 500 M.: n. 491 B., slope of the Quill near Bengalen 200 M.: 11. 7015; (tall 2566, 25tio, 2501 ; locality unknown: ก. $174 \mathrm{G}$.

Saba: Lower part of the Bottum Mountilin 300 M.: 11. 1439 13, Bottom 200 M.: n. 1451 B.; Laddergut 0-200 M.: n. 1964 B.; gut botween Misticgut and Hellsgate $300-400 \mathrm{ML}:$ 11. 2061 ; ('respeen Rendez-vous 400 M.: n. 2511; Hellsgategut: n. 2569.

St. Martin: Hills on the northern side of ( ul de sac 100)-200 M.: 11. 2981 B.; Nount Paradis 300-400 M.: n. 3219 B.

St. Croix ex Millspaugh.

Antill., Mexico, Costarica, Panama, Columbia, Venezuela, Bras. (Symb.).

Piper marginatum Jacq. Ic. rar. II (1786) 2 t. 215.

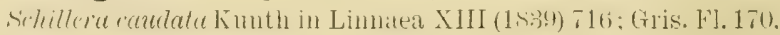

Saba: locality unknown: n. $159 \mathrm{~L}$.

Antill., Panama, Surinim., Guiana gallica, Venezueli, Bras., Peru. (Syml).).

Piper medium Jacq. Ic. rar. I (1781) 2 t. 8.

Piper ceunothifolium Kunth, in H. B. K. Nov. Gen. I (1815) 26; Duss 177.

Linckea Sieberi Gris. Flor. W. I. (1859) 177 (p. p.) (Symlı.).

St. Eustatius: Quill: n. 459 B.

Antill., Amer, cont. (Symb.). 
Piper peltatum Linn. Spec. (1753) 30.

Heckeria peliate Kunth in Linnaea XIII (1839) 565.

Pothomorphe peltata Miq. Comm. phyt. (1838) 37; Gris. Fl. 169; Duss 175; Millspaugh 481.

Saba: Mountain 500 M.: n. 899; Gainbygut: n. S88, 889, 895. Antill., Amer, cont. centr, and austral. (Syml.).

Piper reticulatum Linn, Spec. (1753) 29.

Enckea smitacifolio Griseb. Flor. W. Ind. (1859) 169. (excl. syn. Willd.) (Symb.).

Piper smilacifolium C. DC. Prodr. XVI, 1 (1869.) 245; Duss 175: - non H. B. K. (Symb.). Wild can e.

St. Eustatius: Inner slope of the Quill 350 M.: n. 423 I3., 459 B.; Bottom of the Quill 300 M.: n. 911 B.; Quill : n. 78 H.; 2553, 2527,925, 2529,922,926,939.

Saba: Boobyhill 300 M. : n. 1617 B.; Springbaygut: n. 2083 B., 2106 B., locality unknown: n. 159 L.

Guadeloupe, Martinique, St. Vincent, 'Trinidad. (Symb.).

1866. Peperomia Ruiz, et Pav.

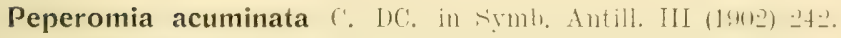
Peperomia glabella Grisel). Flor. W. Ind. (1859) 165. (Symb.).

Peperomia glabella var, nigropunctata Duss Fl, phan. (1897) 172. (Symb.).

St. Eustatius: Top of the Quill 400 M.: n. 178 B., 317 B., near Maryglory 150 M. : n, 1160 B.; Missionhouse road: n. 551.

Saba: Greathill 400 M.: n. 1382 B.; Paris n. 315, 321; John Ednyhill: n. 302; locality unknown: n. $103 \mathrm{~L}, 171 \mathrm{~L}$.

St. Martin: Nakedbuyhill : 17. 2.5 B.; Mountain between Mrnt des Accords and Centryhill 200-300 M.: n, 2898 B.

Antill., Columbia, Guiana. (Symb.).

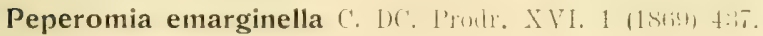

Peperomia exilis Griseb. Flor. WV. Ind. (1859) 164. (Symb.); Duss 170.

Saba: Mountain 800 M. : 11. 1813 B; Mount:in 6-800 M. : n. 19.

Cuba, Jamaica, Hispaniola, Portorico, St. Kitts, Guadeloupe, Martinique, St. Vincent, Trinidad. (Symb.).

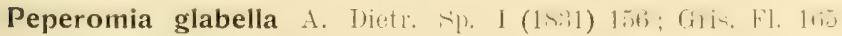
p.p. (Symb.). orehidspurslane. 
St. Eustatius: 'Top of the Quill 400 M. : n. 180 B., 334 B.;

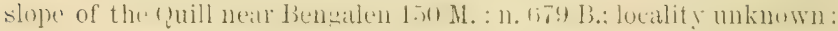
n. 1023 B., 193 G.; Quill: n. 646 , 593, 620, 633, 1054; Boven: ก. 10.:).

Saba: Along roats hetween lanttom and Trindwardside ano M.:

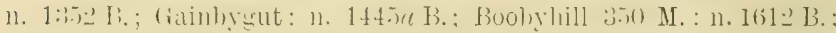

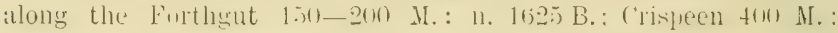

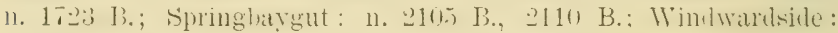
11. 2321 B.; Peperpot: n. 1052.

St. Martin: Inuntain between Mont les Accords and Centry hill 200-300 M.: 1]. $2886 \mathrm{~B}$; ; top of the hill on the northern side of Cul de Sac 200-300 M.: n. 3008 B., Milldrumhill $300-400$ M. : 11. 3135 B.; Mount Paradis 150-400 M.: n. 3192 B., 3196 B., $3340 \mathrm{~B}$.

I also saw Peperomat glabella on Nakedboyhill $100-200 \mathrm{M}$.

Antill., Costarica, Amer-austr. (Symb.).

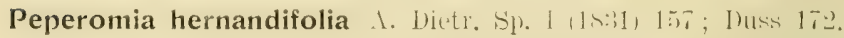

Saba: Mountain 500 M.: 11. 1808 B.; Mountain 6-800 M.: n. $63 \pm$, 140; locality unknown: n. $174 \mathrm{~L}$.

Antill., Costarica, Venezuela. (Symb.).

Peperomia Langsdorffii Miq. Syst. (1843) 116.

St. Eustatius: Jop of the Quill 380 M.: n. 278 B; lower parts of the hills near Concordia: n. $974 \mathrm{~B}$.

St. Martin: Nakedboyhill 200-265 M.: n. 2717 B., along roarls near Colebayhill: n. 3041 B.; gut from Bethlehem to Cul de Sac 0-100 M.: n. 3414 B.; Milldrumhill 100-200 M. : n. 3117 B.: Colebayhill : n. 141 .

St. Croix. (Symlo).

Haïti, St. Domingo, Portor'ıco, St. 'I'homis, St. Jan, 'Tortola, Antigua. (Symb).).

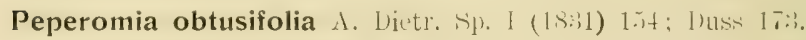

Peperomia magnoliacfolia A. Dietr. Sp. I (1S:31) 158; Duss 173. (Symb.).

St. Eustatius: 'I'op of the Quill 400 M.: n. 242 B., 353 B.: Quill: n. 277, 298, 279, 278, 291, locality unknown: n. 290.

Saba: Mountain 600 M. : 13. 1757 B.; Springluaygut : n. 2102 B.; Milldrumbill 300-400 M.: n. 3146 B.

St. Croix. (Symb.).

Antill., Amer. cont. trop., (F'lorida, Mexico, Vonezuela.) (Symb.). 


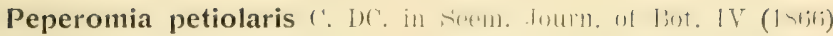
138. IV a 11 p urs 1 a 11 e.

St. Eustatius: In a garden in Oranjestad: n. 63 B.; at the seashore near T'umbledowndickbay: n. 155 B.; on walls in Oranjestad: 11. 69 (i.

Saba: Along roads betwcen Bottom and Windwardside 200 M.: n. 1341 B.; lower part of the Bottom Mountian 250 M. : n. 1410 B.; Windwardside: 11. 1893 B.; Bottom: 11. 2245 B.; locality unknown: n. $39 \mathrm{~L}$.

Cula, Paraguay. (Symb.).

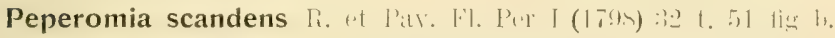
Peperomia repens Tith. in H. B. K. Nov. gen. I (1815) 65; Giris. Fl. (1859) 165. (Symb).

St. Eustatius: Buttom of the Quill 800 M.: n. 434 B. Antill., Amer. cont. austral. (Symb.).

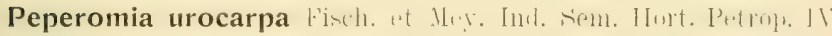
(1837) 42.

Saba: Mountain 800 M.: 11. 1825 B., 594, 647.

Antill., Amer. cont. trop. (Symb.).

\section{Batidaceae.}

2362. Batis Linn.

Batis maritima Linn. Syst. $X$ ed. II (1759) 1380; Gris. F'l. 61: Duss 92; Nillspaugh 486. Tr ild ba na nat.

St. Martin: near Filipshurg: n. 2388 B.; road near Fort Amsterdam: n. 47.

I also saw Batis maritima : near Simsonshay village; Oysterpond; Green Key.

Anguilla Boldingh.

St. Croix ex Millspaugh.

Florida, Baham., Antill., Amer. cont. trop. orient., Californ., Ins. Sandw. (Symb.).

\section{Ulmaceae.}

1898. Celtis Iimn.

Celtis Iguanacea Sarg. Silva VII (18\%5) 64.

Celtis aculeata Siv. Prodr. (1788) 53; Gris. Fl. 149; Duss 152; Millspaugh 48\%. S n a $\mathrm{k}$ y. 
St. Eustatius: Top of the Quill 400 M. : 11. 21!) B.; Botton of the Quill 3010 M.: 11. 4.9.) B.: tol of Signalhill 2.25 M.: 1. 1029 B.; Red Cliff-'T'umbledowndickbay: n. 229.

Saba: Bottom - Marypoint 300 M.: 11, 1476 B.: Windwardside: n. $1950 \mathrm{~B}$; springbaygut: n. $2116 \mathrm{~B}$.

St. Martin: from lilipshurg to frumabas: 11. 2.349 B., .2:99 B.; from Filipsburg to Belvelere: 11. 25-2 B.; Nakulloyhill 50-200 M.: 11. 2680 B.; between Belvedere and Oysterpond: n. 3079 B.; Mount Paradis $100-200$ M.: n. $3293 \mathrm{~B}$.

Anguilla Boldingh.

St. Croix ex Millspaugh.

Antill., Amer. cont. trop. (Symb.).

\section{Trema Lour.}

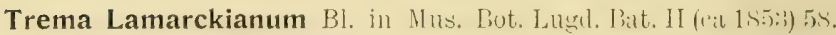
Sponia Lamarclitana Dene. in Nouv. Ann. Mus. Paris III (1834) 498; Gris. Fl. 150; Duss 153.

St. Eustatius: Gilboohill 150 M. : n. 1123 B.

Saba: Hellsgato-Marypoint: 11. 2040 B.

Bermud., Baham., Antill. (Symb.).

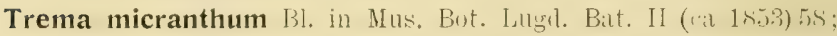
Millspaugh 482 (?).

Sponia miciontha Dene. in Nour. Amm. Nus. Paris III (1s:it) 498: Gris. 1'l. 150; Duss 152.

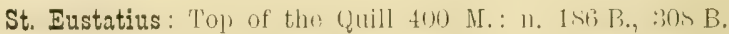

Saba: Gainbygut: n. 292, 293; Laddergut: n. 130, 294.

St. Croix ex Millspaugh.

Florida austr., Antill., Amer: cont. trop. (Symb.).

\section{Moraceae.}

1981. Dorstenia Linn.

Dorstenia contrajerva Liml. Fpec. (17-ij) 1:1; filis. Fl. 15t; Duss 15!).

St. Martin: Mount Paradis 300- 400 M.: 17. 3344 B.

Antill., from Mexico to P'eru. (Symb.).

1946. Artocarpus Linn.

Artocarpus incisa Linn. f. Suppl. (1781) 411.

Artocarmus communis Forst. Char. Gen. (1776) 100. B read fir 1 it t ree .

Cultivated. [Ins. Pacif.] (Symb.). 


\section{Ficus Linn.}

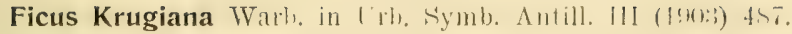

Ficus laurifolia Duss Fi. Ant. frane. (1897) 158. (haud Iam.). (Symb.).

St. Eustatius: 'I'op of the Quill 400 M.: n. 207 B.; bottom of the Quill 300 M.: n. 907 B.

Saba: Bottom: n. 1875 B.

Guadeloupe, Dominica, Martinique, St. Vincent. (Symb.).

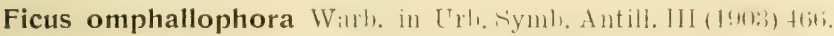
Ficus pertusa Gris. (haud L. f.) Flor. W. I. (1859) 152 (p. p.); D) 155 .

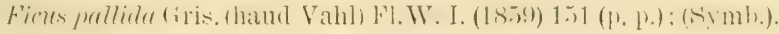

Saba: 'Top of the Mountain 800 M.: n. $1827 a$ B.

sit. Eustatius, st. Kitts, Fualeloupe, Tominica. Matrinirur, St. Vincent, Grenadit. (Symb.).

Ficus populnea Willd. Sp. Pl. [Y (1806) 1141; Gris. I]. 151; Millspaugh 483.

Ficus laevigata Vahl, Enum. Il (180.j) 183; Gris, Fl, 151; Millspaugh 483.

St. Eustatius: Flope of the lower latrt of Signalhill: 11, 1(1:) P.: top of the Quill: 11. 207 B., 371 B., 378 B.; along roals in Oranjestad: 11. 914 B.; along roads near Concordia: n. 1091 B.; near Boven 200 M.: n. 1247 B,; Quill: n. 732, 846, 883; top of the Quill: n. 135, 733; Clitt: n. 738, 788.

Saba: Bottom: n. 2283 B.; from Windwardside to Hellsgate: 1). $1681 \mathrm{~B}$.

St. Martin: in a garden in Filipsburg: 11. 2552 B.; from Filipsburg to Belvedere: 17. 2577 B.; Marigotroad near Marigot: 1. 2820 B.; Lowlands: n. 328t B.; Mount Paradis $100-200$ M.: n. 3297 B.; $300-400$ M. : 11. 3324 B. ; Nary's Fancy : n. 705 ; Centryhill: n. 888 ; Prince quarter: n. 838.

St. Croix ex Millspaugh.

Baham., Antill. (Symb.).

Ficus Urbaniana IVarl, in (Th), Symb. Antill. III (1!)(1:) f:i!.

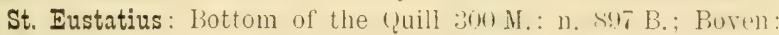
n. 23:1, 2371 ; (quill: n. 2322; locality unknown: n. 2395.

Saba: Gut between Hellsgate and Masticgut 300- 400 M.: 11. 2026 B.; Springbaygut: n. 2080 B.

St. Martin: Point Blanche (coll. George): 11. 2495 B.; Mount. Paradis 150--400 M.: n. 3200 B.; gut from Bethlehem to Cul de Súa 100-300 H.: n. 3423 B,; John Ednyhlll: 1. 2347. 
St. Croix. (Symb.).

Guadeloupe, Martinique, St. Vincent. (Symb.).

\section{Cecropia Linn.}

Cecropia peltata Jinn. Syst. X. al. II (1759) 12st; firis. li. 153; Millspaugh 498. Wild papav.

St. Eustatius: Quill: n, 2.54 B.: Bottom of the Quill 300 Mr.: n. 904 B., 955 B., 105 H. ; Quill: n. 243, 219.

Saba: Mountain near Windwardside 600 M.: n. 2158 B. locality unknown: n, $192 \mathrm{~L}$.

St. Croix ex Millspaugh.

Antill, Venezuela, Guiana. (Syml).).

\section{Urticaceae.}

1978. Urera Gaudich.

Urera caracasana (iri.. Flor. W. I. (1859) 154; Duss 1131.

Uiera alceaefolia Gaudich. in Freyc. Voy. (1826) 479.

St. Eustatius: Buttom of the quill :00 M.: 11. 4., B., 90:3 B., $909 \mathrm{~B}, 130 \mathrm{H} ., 222 \mathrm{G}$.

Saba: Mountain 6-800 M.: n. 1949; Hellsgategut: n. 1950; locality unknown: n. $194 \mathrm{~L}$.

Antill., Amer, cont. trop. (Symb.).

\section{Fleurya Gaudich.}

Fleurya aestuans Faulich, in Freve. Vuy. (1826) thi: Gris. Fl. 154; Duss 160; Millspaugh 483. Stinging nettle.

St. Eustatius: Glissgut near Onmiestad: n, ti 13, along roads in Oranjestad: n. 1079 B.; Oranjestad: n. 2832 ; locality unknown n. $135 \mathrm{G}$.

Saba: neal the Sulphurmines: 11. 1703 B.; locality unknown: 11. $154 \mathrm{~L}$.

I also saw Fleurya aestuans: Windwardside.

St. Martin: Milldrumhill in plantations 300 M.: n. 3132 B.

St. Croix ex Millspaugh.

Baham., Antill., Amer. cont. trop., Afr. trop., Ind. Sundan. (Sym7).).

\section{Pilea Linill.}

Pilea microphylla Liehm. in Tillensk, Selsk. Skir. Koprenh. V. Ser. Vol. II (1851) 2S6; Gris. Fl. 155; Duss 161. 


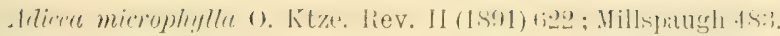
Pilec muscosa Lindl. Coll. Bot. (1821.) t. 4. L a ce plant.

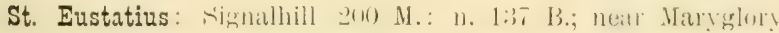
150 M.: n. 1156 B.; in a garden in Oranjestad: n. 1269 B.; Cultuurvlikte: n. 105!); Signalhill: n. 1037; Boven: n. 105s; locality unknown: n. $150 \mathrm{G}$., $301 \mathrm{H}$.

Saba: along rouls betwen buttom and Windwardside son M. n. 1330 B.; slope of the Mountain near St. Johns 250 M.: n. 17:33 B.; Windwardside: n. 1900 B.; Laddergut 0-200 M.: n. 2020 B.; between Hellsgite and Mastiegut: n. Dote B.: Nonntain near Wind. wardside 600 M.: n. 2173 B.; Ladder: n. 1038; Greathill: n. 1039; Crespeen-Rendez-vous 400 M.: n. 1026; locality unknown: n. $142 \mathrm{~L}$.

St. Martin: Jountinin letween Mont de's Aceords and rentry. hill 200-300 M.: n. 2900 B.; Mount Paradis 300-400 M.: n. $32: 5 \% \mathrm{~B}$.

St. Croix ex Millspaugh.

Florida austr., Permud, Baham., Antill., Amer. cont, trop. (Symh.).

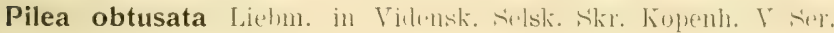
Tol. II (1851) 300

Piled mbescens Gris. Flor. IV. I. (1859) 159 (non Lielmm.): (Symb.).

Saba: T'op of the Mountain 800 M.: n. 17 $89 a$ B., 1786a B., $2218 a$ B.

l.ortorico, Montserrat, Martinique. (Symb.).

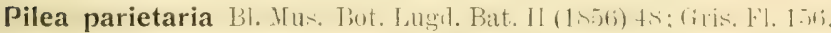
Pilea ciliaris Wedd. in Ann. Sc. nat. III Sér. XVIII (18.52) 20(); Gris. Fl. 156,

Saba: Top of the Mountain 800 M.: n. 1800 B.

Antill., Guatemala, Venezuela. (Symb.).

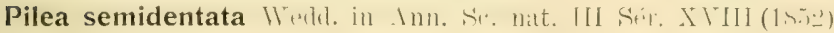
215 ; Gris. Fl. 157.

Pilea clegans Wedd. in Ann. Sc. nat. III Sér. XVIII (1852) 215; Gris. Fl. 157.

St. Eustatius: Top of the Quill 400 M.: 11. 175 B., 340 B., 351 B., 368 B.; slope of the Quill near Bengalen 150 M.: 11. 685 B.; Quill: n. 24t, 1102, 192 G., 273 H.

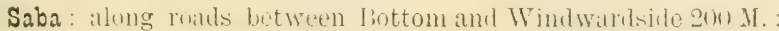
n. 1338 13.; from Marypoint to Bottom: 1]. 2063 B.; Gainbygut: n. 1190 . 
Hispaniola, Portorico, St. Thomas, Gualelompe, Dominica, Martinique. (Symb.).

1990. Boehmeria Jaer.

Boehmeria ramiflora Jacy. Enum. I'l. carib) $(1630)$ :31; (iris. Fl. 160; Duss 163.

St. Eustatius: Bottom of the Quill 300 M. : n. 447 B.; Ben. galen : 1]. 1152, 1172, 1202, 1222, 1242; Quill : n. 1192, 1225, 1233.

Saba: Mountain 800 M.: n. 2219 B.

Guadeloupe, Dominica, Martinique, st. Vincent, Grenada, Barbalos. (Herb. Krug et Urban.).

\section{Rousselia Gautlich.}

Rousselia humilis Urh, in Symh, Antil. I (1905) 20:5.

Rousselia lapmelacen Gaudich. in Bot. Freyc. Voy. (1826) 503; Gris. Fl. 160.

Saba: from Hellsgate to the Sulphurmines: n, $1669 \Omega \mathrm{B}$.

Bahum., Antill. (Symb).).

\section{Loranthaceae.}

\section{Phoradendron Nutt.}

Phoradendron trinervium Gris Flor. W. I. (1)rif) 314; Duss :32.S.

St. Eustatius: Signalhill 200 M.: n. 135 B., 1048 B.; slope of the Quill near Bengalen 100 M. : 11. 672 B.; gut near Fort de Wind 50 M.: 11. 813 B.; Cultuurvlakte: n. 26; Red Cliff: 11. 14; White Wall: n. 13; locality unknown: n. $209 \mathrm{G}$.

Saba: Paris 400 M.: n. 1371 B.; Saddle: n. 1660 B.; Paris 2-400 M. : n. 27.

St. Martin: Mountain hetween Mont les Accords and Centry. hill 200-300 M.: n, 2888 B, : hills on the eastern side of Cul de Sac: n. 3448 B.

Antill., Costarica. (Symb.).

\section{Olacaceae.}

\section{Schoepfia Schreb.}

Schoepfia Schreberi J. F. Gmel. Syst. (1791). 376.

St. Eustatius: Top of the Quill 400 M.: n. 201 B., 364 B., 375 B., 377 B.; inner slope of the Quill 350 H. : n. 1226 B. 
Saba: Botlom 200 M. : 11. 1447 B.; Springhaygut: 11. 2085 B., 2125 B. ; Wollgut: 11. 31 .

Antill., Mexico? San Salvador"? Venezuela. (Symb.).

\section{Aristolochiaceae.}

2174. Aristolochia Linn.

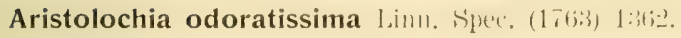

Cultivated. [Jamaica.| (Ind. Kew.).

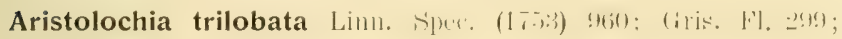
Duss 314; Millspaugh 484.

St. Martin: Mount Paradis 300-400 M.: n, $33: 37$ B.

St. Croix ex Millspaugl.

Antill., Amer. cont, cent. and austr, trop. (Symb.).

\section{Polygonaceae.}

\section{Coccoloba Lin11.}

Coccoloba diversifolia Jacı. Lutum. Syst. (17611) 15; Millspatugh tist. Coccolobe munctata Gris. (non L.) Flor. W. J. (1859) 163.

Coccoloba barbadensis. Jac . Enum. Syst. (1760) 37; Duss 166.

Coccoloba diversifolia Gris. (non Jacy.) pr. 1). Flor, W. I. (1859) 163 (Symb.). Redw ood.

St. Eustatius: Top of the Quill 400 M.: n. $179 \mathrm{~B}, 210 \mathrm{~B}$, 312 B.; slope of the Quill on the side of White Wall 200 M.: $n$. 1172 B.; Boven: n. 37; Little Mountain-Red Clitf: n. 36, 38, 156; top of the Quill: n. 28; Gilboohill: 11. 25.

Saba: lower part of the Bottom Mountain 250 M.: n. 1418 B.; Windwardside-Hellsgate: n. 1665 B.; St. Giles: n. 1860 B.; Mountain near Windwardside 600 M.: n. 2175 B.: Paris-Greathill: 1]. 29, 30, 31; Peperpot: n. 24; locality unknown: n. 340 L.

St. Croix ex Millspaugh.

Baham., Antill., Curaçao. (Symb.).

Coccolobà Krugii Lindau Mon. Cocc. (1S92) 145.

St. Martin: near Mulletpond: n, 2756 B.

Anguilla Boldingh.

Baham., Portorico, Barlsuda. (Symb.).

Coccoloba nivea Jacy. Linum. Syst. (1661) 1!! (iris. 11. 16:3; Millspaugh 484.

Coccoloba excoriata Linn. Spec. (7662) 524; Duss 168. Sugarygrape. 
St. Eustatius: Quill: 11. 142; from Sigualhill to Venusbay: 11. $964 \mathrm{~B}$.

Saba: Boobyhill 300 M.: n. 1582 B.; Windwardside-Hellsgate: n. 1688 B.; Springlaygut: n. 2087 B.; Windwardside: n. 2:316 B. ; Bottom: 11. 187, 188, 189; locality unknown: n. $101 \mathrm{~L}$.

St. Martin: from Filipsburg to Belvedere: n. 2579 B.; lower part of the hills between Mont Chambord and Mount Vermon: 10. $2961 a$ B.; top of the hill at the northern side of Cul de Sac 200-300 M.: 13. 2943 B.; Mount Paradis 300-400 M.: n. 3228 B., :319 B.; sut from Bethlehem to 'ul de siac 100 -200 M.: 11. 34:0 1.; John Ednylill: 11, 3.

I also saw Coccoloha nivea: along roads near Grande Case.

St. Croix ex Millspaugh.

Jamaica, Hispaniola, Portorico, St. 'Thomas, St. Barthélemy, Antigua, (iualeloupe, Mirtinique, Barbatos (cultal, Gronada, Tobago, Trinidad. (Symb).

Coccoloba obtusifolia Jacq. Enum. Syst. 19.

Coccoloba menctata Griseb. (non L.) pr. p. Flor. W. I. (1859) 163.

Coccoloba punctata Griseb. $y$ microstachya in F'lor. W. I. (1859) 163; (Symb.).

Saba: Boobyhill: n. 1569 B., 1571 B.

Baham., Antill. (Symb.).

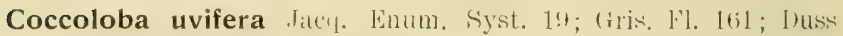
165; Millspaugh 484. Sea grape.

St. Eustatius: Seashore neal (1rajestul: 1. 17 B.; top of the Quill 40 M.: n. $263 \mathrm{~B}$; slope of the lower part of the Quill near Bengalen: n. 717 B.; along roals in Oranjestad: 11. 1057 B.; near Whito Wall: n. 1257 B.; Concordiabay: n. 183; Venusbay: 185; locality unknown: n. $120 \mathrm{H}, 198 \mathrm{G}$. Wall.

I also saw Coccoloba urifera: slope of the Quill on the side of White

Saba: l'aris 400 M.: 11. 1370 B.; Bottom: n. 1988 B.; Ladder: n. 186; locality unknown: n. $360 \mathrm{~L}$.

I also saty Coccololat uvifera: Green Island.

St. Martin: Guanabay: 11. 2432 B.; at the seashore near Simsonsbay village: $\mathrm{n}$. 3030 B.

I also saw Coecoloba uvifera in Lowlands; Marigothill to Marigot.

St. Croix ex Millspaugh. 
Bermud., Florida, Baham., Intill., Mexico, Amer. contr., Ve. noznclil, Guiana. (Symb.).

\section{Antigonon Endll.}

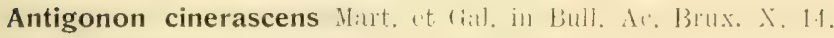
Cultivated, [from Mexico to Guatemala.] (Symb.).

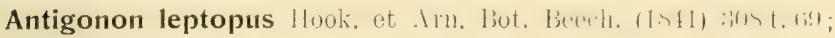
(Yris. F]. 16t; Duss 612. Coralita.

St. Eustatius: in al gillelen in Oranjestad: 11. 1071 B., 1is H., „21 (i.

Saba: Windwardside: 11. 1931 B.; Bottom: 17. 2242 B.; locality unknown: n. $46 \mathrm{~L} ., 299 \mathrm{~L}$.

St. Martin: near Bethlehem: n. 2670 B.

Jamaica. (Gris. Fl.) Cultivated and escaped.

\section{Chenopodiaceae. 2323. Chenopodium Linn.}

Chenopodium ambrosioides Lims. Suec. (175:3) Ll!); (iris. Fl. 150; Duss 51; Millspaugh 485 . W o r m b u h.

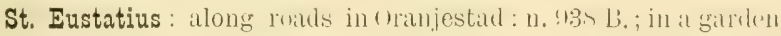
ill Oranjestad: n. 1085 B. ; locality unknown : n. $5 \mathrm{G}_{0}, 5 \mathrm{H}$.

Saba : along roads between Bottom and Windwatisile 20) H.: n. 1921 B., 1301 B.; Bottom: n. 1846 B. ; Laddergut 0-200 M. : n. $1955 \mathrm{~B}$; ; seashore near Forthbaylanding: n. 2149 B.; Paris: 11. 223 ; Bottom : n. 224; locality unknown: n. $235 \mathrm{~L}$.

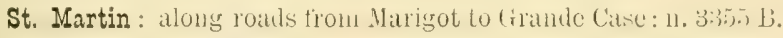

St. Croix ex Millspaugh.

Trop. and warmer countries of both hemisph. (Symb.).

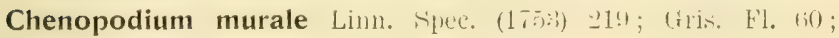
Duss 51 ; Millspaugh 485 . Sp ra in b u s h.

St. Eustatius : along roals in Granjestad: 11. in B.; locality unknown: n. () G., 9) H.; seashore: n. 244; Village: n. 243.

St. Martin: from Filipsburg to Guanabay: 11. $2344 a$ B.

St. Croix ex Millspaugh.

Baham., Antill., indig. in the Old world. (Symb.).

\section{Salicornia Linn.}

Salicornia herbacea Linn. Spec. (1762) 5.

St. Martin: near Mulletpond: n. $27+9$ B.

Anguilla Boldingh.

Amer. sept., Europa, Afr., Asia. (Symb.). 


\section{Amarantaceae.}

\subsection{Celosia Linn.}

Celosia argentea Linn. Spec. (1753) 205.

Cultivated. [Antill., Amer., Afr., Asia trop.] (Symb.).

Celosia nitida Vahl symb. II (17.11) 44; (iris. Fl. ti2; Duss 53); Millspaugh 485.

St. Martin: near Bethlehem: 11. 26666 B.; Marigotroad: n. 2803a B.

Florida austral., Antill., Mexico, Calif. peninsula, Amer. contr. (Symb.).

\section{Amarantus Linn.}

Amarantus gracilis Desf. 'Tabl, I ed. (180t) 43.

Euxolus candatus Moq. in DC. Prodr. XIII. II (1849) 247; Gris. Fl. 68; Millspaugh 486 .

Amarantus caudatus Linn. Spee. (1753) 990.

St. Eustatius: in it garden : 11. 547.

St. Martin. (Symb.).

St. Crois ex Millspaugh.

Baham., Antill.; Bonaire, Curaçao, trop. countries of both hemisph. (Symb.).

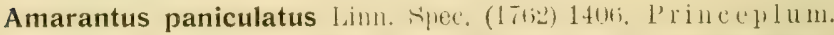
Cultivated. |Haham., (rmana., trop. comntric's of loth hemisph.] (Gris. F'l.).

Amarantus polygonoides Linı. L'l. Jamm. P’ugil. II. (17in!n 27; Millspaugh $4 \$ 66^{\circ}$.

Amblogyme polygonoides Raf. lil. 'T'ellur III (1836) 42; Gris. Fl. 68; Dues 58. Wh it o polly.

St. Eustatius: near Maryglory 150 M. : n. 1135 B.; locality unknown: n. $19 \mathrm{G}$.

Saba: Bottom: n. 559 .

St. Martin: from Filipsburg to Guanabay: 11. 2406 B; in an orchard near Bellevue: n. 2839 B.; between Mount Vernon and Orientbay: n. 2931 B., 3360 B.; Tintamarre: n. $3384 a$ B., 3393 B.; near Filipsburg: 11.595.

St. Croix ex Millspaugh.

Florida, Key, Baham., Antill., Aruba, Curaçao. (Symb.).

Amarantus spinosus linn. Apece (17.,3) !91; (iris. l'l. 168; Duss 58; Millspaugh 486.

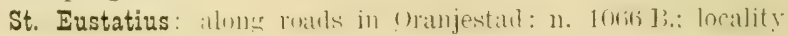
unknown: $\mathrm{n} .18 \mathrm{G}$. 


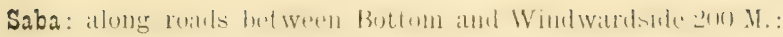
11. 1323 B.; Bottom 200 M. : 11. 1454 B.; Windwarlside: 11. 1918 B., 2327 a B. ; Bottom: n. 568, 569; locality unknown: 11. $252 \mathrm{~L}$.

St. Martin: Hount Paradis $100-200$ M. : 11. 3295 B.

St. Croix ex Aillspaugh.

Bermul., Baham., Amer. cont., Afi: trop., Asia trop. to dapon. and N. Guinea. (Symb.).

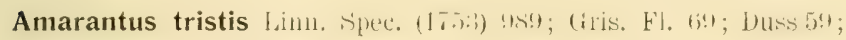
Millspaugh 486 . Ca 1 a $10 \mathrm{e}$.

Amarantus gangeticus linn. śyst. ed. X. (1759) 1268.

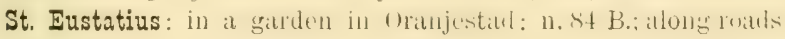
in Oranjestad: 11. $551 \mathrm{~B}$; Cultuurvlakte: 11. 576 ; in a garden: 11 . 575 ; locality unknown: 10. $16 \mathrm{H}, 17 \mathrm{G}$.

Saba: between Bottum and Maryloint 10in-zon M[. : 13. 14ti-2 B.: Windwardside: n. 1919 B.; Rendez-vous 400 M.: n. 580; Bottom: 11. 577.; locality unknown: 11 . $33 \mathrm{~L}$.

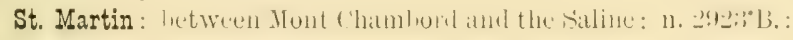
lower part of the Milldrumhill: 11.3163 13.; Marigotroal: 11. 552.

St. Croix ex Millspaugh.

Antill, Chile. (Syml).).

\section{Achyranthes Lim.}

Achyranthes obtusifolia Lanl. Enc. I (1783) 545.

Arlyranthes aspera Linn. vor', obtusifolia Gris. Flor. W. I. (1860) 220; Millspaugh 485. M a n better man.

St. Eustatius: in a garclen in Oranjestad: 13. 82 B.; along roads in Oranjestad: n. 1067 B.; lowel part of the Quill: n. 515; Cliff at the seashore: 11. 514; English quarter: n. 51:3; along roarls in Orinjestad: n. jou B.; locality unknown: n. $40 \mathrm{a}$ B., 93 G., $176 \mathrm{H}$.

I also salw Achyranthes ohtusifolia on the seashore near Oranjestad; slope of the Clitr.

Saba: Winclwardside-Hellsgate: 1. $1671 \mathrm{~B}$; Windwardside: n. 1885 B.: locality unknown: 1, 2.5. L.

I also saw Achyranthes obtusifolia: Latddergut.

St. Martin: from Filipsburg to Belvedere: 11. 2583 B.; Marigutroat: 11.516.

I also silw Achyranthes obtuxifolia in Cul de Sac; Marigothill-Marigot: in pastureground near Bellevue.

Anguilla Boldingh.

St. Croix ex Millspaugh.

Antill., Afr., Asia-trop., Ins-Paul. (Symb.). 
2335. Alternanthera Forsk.

Alternanthera crucis (omb. Nov.

Telanthera crucis Moq. in DC. P'rod. XIII. Il. (1819.) 362; (iris. li. 1i7.

St. Martin: Pointe Blanche: n. 3407 B.

St. Croix. (Herb, Krug et Urban.).

Alternanthera repens O. Ktze Rev. II (1s91) j40.

Alternanthera achyrantha R. Br. Prodr. I (1810) 417 ; Gris. H. 67 ; Duss 57 ; Millspaugh 485.

St. Eustatius: in a garden in Oranjestal: n. 83 B.; Cultuurvlakte: n. 522; in a garden: n. 521 ; locality unknown: $n .520$.

Saba: letween Bottom and Maryloint 100-2(10) II. : 1. 14:5 B.: Windwardside: 11. 1892 B., 1897 B.; Bottom: n. 524, 525.

St. Martin: along ruals from coleblity to Simsonsbay: 11. 3246 B.; from Filipsburg to Point Blanche: 11.3407 B.; gut from Bethlehem to Cul (le Sac 100-200 M.: n. 3421 B.; Colebayhill: ก. 523.

Anguilla Boldingh.

St. Croix ex Millspaugh.

Baham., Antill., Amer. cont, Ins, Canar., Hispania. (Symb.).

\section{Gomphrena Linu.}

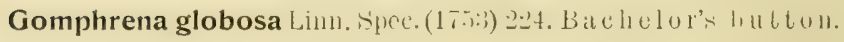
Cultivated. [Indig. Asia orient, et austr. from Ins. Sundaic, to Nova Guinea.]. (Symb.).

\section{Iresine Linn.}

Iresine elatior L. (']. Rich. ilf. Willd. Spes. IV (1h06) itbo; (iris. Fl. (it); Duss 55; Hillspaugh 485. Wh ite snow plant.

St. Eustatius: Cultuurvlakte: 11. 591; road near Sugarloaf: 11. 594; locality unknown: n. 1118 B.

Saba: Peperpot: n. 592.

St. Martin : Colebayhill : n, is:) ; trom l'ilipsbures te (iuanaluy : 11. 2:31; B.; Nakedboyhill 100 M.: 11. 269.2 B.

St. Croix ox Millspaugh.

Antill., Venezuela, Nova Granata. (Symb.).

Iresine paniculata 0 . Ktze. Rev. II (1891) 542.

Iresine celosioides Linn. spec. (1763) 145t; Gris. Fl. 64; Duss 55. 


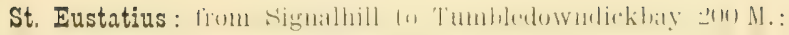

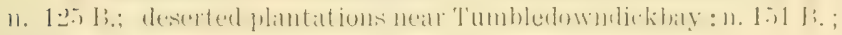
near Maryglory 150 M.: n. 1164 B.; slope of the Quill: n. 585 ; Boven: 11. 58t.

Saba: Bottom: 11. 1455 B.; Boobyhill 300 M.: 17. 1577 B.; Hellsgate: n. 1693 B.; Ladder: n. 590 ; Greathill 2-400 H.: 11. 589; Rendez-vous: n. 588 ; locality unknown: 17. 146 L.

St. Martin: Marigotroal near Marigot: n. 2817 I3. ; lower part of the Milldrimhill: $11,3176 \mathrm{~B}$; ; Centryhill: n. 587 .

Baham., Antill., Amer, cont. from Un. Stat, to Argentinia. (Syml).).

\section{Philoxerus li, $\mathrm{Br}$.}

(f. 2339. Iresine linn, sect. 1.

Philoxerus vermicularis Li, Br. ['rodr. I (lilo) flu; (iris. Fl. (i.); Duss 56.

Lithophila vermiculatu Uline in Field Col. Mus. Bot. II (1900) 39; Millspaugh. 485.

St. Martin: Oysterpond: n. !) locality unknown: n. 2523 B.

St. Croix ex Millspaugh.

Florida austr., Key ins., Baham., Antill., Amer. cont. trop., Afr. occil. (Symb.).

\section{Lithophila SW.}

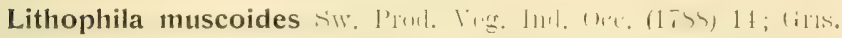
1.]. 66; Duss 56; Millspaugh 485.

Alternanthera muscoides Benth et Hook, f, Gen. III (1880) 39.

St. Eustatius: Seashore neall Oranjestad: 11. 20 B.; leserted plantations near 'Tumliledowndickbay: n. 167 B.; Concordiabay: n. 53t; seashore: 17.533 , 5\$ 6 a $\mathrm{B}_{0}$; Cliff near Fort Amsterdam: 11. 532; locality unknown: $11.408 a$ B.

Saba: along the seashore near Forthbaylanding: n. 16to B.; Laddergut: 11. 1977 B.; seashore near Flat, Point: 11. 2136 B. ; Ladiler: n. $530,531$.

St. Martin: from Filipsburg to Guanabay: 11. 2376 B.; diong the saltpond near Filipslurg: n. 2449 B.; Green Key: n. 3368 B; Filipsburg-Fort Amsterdam: n. 586; Guanabay: n, 585.

St. Croix ex Millspaugh.

Baham., Antill., Bonaire, Curaço, Aruba. (Symb.). 


\section{Nyctaginaceae.}

\section{Mirabilis Linn.}

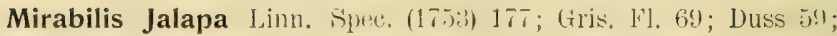
Millspaugh 486. Fo ur o'clock.

St. Eustatius: in a garden in 0ranjestad: n. $1253 \mathrm{~B} .988$, $153 \mathrm{H}$, $154 \mathrm{H} ., 239 \mathrm{G}$., $240 \mathrm{G} ., 247 \mathrm{G}$.

Saba: Lowerpirt of the Bottom Mountain 25) M.: 11. 1417 B.; Peperpot: 11. 34, s7; Bottom: n. 333 ; locality unknown: n. $112 \mathrm{~L}$.

St. Martin. (Symb.).

Anguilla Boldingh.

St. Croix ex Millspaugh.

Baham., Antill., Amer. cont, trop. (Symb.). Cultivated and uscaped.

\section{Boerhaavea Linn.}

Boerhavea erecta Linn. Spec. (175i) 3; (iris. fll, (39; Duss 60); Millspaugh 486 .

St. Eustatius: Uranjestaul: 11. 25, 215,27 ; lucality unkiluwn: 11. 601 B., 86 G., $223 \mathrm{H}, 228 \mathrm{G}$.

St. Martin: from Filipslurg to (iuamabay: 11. Jf(1.) B.

St. Croix ex Millspaugh.

Bermud., Baham., Antill., Amer. cont. (Symb.).

Boerhaavea hirsuta Willd. Phytogr. (17!4) 1; (iris. Fl. 6!); Duso 60. Potta potta, Hog meat.

Boerhavic repens Linn. Spec. (1753) 3.

St. Eustatius: Suashore near Orinjestiul: 11. 411 13.; in a garden in (1)anjestad: 11. 85) B.; ('ultumrvlakte: 11. 20, 21 ; locality unknown: 11. $87 \mathrm{G} ., 175 \mathrm{H}$.

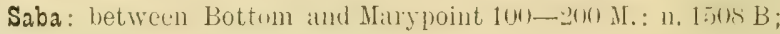
Laddergut 200 M.: 11. 1995 B., 22.

St. Martin; along roads from Grande Ciase to Narigot: 11.3098 b.

St. Croix (Symb.).

Antill., Amer, sept, and centr. (Symb.).

Boerhaavea paniculata L. ('l. Lich. in Act. Ifist. Natt. l'al. I (1792) 105; Gris. Fl. 69; Duss 60; Millspaugh 487.

St. Eustatius: in a garden in (ranjestid: 11. 524 B.; Bengalen: n. 632 B.; along roads near Glassbottle: n. 872 B.; Fairplay: n. 61; Oranjestad: n. 3; locality unknown: n. $1011 \mathrm{~B} ., 1019 \mathrm{~B}$, $62,6+$.

Saba: along roarls between Buttom and Windwardsile $200 \mathrm{M}$.: 11. 1:347 B.; along roakls 200 M.: n. 1384 B.; between Bottom and 


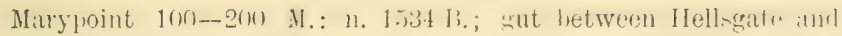
Masticgut 300-400 M.: n. $2056 \mathrm{~B}$.; locality unknown: n. $31 \mathrm{~L}$.

St. Martin: Fort Willem 0-50 M.: n. 2537 B.; Fort Amsterdam: 17. 39; road to Guanabay: 11. 4; locality unknown: n. 2305 B.

St. Croiz ex Milispaugh.

Baham., Antill., Amer. centr. and Austral. (Symb.).

Boerhaavea scandens Linn. Apece (175:3) :3; Gris. Fi. (is?.

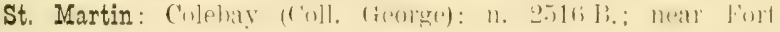
Amsterdam: n. 10 .

Anguilla Boldingh.

Baham., Antill., Curaçao, Amer. cont, from Arizona to Pertu. (Syml.).

\section{Pisonia Linn.}

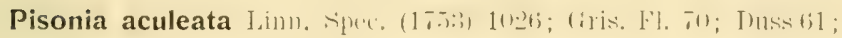
Millspaugh 48\%. Cockspur.

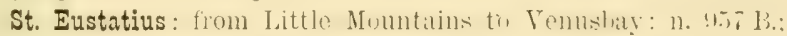
along roats near Oranjestad: n, 1002 B.; slone of the hills near Concordiabay: n. 89 ; Venusbay: n. 35.

Saba: lower part of the Bottom Mountain 300 M.: n. 1425 B.; Boobyhill: n. 1610 a B.; Rendez-vous: 1. 90; Quarter 300 M.: 11. 36 : locality unknown: n. $148 \mathrm{~L}$.

I also saw Pisonia aculeata: Springlaygut; Flat Point.

St. Martin: Princequarter (Coll George): 17. 2467 B.; from

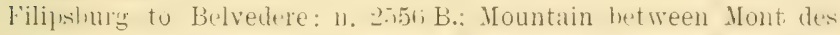

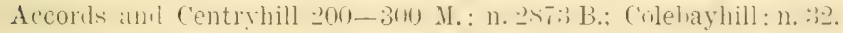

I also saw Pisonia aculeata in: Lower part of Milldrumhill; Mount P'aradis: along roads near Nakedhoyhill.

St. Croix ex Millspaugh.

Florida austral., Baham., Antill., trop. contr. of both hemisph. (excl. Afr. cont.) (Symb.).

Pisonia fragrans Dumont Cours. But. Cult. II ed. vol. Til (1814) 114.

Pisonia obtusata Sw. Flor. III (1806) 1960 (non Jaç. 1798); Gris. F'l. 71 (p. p.) (Symb.); Duss 62.

Pisonia inermis Gris. Flor. W. I. (1859) 7. (p. p., non Jacq.) (Symb.); Millspaugh 487. Black Loblolly tree.

St. Eustatius: along the foul from wranjestal to White Wall: n. \pm B.; top of the Quill 400 M.: 11. 337 B.; 450 M.: n. 357 B., 
362 13, ; immer slope of the Quill 350 M.: 4.5 B; tols of the (puill 500 M.: n. 478 B., 489 B.; slope of the Quill near Bengalen: n.

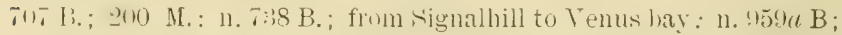
near Boven 200 M.: n. 1236 . B.; Cultuurvlakte-Pango: n. 9; Cultuurvlakte: n. 20; locality unknown: n. 180 G., 17.

Saba: Marypoint: n. 37.

St. Martin: Marigothill-Marigot 0-200 M.: n. 2ntit9 B.; Nalkerboyhill $50-200$ M.: 11. - -7) B. ; luwer part of the hills betwen

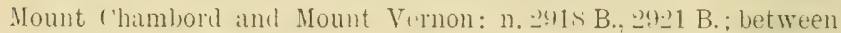
Mount Ternon and ()rienthay: n. 2927 B.; along rouls from Grande Case to Mrarigot: 11 . :3091 B.; Millelumlill :3(t)-400 M.: n. :31S63 B.; Mount Paralis zon-:300 M.: 11. :3:2 B.; gut from Bethlehem to Cul de Sac 100-200 M.: n. 341S B.; near Fort Amsterdam: n. 1; John Ednyhill: 1. 23.

I also saw Pisonia fragrans on the hill between Mont des Accords and Centryhill; near Saline.

St. Croix ex Millspaugh.

l'lorida austr., Antill., Amer, centr., Columbia, Guiama. (Sym7).).

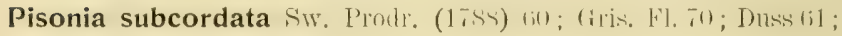
Millspaugh 487. M a p poo.

St. Eustatius: Feathore near (Mranjutad: 11. 25 13. : Tup of the Quill 400 M.: n. 185 B., 370 B.; in deserted plantations at the seashore near Bengalen: 11. $596 \mathrm{~B}$.; slope of the lower part of the Quill near Bengalen: n. 714 B.; locility unknown: n. 181 G., $322 \mathrm{H}$.

I also siw Pisonia sulıcordata: in Signalhill $200 \mathrm{M}$. ; top of the Clitt between Oranjestad and White Wall; gut near Fort de Wind; T'umbledown. dickbaty-Venusbay; Bergje.

Saba: Between Bottom and Marypoint 350 M.: n. 1498 B.

I also saw Pisonia subcordata: Forthbaygut; 'Tentgut; Sadlle; Cris. peenhill; Bottom-Windwaralsile; Gilesgnt; Springbaygut; Flatpoint.

St. Martin: from Filipsburg to Guanabay: 11. 2373 B. ; Prince quarter (Coll. George.): n. $2471 \mathrm{~B}$.

I also satw Pisonia subcordata: along roads near Bethlehem; along raads from Filiphurg to Nakenlhoshill: Marigothill-Matrgot; Nakedboyhill: Fort Willem; hills on the northem side of Cul de Sac; along roarls from Filipsburg to Simsonsbay : along roals near Grande Case; Yallée des Pères; lower part of Milldrumhill; Mount Paradis $300-400$ M.; Lowlands.

Anguilla Boldingh. 
St. Croix ex Millspaugh.

Portorico, St. Thomas, St. Barthélemy, St. Kitts, Antigna, Guadeloupe, Désirade, Martinique. (Symb.).

\section{Phytolaccaceae.}

\section{Rivina Linn.}

Rivina humilis Limu. Spec. (175) 121; Millspaugh 486.

Rivina laexis Limn. Nant. I (1767) 41; Gris, W. 59; Duss 49. Ju m b y pe p per.

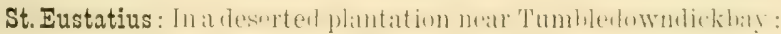
n. $170 \mathrm{~B}$; Bengalen: n. 611 B., $636 \mathrm{~B}$; slope of the Quill near Bengillen 50-200 M.: N. 646 B.; along roads in Oranjestid : 11. 939 T3.: Village: 11. 176; locality unknown: n. $161 \mathrm{G} ., 211 \mathrm{H}$.

Saba: along roads between Bottom and Windwardside 200 M.: 11. 1339 B.; along roads 200-300 M.: n. 1713 B.; Laddergnt 200 M.: n. 1998 B.; Peperpot: n. 173; Bottom: n. 174, 175; locality unknown: 11. $32 \mathrm{~L}$.

St. Martin: Mountain between Mont des lecords and cintry. hill 200-300 M.: N. 2904 B.; along roads in Cul de Sac: n. 2997 B.

I also saw Rivina humilis in Mount Paradis 100-200 M.; gut from Bethlehem to Cul te Sac.

St. Croix ex Millspaugh.

Florida austr., Baham., Antill., Amer, cont. trop. et subtrop. (Symb.).

\section{Villamilla R. et $P$. \\ ef. 2:370. Rivina Linn. Sect, 2 .}

Villamilla octandra Ilmk í, in Penth. et IIouk. (irn. Plant III (1880) $\$ 1$; Millspaugh $4 \$ 6$.

Rivina octandra Linn. Cent. plant. II (1756) 9; Gris. I']. 59; Duss 50. White hoop.

St. Eustatius: leserted blantations near 'Tumbledowmlickbay: n. $169 \mathrm{~B}$; slope of the hills near Concordiabay: n. 191, 192, 194; lied ('lift-l'umberdowndickbay: 11. 19:3: locality unknown: n, 32:: 11.

Saba: T'op of the Cliff near the seaside of Boobyhill : n. 1610 B.: Hellsgate: n. 1698 B.; gut hetween Hellsgate and Masticgut 300-400 M.: n. 20.33 B.; Crispeen Rendez-vous: n. 127; Hellsgate n. $177,178,179$; locality unknown: n, 236 L.

St. Martin: Cul de Sac along roads: n. 2966 B.; Centryhill: n. 126; French frontier: n. 190.

St. Croiz ex Millspaugh.

Antill., Amer. cont, trop. (Symb.). 
2371. Petiveria Linn.

Petiveria alliacea Limm. Snec. (17.):3) :34; (iris. Fl. 5!) Duss 50; Millspaugh 486 .

St. Eustatius: ncitr 'tumbledowndickbay: n. 1:3!n B.; locality unknown: n. $238 \mathrm{H}, 260 \mathrm{G}$.

Saba: Windwardside: n. 1591 B; Laddergut: n. 2001 B; Peperpot: n. 152, 153; Rendez-vous 400 M.: 1. 151; Bottom: 11. 150; locality unknown: n. $72 \mathrm{~L} ., 328 \mathrm{~L}$.

St. Martin: Marigothill: n. 2615 B.; gut from Bethlehem to Cul de Sac: 11. 3422 B.

St. Croix ex Millspaugh.

Florida austr., Antill., Amer. cont. trop. (Symb.).

\section{Microtea Sw.}

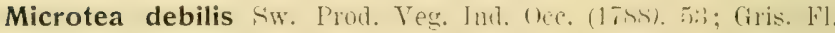
59; Duss 4!); Millspaugh 486.

St. Eustatius: in a garlen in Mranjestad: 11. 59 B.; along mads in Oranjestad: 11. $569 \mathrm{~B}, 91086 \mathrm{~B}$; Oranjestad: 11, 195; locality unknown: n. $48 \mathrm{G}, 22$.

St. Martin: From Filipsburg to Belvedere: n. 2585 B.

St. Croix ex Millspaugh.

Antill., Panama to Peru, Bras. equat. and Guiana. (Gris. Fil.). 2380. Phytolacca Jimn.

Phytolacca icosandra Linn. Sist. X. exl. If (17.)!h 1010; (iris. F1. 58: Duss 49.

Saba: Mountain 800 M.: n. 2214 B.; locality unknown: 1. 169 J., 175 L., $209 \mathrm{~L}$.

St. Martin: Mount Paradis $300-400$ M.: n. 3211 B.

Biham., Antill., Amer, cont. trop. (Symb.).

\section{Aizoaceae.}

\section{Mollugo Linn.}

Mollugo verticillata Limn. Spece. (175:3) s!n; (iris. FI. p. 5i.

St. Eustatius: along roads in Oranjestird: n. 1098 B.; slope of the Quill near Bengalen: n. 19ri.

Antill., Amer, cont. (Symb.).

2394. Sesuvium Linn.

Sesuvium portulacastrum Linn. Sịst. X el. II (17.5(1) 10.s; (iris. Fl. 57 ; Duss 47 ; Millspaugh 487. 
St. Eustatius: Seashore ntar Bengalen: 11. 55, 13.; neal White Wall: n. 851 B., 855 B.; near Maryglory 150 M.: n. 1162 B.; seashore near Turtlebay: 11. 93, 94.

Saba: near the Sulphurmines: n. $1703 a$ B.; seashore noar Lasldergut: B. 2132 B.

St. Martin: near Filipsburg: n, 2386 a B, near Mulletpond: n. 2763 a B. ; Tintamarre: n. 3389 B.

St. Croix ex Millspaugh.

Bermud., Baham., Antill., Curaça, trop. and subtr. countries of both hemisph. (Symb.).

\section{Trianthema Linn.}

Trianthema portulacastrum Linn. Spec. (175:3) 2:3:).

Trianthema monogymum Linn. Mant. I (1767) 69; Gris. F'. 58; Duss 48; Millspaugh 487.

St. Eustatius : in a garden in (ramjestarl : n. 111.58 B.. 10t, (1) (i.

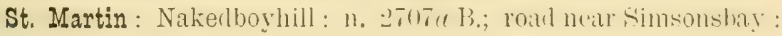
n. $3247 a$ B.; Colebayhill: n. 108.

St. Croix ex Millspaugh.

Key, Antill, Curaçao, Amer, cont, trop., Afr., and Aslit trop. (Symb.).

\section{Portulacaceae.}

\section{Talinum Adans.}

Talinum paniculatum Giirtn. Fruet. II (1791) 219 t. 128.

Talinum patens Willd. Spec. II (1800) 863 (var «) (Symb.); Gris. Fl. 57 ; Duss 46; Millspaugh 487.

Saba: Laddergut 0-200 M.: 11, 1952 B.; Peperpot: n. 40; locality unknown: n, $124 \mathrm{~L}$.

St. Croix ex Millspaugh.

Antill., Amer, cont. trop. (Symb.).

Talinum triangulare Wille. Sper. II (1800) 862; Ciris. Fl. in; Duss 46 ; Millspaugh 487.

St. Eustatius: Bengalen: 11. 1i12 B. ; along roads in Oranjestiul: 1. 1132 B.; near Boven 200 M. : n. 1241 B.; locality unknown: n. $63 \mathrm{G}$.

Saba: near the Sulphurmines: n. 1701 B.; Mountain 400 M. : n. 1768 B.; Bottom: n. 2232 B. ; locality unknown: n. 256 L.

St. Martin: from lilipsburg to Belvedere: 11. 2.jtig B.; from Belvedere te Oysterpond: n. 3044 B.; Prince quarter: 11. 42; Cul do Sac: n. 41; locality unknown: n. 3613 B. 
St. Croix ex Millspaugh.

Antill., Amer, austr. (Syml).).

\section{Portulaca Linn.}

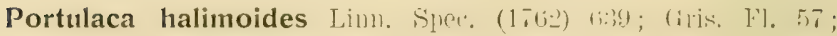
Duss 47; Millspaugh 488. Silk cotton purslane.

St. Eustatius: in a garden in Oranjestad: n. $86 \mathrm{~B}$; along ruals in (ramjestad: n. its B, 10.51) B.; reashore neal White Wall: n. 853 B.; old Fort: 11. 67; White Wall: 1. 66; locality unknown: n. $389 \mathrm{H}$.

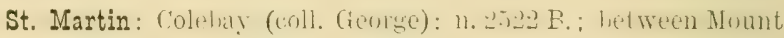
Vernon and Orientlay: n. 2943 B.; Green Key: 11. 3362 B.; Fort Amsterdam: n. 57; Guamabay: 11. 58.

Anguilla Boldingh.

St. Croix ex Millspaugh.

Antill., Mexico. (Symb.).

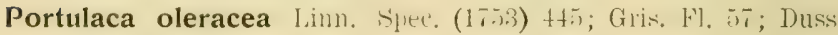
46 ; Millspaugh 487.

St. Eustatius: Seashore near (1)anjestar: n. 41 B.; in a garden in Oranjestad: n. 88 B., 521 B ; Bengalen: n. 635 B.; slope of the lower part of the cuill near Bengalen: n, i2n B.; seathore near Venuslay: n. 966 B.; near Boven: n. 200 M.; seashore: 11.72,73; locality unknown: n. $28 \mathrm{G} ., 29 \mathrm{G}$.

Saba: along roals between Buttom and Winlwardside $200 \mathrm{MI}$ : 11. $13: 9$ B.; Windwardside-I [ellsgate: n. $166+$ B. ; near l'lat Point: n. $21: 30$ B.; Windwardsian: n. 2:320 B.; locality unknown: 11. 80 L.

St. Martin: from Filipsburg to (illumal ar: n. $2+13 \mathrm{~B}$; along roads near Prince quarter: 11. 2912 B.; between Mount Vernon and ()ientbay: 11. 2y 40 B.; from Belvedere to Oysterpmd: 11. 3052 R.; Tintamarre: n. 3387 B.; locality unknown: n. 68. loyhill.

I also saw Portulaca olericea : in pastureground near Belvedere; Naked.

Anguilla Boldingh.

St. Croix ex Millspangh.

Bermud., Baham., Antill., trop, and warmor countries of both hemisph. (Symb.).

Portulaca pilosa Limm, Spee. (1753) t45; (iris, IV. $\pi 7$; Duss 47 ; Millspaugh 487.

Saba: along roads 200 M.: n. 1383 B.; Bottom : 11. 2264 B., $2299 \mathrm{~B}$. 
St. Martin: 1 also saw Portulaca pilosa: along roads near Bethlehem.

St. Croiz ex Millspaugh.

Llorida, Antill, Mexico. (Symb.). Also cultivated.

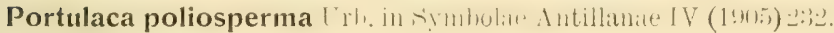

St. Martin: Lowlands: n. $3286 a \mathrm{I}$.

Portorico. (Symb.).

Portulaca quadrifida Limn. Nint. I (1767) 73.

St. Eustatius: along roads in Oranjestad: 11. 559 B.; locality unknown: n. 1022 B.

St. Martin: along roads from Bethlehem to Mont Chamlord: n. 2917 B.; lower part of the hills between Mont Chambord and Jount Vernon: n. 2917 B.; Marigotroad: n. 79.

'l'rop. countries of the Old world. (Jnd. Kew.).

\section{Basellaceae.}

2427. Boussingaultia H. B, K.

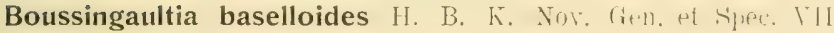
(1825) 196 t. 645 .

Cultivated. [Amer, trop.] (E. \& P.).

\section{Caryophyllaceae.}

2452. Drymaria Willd.

Drymaria cordata Willd. all. Li. ot Sich. Sist. I (lil!1) flit; ; Gris. F1. 56; Duss 45; Millspaugh 468. White snow.

Saba: along roads between Bottom and Windwarlside $200 \mathrm{Mr}$ : n. 1287 B.; Windwardside-Hellsgate: n. 1670 B, ; Windwardside: n. 2306 B.; Botton: n. 200; Peperpot: n. 198; Crispeen-Rendezvous 400 M.: n. 199.

I also saw Drymaria cordata: Laddergut.

St. Croix ex Millspaugh.

Antill., Amer, cont. trop., Afr. and Asia trop. (Symb.).

\section{Nymphaeaceae.}

2513. Nymphaea Linn.

Nymphaea spec.

Cultivated. 


\section{Menispermaceae. \\ 2574. Cissampelos Linn.}

Cissampelos Pareira limn. Fine. (17.;.) 10.31: liris. Fl, 10; Duss 7; Millspaugh 488.

St. Eustatius: along roaus in Oranjestad: 11. 57t B.; slope of the Quill near Bengalen 100 M.: n. ti67 B.; slope of the lower jart of the Quill near Bengalen: n. i10 B.; seashore near White Wall: n. s4S B.; slope of the Quill: 11. 55, 56; locality unknown: n. $218 \mathrm{H}$.

Saba: near the Sulphurmines: n. $1702 \mathrm{~B}$.

St. Martin: Mountain hetween Mont des Accords and Centry. hill $200-3001$ M.: n. 2455 B.; Mount Paradis 150 - 400 M. : n. 3191 B.; locality unknown: n. 3620 B.

St. Croix ex Millspaugh.

Antill., Amer. cont. trop., Afr., Asia trop. (Symb.).

\section{Hyperbaena Miers.}

Hyperbaena domingensis Benth. in .Turn. Linn. Sne. T. Suppl. II (1861) 50.

Cocculus domingensis DC. Syst. I (1818) 528: Gris. Fl. 10; Duss 7 .

Pachygone domingensis Eichl. in Flora XLVII (1864) 394.

St. Eustatius: Bottom of the Quill 300 M.: n. 421 B., sit B., 896 B.; top of the Quill 500 M.: n. 486 B.; slope of the lower part of the Quill near Bengalen: n. 708 B.; seashore: 11. 57; Quill; n. 58, 73.

Saba: Springbaygut: n. 2092 B.

Cuba, Jamaica, Hispaniola, Portorico, St. Thomas, Guadcloupe, Dominica, Martinique. (Symb.).

\section{Anonaceae.}

2729. Anona Linn.

Anona montana Macf. Flor. Jam. I (18:3i) 7 ; Gris Fl. 4.

St. Eustatius: nea Bengalen: n. 1188 B., locality unknown: n. $274 \mathrm{H}$. ; slope of Signalhill: n. 21.

Saba: along the Forthgut n. 1638 B.; Ladder: n. 20. Antill., Guiana. (Symb.).

Anona muricata Limn. Spec. (1753) J336; Gris. Fl. 4; Duss 2; Millspaugh 488. So u $\mathrm{r}$ s a $\mathrm{p}$. 


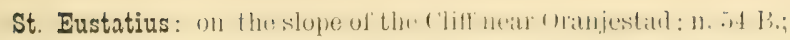
itlong the lower part of Signalhill: 11. $121 \mathrm{~B}$; lower part of the slope of the Quill near Bengalen: 11. $745 \mathrm{~B}$; along roads in Oranjestad: n. $1279 \mathrm{~B}$; locality unknown: n. $89 \mathrm{H} ., 173 \mathrm{G}$.

I also saw Anona muricata on the slope of the Quill on the side of Wliite Will.

- Saba: lower part of the Bottom Mountain 300 M.: n. 1438 B.; Bottom: 11. 1st+ B.; Springbaygut: 11. 20\%5 B.; Marypoint: 12. 22; locality unknown: n. $3 \mathrm{~L}$. Gilesgut.

I also saw Anoni muicata: between Bottom and Marypoint; Fortbaygut;

St. Martin: Prince quarter (coll. George): n. 2463 B.; from Filipsburg to Belvedere: 11. 259:3 B.: Hountain hetween Mont des Accords and Centryhill 200-300 M.: n. 2880 B.; lower part of Nount Paradis: n. $335+\mathrm{B}$.

I also saw Anona muricata: Marigothill-Marigot; Nakedlurylill; hill on the northern side of $\mathrm{Cul}$ de Sac; from Belvedere to Oysterpond; one of the islands in Simsonsbaylagoon; Top of Mount Paradis 400 M.

St. Croix ex Millspaugh.

Antill.; cult. in Amer. trop. Asia trop. and Afr. occ. (Symb.). Also cultivated.

Anona reticulata Linn. Spree. (175i) 7.37; Gris. lil. 5; Duss 4; Millspaugh 488. Custard apple.

St. Eustatius: Slope of the Quill 350 M.: n. 1219 B.

Saba: Boobyhill 300 M.: n. $1632 a$ B.; Bottom: 11. 1812 B.

I also saw Anona reticulata: Laddergut.

St. Croix ex Millspaugh.

Antill.; cult. in Amer., Afr. and Asia trop. (Symb.).

Also cultivated.

Anona squamosa Liun. Syee. (1753) 737; (tris. Fl. 5) Duss 3; Millspaugh 488.

St. Eustatius: in il garden in Oranjestal: 11. 525 B.; in deselted plantations at the seashore near Bengalen: $11.595 \mathrm{~B}$; locality unknown: n. $88 \mathrm{II}$, $91 \mathrm{G}$.

I also saw Anona squamosa in Signalhill.

Saba: Bottom: n. 1848 B., 2256 B.

I also saw Anona squamosa: Laddergut, Forthbaygut. 
St. Martin: from Filipsburg to Guanabay: 11. 2347 B.; Mountain between Mont des Accords and Centryhill: 13. 2869 B.; from Belvedere to Oysterpond: 11. $30 \pm 9$ B.; Mount Paradis 200-300 M.: n. $3309 \mathrm{~B}$.

I also saw Anona squamosa: along roads from Filipsburg to Nakedboyhill; Margothill-Marigot; near Bellevue; lower part of Milldrumhill; Vallée des Pères; one of the islands in Simsonsbaylagoon.

St. Croix ex Millspaugh.

Antill, ; cult. in Amer. cont. trop., Afr., Asia trop. (Symb.).

Also cultivated.

\section{Lauraceae.}

2783. Persea Gïrtn.

Persea americana Mill. Gard. Dict. VIII ed. (1768).

Persea gradissima Gärtn. f. Fruct. III (1807) 222 tab. 221; Gris. Fl. 280. Duss 298.

Persec Persea Cock. in Bull. 'Lorr. Bot. Club. XIX (1892) 95; Millspaugh 489. Pear tree, Avocado.

St. Eustatius: in deserted Hantitions near Tunbledorndickbay: n. 141 B., 146 B.; top of the Quill 400 M.: n. 223 B.; slope of the Quill near Bengalen 50-200 M.: n. 652 B.; in a garden near Glassbottle: n. 1210 B. ; locality unknown: n. 112 H., 146 G.

Saba: Windwardside: 11. 1924 B.; Mountain near Windwardside 600 M.: n. 2153 B.; locality unknown: n. 1565 B., 149 L.

I also saw Persea americama in Laddergut.

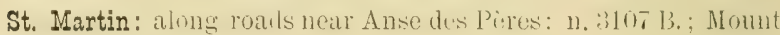
Paradis $150-400$ M.: n. $3193 \mathrm{~B}$.

Anguilla Boldingh.

St. Croix ex Millspaugh.

Baham. Antill., trop. countries of both hemisph., Ind. in Mexico. (Symb.). Also cultivated.

\section{Phoebe Nees.}

Phoebe elongata Nees. Syst. (1836) 116; Duss 298.

St. Eustatius: Quill: n. $256 a$ B., 410 B., 898 B.

Cuba, Hispaniola, Portorico, Guadeloupe, Martinique, St. Vincent, Grenada, Tobago, Trinidad. (Symls.).

\section{Nectandra Roland.}

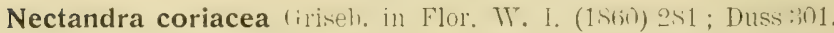
Nectandra Wildenoviana Nees. Syst.(1836) 321. Sweet wood. 
St. Eustatius: Top of the Quill 400 M.: 11. $221 \mathrm{H3}$, $256 \mathrm{~B}$. $272 \mathrm{~B}$., $288 \mathrm{~B}$., $289 \mathrm{~B}$; ; slone of the Quill 350 M.: 11. $410 \mathrm{~B}$.; slope of the Quill near Bengalen 50-200 M.: 1. 648 B., 680 B., $696 \mathrm{~B}$., $728 \mathrm{~B}, 751 \mathrm{~B}$; B Bottom of the Quill $300 \mathrm{MI}$ : n. $893 \mathrm{~B}$, $902 \mathrm{~B}$; lower part of the Hills near Concordia: n. 9si B.; Glassbottlo 150 M.: n. $111+$ B.; top of the (quill: 11. 248; Clitf: n. 243; slope of the Quill: n. 247; Quill: n, $168 \mathrm{G}, 116 \mathrm{H}$; locality unknown: 11. 244,245 .

Saba: Paris 2-400 M.: n. 242; Windwardside: n. 1947 B.

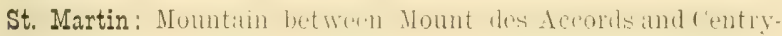
hill 200-300 M.: n. 2855 B., 2898a B.; Milldrumhill 300-400 M.: 11. 3125 B.; Mount Paradis 200-300 M.: n. 3306 B., 3320 B.; 10. callity unknown: 11. $3584 \mathrm{~B}$.

St. Croix. (Symb.).

Florida, Baham., Antill., Yucatan. (Symb.).

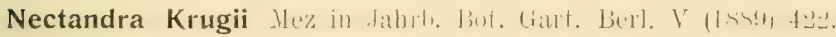
Nectandra discolor Gris. Flor. W. I. (1860) 282 non Necs. (Symb.). Black sweet wood.

St. Eustatius: Top of the Quill 500 M.: 11. 477 B., $487 \mathrm{~B}$.; Quill: $\mathrm{n}, 1,2,3, \pm, 16,19,20,22$; locality unknown: 11. 17, 18, $23,24,25,215,246$.

Saba: Boobyhill 300 M. : n. 1575 B.; Windwardside: n. 1946 B.; near Devilshand: n. 2074 B. ; near Flat Point: n. 2140 B.

Portorico, Guadeloupe, Dominica. (Symb.).

\section{Papaveraceae.}

\section{Argemone Linn.}

Argemone mexicana Limn. Alee. (17.5) 50S: (iris. Fl. 1:3; Lusin 8; Millspaugh 489. Th istle.

St. Eustatius: Seashore neal (1)injestarl: n. 4:3 B.; Cultumvlakte: n. 124 ; locality unknown: n. $20 \mathrm{G} ., 17 \mathrm{H}$.

Saba: Bottom: n. 2271 B.; locality unknown: n. 155 L.

St. Martin: I saw this plant: near Filipsburg and in pastureground near Bellevue.

Anguilla Boldingh.

St. Croix ex Millspaugh.

Bermud., Baham., Antill., Amer. cont. trop, and from there in trop. countries of the Old world. (Symb.). 


\section{Cruciferae.}

\section{Lepidium Linn.}

Lepidium virginicum Linn. Speec. (175.3) 645; (tris. Fl. 14; Duss 9; Millspaugh 489.

St. Eustatius: along ruads near (ilassbottle: n. S5:) B.; slope of the Quill: n. 340.

Saba: along roals letween Bottom and Windwardside 200 M.: 1). 1292 B., $136:$ B.: Mountain near Windwardside 600 M.: n. 2164 B.: Ladder: n. 342.; Peperpot: n. 341; locality unknown: n. 123 L.

St. Martin: Mountain between Mont des Accords and Centryhill 200-300 M.: 11. 2901 B.; hill on the northern sirle of Cul do Sac 0-100 M.: 11. 3004 B.; Milldrumhill :200-300 M.: 11. 3121 B.

Anguilla Boldingh.

St. Croix ex Millspaugh.

Bermud., Baham., Antill., Amer, seft. from Citnatial to 'l'exas. (Symb.).

\section{Cakile Juss.}

Cakile lanceolata (). E. Schulz. in UTrb. Symb. Antill. III (1903) 504.

Cakile aequalis L'Hérit ap. DC. Syst. II (1821) 430: (4ris. Fl. 14; (Symb.); Duss 10; Millspaugh 489.

St. Eustatius: Turtlebay: n. 328.

Saba: between Hellsgate and Marypoint: 13, 2044 B.

St. Croix ex Millspaugh.

Bermud., Baham., Antill., Columbia. (Symb.).

\section{Brassica Linn.}

Brassica integrifolia O. E. Sehulz in ('1\}. symb), Antill. III (1903) 509.

Sinapis brassirata Gris. Flur. W. I. (1859) 14; Millsjatugh 489.

Sinapis juncea Eichl. in Mart. FJ. Bras. XIII. 1. (1865) 306. (non L.). (Symb.); Duss 10. Mustard.

St. Eustatius: locality unknown: n, $141 \mathrm{H}, 292 \mathrm{G}$.

Saba: Windwardside: n. 1884 B.; Rendez-vous 400 M.: n. 3๖u, 326; locality unknown: n. $26 \mathrm{~L}$.

St. Croiz ex Millspaugh.

Antill.; Indig. in Asia austr. and orient. (Symb.). 


\section{Capparidaceae.}

3087. Gynandropsis DC.

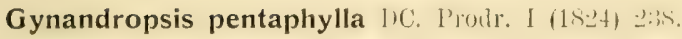

Cleome pentaphylla Linn. Spec. (1763) 938; Gris. Fl. 15; Duss 11; Millspaugh 489. Massamby.

St. Eustatius: along roads in Oranjestaul: $17.547 \mathrm{~B} .933 \mathrm{~B}$. Cultuurvlakte: n. 205, 185 ; locality unknown: n. 29 H., 33 G.

Saba: Bottom: n. $18+1$ B; locality unknown: n. $153 \mathrm{~L}$; Bottom: n. 186.

St. Martin: Colebayhill: n. 188; Prince quarter: n. 189.

I also saw Gynandropsis pentaphylle: Momnt Paradis 100-200 M.

Anguilla Boldingh.

St. Croix ex Millspaugh.

Baham., Antill., Amer, cont. calid.; Indig. in $\Lambda \mathrm{fr}$, aud Asia trop. (Symb.).

\section{Polanisia Raf.}

Polanisia viscosa DC. Prod. I. (1824) 249.

Cleome graveolens Rafin, in Journ. phys. (1819) 98.

Cleome viscosa Linn. Spec. (1758) 672; Gris. FJ. 16; Duss 12.

Polemisice icosandre Wight et Arm. Prod. (1834) 22 ; Millspaugh 490. Wild inassamby.

St. Eustatius: along ruals in Oranjestad: 11. .3.5 B.; locality unknown: n. $28 \mathrm{H} ., 32 \mathrm{G}$.

I also saw Polanisia viscosa along the seashore near Oranjestad.

Saba: lower part of the: Bottom Mnuntain 2.50 M.: n. 1:39:3 b.

St. Martin: from Filipshurg to (itanaluay: 11. Zto1 B; from Filipsburg to Belvedere: 11. 2558 B.; Colebayhill: 11. 190.

St. Croix ex Millspaugh.

Antill.; introrluced from Sonth. (hina and sonesamb. ((iris. Fl.).

\section{Capparis Linn.}

Capparis Breynia Jace. Sel, Amer. (Ititi) 1til t. 103; Duss 13. Capparis amygdalina Lam. Enc. I (1783) 608; Gris. Fl. 17.

St. Eustatius: fiut neall fort de Wind in M.: n. 8:32 B.: from little Mountain to Venusbay: 11. (19.) B.; along loads in (ranjestall: 11. 1068 B.: slope of the Quill on the side of White Wall 200 M.: n. 1178 B.; P'angohill: n. 16s'; Little Mountain-Boten: n. 166,167 .

I also saw Capparis Breynia on Signalhill, 
Saba: between Bottom and Marypoint: n, 1466 B.; Springbaygut: n. $2120 \mathrm{~B}$; Windwardside: n. $2319 \mathrm{~B}$.

St, Martin: Prince quarter (coll. George.): n. 2460 B., 2481 B.; Nakedboyhill 50-200 M.: n. 2695 B., $200-265$ M.: n. 2730 B., 2731 B.; Marigotroad near Marigot: 11. 2815 B.; Mountain between Mont des Accorls and Centryhill 200-300 M.: n. 2889 I3.; gut from Bethlehem to Cul de Sac: 11. 3433 B.: near Fort Amstêrdam: n. 143 ; behind Centryhill: n. 144 .

I also sam Capparis Breynia on Mount Paradis 100-200 M.

St. Croix. (Symb.).

Antill., Amer. centr., Nova Granati, Venezuela. (Symb.).

Capparis coccolobifolia Mith, ap. Eichl, in Mart. Fl. Wiks, N11J. 1. (1865) 284.

Capparis cmophallophore var. latifolia Griseb. Flor. W. I. (1859) 18 (p.p.) (Symb.).

St. Eustatius: in heserted plintations natr Tumbledummlickbay: $11.145 \mathrm{~B}$.

St. Martin: Prince quarter (coll. George): n. 2478 B.; Milldirumhill 300-400 M[.: n. 3147 B.; near Fort Amsterdam: n. 109: Centryhill: n. 129; Guanaluay: n. 108; locality unknown: n. 3612 B.

St. Croix. (Symb.).

Antill., Curaçao, Venezuela. (Symb.).

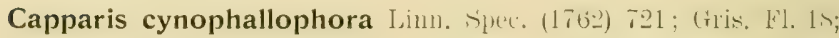
Duss 13; Millspaugh 490. Mustard tree, Man of war bush.

St. Eustatius: Slope of the lower part of signallill: $11.11 \mathrm{~s} \mathrm{~B}$; seashore near Orinjestad: n. 27 B.; slope of the Quill near Beligillen $200 \mathrm{MI}$ : n. $691 \mathrm{~B}$, 695 B., 73+ B.; along roads in Oranjestad: n. 925 B.: frum Little Mountain to Venusiay: 11. 9S5 B.; Bengalen: n. 749 B.; hear Naryglory 150 M.: n. 1145 B; Seinpost: n. 124; Red Cliff: n. 125 ; Boven: n. 127; locality unknown: n. $46 \mathrm{H}$, $191 \mathrm{G}$.

I also saw Capparis cynophallophora on Signallill; in at garden in Oranjestad.

Saba: along ruads letween Bottom and Windwardside 200 M. n. $1305 \mathrm{~B}$; ; along the Forthgut 150-200 M.: M. $1626 \mathrm{~B}$.; along roads 200-300 M.: n. 1714 B.; slope of the Mountain near St. John's: n. 1736 B.; St. Giles: n. 1859 B.; Bottom: n. 1882 B. ; gut letween H(ilsgate and Mastiegut $3(10-400)$ M.: n. 20.99 B.; Windwardside: n. 2310 B. ; Great Hill: n. 202; Quarter: n. 128.

I also saw Capparis cynophallophora in Springhaygut. 
St. Martin: Cul de Sac: 11. 2606 B.; ncal Mulletpond: 11. 277. B.; hills on the easterm side of Cul de Sac: 11 . $3436 \mathrm{~B}$.

I also saw Capparis eynophallophora: Cul de Sac: Marigothill-Marigot; Nakedboyhill 100-200 M. near Saline; hills on the nortlern sile of Cul de Sac; along roads from Filipsburg to Simsonsbay; from Belvedere to Oysterpond; Jower part of Mild rumhill; Mount Paralis 100-400 M.

Anguilla Boldingh.

St. Croix ex Millspaugh.

Florida austral., Antill., Panama, Amer, austr. (Symb.).

Capparis frondosa Jaley. Enum. (1760) zt; Grin. Fl. 1!); Dusi14; Millspaugh 490. Ch u r ch hlos s o m.

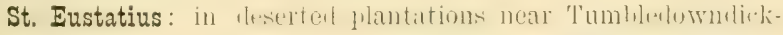
bay: n. 140 B.; Bottom of the Quill 300 MI.: n. 445 B.; slope of the Quill near Bengalen 50 M.: n. 655 B.; hills near Concordia: n. 967 B.; slope of Signalhill: n. 157; Cultuurvlakte: n. 158; neal Seinpost: 1. 159; Red Cliff: n. 160; Quill: n. 161, 162, 201; Waterrock: n. 162; locality unknown: n. 1S! G.

Saba: along roads between Bottom and Windwarclside 200 M.: n. 1334 B.; lowel part of the Bottom Nountain 300 M. : n. 1443 B.; Laddergut 200 M.: n. 2007 B.; Peperpot: n. 148, 163; Cresp.Rendez-vous: n. 161; Marypointgut: 11. 1 อั2.

I also saw Capparis frondo-a in Forthbaygut and Springbaygut.

St. Martin: from Filipsburg to Guanabay: n. 2383 B.; Princequarter (coll. George): n. 2461 B., 2482 B.; lower part of the liills letween Mont Chambord and Nount Vernon: n. 2961 B.; from Belvedere to Oysterpond: n. 3058 B.; Centryhill: 11. 145; John Ednyhill: n. 146.

I also saw Capparis frondusa along roads near Bethlehem; Mount I'aradia 100-200 II.; lowexpart of Milldrumhill; Nakedboyhill.

St. Croix ex Millspaugh.

Antill., Amer. centr., Nova Granata, Venezuela. (Symb.).

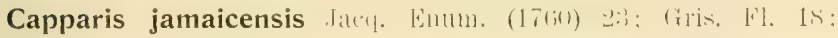
Duss 13; Millspaugh 490.

St. Eustatius: Slope of the quill above White Wall 354 M.: n. $3 \mathrm{~B}$; slope of the lower part of Signallilll: n. $105 \mathrm{~s}$; top of the Quill 400 M.: n. 305 B.; top of the Quill 500 MI.: n. 488 B., 494 B.; near Maryglory 150 M. : n. 1139 B.; neal White Wall: n. $1256 a$ B.; Boven: n. 133, 134, 135; Greatgut: n. 174. 
Saba: Boobyhill 300 M.: n. 1621 B.; St. Giles: 11. $1862 \mathrm{~B}$; Windwardside: 11. 15.; Wellidge: n. 179. 1s0; Bottum: n. 222; locality unknown: n. $48 \mathrm{~L}$.

St. Martin: from Filipsburg to fiuanabay: n. 2368 B., 2352 B., 2412 B., 2417 B.; Prince quarter (coll. George): n. 2246 B., -2476 B., 2480 B.; Furt Wiliem 0-51) MI.: 11. 2.589 B.; Nakerlhoyhill 50-200 M.: n. 2712 B.; near Mulletpond: n. 2771 B.; near Fort Willem: 11. 2797 B.; lower part of the hills between Mont Chambord and Mount Yernon: 11. 23m0 B., 296.) B.; between Belvedere an Oysterpond: 17. 3075 B., 307s B.: Milldrumhill $300-400$ M.: n. 3136 B. Contryhill: 1). 178, 170; Oysteruond: n. 177; Prince quarter: n. 171; John Ednyhill: n. 175; road to Guanabay: n. 176.

I also satw Capparis jamaicensis along roads from Filipsburg to Simsonsbaty, in the Lowlands and near Saline.

Anguilla Boldingh.

St. Croix ex Millspaugh.

Florida austral., Baham., Antill. (Syml).).

\section{0.t. Morisonia Linn.}

Morisonia americana Limn. Spec. (1753) .03. (1)is. Fl. 19; Duss 14; Millspaugh 490. Wild $\mathrm{misple.}$

St. Eustatius: slope of the lower part of signalhill: n. $114 \mathrm{~B}$; from Little Mountain to Venusbay: 11. 96.) B.; Little Mountain: n. 195; Boven: n. 218; locality unknown: n. 217.

Saba: letween Bottom and Marypoint 100-200 M. : 11.1556 B.; Cow pasture: n. 196.

St. Martin: Prince quarter (coll. George): 11. $2483 \mathrm{~B}$; from Filipshurg to Belvedere: 11. 2568 B.; Colohayhill: 11. $219,197$.

St. Croix ex Millspaugh.

Antill., Nova Granata. (Gris. Fl.).

\section{Moringaceae.}

\section{Moringa Juss.}

Moringa oleifera Lam. Enc. I (1783) 398.

Moringa pterygosperma Gärtn. Fruct. II (1791) 314 t. 147 f. 2 ; Gris. Fl. 16; Duss 12.

Muringa moringa Nillspl, in Field. Col. Mus. But. I (1902) 490 . Millspaugh 490. Moringo.

St. Eustatius: in a garden in Oranjestad: 11. 66 B., $227 \mathrm{G}$.

Saba: Bottom: n. 1878 B.

St. Martin: from Filipsburg to Belvedere: n. 2576 B.

I also saw Moringat uleifera along roads from Filipsburg to Nakedboyhill. 
St. Croiz ex Millspangh.

Baham., Antill., trop countries of both hemisph. (Symb.). Inclig. in India nrient. Also cultivated.

\section{Crassulaceae.}

\section{Bryophyllum Salisb.}

Bryophyllum pinnatum \&. Kur\% in , Tourt. As. Sim. Bumg. Xi. (1871) If 52; Millspaugh 490.

Bryopluyllum calycinum Salisb. Parad. Lond. (1805) t. 3; (tris. Fl. 303; Duss 319. Leaf of live.

St. Eustatius: along the lower part of Signalhill: 11. 1:me B. Cultuurvlakte: n. 335, 336, 287 H., $297 \mathrm{H}$.

Saba: Great Hill 400 M.: n. 1379 B.; top of the Cliff near the seaside of Boobyhill: n. 1607 B.; Peperpot: n. 387; locality unknown: n. 28 s I.

I also saw Bryophyllum pinnatum: gut between Hellsgate and Mits. tiegut; Crispeênhill; Windwardside; Laddergut; Springbaygut.

St. Martin: I did not collect the plant on this island, but I saw it:

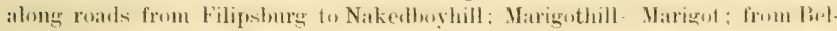
vedere to Oysterpond; Mount Patadis $400 \mathrm{M}$.

Anguilla Boldingh.

St. Croix ex Millspaugh.

Bermud., Baham., trop. and subtrop. countrius of hoth hemisuh. (Symb.).

\section{Rosaceae.}

3396. Prunus Linn.

Prunus myrtifolia Urh, in Symholat Antillanae V (1904) 9:3. Prumes sphaerocarpa Sw. Prodr. (1788) 80; Gris. Fl. 231.

St. Eustatius: Slope of the Quill 400 M.: n. $272 a$ B. Antill., Brasilia. (Symb.).

\section{Chrysobalanus Linn.}

Chrysobalanus icaco Limm. Spece (175.3) .,13: (iris. Fl. 20.9: I mes 257; Millspaugh 490. Cocoa plum, Fat pork.

St. Eustatius: Top of the Quill 400 M.: n. 839 B.; slope of the Quill 300 M.: n. 880 B.; slope of the Quill 150 M.: n. 232, 247; slope of the Quill: 11. 25), 25ti, 257, 258; Grandgut: 11. 258: locality unknown: 11. 254, $73 \mathrm{H} ., 155 \mathrm{H}, 224 \mathrm{G} ., 246 \mathrm{G}$. 
Saba: between Bottom and Marypoint 350 M.: n, 1491a B.; Mountain 600 M.: n. 1739 B.; Bottom: n. 2290 B.; Long peak: 11. 249 ; locality unknown: n. $18 \mathrm{~L}$., $335 \mathrm{~L}$.

St. Martin: Colehay (coll. George): n. 2514 B.; seashore near Simsonsbay: n. 3036 B.; Lowlands: n. 3273 B.

St. Croiz ex Millspangh.

Florida austral., Baham., Antill., Amer, austr., Afr. austr. (Symb.).

\section{Hirtella Linn.}

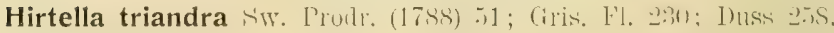
Hairy plum.

Saba: Mountain near Hellsgate 600 M.: n. 2277 B.

Antill., Amer: austr. (Symb.).

\section{Leguminosae.}

3436. Inga Willd.

Inga laurina Wille. Apec. IV. 2 (1S06) 1018; Gris. F'l. 6.27: Dust 255; Millspaugh 491. Red wood.

St. Eustatius: Top of the Quill 400 M.: n. 183 B., 205 B., $380 \mathrm{~B} ., 481 \mathrm{~B}, 483 \mathrm{~B}, 498 \mathrm{~B}, 963 a \mathrm{~B}, 367 \mathrm{~B} ., 395 \mathrm{~B}$; ; slope of the Quill near Bengalen: n. 726 B.; Kant: 11. 312, 313, 314; lncality unknown: 11. 51, 100.

Saba: lower part of the Bottom Mountain 250 M.: n. 1421 B.; Boobyhill 300 M.: n. 1578 B.; Windwardside: n. 1611 B., 2142 B.; Peperpot: $n .79,80$.

St. Martin: Mount Paradis: n. 3214 B., 3226 B., 3827 a B.

St. Croix ex Millspaugh.

Hispaniola, Portorico, St. 'Thomas, St. Jan, St. Kitts, Antigua, (inaleloupe, Dominica, Martinique, it. lueia, St. Yintent, Barbados, Grenada, Trinidad. (Symb.).

\section{Enterolobium Mart.}

Enterolobium Timbouwa Mart. Flora XX (1597) 1.55.

Cultivater. [Antill., Amer, austr.] (E. \& P.).

\section{Pithecolobium Mart.}

Pithecolobium unguis-cati Benth. in Hook. Loml. Tourn. But. III (1814) 200; Gris. Fl. 226; Duss 254; Millspaugh 491. Cra bwood. 
St. Eustatius: Slope of the lower part of Signalhill: 13. 106 B.; betweon Oranjestad and Fort de Wincl on the top of the Cliti 40 M. : n. 768 B.; Simnalhill 200 M.: n. 1035 B.; Cultumrlakte: 1). 1250; slope of the Quill: 1. 1369; Boven: n. 1363; Gilboohill: n. 1379; road to English (quarter: n. 1372; locality unknown: 11. 28111.

Saba: between Bottom and Marypoint 100-200 M.: 11. 15 41 B.; gut between Hellsgate and Nastigut 300-400 M.: n. 2054 B.; Hellsgategut: 1 . 1361, 1370; pasturo: n. 1368.

St. Martin: From Guanalay to Filipsburg: n. $239+$ B.; from Filipsburg to Belvedere: n. 2569 a B.; Nakedboyhill: n. 2681 B.; near Mulletpond: n. $275+$ B.; hill on the northern side of Cul de Sire 0-100 M.: 17. 2971 B.

I also saw Pithecolobium unguis.cati: Marigothill-Marigot; from 3el. vedere to Oysterpond; along roads near Grande Case; Lowlands: Mount I'aradis 300-400 M.; Tintamarre; gut from Bethlehem to Cul de Sac.

Anguilla Boldingh.

St. Croix ex Millspaugh.

Florida austr., Key Ins., Antill., Venezuela, Nova Gramata. (symb.).

\section{Albizzia Durazz.}

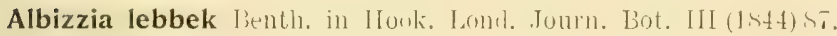
Cultivated. [Baham., Antill., Curacao, Asia austr. and orient. Afric. trop.] (Symb.).

\section{Acacia TVilld.}

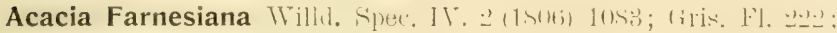
Duss 250; Millspaugh 491. Cas li a.

St. Eustatius: Red Cliff: n, 390.

Saba: between Bottom and Marypoint 100 -200 M.: n. 1460 B.; St. Giles: n. 1864 B.; Paris 2-400 M[.: 17. 372 ; Bottom: n. 414.

St. Martin: from Guanabay to Filipsburg: n. 2434 B.; Fort Willem 0-50 M.: n. 2545 B.; near Mulletpond: 11. 277s B.; Green Key: 11. 3365 B.; Filipsburg: n. 251; Fort Amsterdain: n. 386.

St. Croiz ex Millspaugh.

Baham., Antill., warmel countries of both hemisph. (Symb.). Acacia macrantha Humlı, et Tompl. E Willd. Siper. Plant. IV (1805) 10s0; Gris. Fl. 221; Duss 249. French casha, Creole casha, Spanish casha.

St. Eustatius: Seashore near Oranjestal:. 1]. 15 B., 3S B.; top 
of the Quill 400 M.: n. $216 a$ B., 318 B.: along roads in Oranjestad: 11. 543 B., 567 B.: Bengalen: 11. 630 B.: between Oranjestad and l'ort de Wind on the top of the Cliff $40 \mathrm{M}$. : n. Ts1 B.; near White Wall: 11. $858 \mathrm{~B}$; near Zeelandia: 11. 25:; slope of the hills neal Concordia: 13. 260; top of Pangohill: 11. 259 ; Seafeathersbay: 11. 268 ; locality unknown: n. $36 \mathrm{H}$., $39 \mathrm{H} ., 50 \mathrm{G}$., $30 \pm \mathrm{G}$.

St. Martin: l'rince yuarter (coll. George): 1]. 2454 B.; from Filipsluurg to Belvedere: n. 5.5. Oysterpond: n. 3076 B.; lower part of Mount Paradis: 17. 9.291 J3.; Colebayhill : n. 49.

Antill., Mexico, Venezucla, Equador, Peru, Buenos Ayres, Galapagos. (Gris. J'l.).

Acacia riparia H. B. K. Nov. Gen. Y I 11,23) 276: Duss 24.

Acacia samentosa Gris. Kar. (1857) 64; (non Desv.) (Symb.); Gris, Fl, 221.

St. Eustatius: near Bengalen: n. $600 a$ B.

St. Martin: Prince prartel (coll. (ieorge): n. -45.) B.: Nakiclboyhill 200-265 M.: n. 2732 B.; Mountain between Mont des Accords and Centryhill 2th-30n M.: n. 28622 B. Colehey-rentry hill: n. 7 is.

St. Croix ex Millspaugh.

Antill., Amer. austr: trop. (Symb.).

Acacia tortuosa Willu. Spec. Plant. IV (1505) 1083; (Gris. F'. 2.32; Millspaugh 491. D u t ch $\mathrm{cash}$.

St. Eustatius: in deserted plantations it the seashore nuar Bengalen: 11. 588 B., 590 B., 600 B.; between Oranjestad and Fort de Wind on the top of the Cliff 40 M.: n. 780 B.; along roads near English Quarter: 11. 1004 R.: Signalhill 150 M.: n. 10:4 B., 1025 B.; slope of the Quill on the sicle of White Wall 200 M.: n. 1175 B.; Cultuurvlakte: n. 383 , 395; seashore: n. $384 ;$ Red Cliff: 11. 378; locality unknown: n. 371 .

St. Croix ex Millspaugh.

Antill., Mexico, Venezuela, Equador, Galapagos. (Gris. Fl.).

\section{Leucaena Benth.}

Leucaena glauca Benth. in Houk. Journ. But. IV (18+2) 416; Gris. Fl. 220; Duss 247; Millspaugh 491. Mimosa, 'I'antan.

St. Eustatius: Seashore near Orinjestar: n. 2:3 B.; slopu of the lower part of the Quill near Bengalen: n. 719 13.; in a garden in ()ranjestal: n. 127+ B.: Cultuurlakte: n. $317,31 \mathrm{~s}$; locality unknown: $11.37 \mathrm{II}, 44 \mathrm{G}$. 
I also saw Lencacna glauca on the slope of the Cliff; Signallitl; deserted plantations near Bengalen.

Saba: Jaddergut: n. 318 ; locality unknown: n. 129 L.n 136 L.

St. Martin: from Filipsburg to Belvedere: n. $2590 \mathrm{~B}$.

I also saw Lencaena glauca: hill between Mont des Accords and Centryhill; near Saline; hill on the northern side of Cul de Sac; along roads near Gramele Case; lower part of Mildrumhill; Mount Paradis 300-400 $\mathrm{ML}$.; hills on the eastern side of Cul de Sac.

Anguilla Boldingh.

St. Croix ex Millspaugh.

Bermud., Baham., Antill., Curagao, "warmer countries of botll hemisph.; in Amer. trop. indig? (Symb.).

\section{Mimosa Linn.}

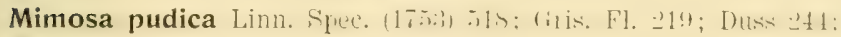
Millspaugh 492. Shame lady shame.

St. Eustatius: Slope of thr !nill on the sile of White Wall 200 H.: n. $118+$ B.; along roads near Bengalen: n. 1192 B.; locality mnknow: n. $216 \mathrm{H}$.

St. Croix ex Millspaugh.

Antill., Amer, cont, trop., Asia trop. (Symb.).

\section{Desmanthus Willt.}

Desmanthus depressus Huml, et Bompl. ex Willa, spec. Plant. IV (1805) 1016; Gris. Fl. 218; Duss $24 t$.

St. Eustatius: Top of the Quill t50 M.: 11. 347 B.; lower part of the slope of the Quill near Bengalen: n. 721 B.; Fort de TWind: 11. 797 a B.; along roads near English Quarter: n. 999 B.; Cultuurvlakte: n. 282 .

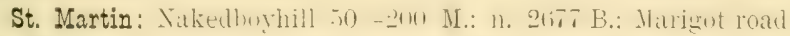
neal Marligut: 13. 2\12 B.; lowel nart of the Milldrumhill: n. :16; B.; T'intamarre: n. 8375 B., 3402 B.

Elorida, Antill., Texas to Peru, Galapagos, (Gris. Fl.).

Desmanthus virgatus Willu. spec. IV. 2 (1stuj) 1414: (iris. Fl. 218; Duss 24t; Millspaugh 492. IV ild tantan.

St. Eustatius: in a garden in Oranjestad: n. $71 \mathrm{~B}$; seashore near 'Tumbledowndickbay: 11. 161 B.; Bengalen: n. 603 B.; slope of the Quill near Bengalen 200 NL: n, $7 \pm 1$ B.; along roads in Oranje. stal: n. $1075 \mathrm{~B}$; near Boven 200 MI: n. 1244 B.; Cultuurvlakte: n. 24 ; locality unknown: n. $162 \mathrm{G} ., 302 \mathrm{G}$.

Saba: Boolyhill 300 MI.: n. 1614 B.; Laddergut 0-200 M.: 11. 1967 B., 2012 B.; Paris 200-400 M.: n. 99. 
St. Martin: from Filipsburg to Guanalsy: n. 2371 B.; Cul de Sac-Marigothill 0-200 M.: n. 2633 B.; between Belvedere and Oysterpond: n. 3050 B.; slope of Centryhill: n. 98; French frontier 11. 295 .

St. Croix ex Millspaugh.

(Bermud.), Baham., Antill, (Amer. cont. trop. and subtrop. Jndia-orient.). (Symb.).

\section{Neptunia Lour.}

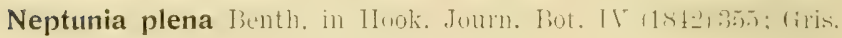
Fl. 218: Duss 243. W a terthistle.

St. Martin: near Marigot: n. $2627 a \mathrm{~B}$.

Antill., Curaça, Amer. austr., Asia trop. (introd.) (Symb.).

\section{Adenanthera Linn.}

Adenanthera pavonina Linn. Spec. (1753) 384.

Cultivated. [Asia trop.] (Symb.).

\section{Hymenaea Linn.}

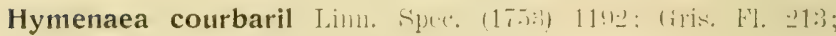
Duss. 238; Millspaugh 492. Locust.

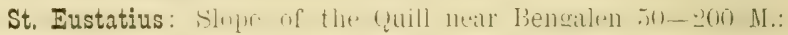
n. 649 B.; slope of the Quill 250 M.: n. Ss9 B.; slope of the Quill: n. 461, 2947; locality unknown: n. 75 H., 175 IT., $216 \mathrm{G}$.

Saba: Windwardside: 11. 2332 B.; locality unknown: 1]. 348 L.

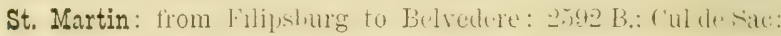
n. 2995 B.; Centryhill: n. 472 ; French frontier: 11.462.

I also saw Hymenaea courbaril on the hills on the northern sirle of Cul de Sac.

St. Croix ex Millspaugh.

Antill., Amer. cont. trop. (Symb.). Also cultivated.

\section{Tamarindus Linn.}

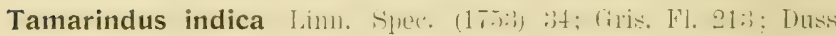
237; Millspaugh 492. T' a marind.

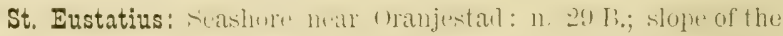
lower part of Signalhill: 1). 112 B.; in a garden in Oranjestad: $n$. 1065 B., $40 \mathrm{H}, 47 \mathrm{G}$.

Saba: between Bottom and Marypoint 100-200 M.: n. 1547 B.: Bottom: n. 1873 B., 1871 B.; locality unlinown: n. $117 \mathrm{~L}$. 
St. Martin: from Filipsburg to Guanalay: 11. 2350 B.; Prince. quarter (coll. George): n. $2468 \mathrm{~B}$.

I also saw T'amarindus indica: Marigothill-Marigot; Fort Willem; near Saline; along roads fron Filipsburg to Simsonstay; Villee des Peres; one of the islands in Simsonsbaylagoon.

Anguilla Boldingh.

St. Croix ex Millspaugh.

T'rop. countries of both hemisph.; Ind. veris. in Africa. trop. (symb.). Also cultivated.

\section{Bauhinia Limn.}

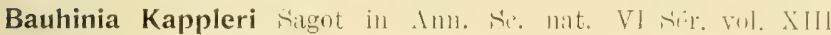
(1882) 317.

Cultivated. [Asia austro-orient.]. (Symb.).

\section{Cassia Limn.}

Cassia alata Linn. Spec. (1753) 378.

Cultivated. [Cosmop. trop.]. (Ind. Kew.).

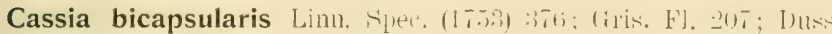
281 ; Millspaugh 492. B l y d o g.

St. Eustatius: Seathore near oranjestar: 11. 203 B., ,0 B.: slopk of the lower part of Signalhill: n. 113 a.; in leserted plantation. at the seatshore near Bengalen: 1r. in B.; betweren Oranjestad amel Fort te Wind on the top of the Cliff 40 M.: 17. 793 B.; gut nen Fort de France: n. 816 B.; along roads near Glassbottle: n, 1214 B.; locality unknown: n. 25 G.; Cultuurvlakte: n. 329; near Seinpost: n. 325.

Saba: Windwardside: n. 1891 B.; Peperpot: n. 330 ; Great. hill: n. 315; locality unknown: n. $28 \mathrm{~L}$.

I also saw Caksia hicapsularis: in Laddergnt.

St. Martin: from Filipsburg to Guanabay: 17. 2370 B.; lower part of the hills between Mont Chambord and Mount Vernon: n. 2955 B.; Cul do Sac: n. 320.; Guanabay: n. 313.

I also satr Cassia bicapsularis: along roads from Filipsburg to Naked.

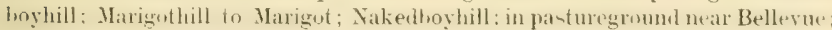
lower part of Milldrumhill; Mount Paradis 300-400 M.; one of the island. of Simsonsbaylagoon; Lowlands.

St. Croix ex Millspaugh.

Bermud., Baham., Antill., Curaçao., Amer. cont. trop., Inquil. in India Orient. and Madeira. (Symb.). 
Cassia fistula Linn. Spec. (1753) 377. Liquorice plant. Cultivated. [Asia trop.]. (Symb.).

Cassia glandulosa Limn. Sy,st. X ed. II (17t) 1017: (iris. Fl. 211; Duss 233.

Chamacrista glandulosa Greene in Pitton. IV (1899) 28; Millspaugh 493. Wild peas.

St. Eustatius: Top of the Quill 390 M.: 13. 499 B.; slope of the Quill near liengalen 100 M.: 17 . (660 B.: betwen Oranjestad and Fort de Wind on the top of the Cliff $40 \mathrm{Mr}$ : n. $787 \mathrm{~B}$.; near White Wall: n. 1262 B.; Cultuurvlakte: n. 740 ; Little Mountain: n. 1768; slope of the Quill: n. 992, 1763; locality unknown: n. 81 H., $99 \mathrm{G} ., 170 \mathrm{G}$.

Saba: along rouls between Bottom and Windwaldside 200 .I. n. $1350 \mathrm{~B}$; : between Windwardside and Hellsgate: n. $1680 \mathrm{~B}$; Windwardside: n. 232+ B. : l'elerpot: n. 17.0, 7.:- r'repeen-liendez-vous: n. 991.

St. Martin: Marigothill--Marigot $11-200$ M.: n. 2051 B.; (4ntryhill: n. 260; Marigotroad: n. 993.

Anguilla Boldingh.

St. Croix ex Millspaugh.

Antill., Amer. austr: (Symb.).

Cassia obovata Collat.; Gris. Fl. 209.

St. Martin: along the saltpond near Filipsburg: n. 2441 B.; Guanabay: n. 274.

Anguilla Boldingh.

From Africa trop. (Gris. Fl.).

Cassia occidentalis Linn., Spee. (175:3) :37.; (iris. Fl. 2017; Duss 235; Millspaugh 492. Bitterroot.

St. Eustatius: Seashore near White Wall: II. $85 ! \mathrm{B}$; along roads neal Glassbottle: 1). $86+\mathrm{B}$; alongr roals in Oranjestad: $n$. $562 a$ B., 1103 B.; Jocality unknown: n. $4 \mathrm{G} ., 4 \mathrm{H}$.

Saba: lower nart of the Bottom mountain 2.80 M.: 13. 1994 B.: Bottom: n. 2243 B.; Gainbygut: n. 1035; locality unknown: n. $162 \mathrm{~L} ., 237 \mathrm{~L}$.

St. Martin: along roads near Fort Willem: n. 2525 B.; Marigotroad neall Marigot: 11. 2\$19 B.; in pastureground near Bellevue: n. 2843 B.; Prince quarter: n. 1034; locality unknown: n. 2524 B.

I also saw Cassia occidentalis: Jowlands.

Anguilla Boldingh. 
St. Croix ex Millspaugh.

Bermud., Florida austr., Babam., Antill., Amer., Africa, Lsia trop. (Symb.).

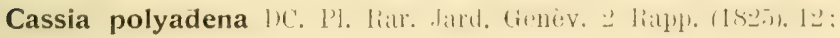
Cris. Fl. 210.

St. Martin: Lowlands: n, 3272 B., 3274 B., 3288 B.

Baham., Cuba. Dumingo, Jamaicil, St. Barthremy, fiuadeloupe. (Herb. Krug et Urban).

\section{5) 43. Krameria Loefl.}

Krameria ixina Linn. (1762) 117; Gris. Fl. 31; Wild senna.

St. Eustatius: Signalhill 200 M.: 17. $126 \mathrm{~T}$; ; between oranjestad and Fort de Wind on the top of the Cliff 40 M.: n. $763 \mathrm{~B}$; near Maryglory 150 M.: n. 1163 B.; near White Wall on the top of the Cliff: n. 1256 B.; Cultuurvlakte near Golden Rock: n. 181; slope of the hills near Fort Amsterdam: n. 164; Signalhill-lied Cliff: n. 163; locality unknown: n. $200 \mathrm{H}$.

Hispraniola, St. Thomas, Antigua, Curaçao, Columbia. (Symb.).

\section{Parkinsonia Linn.}

Parkinsonia aculeata Lim. Spee. (17.3:) :37.); (iris. l't. 2044; Duss 227; Millspaugh 493. W onder tree, Jeruzalem thorn.

St. Eustatius: along roads in Oranjestad: 11. 915 B., 63, 73, $150 \mathrm{H} ., 73 \mathrm{G}$.

St. Martin: near Filipsburg: 11. $2340 \mathrm{~B}$.

I also saw Parkinsonia aculeata on Mount Paradis 100-200 M.

St. Croix ex Millspaugh.

Key ins., Antill., Amer. cont. trop.; escaped in Afr. and Asia trop. (Symb). Also cultivated.

\section{Haematoxylon Linn.}

Haematoxylon campechianum Limn. Since. (1753) 384; (iris. 1\%). 204; Duss 226; Millspaugh 493. Log wood.

St. Eustatius: along a roid near Granjestat: 11. 1254 B: lncirlity unknown: n. $291 \mathrm{H}$.

St. Croix ex Millspaugh.

Baham., Antill., Amer. cent. et Austr. sept. (Symb.).

3556. Poinciana Linn.

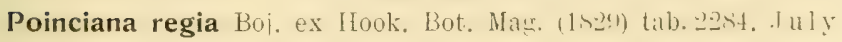
tree, Flamboyant.

Cultivated. [Madagascar.]. (Symb.). 


\section{Caesalpinia Linn.}

Caesalpinia ciliata L'b. in Symhlae Antillanwe VI (1!U0) 275. Guillandina Bonduc Gris. Kar. (1857) n. 466 p. 60 et quoad specim. Guad. Flor. p. 240; non Linn. (Symb.).

Guillandina Grisebachiana $\mathrm{Kr}$. et Urb. in Duss Flore. Ant. franc. (1897) 229.

St. Eustatius: Seashore near Oranjestad: 12. 21 B.; seashore neir Thumbledownlickbay: $11.156 \mathrm{~B}$; along roads in Oranjestad: n. 1090 B., $69 \mathrm{H}$.

Saba: 0. Kuntze (Symb.).

St. Martin: Colebay (coll. George): 11. $₫ 501$ B.; near Mulletpond: n. $2756 \mathrm{~B}$.

Barthelemy, Antigui, Guadeloupe, Desirade, Marie Galante, Martinique. (Symb.).

Caesalpinia coriaria Wilkl. Spec. Plant. II (1799) 538; Millspaugh 493.

Lebidibic coriario Schlecht. in Linnaca V (1830) 193; Gris. Fl. 231; Duss 231. Divi divi.

St. Eustatius: in a garden in Oranjestad: n, 64 B, $245 \mathrm{G}$.

Saba: locality unknown: n. $301 \mathrm{~L} . ;$ Windwardside: n. $2331 \mathrm{~B}$.

St. Martin: along roarls from Colebayhill to Simsonsbay: n. 3016 B., 3017 B.

St. Croiz ex Millspaugh.

Baham., Antill., Curaçao, Mexico, Venezuela, Nova Granata. (Symb.).

Caesalpinia Crista Limm. Splec. I ed. I (175:3) 380; Millspatugh 493. Guillandina glabra. Griseb. Flor. W. I. (1860) 205 (quoad. specim. Martin. a. Cl. Siagot. non Duchassaing missum.); Duss 22s; (Symb.).

St. Eustatius: Top of the Quill 400 M.: n. 306 B.; slopo of the Quill 50 M.: n. 635 ; locality unknown: n. $160 \mathrm{G}$.

Saba: lower part of the Bottom Mountain 250 M.: 11. 1409 B.; Bottom: n. 634 ; locality unknown: 1]. $354 \mathrm{~L}$.

St. Martin: near Mulletpond: n. $2788 \mathrm{~B}$.

St. Croix ex Millspaugh.

Bermuda., Key ins., Baham., Antill., Amer. cont. trop., Asia austro-orient., Nova Guinea, Australia. (Symb.).

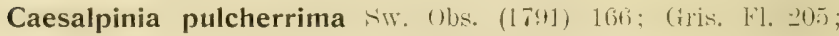
Duss 230; Millspaugh 493. Pride of Barbados.

St. Eustatius: in a garden in Oranjestad: n. $1088 \mathrm{~B} ., 67 \mathrm{H}$. $68 \mathrm{H} ., 74 \mathrm{G} ., 75 \mathrm{G}$. 
Saba: St. Giles: 11. $1871 \mathrm{~B} . ;$ locality unknown: $223 \mathrm{~L}$.

St. Martin: from kilipsburg to Belvedere: 11. $2598 \mathrm{~B}$.

I also saw Caesalpinia pulcherrima on Marigothill near Bellevue.

Anguilla Boldingh.

St. Croix ex Millspaugh.

Key ins., Baham., Antill., Amer. cont. trop., Afl. trop., Asia trop. Patria ignot. (Symb.). Also cultivated.

\section{Crotalaria Linn.}

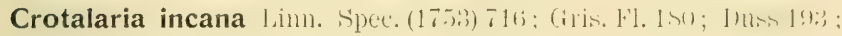
Millspaugh 49t. Sma 11 y e 110 w poplousl.

St. Eustatius: along roads: n. $256 \mathrm{H}:, 596 \alpha \mathrm{B}$.

Saba: Windwardside: n. 2144 B.

St. Martin: Mountain leetween Mont des Arourds and f'entry. hill 200-300 M.: n. 2872 B.; roads from Marigot to Filipsisurg: 12. 3110 B.; French frontier: 1]. 2294.

St. Croix ex Millspaugh.

Florida austral., Antill., warmer countries of both hemisph. (Synib.).

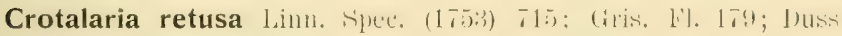
193; Millspaugh 494. Big y o $110 \mathrm{w}$ p o p) b us h.

St. Eustatius: 'Top of the Quill 400 M.: 1). 231 B.; White Wall: 11. 810 B.; along roads near Bengalen: n. 1187 B.; Fairplay: n. 625 ; Cultumblakte: 11. tis.); slope of the (quill: 11. 64.); along ruads: 11. $285 \mathrm{G} ., 286 \mathrm{H}$.

Saba: lower part of the Bottom Mountain 250 M.:n. 1387 B.; Mountain 600 M.: n. 1753 B.; Laddergut 0-200 M.: n. 1961 B.; locality unknown: 11. $66 \mathrm{~L}$.

St. Martin: from Guanabay to Filipsburg: 11. 2436 B.; Point Blanche (coll. George): 11. 2491 B.; from Filipslourg to Belvedere: 11. $2600 \mathrm{~B}$; in an orcharcl near Bellevue: n. $2848 \mathrm{~B}$.

St. Croix ex Millspaugh.

Baham., Antill., Amer. cont. trop., Afr. trop., Asia austroorient., Austral. (Symb.).

Crotalaria verrucosa Limn. Fiece (17.3) 71.): (ilis. li. 175: Duss 192; Millspaugh 494. Purple pop bus h.

St. Eustatius: along roads in Oranjestad: n. 923 B.; Fairplay : 11. 1634 ; slope of the Quill: n. 1614, 1624; locality unknown: n. $285 \mathrm{H}$.

St. Martin : along roals from Granule (ase to Mirignt: 11, :310s b. Antill.; from the East Indies. (Gris. Fl.). 


\section{B688. Medicago Linn.}

Medicago sativa Linn. Spec. (1753) 778.

Cultivated. [Europa]. (Ind. Kerv.).

\section{Indigofera Linn:}

Indigofera suffruticosa Mill. Game. Dict. VIII ext. (1768) 11. ㄹ.

Indigufore anil Linn. Mant. II (1761) 272; Gris. Fl. 181: Diss 134; Millspaugh 494. Wild indig o.

St. Eustatius: in a galden in Oranjestad: n. 73 B.: Bengilen: 11. (10) B.; along roads near Luglish Quarter: n. 1001 B.; Fairplay: n. 290; Cultuurvlakte: n. 221; slope of the Quill: n. 231, 241; locality unknown: n. $172 \mathrm{G} ., 157 \mathrm{H} ., 219 \mathrm{G}$.

Saba: between Bottom and Marypoint 100-200.: n, 1524 B.; along roads 200 -300 M.: n. 1709 B.; St. Giles: 11. 1867 B.; Hellswate-Marypoint: 11. 2047 B.; gut near Devilshand: 11. $2077 a$ B.; Ladder: n. 251; locality unknown: n. 87 L., 251 L.

I also saw Indigofera suffiruticosa on Crespeentrill.

St. Martin: along roads near Filipsburg: n. 2528 B.

I also saw Indigofera suffruticosa near Grande Case.

St. Croix ex Millspaugh.

Baham., Antill., Amer. cont. caliclior; inq. in the trop. Old world. (Symb.).

\section{Tephrosia P'ers.}

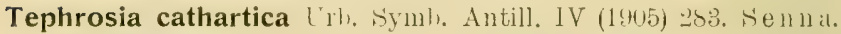

St. Eustatius: Seashore near English Quarter: 11. 1010 B.; English Quarter: 17. 14!\%, 150s; roal to English Quarter: 11. 1493; locality unknown: n. $158 \mathrm{G} ., 240 \mathrm{H}$.

Saba: Bottom: n. 2236 B.; locality unknorvn: n. $91 \mathrm{~L}$. Baham., Antill., Bay of Honduras, Cartagena. (Symb).

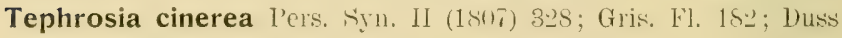
195. Senna.

St. Eustatius: Fort de Wind: n. so1 B.; White Wall : 11. \$1: B., 814 B., 821 B.; near Boven: 200 MI.: n. 1243 B.; slope of the Quill: 11. 1580.

St. Martin: Point Blanche (coll. George): n. 2478 B.

St. Croix (Symb.).

Baham., Antill., Curaçao, Amer. cont. trop. (Symb.). 
3745. Cracca Benth.

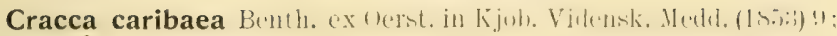
Gris. F๋l. 183: Duss 195.

Benthamanthe caribaca O. Ktze. Rev. III 2) (1898) 53: Millspaugh 494 .

St. Eustatius: along roads in Oranjestad: n. 920 B.; near Naryglory 150 M.; n, 1151 B.

St. Martin: Marrigot roul near Marigot: n. -1s1:3 B.; lower part of Mount Paradis: n. $3290 \mathrm{~B}$; hills on the eastern side of Cul de Sac: n. 3444 B.; road near Fort Amsterdam: n. 1133 B; Colebay. hill: n. 1143; Prince quarter: n. 1098; road to Guanabay: n. 1123.

St. Croix ex Millspaugh.

Antill., Amer. centr., Venezuela, Equador. (Symb.).

\section{Sesbania Scop.}

Sesbania grandiflora P'ors. Sirn. II (1\$ Cultivated. [India orient., Austral. sept.]. (Symb.).

Sesbania sericea F. D.C. Proth. II (1.25) $2666^{3}$ (tris Fl. 147; Duss 196.

St. Martin: Colebay (coll. George): n. 2520 B.; near Simsonsbaylagoon: n. $3037 \mathrm{~B}$.

1 also saw Sesbania sericea near Marigot along a Saltpond.

Baham., Antill. (Symb.).

\section{Stylosanthes Sw.}

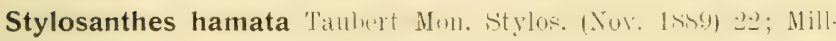
spaugh 495 .

Stylosanthes procumbens Sw. Prodr. (1788) 10S; Gris. F'. 188; Duss 202. Wild clover.

St. Eustatius: Signalhill 200 M.: n. 130 B.; in a garden in Oranjestad: n. 755 B.; between Oranjestad and Fort de Wind on the top of the Cliff 40 M.: 1]. 758 B.; hills near Concordia $100 \mathrm{M}$.: n. $95+$ B.; lower part of Gilboohill: n. 1306; near Sugarloaf: $n$. 1310; linglish Quarter: n. 1386 ; locality unknown: n. $174 \mathrm{H} ., 179 \mathrm{H}$, $252(\mathrm{x}, 300 \mathrm{G}$.

Saba: along Forthgut 150-200 M.: n. 1628 B.; along roads 200-300 M.: n. 1716 B.; seashore near Flatpoint: n. 2138 B.; Marypoint: n. 1328.

St. Martin: from Filipsburg to Guanaloay: n. 2358 B.; Point

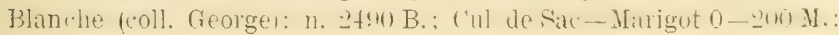


11. 2693 B.; in an orchard near Bellevue: 11. $2449 a \mathrm{~B}$; between Mount Vernon and Orientbay: n. 2939 B.; Colebayhill: 11. 1308; road to Guanabay: 11. 1316.

Anguilla Boldingh.

St. Croix ex Millspaugh.

Baham., Antill., Amer. sept., Mexico, Amer, centr., Nova Grenata. (Symb.).

\section{Arachis Linn.}

Arachis hypogaea Linn. Spee. (175i) 741; (Aris. F'l. 189; Duss 203; Millspaugh 495. Pea nut.

Saba: Mountain 600 M.; 13. 1778 B.

St. Croix ex Millspaugh.

Cult. in tropical countries. Patria ignota. (Syml.).

\section{Zornia Gmel.}

Zornia diphylla Pers. syn. II (1,817) 318; (iris. Fl. 145; Duss 1!h.

St. Eustatius: along roarls near Bengalen: 11. $1186 \mathrm{~B}$.

St. Croix (Symb.).

Antill., warmer countries of both hemisph. (Symb.).

\section{Desmodium Desv.}

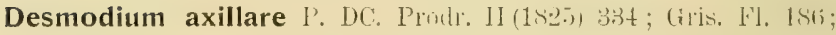
Duss 200.

Meibomia axillaris 0. Ktze. Rev. I (1891) 195; Millspaugh 495.

Saba: luetween Bottom and Marypoint 100-200 M.: 11. 1501 B.; Mountain s00 M.: n. 1.507 R.; Mountain neall Windwallside $6(0)$ MI. : 11. $218+$ B.; Mountain 600-800 M.: n. 2011; Gainbygut: n. 2018; locality unknown: n. $201 \mathrm{~L}$.

St. Croix ex Millspaugh.

Antill., Costarica, Venezuela, Nova Granata, Chili, Guiana, Brasil. (Symb.).

Desmodium scorpiurus Dest. lourn. Bot. I (1S13) 12): (iris. Fl. 187; Duss 201.

Meibomic scorpiumes O. Ktze. Rev. I (1891) 198; Millspaugh 495.

St. Eustatius: Fairplay: n. 1346; seashoro: 17. 1345; Cultuurvlakte: $\mathrm{n}, 1314$.

St. Croix ex Millspaugh.

Antill., from Mexico to Peru. (Symb.). 


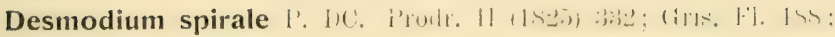
Duss 201;

Meibomia spiralis O. Ktze. Rev. I (1891) 197; Millspaugh 495.

Saba: along reads between Bottom and Windwardside 20) M. : 11. 1327 B.; Laddergut 0-200 M.: n. 1969 B.; locality unknown: 17. $262 \mathrm{~L}$.

St. Martin: hills on the eastern side of cul de Sac: n. 3446 B., $3445 a$ B.

St. Croix ex Millspaugh.

Antill., Amer, cont, trop., Afr, trop., Ind, or, and Ins. pacif. (Symb.).

Desmodium supinum P. DC. Prodr. II (1825) 332.

Desmortium incanum DC. Prodr. II (1825) 332; Gris. F'l. 186; Duss 200. Wild peas.

Meibomia incana Ktze. Rev. I (1891) 197; Millspaugh 495.

St. Eustatius: Top of the Quill 380 M.: n. $273 \mathrm{~B} ., 278 \mathrm{~B}$; slope of the Quill near Bengalen 50-200 M.: n. 642 B., n. $675 \mathrm{~B}$; ; slope of the lower part of the Quill near Bengalen: 11. 725 B.; along roads in Oranjestad: D. 1093 B.; near Maryglory 150 M.: n. 1142 B.; slope of the Quill: 11. 1786, 1807, 1817; Cultuurvlakte: n. 1778; locality unknown: n. $329 \mathrm{H}$.

Saba: along roals between botton and Windwardside $300 \mathrm{M}$. 11. $1281 \mathrm{~B}$; ; between Bottom and Marypoint: 100-200 M.: $n$. $1517 \mathrm{~B}$; t top of the Cliff near the seaside of Boobyhill: n. 160. B.; Mountain near Windwardside 600 M.: 11. 2166 B.; Ladder: 11. 1776, 1796; Rendez-vous 400 M.: n. 1806, 1848; Gainbygut: n. 1816; locality unknown: $25 \mathrm{~L}$.

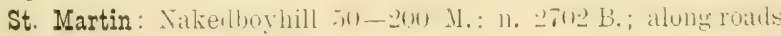
in Cul de Sac: n. 2998 B.; top of the hill on the northerin sicle of Cul de Sae 200-300 M. : n. 3012 B.: Mount Paradis 150-400 M. n. 3199 B., 3343 B.; Colebayhill: n. 1777; French frontier: 11. 1787; road to Guanabay: n. 1797.

St. Croix ex Millspaugh.

Baham, Antill., Amer, cont, trop., Afr. trop. (Symb.).

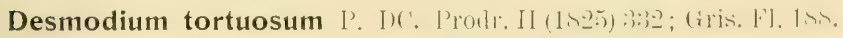

Saba: Ladder: 11. 171, 181; Gainbygut 191.

Antill., Amer. cent. and austr. sept. (Symb.).

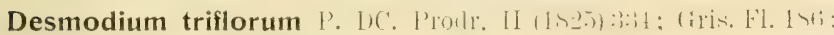
Duss 198. Sisternweed.

Meibomia triflora 0. Ktze. Rer. I (1891) 197; Millspaugh 495,

St. Eustatius: locality unknown: n. $249 \mathrm{H}$. 
St. Martin: Mnuntain between Mont les Acourds and Centrylill 200-300 M.: n. 2875 B.; Mount Paradis 150-400 M.: 11. $3198 \mathrm{~B}$.

St. Croix ex Millspaugh.

Antill., Amer. cont. trop., Afric. trop., Asia austr.orient. (syml).).

Desmodium uncinatum I'. DC. I'rorly. II (1895) 325. (iris. Fl. 15.

Saba: from Marypoint to Bottom 100-200 M.: n. $150 t a$ B.

Cuba, Jamaica, Hispaniola. (Herb. Krug et Urban). Sonora to Bolivia, Venezuela to Uraguay. (Gris. Fl.).

\section{Alysicarpus Neck.}

Alysicarpus nummularifolius I'. LC. I'rodr. II (15.5.) 35.3.

Alysicarpus vaginalis P. DC. Prodr. II (1825) 353; Gris. F'. 185; Duss 199; Millspaugh 494. Sistern peanut.

St. Eustatius: locality unknown: n. $250 \mathrm{H}$.

Saba: hetween Bottom and Marypoint 100-200 M.: 11. 1549 bi., $1550 \mathrm{~B}$; seaside of 'T'entguthill 100 M. : n. $1653 \mathrm{~B}$.

St. Martin: from Filipsburg to Guanabay: n, 2.359 B.; lowel part of Milldrumhill: n. 3115.

St. Croix ex Millspaugh.

Antill.; Patria Ind. or. (Symb.).

\section{Lonchocarpus H. B. K.}

Lonchocarpus violaceus II. B. K. Nov. (icm. et Sp. VI (1 N2:3) 383; Gris. Fl. 200; Duss 219.

St. Eustatius: Top of the Quill 400 M.: n. 176 B., 345 B.; Quill: $n .45$; slope of the Quill: n. $888 \mathrm{~B}$.

Amer. austr. (Ind. Kew.), Antill. (Herb. Krug et Urban).

\section{Piscidia Linn.}

Piscidia piscipula sirg. in Gard. and Fur. IV. (1s!11) t36.

Piscidia erythrine Linn. Syst. I ed. II (1759) 1115; Gris. Fl. 200: Duss 220.

Ichthiomethia piscimula Hitchc. in Gard. and For. IV. (1891) 472; Millspaugh 495. D o g w o od, S t in k w ood.

St. Eustatius : from 'Tumbledowndickbiay to Signalhill: $11.16+$ B.; White IVall: n. $823 \mathrm{~B}$; slope of the hills near Concordiabay: n. 705; Seinpost: n. 715; Boven: n. 706, 725; locality unknown: n. $210 a$ G., 314 H., 724. 
St. Martin: Prince quartor (coll. George): n. $2477 \mathrm{~B}$; Fort Willem: 11. 25 14 B.; gut from Bethlehem to Cul de Sac 100-200 M. : n. $3424 \mathrm{~B}$.

I also saw I'iscidia piscipula: Marigot to Marigothill; Nakedboythill; hill on the northern side of Cul de ar: near Saline: one of the i lands in Simsonsbaylagoon

St. Croix ex Millspaugh.

Florid. austr., Key ins., Baham., Antill., Mexico austr. and Amer, austro-sept. (Syml).).

\subsection{Andira Lam.}

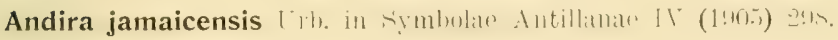
Andire inermis H. B. K. Nov. Gen. II (1823) 385 in obs.; Gris Fl. 202; Duss 224.

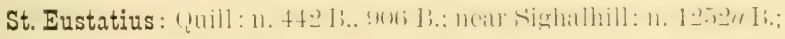
locality unknown: n. 42 .

Antill., Amer. cont. trop., Afr. occ. (Symb.).

\section{Abrus Linn.}

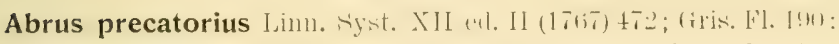
Duss 204; Millspaugh 496. Jumb b beans, Liquorice plant.

St. Eustatius: on the slope of the Cliff near Oranjestad: n. 55 B.; along roads in Oranjestad: n. 572 B.; slope of the Quill near Bengilen 250 M.: n. 702 B.; along roads: n. 26; slope of the Quill: 11. 24; locality unkuown: 11. 23, $26 \mathrm{H}$., $285 \mathrm{G}$.

I also saw Abrus precatorins: in deserted plantations near Bengalen; Signaltill $100 \mathrm{MI}$.

Saba: Bottom 200 M.: n. 1450.; Laddergut 200 M.: n. 2006 B.; locality unknown: n. $312 \mathrm{~L}$.

St. Martin: Prince yuarter (coll. Georgt'): 11. 24.is B.; Marignthill-- Natrigot 1)-200 M.: n. -2fitu B.; along rokds from Bethlehem to Mont Chambord: n. 2910 B.; Colebayhill: n. 25 ; locality unknown: n. $3619 \mathrm{~B}$.

I also saw Abrus precatorius on Nakedboyhill; top of Mount Paradis $300-40)$ H.

St. Croix ex Millspaugh.

Baham., Antill., Amer. cont. calid., Africa, Asia calidior. (Symb.).

\subsection{Clitoria Linn.}

Clitoria ternatea Linn. Spec. (1758) 753.

- Cultivated. [Afric. orient.]. (syml).). 


\section{Centrosema Benth.}

Centrosema virginianum Fenth. in Ann. Wien. Nus. II (1sis) 120; Gris. Fl. 193; Duss 209.

Bradburya virginianum O. Ktze. Rev. I (1891) 164; Millspaugh 300.

St. Eustatius: Top of the (quill :3,0 N.: 11. an B.; Blope of the Quill near Bengalen 50--200 M.: n. 639 B., 661 B., 683 B; between Oranjestad and Fort de Wind on the top of the Clift 40 M.: $n$. $777 \mathrm{~B}$; gut near Fort de Wind 50 M.: n. 844 B.; slope of the Quill 200 M.: n. 878 B; lower part of the hills near Concordia: n. $979 \mathrm{~B}$; along roads in Oranjestad: 11. $1131 \mathrm{~B}$; along roads near Bengalen: 1. 1193 B.; Cultuurvlakte: n. 151, 161; slope of the Quill: n. 211 ; locality unknown: n. $104 \mathrm{H}$., $236 \mathrm{G}$.

Saba: lower part of the Bottom Mountain 250 M.: n. 1414 B; Bottom 200 M.: n. 1452 B., 152; locality unknown: n. $194 \mathrm{~L}$.

I also saw Centrosema rirginianum in Latdergut.

St. Martin: from Filipsburg to Guanabay; n. 23s B B.; Point Blanche (coll. George): n. 2494 B.; from Filipsburg to Belvedere: 11. 2574 B.; Nakedboyhill: $2682 \mathrm{~B}$; ; lower part of Milldrumhill: 1 . 3172 B.; gut from Bethlehem to Cul de Sac 0-100 M.: 11. 3412 B.; Cul de Sac: n. 213; Centryhill: n. 162.

St. Croix ex Millspaugh.

Bermud., Baham., Antill., Amer, cont, from the South U. S. to Argentin., Afr. trop. occ. (Symb.).

\section{Teramnus Sw.}

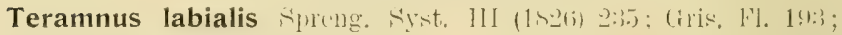
Duss 209; Millspaugh 496.

St. Martin: in pastureground near Bellevue: n. $282+\mathrm{B}$; from Filipsburg to Belvedere: n. $2563 \mathrm{~B}$; locality unknown: n. $3500 \mathrm{a}$ B.

St. Croix ex Millspaugh.

Antill., Africa trop. to the Cape, East Indies. (Gris. l'l.).

\section{Erythrina Linn.}

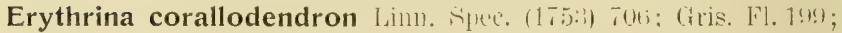
Duss 217; Millspaugh 496.

Saba: lowel part of the Bottom Mountain 250 M.: n. 1411 B.; St. John's 250 M.: n. 1729 B.

St. Martin: Marigothill-Marigot $0-200$ M.: n. 2653 B.

St. Croix ex Millspaugh.

Bermud., Antill., Mexico, Amer. contr. and austro sept. (Symb.). 
3877. Mucuna Adans.

Mucuna pruriens P. I)C. I'rodr. II (1825) 405.

Cultivated. ftrop. countries of hoth hemisph.] (E. a. l'.).

\section{Galactia Atans.}

Galactia dubia L. DC. l'rodr, II (1825) p. 248.

Galactia filiformis Grisol). lilor. W. I. (1860) 194 (p. p.). (Syml).); D) Uss $210(p, p$.$) .$

St. Eustatius: T'op of the hills near Concordia 100 M.: n. 943 13.; between Oranjestad and Fort de Wind on the top of the Cliff $40 \mathrm{M}$. : 11. 789 B.; Fort de Wind : n. $795 a$ B.; White Wall: n. $818 a$ B.; gut near Fort de Wind ro M.: 1]. 827 B., 850 B.;

St. Martin: near Mullet pond: 11. 2779 B.; along roats in Cul de Sic: 17. 2999 B.

Anguilla Boldingh.

Portorico, St. Thomas, Guadeloupe, Antigua, Desirade, Marie. Galante. (Symb.).

Galactia longifolia Benth. in Amm. Wien. Mus. II (15;3) 1:27.

Galactia angustifolia Gris. Flor. W. I. (1860) 194; Duss 210; non Kth. (Syml).).

Galactia tenuifora Wight et Arn. var. Bentb. in Mart. Flor. Bras. XV. I (1859) 143. (Symb.); Millspaugh 495.

St. Eustatius: Fairplay: 1). 300 ; slope of the Quill: n. :301, 27.55; Cultuurvlakte: n. 302; locility unknown: n. 303.

St. Croix ex Millspaugh.

Antigua, Guadeloupe, Marie-Galante. (Symb.).

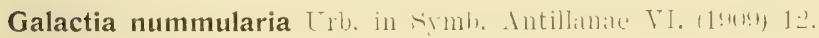

St. Martin neal Guanabay: n. 2428 B.

Galactia rubra Urb. in Symb. Antillanae. II. (1900) 309.

Galactia longiflora Gris. Flor. Wr. I. (1S60) p. $19 \pm$ (p.p.); Duss 210, - non Aru. (Symb.).

St. Eustatius: Slope of the Quill on the side of White Wall 200 M.: n. $117+$ B; Tree gut: n. $20 \pm$ H., 282 G.

Saba: between Bottoin and Marypoint 350 M.: n. 1496 B.; slope of the Mountains near St. John's: 11. 1737 B.; along roads 200-300 M.: 11. 1715 B.; Hellsgate-Marypoint: n. 2042 B. ; Laddergit: 1 . 2760 .

St. Martin: Top of the hill on the northern side of Cul de Sac 200-300 M.: 1). 2994 B.; French frontier: n. 76, 78.

St. Kitts, Antigua, Guadelonpe, Dominica, Martinirue. (Symh.). 
3891. Canavalia P. DC.

Canavalia obtusifolia L'. DC. I'rodr. II (1S25) to)t: (tris. Fl(1)1. 1!17; Duss 215; Millspaugh 496. Sea peas.

St. Eustatius: Feashore near (ranjestal: n. 2.. B., 3., B.: near Boven 200 M.: n. 1231 B.; Cultuurvlakte near Golden Rock: n. 381 ; locality unknown: n. $119 \mathrm{H}$.

I also saw Canavalia obtusifolia on the top of the Cliff near Gallowsbay.

Saba: I saw Canavalia obtusifolia near Flat Point.

St. Martin: Point E3lanche (coll. (ieorget 11. 2484 13,: seashore nenr Mulletpond: 2767 B.

St. Croix ex Millspaugh.

Florida, Bermul., Baham., Antill., warmer countries of both hemisph. (Symb.).

Canavalia spec.

St. Eustatius: n. 244 (;.

38!2. Cajanus P. DC,

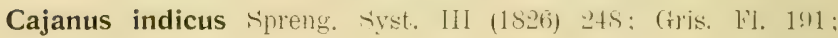
Duss 205.

Cajanus cajan Millsp. in Field. Col. Mus. Bot. II (1900) 53; Millspauch 497.

St. Eustatius: locality unknown: 1n. 55 G., $210 \mathrm{H}$.

Saba: Hellsgate: n. 1696 B.; locality unknown: n. $16 \mathrm{~L}$.

I also saw Cajanus indicus: Laddergut.

Anguilla Boldingh.

St. Croix ex Millspaugh.

Warmel countries of both. hemisph. (Symb).: Also cultivated.

3897. Rhynchosia Lour.

Rhynchosia minima P. I) Prodt. II (1825) 35.5: Gris. Fi. 190; Duss 20 5.

Dolirhlu: minimus Hedir. Vorles. Churgf. Phys. Ges. II (17si) 351; Millspaugh 496.

St. Eustatius: Top of the Quill 450 M.: n. 354 B.; slope of the Quill: n. 1429, 1449; Cultuurvlakte: n. 1439.

Saba: between Botton and Marypoint 100-2001 M. : n. 1504 B.

Anguilla Boldingh.

St. Croix ex Millspaugh.

Warmer countries of both hemisph. (Symb.). 


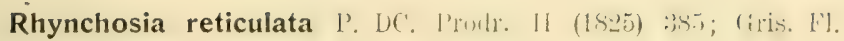
(1859) 190.

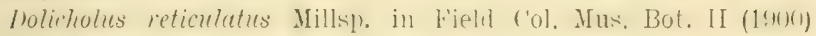
53; Millspaugh 496. $\mathrm{P} \mathrm{c}$ a withe.

St. Eustatius: Top of the (luill 400 M.: 11.215 B., 276 B,; slopo of the Quill near Brengalen 50 M.: 11. 6r50 B.; hills near ('oncordia 100 M.: n. 944 B.; along roads near English Quarter: 11. 100; B.; Red Cliff-T'umblerlowndickbay : 11. 15.49: slope of the Quill : n. 15.2!, $1552,1539$.

Saba: along roals between Bottom and Windwarlside eno M.:

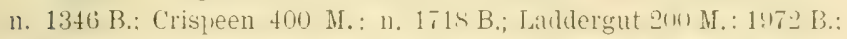

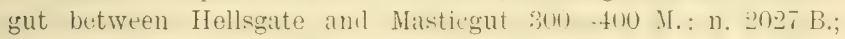
Windwarisile: 11.2311 B.: Latder: 11. 15.2., 1561 : l'allis 2-400 M.: n. 1542; Mountain 400-800 M.: n. 1542.

St. Martin: Prince quarter (coll. George): 11. $2 \pm 70$ B; Cul de

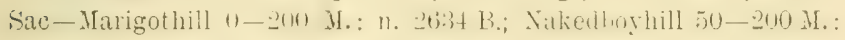
n. 2693 B.; Mountain betreen Mont des Accords and Centryhill 200-300 M.: n. 2870 B.; lower part of the hills between Mont Chambord and Ifunt Vernon: n. 294. B.; lower part of the Mill. drumhill: n. $3173 \mathrm{~B}$; hills on the eastern side of Cul de sac: $n$. 3439 B.; French frontier: 11. 1512.

St. Croix ex Millspaugh.

Antill., Amer. austr. (Symb.).

\section{Phaseolus Linn.}

Phaseolus lathyroides Jinn. Spec. (1763) 1018.

Phaseolus semierectus Linn. Mant. I (1767) 100; Gris. F1. 197; Duss 214; Millspaugh 497.

St. Eustatius: in a garden in Mranjestad: n, 1111 B.: Fiarplay: n. 590; Cultuurvlakte: $11.472,580$.

St. Croix ex Millspaugh.

Baham., Antill., Curaçao, Amer. centr. et austr., India orient., Malaya. (Symb.).

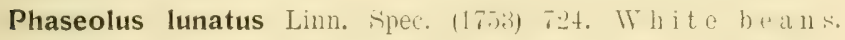
Cultivated. [All. trop. countries.] (Symb.).

Phaseolus vulgaris Limm. Slece (17.53) 72:s, s n ij boont jes. Cultivated. [Everywhere.] (Ind. Kew.).

\section{Vigna Savi.}

Vigna repens U. Kitze. Rer, I (1.4!1) 212; Millspaugh 4t4 Vigna luteola Benth. in Mart. Flor. Bras XV. I (1859) 194 t. 50 f. II; Gris. Fl. 195 ; Duss 211. 
Trigna glabra Savi. Mem. Phas. III (1882) 8.

St. Eustatius: Slope of the lower part of the Quill near Bengalen: $n, 716 \mathrm{~B}$; locality unknown: $n, 1 \mathrm{G} ., 66 \mathrm{H}$.; slope of the Quill: n. 1548, 1549, 2005, 2015.

St. Croix ex Millspaugh.

Bermud., Antill, Amer. calid. from U. S. to Argentin., Afr. trop., Asia trop., Australia. (Symb.).

\section{Lablab Adans.}

Lablab vulgaris 'Savi Diss. (1821) 19 t. 19 f. 8.

Dolichos lablab Linn. Spec. (1758) 725; Gris. Fl. 196; Duss 312; Millspaugh 497.

St. Eustatius: Cultuurvliktr: 11. 18!11; slope of the Quill: n. 1937 .

Saba: Windwardside: n. 1887 B.; Bottom: 11. 2240 B.; CrispetnRendez-vous: n. 1927 ; locality unknown: n. $167 \mathrm{~L} ., 304 \mathrm{~L}$.

St. Croix ex Millspaugh.

Trop. countries of both. hemisph.; Ind. veris, in Afr. trop. (Symb.). Also cultivated.

\section{Oxalidaceae.}

3936. Oxalis Linn.

Oxalis corniculata Linn. Spec. (1753) 435; (iris. Fl. 183; Duss 134; Millspaugh 497.

St. Eustatius: Slope of the (yuill near Glassbottle: n. $1206 \mathrm{~B}$; slope of the Quill: n. 131, 132.

Saba: lower pilt of the Bottom Muantain 250 M.: n. 1400 B.; letween Bottom and Maryloint 100-200 M.: n. 1515 B.; Boobyhill 300 M.: n. 1620 B.; locality unknown: n. 50 L.; CrespeenRendez-vous: n. 130; St. John's: n. 134; Hellsgate: n. 133.

St. Martin: Mountain between Mont des Accords and Centry. hill 0-200 M.: n. 2Slis B.; hill on the northern sicle of Cul de Sac $0-100$ M.: n. 3001 B.

I also saw Oxalis corniculatit on the top of Mount Parardis 300-100 M.

St. Croix ex Millspaugh.

Warmer countries of both hemisph. (Symb.).

Oxalis Martiana Zuee, in Denksthr. Akad. Münch. IX (1s2824) 144.

Cultivated. [Madagascar]. (Ind. Kew.). 


\section{Erythroxylaceae.}

3956. Erythroxylum Linn.

Erythroxylum brevipes [M: I'rodr. I (18-4) 573; Millspaugh 497.

St. Martin: (olebayhill: n. 2(1)1t; I'rince puarter: 11. 2015: John Ednyhill: n. 2015.

St. Croix (Symb.).

Cuha, Hispaniola, st. Thomas, St. Barthilemy; Cinadelounc. (Symb.).

Erythroxylum havanense Jacq. Enum. 1!. Carib. (1760) 21.

Erythroxylum obtusun DC. Prodr. I (1824) 574; Gris. Fl. 113. p. p. (Symb.). Bracelet.

St. Eustatius: Top of the Quill 400 M.: n. 217 B., 329 B.; 500 M.: n. 495 B.; from Little Mountains to Venusbay: n. 958 B.

Saba: between Bottom and Marypoint 300 M.: n. 1478 B.; Springbaygut: n. 2118 B; Laddergut: n. 2017.

St. Martin: Milldrumhill 300-400 M[.: 1]. 3155 B; Hount Paradis 200-300 M.: n. 3314 B.

Cuba, Isla de Pinos. (Symb.).

\section{Zygophyllaceae. \\ 3968. Guaiacum Linn.}

Guaiacum officinale Limn. Spec, (175), 3>1: (tris. Fl. 134; Thss 136; Millspaugh 498.

St. Martin: Tintamarre: n. 3370 B., 3378 B.

St. Croix ex Millspaugh.

Baham., Antill., Amer, austr., (Symb.).

\section{Tribulus Linn.}

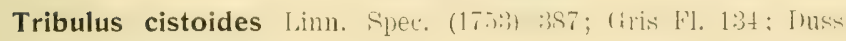
136; Millspaugh 498.

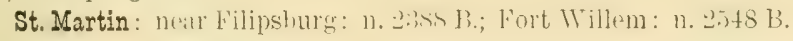

St. Croix ex Millspaugh.

Florida austr., Baham., Antill., trop. countries of both hemisph. (Symb.).

\section{Kallstroemia Scop).}

Kallstroemia maxima Wight e.t inn. l'rouls, I (1s:it) 14., Mill. spaugh 498.

Tribulus maximus Linn. Spec. (1753) 386; Gris. Fl. 13t; Duss 136 . 
St. Eustatius: in a garden in Oranjestad: n. $511 \mathrm{~B}$; Bengalen: n. 616 B.; Cultuurvlakte: n. 243, 248, 249; locality unknown: n. $30 \mathrm{G}$.

Saba: Windwardside: n. 1951 B; Bottom: n. 247.

St. Martin: from Guamaliay to Filipsburg: n. 2437 B.; along roads neall Filipsburg: n. 2530 B.; Centryhill: n. 246; Marigot. road: n. $244,245$.

St. Croiz ex Millspaugh.

Key Ins., Baham., Antill., Amer. from the Southern U. S. to Bolivia. (Symb.).

\section{Rutaceae.}

3991. Fagara Linn.

Fagara flava Kr. et Urb. in Lngl. Jilurb. XXI (1র96) $5 r 1$.

Xanthoxylum flavum Vahl. Eclog. III (1807) 48; Duss 140; Millspaugh 499. Yellow saunder.

Anguilla Boldingh.

St. Croix ex Millspaugh.

Bermud., Key Ins., Baham., Antill. (Symb.).

Fagara martinicensis Lam. Ill. I (1791) 3:34 n. 16:59 t. 811 f. 1. Ianthoxylum clava Herculis Sw. Obs. (1791) 375 (non Linn.); Gris. Fl. 138; Millspaugh 499.

Xanthoxylum martinicense P. DC. Prodr. I (1824) 726; Duss 141. Yellow prickle.

St. Eustatius: Quill: n. 190 B., $416 a$ B., 1.

Saba: Windwardside: 11. 1942 B.; Mountain near Hellsgate: 11. $2279 \mathrm{~B}$.

St. Martin: along roaits in Cul de Sac: n. $2989 a$ B.

St. Croix ex Millspaugh.

Cuba, Jamaica, Hispaniola, Portorico, St. Thomas, St. Jan, Tortola, Montserrat, (iuadeloupe, Desirade, Martinique, St. Lucia, Trinidad. (Symb.).

Fagara spinifex Jacq Fragm. (1809) p. 10 t. 6 f. 2.

Fagara microphylla Desv. Tabl. I ed. (1804) 200; Gris. Fl. 137 (excl. syn. .Br. et L.). (Symb.); Duss 139; Millspaugh 499.

Xanthoxylum spinifex P. DC. Prodr. I (1824) 728.

St. Martin: between Mount Vernon and Orientbay: n. 2!:28 J3; from Belvelere to Oysterpond: 11. 306: B.; Mount Paradis 100200 M.: 11. 3296 B.; gut from Bethlehem to Cul le sac $100-200 \mathrm{M}$.: n. 3425 B.; Prince quarter: n. 2, 3. 
Anguilla Boldingh.

St. Croix ex Millspaugh.

Antill., Venozuela. (Symb.).

Fagara trifoliata Sw. Prodr. (1788) 33.

Tobinia ternata Desv. in Ham. Prodr. (1825) 57; Gris. Fl. 136.

Tobinia punctata Gris. klor. W. I. (1859) 137; Duss 138; Millspaugh 499 .

Xanthoxylum ternatum Siv. H'lor. I (1797) 570.

St. Eustatius: Top of the Quill 400 M.: n. 204 B.

St. Martin: from Filipsburg to Guanabay: n, 2386 B.; Point Blanche (coll. George): 11. 2492 B,; Nakedboyhill 50-200 M.: n. 2708 B.; lower part of the hills between Mont Chambord and Mount Vernon: n. 2953 B.; from Belvedere to Oysterpond: n. 3056 B., 3074 B., 3081 B., 3082 B.; Prince quarter: n. 4, 10, 11, 12, 13; Centryhill: n, 15.

Anguilla Boldingh.

St. Croix ex Millspaugh.

Jamilica, Hispanioli, Portorico, st. Barthilemy, Antigua, Montserrat, fiudeloupe, Marie-Cialante, Desirale, Les hiantes, Dominica, Martinique, st. Lucia, St. Vincent, Barbados, Trinidad. (Symb.).

\section{Amyris Linn.}

Amyris elemifera Limn. Syst. I el II (1759) 10100; Duss 183.

St. Martin: Tintamarre: n. 3391 B., 3395 B.

St. Croix (Symb.).

Baham., Florida austr., Key West, Antill. (Symb.).

\section{Murraya Linn.}

Murraya exotica Linn. Nant. II (1771) 563; Duss 1:2; Milispaugh 499. Boxw ood.

St. Eustatius: in a garden in Oranjestad: n. 937 B., $40 \mathrm{G}$.; Little Mountain-Boven: n. 14.

Saba: Windwardside: n. 1908 B.; locality unknown: n. $281 \mathrm{~L}$.

St. Martin: Hills on the eastern side of Cul le silc: 11. 3446 B.

St. Croix ex Millspaugh.

Antill.; Ind. in Asia austro-orient., Austral. sept, Polynes. (Symb.).

4094. Triphasia Lour.

Triphasia trifoliata P. DC. Proull. I (1 24$)$ 536; I)uss 132; Millspaugh 499. Myrtle lemon. 
Triphasia aurantiola Lòr. Fl. Cochinchin. (1790) 153.

St. Eustatius: in a garden in Oranjestad: 11. 53 B. ; Bengalen: 1. 613 B.; seashore: n. 24, 26; locality unknown: n. $226 \mathrm{G}$.

Saba: Bottom: n. $1856 \mathrm{~B}$. ; locality unknown: n. $97 \mathrm{~L}$.

St. Martin: Cul de Sac-Marigot $0-200$ M.: n. -622 B. ; hills on the eastern side of Cul de Sac: n. 3447 B.

St. Croix ex Millspaugh.

Antill.; Indig. in India orient. (Symb.). Also cultivated.

\section{Citrus Linn.}

The author was not yet able to find out with a security so great, to make it possible to mention it in this Flora the names of the different species of Citrus that are cultivated in the gardens.

Only one plantation of Citrus Limonum Risso in Saba be mentioned.

\section{Simarubaceae.}

\section{Suriana Linn.}

Suriana maritima Linn. Spec. (175) 25t; (iris. ll. 5s; Duss 48; Millspaugh 499 .

St. Eustatius: Scashore near Concordiabay: 11. 36; near Seafeathersbay: n. 35 ;

I also saw Suriana maritima at the seashore near English Quarter.

St. Martin: Colebay (cull. (reorgt): 11. 164:) B; from Belveciere to Oysterpond: n. 3043 B.

I also saw Suriana maritima near Mulletpond.

Anguilla Boldingh.

St. Croix ex Millspaugh. (Symb.).

Bermud., Baham., Antill., trop. countries of both hemisph.

\section{Picrasma Blume.}

Picrasma antillana LT. in Srmbolat Antillanae $\mathrm{I}^{\top}(1908) 378$.

Picracna cxcelsa Gris. in Flor. W. I. (1859) 140 (p. p.); Duss 143; (Symb.).

St. Eustatius: (quill: 11. 148 P.. 41 la B.; locality unknown: n. $210 a \mathrm{G}$.

Saba: from Bottom to Marypoint 100-200 M.: n. 1t63a B.; near Hellsgate: n. J $1997 a$ B. : locality unknown: 11. $2073 a$ B., 341 L.; Cresp.-Rendez-vous 400 M.: n. 44.

St. Martin: Mount Paradis: n. $3219 a$ B. 
St. Croix (Symb.).

St. Thomas, St. Jan, St. Kitts, Antigua, Guadeloupe, Martinique, St. Vincent, Barbados. (Symb.).

\section{Picramnia Sw.}

Picramnia pentandra siv. Flor. I (17!ti) 201); (iris. Fil. 140; Duss 142. Wild coffee, Bastard bough.

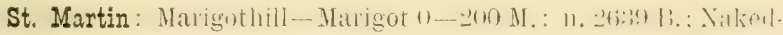
boyhill 50-200 A.: n. 2690 B., 2697 B., 2714 B, Mountain between Mont des Accords and Centryhill 200-300 M.: n. 2878 B.; lower part of the hills between Mont Chambord and Mount Vernon: n. 2956 B., 2962 B.; top of the hill on the northern side of $\mathrm{Cul}$ de Sac 200-300 M.: n. 3010 B.; Milldrumhill 300-400 M.: n. 3184 B.; Mount Paradis 150-400 M. : n. 3197 B., 3299 B., 3345 B.; Centryhill: n. 92, 93; Prince quarter: n. 90; French frontier: $n$. 37, 38, 39; locality unknown: n. 3621 B., 91.

I also sitw Picramnia pentandra: from Belvedere to Oysterpond.

Florida austr., Key Ins., Baham., Antill. (Symb.).

\section{Burseraceae.}

\section{Bursera Linn.}

Bursera simaruba Sarg. (iitrd. and For. III (1890) 2(it).

Burserce gummiferce Jacq. Sel. (1763.) $9 \pm$ t. 65; Gris. Fl. 173; Duss 181; Millspaugh 500. G um tree, Balsam tree, Lime tree.

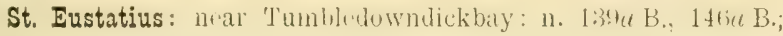
top of the Quill 400 M.: 13. 202 B.; slope of the Quill on the side

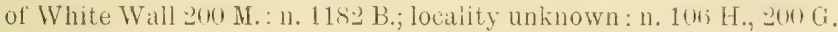

I also saw Bursera simaruba in a gut near Fort de Wind.

Saba: Seashore near Saddle: n. $1652 \alpha$ B; gut near Devilshand: n. $2078 a$ B.; locality unknown: n. $290 \mathrm{~L}$.

I also saw Bursera simaruba: in Hellsgate, T'entgut, Springbaygut.

St. Martin: Marrigotroad: 11. $2819 a$ B, French frontier: n. 71, 72.

I also saw Bursera simaruba: Top of Nakedboyhill; near Saline; along roads from Filipsburg to Simsonsbay; Vallée des Pères; Lowlands.

Anguilla Boldingh.

St. Croix ex Millspaugh.

Florida, Koy Ins, Baham., Antill., Curaçao, from Mexico to Nova Granata and Venezuela. (Symb.). 


\section{Meliaceae.}

\section{Swietenia Jacq.}

Swietenia mahagoni Jacy. Enum. (1760) 20; (ilis. Fl. 131; Duss 130; Millspaugh 500. M a hag o n y.

St. Eustatius: Bottom of the Quill 300 M.: n. 216 B.; in a garden in Oranjestad: n. 1072 B.; locality unknown: n. $82 \mathrm{H}$.

Saba: Laddergut 0-200 M.: n. 1978 B.; Greathill: n. 92 ; locality unknown: n. $12 \mathrm{~L}$.

I also saw Swietenia mahagoni: near Marypoint.

St. Croix ex Millspaugh.

Key Ins., Baham., Antill., Mexico, Amer. centr., Peru. (Symb).). Also cultivated.

\section{Melia Linn.}

Melia azedarach Limn. Spec. (1753) 884; Mtillspaugh 500.

Melia sempervirens Sw. Prodr. (1788) 67; Gris. Fl. 128; Duss 126. Lila c.

St. Eustatius: in a garden in Oranjestad: n. $44 \mathrm{H}, 139 \mathrm{G}$.

I also saw Melia azedarach in the slope of the Cliff.

Saba: Crispeen 400 M.: n. 172: B.; Mountain near Windwardside 600 M.: 11. 216:3 13.; Peperpot: n. 93; Crispeen-Rendez-vous 400 M.: n. 92; locality unknown: 11. 125 L.

St. Martin: near Bethlehem: n. $2661 \mathrm{~B}$.

St. Croix ex Millspaugh.

Bermud., Baham., Antill, warmer countries of both hemispil. (Symb.). Also cultivated.

\section{Malpighiaceae.}

\section{Heteropteryx Juss.}

Heteropteryx purpurea II. B. K. Nor. Gen. V (1S21) 1633; Duss 116; Millspaugh 498 .

St. Eustatius: Hills near Concordia $100 \mathrm{M}$.: n. 550 B.; from Little Mountains to Venusbay: 11. 990 B.; slope of the Quill on the sicle of White IVall 200 M.: 11. 1179 B.; Pongo-Signalhill: n. 1965; Red Cliff: n. 1969; Sugarloaf: n. 1966, 1967, 1968.

St. Croix ex Millspaugh. Antill., Venezuela, Colombia. (Symb.). 


\section{Stigmatophyllon Juss.}

Stigmatophyllon ciliatum A. Juss, in $\mathrm{A}$, At. Hil. Fl. Mials. Her. III (1827) 49.

Cultivated. [Jamaica, Brasil.]. (Gris. Fl.).

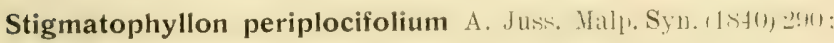
Gris. Fl. 119; Duss 116; Millspaugh 498. Go a t bus h.

St. Eustatius: Seathore nur Oranjestal: 11. 31 B.: in a garden in Oranjestad: n. $96 \mathrm{~B}$; dested plintation near 'Tumbledowndickbay: 11. 142 B., 171 B.; top of the Quill 400 M.: n. 191 B., 212 B.; slope of the Quill near Bengalen 150) M.; 11. 6ist; B.; between (1)anje. stad and Fort de Wind on the top of the Cliff 40 M.: n. 791 B.; along roats in oranjestanl: 11 , $193 \mathrm{~B}$.; lower part of the hill neal Concordia: 11. 980 B.; Signalhill 150 M.: 17. 1042 B., 105. B.; along

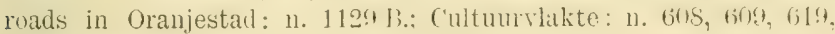
630; locality unknown: n. $137 \mathrm{G} ., 144 \mathrm{H}$.

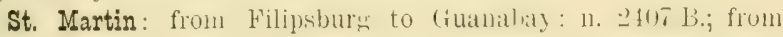
Filipsburg to Belvedere: n. 2557 B.; Cul de Sac-Marigothill 0-200 M.: n. 2629 B; lower part of the Millurumhill: n. 3171 B.; Tintamarre: 3398; Vineyardhill: n. 618; Cul de Sac: n. 639; Colebayhill: n. 94 .

I also saw Stigmatophyllon periploeifolium on: Marigothill-Marigot; Nakedboyhill.

Anguilla Boldingh.

St. Croix ex Millspaugh.

Cuba, Jamaica, Hispaniola, Portorico, St. Thomas, St. Jan, Antigua, Martinique, St. Lucia. (Symb.).

Stigmatophyllum sericeum Wrisht x. (iris. (at. Pl. Cul., (1stib) $4:$. Stigmatophyllum diversifolizm A. Juss, in Arch. Mus. Par. III (1843) 381.

St. Martin: Lowlands: n. 3286 B.

Anguilla Boldingh.

Cuba (Herb. Krug et Urban).

\section{Galphimia Cav.}

Galphimia gracilis Bartl, in Limmea XIH (1:34:4) 5.5.

Cultivated. [Mexico.]. (Symb.).

\section{Malpighia Linn.}

Malpighia angustifolia Linn. Spec. (176i2) (i11); (iris. Fl. 11?: Duss 113. Bastard cherry. 
St. Martin: lwwel bat of the lills between Mont rlamboril

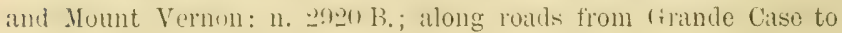
Harigot: n. $3090 \mathrm{~B}$.

I also saw Malpighia angustifolia near Saline.

St. Thomas, St. Jan, Antigua, Guadeloupe, St. Lucia. (Niedenzu 1899).

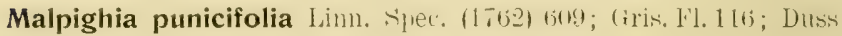
113. Cherry.

St. Eustatius: Seashore near Oranjestad: 11. 败 B.; slope of the lower part of Signalhill: n. 107 B.; Oranjestad: n. 97; Gilloohill: n. 38 ; along, the road to Sugarloaf: n. 87; locality unknown: n. $63 \mathrm{H}, 78 \mathrm{G}$.

I also saw Malpighia punicifolia: top of Signalhill; top of the Cliff hetween Oranjestad and White Wall.

Saba: hetween Bottom and Marypoint 100_-200 M.: 13. 15+6 13.; locality unknown: n. 1477 B., $334 \mathrm{~L}$.

St. Martin: from (iuanabay to Filipsburg: 11. -24:5 B.; Malligotroad near Marigot: n. 2son B., zs10 B.; P'rince yuarter: n. 4 r. $57,58$.

Anguilla Boldingh.

St. Croix (Symb.).

St. Thomas, Antigua, st. Barthelemy, cruadeloupe, Martinipue, Trinidad, Margarita, Bonaire, Curaçao. (Symb.).

\section{2อั2. Bunchosia L. Cl. Rich.}

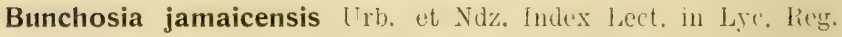
Ilos. Brunsb. de Genere Bunchosia (1898) 10.

Bunchasia Linderiana Gris. Flor. W. I. (1860) 115.

Saba: Seaside of tentguthill 104 M.: n. 16jõ B.; Windward. side: 11. $1943 \mathrm{~B}$.

Antill., Moxico, Costarica, Guima. (Gris. Fl.).

Bunchosia nitida I. (1. Rich, ap. Juss, in Ann. Mus, Par. XVIII (1811) 481.

St. Martin: between Mont ('hamborl and the Saline: 11. 2924 B.: lower part of the hills between Mont Chambord and Mount Vernon: n. 2963 B.; near Simsonshayvillage: n. 3257 B.; Mount Paradis $300-400$ M.: n. 3322 B.

Hispaniola, Montserrat, Guadeloupe, Martinique, St. Vincent, Bequia, Barbados, Grenada. (Symb.). Surinam. (Pulle). 


\section{Byrsonima L. Cl. Rich.}

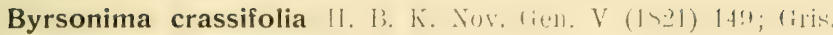
Fl. 114. (rel. - 13. martinicensis Kre et Urb.?)

St. Martin: Lowlands: 11, 2381 ( B.

Antill., Amer, austr, sept. (Symb.).

Byrsonima lucida L. Cl. Rich. ap. Juss, in Ann. Mus, Par. XVIII (1s11) fs1; (ilis. Fil. 115; Inus 111.

St. Martin: Lowlands: n. $3621 \mathrm{~B}$.

Anguilla Boldingh.

Key Ins., Baham., Antill. (Symb.).

Byrsonima spicata L. Cl. Rich. ap. Iuss. in Inn. Alus. I'al, vol. XVIII (1811) 481; Gris. Fl. 115; Duss 111; Millspaugh 497. Holly hock.

St. Eustatius: T'op of the Quill S3:1 M.: 11. 2.3: B.; inner slope of the Quill 350 N.: n. 1229 B.; Quill: n. $80 \mathrm{H}$.

Saba: Windwardside-Hellsgate: n. 1679 B.; Windwardside: n. 1944 B.

St. Croix ex Millspaugh.

Antill., Panama, Venezueia, Guiana, Brasilia. (Symb.).

\section{Euphorbiaceae.}

4299. Phyllanthus Linn.

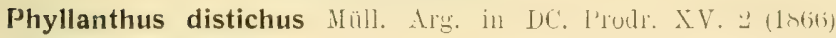
413; Millspaugh 500.

Cicca disticha Linn. Mant. I (1767) 124; Gris. Fl. 32; Duss 20.

St. Eustatius: on the slope of the Cliff near Oranjestad: 11. $52 \mathrm{~B}$; in a garden in Oranjestat: $\mathrm{n} .90 \mathrm{H}, 148 \mathrm{G}$.

Saba: Bottom: n. 1876 B.; locality unknown: n. 116 L., $238 \mathrm{~L}$.

St. Martin: Top of the liill on the northern sile of Cul de Sac 200-300 M.: n. 2992 B.

St. Croix ex Millspaugh.

Antill.; Indig. in India orient? (Symb.). Also cultivated.

Phyllanthus epiphyllanthus Linn. Spec. 1 1763) 13:1-2; I)

Phyllanthus falcatus Sw. F'lor. II (1800) 1115; Gris. F'l. 35.

St. Martin: Colebay (coll. George): n. 2502 B.; shore of the Simsonslaylagoon near Cul Piquard: n. $27+4$ B. : near Mlulletpond: n. $2785 \mathrm{~B}$.

I also saw Phyllanthus epiphyllanthus: Lowlands.

Anguilla Boldingh.

Baham., Antill. (Symb.). 
Phyllanthus niruri Limm. Spec. (175.3) 981; Gris. Fl. 34; Duss 23; Millspaugh 501. Surinam bitter.

St. Eustatius: in a garden in Otanjestad: 11, i6 B. : along roads in Oranjestad: n. 579 B.; along roads near Glassbottle: n. 871 B.; locality unknown: n. $41 \mathrm{G} ., 3 \pm \mathrm{H}$.

Saba: lower part of the Bottom Mountain 300 M.: n. 1429 B.;

St. Martin: from F'ilipsburg to Guanabily: 11. 2408 B.; lower part of the Milldrumhill: n. $3166 \mathrm{~B}$; ; in a plantation of Cotton near Bethlehem: n. 3410 B.

I also saw Phyllantlus niruri in pastureground near Bellevue.

St. Croix ex Millspaugh.

Bermud., Baham., Antill., almost all warmer countries of both hemisph. (Symb.).

Phyllanthus nobilis Müll. Arg. in Le' P'rodr. XV 2 (186ti) 114 ; Millspaugh 501

Cicce antillana A. Juss. T'ent. Euphorb. (182t) 108 t. 4 f. 13 B.; Gris. II. 33 ; Duss 21. Gooseberry.

Saba: Gainbygut: n. 2074; locality unknown: $43 \mathrm{~L}$.

St. Martin: Nakedloyhill: n. $273 \%$ B.; Monnt Paralis: 11. 3202 2 B.

St. Croiz ex Millspaugh.

Antill., Guiana. (Symb.).

\section{Croton Linn.}

Croton astroites Dryand. in Ait. Hort. Kew. I ed. vol. III (17s!n) 375; Gris. Fl. 42; Duss 33; Millspaugh 501. Wild marrow.

St. Eustatius: Wlope of the Quill ahove White Wall: n. 2 B.; slope of the lower part of White Wrall: 11. 97 B.; Signalhill $200 \mathrm{M}$.: n. 128 B.; gut near Fort de Wind 50 M.: n. 826 B.; along a road in Oranjestad: n. 930 B.; hill near Concordia 100 M.: n. 949 B.; top) of Signalhill 2.5 M.: 1]. 1080 B.; Signallill 200 M.: n. 1047 B.; near Maryglory 150 M.: n. 1147 B; along roads near Bengalen: 11 . 1194 B.; near Boven 200 M.: n. $1239 a$ B.; Cultuurvlakte: n. 125; Pangohill: n. 122, 123; Gilboohill: n. 124; Sugarloaf: 11. 128; locality unknown: n. 150 a B., 269 B., 171 G., 3577 B.

St. Martin: from Filipshurg to Guanabay: 11. $2362 \mathrm{~B}$; Fort Willem 50 M.: n. 2536 B.; locality unknown: n. 3611 B.; near Fort Amsterdam : n, 126, 127.

I also saw Croton astroites: Cul de Sac; Nakedboyhill

St. Croix ex Millspaugh.

Portorico, St. Thomas, St. Jan, St. Barthélemy, St: Kitts, Antigua, Guadeloupe, Desirade. (Symb.). 


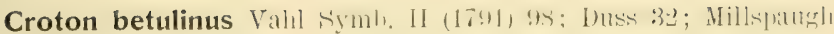
501. Black sage.

St. Martin: Marigotrout neal Mariunt: 11. 2sole B, in pasture.

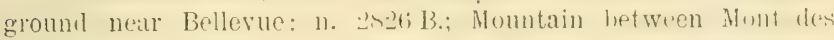
Accords and Centryhill: n. 2865 B.; along roads near Colebay: n. 3025 B; shore of the Simsonsbaylagoon near Cul Piquard: $\mathrm{n}$.

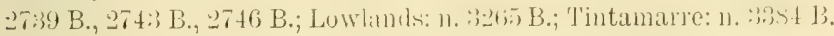

I also saw Croton betulinus: one of the islinds in Simsonsbaylagoon.

Anguilla Boldingh.

St. Croix ex Millspaugh.

Florida austr., Antill. (Symb.).

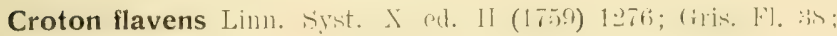
(excl. syn. Willd.). (Symh.); Millspaugh 501. Malrow, Y ellow balsem.

Croton balsamifer Jacq. Inum. Pl. Carib. (1760); Duss 31.

St. Eustatius: on the slope of the Clift' near Gallowsbay: 1]. $61 \mathrm{~B} . ;$ in deserted plantations at the seashore near Bengalen: n. 582 B., 584 B., 594 B., 597 B., 599 B.; slope of the Quill near Bengalen 200 M.: n. 737 B.; hetween Oranjestad and Fort de Wind on the top of the Cliff $40 \mathrm{M} .:$ n. $775 \mathrm{~B}, 778 \mathrm{~B}$., $785 \mathrm{~B}$., $792 \mathrm{~B}$; Fort de Wind: n. $795 \mathrm{~B}$; seashore near Venusbay: n. $968 \mathrm{~B}$; Gilloohill 150 M.: n. 1124 B.; along roads near Bengalen : n. 1195 B.; slope of the hills near Concordiabay: n. 131; Cultuurvlakte: n. 130, 132; Tumbledowndickbay: 11. 133; Venusbay: n. 134; Sugarloaf: n. 140 ; locality unknown: n. $5 \pm \mathrm{H}, 120 \mathrm{G}$.

Saba: along roads hetween Bottom and Windwariside gno Mr.: n. $1321 \mathrm{~B}$; along the Forthgut 150-200 M.: 11. $1627 \mathrm{~B}$; along the lower part of the Forthgut: n. $1643 a$ B.; St. Giles: n. 1865 B.; Laddergut 0-200 M.: n. 1971 B.; Ladderlanding: n. 135, 136; Greathill 200 M.: n. 141; Quarter 300 M.: n. 249; Marypoint: n. 250 ; locality unknown: n. $315 \mathrm{~L}$.

St. Martin: from Filipsiurg to Guanabay: i. 2351 B.; Guanabay: 1n. 2431 B.; Prince quarter (coll. George): 11. 2469 B.; Fort Willem 0-50 M.: n. 2531 B., 2543 B.; near Bethlehem: n. 2662 B., shore of the Simsonsbaylagoon near Cul Piquard: n. 2741 B.; road near Fort Willem: n. 2796 B.; between Mount Vernon and Orientbay: n. 2937 B.; near Simsonbayvillage: n. 325 B.: Green Key: n. 3364 B., 3867 B.; 'Tintamarre: n. 3882 B.; Filipsburg: n. 137; Centryhill: n. 138; Vineyardhill: 12. 139; locality unknown: ก. 37 . 
I also saw Croton flavens: from Belvedere to Oysterpond; between Mount Vernon anil Saline; Nakedboyhill.

Anguilla: Boldingh.

St. Croix ex Millspaugh.

Ilispaniola, Portorico, St. Thomas, St. Jan. (Symb.). Baham. (Herb. Leiden).

Croton lobatus Liml. Sipec. (1750) 1005; firis. Fl. 12: Duss 33: Millspaugh 501. L i l a c b u sh.

St. Eustatius: in a garden in uranjestad: n. it B., $1276 \mathrm{~B}$; along roads in Oranjestad: n. 564 B.; Fairplay: 12. 167; slope of the Quill: n. 164, 165, 166; locality unknown: n. $92 \mathrm{G} .177 \mathrm{H}$.

Saba: Bottom: n. 1839 B., 1843 B.

St. Martin: Colebayhill: n, 161.

I also saw Croton lohatus near Belvedere.

Anguilla Boldingh.

St. Croix ex Millspaugh.

Antill., Bonaire, Curaçao, Aruba, Amer, cont. trop. (Symb.).

\section{8, Ditaxis Vahl.}

Ditaxis fasciculata Vahl ap. A. Juss. Tent. Fuph. (1+24) p. $110 \mathrm{t}$. 7 f. 24 ; Gris. Fl. 44; Millspaugh 501.

St. Martin: Prince quarter: (coll. George) n. 2457 B.; near Nakedboyhill: 17. 2566 B., 2733 a B.; Point Blanche: 11. 3405 B.; Vineyardhill: n. 769 ; locality unknown: n. 21.

St. Croix ex Millspaugh.

Cuha, Portorico, St. Thomas, St. Jan. (Symb.).

\section{Argithamnia Sw.}

Argithamnia candicans sw. L'rolr. (178s) 34: Gris. Fl. 4t: Millpaugh 502 .

St. Martin: Fort Willem (1-50) M.: 11. 2-440 B.; Nikedboyhill 50-200 M.: n. 2700 B.; lower part of the lills between Mont Chambord and Mount Yernon: 11. 2959 B.; Mount Paradis 200300 M.: n. 3317 B.; T'intamarre: 11. 3401 B.; Colebayhill: n. 2075; Centryhill: n. 767.

Anguilla Boldingh.

St. Croix ex Millspaugh.

Baham., (Hitchc.), Antill. 


\section{Bernardia Litans.}

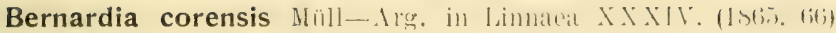
173: Duss 35.

Polyboea corensis Krlotseh. in End. Gen. Suppl. IV. III (1850) 88. Giris. Fl. 46.

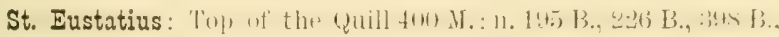
$104 \mathrm{~B}$; ; slope of the Quill near Bengalen 100 M. : 17. (665) B.; Sig. malhill $200 \mathrm{M}$.: n. $1050 \mathrm{~B}$.

St. Martin: Mount Paradis 300- 400 M.: n. 32224 B.: Mount Paralis 200-300 M.: n. 3303 B.

Haiti, st. Barthelemy, Martinique, Dominiea, Guadeloupe, Grenada, St. Vincent, Curaçao. (Herl). Krug et Urban).

\section{Adelia Linn.}

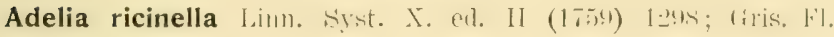
45; Millspaugh 502.

St. Martin: Filipsburg near the silltputs: n. .:35i [3, : from Filipsburg to Furt Amsteriam: n. 2.j6ti 13.: Prince unarter (coll.

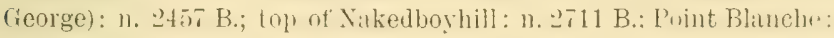
n. 3405 B.; near Fort Amsterdam: n. 750; Colebayhill: 11. 751; Prince quarter: n. 752.

St. Croix ex Millspauch.

Cuba, Jamaica, Hispaniola, Portorico, St. Thomas, St. Jan. (Symb.).

\section{Acalypha Linn.}

Acalypha chamaedrifolia Hüll. Arg. in Dr. Prodr. X T. 2, (1siti) 879: Duss 35).

Acalyphe reptans Sw. Prodr. (1788) 99; Gris. Hel. 48; Millspaugh 502. Bastard nettle.

St. Eustatius: along roals in (1)anjestarl: 11 . 5.2 B., (1) 1 F.

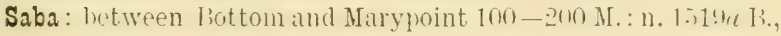
1520 B., 1540 B.; Hellsgate: n. 1692 B; Laddergut 0-200 M.: 11. 1962 B.; Windwardside: n. 2334 B.

St. Martin: in an orchard near Bellevue: n. 2850 B.; from Grande Case to Narigot: n. 3092 B.; lower part of Milldrumbill: n. 8159 B.

St. Croix ex Millspaugh.

F'lorida austral., Antill. (Symb.).

Acalypha Poiretii Spreng Syst, III (1826) 879.

St. Martin: near Grande Case: n. 3092 B. 
Anguilla Boldingh.

St. Thomas, Curaçao. (Herb. Krug et Urban).

Acalypha Wilkesiana Müll-Arg. in DC . Prodr. XV. 2. (1866) 817.

Cultivated. [Ins. Pacif.]. (Symb.).

\section{Tragia Linn.}

Tragia volubilis Linn. Spee. (17.73) 981); (iris. Fl. 48; Duss 36; Millspaugh 502.

St. Eustatius: Top of the Quill 400 M.: 11. 2.25 B., 25.2 B., 28.2 B.; inner slope of the Quill near Bengalen 350 M.: 11. $703 \mathrm{~B}$.

Saba: letween Bottom and Narypoint 100-200 M.: n. 1467 B.; Mountain 600-800 M.: N. 1791 B.; Laddergut (1-200 M.: 11. 2011 B.

St. Martin: Nikedboyhill 50-200 M.: 11. 2710 B.; Mountain

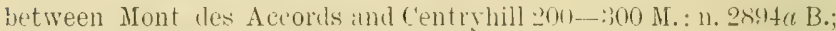
Mount l'aradis 200-300 M.: n. 3308 B.

St. Croix ex Millspaugh.

Antill., Curaçao, Amer. cont. trop., Afr. oce. (Symh.).

\section{Dalechampia Linn.}

Dalechampia scandens Lim. Spee. (175:3) 1054; liris. lil. 51; Duss 40 ; Millspaugh 502.

St. Eustatius: neal Maryglory 150 M.: 1). 1143 B.

St. Martin: Nakedboyhill 280 M.: 17. 2735 B.

St. Croix ex Millspaugh.

Antill., Amer. cont. trop., (Afr, trop. and Ind, or.) (Symb.).

\section{Ricinus Linn.}

Ricinus communis Limm. Spec. (1753) 1007; (iris, lil. 37; Duss 30; Millspaugh 502. Castoroil plant.

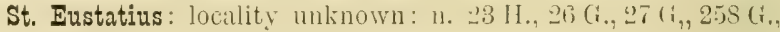
$288 \mathrm{H}_{\text {, }}, 289 \mathrm{H}$.

I also saw Ricinus communis: Seashore near Oranjestad.

Saba: Boobyhill 300 M. : n. 1.566 B.; locality mknown: n. 64 I. ; I also saw Ricinus communis: Laddergut.

Anguilla Boldingh.

St. Croix ex Millspaugh.

Indig. veris, in Africa. (Symb.). Also cultivated. 


\section{Aleurites f'orst.}

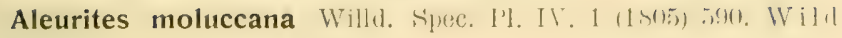
date.

Cultivated. Asia austr. orient, and Ins, pacific.] (Symb.).

\section{Jatropha Linn.}

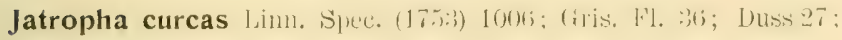
Millspaugh 502. Grave physic nut.

St. Eustatius: between Granjestad and liort de Wind on the top of the Cliff 40 M.: 11. 771 B.; along roads near Glassbottle: 11. 1209 B.; seashore: n. 242, 2073; Cultuurvlakte: n. 24t; locality unknown: 17. $52 \mathrm{G} ., 168 \mathrm{H}$.

Saba: Bottom: n. 243.

I also saw Jatropha curcas in Forthbaygut; Hellsgategut.

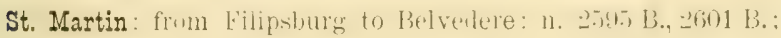
along roads from Grancle Case to Marigot: n, 3106 B.; Cul de Sac: 11. 245 .

St. Croix ex Millspaugh.

Bermud., Antill., Amer. cont. trop. (Symu.).

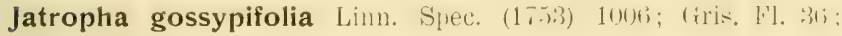
Duss 25; Millspaugh 502. Physic nut.

St. Eustatius: Seashore near Ormjestad: n. 19 Ii, along roakls in Oranjestad: $n .570$ B., 558 B., 1126 B.; in deserted plantations near Bengalen: n. 589 B.; seashore: n. 247, 249; Cultuurvlakte: n. 246; locality unknown: n. $132 \mathrm{G}$, $133 \mathrm{G} ., 196 \mathrm{H} ., 197 \mathrm{H}$.

I also saw Jatropha gossypifolia: on Signallill 200 M.; top of the Cliff between Oranjestad and White Wall.

Saba: alung roads hetween Bottom and Windwardsile -2m M. : n. $1360 \mathrm{~B}$; ; along the Forthgut 0-150 M.: 11. $1639 \mathrm{~B}$.; locality unknown: n. 241 L., $313 \mathrm{~L}$.

I also saw Jatropha gossypifolia in: Laddergut.

St. Martin: lower part of Milldrumhill: n. 3113 B.

I also saw Jatropha gossypifolia: along roads from Filipsburg to Nakedboyhill; in pastureground near Bellevue.

St. Croix ex Millspaugh.

Key Ins., Baham., Antill., Curaçao, Aruba, Amer. cont. trop. Africa occ. (Symb.).

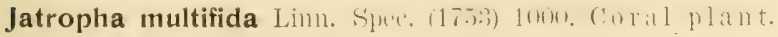

Cultivated. [Amer, cont, trop.] (Symb.). 
4444. Manihot Allans.

Manihot utilissima Pohl Plant. Bras. I (1527) p. 32 t. 24. Cassave.

Cultivated. [Amer. cont. trop.]. (Symb.).

\section{Codiaeum A. Juss.}

Codiaeum variegatum Bl. Bijur. XII (1895) 606. Croton. Cultivated. [Ins. Sundalic.]. (Symb.).

\section{Sapium Jacq.}

Sapium spec. (without flowers or fruits).

Saba: T'op of the Mountain 600 M.: n. 2189 B.

\section{Hippomane Linn.}

Hippomane mancinella Linn. Spec, (1753) 1191.; Gris. Fl. 50 ; Duss 37; Millspaugh 503. N a n gen iel tree.

St. Eustatius: Seashore near Granjestad: n. 16 B.; Concordiabay: n. 184; seashore: n. 182; locality unknown: n, 1014 B., $324 \mathrm{H}$.

I also saw Hippowane mancinella: seashore near Tumbledowndickbay.

Saba: Seashore near Flat Point: n. 2133 B.

St. Martin: from Filipsburg to Guanabay: n. 2845 B.; near Fort Willem: n. $2799 \mathrm{~B}$.

I also saw Hippomane mancinella: along roads from Filipsburg to Nakedboyhill; Cul de Sac; Fort Willem; near Mont Chambord; near Saline; near Grande Case; Vallée des P'eres; one of the islands in the Simsonbay. lagoon; Lowlands; Green Key; Tintumarre.

St. Croix ex Millspaugh.

Florida austr., Baham., Antill., Curaça, from Hexico to Venezuela. (Symb.).

\section{Actinostemon Mart. ex Klotzsch.}

Actinostemon caribaeus (iris. in (imett. Abh. III (1857) 168. Excoecaria caribaed Gris. Flor: W. I. (1859) 51; Duss 40.

St. Eustatius: Great gut: n. $748,749$.

St. Martin: Milhrumhill: 11. $314: 3 a$ B., 814.4 B.; John Eduylhill: n. $345,346,347,348,2051$.

Antill. (Herl). Krug et Urban); Amer, cont. (Herb. Leiden).

\section{Gymnanthes Sw.}

Gymnanthes lucida Siw. Proulr. (1788) 96: Millspaugh 503.

Excoccaria lucida Sw. Flor. II (1800) 122; Gris. Fl. 50; Duss 39. 
St. Eustatius: Top of the Quill t50 M.: n. 348 B., 372 B.; ,ignalhill: 11. 1036i B.: near Maryentory 150 M.: 11. 1152 B., 116il B.: locality unknown: n. $333 \mathrm{H}$.

St. Croix ex Millspaugh.

Florida austr., Baham., Antill. (Symb.).

\section{Hura Linn.}

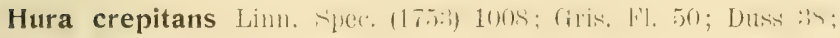
Millspaugh 503. Sandbox tree.

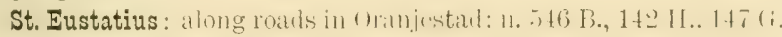

St. Martin: from Filipsburg to Belvedere: n. 2596 B.

St. Croix ex Millspaugh.

Baham., Antill, Amer. cont. trop. (Symb.).

4098. Euphorbia Linn.

Euphorbia articulata l3urm. ex Aub]. Pl. (illi. I (17.5) 4 Si): Gris. H1. 53; Millspaugh 503.

Einjhorbia linearis Retz. Obs. III (1791) 32; Duss 41 .

St. Eustatius: lutween cranjestinl and Fort de Wind on the top of the Cliff to M.: n. 767 B; gut near Fort de Wind 50 M.: n. 847 B.; lower part of the hills near Concordia: n, $976 \mathrm{~B}, 2071$; l'umbledowndickbay: n, 2070; White Wall: n. 2068, 2069.

Saba: Green Island: n. 2131 B.

St. Croix ex Millspaugh.

Portorico, St. Thomas, Culebra Island, St. Kitts, Guadeloupe, Martinique, St. Vincent. (Herb. Krug. et Urban).

Euphorbia buxifolia Limm. Enye. II (1-isti) 421: (iris. Fl. Ais; Duss 42; Millspaugh 503.

St. Eustatius: White Wall: n. 811 B.; seashore near White Wall: n. 854 B.; seashore: 11. 2065; Seafeatersbay: n. 2076, 2077; White Wall: n. 2064.

St. Martin: Colebay (coll. George): n. 2517 B.; Green Key: n. $3361 \mathrm{~B}$.

Anguilla Boldingh.

St. Croix ex Millspangh.

Bermud., Florida and Mexico and throughout the West Indies (Hemsley 1884).

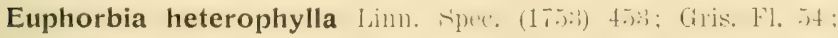
Duss 44.

Euphorbia cyathophore Nurr. Comm. Gotting. VII (1786) 81; Millspaugh 503. Joseph's coat. 
St. Eustatius: Glassgut at the seashore near Oranjestad: $n$. $49 \mathrm{~B}$; in a garden in Oranjestad: n. $576 \mathrm{~B}$., 2096, $32 \mathrm{H} ., 36 \mathrm{G}$.; Oranjestad: n. 2098; Cultuurvlakte: 11. 2097; locality unknown: n. 2078 .

St. Martin: near Bethlehem: 11. 2668 B.; along roads from Grande Case to Marigot: n. 3095 B.

St. Croix ex Millspaugh.

Bermudas, Eastern Nortll-America, from Illinois southward and through the TVest Indies and Mexico to Peru and Brazil. (Hemsley 1884).

Euphorbia hypericifolia Linn. Spee. (175i) 4jt; (iris. Ll. it; Duss 43; Millspaugh כ03.

St. Eustatius: Bengilen: 11. (60) B.; along roals near (1)assbottle: n. 869 B.; slope of the Quill: n. 2079, 2080, 2095.

Saba: lower part of the Bottom Mountain 250 M.: 11. 1399 B.; Laddergut 200 M.: n. 2008 B.; Peperpot: 13. 2081; Crespeen-

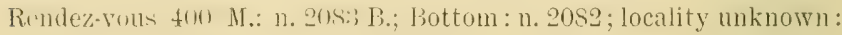
n. $128 \mathrm{~L}, 161 \mathrm{~L}$.

St. Martin: Marigothill-Marigot $0 \longrightarrow 200$ M.: n. 2654 B.

St. Croix ex Millspaugh.

Bermudas, Eastern States of North-America, West Indies, Mexico and South-America. (Hemsley 188t).

Euphorbia petiolaris Curt. Bot. Mag. (1795) 853: Millspaugh 50?.

Euphorbia verticillata Poir. Encyc. Supp. II (1811) 611; Duss 45. Black mangeniel.

St. Martin: near Filipsburg: n. 3341 B.; from lilipsburg to Belvedere: 11. 2567 B., 2573 B.; Marigothill-Marigot 0-200 M.: 11. 2632 B.; near simsonsbalylagoon: n. 30:3i) B.; along roads from Colebay to Simsonsbay: n. 32.53 B.; Filipslurg: 11. 20131; Vineyarrhill: n. 2032; Cul de Sac: n, 2033, 2034.

I also saw Euphortia petiolaris: along roads near Bethlehem; along roads from lilipshurg to Nakedboyhill: Nakerlboyhill; lill between Mont. des Accords and Centryhill; near Saline; along roads from Belvedere to Oysterpond; near Grande Case; lower part of Milldrumhill; Lowlands; lower part of Mount Paradis; top of Mount Paradis.

St. Croix ex Millspaugh.

Portorico, St. Thomas, St. Jan, Guadeloupe. (Herb. Krug. et Urban).

Euphorbia pilulifera Linn. Spec. (17.53) 4.74; (iris. Fl. i1; Thus 42; Millspaugh 503. Chickon weed. 


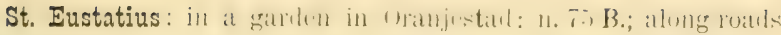
in Oranjestad: n. 512 B., 577 B.; along roarls near Glassbottle: n. 875 B.; locality unknown: n. 8 G., $8 \mathrm{H}$.

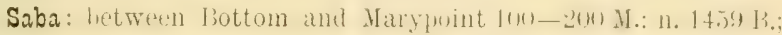

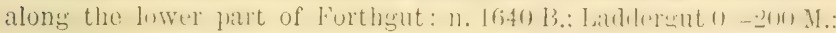
n. 1963 B.; Crespeen-Rendez-vous: n. 2037; locality unknown: n. $239 \mathrm{~L}$.

St. Martin: in an orchard near Bellevue: n. 2840 B; Centryhill : 17.349.

Anguilla Boldingh.

St. Croix ex Millspaugh.

All tropical countries. (Gris, Fl.).

Euphorbia prostrata Ait. Hort. Kew. idl. I. II. (17) 1:3:; Gris. Fl. 53; Duss 42; Millspaugh $50 t$.

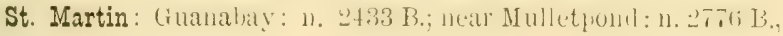
2780 B.

St. Croix ex Millspaugh.

Bermulas, Loulisina and Texas southwad to l'eru and brazil: also in tropical Africa and naturalised in Sicily and elsewhere. (Hemsley 1881).

Euphorbia pulcherrima Wille ex klotsich, in (ut) ef lietl. Allg. Gartenz. Il (1834) 27. Stal of Bethlohem.

Cultivated. [Mexico.] (Ind. Kew.).

Euphorbia thymifolia Limn. Spec. (175)it t5t; Duss t2; Mfilspaugh 501; Children chickenweed.

St. Eustatius: locality unknown: n. 229 H., 25: (i.

St. Martin: Lowlands near Cherrycap: n. 3259 B.

All tropical countries. (Gris. Fl.).

\section{Pedilanthus Neck.}

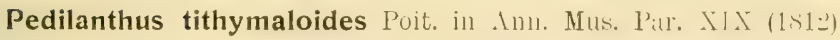
390. t. 19; Gris. Fl. 52; Duss 41 ; Millspaugh J04. Parakiet.

St. Eustatius: along the lower patt of signallitl: 11. 12:; $\mathrm{B}$.: gut near Fort de Wind 50 Mr. : 11. 842 B.; along roads near Signalhill: n. 1128 B.

Saba: Bottom: n. 2250 B.

St. Martin: from Filipsburg to (intand aty: 11. 237- B.; Prince. quarter (coll. (ieorge): n. 24 ti. B.; from Belvelere to oysterponst: n. 3054 B; near Fort Amsterdam: n. 2072. 
I also saw Pedilanthus tithymaloides: along roals near Bethlehem; Marigothill to Marigot: Nakedboyhill: near Bellewe; hill between Mont de's Aceords and Centryhill; near Saline; hill on the northem side of Cul de Sac: Mount Paradis 300-400; Lowlands; lower part of Mount Paradis; Tintamarre: hills on the eastern side of Cul de Sac.

Anguilla Boldingh.

St. Croix ex Millspaugh.

Portorico, Guadeloupe, Martinique, St. Vincent, Barbados, Grenada, Tobago. (Herb. Krug et Urban.).

\section{Anacardiaceae.}

\section{5ั45. Mangifera Linn.}

Mangifera indica Linu. Spec. (175:3) 200; (iris. Fl. 176; Duss 157: Millspaugh 50t. Man gotree.

St. Eustatius: in a garden in Oranjestad: n. $57 \mathrm{H}, 130 \mathrm{G}$, $3590 \mathrm{~B}$.

Saba: locality unknown: n. 156 L., 196 L., 302 L.

1 also saw Mangifera indica: Latdergut; Bottom; Marypoint.

St. Martin: I sitw Maugiferat indical: Matrigothill-Marigot; from ('ul de Sac-Marigot; Vallée des Pères; lower part of Milldrumhill; Mount Paradis 300-400 M.: Lowlands.

St. Croix ex Millspaugh.

India orient, Malaya, Mexico, Amer. centr. et merid-sept. Antill. (Engler 1883). Also cultivated.

\section{Anacardium Rotböll.}

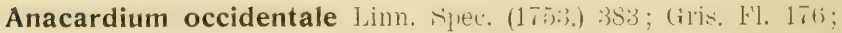
Duss 189; Millspaugh 505. Cas hew, Cherry.

St. Eustatius: Slope of the lower part of the quill near Bengalen: n. 718 B.; locality unknown: n. 45 H., $207 \mathrm{G}$.

I also saw Anacardium occidentale: on the slope of the Quill on the side of White Wall; top of the Quill; top of Signalhill.

Saba: locality unknown: 11. $227 \mathrm{H}$.

I also saw Anacardium occidentale: Laddergut; near Marypoint.

St. Martin: from Filipshurg to Belvedere: 11. 2597 B.; Low. lands: n. $3267 \mathrm{~B}$.

I also saw Anacardium occidentale: along roads from Filipsburg to Nakedhoyhill; Nakedboyhill; lower part of Milldrumhill.

St. Croix ex Millspaugh. 
In all warmer countries of America. (Engler 1885). Also cultivated.

\section{1う.2. Spondias Limn.}

Spondias lutea Limn. Spec. (1753) 613. Y e $110 \mathrm{w}$ p l u $\mathrm{m}$.

St. Eustatius: Signalhill: n. $125 a$ B.; in a garden in Oranjestad: 11. 1016 B.

Saba: Bottom: 11. $1873 a$ B., 1877 B., 2234 B.

Antill., Mexico, Amer. cont. austr., Malay. (Englor 1883). Also cultivated.

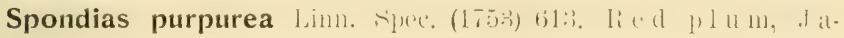
ma i c a plum.

Cultivated. [Antill., Mexico, Peru, Nova Granata.] (Engler 1883).

\section{Comocladia Linn.}

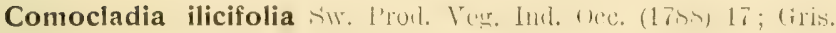
Fl. 176; Duss 184; Milspaugh 504. Led man, Centepee p I a is t.

St. Eustatius: White Wall: n. 822 B.; Signalhill 150 M.: n. 1041 B., 99; Gilboohil: n. 100; locality unknown: n. 111 H., $332 \mathrm{H}$.

Saba: Bottom: n. 1852a B.; Springhaygut: 11. 2096 B.

St. Martin: from Filipsburg to Guanabay: n. $2369 \mathrm{~B}$; Centry= hill: 11. 101, 102; near Point Blanche: n. 104 ; near Guanabay : n. 103.

I alsu satw Comocladia ilicifolia: along roads near Bethlehem; along

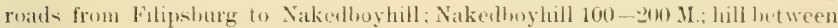
Iont des Accords and Centryhill; near Saline; from Belvedere to Oysterpond; lower part of Mount Paradis.

Anguilla Boldingh.

St. Croix ex Millspaugh.

Portorico, St. 'Thomas, St. Domingo, Guadeloupe, Antigua, St. Lucia. (Engler 1885).

\section{I58. Celastraceae.}

4626. Maytenus Molina.

Maytenus elliptica Kr. et [rh. in Duss li]. Ant. franu. (1S:1) 145 (excl. syn. Grisel).).

St. Eustatius: Top of the Quill 400 M.: n. 233 B., $344 a$ B., 369 B.; Quill: n. $412 a$ B.; slope of the Quill neal Bengalen: $n$. $744 a$ B. ; near Signalhill : n. $952 a$ B. 


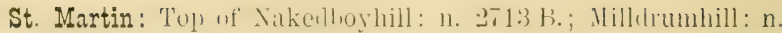
3152 B., 3182a B.

St. Croix. (Symb.).

St. Domingo, Portorico, St. Thomas, St. Jan, Montserrat, Guadeloupe, Marie dialantc, Martiniøue, st. Vincent, Beçuia. (Symb.).

\section{Gyminda Sarg.}

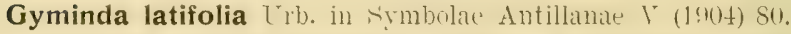

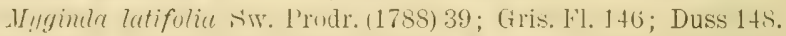

Myginda pallens Sarg. Forest Trees N. Amer. IX:38; Duss 147 (non Sm.); (Symb.).

Rhacoma latifulic Loesener in Engl.-Prantl. Nat. Pflanzenfam. III. 5 (1892) 217.

St. Eustatius: Top of the Quill $f(x)$ M.: n. 37. $\mathrm{a}$ B., $393 \mathrm{~B}$.

St. Martin: Colebay (coll. ('eorge): 11. 2512 B.; near Simonshay: n. $3035 a$ B.

Key ins., Antill., Mexico. (Symb.).

\section{Rhacoma Linn.}

cf. 4649. Myginda Jacq. syn.

Rhacoma crossopetalum Linn. Syst. X. ed. II (175!1) s!M.

Myginde rhacoma Sw. Prodr. I (1788) 39; Gris. Fl. 146; Duss 147.

Myginda pallens Sinith in Rees Cycl. XXV (1813) n. 4; Gris. F'1. 146; Millspaugh 505.

St. Eustatius: Signalhill !00 M. : n. 12!n B.; (leserted plantations near Tumbledowndickibay: 11. 144 B.; White Wall: 11. $\$ 16$ B.; gut near frort de Wind 50 M.: n. 841 B.; seashore near White Wall: 11. 860 B.; rignalhill 50 M.: 11. 1028 B.; Berg Amstertam: n. 115; top P'ongo: n. 11:, 114; Pongo-Signalhill: n. 11:; White Wall: n. 111; Crookwell: n. 110; Little Mountain-Boven: n. 116; Sugarloaf: n. 117.

Saba: lower part of the Bottom Nountain 250 M.: n. $1391 \mathrm{~B}$; gut hetween Masticgut and Hollsgate $300-400$ M.: 1. $2029 \mathrm{~B}$.; Paris: 11.211.

St. Martin; neal Mulletpond: n. 2751 B., 2753 B.; near Bethlehem: 11. $2600 a$ B.; Tintamarre: n. 3397 B., 3400 B.

Anguilla Boldingh.

St. Croix ex Millspaugh.

Florid. austral., Key, Baham., Antill. (Symb.). 


\section{Schaefferia Jikr.}

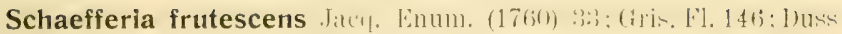
148; Millspaugh 505. Boxwood.

St. Eustatius: lexertel plantations near Tumblesluwnlickbis: n. 147 B.; from Little Mountain to Venusbay: n. 962 B.; Boven: ก. 215 .

Saba: along the Forthgut 150-200 M. : n. 1636 B.

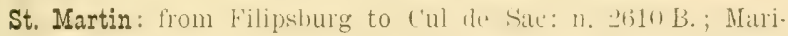
gothill-Marigot ()-200 M.: 11. ¿617 B.; Mountilin letween Mont des Accords and Centryhill 200--300 H.: n. 2859 B.; lower part of the hills between Mont Chambord and Mount Vernon: n. $296+$ B.; Colelaylill: 11. 210 ; Centrylill: $11.1,2,3$; Frencl frontier: n. 5 ; locality unknown: n, 4, 7 .

St. Croix ex Millspaugh.

Florid. austral., Key, Baham., Mexico, Nova Granata, Antill. (Symb.).

\section{Sapindaceae.}

\section{Cardiospermum Linn.}

Cardiospermum halicacabum Linn. Spee. 117.) 3titi; (iris. l'l. 122; Duss 118; Millspaugh 505. Sprainbushwine.

St. Eustatius: Bengalen: 11. 61^ B.: allong roads in (1ranjestad: n. 1106 B; near the seashore: n. 146; slope of the Quill: n. 148; Cultuurvlakte: 11. 147; locality unknown: n. $154 \mathrm{G} ., 294 \mathrm{H}$.

Saba: St. Giles: n. 1868 B.; Windwardside: n. 2307 B.

St Martin: from lilipsburg to (iuamabay: n. .4-121 B.; l'oint

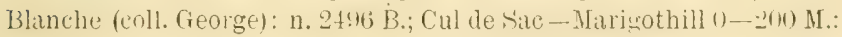
n. $2632 \mathrm{~B}$; along roads from Marigot to Filipsburg: n. 3112 B.; Mount Paradis 2010-301) M.: n. :3:316 B ; Marigotroad: 13. 1.50, 151, 15.2.

St. Croix ex Millspaugh.

'l'rop. and warmer countries of both hemisph. (E. a. P.).

\section{Allophylus Linn.}

Allophylus occidentalis Radlk. in Sitzungsb. jhyss. math. (1). Bayer. Acad. Wiss. XX (1890) 230.

Schmidelic occidentalis Sw. F'l. Ind. Occ. II (1800) 6tō; Gris. F1. 126; Duss 122; Millspaugh 506.

St. Eustatius: Top of the (yuill thin M.: 11. $21:$ ) B.; inner slope of the Quill :350 M.: 11. 45.5 B., 1215 B., 3.9! H.; locality unknown: n, $50,51,52$. 
Saba: Boobyhill 300 M.: n. 1591 B.; gut between Hellsgate and Masticgut 300 M.: n. 2057 B.

St. Croix ex Millspaugh.

Antill., Cula to Minaes Geraes. (Gris. WI.).

\section{Melicocca Linn.}

Melicocca bijuga Linn. Spec. (176-) 49.5; (iris. Fl. 127; Duss 12:3; Millspaugh 505. Kinnuptree.

St. Eustatius: Signalhill ?(k) M.: N. 136 B., 16, B.: Bengalen: 13. 633 B.; lower part of the hills near Concordia: n. 978 B.; along the slope of the lower part of Signallill: n. 143; Oranjestad: n. 144, $86 \mathrm{H} ., 204 \mathrm{H}$.

Saba: Bottom: n. 1872 B; locality unknown: n. $1 a \mathrm{~L}$.

1 also saw Melicocca bijuga in Laddergut.

St. Martin: I saw Melicocea bijuga: along roads from Filipsburg to Nakedboyhill; near Bellevue.

St. Croix ex Millspaugh.

'Triniclad, Jamaica, Guiana, Nova Granata. (Gris. Fl.).

Also cultivated.

\section{Dodonaea Lim.}

Dodonaea viscosa Linn. Mitut. (17tii) as: (iris. Fl. 12:- Duss 123; Millspaugh 506.

St. Eustatius: Slope of the (quill above White IVall :350 M.: n. $1 \mathrm{~B}$; between Oranjestad and Fort de Wind on the top of the Cliff 40 M.: n. 766 B.; White Wall: n. $80 \pm$ B.; gut near lort de Wind: n. s2S B., s35a B.; slope of the Quill on the side of White Wall 200 M.: n. 1199 B.; near Sugarloaf: n. 154, 155, 162; near White Wall: n. 156, 157, 163; locality unknown: n. 3635 B.

St. Martin: at the seashore near Simsonshayvillage: 11. 3031 B.; Colebay (coll. George): n. 2504 B.; Simsonsbay: n. 159, 161.

St. Croix ex Millspaugh.

In almost all warm countries. (Hemsley 1884).

\section{Hypelate Swartz.}

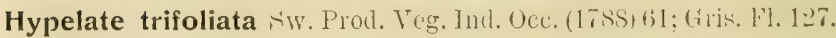
St. Martin: near Simsonsbaylagoon: n, 2738a B. Anguilla Boldingh. Baham., Antill. (Herb. Krug et Urban). 


\section{Balsaminaceae.}

4856. Impatiens Linn.

Impatiens Balsamina Linn. Spec. (1753) 988.

Cultivated. [India orient?]. (Incl. Kew.).

\section{Rhamnaceae.}

\section{Zizyphus Adans.}

Zizyphus Jujuba Lam. Encycl. III (1789) 318; Duss 94.

St. Martin: from Filipsluurg to Guanabay: n. $2393 a$ B.; near Filipsburg: n. $2337 a$ B.

India. orient., Malay. (Ind. Kew.).

\section{$4875 \%$. Krugiodendron Urb.}

Krugiodendron ferreum $(1 \%$, syml. Ant. III (190:) :31t; Mill. spangh 506 .

Condatia ferrea Gris. Flor, W. I. (1859) 100; Duss 92. Ironherry.

St. Eustatius: from Little Mountain to Venusbay: n. 963 B.; Boven: n. 192; locality unknown: n. $337 \mathrm{G}$.

St. Martin: near Mulletpond: n. 2773 B.; Mount Paradis: n. 3313 B.; French frontier: n. 191.

St. Croix ex Millspaugh.

Florida, Key Ins., Baham., Antill. (symb.).

4882. Colubrina L. Cl. Rich.

Colubrina ferruginosa Brong. in Ann. Se. Nat, Sil. I X (1527) 386. t. 15 f. 3; Gris. Fl. 100; Millspaugh 506.

Saba: St. Giles: n. $1866 a$ B.

Florida, Antill. (E. a. P.).

\section{Gouania Jacq.}

Gouania domingensis limm. Spece (17633) Ltifi:\}: Gris. Fl. 101: Duss 93; Millspaugh 506. White root.

St. Eustatius: from Tumblemownickbat to signalhill; n. 16is E. ; near Maryglory 150 M.: 11. $1136 \mathrm{~B}$.

Saba: lower part of the Bottom Mountain 300 M.: n. 1444 B.; Hellsgate: 11. 189. 
St. Martin: Milrignthill-Marigot 11-2001 M. : 11. 26:3ti 13.; Marigotroall near Marigot: n. 2811 B.; lowel part of the hills between Mont Chambord and Mount Vernon: n. 2954 B.; lower part of Colebayhill: n. 153, 190.

St. Croix ex Millspaugh.

Amer. trop. (E. a. P.).

\section{Vitaceae.}

\section{Cissus Linn.}

Cissus obovata Vahl symb. Bot. III (176t) 16; (iris. l'l. 10:.

St. Martin: 'I'op of Mount Paradis: n. 3236 B., 3350 B.

St. Croix. (Planchon 1883).

Portorico. (Planchon 1883).

Cissus sicyoides Limn. Syst. ed. X. (175!1). 897 ; (iris. Fl. 103; Duss 95; Millspaugh 506. P udding withe.

Vitis sicyoides Miq. in Ann. Mus. Bot. Lugd. Bat. I. (1863) 83.

St. Eustatius: Suashore near Uranjestarl: 11. It B.; slope of the hills near 'Tumbledowndickbay: n. $153 \mathrm{~B}$; ; top of the quill S.41) M.: 11. 284 B.; slope of the Quill near Bengalen 100 M. : n. 668 B., 709 B., 727 B.; Cultuurvlakte: n. 5; seashore: n. 6, 7; locality unknown: n。 $129 \mathrm{H}$., $155 \mathrm{G}$.

Saba: along roads between Bottom and Windwardside $200 \mathrm{M}$.: n. 13+4 B.; letween Bottom and Marypoint 190-200 M.: 11. 150k B.; gut letween Hellsgite and Masticgut $300-400$ M.: 1. 20.̃1 B., 2076 B.; Springbaygut: n. 2107 B. ; Bottom: n. 14, 15; locality unknown: n. $104 \mathrm{~L}$.

I also saw Cissus sicyoides in Laddergut.

St. Martin: from Filipsburg to (iuamahary: 11 . 23sst B.; along the siltpond near Filipshurg: 11. 2443 B.; from Filipsburg to Belvelere: $11 .-2589 \mathrm{~B}$; gut from Bethlehem to Cul le Sac: 11.3434 B.; hills on the eastern side of Cul de Sac: n. $3437 \mathrm{~B}$; Centryhill: n. 17 ; Cul de Sac: n. 16.

I also saw Cissus sicyoides: in Marigothill-Marigot; Nakedboyhill; in pasturesround near Belvedere; hill between Mont des Aecords and Centrylill; hill on the northern side of $\mathrm{Cul}$ de Sac; from Belvedere to Oysterpond; Mount of Paradis 300-400 M.

Anguilla Boldingh.

St. Croix ex Millspaugh.

Americ. trop. (Planchon 1887). 


\section{Tiliaceae.}

\section{Corchorus Linn.}

Corchorus acutangulus Limn. Encye. (17ifi) II 101: (iris. lil. 97: Millspaugh 507.

St. Eustatius: Bengalen: n. 607 B.; Fort de Wind: n. 808 B.

St. Croix ex Millspaugh.

In all tropical countries (E. a. P.).

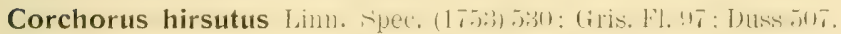

St. Eustatius: Slope of the Quill near Bengalen: n. $656 \mathrm{a} \mathrm{B.;}$ from Little Mountains to Venusbay: n. 994 B.; Signalhill 200 M. : 11. 1044 B.; in a garden in Oranjestad: 11. 1273 B.

St. Martin: (coll. George:) n. $2495 a$ B.; near Mulletpond: n. 2762 B.; Mount Paradis 300-400 M. : n. 3245 B.; Fort Amsterdam : n. 252 ; French-frontier: n. 225.

Anguilla Boldingh.

St. Croix ex Millspaugh.

Baham., Antill., Aruba, Bonaire. (Herb. Krug et Urban).

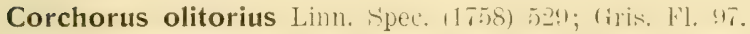

St. Eustatius: locality unknown: n. $269 \mathrm{G}$.

In all tropical countries. (E. a. P.).

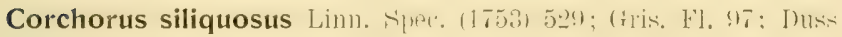
89; Millspaugh 507.

St. Martin: Top of the hill on the nutherle siste of rul de sile: n. 3005 B.; Marjgotroad: 17. 248.

Anguilla Boldingh.

St. Croix ex Millspaugh.

Antill., F'lorida to T'exas, Panama, Nova Granata. (Gris. Fl.).

\section{Triumfetta Linn.}

Triumfetta rhomboidea Jacy. Linum. I'l. (arib, (1760) 2.2: (iris. Fl. 96; Millspaugh 507. Wild maho.

St. Eustatius: locility unknown: 11. 15.1 H.. 210.; slope of the Quill: n. 247.

Saba: Ladder: n. 246.

St. Croix ex Millspaugh.

In both hemisph. (E. a. P.).

Triumfetta semitriloba Jacf. Enum. Pl. Carib. (1760) 2.); (iris. Fl. 96; Duss 89; Millspaugh 506.

St. Eustatius: (liff neil (Milnjestall: 11. if B.; top) wf the (ynill $400 \mathrm{M}$.: n. $198 \mathrm{~B}$.; slope of the Quill near Bengalen 50-200 M.: 
n. 645 B.; 711 B.; Cultuurvlakte: n. 155 ; slope of the Quill : n. 229, 230, 231, 232; Quill: n. 243, 245; locality unknown: n. 244.

Saba: lower part of the Bottom Mluutain 250 M.: n. 1395 B.,

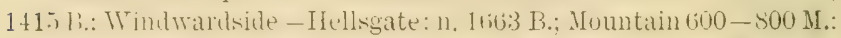
11. 17.2 13.: wht betwern Helisgate and Misticgut 300-400 N.: n.

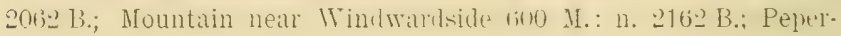
pot: 1., 236; Gainbygut: 235.

St. Martin: Cul de Sac-Marigothill 0-200 M.: n. 2635 B.;

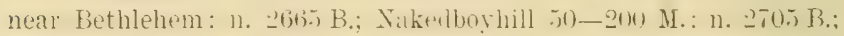
Mount Paradis $300-400$ M.: 11. 3215 B., 3243 B; Centryhill : n. 23t; Marjgotroad: n, 233.

St. Croix ex Millspaugh.

In both hemisph. (E. a. P.).

\section{Malvaceae.}

\section{Abutilon Adans.}

Abutilon crispum swet. Hort. Brit. exl. I(1S2) 53; (iris. Fl. 79; Duss 68.

St. Eustatius: English Quarter: n. 606, 616, 626, 636.

St. Vincent, New Mexico, Texas, Cuba and Mexico to Venezuela, East Indies. (Gris. Fl.).

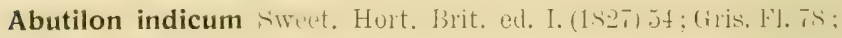
Duss 67; Millspaugh 507.

St. Eustatius: Bengalen: n. Ni2- B.. 115s B.; along roalds in Oranjestad: n. 932 B; Concordia: n. 255; English Quarter: 13. 287; locality unknown: n. $201 \mathrm{H}$.

Saba: locality unknown: n. $75 \mathrm{~L}$.

St. Martin: along roitds from Bethlehem to Mlont C'hambond: n. 2915 B.; Mount Paradis 200-400 M.: n. 3204 B.

I also saw Abutilon indicum: along roads from Filipsburg to Nakeibnyliill; hill between Mont des Accords and Centryhill.

Anguilla Boldingh.

St. Croiz ex Millspaugh.

Antill., Panama, Niger, Nubia to Mozambique, Last Indies. (Gris. Fl.).

\section{Althaea Linn.}

Althaea officinalis Linn. Spec. (1753) 686.

Cultivated. [Europa orient.] (Ind. Kew.).

4995. Malvastrum A. Gray.

Malvastrum spicatum A. (tray. in M[em. Am. Acall. N. A. I (18t9) 22; Gris. Fl. 72; Duss 62; Millspaugh 508. 
St. Eustatius: in a garden in Oranjestad: n. 90 B.; Fort de Wind: n. $799 \mathrm{~B}$; locality unknown: n. $256 \mathrm{G}$.

Saba: along roads between Bottom and Windwardside $200 \mathrm{M}$.: n. $130+$ B.; Hellsgato-Marypoint: 11. 2046 B.

St. Martin: from Filipsburg to Guanalaly: n. 2346 B.; near Bethlehem: n. $2663 \mathrm{~B}$.

St. Croix ex Millspaugh.

Antill., Texas to Brazil., Cape Verle Islands, East Indies, New Hollind. (Gris. Fi.).

Malvastrum tricuspidatum A. (iliy P. Wright I (1)ie) l6: Gris. Fl. 72; Duss 63.

Malvastrum coromandelianum Garcke Bonplandia V (1857) 297; Nillspaugh 508.

St. Eustatius: Quill: n. 901 B.

St. Croix. (Symb).).

Cosmop. trop. (Ind. Kew.).

\section{Sida Linn.}

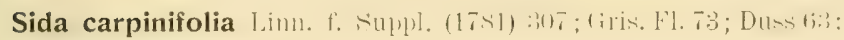
Millspaugh 508.

Sida acute Burm, f. Fl. Ind, (1768) 147. S o a p b us l..

St. Eustatius: in a garden in Oranjestad: n. 91 B., 568 B.; Bengalen: n. 620 B.; Fairplay: n. 1505; Cultuurvlakte: n. 1502 ; slope of the Quill: n. 1506; locality unknown: n. 1507, 15 H., $16 \mathrm{G}$.

Saba: along roads between Botton ame Windwardsirle eng .I.:

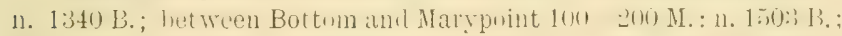
top of the Clity near the seaside of Boolyhill: n. 1til) B.: Windwark. side: 11. 1901 K.; Ladhlergut U-200 M.: 11. 201.s B.; grnt between Hells-

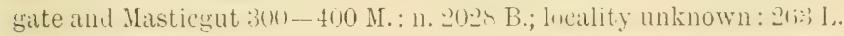

St. Martin: from Guanabay to Filipsburg: n. 2439 B; lowel part of Milldrumhill: n. 3170 B.

I also saw Sida carpinifolia on the hill between Mont des Accords and Centryhill; between Mount Vernon and Saline; near Grande Case; Mount Pitradis $300-400 \mathrm{M}$.

St. Croix ex Millspaugh.

Antill., Florida, Bermud., Cuba and Guatemale to Nova Gramata and Brazil, trop. Afr., Canary Islands, East Indies. (Gris. Fl.).

Sida ciliaris Linn. Sist. Lil. X $(1-2.9) 114$.$) ; (iris. Fl. T3; Dusion$ 64; Millspaugh 509.

St. Eustatius: on the top of the ('litt' letween Oranjestall aml Fort do Wind: 11. 756 B.; near Maryglory 150 M.: n. 1153 B.; near Boven 200 M.: 11. 1245 B. 
St. Martin: from Filipsburg to Bulvedere: 11. 2562 B.; between Mount Turnon and orientbay: n. gatis B.; lower part of Colebayhill: n. $470 \mathrm{~A}$.

Anguilla Boldingh.

St. Croix ex Milispaugh.

Antill., Venezuela. (Gris. Fl.).

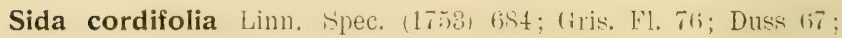
Millspaugh 509. Mash mellow.

St. Eustatius: Bengilen: 13. 4i23 13; Cultururvlakte: n, .11, 524; English Quarter: n. 510; locality nnknown: n. 3582 B., 182 G.

Saba: Windwardside-Hellsgate: n. 1662 B, Hellsgate: n. $1695 \mathrm{~B}$.

St. Martin: Prince quarter (coll. George): n. 2462 B.; from Filipsburg to Belvedere: 11. 2.5.) B.: Nalkellhoyhill 50-200 M.: 11. 2679 B.: from Belvedere to oysterpond: n. :304.s B.; along roilds from Grande Case to Marigot: n. 3094 B; locality unknown: n. $2597 \mathrm{~B}$.

St. Croix ex Millspangh.

Antill., Cuba and Mexico to Peru and Brazil., Cape Verde Islands, Niger, Nubia, Cape, East Indies, Canton. (Gris. Fl.).

Sida glomerata (ily. Diss. I (1755) 1s t. 2 f. li.; (tris. Fl. 7.:; Duss 64.

St. Eustatius: locality unknown : 13. $275 \mathrm{H}$.

Bermud., Antill., Mexico. (Hemsley 1884).

Sida rhombifolia Linn, spee. (175:3) 484; (iris. Fl. it; Inss 75; Millspaugh 50 ?.

St. Martin: from Flat Point to Windwardside: n, $2148 \mathrm{~B}$; Mount Paradis: n. 3203 B.

Trop. countries of both hemispl.. (Ind. Kew.).

Sida spinosa Linn. Spee. (175) tisi); (iris. Fl. it; Duss 64; Millspaugh 509.

St. Eustatius: Bengalen: n. (il) B.; hill near Concordia 100 M.: n. 945 B.; Cultuurvlakte: n. 131, 205.

St. Martin: Cul de Sac: 11. Dol6 B.; near Bethlehem: 11. 26rit B.

St. Croix ex Millspaugh.

Afr., Asia, America. (E. a. P.).

Sida supina L'Hérit. Stirp. Nov. (17Rt) 109bis, t. 50; (ilis. Fl. 75; Millspaugh 509.

St. Eustatius: along roads in Oranjestad: n. 918 B.; near Maryglory 150 M.: n. 1155 B. 


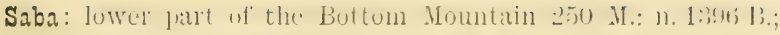

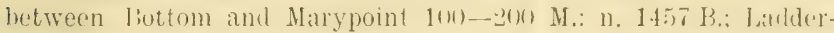
gut 200 M.: n. 2005 B.; l'eperpot: n. 658.

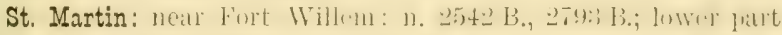
of the hills letween Wont Chambord and Hount Vernon ; n. 2.94s 13.; along roads in Cul de Sac: n. $2967 \mathrm{~B}$.; lower part of the Milldrumbill: n. 3168 B.

St. Croix ex Millspaugh.

Antill., Haiti and Mexico to Venezuela and Lima, Galapagos Islands. (Gris. Fl.).

\section{Urena Linn.}

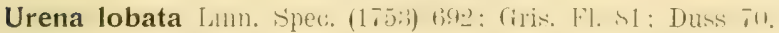

Urena americana Linn. Pl. Surin. II (1775); Millspaugh 509.

Saba: between Bottom and Marypoint 300 M.: n. 1559 B., 1472 B.; Mountain 800 M.: 11. 1749 B., 1803 B., 19, 31.

St. Croix ex Millspaugh.

In all tropical countries. (E. a. P.).

\section{Pavonia Cav.}

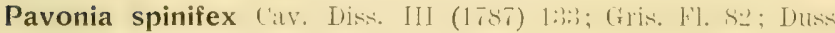
72; Millspaugh 508. Ginger bush.

Saba: lower part of the Bottom Mountain 250 M.: n. 1401 B.; Windwardside: n. 1592 B., Crespeen-Rendez-vous 400 M.: n. 29; Middle island: $\mathrm{n}, 30$.

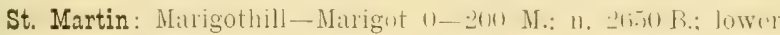
part of Mount Paradis: n. 3188 B., 3302 B.

St. Croix ex Millspaugh.

Bermud., Antill., Amer. merid. (Hemsley 1884).

\section{Hibiscus Linn.}

Hibiscus esculentus Linn. Spec. (1753) 696.

Cultivated. [In all trop. countries]. (Ind. Kew.).

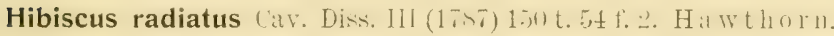
Cultivated. [Asia and Austral. trop.]. (Ind. Kew.).

Hibiscus Rosa-sinensis Lim. Apec. (17.7i) ti!t. H ibisı.

Cultivated. [Trop, countries of the old world]. ([nd, Kerw.).

Hibiscus Sabdariffa Linn. Spec. (1753) 695.

Cultivated. ['Trop. countries of the old world]. (Ind. Kew.). 
Hibiscus schizopetalus Hook. f. Bnt. Mag. (1880) t. 6524. Basket hibisc.

Cultivated. [Afr. trop.]. (Ind. Kerv.).

Hibiscus tiliaceus Linn. Spec. (1753) 694.

Paritiam titiaceum St. Hill kl. Bras. Mer. 1 (1827) 256; Gris. F1. 86; Duss 79; Millspaugh 508.

St. Eustatius: at the seamore near Concordialiay: n. 1089 B.

St. Martin: Mount Paradis $300-400$ M.: n. 3326 B.

St. Croix ex Millspaugh.

All tropical countries. (E. a. P.).

\section{Abelmoschus Médik.}

Abelmoschus moschatus Mélik. Malv. (178i:) 46.

Cultivated. [Trop. countries of the Old world]. (Ind. Kew.).

\section{Thespesia Soland.}

Thespesia populnea Solankl. ex Correa in Ann. Mus. Pitr. IX (1807) 290; Gris. Fl. 87; Duss 79; Millspaugh 509.

St. Eustatius: Seashore near ()ranjestal: n. 30 B.; seashore near T'umbledowndicklay: rı. 15. B.; slope of the (quill 50 M.: n. 35.

Saba: Bottom: n. 2246 B., 36; locality unknown: n. $233 \mathrm{~L}$.

St. Martin: Cul de Sac-Marigothill 0-201) M.: n. $2625 a$ B.

St. Croix ex Millspaugh.

Antill., East Indies, North coast of Austral., New South Wales, Senegambia. (Gris. Fl.).

5020. Gossypium Linn.

Gossypium barbadense Jinn. ipee. (175i3). ti!3. (1) rele cotton.

Cultivated. [Amerika]. (H.a. P.).

\section{Bombacaceae.}

5023. Adansonia Linn.

Adansonia digitata Limn. Sper. (1753); 1190. (i ul in e a ta mar. in t.

[Africa]. (E. a. P.). 
5026. Ceiba Médik.

Ceiba pentandra Gïrtn. Fruct. II (1791), 244 t. 133.

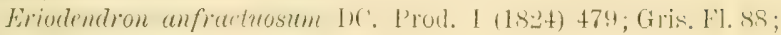
Duss 80; Millspaugh 510. Silk cotton tree.

St. Eustatius: 'Tol' of the Quill $4(x)$ M.: 1]. $22 \pm$ B.; in a garlen in Oranjestad: n. $321 \mathrm{H}$.

I also saw Ceiba pentandra; at the seashore near Oranjestad: Bottom of the Quill.

St. Martin: Cul de Sac: n. 2619 B.

I also saw Ceiba pentandra: near Grande Case; Villée des Pères.

St. Croix ex Millspaugh.

Nexico, Antill., Guiana, Afi., Malay, (E. a. P.).

Also cultivated.

\section{Quararibea Aubl.}

Quararibea turbinata Poir. Eneyel. Supp]. IT. (5:31.

Myrodia twrbinate Siv. Prod. Veg. Ind. Oce. (1788) 102; Gris. F1. 88; Duss 82; Millspaugh 510. Mi 11 e w o o d.

St. Eustatius: Bottom of the quill: n, tute B., sinke 13, 11:3 H, $217 \mathrm{G} ., 118,119,120,121,122$.

St. Croix ex Millspaugh.

Brazil. (Ind. Kew.).

\section{Sterculiaceae.}

\section{Melochia Limn.}

Melochia nodiflora Sw. ['rou. Tex. Int. (1)e. (17)s, $: 17$; (iris. Fl. 94; Duss 87; Millspaugh 510.

St. Martin: Top of the hill on the northern silte uf cul ile sat. 200-300 M.: n. 2989 B.: Tintamarre: n. 3392 B.

St. Croix ex Millspaugh.

Antill., Mexico, Panama. (Gris. Fl.).

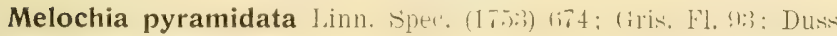
86; Millspaugh 510.

St. Eustatius: along roarls in Oranjestad: n. 11106 B.; slope uf the Quill: n. 198, 199; Cultuurvlakte: 11. 197, 200; in a gurden in Oranjestad: n. 200, 39 G.

St. Croix ex Millspaugh.

Baham., Antill., Mexico, Texas, Venezuela, Guayaquil, Brazil. (Gris, Fl.). 
Melochia tomentosa Lium. Syst. eu. X (1759) 1140) (iris. Fl. 93; Duss 86; Millspaugh 510. Black toa reh.

St. Eustatius: Nlope of the lower part of Signalhill: n. 102 B.; Signallill 200 M.: 11. 1:27 B.: along rouds in Oranjestad: 11. 9:2 B.; along ruats near Concordiabay: n. 975 B; Cultumvlakte: 12. 2114; along roads: n. $232 \mathrm{H}$.

Saba: I saw Melochia tomentosa on Crispeenlill.

St. Martin: from Filipsburg to ciuanabiy: 11. 235t B.; l'rince suarter (coll. George): 11. 2472a B.; Fort Willem 0_50 M.: 25.22 B., $2795 \mathrm{~B}$; lower part of the hills between Mont Chambord and Mount Vernon: n. 2945 B.; Colebayhill: n. 205; Prince quarter: ก. 116 .

I also saw Melochia tomentosa: along roads from Nakedboyhill to Filipslurg; Nakedboyhill; from Belvelere to Oysterpond; Tintamare.

Anguilla Boldingh.

St. Croix ex Millspaugh.

Antill., Cuba and Mexico to Tenezuela and Brazil. (Gris. Fl.).

\section{Waltheria Limn.}

Waltheria americana lim. Spec. (175:3) 673: (1)is. Fl. 95; Duss ti. ITaltheria indica Linn. Spec. (1753) 673; Millspaugh 511. Mash mellow.

St. Eustatius: Seashore nuar Orinjestarl n. it B.; top of the Quill 400 M.: n. 503 B.; in a garden in Oranjestad: 17. 509 B.; Bengalen: 1]. $619 \mathrm{~B}$; slopo of the lower part of the Quill near Bengalen: 11. T:O 13; on the Cliff hetween oranjestad and Fort de Wind: n. 757 B., $763 a$ B.; Pongohill: 11. 43; slope of the Quill: n. $44,45,46$; Cultuurvlakte: 11. 42, 47; along roads in Oranje. stad: n. 1 is $1 \mathrm{H}$.

Saba: between Bottom and Marypoint 100 -200 M.: 11. 1528 B.; Windwardside-Hellsgate: 11. $1685 \mathrm{~B}$.

St. Martin: near Fort Willem: n. 2794 B.; in an orchard near Bellevue: n. 2844 B.

Anguilla Boldingh.

St. Croix ex Millspaugh.

In tropical and subtropical countries. (Hemsley 1884).

\section{Ayenia Loefl.}

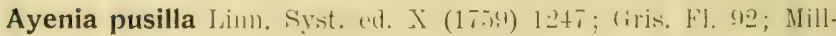
spaugh 510 . 
St. Eustatius: 'Top of the Clifl between Gallowshay and White Wall: n. $761 \mathrm{~B}$.

St. Martin: from Filipsburg to Point Blanche: n. 3400a B.

St. Croix ex Millspaugh.

Antill., Peru. (Ind. Kew.).

\section{Theobroma Lim.}

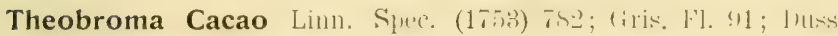
85; Millspaugh 510. Coco

St. Eustatius: Bottom of the Quill 350 M.: 1]. 446 B., 1217 B.; Quill : n. 12\%, 124, 125, $102 \mathrm{H} ., 214 \mathrm{G} ., 221 \mathrm{G}$.

Saba: Windwardside: n. 1937 B.

St. Croix ex Millspaugh.

Amer. cent, and merid, Curaçao(?) (E. a. I'.).

\section{Helicteres Linn.}

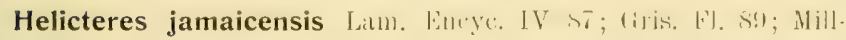
spaugh 510.

Helicteres Isora Linn. Spec. (1753) 963.

St. Martin: Prince quarter: n. 501.

St. Croix ex Millspaugh.

Antill, Asia et Austral. trop. (Incl. Kew.).

\section{Ochnaceae.}

\section{Sauvagesia Linn.}

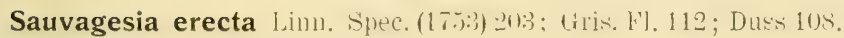

Saba: Mountain 800 M.: n. 1821 B., 2209 B., 134; gut between Hellsgate and Masticgut 300-400 M.: n. 2025 B.

In all tropical countries. (E. a. P.).

\section{Marcgraviaceae.}

\section{Marcgravia Linn.}

Marcgravia umbellata linn. Spee, (175), s13; (iris. l\%l. 110; Duss 106.

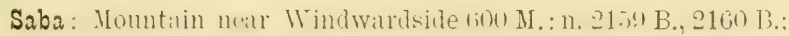
Mountain 800 M.: n. 2207 B., 58, 59; Mountain near Hellsgate 500 M.: n. 2275 B., 2276 B.

Antill., Guiana and Columbia. (Urban 1896.). 


\section{Theaceae.}

5135. Ternstroemia Mutis ex Linn. f.

Ternstroemia peduncularis D(: in Mem. Suc. P'hys. Genèv. I (1822) 409.

Ternstroemia ellipkica Sw. Prod. Veg. Ind. Occ. (1788) 81; Gris. Fl. 103; Duss 96; Millspaugh 511.

St. Eustatius: Top of the Quill 400 M. : 11. 5 B., 22s B., 248 B.. $26+$ B., 323 B., 359 B., 401 B., 504 B., 222, 22:3, 22t, $225,227,228$ : inner slope of the Quill 350 M.: 11. 12.2S B.; locality unknown: n. $130,226$.

St. Martin: Lowlands: n. $3271 \mathrm{~B}$.

St. Croix ex Millspaugh.

Antill., Mexico, Panama. (Gris. Fl.).

Freziera Sw.

ef. 5157. Eurya 'thunb. subg. II.

Freziera undulata Wille. S1). Plant. II. (17!9) 1179; (His. Fl. 104: Duss 97.

Saba: Mountain S00 M.: n. 2211 B., 6t, 65.

St. Kitts, Montserrat, ciuadeloupe, Dominiea, Martinique, st. Vincent, St. Lucia, Grenada, Trinidad. (Urban 1896).

\section{Guttiferae.}

\section{4 . Marila Swartz.}

Marila racemosa siw. Prod. Veyg. Ind. Oce, (17sis) st; (iris. Fl. 111; Duss 107.

Saba: Mountain 800 M.: n. 1829 B.

frualeloupe, Martini(ye, Dominica, it. Vincent. (Herl). Krug et Urban).

\section{Mammea Linn.}

Mammea americana Limn. Spec. (1758) 512; (iris. Nl. 108; Duss 102; Millspaugh 511. Ma mie.

St. Eustatius: along the lower part of Kignalhill: n. $120 \mathrm{~B}$; inner slope of the (uuill 3.0 M.: n. 461 B.; slope of the Quill near Bengalen 150-200 M.: n. (6.51 13,; Quill: n. 9; locality unknown: n. $70 \mathrm{H}, 184 \mathrm{G}$.

Saba: Boohyhill 3100 M.: n. 1619 B.: locality unknown: n. 114 I. I also saw Mammea americana: Hellsgate. 
St. Martin: I saw Mammea americana: Mount Paralis $300-400$ M.

St. Croix ex Millspaugh.

Antill., Guiana, Nova Granata; in warmer parts of Amer. Afr. ind Asia cultivated. (Vesque 1893).

Also cultivated.

\section{Clusia Linn.}

Clusia alba Jacq. Enum. Pl. Carib. (1760) 31; Gris. Fl, 107; Millspangh 511. Wild mamie.

St. Eustatius: Top of the Quill 400 M.: n. 193 B., 376 B., 8, $10,100 \mathrm{H} ., 185 \mathrm{G}$.

Saba: between Bottom and Marypoint 350 M.: n. 1489 B;

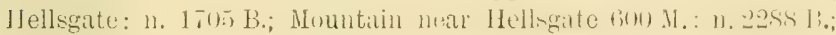
locality unknown: $11.357 \mathrm{~L}$.

I also saw Clusia alba: on Gates Hill; in Gut between Ifellsgate and Masticgnt.

St. Croix ex Millspangh.

St. Kitts, Martinique, Dominica, Guarluloupe. (Vesque 15\%3).

Clusia rosea Jacq. Enum. Pl. Caril) (1760) 34; Gris, FJ. 107; Duss 99; Millspaugh 511.

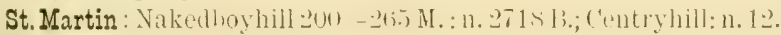

St. Croix ex Millspaugh.

Baham., Antill., Venezuela, Panama, Carolina. (Vessue 1893).

Clusia spec. nov. without flowers and fruits.

Saba: Greathill: n. 11.

\section{Bixaceae. \\ 5249. Bixa Linn.}

Bixa Orellana Limu, Fipece. (175:) 512; (iris. Fl. 20; Duss 14; Millspaugh 511. Roucou.

St. Eustatius: in a garden in uranjestad: 11. $533 \mathrm{~B}$ B., $140 \mathrm{H.}$ $231 \mathrm{G}$.

Saba: lower part of the Bottom Mountain 300 M.: n. 1426 B.; Bottom: n. 1850 B., 2241 B.; locality unknown: n. 245 L., $292 \mathrm{~L}$.

St. Croix ex Millspaugh.

Indig. in Amer. trop. (E. a. P.). Also cultivated.

\section{Canellaceae.}

5254. Canella Swartz.

Canella aiba Murr. Syst. et. XIV (175t) 44:3; (iris. F. 109; Duss 103; Millspaugh 511. Pepper cinnamom. 
St. Martin: Colebay (coll. George) : n. 2503 B., 2505 B.; shore of the Simsonsbaylagonn near Cul P'ipuard: n. 27+1) B.; near Mulletpond: 13. 27til B.; near Tischrijver: n. 2926 B.: ncar Simsonsbaylagoon: 11. 303\$ B.: Millụmhill $8001-400$ M.: n. 3126 B.; Lowlands: n. 3282 B.

St. Croix ex Millspaugh.

Florida, Antill. (E. a. P.).

\section{Flacourtiaceae.}

\section{Prockia Linn.}

Prockia Crucis Linn. Syst. ed. X (1759) 107 \&.

Cultivated. [Amer. trop.]. (E. a. P.).

\section{Xylosma Forst.}

Xylosma buxifolium A. liray in Mrom. Am. Acall. N. S. VIII. (1860) 155.

Myroxylon burifolium Krug et Urbin in Additamenta ad ('ognitionem Florae Inliac occidentalis I (189:3) 300; Duss $1 \mathrm{5}$. Cockspur.

St. Eustatius: Top of the Quill 450 M.: n. 373 B., 43. Baham., Antill. (Urban 1893).

\section{Samyda Linn.}

Samyda serrulata Linn. Spee. (1762) 55s; (iris. l'l. 25); Duss 17 ; Millspaugh 512. Banso, Wild guave.

St. Eustatius: Mignalhill 1.in M.: 11. 1025 B.; near Maryglory 150 M.: n. 1146 B.; slope of the Quill on the side of White Wall 200 M.: n. 1169 B.

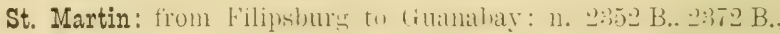
2390 B.; Prince quarter (coll. George): n. $2425 \alpha$ B., 2458 B.; Fort Willem: n. $2532 a$ B.; Cul de Sac: n. 2605 B.; Nakedboyhill 50-200 M.: n. 2687 B.; 200-265 M.: n. 2723 B.; lower part of the hills hetween MInt ('hambosd and Mount Vernon: 11. 2951 B.; Mount Paradis 200-400 M.: n. 3207 B.; 100-200 M.: n. 3301 B.; 200-300 M.: n. 3305 B.; locality unknown: n. $361+$ B.; Centryhill: n. 30, 88; Colebayhill: n. 32; Vineyardhill: n. 31, 33; French frontier: n. 89 .

I also saw Samyda serrulata: near Saline; from Belvedere to Oysterpond; along roads near Grande Case; Lowlands.

St. Croix ex Millspaugh.

St. romingo, Portorico, st. 'Thomas, Antigua, st. Billthélemy, Trinidad. (Herb. Krug et Urban). 
5343 . Casearia Jace.

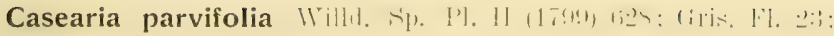
Duss 16; Crack open.

St. Eustatius: Top of the Quill 400 M.: n. 220 B., 236 B., $299 \mathrm{~B}$; ; top of the Quill $500 \mathrm{M} .: \mathrm{n} .496 \mathrm{~B}$; ; slope of the Quill near Bengalen 150 M.: n. 677 B., 682 B.; slope of the Quill near Glassbottle: n. 867 B.; Bottom of the Quill 300 M.: n. 892 B.; top of the Quill: n. 160, 166; locality unknown: n. $196 \mathrm{G}$.

I also saw Casearia parvifolia: slope of the Quill on the side of White Wall.

Saba: along roals between Buttom and Windwatride end M. : n. 1345 B.; Greathill 2-400 M.: n. 163; Paris: n. 161, 162; Gainbygut: 11.164 ; Cowpasture: n. 165; locality unknown: n. $327 \mathrm{I}$.

I also saw Casearia parvifolia: Forthhaygut; gut between IIellsgate and Hastiegut; Springbaygut.

St. Martin: Marigothill-11arigot (1)-20) M. : n. Mountain between Mont des decorils and Centryhill 200-300 .I.: n. 2894 B.; Mount Paradis 200-400 M.: n. 3310 B.; Centryhill: n. 29; John Ednyhill: n. 28; French frontier: n. 27.

I also saw Casearia parvifolia: on the hills on the northern side of Cul de Sac; from Belvedere to Oysterpond.

St. Croix ex Millspaugh.

Antill., Guiana, Pernambuco. (Gris. Fl.).

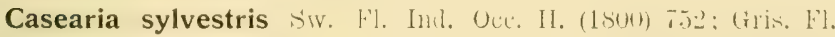
2:3; Millspaugh 512.

St. Martin: Hill on the northern side of Cul de Sac 0-100 Nf: n. 3005a B.; 200-300 M.: n. 3011 B.; Hount Paradis 300-400 M.: n. $3201 \mathrm{~B} ., 3241 \mathrm{~B}$.

St. Croix ex Millspaugh.

In tropical America. (E. il. P.).

\section{Turneraceae}

5357. Piriqueta Aubl.

Piriqueta villosa Aub]. Pl. Gui. I (1775) 298 t. 117.

Piriqueta cistoides G. F. W. Mey ex Steud. Nom. ed. II (1S41) 344; Gris. FI. 298; Duss 314.

St. Eustatius: along roads near English Quarter: n. $997 \mathrm{~B}$, $998 a$ B.; slope of the Quill on the side of White Wall 200 M.: n. $1185 \mathrm{~B}$; ; slope of the Quill: 13. 237, 238; locality unknown: n. 236 .

Antill., Cuba and Panama to Brazil. (Gris, Fl.). 


\section{Turnera Linn.}

Turnera ulmifolia Linn. Fipece. (17) spaugh 512.

St. Eustatius: White Wall: 11, sos I); gut near Fort le WVind: n. 835 B.; near Boven 200 M.: n. 1237 B.; White Wall: n. 240; Greatgut: n. 241.

Saba: Flat Point: n. 2134 B.

St. Martin: between Mount Fermon and orientlay: n. 20.9.) B.: Tintamarre: n. 3403 B.

St. Croix ex Millspaugh.

From Mexico and the Antill. to Argentinia. (E. a. P.).

\section{Passifloraceae.}

\section{Passiflora Linn.}

Passiflora foetida Limn. Flec. (175:3) 95: (iris. l'l. 294 : Duss 313; Millspaugh 512.

St. Eustatius: lairplay: n. 2305; seashore: n. 1974, 2331; road near Sugarloaf: n. 2397.

Saba: Gut near Marypoint: n, 2048 B.

St. Croix ex Millspaugh.

Antill., Cuba and Mexico to Brazil. (Gris. Fl.).

Passiflora laurifolia Linn. Fivec. (175.3) 956; Gris. H'l. 293; Duss 312; Millspaugh 512. Bell apple.

St. Eustatius: T'op of the Quill 400 II.: 11. 249 B., $290 \mathrm{~B}$; ; in a garden in Oranjestad: n. $526 \mathrm{~B}$.; slope of the Quill near Bengalen 250 M.: n. 701 B.; Cultuurvlakte: n. 1439; slope of the Quill: n. 1426; Boven: n. 85; locality unknown: n. 1429, 1442, $1443,24 \mathrm{G} ., 79 \mathrm{H}, 144 \mathrm{G}$.

Saba: Bottom: n. 2272 B.; Jenkygut-Hellssate: n. 87; locitlity unknown: n. 2 L., $170 \mathrm{~L}$.

St. Martin: Mount Paradis 300-400 M.: n. 3333 B.

St. Croix ex Millspaugh.

Antill., Nova Granata, Guiani, Brazil. (Gris. Fl.). Also cultivated.

Passiflora maliformis Linm. Apec. (175.3) 45ti; Gris. Fl. 293; Duss 313.

Saba: Mountain 200 M.: n. 1904; locality unknown: n. 305 L. Antill., Haiti to Nova Granata (Gris. Fl.). 


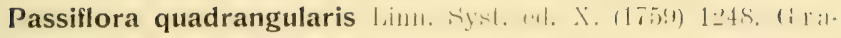
nadilla.

Cultivated. [Antill., Panama, Peru, Brasil.]. (Gris. Fl.).

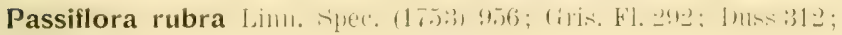
Millspaugh 512. Snakeberry wine.

Saba: between Bottom and Harypoint 300 M.: n. 1488 B., 1494 B.; Crispeen 400 M.: n. 1764 B.; gut between Hellsgate and Masticgut 300-400 M.: 11. 2024 B.; Mountain 500 M.: n. 2217 B.

St. Martin: lower part of Mount Paradis: n. 3292 B.

St. Croix ex Millspaugh.

Antill., Panama, Venezuela, Nova Granata. (Gris, Fl.).

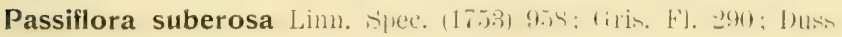
311; Millspaugh 512.

St. Eustatius: T'op of the Quill 400 M.: n. 192 B., 313 B.,

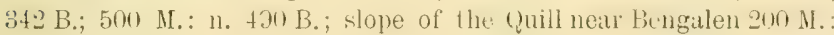
n. $690 \mathrm{~B}$.; slope of the lorrer part of the Quill near Bengalen: n. $713 \mathrm{~B}$; gut near Fort de Wind: 50 M.: n. 837 B.; slope of the Quill: n. 2417; locality unknown: n, $188 \mathrm{H} ., 271 \mathrm{G}$.

Saba: Windwardside-Hellsgate: 17, 1665 B.: Bottom: n. 254t5, 2571; locality unknown: n. $314 \mathrm{~L}$.

St. Martin: Nakedboyhill 200-260 M.: n. 2721 B., 2734 B.; near Mulletpon?: n. 2757 B., 2int I3.; in an orchard near Bellevue: 11. 2si3t B.; Mountain between Mont des Accords and C'entryhill $200-300$ M.: n. 2871 B.

Anguilla Boldingh.

St. Croix ex Millspaugh.

Antill., Panama, Venezuela. (Gris. Fl.).

\section{Caricaceae.}

5377. Carica Linn.

Carica Papaya Lim. Spec. (1753) 1026. Pa pao.

Cultivated. [Amer. trop.]. (E. a. P.).

\section{Begoniaceae.}

5397. Begonia Linn.

Begonia domingensis DC. in Ann. Sie. Nat. Sil IV. II (1S5!n) 124 (vel. affin.); Gris. Fl. 304.

St. Eustatius: Top of the Quill 450 M.: n. 382 B., 472 B.; Bengalen: n. 193, 194; Quill: n. 192. 
Saba: Mountain 600-800 M.: n. 1787 B., 1809 B., 2212 B., $169,189,190,191$; locality unknown: n. $173 \mathrm{~L}$.

Dominica, Haiti. (Gris. kl.)

\section{Cactaceae.}

\section{5 t01. Cereus Mill.}

Cereus grandiflorus Mill. Gard. Dict. ed. VIlI (176is) 11. 11: Gris. Fl. 301; Duss 317; Millspaugh 513.

St. Eustatius: Jenkinsbay: n. $1253 a$ B.

Saba: Bottom: n. 2242a B.

St. Croix ex Millspaugh.

Jamaica, Caraiben, Haiti, Vera Cruz, Mexico, Sonora. (Scluumann 1903).

Cereus Peruvianus Mill. (iard. Diet. el. VIII 11768$)$ n. 4.

St. Eustatius : in a garden in Oranjestad.

St. Martin: near Bethlehem: n. $3438 \alpha$ B.

Antill., Mexico, 13ras., Guiana. (Schumann 1903).

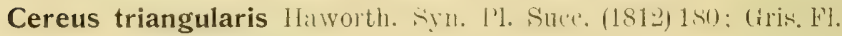
301; Duss 317; Nillspaugh 513.

Cereus compressus Mill. Gard. Dict. ed. VIII (1768) n. 10. Slingercactus, Straw bery.

Saba: St. John's: n. $1871 a$ B.; Windwardside: n. $1917 a$ B.; near Devilshand: n. $2097 a$ B.

St. Martin: near Oysterpond: n. 30S\%) B.; Nount Paralis: n. $3322 a$ B.

St. Croix ex Millspaugh.

Antill., Florida, Mexico, Rio de Janeiro, Asia. (Schumann 1903).

\section{Cereus spec.}

St. Eustatius: n. 141, 153 .

Saba: locality unknown: n, $1660 a$ B.

\section{Melocactus Link et Otto.}

Melocactus communis Link et otto in Verh. P'reluss. Vur. (iartenb. III (1827) +17 t. 11; (iris. Fl. 300; 1)nss 317; Millspaugh 51\%. l'opehead.

St. Eustatius: I saw Melenactuc rommmuis: Top of Signallill ; near Tumbledowndick bay; Gilboohill.

Saba: I saw Melocactus communis: near the Sulphurmines.

St. Martin: 1 saw Melocartus eommunis: near Filipshurg; near (iuanahay; along roads from Filipshurg to Nakedhoylill; top of Nakedboyhill: Lowlands; Green Key. 
St. Croix ex Millspaugh.

Antill. (Schumann 1903).

\section{Opuntia Mill.}

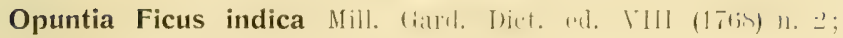
Gris. 11. 302; Sweet prickle.

St. Eustatius: along roads: n, $151 \mathrm{G}$.

I saw Opuntia Fiens indiea: in deserted plantations near Bengalen; roads allong the lower part of Signalhill; Signalhill.

Saba: I saw Opuntia Fiens indica: St. Giles; Latdergut.

St. Martin: I saw Opuntia Ficus indiea; near Filipsburg; Nakedhoyhill; Fort Willem; Green Key.

Mexico, warmer countrios of hoth hemisph. (Schumann 19n3). Opuntia spinosissima Mill. Ciaril. Dict. ed. Ifll (17tis) n. 8; Gris. Fl. 302; Duss 318; Millspauch 513.

St. Eustatius: I saw Opuntia spinosissima: on the top of the Clitf near Signalhill; on the White Wall.

St. Martin: I saw Opuntia spinosissima: Top of Nakedboyhill; Tintamarre.

St. Croix ex Millspaugh.

Ind. Occid. (Schumann 1903). Baham. (Hitchc.)

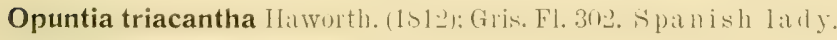

St. Eustatius: English Quarter: n. 1004a B.; Gilboohill: n. $1126 a$ B.; Jenkinsbay: 11. 1230 a B.

I saw Opuntia triacantha: Slope of Signallill; roads along the lower part of Signallitl; top of the Cliff between Oranjestad and White Wall.

Saba: Paris: 1n, $1384 a$ B.

I also saw Opuntia triacantha: Laddergut.

St. Martin: near Fort Willem: n. 2551a B.; near Nakedboyhill: n. $2577 a$ B.

I also saw Opuntia triacantha: near Filipsburg; along roads near Beth. lehem; one of the ishands in Simsonsbaylagoon.

Ind. Occid. (Schumann 1903).

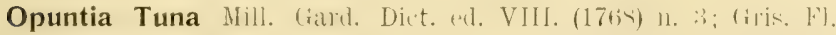
302; Duss 318; Millspaugh 513. Sour prickle.

St. Eustatius: along roads: n. 152. Island.

Saba: I saw Opuntia Tuna: Giles gut; near the Sulphurmines; Green

St. Martin: I saw Opuntia Tuna: Nakedboyhill; Fort Willem: one of the Islands in Simsonsbaylagoon.

Indial Oceil., Californiat: (Schumann 1903). 
5418. Nopalea Salm-Dyck.

Nopalea coccinellifera Salm-Dyck Cath. Hort. Dỵtk. eu. II (1850) 64. Curaçao prickle.

Cultivated. [Mexico]. (Schumann 1908).

\section{Peireskia Milll.}

Peireskia aculeata Mill. (iarl. Dict. ed. VIII (176s).

Cultivated. |warmer countries of Amerika|. (Nehunramn 190:3).

Peireskia bleo DC. Prod. III (1828) 475.

Cultivated. [Nova Granata, Brasilia]. (Schumann 1903).

\section{Thymelaeaceae.}

\section{Daphnopsis Mart. et Zuc.}

Daphnopsis caribaea Gris. Flor. W. I., (1860) 278; Duss 297. Malio.

St. Eustatius: Top of the Quill 400 M.: n. 197 B., 251 B., $397 \mathrm{~B}$; s slope of the Quill on the side of White Wall $200 \mathrm{M}$ : 11. 1177 B.; Quill: n. $15 t$.

Saba: along rouls between Bottom and Windwardside $200 \mathrm{~N}$.: 11. 1350 B.; (ireathill $400 \mathrm{M}$.: 3. 1386 B.; seaside of 'T'ntguthill 101) M.: n. 1654 B.; Hellswate: n. 1699 B.: Springbaygut: n. 2077 B.; Paris 2-400 M.: n. 233, 234, 235.

I saw Daphmopsis carihaea: Gut hetween Hellsgate and Masticgut; Saddle.

St. Martin: Cul de Sac: 11. 2604 B.; Milldrumhill 200-300 M.: n. 3119 B., 3124 B.; Mount Paradis 300-400 M.: n. 3240 B.

I also saw Daphnopsis caribaea: Top of Nakedboyhill; near Saline.

St. Croix. (Symb.).

Portorico, St. Thomas, St. Kitts, Martinique, Guadeloupe, Margareta, Dominica. (Herb, Krug et Urban).

\section{Lythraceae.}

\section{Lagerstroemia Linn.}

Lagerstroemia indica Limn. Spec. (176-2) 7ist. Queen of Crib. flower.

('ultivated. Japan, ('hina, Molukk., Australiat. (Koehme 14031.

\section{Lawsonia Linn.}

Lawsonia inermis Linn. Spec. (1753) 349. M iminet. Cultivated. [Africa, Asia, Australia]. (Koehne 1903). 


\section{Punicaceae.}

5501. Punica Limu.

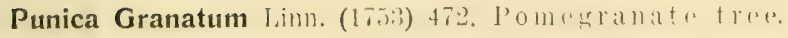

Cultivated. [Balkan and Himalaya]. (E. a. P.).

\section{Rhizophoraceae.}

5523. Rhizophora Linn.

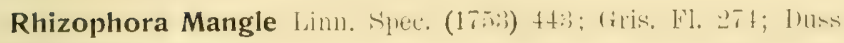
292; Millspaugh 514.

St. Martin: near Colebay (coll. George): n. 2498a B.; from Filipslurg to Simsonsbay: n. 3027 B.

I saw Rhizophora Mangle: at Simsonshaylagoon; Oysterpond.

St. Croix ex Millspaugh.

America. (E. a. P.).

\section{Combretaceae.}

5541. Quisqualis Linn.

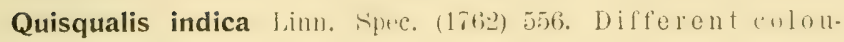
red jessamine.

Cultivated. IInd. orient., Ins. Philipp., Afr. trop. orient., Guinea). (E. a. P.).

5544. Terminalia Linn.

Terminalia Catappa Linn. Mant. II (17-1) 51!. A I mond tree. Cultivated. |Madagascar, Malay arch., Nova (inineal. (E. a. l’.).

\section{Conocarpus Gärtn.}

Conocarpus erectus Limn. Spec. (175:3) 76; Cris. Fl. 277; Dust 295; Millspaugh 516. Button wood.

St. Eustatius: Seashore at Venusbay: 1, $970 \mathrm{~B}$.

St. Martin: fiom Filipshurg to (inumilay: 11. 237s B.; Coluhay (coll. George.): n. 2515 B.; shore of the Simsonsbaylagoon near Cul Piquard: n. 2747 B.; near Simsonsbayvillage: n. 3029 B., 3029 B.; Simsonsbay: n. 160.; Lowlands: n. 3266 B.

Anguilla Boldingh. 
St. Croix ex Millspaugh.

Antill., Florida to Brazil., (ialipagos, Marianne Islinds, trop. coast of Africa. (Gris. Fl.).

\section{5̆1. Laguncularia Gürtn.}

Laguncularia racemosa (iartn. f. Fiuct. III (1505) $209 \mathrm{t}$. 2) 17 f. 2; Gris. F'. 276; Duss 295̄ Millspaugh 516.

St. Martin: Colehay (coll. George): 11. $24 ! 7$ B.; from kilipshure to Cul de Sac along the Saltpond: n. $2612 \mathrm{~B}$; shore of the fimsonsbaylagoon near Cul l'ipuard: 11. 궁 B.: along the Simsons-

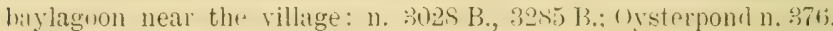

Anguilla Boldingh.

St. Croix ex Millspaugh.

Amer: trop. to Florida, trop. Africa. (E. a. P.).

\section{Myrtaceae.}

5559. Psidium Linn.

Psidium Guajava I.inn. Snec. (175) t7u; Gris. I'l. 241; Duss 261; Millspaugh 515. Guava tree.

St. Eustatius: in a garden in Oranjestad: 11. $512 \mathrm{~B} ., 41 \mathrm{H}$, $48 \mathrm{G} . ;$ slope of the Quill: n. $62,63,64$; road near English Quarter: 17. 41.

Saba: Boobyhill 350 M.: 17. 1615 B; Ladder: n. 40; Longpeak: n. 61 ; locality unknown: 1. $119 \mathrm{~L}$.

1 also silw Psidium Guajava: in a gut between Hellsgate and Mastiegut: between Bottom and Marypoint; Crespeenhill.

St. Martin: from libipsburg to Belvelere: n. ass B.; cul de Sac: n. 60.

I also saw Psidium Guajava: Marigothill to Matigot; Nakcdboyhill; lower part of Milldrumhill; Mount Parulis 3-400 M.; Lowlinds.

Anguilla Boldingh.

St. Croix ex Millspaugh.

Baham., Antill. from Mexico to Brasilia. (Urban 1895).

\section{Amomis Berg.}

cf. 5566. Pimenta Lindl, sect. I.

Amomis caryophyllata Kl. et [ $H h$, in Lrbin. Ailditamentil ad

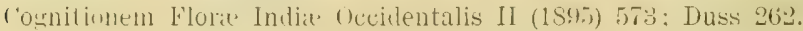

Pimenta acris Kostel. Allg. med. pharm. Fl. IV (1835) 15226; Gris. 11. 241; Millspaugh 515. Cinnamom tree, Bay berry. 
St. Eustatius: Top of the Quill 500 M.: n. 493 B., 56, 57 ; Bengalen: 11. 53.

Sapa: between Bottom and Marypoint 300 M.: $n, 1479$ B., 1557 B.; Booly hill :300 N1.: 13. 1.).4; B.; Botton: 11. 19.97 B.; Greathill: n. 54; Bottom: n. 51, 52; Masticgut: n. 55.

St. Martin: Millưumhill $300-400$ M.: n. 3156 B.

St. Croix ex Millspaugh.

Venezuela, Guiana; cult, in Baham. and Ind. Orient. (Urb. 1895).

\section{Myrcia DC.}

Myrcia paniculata Kr. et lirb), in (Yhan Alditimenta all cognitionem Florae Indiae Occidentalis II (1895) 577; Duss 263.

Myrcic coriaced DC. Prodr. IIJ (1828) 243; Gris. Fl. 23t; Millspaugh 515. Redwood, Birdsberry.

St. Eustatius: Top of the Quill 400 M:: n. 173 B., 188 B., 260 B., 346 B., 505 B.; locality unknown: n, $114 \mathrm{H}$.

Saba: Puris 400 M.: n. 1369 B.; Mountain near Windwardside 600 M.: 11. 2180 B.; locality unknown: n. 1568 B., 140 L.; Wellgut: $n, 43$.

St. Martin: Nakedboyhill 50-200 M.: n. 2715 B; lower part of Mount Paradis: n. 3189 13.: Mount Paradis $300-400: 11$. 3221 F., 3352 B.; Lowlands: n. 3268 B.

St. Croix ex Millspaugh.

Antill., Brazil. (Urb. 1895).

Myrcia splendens DC. P'rodr. III (1.24) 2utt; (iris. Fl. 234: Duss 265. Surinam cherries.

St. Eustatius: Top of the Quill 400 M.: n. 200 B., 285 B., 3.) B.; inner slone of the Quill 350 M.: n. 4:0 B., 1218 B., 12.2 B.; slope of the (quill near Bengalen in-200 M.: 14. (i.st B.; slope uf the Quill neat Bengalen 150 M.: n. 684 B.; slope of the Quill 150 M.: n. 1112 B; Quill: n. 44, 46, 47; slope of the Quill: 11. 45; Little Mountain: n. 44; locality unknown: n. 177 G., 213 G.

Saba: along roads 200-300 M.: n. 1711a B.; Windwardsicle: n. 1945 B; Hellsgate-Marypoint: n. 2043 B*; Crespeen-Rendezvolus: 1 . 48 .

St. Martin: Mount Paradis 150-400 M.: n. 3195 B.; Tintamarre: n. $3396 \mathrm{~B}$.

Cuba, Jamaica, Haiti, St. Domingo, Portorico, St. Thomas,

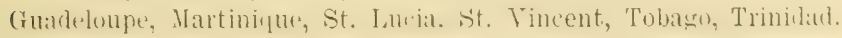
(Urban 1895). 
5575. Calyptranthes Swartz.

Calyptranthes Boldinghii $\mathrm{TH}^{\mathrm{H}} \mathrm{H}$, in Symbolie Antillana VI (1909) 2:2.

St. Martin: Lowlands: n. 3270 B.

\section{Eugenia Linn.}

Eugenia axillaris Willd. Spec. II (1s00) 9io; (ris. Fl. 236; I) 270; Millspaugh 514. Choakyberry, Pigeonberry.

St. Eustatius: Slope of the duill near Bengalen , in-200 M.: n. 641 B.; Gilboohill 150 M.: n. 1120 B.; Boven: 11. 19, 21; top of the Quill: n. 20; locality unknown: n. $55 \mathrm{H}$.

Saba: T'op of the Cliff near the seaside of Boolyyhill: n. 1607a B.; Mountain 400 M.: n. 17t0 B.; Windwardside: n. iss:3 B.; Ladedergut: 11. 2000 B.; Springbaygut: n. 2117 B.; Greathill: n. 22.

St. Martin: from Filipsturge to liuallibay: n. 2392 B.; from Belvedere to Oysterpond: 11. 30.5; B., 3072 B.; Lowlands: 11. 3263 B.; Tintamarre: n. 3388 B,; top of Centryhill: n. 39.

Anguilla Boldingh.

St. Croix ex Millspaugh.

Bermud., Florida, Key, Bahain., Antill. (Urban 1895).

Eugenia cordata DC! Prohl. III (I 298$)$ 27:; rar. Sintensii Kr. et Urb. in Additamenta ad Cognit. Hl. Ind. Oce. (1895) 656.

Éugenia sessiliflora DC. Prodr. III (1828) 273; Gris. Fl. 236.

St. Eustatius: from Signalbill to Venusbay: n. 9t0 B.: Gilboohill: n. 1121 B.; near White Wall: n. 1266 B.

St. Croix. (Symb.).

St. Domingo? Portorico, St. Thomas, St. Jan, T'ortola, Antigua, Martinique, st. Lucia, S't. Vincent, Mustique Island, Becquia Island. (Urban 1895).

Eugenia floribunda IVest st. Croix (1793) 290); Gris. FJ. 239; Duss 273; Millspaugh 514. Guaveberry.

St. Eustatius: locality unknown: n. $200 a$ G.; 40.

St. Martin: in an orchard near Bellevue: n. 2831 B.; Mount Puradis $300-400$ N.: n. $3320 a \mathrm{~B}$.

St. Croix. (Urban 1895).

Antill., Guiana, Brazil. (Urban 1895).

Eugenia fragrans Willd. Spec. (1800) 964.

Anamomis fragrans Gris. Flor. W. I. (1860) 240.

Anamomis punctata Gris. Flor. W. I. (1860) 240.

St. Martin: Top of Nakedboylill: n. 2715 B.

F'orida, Antill. (Urban 1895). 
Eugenia Jambos Linn. Spec. (1753) 470.

Jambosa vulgaris DC. Prod. III (1828) 286; Gris. Fl. 235; Duss 274.

Jambosa Jambos Millspaugh 515. Plum rose.

St. Eustatius: Slope of the (quill neial Bengalen 50-2010 M.: n. 644 B., 26 ; locality unknown: n. $103 \mathrm{H}$.

Saba: Crespeen-Renclez-yous 400 M.: 11. 27 ; locality unknown: n. 109 L., $321 \mathrm{l}$.

I also saw Lugenia Jambos: Bottom; Laddergut.

St. Martin: Mount Paradis 300-400 M.: n. 3347 B.

St. Croix ex Millspaugh.

Indig. in Asia austro-orient. and Australia. (Urban 1895).

Also cultivated.

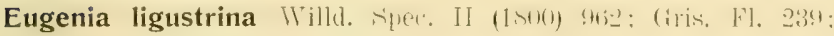
Duss 268; Millspaugh 514. Blackbery, Blackcherry.

St. Eustatius: deserted plintations near Tumhledowmickhay: 11. 148 B.; slope of the Quill 350 M.: n. 392 B.; top of the Quill 500 M.: n. 473 B., 497 B.; hill near Concordia 100 M.: n. 952 B.; from Little Mountain to Venuslay: n. 986 B., 992 B.; slope of Signalhill: n. 28; Quill: n. 29; Venusbay: n. 30; top of the Quill: n. 31,32 ; locality unknown: n. $109 \mathrm{H}$.

St. Martin: from lilipsburg to finanabay: n. 2411 13,; Prines unarter foll. (ieorgel: n. ats+ B.: from Filipshurg to Belvedere: n. 2584 B.; Cul de Sic: n. 2607 B.; lower part of the hills between Mont Chambord and Mount Vernon: n. $29 \pm 6$ B., 2952 B.; Milldrumhill 300-400 M.: 1. 3137 B.; Lowlands: n. 3280 B.; near Fort Amsterdam: n. 36; Vineyardhill: n. 33, 35; Prince çuarter: 1.34 ; Guanabay: n. 34 .

St. Croix ex Millspaugh.

Baham., Antill., Brazil. (Urban 1895).

Eugenia monticola DC. Prodr. III (1828) 275.

Engenia Poiretii Berg, in Linnaea XXVII (1856) 186 (excl. syn. Cand., Poir., Spreng.); Gris. Fl. 236; (ex descr.).

Eugenia obtusata Willd. ex Berg. in Linnaea XXVII (1856) 240; Gris. 11. 237 ; (ex deser.).

Eugenia buxifolia Gris. Flor. W. I. (1850) 236; (excl, syn.), - non Willd.

Eugenia pallens Gris. Flor. W. I. (1860) 237; (specim. Guad.), - non DC. (Symb.). Duss 269; Millspaugh 515. Birdsberry. 
St. Martin: Filipsburg near the Saltpond: n. 2:365 B.; Nakedboyhill 200 M.: n. 2585 1.: Marigothill: n. 2609 B.; neal simsonsbayligoon: 11. 2745 13.; near Mulletponat: 1]. 2itis B.; lill between Mont des Accords and Centryhill: n. 2863 B.; near Oysterpond: n. 3073 B.; Milldrumbill: n. 3116 B.; Mount Paradis: 11. 3220 B , 3330 B. ; Lowlands: 11. 3283 B.

Anguilla Boldingh.

St. Croix ex Millspaugh.

Baham. (Hitchc.); Antill. (Urban 1899).

Eugenia procera [oir, in Lam. Enc. Suppl. III (1S13) 124: Gris. Fl. 238; Millspaugh 515. Bastard black-berry.

St. Eustatius: deserted plantations near Thmbledownlickbay: n. 149 B. ; Signallhill 200 N.: n. 1037 B.

Saba: along roads betwern Bottom and Windwardside 200 M. : n. 1356 B.; Springloaygut: n. 2098 B.; Peporpot: n. 38.

St. Martin: along roals frum Cirande Case to Marigot; 1 . 3100 B. : Mount Paradis 300-400 M.: n. 3321 B.; Colebayhill: n. 37.

St. Croix ex Millspaugh.

Cuba, Jamaica, Haiti, St. Domingo, Portorico, St. Thomas, St. Jan, Antigua, Martinique, Barhados. (Urban 1895).

Eugenia rhombea Kr. et Urh. in UTrban. Adkitamenta ad Cognitionem Florre Ind. Occid. JII (1895) 665.

St. Eustatius: Seinpost: n. 39; Wittehoek: 11. 40. Florida, Key West, Babam., Antill. (Urban 1895).

Eugenia uniflora Limn. Spec. (175:3) 470; Gris. Fl. 2:3!. Honeyberry, Surinam cherries.

St. Eustatius: Flope of the lower part of Signalhill: n. $117 \mathrm{~B}$; deserted plantations near Tumlledowndicklay: 11. $143 \mathrm{~B}$; top of the Quill 400 M.: n. 172 B., 177 B., 326 B.; in a garden in Oranjestad: 11. $861 \mathrm{~B}, 55 a \mathrm{~B}, 76 \mathrm{H}$.

Saba: lower part of the Bottom Mnuntain 2.00 M.: 11. 1424 B.; Crispeen 400 M.: n. 1720 B.; locality unknown: n. 70 L.

St. Croix ex Millspaugh.

Floricla, Antill. (Urban 1895).

\section{Melastomataceae.}

5633. Tibouchina Aubl.

Tibouchina strigosa Cugn, in Monogr. Phan. VII. (18!1) 266.

Chaetogastra strigosa DC. Prod. III (181S) 134; Gris. Fl. 266. 
Chactorgestra chironioides Gris. Flor. W. I. (1860) 267 (p. p.); Duss 288.

Tibouchina ornate Baill. Adansonia XII (1877) 74.

Saba: 'Top of the Mountain 800 M.: 11. 18,

Antill., British Guiana. (Cogniaux 1891).

5749. Charianthus D. Don.

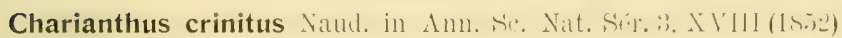
III; Gris. H1. 264.

Charianthus noclosus 'Triana Melastom. in 'I'rans. Linn. Soc. XXVIII (1871) 99, var crinitus Naud; Duss 286.

Saba: Nountain 600-800 M.: n. $178+$ 13.; 187 L., 197 L., 203 L. $1,2,3$.

Antill. (Cogniaux 1891).

\section{Tetrazygia Rich.}

Tetrazygia discolor DC'. I'rod. IIl (152s) 17.2; (iris. Fil. 2.5. Duss 280.

St. Eustatius: 'l'op of tho Quill: 11, 16, 17.

Saba: lower part of the Bottom Mountain 300 M.: n. 1436 B.; between buttom ans Marypoint 110-gon M.: n. 1551 13.; (iainbygut: $11,14,15$.

Antill., French Guiana (Cogniaux 1891).

\section{Miconia Ruiz. et Pav.}

Miconia impetiolaris D. Don. in Mem. IVern. Suc. IV (1823) 316 ; Gris. Fl. 256; Duss 28t; Millspaugh 516.

St. Eustatius: Top of the (Uuill 400 M.: 11. :310 B.: Botton of the Quill 300 M.: n. 469 B.

St. Croix ex Millspaugh.

Antill., Bolivia, Panama, Costarica, Honduras, Guatamala, Mexico. (Cogniaux 1891).

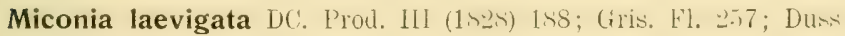
281; Millspaugh 516.

St. Eustatius: 'T'op of the Quill 400 M.: n. 208 B., 262 B.,

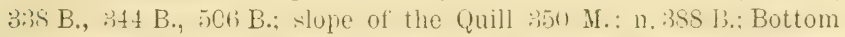
of the Quill :300 II.: 11. t52 B.; slope of the Quill near Bengalen 200 M.: n. 742 B.; slope of the Quill: n. 9, 10, 11.

Saba: lower part of the Bottom Mountain 250 M.: n. 1406 B.; Windwardsike-Hellkgate: n. 1652 B., 164:3 B.; Wountain S00 M.: n. $1825 a$ B., 2216 B.; Springhaygut: n. 2119 B.; Peperpot: n. 8 ; 
Nountain: 17. 5; Crespeen--Rendez-vous 400 M.: n. 6, 7; locality unknown: n, $41 \mathrm{~L}, 216 \mathrm{~L}, 306 \mathrm{~L}$.

St. Martin: Top of the hill on the northern side of $\mathrm{Cul}$ de Sac 200-300 M.: n. 3006 B.; along roads from Grande Case to Marigot: 11. 3100 B.; Millummhill: 11. 3180 B.; Mount Paranlis $300-400$ M. : n. 3208 B., 3341 B.

St. Croix ex Millspaugh.

Antill., Mexico, Cruatanala, Costarica, Venezueli, Surinam, Guian., Brazil. (Cogniaux 1895).

\section{Araliaceae.}

5883. Panax Linn.

Panax fruticosum Linn. Spec. (1763) 1513.

Cultivated. [Asia trop., Malaya, Polynes.] (Ind. Kew.).

\section{Umbelliferae.}

5923. Eryngium Linn.

Eryngium foetidum Limn, spec, (175:3) 23:2 Gris. 1'l. 308; Duss 3:4.

Saba: between Botton and Maryloint 100-21)( MI.: 11. 1537 B.; Mountain near Windwardside to0 M.: n. 2176 B.: locality unknown: n. $37 \mathrm{~L}$.

St. Martin: Top of the hill on the northern side of ('ul the Sac $200-300$ M.: 11. 298; B.; Mount Paradis 200-400 M.: n. $320 z$ B.

Antill., Cuba and Panama to Nova Granata and Brazil. (Gris. Fl.).

6062. Foeniculum Adans.

Foeniculum vulgare Mill. Garil. Dict. wi. VIIl (ITis) n. 1. Cultivated. [Europa]. (Ind. Kew.).

\section{5a. Theophrastaceae.}

\section{Jacquinia Linn.}

Jacquinia armillaris Jacu. Enum. 1'l. Ciril. (1760) 15; (iris. L'l. 397; Duss 383; Millspaugh 517. Picrous bark.

St. Eustatius: White WVall: 1). su- B, hill near Concordia: n. 953 B.; seashore near Venusbay: 969 B., 284, 285.

St. Martin: from Filipsurg to fuanalay: 2418 13.; Prince uzuarter (coll. (itorge): n. 2.098 B.; near Mulletpond: 11. 27rt B.; Mountain between Mont des Accords and Centryhill: 11. 2908 B.; between Mount Vernon and Orientbay: 11. $2929 \mathrm{~B}$; from Belvedere to 
Oysterpond: 11. 3061 B.; Mount Paradis 200-300 M.: 11. 3304 B.; near Colebayhill: n. 65, 137; Prince quarter: 1). 606; Guanabay: n. 63,192 .

St. Croix ex Millspaugh.

Cuba, Jamaiea, St. Domingo, Portorico, St. 'Thomas, St. Jan,

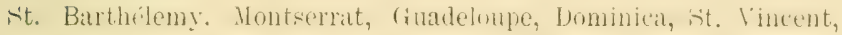

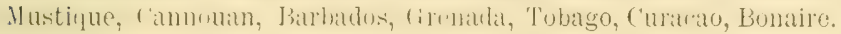
(Symb.).

Jacquinia Berteri Spreng. Syst. I (1825) 668.

St. Martin: near Mulletpond: n. 2781 B.; Lowlands: n. $3283 a \mathrm{~B}$.

Anguilla Boldingl.

Baham., Antill. (Syml).).

\section{Myrsinaceae.}

6285. Ardisia Swartz.

Ardisia coriacea Sw. Prod. (1788) 48; Millspaugh 517.

Ardisia guadalupensis Duchass. et Walp. apud Gris. Flor. W. I. (1861) 396; Duss 38\%. Bastard cinnamom.

St. Eustatius: T'op of the Quill 400 M.: 11. 196 B., 244 B., 399 B., 44 ; locality unknown: 1]. 30, 45.

Saba: Hellsgate: 11. T711 B,; gut hetween Masticgut and Hells. gate 300-400 M.: n. 2032 B.; Jenkygut: n. 46 ; Hellsgate: n. 43 ; locality unknown: n. $76 \mathrm{~L}$, $183 \mathrm{~L}$.

St. Croix ex Millspaugh.

Portoricr, st. Thomas, st. Jan, Tortula, (fuadelulupe, Drminica. Martinique, St. Lucia. (Symb.).

\section{Rapanea Aubl.}

Rapanea ferruginea Me\% in Symholat Antillanlte II (1901) 429\%. Myrsine laeta Gris. Flor. W. I. (1861) 392.

Saba: T'op of the Mountain 800 M.: n. 1836 a B; Rendez-vous Mountain (5) M.: 11. J150 B.; Mountain near Hellsgate: 11. 227 B. Antill. Amer, trop. from Mexico to Bolivia. (Symb.).

\section{Plumbaginaceae.}

6312. Plumbago Linn.

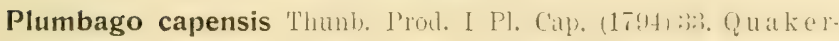
blossom.

Cultivated. [Afr: australis]. (Ind. Kew.). 


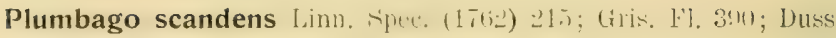
380; Nillspaugh 517. Blister bush.

St. Eustatius: Feashore near (1)anjestad: 11. 1:3 B., t2 B.; along roads in Oranjestad: n. 561 B.; in deserted plantations at the seashore near Bengalen: n. 592 B.; gut near Fort de Wind: $n$. S45 B.; Cultuurvlakte: 354 ; locality unknown: n. 31 H., 35 G.

I also saw Plumbago scandens: along the lower part of Signallill; top of Signalhill,

Saba: Bottom: n. 1852 B.; Laddergut 200 M.: n. 1995 a B.; Peperpot: 1). 149, 356: Ladder: 11. 35:; locality unknown: 11. $319 \mathrm{~L}$.

I also saw Plumbago scandens: Forthbaygut.

St. Martin: along roads in Prince quarter: n. 2933 B.

St. Croix ex Millspaugh.

Antill., Mexico to Brazil and Chili, Galapagos, (Gris. Fl.).

\section{Sapotaceae.}

\section{Achras Linn.}

Achras sapota Limn. Syst. I ed. II (175!1) ISS; Millspitugh 517. Sapota Achras Mill. Gard. Dict. VIII ed. (1768) n. 1; Gris. Fl. 399; Duss 385. Mispel, Sapodille.

St. Eustatius: lower part of Signalhill: 11. $115 \mathrm{~B}$.

St. Croix ex Millspaugh.

Baham., Antill., Curaçao from Mexico to Guiana. (Symb.). Also cultivated.

6363. Lucuma A. DC.

Lucuma multiflora A. DC. Prodr. VIII (1St4) 168.

Saba: near Hellsgate: n. 1675 B.; Windwardside: 11. 2313 B.

St. Croix. (Symb.).

Portorico, St. Thomas, St. Kitts, Montserrat, Guadeloupe, Martiniıue. st. Lucia, it. Vincent, (irenata, Trinidad. (Fymb.).

\section{Sideroxylon Linn.}

Sideroxylon foetidissimum Jaç. Enum. (17ti(0) 15.

Sideroxylon mastichodendron Jacq. Coll. II (1788) p. 253 t. 17

f. 5; Gris. Fl. 399; Duss 386; Millspaugh 518. Mastictree.

Saba: Hellsgate: 1). 304! R.; Masticgut: 1) t4 : Jenkygut: 11. 41).

St. Croix ex Millspaugh.

Florida, Key, Baham., Antill. (Symb.). 
6373. Dipholis A. DC.

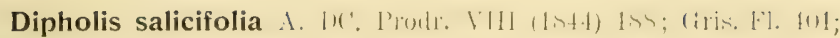
Duss 38S; Millspaugh 518.

St. Eustatius: Slope of the Quill on the side of White Wall 200 М.: n. 1168 B.

St. Croix ex Millspaugh.

F'lorida, Key, Baham., Antill., Yucatan. (Symb.).

\section{Bumelia Sw.}

Bumelia obovata A. DC. Prodr. VIII (1844) 191.

Bumelia cuneato Sw. F'lor. I (1797) 496; Gris. Fl. 401; Duss 613; Millspaugh 517.

St. Eustatius: from Little Mountain to Venushay: (SS:) B.; locality unknown: n. $338 \mathrm{H}$.

Saba: lietween bottom and Marypoint 100--206) M.: 11. 1535) B.

St. Martin: near Mulletpond: 11. U76.. B.; at the seatshore of Simsonsbay: n. 3035 B.; Colebayhill: n. 49.

St. Croix ex Millspaugh.

Jamaica, St. Domingo, Portorico, St. 'l'homas, T'ortola, St. Barthelemy, Antigul, Montserrat, (iuadeloun, Desirade, Martinique, St, Lucia. (Symb.).

\section{Chrysophyllum Linn.}

Chrysophyllum argenteum .July. Entum. (1760) 1.i; Duss ins.).

Chrysophyllum glabrum Jacq. Enum. (1760) 15; Gris. Fl. 398; Duss 385.

Saba: Gut near Devilshand: n. 2115 B.

Cuba, Haiti, St. Domingo, Portorico, Antigua, Montserrat, Guadelonpe, Marie-Galante, Dominica, Martinique, St. Vincent. Barbados, Grenada, Trinidad. (Symb.).

\section{Symplocaceae,}

6118. Symplocos. Jacq.

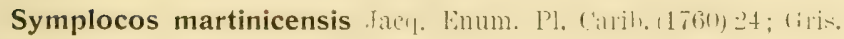
H.l. 403; Duss 389. Blue berry.

St. Eustatius: 'l'op of the Quill 400 M.: n. 297 B.; top of the Quill 500 M.: n. 474 B.; locality unknown: n. 126, 127, 128, 129.

Saba: Mountain near Windwardside 600 M.: n. 2152 B.. $2185 \mathrm{~B}$. 
Portorico, St. Thomas, St. Kitts, Montserrat, Guadeloupe, Do. minica, Hartiniciue, St. Lucia, St. Vincent, Grenada, 'Trinidad. (Brand 1901).

\section{Oleaceae.}

\section{Forestiera Poir.}

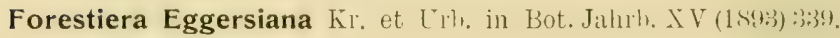
St. Martin: Prince quarter: n. 1, 2.

St. Thomas. (Urban 1893).

\section{Linociera Swartz.}

Linociera compacta R. Br. Prod. (1810) 523; (Hris. Fl. $f(15$. Bridgo tree.

Mayepea caribute O. Ktze Rev. Gen. (1891) 411; Duss 391; Millspaugh 518.

St. Eustatius: Top of the Quill 400 M.: 1]. 181 B., 261 B., $301 \mathrm{~B} ., 343 \mathrm{~B} ., 352 \mathrm{~B}$; ; slope of the quill near Bengalen 250 M.: 11. $700 \mathrm{~B}$; ; slope of the Quill near Glassbottle 150 M.: 1. $1113 \mathrm{~B}$, 1117 B.; Quill: n. 345 ; slope of the Quill: n. 343,344 ; top of the Quill: n. 336, 337; locality unknown: n. $77 \mathrm{H} ., 169 \mathrm{G} ., 40$.

I also saw Linociera compacta: Gilboohill.

Saba: lower part of the Bottom Hountain 250 M.: n. 1419 B., 1422 B; Jenkeygut: $n$. 340.

St. Martin: Milldrumhill: 11. $3151 \mathrm{~B}$.

St. Croix ex Millspaugh.

Antill., Guiana. (Urban 1893).

\section{Jasminum Linn.}

Jasminum azoricum Linn. Spec. (1753) 7.

Cultivated. [Ins. Azor, and Madeira]. (E. a. P.).

Jasminum pubescens Willd. Sp. Pl. I (1798) 37.

Cultivated. [Asia trop.] (Ind. Kerw.).

Jasminum undulatum Ker. Gawl. in Bot. Reg, t. 4t3t, Glebiatn jessamine.

Cultivatel. [Himalaya, China]. (Ind. Kew.).

\section{Loganiaceae.}

6453. Spigelia Linn.

Spigelia Anthelmia Linn. Spee. (17.53) 149; (1ris. 1\%. 331; Duss 334t. St. Eustatius: I saw Spigelia Anthelmia at Bengalen. 
Saba: lower part of the Bottom Hountain $300 \mathrm{MI}:$ 11. $1427 \mathrm{~B}$; between Bottom and Harypoint 100-200 M.: n. 1522 B., 1529 B.;

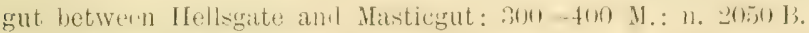

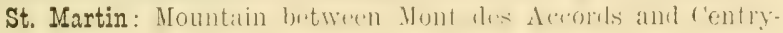
hill 200-300 M.: n. 2876 B.

Anguilla Boldingh.

Antill., Amol. austral. (E. a. P.).

\section{Gentianaceae.}

6541. Leiphaimos Cham. et Schlecht.

Leiphaimos aphylla (iilg in Engl, u. I'rantl. Nat. I'll. fiul, IV. 2. (1895) 104.

Voyria uniflora Pers. Syn. I (1805) 284; Gris. Fl. 425; Duss 402.

Voyria aphylla Pers. Syn. I (1805) 284.

St. Eustatius: 'Top of the Quill 400 M.: n. 296 B.; 450 M.: n. 365 B., 402 B.; top of the Quill: n, 40.

Saba: Mountain 800 M.: n. 1831 B., 2226 B.

Antill., Guiana, Brazil. (E. a. P.).

\section{Apocynaceae.}

6560. Allamanda Liun.

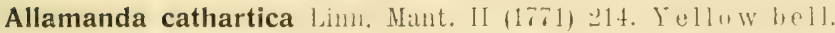
Cultivated. [Antill., Amer. centr. Brazil.] (E. a. P.)

\section{Plumiera Linn.}

Plumiera alba Limn. Ápec. (17.5) 210; Gris. l'l. 411; Duss 395; Millspaugh 518. White frangepane, Pigeonwood.

St. Eustatius: in a garden in Oranjestad: n. $49 \mathrm{H}, 67 \mathrm{G}$.

Saba: along roark hetween Bottom and Windwarside 2010 M.: n. $1354 \mathrm{~B}$.

St. Martin: from Filipsburg to Guanabay: 11. 237t B.; I'rince. quarter (coll. George): 11. 2464 B.; fort Willem: n. 2534 B.; near Fort Amsterdam: n. 237, 238.

I also saw Plumiera alba: along roads from Filipsburg to Simsonsbay; from Belvedere to Osaterpond; lower part of Milldrunhill: Lowlands: Tintamarre; gut from Bethlehem to Cul de Sac.

Anguilla Boldiugh.

St. Croix ex Millspaugh.

Portorico, St. Thomas, Guateloupe, Martinique, St. Lucia, Grenada. (Herb. Krug et Urban). 


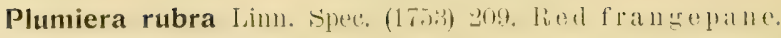
Cultivated. [Mexico and Venezuela]. (k. a. P.).

\section{Lochnera Reichb.}

Lochnera rosea lieichb. ('omp). (1828) 134.

'ince rusea Limn. syst. ed. I (1759) 9tt; Gris. I']. 410; Duss 395; Millspaugh 519. Churchyard blossom.

St. Eustatius: Sirashore near Oranjestad: n. 36 B.; along roads in Oranjestan: 11. 5il B.; slope of the Quill: 11.252 ; in a garden. 11. 34; locality unknown: 11. $22 \mathrm{G.,} 60 \mathrm{H} ., 330 \mathrm{H}$.

Saba: along roads letween Bottom and Windwardside 2110 M.: n. 1349 B.; Springbiygut: 11. 2104 B.; seashore near Forthlaylanding: n. 2146 B.; Ladder: n. 254; locality unknown: n. 74 L., 90 L.

St. Martin: along the saltpond near Filipshurg: n. 2440 B.; Colebay (coll. George): n. 2510 B.

I also satw Lochuera rosea: along roads near Bethlehen; along roads near Cirande Case.

St. Croix ex Millspaugh.

In all trop. countries. (E. a. P.).

\section{Tabernaemontana Limn.}

Tabernaemontana citrifolia Jaci]. Sel. Am. St. Hist. (176i3) t. 175 f. 13. p. 38; Gris. Fl. 409 ; Duss 395 . Milky tree.

St. Eustatius: 'l'op' of the Quill 40() M.: n. 23. B.; immer slope

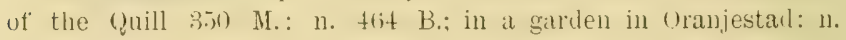
527 B.; slope of the Quill 300 M.: n. 886 B.; slope of the Quill near Glassbottle: n. 1207 B.; top of the Quill: n. 244, 245, 246.

Saba: along roads betwet'n Bottom and Windwardside 200 M.: 11. 1:57 B.; Windwardside-11ellswate: 11. 11i76 B.; Springbaygut: 11. 21:1 B.; Crispeen-Rendez-vous 410 M.: 11. 2+1; Hellsgategut: 11. 243; Malypointgut: n. 242.

St. Martin: neil Bethlehem: 11. 2671 B.; Mountain between Mont des Accords and Centryhill 0- 200 M.: 11. 29ti7 13; Mount Paradis $300-400$ M.: n. 3213 B., 3325 B.

Antill., Mexico. (Gris. Fl.).

Tabernaemontana coronaria IViliıl, Enum. Hort. Berol. (1814) 97.). Cultivated. [India orient.] (Ind. Kew.).

Tabernaemontana Cumingiana A. I)(. Prut. VIII (1844):373. Cultivated. [Ins. Philipp.] (Ind. Kew.). 


\section{Rauwolfia Iinn.}

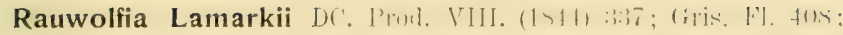
Duss 393; Millspaugh 51:. Snakeberry tree, Billy ache, Antigua loalsam.

St. Eustatius: T'op of the Quill 500 M.: n. 480 B.; slope of the Quill near Bengalen 50-200 M.: n. (5+7 B., 739 B.; gut near Fort de Wind 50 M.: n. 840 B.; along roads in Oranjestad: 11. 935) B., 1069 B.; near Boven: 200 M.: 1. 1235 B., 240 ; slope of the Quill 200 M.: n. 239 ; near Roundhill: 11. 37 ; locality unknown : n. $27,237 \mathrm{G} ., 208 \mathrm{H}$.

Saba: between Bottom and Marypoint 300 M.: 17. 1468 B; locality unknown: n. $270 \mathrm{~L}$.

St. Martin: from Filipslourg to Guanabay: n. 2861 B,; Fort

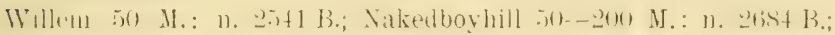
in pastureground near Bellevue: n. $2812 a$ B., 2825 B; lower part of Milldrumhill: n. $316+$ B.

I also saw Rauwolfia Lamarkii: along roads from Filipsburg to Nikedboyhill; near Saline; in pasture ground near Belvedere; Lowlands; Momnt I'atralis $100-200$ M.; Tintamarre.

St. Croix ex Millspaugh.

Antill., Eqquador. (Gris. Fl.).

66832. Thevetia Adans.

Thevetia neriifolia Juss. ex stemu. Nom, Ed, Il, II (1)it1) tiso. Cultivated. [Antill. and Mexico to Brazil.] (E. a. L').

\section{Urechites Müll.-Arg.}

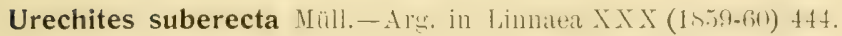
Echites suberecta Jacq. Enum. Pl. Carib. (1760) 13; Gris. Fl. 415; Millspaugh 518. Bitterpot.

St. Eustatius: in the tnip of the Clitl between hranjestal and Fort de Wind: 17. 759 B., 776 B.; Signalhill 200 M.: 1]. 1045 B.; Cultuurvlakte: n. 248; near Brookswell: n. 247; near Sugarloaf: n. 249 ; locality unknown: n. $181 \mathrm{H}$.

Saba: lower part of the Bottom Mountain 250 M.: 1. 1395 B.

St. Martin: Fort Willem 50 M.; 11. 25t!l P.; near Furt Amsterdam: 17. 250.

I also saw Urechites suberecta: Lowlands.

Anguilla Boldingh. 
St. Croix ex Millspaugh.

Cuba, Jamaica, Hispaniola, Portorico, St. Thomas, St. Kitts. (Herb. Krug et Urban).

\section{Nerium Linn.}

Nerium Oleander Linn. Spec, (175:3) 209. Oleander. Cultivated. [From Portugal to Mesopotamia]. (E. a. P.).

\section{Asclepiadaceae. \\ 6736. Cryptostegia $\mathrm{R}, \mathrm{Br}$.}

Cryptostegia grandiflora R. Br. in But. Reg. t. 4:3.) (181!).

Cultivated. [Patria ignot.] (Symb.).

\section{Asclepias Linn.}

Asclepias nivea Linn. Spec. (1753) 215; Gris. Fl. 419.

Asclepias Curassavica Linn. Spec. (1753) 215; Gris. Fl. 419; Duss 398; Millspaugh 519. I pecacuanha.

St. Eustatius: Locality unknuwn: n. $111 ., 134$ (i.; slope of the Quill: n. 52, 222, 223.

Saba: hetwern Bottom and Mirrypoint 100-200 M.: 18. 1517̈l B.; Paris 200-400 M.: n. 221; locality unknown: n. 160 L.

St. Martin: Milldrumhill 200-300 M.: n. 3120 B.; Mount Paradis 300-400 M.: n. 3329 B.; Mary's Fancy: n. 220.

St. Croix ex Millspaugh.

Baham., Antill.; widely dispersed in both hemisph. (Symb.).

6792. Calotropis $\mathrm{R} . \mathrm{Br}$.

Calotropis procera R. Br. in Ait. IJurt. Kew. Ed. '2 (1811) II 78; Gris. Fl. 420; Duss 399; Milspaugh 519. Liberty tree, Sprain leaf.

St. Eustatius: Top of the Quill 500 M.: 11. 475 B.; in deserter plantations near English (unarter: n. 198 B.; lneality unknown: n. $43 \mathrm{H}, 54 \mathrm{G}$.

Saba: along the seashore near forthbaylanding: n. 16iti B.; locality unknown: n. $113 \mathrm{~L}$.

I also saw Calotropis procera: Flat Point.

St. Martin: near Simsonshayvillage: n. 3255 B.; locality unknown: n. $3610 \mathrm{~B}$.

1 also saw Calotropis procera: on Mount Paradis 100-200 M. 
Anguilla Boldingh.

St. Croix ex Millspaugh.

Indig. in Africa sept. and Ind. Orient. (Symb.).

6798. Metastelma R. Br.

Metastelma parviflorum Ii. [3r. in Wern. fore. I (1.40!9) i); Duss 398.

Metastelma Paralias Done in DC. Prodr. VIII (1844) 514: Gris. Fl. 417 .

Metastelma Schlechtendalii Gris. Flor. IV. I. (1861) 417; (nec Dene); (Syml).); Millspaugh 519.

St. Martin: juxta salinas propo Filipsbourg, Febr. 1821: PJeri; (Symb.).

Lowlands: n. $3267 a \mathrm{~B}$.

Baham. (Hitche.); Antill. (Symb.).

6907. Hoya R, Br.

Hoya carnosa R. Br, in Jém. Wern. I (1809) 27.

Cultivated. [Ins. Malay.| (Symb.).

\section{Ibatia Dene.}

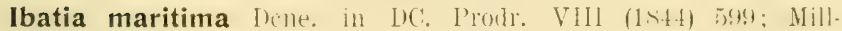
spaugh 519.

Ibatic muricate Gris. Flor. W. I. (1861) 421; Duss 400.

Lachonostoma maritimum Nichols. Dict. Gardn. Il 236. Mariapompoen.

St. Eustatius: Bengalen: 1n. 6i:34 B.; near Boven :200 M.: n. 1232 B.; locality unknown: n. 225 If, $277 \mathrm{G}$.

St. Martin: from Filipsburg to Guanabay: n. 2400 B.; near Fort Willem 50 M.: n. 2789 B.; Mountain between Mont des Accords and Centryhill 200-300 M.: n. 2861 B.

I also saw Ibatia maritima: Mount Paradis 100-200 M.

St. Croix ex Millspaugh.

Huiti, St. Domingo, St. 'I'homas, Antigua, Guadeloupe, Dominica, Martinique, St. Vincent. (Symb).

\section{Convolvulaceae.}

6968. Cuscuta Linn.

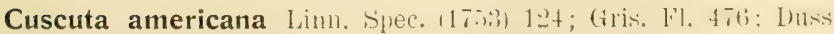
443; Millspaugh 519. Love wille. 
St. Eustatius: in deserter plantations at the seashore near Bengalen: 5si B.: Cultuurvakto: 11. 141 : seishure: n. 140 ; locality unknown: n. $99 \mathrm{H}, 186 \mathrm{G}$.

Saba: lower part of the Bottom Mountain 250 M.: n. 1413 B; locality unknown: n. $106 \mathrm{~L}$.

I also saw Cuscuta americana in Ladilergut.

St. Martin: from Filipsburg to (inamabay: 11. 234!) B.; from Filipshurg to Bulvelere: 11. 257s B.: butween Nont Chambord anil the Saline: n. 2922 B.: Cul de Sac: n. 295.

St. Croix ex Millspaugh.

Antill., Cuba and Mexico to Brazil. (Gris. Fl.).

Cuscuta umbellata H. B. ct K. Nos. (ien. ot ipee. III (1s1s) 121 ; Gris. H1. 476.

Saba: near Vischvijver: 11. $2922 \mathrm{~B}$.

Jamaica, New Mexico to Brazil (Gris. Fl.).

\section{Evolvulus Linn.}

Evolvulus argyraeus Choisy Conv. Rar. (1838) 153.

St. Martin: T'intamarre: 13. 3371 B.

Equador. (Ind. Kew.).

Evolvulus glaber Spreng. Syst. I (1825) 862.

Evolvulus mucronatus Sw. ex Wikst. in Vet. Acad. Handl. Stockholm (1827) 61; Gris. Fl. 475; Millspaugh 520.

St. Martin: along roals from Bethlehem to Mont Chamburd: n. 2916 B.; between Belvedere and Oysterpond: n. 3066 B.; near Fort Amsterdam: n. 150; locality unknown: n. 151.

St. Croix ex Millspaugh.

Baham., Antill., Portorico to Peru. (Gris. Fl.).

Evolvulus sericeus Sw. I'roul. Teg. Ind. Oec. (17ss) 5.); (iris. F'. 475 ; Duss 442.

Evolvulus incamus Pers. Syn. I (1805) 288.

St. Eustatius: I'op of the Quill: n. 380 B.; top of the Clifi between Oranjestad and Fort de Wiml: 1]. Tio B.; White Will : 11. ti?.

St. Martin: along roads from Colelatyhill to Simsonsbay: 11. 3014 B; Fort TVillem: n. $2793 a$ B ; 'Tintamare: 11. 3371 B.

Baham., Antill., 'Texas to Montevideo. (Gris. Fl.).

6986. Porana Burm.

Porana paniculata Roxb. Hort. Beng. (1\$1t) 13. II h it e coralita. Cultivated. [India orient. Malay.] (Ind. Kew.). 
6991. Jacquemontia Choisy.

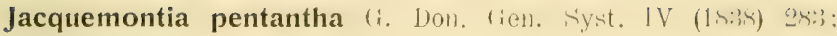
Millspaugh 521.

Jacquemontia violacea Choisy in Mém. Soc. Phys, Genèv. VIII 1. (1S38) 61; Duss 442.

Convolvulus pentanthus Jacq. Coll. IV. 210; Gris. Fil. 474.

St. Eustatius: Seashore near White Wall: n. 852 B.; near

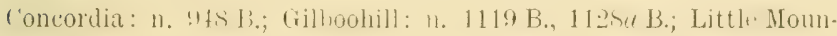
tain: n. $1163 a$ B.; Golden Rock: n. 55 ; locality unknown: n. $222 \mathrm{H}$.

Saba: Boobyhill: n. 1576 B.

Portorico, Becquia, St. Thomas, Barbuda, Gundeloupe, Marti. nique, St. Vincent, Grenada, Barbarlos. (Herb. Krug et Urban).

\section{Operculina Silva.}

Operculina ventricosa l'eter in Engl. 11. P'rantl. Nittïl. Pflan. zenfam. IV. 3a. (1891) 32. Hoofdpijn blad.

Cultivated. [Antill.] (Herb. Krug et Urban).

\section{Ipomoea Linn.}

Ipomoea arenaria Steud. Nom. Ed. II. 1. (1841) 81 J.

St. Martin: noar Mulletpond: n. 2777 B.; Lowlands: n. 8264 B.

St. Croix (Herb. Krug et Urian).

Cuha, Portorico, St. Thomas. (Herb. Krug et Urban).

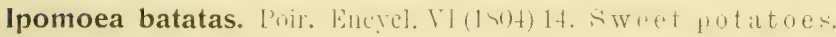
Cultivated. [Amer. centr.] (L. a. P.).

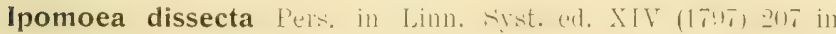
nota; Fl. 467 ; Duss 435.

Ipomoea sinuatu Orteg. Hort. Matr. Dec. (1800) 81; Millspaugh 520.

St. Eustatius: Slope of the Quill on the side of White Wall 200 M.: n. 1183 B; Fairplay: n. 1, 3; locality unknown: n. 164 G.

Saba: Bottom: n. 4.

St. Martin: Cul de Sac-Narigothill 0-200 M.: n. 2628 B.: French frontier: n. 2; locality unknown: 11. 3598 B.

St. Croix ex Millspaugh.

All trop. countries. (Gris. Fl.).

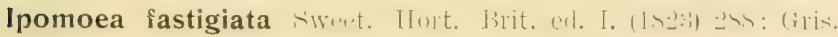
W1. t68; Duss 436; Millspaugh 520. Wild potatowine.

St. Eustatius: Slope of the Quill near Bengalen 200 M.: $n$. $689 \mathrm{~B}$; slope of the Quill: n. 177; locality unknown: n. $220 \mathrm{H}$., $226 \mathrm{G}$... $270 \mathrm{G}$. 
Saba: luwel part of the Bottom Mountain 300 M. : 11. 1445 B.; between Bottom and Marypoint 1(n)-20n M.: 11. 1464 B.: Mountain 600 M.: 11. 1745 B.; Laddergut $0-200$ M.: n. 2010 B; Windwardside: n. 2281 B.; Crespeen-Rendez-vous; n. 179; Rendez-vous 400 M.: 11. 178; locality unknown: n. 68 L., 218 L.

St. Martin: from Filipshurg to Gutnabay: 11. 2:391 B; along roads in Prince quarter: n. 2913 B.

St. Croix ex Millspaugh.

Antill., Cuba and Mexico to Brazil. (Gris, Fl.).

Ipomoea Nil liotl. Catalect. Fisc. I (1797) :36; (iris. l'l. 47:3; Duss 441.

Pharbilis Nil Choisy in Mem. Soc. Phys. Genèv. VI (1833) 441; Millspaugh 521.

Ipomoea hederacea Jacq. Coll. I (1786) 124.

St. Elastatius: inner slope of the Quill 350 M.: 11. 458 B.

Saba: Slope of the Hountain near tit. .'ohn's 300 M.: 11. 17:32 B.

St. Martin: Mount Paradis 100-200 M.: 1). 3294 B.

St. Croiz ex Millspaugh.

In warmer countries of both hemisph. (Hemsley 1884).

Ipomoea pentaphylla Jacy. ('oll. II (17.4.) 207\% (iris. l'l. 467 ; Duss 436; Millspauch 520. Noyeaux.

St. Eustatius: Cultumrvlikte: n. 196; focality unknown: n. 19) $11 ., 2) 1 \mathrm{H}$.

I also saw Ipomoea pentaphylla: on the slope of the Cliff.

Saba: Locality unknown: n. 30 L., 363 L.

St. Croiz ex Millspaugh.

Antill., Venezueli, Equador, Pacif. Isl., Asia trop. and Africa. (Gris. Fl.).

Ipomoea pes caprae lioth. Nor. ['. Sp. (18:1) 109; (iris. Kl. 470; Duss 438; Millspaugh 520.

Ipomoea biloba Forsk. Fl. Aegypt. Arab. (1775) 44. Sea wine.

St. Eustatius: Seashore near Oraujestald: 11. 10 B., 诖 B., 1.)2 H. ; seashore near White Wall: n. 857 B.; seashore: n. 162, 163.

I also saw Ipomoea pes caprae: Seashore near Tumbledowndickbay.

St. Martin: Guanabay: n. 2425 B.; near Fort Amsterdam: 1. 164 .

I also saw lpomoea pes caprite: at Simsonsbay.

St. Croix ex Millspaugh.

In all trop. and subtrop, countries. (IIemsley 18S4). 
Ipomoea sphenophylla Uib. in symulrolik Intillaniu I' (1908) 47.4.

St. Eustatius: Signalhill: n. 1038 B.

St. Eustatius. (Symb.).

Ipomoea violacea Linn. Spec. (1753) 161.

St. Martin: Tintamarre: n. 3374 B.

Venezuela, Antill. (Herb. Krug et Urban).

\section{Calonyction Choisy.}

Calonyction speciosum Choisy ('onv. ()r. (1sist) $5 \%$.

Ipomoea bona nox Linn. Spec. (1762) 288; Gris. Fl. 466; Duss 434; Millspaugh 520. Lilac bell.

St. Eustatius: slope of the (quill: n. 16:): locality unknown: n. $280 \mathrm{H}$.

St. Croix ex Millspaugh.

Amer, trop. (Ind. Kew.).

Calonyction tuba Colla. Nov. Spec. Calon. (1840) 15.

Ipomoea tuba G. Don. Gon. Syst. IV. (1838) 271; Gris. Fl. 467; Duss 434; Millspaugh 521.

Ipomoea grandiflora Lam. Tabl. Encyc. I (1791) 467.

St. Eustatius: on the slope of the Cliff near Oranjestad: n. $59 \mathrm{~B}$, ; seashore near Tumbledowndickbay: n. $163 \mathrm{~B}$.

St. Croix ex Millspaugh.

Antill., Guiana. (Gris. Fl.).

7005. Quamoclit Moench.

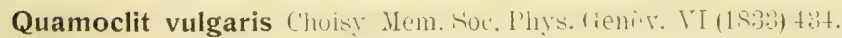
Cultivated. [India orient.] (E. a. P.).

\section{I. Hydrophyllaceae.}

\section{Hydrolea Linn.}

Hydrolea jamaicensis Raeusch. Nom. El. III (17iti) iti.

Nama jamaicense Linn. Spec. ed. X (1759) 950; Gris. Fl. 477; Duss 444 ; Hillspaugh 521.

St. Eustatius: near English Quarter: n. 1021 B.

St. Croix ex Millspaugh.

Bermud., Florida, 'lexas, Mexico, Antill. (Hemsley 1884).

\section{Borraginaceae.}

\section{Cordia Linn.}

Cordia salvifolia Inss. in l'oir. Encye. III (1suk) th: (iris. lil. 480 ; Duss 447. 
St. Eustatius: Littlo Mountain: 11. 1141 B., 115t B.; slope of the Quill above White Wall: 11. 1173 B., 1176 B.

Saba: Hellsgate: n. 1686 B.; St. Giles quirter: n. 1861 B. ; gut near Devilshand: 1]. 2088 B.

Guadeloupe, Martinique. (Herb. Krug et Urban).

Cordia Sebestena Linn. Spec. (175:3) 190. Searlet accoldia.

Cultivated. [Baham., Antill., Cuha to Guiana and Nosa Granata]. (Gris. Fl.).

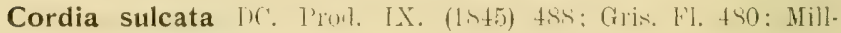
spaugh 522. Manjack.

St. Eustatius: Quill: n, 93.

Saba: between Bottom and Marypoint 300 M.: n. 1477 B.,

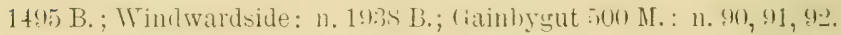

St. Croix ex Millspaugh.

Antill., Cuba to French Islands. (Gris. Fl.).

Cordia tremula Gris. Flor. W. I. (1861) 479.

Saba: from the Sulphurmines to Marypoint: n. 2030 a B.

Antigua, Martinique, St. Vinernt, Cirenada, Barbados, Tobago. (Herl. Krug et Urban).

\section{Beureria Jacq.}

Beureria succulenta Jact. Enum, Pl. Carib. (1760) 14; (itis. Fl. 481; Duss 449.

Ehretia Bourreria Linn. Spec. (1762) 275; Millspaugh 522. White chank.

Saba: 13ottom: 11. 1!183 B.: from íulphurmines to Marypoint: n. $2031 a$ B.; gut near Devilshand: n. $2093 a$ B.; locality unknown: ก. $307 \mathrm{~L}$.

St. Martin: Marigotroad near Marigot: 11. 25(1.) B., -2407 B.;

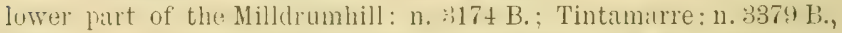
3350 3.; sut from bethlehem to ral de Sar (1-10n M.: n. 34t:3 B.; Colebayhill: n. 63 .

Anguilla Boldingh.

St. Croix ex Millspaugh.

Baham. (Horb. Leiden), Antill., Cuba to French Islands, Curaçao. (Gris. Fl.).

7045. Rochefortia Swartz.

Rochefortia acanthophora (iris. Flol: IV. I. (1.Sil) 4.52; Millspaugh 523.

St. Eustatius: Little Mountain: n. $1140 a$ B. 
St. Martin: Filipsturg near the saltponds: 11. 2356 a B. Mount Paradis: 17. $3244 \imath \mathrm{B}$.

St. Domingo, Portorico, St. Jan. (Herb. Krug et Urban).

\section{Tournefortia Linm.}

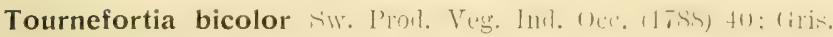
Fl. 483 ; Duss 451.

Saba: Bottom: n. 2237 B; Quarter: 11. 147; Gainhy gut: ก. 146 .

Antill., Cuba and Mexico to Brazil. (Gris. Fl.).

Tournefortia caribaea Gris. Flor. W. I. (186i1) 484.

Saba: Hellsgilte: n. 148, 149.

Dominica, Martinique, St. Vincent, Barbados. (Herb. Krug et Urban).

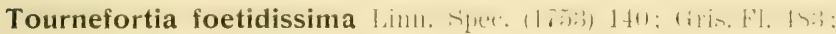
Duss 450; Millspaugh 523.

St. Eustatius: Bottom of the Quill: 300 M.: n. 443.

Saba: lorver part of the Bottom Mountain 300 M. : n. 1431 B.; Mumntain son H.: n. InI0 B.: gut between Hellogate and Hasti. gut 300-400 M.: 11. 2030 B.; Gainbygut: n. 150, 151; locality unknown: n. $186 \mathrm{I}$.

St. Martin: Marignthill: n. 2tit9e B. : Marigotruall: 11. 2sin; B.; hill on the northern side of Cul de Sac: n. 2996 B.; Mount Pa. radis: n. $3317 a$ B.; gut from Bethlehem to Cul de Sac: n. $3 \pm 11$ B.

St. Croiz ex Millspaugh.

Hispaniolih, Porturicu, Duminica, (ivanleloupr. Martinipue. (Herh. Krug et Urban).

Tournefortia gnaphalodes Li. Br. Prod. (1S10) 49t; Miris. Fl. 483; Duss 450; Millspaugh 523. White lavendel, Sea purs. lane.

St. Eustatius: White Wall: n. 809 B.; Concordiabay: 11. 15t; seashore: $n .267 \mathrm{H}$.

Saba: Bay: n. 66 .

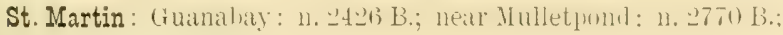
near Fort Amsterdam: n. 152.

I also saw Tournefortia gnaphalodes: Green Key.

Anguilla Boldingh.

St. Croix ex Millspaugh.

Bermud., Florida and throughout the Wrest Indies. (Hemsley 1884). 


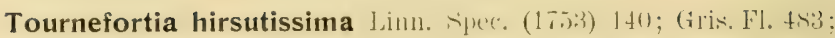
Duss 450 ; Millspaugh 523.

St. Martin: Hill on the northem sicle of Cul le sac 0-100 M.: n. $2976 \mathrm{~B}$; top of the hill on the northern side of Cul de Sac 200 -300 M.: n. 2990 B.; Hount Paradis $300-400$ M.: n. $33: 30$ B.

St. Croix ex Millspaugh.

Antill., Cuba and Mexico to Brazil. (Gris, Fl.).

Tournefortia volubilis Linn. Apec. (1758) 140: (iris. Fl. 484; Duss 451; Millspaugh 523.

St. Eustatius: hetween (ranjestad and Fort de TVind on the top of the ('liff 40 M.: 11. 76.2 B., 7א. B.; near Maryglory 150 M.: n. 1144 B.

St. Martin: from Filipsıurg to Belvelere: n. 1565 B., 2566 a B.: near Fort Willem: n. $2791 \mathrm{~B}$; from Belvedere to Oysterpond: 1 . 3064 B.; Tintamarre: 11. 3381 B.; locality unknown: n. 164; 165.

Anguilla Boldingh.

St. Croix ex Millspaugh.

Antill., Venezuela to Brazil. (Gris. Fl.).

\section{Heliotropium Linn.}

Heliotropium curassavicum Linn. Spee. (175:) [30: (iris. H. 486; Duss 453; Millspaugh 523. Sealavendel.

St. Eustatius: Seashore near Oranjestad: n. ") B; strashure near Tumbleduwndickbay: 11. 166 B.; Signalhill: n. 105.) B.; seashore near Fairplay: n. 97.

St. Martin: Guamabay: 11. 2422 B.; near Fort Amsterilam: ก. 59.

I alsu saw Heliotrnpium curassavicum : at Simnunsbaywillage; (Oysterpond.

St. Croix ex Milispaugh.

A mer. trop. and subtrop. Matrokko, the Cape, Australia. (k. a. P.).

Heliotropium indicum Liml. Spee. (1758) 130; Gris. Kl. 455; Duss 452; Millspaugh 523. Eye bright.

St. Eustatius: Bengalen: n. $631 \mathrm{~B}$; along roats near (ilassbottle: n. 887 B.; slope of the Quill: n. 115; English Quarter: n. 119; locality unknown: n. $14 \mathrm{H}, 15 \mathrm{G}$.

Saba: Crispeen 400 M.: n. 1726 B.

St. Martin: near Bethlehem: 11. 26i2 B; hetween Mfomt Velnon and Orientbay: n. 2941 B.; Mary's Fancy: n. 74; locality unknown: n. 73. 
1 also saw Heliotropium indieum: Mount Paradis

St. Croix ex Millspaugh.

All trop) countries. (E. a. P.).

Heliotropium microphyllum sif. ex Wikstl, in Vet. Acal. Handl. Stockh. (1827) 58; Gris, Fil. 486.

St. Martin: in drerterl blantitions neall Simsonshilyshore: $n$. 3034 B.; 'l'intamar'e: 11. 3372 B.

Anguilla Boldingh.

Antigua, Guadeloupe. (Herb. Krug et Urban). Baham. (Hitchc.).

Heliotropium parviflorum Linn. Hant. II (17i1) 2011; (iris. Fl. 485; Duss 452; Millspuugh 523. Ey e brigh t.

St. Eustatius: in a garden in Oranjestad: n. S0 13.; along roads in Oranjestad: n. 517 B.; Bengalen: 11. 622 B.; slope of the lower part of the Quill near Bengalen: n. 732 B.; slope of the Quill: n. 72 ; in a garden: n. 129; locality unknown: n. $7 \mathrm{G} ., 7 \mathrm{H}$.

Saba: alung roads between Buttom and Windwardaide and M.: n. 1361 B.; Crispeen 100 M.: n. 1721 B.; Laddergut 0--200 M.: n. 1956 B.; Bottom: n. 130.

St. Martin: from Filipsburg to Guanabay: 11. 2ut4 B.; Prieregut: n. 128.

I also sitw Heliotropium parviflorum: on Nakedboyhill.

Anguilla Boldingh.

St. Croix ex Millspaugh.

Florida and Texas to Peru and Brazil. (E. a. P.).

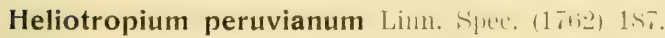

Cultivated. [Peru]. (Ind, Kew.).

\section{Verbenaceae.}

7138. Verbena Linn.

Verbena chamaedrifolia Juss. in Anu. M[us. Par. TII (1s06i) $7: 3$. Lilac verbena.

Cultivated. AArgentin. and South Brazil.] (L. a. P.).

\section{Lantana Linn.}

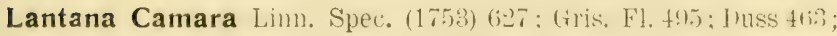
Millspaugh 524. Sage.

St. Eustatius: Top of the Quill ton M.: 11. 20!1 B., 267 B.; neal Maryglory 150 M.: 11. 1159 B.; slope of the Quill on the side of 
White Wall 200 M. : 11. 1166 B.; Oranjostad: 11. 165, 166; Cultuurvlikte: 11. 167, 1tis, 16!1, 170; locality unknown: n. 171, s. (i., $110 \mathrm{~J}$.

Saba: along roads between Bottom and Windwardside $200 \mathrm{M}$. n. 1293 B.; Greathill 400 M.: n. 1380 B.; along the Forthgut 150-200 M.: 12. 1624 B.: Laddergut 200 M.: 11. 2002 B.; Bottom: n. 2255 B.; Ladder: n. 172; Bottom: n. 173; locality unknown: n. $253 \mathrm{~L}$.

St. Martin: from Filipslumg to (iuanahay: n. 2364 B.; Princequarter (coll. (ieorge): 11. 247.2 B.; Fort Willem 0-50 M. : 11. 2535 B.; from Filipsburg to Belvedere: n. 2564 B; Mountain letween Mont des Accorủs and C'entryhill 200-300 M.: 11. 2S81 B; from Belvedere to Oysterpond: 11 . :31) 16 B., :3083 B.; along roals firom (trande Case to Marigot: 11. 3105 B.: Vineyardhili : 1. 175; Guanabay : 11. 174.

I also saw Lantana Camara on: Nakedboyhill; lower part of Mildrum. lill; hills on the eastern side of Cul de Sac.

St. Croix ex Millspaugh.

In all warmer countries of America; also in the old world. (Hemsley 1884).

Lantana involucrata Linn. Cent. Pl. II (17.)6) : 20 ; (iris. Fl. 496; Duss 464; Millspaugh 524. Sage.

St. Eustatius: Seashore near (Manjestad: 11. 1t B.: slope ol the lower part of Signalhill: 11. 101 B.: stashore near T'umhledown. dicklay: n. 160 B.; top of the Quill 400 M.: 11. 218 B., 508 B.; along roads in Oranjestad: $11,580 \mathrm{~B}, 1100 \mathrm{~B}$.: slope of the Quill

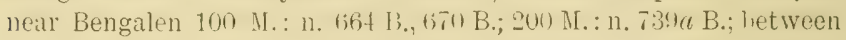
Oranjestad and Fort de IVind on the top of the Cliff 40 M.: n. 773 B., $7 \%$ B., 786 B.: slope of the (Quill: $11.194,19.5,196$; Cultumvlakte: n. 197, 198; English Quartor: n. 199, 200; locality un. known: n. $56 \mathrm{H} ., 89 \mathrm{G}$.

Saba: along the Forthgut 150-200 Y.: n. 16:3i B.: HellsgitteMarypoint: n. 2036 B.; Marypoint: n. 201.

St. Martin: Prince quirter (coll. George.): n. 2556 B., 2485 B.; Fort WVillem 0-50 M.: 11. 2533 B.; from Filipsburg to Belvedere: n. 2560 B.; shore of the Simsonsbaylagoon near Cul Pi(juard: $n$. $27+2$ B.; near Mulletpond: 11. 2761 B.; Mountain letween Mont des Accords and Centryhill 26m-3010 M.: n. 28S2 B.; letween Mount Vermon and orientbay: n. 2!30 B.; Mount Paradis 100_-200 M.: n. 33.; B.; Green liey: n. 336:3 B.; Tintamarre: 11. 3394 B.; hills on the eastern side of Cul de Sac: n. 3440 B.; near Fort Amsterdam: n. 202; Colebayhill: n. 203; French frontier: n. 204, 212.

I also saw Lautana involucrata: on one of the Islands in Simsonsbay lagoon. 
Anguilla Boldingh.

St. Croix ex Millspaugh.

Bermud., Florida and Antill., Mexico and northern part of Amer. austr. (Hemsley 1884).

\section{Lippia Linn.}

Lippia reptans $\mathrm{H}$. B. et $\mathrm{K}$. Nov. Gell. et Sp. II (1817) 263; Gris. Fl, 495; Duss 462.

St. Martin: neal Filipsburg: n. 2336 B.; from Filipsburg to Guanabay: n. 2419 B. ; Guanabay: n. 186.

Anguilla Boldingh.

Antill., Mexico to Brazil. (Gris. F'l.).

\section{Bouchea Cliam.}

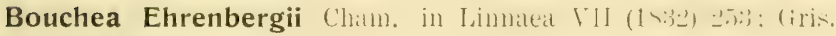
Fl. 493; Millspaugh 523. Vervine.

St. Eustatius: Locality unknown: 11. $299 \mathrm{G}, 228 \mathrm{H}$.

Saba: Windwardside: n. 1922 B.

St. Crolx ox Millspaugh.

Amer. and Afric. (E. a. P.).

\section{Stachytarpheta Vahl.}

Stachytarpheta jamaicensis Vahl. Linum. I (lis)t) g(li: (iris. F']. 494; Duss 461.

Stachytarpheta indica Vahl. Enum. I (180t) 206.

Valerianoides jamaicensis Medic. Phil. Bot. I (178y) 177; Mill. spaugh 525. Purple wormbush.

St. Eustatius: in a garden in (ranjestad: 11. 11-2 B.; Bengalen: 1. $605 \mathrm{~B}$; ; slope of the Quill near Bengalen $150 \mathrm{M}$; ; n. $685 a \mathrm{~B}$; White Wall: n. 817 B.; Cultuurvlakte: n. 227, 229; slope of the Quill: n. 228; locality unknown: 11, $293 \mathrm{H} ., 305 \mathrm{G}$.

Saba: along loads between Bottom and Wrindwardside and M. 11. $13: 31 \mathrm{~B}$; t top wf the Clift near the seasile of Booliyhill : 11. 1tiof B.; Windwardside: 11. 2:32:) B.; Liaklet: n. 2:30) locality unkmown: n. $346 \mathrm{~L}$.

I also saw Stachytarpheta jamaicensis: Crispeen.

St. Martin: from Filipsburg to Belvedere: n. $2581 \mathrm{~B}$.

I also saw Stachytarpheta jamaicensis: Margothill-Marigot; in pasture. ground near Bellevue: hill luetween Mont des Areords and Centrybill: luser part of the Milldrumhill; Lowlands. 
Anguilla Boldingh.

St. Croix ex Millspaugh.

Bermud., Florida and Mexico to Brazil.; also in Afr, trop. and Asia. (Hemsley 1884).

\section{Priva Arlans.}

Priva echinata fuss. in Ann, Mus. Par, VII (1.S06i) 6i9; (itis. F'l. 493 ; Duss 461.

Priva lappulacea P'ers. Syn. II (1807) 139; Millspaugh 524.

St. Eustatius: along roads in Oranjestad: n. $557 \mathrm{~B}$; along roads near Glassbottle: n. $873 \mathrm{~B}$; in a garden in Oranjestad: n. 1275 B.; Quill: n. 219; locality unknown: n. 220, 221, 222, $38 \mathrm{G}$.

Saba: Boobyhill: n. 1 ø̃94 B.; Laddergut 0-200 M.: n. 2019 B. 223; Peperpot: n. 224; Bottom: n. 225, 226.

St. Martin: Marigotroad near Marigot: n. 2814 B.

St. Croix ex Millspaugh.

Bermud., Florida and Mexico to Brazil. (Hemsley 1884).

\section{Citharexylum Mill.}

Citharexylum quadrangulare Jakl. Enum. I'l. ('ariib. (1760) «ti; Gris. Fl. 497; Duss 465; Millspaugh 524. Susann a berry.

St. Eustatius: on the slope of the Cliff neall Oranjestad: 11. 53 B.; scashore: n. 152; Quill: n. 153; top of the Quill 400 M.: n. $271 \mathrm{~B} ., 641 a \mathrm{~B}$; slope of the Quill near Bengalen: n. $7 \pm 6 \mathrm{~B}$.; hills near Concordia: 11 . Ista B.; locality unknown: n. $107 \mathrm{H}, 121$ (i.

Saba: along roads between Bottom and Windwardside $200 \mathrm{M}$.: n. 1:314 B.; hetween Buttom and Marypoint 100-200 M.: 11. 1527 B.; Paris: 11. 154 ; locality unknown: n. $182 \mathrm{~L} ., 190 \mathrm{~L} ., 336 \mathrm{~L}$.

St. Martin: Prince quarter (coll. (ieorge): n. 2473 a B.; from Filipwburg to (iumabay: 11. 2355 B., 2409 B.; Marigotroald near Marigot: n. 2818 B.; Centryhill : n. 155.

St. Croix ex Millspaugh.

Antill., Guiana. (Gris. Fil.).

\section{Duranta Linn.}

Duranta repens Linn. Spec. (1753) 6:37; Millspiugh i.24.

Duranta Plumieri Jacq. Select. Am. (1763) 186 t. 176 f. 76; Gris. Fl. 498; Duss 465. Adonis.

St. Eustatius: in a garken in Oranjestad: n. 10.58 B.; near Maryglory 150 M.: n. 1137 B., 1157 B.; locality unknown : n. 139 H.. 298 G., 1013 B. 
Saba: Springbilygut: 11, 2095 B.; Windwardside: 11. 2308 13; locality unknown: n. $364 \mathrm{~L}$.

St. Martin: Point Blanche (coll. George): n. 2488 B; along roads from Grande Case to Marigot: n. 3097 B.

Anguilla Boldingh.

St. Croix ex Millspaugh.

F'rom Bolivia and Brazil. to Mexico and the Antill. (E. a. P'.). Also cultivated.

\section{8: Aegiphila Jacq.}

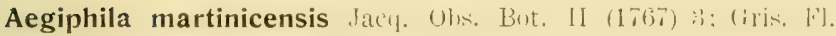
500 ; Duss 466; Millspaugh 523. Bastard white root.

St. Eustatius: Slope of the Quill: n. 246; top of the Quill: n. $386 a$ B., Quill: n. 894 B.; locality unknown: n, $137 \mathrm{H}, 212$.

St. Croix ex Mlillspaugh.

Antill., Cuba and Panama to Guiana. (Gris. Fl.).

\section{Clerodendron Linn.}

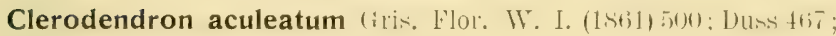
Millspaugh 524. Haguebush.

St. Eustatius: Seilshore near ()ranjustal: 11, -2 B., 151 B.; Bengalen: n. 637 B.; Cultuurvlakte: n. 158, 159, 161; Clift near Oranjestad: n. 162; locality unknown: n. $95 \mathrm{G} ., 147 \mathrm{H} ., 226 \mathrm{H}$.

Saba: along roads hetween Bottom and ITindwardside 2 (n) 11 . n. $1351 \mathrm{~B}$.

I also satw Clerodendron aculeatum: along Forthbaygut

St. Martin: Fort Willem 0-500 M.: n. 2547 B., 2792 B.; liort Amsterdam: n, 160 .

I also saw Clerodendron aculeatum near Mont Chambord.

Anguilla Boldingh.

St. Croix ex Millspaugh.

Amer. trop. (E. a, P.).

Clerodendron fallax Lindl. Bot. Reg. (1844) t. 19.

Cultivated. [Java]. (Ind, Kew.).

Clerodendron fragrans Willd. Enum. ITort. Berol. (1 Sis!is tis?! Cultivated. [China]. (E. a. P.).

Clerodendron Thomsonae Balf. Elinh. New. I'hil. . loum. X... XV (1862) 233.

Cultivated. [Afr. trop.] (Ind. Kew.). 


\section{Avicennia Linn.}

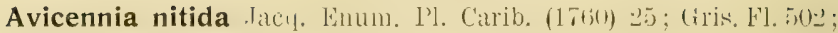
Duss 470 ; Millspaugh 523. Saltpond tree.

St. Martin: Colebay (coll. George): 11. 2498 B; allong roads from Bethlehem to Mont Chambord: n. 2911 B.; along Simsons. halylagnon near the village: n. 3026 B.: (Jysterpond: n. 135); Prince quarter: n. 136 .

I also saw Avicennia nitida: near Fort Amsterdam; one of the Islands in Simsonsbaylagoon.

St. Croix ex Millspaugh.

Antill., Cuba to Brazil., Afr. trop. (Gris. Fl.).

\section{Labiatae.}

7264. Leonotis R.Br:

Leonotis nepetaefolia li. Br. in Ait. Hurt. Kew. ex. II. III. (1811) 409; Gris. Fl. 492; Duss 460; Millspaugh 525. A don is abbot.

St. Eustatius: in a garden in Oranjestad: n. 93 B., 1105 B.; Bengalen: 1). 1i21 B.: ('ulturvlakte: n. 5it; locality unknown: n. $138 \mathrm{G}$., $172 \mathrm{H}$.

Saba: along roads between Bottom and Windwardside 2010 M.:

n. 1300 B.; Ladder: 11. 216; Bottom: n. 57; locality unknown: ก. $247 \mathrm{~L}$.

St. Martin: along roads near Filipsburg: n. 2529 B.

St. Croix ex Millspaugh.

Antill. to Brazil. Afr, and Asia trop. (Gris. Fl.)

\section{Leucas $\mathrm{R} \cdot \mathrm{Br}$.}

Leucas martinicensis R. Br. l'rodr. (1810) .)(0t; (iris. Fl. t!) ; Duss 459; Millspaugh 525.

Saba: Bottom: n. 61 .

St. Martin: from Filipsburg to Guanabily: n, 2342 B.

St. Croix ex Millspaugh.

Antill., Haiti to Brazil., Afr. trop. to the Cape and East Indies. (Gris. Fl.).

7373. Leonurus Linn.

Leonurus sibiricus Limn. Spec. (1753) 584 ; Gris. F'l. 525 ; Duss 45!); Millspaugh 525. 
St. Eustatius: in a garden in Oranjestad: $\mathrm{n}, 94 \mathrm{~B}$; along roads near (hlassbottle: n. $865 \mathrm{~B}$; $\mathrm{n} .865 \mathrm{~B}$; seashore: n. 58 ; locility unknown: n. $136 \mathrm{G}$., $194 \mathrm{H}$.

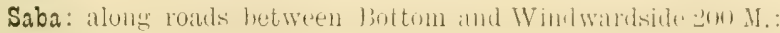
n. 1312 B.; Ladder: n. 60.

St. Martin: from Eilipsburg to Belvedore: n. 2587 B.; Mount Paradis 300-400 M.: 1]. 3238 B.; l'rince quarter: n, 214.

St. Croix ex Millspaugh.

Luropa and Siberia and naturalised in many other countries. (Hemsley 1884).

\section{Salvia Linn.}

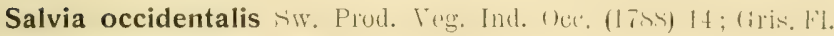
490; Duss 458; Millspiugh 526. Rah bit meat.

St. Eustatius: along roals in Granjestiul: n. .i3ti B.: rultuurvlakte: n. 74, 75, 78; Quill: n. 76, 77; Oranjestad: n. 72, 73; locality unknown: n. $292 \mathrm{H}$.

Saba: alung roads between Bottom and Windwardside son M.: n. 1313 B.; between Bottom and Marypoint 300 M.: n. 1480 B.; gut between Hellsgate and Masticgut 300-400 M.: n. 2065 J3.; Peperpot: n. 80; Crespeen--Rendez-vous 400 M.: n. 79; locality unknown: n. $35 \mathrm{~L}$.

St. Martin: I saw Salvia necidentalis: hill hetween Mont des Arentis and Centryhill; Mount Paradis $300-100 \mathrm{M}$.

St. Croix ex Millspaugh.

Bermud., Florida, Antill., and Mexico to Peru.(Hemsley 1881).

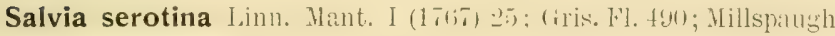
526. Cat nip.

St. Eustatius: in a garden in Oranjestad: n. $95 \mathrm{~B}$; Cultuurvlakte 1 . 81; locality unknown: n. $33 \mathrm{H} ., 37 \mathrm{G}$.; along roads near Glassbottle: n. $868 \mathrm{~B}$.

Saba: Seiside of Tentguthill 100 M.: 11. 1652 B; Springlaygut: n. 2103 B.; Bottom: n, 2257 B.; Greathill: n. 83; locality unknown: 17. $35 \mathrm{~L}, 320 \mathrm{~L}$.

St. Martin: along roads to Fort Willem: 11. 2526 B.; Simsonshayvillage: n. 2838 B.; near Fort Amsterdam: n. St; Prince quarter: n. 82 ;

I also saw Salvia serotina: on a lill between Ifont des Aceords and Centryhill.

Anguilla Bollingh. 
St. Croix ex Millspaugh.

Bermud., Florida austr. and throughout the West Indies. (Hemsley 188t).

\section{Hyptis Jacq.}

Hyptis pectinata l'oit. in Amm. Mus. I'ar. VIl (1806) tit t. :30; Gris. Fl. 489; Duss 457.

Mesosphaerum pectinalum 0. Ktze. Rév. Gen. (1891); Millspaugh 525. Holly stalk.

St. Eustatius: Bengalen: n. 929 B.: Cultuurvlalite: n. 42 ; slope of the Quill: n. 43,2010 ; locality unknown: n. $241 \mathrm{H}$.

Saba: between Bottom and Marypoint 300 M.: n. 1481 B., 100 -2014 M.: n. 153t; B.; St. (iiles: n. $185 \mathrm{~s}$ B.; gut between Hellsgate and Mastiogut $300-400$ M. : 11. 20.52 B; Ladder N. 44; (Gainoy. gut: 1 . 45 .

I also saw Hyptis pectinata at Flat Point.

St. Martin: from Filipsburg to Belvedere: 11. 260:3 B.; hills on the northern side of Cul de Sac $0-100$ M.: n. 2980 B.; between Belvedere and Oysterpond: n. 3088 B.; locality unknown: n. 3599 B.

St. Croix ex Millspaugh.

From Mexico to Brazil., and in the Old world. (E. a. P.).

\section{Coleus Lour.}

Coleus amboinicus Lour. Fil, Cochinch. II (1790) :372; Ciris. Fl. 487; Duss 45.; Millspaugh 525.

Coleus aromaticus Benth. in Wall. Pl. As. Rar. II (1832) 16. Stinging thyme.

St. Eustatius: on the roild along the lower part of signalhill: 11. 119 B., 1133 B., 37; Cultuurvlakte: 1. 38.

Saba: lower part of the Bottom Mountain 250 M. : n. 1392 B. ;

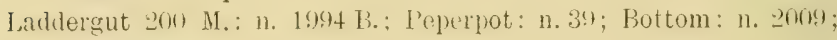
locality unknown: n. $286 \mathrm{~L}$.

St. Martin: along roads near Colebayhill: 1. 3042 B.

Anguilla Boldingh.

St. Croix ex Millspaugh.

Indig. in the East Indies. (Gris. Fl.).

Coleus scutellarioides Benth, in Wall. Pl. As. Rar. II (1893) 16. Cultivated. [Malaya, Australia]. (Ind. Kew.). 


\section{Ocimum Linn.}

Ocimum basilicum Linn. Spec. (1753) 597. Basil. Cultivated. [Asia occid, and trop.] (Ind. Kew.).

Ocimum gratissimum Linn. Spec. (1753) 597.

Cultivated [India orient.] (Ind. Kew.).

Ocimum micranthum Willd. Enum. Hort. Berol. (1.1:3) 1:301: Gris. Fl. 487; Duss 454; Millspaugh 525. Mosquito basil.

St. Eustatius: White Wall : 11. S0.; B.; slope of the (2nill ono 11 . n. $877 \mathrm{~B}$; ; in a garden in Oranjestad: $1.1271 \mathrm{~B}$; slope of the Quill: 11. 62 ; locality unknown: n. 63, 64, $131 \mathrm{G} ., 233 \mathrm{G}, 233 a \mathrm{G}, 296 \mathrm{H}$.

Saba: Liddergut 0-200 M.: n. 2016 B.; Bottom: n. 2254 B.; locality unknown: 17. $327 \mathrm{~L}$.

St. Martin: Cul de Sae: 2613 B.; Nakedboyhill 50-200 M.,

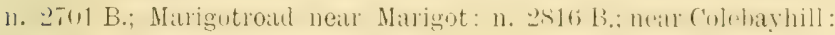
ก. 66 ; Milrigotioad: $\mathrm{n}, 65$.

St. Croiz ex Millspaugh.

Antill., Cuba and Mexico to Brazil. (Gris, Fl.).

\section{Solanaceae.}

7379. Lycium Linn.

Lycium americanum Jacq. Stirp. Am. (1763) 50.

St. Martin: Guanabay: n. 2430 B.. 2433 B.; neall fort Willem: n. 2790 B.; near Fort Amsterlam: n. 105, 106.

Anguilla Boldingh.

Hispaniola, Cula, St. Domingo. (Herb. Krug et Urhan).

\section{Acnistus Schott.}

Acnistus arborescens Schlecht. in Linnaeit VII $(1,832)$ ti $\overrightarrow{7}$; (ilis. Fl. 435; Duss 410.

Saba: along roads hetween Bottom and Windwardside :- (16) M.: 11. 1297 B.: Rendez.vous 400 M.: n. 107. 108.

Antill., Mexico, Venezuela, Nova Granata, Brazil.. Peru. (Gris. k'l.).

\section{Physalis Linn.}

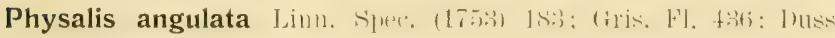
411; Millspaugh 527.

St. Eustatius: Slope of the Quill: 11, 206, 207.

St. Martin: near Bethlehem: 11. 2659 B.; between Belvedlere and Oysterpond: $\mathrm{n} .3065 \mathrm{~B}$. 
St. Croix ex Millspaugh.

Antill., from U. S. A. to Brazil., East Indies. (Gris. Fl.).

Physalis pubescens Limn. Siec. (1753) 153; (iris. F'l. 435: Mill. spaugh 527.

Saba: Bottom: n. 205; from Windwardside to Bottom: n. 2058 a B.; gut near Bottom: n. $1430 a$ B.

St. Martin: Marigotroad: 1. 208.

St. Croix ex Millspaugh.

Amer. (natural. in other trop. countries). (Gris. Fl.).

\section{Capsicum Linn.}

Capsicum ceratocarpum Fing. Monogr. (1, 2) 22.

Cultivated. (Patria ignot.).

Capsicum dulce Ifurt. (X I)m, in DC: Prod. XIII. I (1,52) $42 \mathrm{n}$. Bird pepper.

Cultivaterl. |Trop countries]. (Ind. Kew.).

Capsicum frutescens Limm, frue (17.5.3) 189; Gris. Fl. t36: Duss 411 ; Millspaugh 526.

St. Eustatius: Locality unknown: n. $51 \mathrm{G}$.

Saba: along roals between Jottom and Windwaldside :On Mr: n. 1836 B.; locality unknown: 11. $7 \mathrm{~L}, 9 \mathrm{~L}$.

St. Martin: lower part of the Milldrumbill: n. 3154 B; Momut Paradis 300-400 M.: n. 3217 B.

St. Croix ex Millspaugh.

Trop. countries. (Gris. Fl.). Also cultivated.

\section{Solanum Linn.}

Solanum aculeatissimum Jacr. (oll. I (1786i) 100: (iris. Fl. 442: Millspaugh 527.

St. Eustatius: Locality unknown: n. 190 H., 263 G.

Saba: along ruasts between Inotom and Windwardside eno MF.: n. 1309 B.; Bottom: n. 1855 B., 209; Windwardside: n. 1923 B.; locality unknown: 13. $244 \mathrm{~L}$.

St. Croix ex Millspaugh.

Widely spread in Amer. trop. and subtrop. (Hemsley 1884).

Solanum lanceaefolium Jiler. ('oll. II (17tss) Lst; (iris. Fil. 440; Duss 41.4 .

St. Eustatius: Top of the Quill $t 00$ M.: n. 227 B., 268 B., 295 B.; slope of the Quill: B. $470 a$ B.; top of the Quill 300 M.: n. 882 B.; Quill: n. 216. 


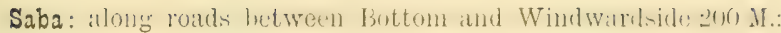

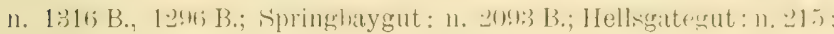
Crespeen-Rendez-vous: n. 129; Hellsgate: n. 218.

St. Martin: Nount Parardis $300-400:$ n. $3216 \mathrm{~B}$. Antill., Venezuela. (Gris. Fl.).

Solanum lycopersicum Linn. Spec. (1753) 185.

Lycopersicum esculentum Mill. Gard. Dict. ed. VIII (1768) n.2; Gris. Fl. 436; Duss 412; Millspaugh 527. T' o mate.

St. Eustatius: Seashore: n. 196; in a gardon in Oranjestad: 11. $96 \mathrm{H}, 143 \mathrm{G}, 227 \mathrm{H}, 230 \mathrm{G}$.

Saba: Locality unknown: n. $145 \mathrm{~L}$.

St. Croix ex Millspaugh.

Indig. in Amer. austral. (L. a. P.). Also cultivated.

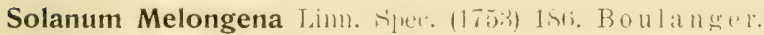

Cultivated. (Patria ignota).

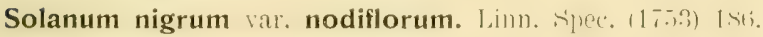

Solanum nodiflorum Dunal Hist. Sol. (1813) 151; non Jacg.; Gris. Fl. 437 ; Duss 412.

Solanum nigrum nodiflorm A. (iray; Millspaugh 527. Wild pe p per:

St. Eustatius: in a garden in Oranjestad: n. 81 B.; along roads in Oranjestad: n. 1078 B; slope of the Quill neal Glassbottle: n. 1202 B.; Culturvlakte: n. 75; slope of the Quill: 11. 219; near Roundhill: n. 220; locality unknown: n. $282 \mathrm{G}$.

Saba: lower part of the Bottom Mountain 250 MI.: n. 1400 a B.; Mountain 600 M.: n. 1779 B.; Windwardside: 11. 1904 B.; Laddergut 0-200 М.: 17. 1957 B.; Bottom: n. 2247 B.; Crespeen-Rendezvous 400 M.: n. 222; locality unknown: n: $251 \mathrm{~L}$.

St. Martin: Marigotroad near Marigot: n. $280+\mathrm{B}$; lill on the northern side of Cul de Sizc 0-100 M.: n. 2979 B., 2!183 B., 2983 B.; Marigotroad: 17.76 .

St. Croix ex MIillspaugh.

All trop. countries. (Gris. Fl.).

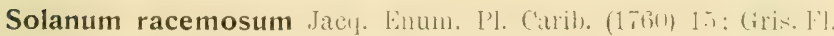
439 ; Duss 414 ; Millspaugh. 527. Can cker ber $r y$.

St. Eustatius: Fashore near Onanjestad: 11. 11 B.: slope ot the lower part of Signalhill: n. 98 B., 113 B.; along roads in Oranje-

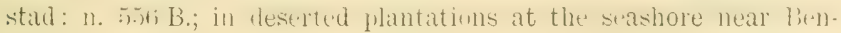
galen: n. j\$6 B., 593 B.; Bengalen: 13. 608 B.; Fort de Wind: 11. 797 B.; hill near Concordia 100 M.: n. 955 a B.; from Little Moun- 
tain to Tenusbay: n. 987 B.; Signalhill 150 M.: 17. 1043 B.; slope of the Quill on the side of White Wall 200 M.: n. 1180 B.; Venusbay: n. 223.

Saba: Feashore near Furthaylameing: n. 1650 B.: Springbaygut: n. 2084 B.

St. Martin: Aillubay: n. 2424 B.; along the saltpond near Yilipsluurg: n. 24.) B.: lowerpart of Milldrumhill : n. :3160 B ; Mount Paradis 300-400 M.: n. 3210 B.

St. Croix ex Millspaugh.

Antill. (Gris. Fl.).

Solanum racemosum Jac\%. var igneum (Limn.) (). E. Schulz. ${ }^{1}$ ) Solanum ignerm Linn. Spec. (1762) 270; Gris. Fl. 440; Millspaugh 527.

St. Eustatius: Teashore near Oranjestad: n. 35 B., t6 B.; along

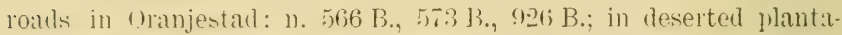
tirns at the seashore near Bengalen: n. 5xs B.; top of the Clifl between Fort de Wind and Oranjestad: 11. $774 \mathrm{~B}$; gut near Fort de Wind: n. s:3; B; ' 'ultuurvlakte: n. 2213; slope of Signalhill: n. 114; seashore: n. 211, 212; locality unknown: n. $46 \mathrm{G} ., 47 \mathrm{H}$.

Saba: betwen Bottom and Marypoint 100_-200 M.: n. 1468 B.; Bottom: n. 1838 B.; Ladder: n. 214; locality unknown: n. $141 \mathrm{I}$., $362 \mathrm{~L}$.

St. Martin: neal Filipshurur: n. 23:3- B.; along the saltpond near Filipsburg: n. 2442 B.; near Bethlehem: n. 2667 B.; near Mulletpond: 1 . 27. 5 B.; in pastureground near Bellevue: 1.2521 B.; lower part of the hills between Mont Chambord and Mount Vernon: n. 2958 B.; along roads from Colebay to Simsonsbay: n. 3..) 1 B.; gut from Bethlehem to Cul de tac 200 M.: n. 34.28 B., 3430 B; Cul de Sac: n. 210.

St. Croix ex Millspaugh.

Antill., Cuba to Guiana. (Gris. Fl.).

Solanum torvum kw. Prod. Veg. Ind. (Oce. (17SS) 47; (ris. Fl. 441; Duss 415; Millspaugh 527.

St. Eustatius: Slope of the Quill: n. 226.

Saba: lower part of the Bottom Mountain : 11. 1402 B.; betwee'n Bottom and Marypoint 100-200 M.: 11. 1533 B.; WindwardsideHellsgate: n. 1678 B.; Laddergut 0-200 M.: n. 1959 B., 224.

St. Martin: Cul de Sar: 11. 2ti1s B.; along roads from Bethle- 
hem to Mont chambord: 11. $2909 \mathrm{~B}$; hills on the eistern side of Cul de Sac: n. 3448 B.; Prince quarter: n. 225.

St. Croix ex Millspaugh.

Bermud., Antill., Cuba and Nexico to Brazil and Equador. liast Indies. (Gris. 1.1.).

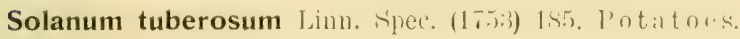

Cultivaterl. [Amer. austr.] (E. a. P.).

\section{Datura Linn.}

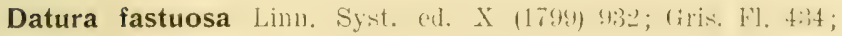
Duss 409; Millspaugh 526. Fire weed.

Saba: Crispeen 400 M.: n. 1766 B.; locality unknown: n. $260 \mathrm{~L}$.

St. Martin: near Bethlehem: 11. $2600 \mathrm{~B}$.

St. Croix ex Millspaugh.

Antill., Afr: trop., East Indies, (Gris. lil.).

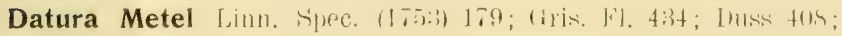
Millspaugh 526. Fire weed.

St. Eustatius: along roaks near Oranjestad: n. $563 \mathrm{~B}$.; in a garden in Oranjestad: n. 1015 B., 1081 B., 190, 12 H., 13 G.

St. Martin: along roads nfall Filipsburg: n. 25) bay: n. 189 .

St. Croix ex Millspaugh.

Antill., Haiti and Mexico to Brazil and in the Old world. (Gris. H'.).

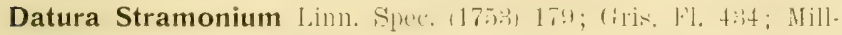
spaugh 526. line weed.

St. Eustatius: in a garden in Oranjestad: n. 542 B.; Cultuurvlakte: n. 194; locality unknown: n. $12 \mathrm{G}, 14 \mathrm{G}, 13 \mathrm{H} ., 3592 \mathrm{~B}$.

Saba: Bottom: 11. 1991 B., 195; locality unknown: 11. 83 L., $260 \mathrm{~L}$.

St. Martin: Mary's Fancy: 11. 193.

St. Croiz ex Millspaugh.

Europa, Asia, Africa and Amer. sept. (E. a. P.).

\section{Cestrum Linn.}

Cestrum laurifolium l'H(il. Stily). Nus. 1175t) rit. t. it; (ilis. F1. 444; Duss 416; Millspaugh 526.

St. Eustatius: Top of the Quill 400 M.: n. 211 B., 239 B., 240 B., 374 B.; Quill: n. 102, 103, 113; Boven: n. 104; ]ocality unknown: n. 3638 B. 
Saba: lower part of the Bottom Yountain 300 M.: n. 1442 B.; aloug roaùs between Bottom and Wrindwardside $200 \mathrm{M}$.: n. 1288 B.: (irispeen fir) M.: 17. 172:3u B.; Springbaygut: 11. 212t B.; Bottom: 17. 2252 B.; Paris 300 M.: n. 109; Greathill 2-400 M.: n. 112; Rendez-vous: 11. 110; (rispeen-Rendez-vous: n. 111; Rendez-vous Mountain: n. 67 ; locality unknown: n. 68.

St. Croix ex Millspaugh.

Cuba, Hispaniola, Portorico, St. Thomas, St. Jan, Antigua, Montserrit, Guadeloupe, Dominica, Martinique, Barhados, Trinidad. (Herb. Krug et Urban).

\section{Nicotiana Linn.}

Nicotiana Tabacum Linn. Spec. (1753) 180.

Cultivated. [Amer, austr.] (E. a. P.).

\section{Petunia Juss.}

Petunia violacea Lindl. Bot. Reg. t. 1626.

Cultivated. [Argentinia]. (Incl. Kew.).

\section{Brunfelsia Linn.}

Brunfelsia americana Limn. Spee. (1758) 191. Lally of the night.

Cultivated. [Antill.] (Gris. Fl.).

\section{Scrophulariaceae.}

7470. Angelonia Humb. et Bonpl.

Angelonia angustifolia Bentls. in DC. Prodr. X (1.846i) 2.) st. Sna pdragon.

Cultivated. [Mexico]. (Gris, Fl.).

\section{Maurandia Ortega.}

Maurandia semperflorens Jici. Hort. Schoenh. III (1,00) 20.

Saba: Gut near Bottom: n. 1388 B.

Antill. [Mexico]. (Ind. Kew.).

\section{Russelia Jacq.}

Russelia juncea Zucear in Floria XY (1א:32) II Beibl, sy?. Bugal plant.

Cultivated. [Mexico]. (Ind. Kew.).

\section{7t. Capraria Linu.}

Capraria biflora Limm. Siee. (17533) 624; (ilis. Fl. 427; I)uss 40:3; Millspaugh 527. Wild tea. 


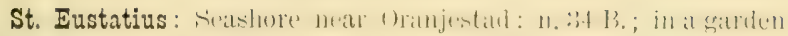
in Oranjestad: 17. 70 B.; seashore near T'umbledowndicklay: n.

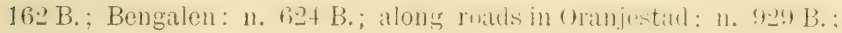
Cultuurvlakte: n. 39; locality unknown: n. $11 \mathrm{G}$., $11 \mathrm{ll}$.

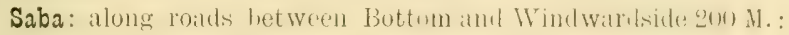
n. 1319 B.; between Bottom and Marypoint 100-200 M.: n. 1510 B.; Laddergut 0-200 M.: n. 1953 B., 54; Bottom: n. 55.

St. Martin: from Filipshurg to fillanibay: 11 . a:s4 4 B.: Filipsburg-Cul de Sac: n. 2611 B.; Mount Paradis 200-400 M.: n. $3202 \mathrm{~B}$.

St. Croix ex Millspaugh.

Bermud., Florida and Moxico to Brazil and l'eru. (Iemsley 1884).

\section{Scoparia Limm.}

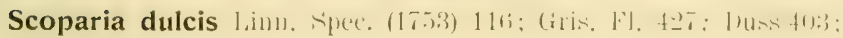
Millspaugh 528. sweet broom.

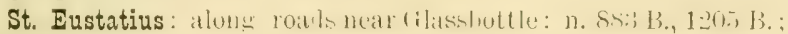
Cultuurvlakte: 11.85 ; slope of te Quill: 1n. 86,87 ; locality unknown: 11. $10 \mathrm{G}, 10 \mathrm{H}$.

Saba: lectween Bottom and Marypoint 1010-2014) M. : 11. 15.2: B.: Ladder: n. 78; Peperpot: n. 79; Mountain: n. 80; Gainbygut: 11. 81.

St. Croix ex Millspaugh.

All tropical countries. (Gris. I'l.).

\section{Bignoniaceae. \\ 7705. Bignonia Limn.}

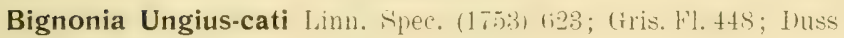
421; Millspaugh 528. Cat claw.

St. Eustatius: 'Iop of the Quill 400 M.: n. 194 B., 281 B., 283 B., 350 B.; Bottom of the Quill 350 M.: n. 454 B.; Red Cliffl'umbledowndickbay: n. 202; Quill: n. 41, 42, 102; slope of the Quill: n. 196, 197; locality unknown: n. $178 \mathrm{G}$., $259 \mathrm{H}$.

I also saw Bignonia Ungius-eati in deserterl plantations near Bengalen.

Saba: Boobyhill 300 M.: 1599 B.; Laddergut 0-200 M.: n. $1966 \mathrm{~B}$; hetween Hellsgate and Marypoint: n. 2071 B.; pastureground: n. 40; Middle Island: n. 149.

St. Martin: Jill on the northern side of ('ul de sar (1)-150 II. 11. 2970 B.; gut from Betlehem to Cul de Sac 200 M.: 11. 3435 B.; John Ednyhill: 13. 198, 199, 200.

I also saw Bignonia Ungins-cati on Mount Paradis 300-400 M. 
St. Croix ex Millspaugh.

Antill. (Gris, Fl.); Amer, cont. (Herb. Leiden.).

Bignonia spec. without flowers and fruits.

St. Eustatius: Quill: n. $438 a$ B.

\section{Tecoma Juss.}

Tecoma capensis Lindl. Bot, Reg. t. 1117.

Cultivated. [Afr. austr.] (Ind. Kew.).

Tecoma leucoxylon Mart. ex DC. Proul. IX (1845) 21!) (iris. Fl. 447; Duss 420; Millspaugh 528. White ceder.

St. Eustatius: in a garden in Oranjestad: n. 72 B.; slope of the lower part of Signalhill: n. 104 B.; top of the Quill 400 M.: 11. 214 B., 331 B., 35.) B.; between Oranjestad and Fort de Wind: n. 782 B.; alons roads in Granjestad: n. 919 B.; along roads near English Quarter: n. 100\% B.: slope of the Quill near Glassbottle:

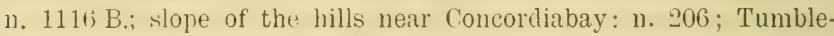
downdickbay: n. ㄴ1t; neal rugarloat': 11. 217; locality unknown: n. $52 \mathrm{H} ., 109 \mathrm{G}$.

I also saw T'ecoma leucoxylon: on Gilboolill; near Jenkinshay.

Saba: between Bottom and Marypoint 100-200 M. : n. 1500 B.: Boobyhill: n. 1.iou B.; (rreen Island: n. 214.) B.; Windwardside: n. 2333 B.; locality unknown: n. $284 \mathrm{~L}$. Hellsgate.

I also saw Tecoma leucoxylon: Sulphurnines; Bottom-Windwardside;

St. Martin: Colebaly (coll. (ieorge): 11. 2513 B.; from F'ilipsburg to Belvedere: n. 2555 B.; Nakedboyhill 50-200 M.: n. 2675 B.

I also saw Tecoma leucoxylon: Marigothill to Marigot; hill between Mont des Accords and Centryhill; lill on the northern side of Cul de Sac: Mount Paradis 300-400 M.: Lowlands.

Anguilla Boldingh.

St. Croix ex Millspaugh.

Baham., Antill., Cuba to Guiana.' (Gris. F'l.).

\section{Stenolobium D. Don.}

Stenolobium stans Seem. Journ. Bot, I (1863) 88 .

Tecoma stans Juss. Gen. (1789) 139; Gris. Fl. 477; Duss 420; Millspaugh 529. Yellow blossom.

St. Eustatius: Gut near Fort de Wind 50 M.: n. 834 B.; hill near Concordia 100 M.: n. 950 B, Cultuurvlakte: n. 204; locality unknown; n. $100 \mathrm{G}_{\text {., }} 178 \mathrm{H}$. 
I also saw Stenolobium stans: slope of the Clisf; slope of the Quill ont the side of White Wall.

Saba: lower part of the Bottom Mountain 800 M.: n. 1440 B.; Laddergut 200 M.: n. 1998 B., 211; locality unknown: n. 134 L.

I also saw Stenolobium stans; between Bottom and Marypoint; Forth. baygut; Bottom-Winclwardside.

St. Martin: Mount Paradis $300-400$ M.: n. 3323 B.; Cul de Sac: 1). 210.

I also saw Stenolobium stans: on Marigothill-Marigot; Nakedboylnill; hill between Mont des Accords and Centryhill; hill on the northern side of Cul de Sac; along roads near Grande Case; lower part of Milldrumhill.

St. Croix ex Millspaugh.

Antill., Cuba and Mexico to 'T'ucuman. (Gris. W'l.).

\section{Crescentia Linn.}

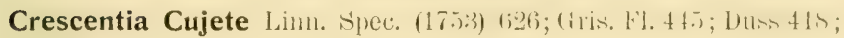
Millspaugh 528. Calabash.

St. Eustatius: in a garden in Oranjestad: 11. 149 G., $255 \mathrm{II}$.

I saw Crescentia Cujete in deserted plantations near English Quarter.

Saba: Windwardside: n. 1920 B.; locality unknown: n. 65 L.

St. Martin: trom Filipsburg to Buvedere: 11. ...) 4 B.; Mount Paradis $300-400$ M.: n. 3338 B.

I also saw Crescentia Cajete: near Saline.

Anguilla Boldingh.

St. Croix ex Millspaugh.

Antill, and Amer. austr. (E. a. P.).

\section{Gesneriaceae.}

\section{Besleria Linn.}

Besleria lutea Limn, spee. (175;3) 619: (ivis. Fl. f6:3; Dusi 4:31.

St. Eustatius: Top of the Quill 500 M.: n. $471 a$ B., $479 a$ B.

Saba: Gut near Bottom: n. $1424 a$ B; Mountain 600 M.: 1 . 1782 B., 41, 42, 43, 208.

Jamaica, Guadeloupe, Dominica, Martinique, St. Lucia, St. Vincent, Grenarla. (Symb.).

\section{Kohleria Regel.}

Kohleria hirsuta Rew. in liegensb. Flor. XXXI (1sti) 2.) (1. Isoloma hirsuta lieg. in Bot. Zeit. IX (1851) 593; Gris. Fl. 4.)9. 
Saba: Locality unknown: n, 60 L., $331 \mathrm{~L}$.

Cultivated?

Trinidad, Venezuela. (Symb.).

\section{Gesneria Linn.}

Gesneria ventricosa Sw. P'rol. Veg. Ind. oce. (17is) s!!.

Pentarhaphia longiflora Lindl. Bot. Reg. sub. t. 1110; Gris. F. 460 ; Duss 430.

St. Eustatius: 'Top) of the (quill 400 M.: n. $471 \mathrm{~B}$; top of the Quill 500 ML.: n. 479 B.; top of the Quill: n. 325, 326, 327, 328.

Saba: Mountain 600 M.: n. 1758 B., 1783 B., 332, 333.

Jamaica, St. Kitti, Montserrat, (ruadeloune, Irominica, Martinique, St. Lucia, St. Vincent. (Symb.).

\section{Lentibulariaceae.}

7901. Utricularia Linn.

Utricularia alpina Jac». Enum. P'. Carilı, $(176()) 11$.

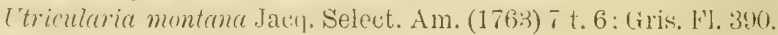

Saba: Mountain 800 M.: 11. 2229 B.

Antill. and Amer. austral. (E. a. P.).

\section{Acanthaceae.}

7914. Thunbergia Retz.

Thunbergia affinis Noore var. pulvinatus Moole in Jumr. Bot. XVIII (1880) 431.

Cultivated. [Africa]. (Herb. Berlin).

Thunbergia alata Boj. in Hook. Exot. Fl. 1823 t. 17; Duss 42 Saba: along roals between Bottom and Windwardside z0n M.: 11. L:311 B.: between Bottom and Marypoint 10(1)--200 M.: 11. 1.its B.; locality unknown: n. 1593 B, 36 L., 10; Village: n. 283.

St. Croix. (Symb.).

Indig.. in Africa orient. (Symb.).

Thunbergia fragans Foxl). 1'l. Corom. I (17!5) 47 t. (iT: (iris. Fl. 458; Duss 428; Millspaugh 530.

St. Eustatius: along roads in (ranjestad: 11. 111.2. B., 15:3 H. Saba: Bottom: n. 284; locality unknown: n. $17 \mathrm{~L}$.

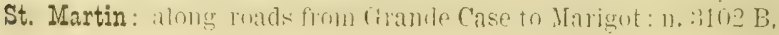


St. Croix ex Millspaugh.

Indig. in Asia trop. (Symb.).

7919. Sanchezia R. et P’av.

Sanchezia nobilis Hook. Bot. Mag. t. 5594.

Cultivated. [Equador]. (Ind. Kew.).

\section{9:30. Blechum Juss.}

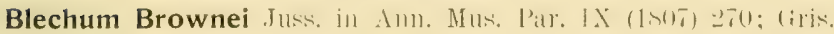
F1. 453; Duss 424.

Blechum Blechum Millspaugh 529.

St. Eustatius: Locality unkmown: 11. 30)+11, slope of the Quill: 1). 258 .

Saba: along roads hetween Bottom and WVindwardwile guo M.: 11. 1295 B.: Ix.tween Bottom and Mirypoint $100-210 \mathrm{M}$. : 11. 1507 13.: Nountain 600 M.; 11. 1744 B.; Bottom: n. 1847 B., 1984 B.; Ladder: n. 260 ; Laddergut: n. 260 bis; locality unknown: 11. $316 \mathrm{~L}$.

St. Martin: Cul de Sac-Marigothill 0-200 M.: n. 2626 B.; in an orchard near Bellevue: n. $28 \pm 6 \mathrm{~B}$.

St. Croix ex Millspaugh.

Amer. trop. (Symb.).

\section{Ruellia Linn.}

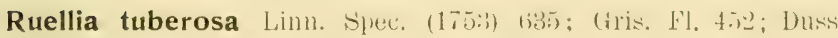
423; Millspaugh 529. Devil's bit, Fever root.

St. Eustatius: along roals near crlasshuttle: n. stid B.: Cultuurvlakte: n. 272; Concordia: n. 274; locality unknown: n. $83 \mathrm{H}$, $129 \mathrm{G}$.

I also saw Ruellia tuberosa on Signalhill.

Saba: between Boltom and Yaryoint 114-201 .1.: n. 151:2 B; ; Hellsgate: n. 1697 B.; locality unknown: n. $297 \mathrm{~L}$; ; Bottom: n. 273.

I also saw Ruellia tuberosa in the Latdergut.

St. Martin: from Filipsburg to Guanabay: 11. 2375 B: from Filipshurg to Belvedere: 11. y.i.? B.: along rouks near Buthlehem: n. $293 \pm$ B. ; Centryhill: n. 12.

I also saw Ruellia tuberosa: on Nakedboyhill; hill between Mont des Acrords and contryhill: in pasturegromul near Belvedere: in pastureground near Bellevue: Lowlands. 
St. Croix ex Millspaugh.

Antill., from Guiana, Venesuclit, Peru and Culumbia to Nexico. (Symb.).

\section{Stenandrium Nees.}

Stenandrium rupestre Nees. in LC: l'rodr. XI. (1847) 28:3.

St. Martin: in an orchard near Bellevue: n. 2835 B.; lower part of the hills on the eastern side of Cul de Sac: n. $3442 \mathrm{~B}$.

I also saw Stenandrimm rupestre on the hill between Mont des Accords and Centryhill.

Baham., Antill. (Symb.).

\section{S012. Graptophyllum Neos.}

Graptophyllum pictum (iritt. Yotul. IV. (1S5) 1:3!!.

Cultivated, [Nova Guinea], (Symb.).

\section{Justicia Linn.}

Justicia sessilis Jacr. Enum. (1760) 11.

Dianthera sessilis Gris. Flor. IV. I. (1861) 455; Duss 426. Prickly balsam.

St. Eustatius: Slope of the lower part of Siynalhill: n. !n! 13.; Inwer jart of the hills near Concortia: 11. 977 B.; neat Maryglory 150 M.: n. 1150 B.; near Boven 200 H. ; n. 1246 B.; Cultuurvlakte:

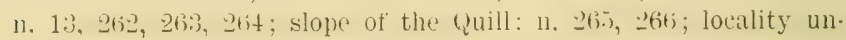
known: n. $74 \mathrm{H} ., 111 \mathrm{G}, 141 \mathrm{G}$.

Saba: Windwardside-Hellsgate: n. $166 \tau$ B.: Laddergut 200 M. : n. $200 \pm$ B.; Paris: n. 267 ;

St. Martin: along roads nea Bellevue: 11. 28+2 B.; Mountain between Mont des Accords and Centrybill 0-200 M.: 11. 2sti6 B.; from Belvedere to Oysterpond: n. $3057 \mathrm{~B}$.

I also saw Justicia sessilis: on the lower part of the Milldrumhill; Mount Paradis

St. Croix. (Symb).).

IIspaniola, Portorico, St. Thomas, Antigua, (ivateloupe. (Symb).

8098. Beloperone Nees.

Beloperone eustachiana Benth. in Benth. of Hook. lien. P'l. II (1876) 1111.

Justicia eustachiana Jacr. Enum (1760) 11; Gris. Fl. 456; Duss 426 . 


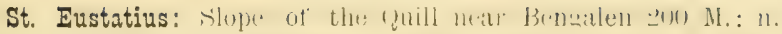

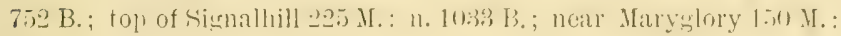
n. 1138 B., 1149 B.; locality unknown: n, $166 \mathrm{G}$., $248 \mathrm{H}$.

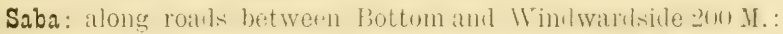

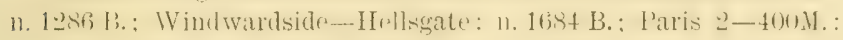
n. 256; Greathill: n. 255; Rendez-rous 400 M.: 11. 257; Bottom: n. 1 ; locality unknown: n. $24 \mathrm{~L}$.

St. Martin: Momntain between Hont des Aceoris and centry. hill 200-300 M.: n. 2860 B.; along roads from Colebay to Simsorsbay: 11. 3248 B.; Green Key: n. 3366 B.

St. Barthélemy, Antigua, Montserrat, Guadeloupe. (Sirmb.).

\section{Myoporaceae.}

8111. Bontia Linn.

Bontia daphnoides Linn. Spec. (1753) 638.

Cultivated. [Antill.] (E. a. P.).

\section{¿69. Plantaginaceae.}

\section{Plantago Linn.}

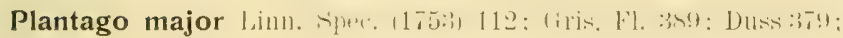
Millspaugh 530 .

Saba: Boolyhill: n. 1566 B.; Windwardside: n. 2327 B.; locality unknown: n. $178 \mathrm{~L}$.

St. Croix ex Millspaugh.

In almost all parts of both hemisph. (E. a. P.).

\section{Rubiaceae.}

\section{Oldenlandia Linn.}

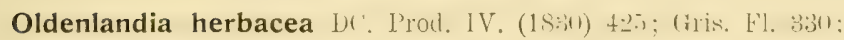
Duss 335; Millspauch 531.

St. Martin: from l-jlipslumg to belvedere: 11, s.jul B.: along roads from Grande Case to Marigot: n. $310 \pm$ B.

St. Croix ex Millspaugh.

Amer. trop., Afric., Asia, Malay, Kaiser Wilhelmsland. (E. a. P.).

\section{Hillia Jacq.}

Hillia longiflora siv. Prod. Veg. Ind. ()ee. (1-4s) sis; (ilik. Fl. 325; Duss 334.

Hillia parasitica Jacq. Enum. Pl. Carib. (1760) 18. 
St. Eustatius: Top of the Quill: 11, 247 B., 68, 69, 70.

Saba: Momntain sor M.: 11. 21!2 B., 2198 B.; locality unknown: n. $127 \mathrm{~L}$.

Antill., Amer. austral. (E. a. P.).

\section{Exostemma I. C. Ricl.}

Exostemma caribaeum Roenn. (t Schult. Syst. Y (1S1!n) 1s; Gris. Fl. 324; Duss 333; Millspaugh 531.

St. Martin: Colubay (coll. (ienrge): n. Ljon B.; Nilkedboyhill 200 - 260 M.: n. 2726 B.; near Mulletpond: n. 2783 B.; Mountain

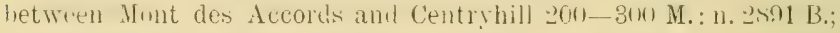
from Belvedere to Oysterpond: n. $3045 \mathrm{~B}$.

Anguilla Boldingh.

St. Croix ex Millspaugh.

Antill. (Herb. Krug er Urban); Key West (Melvill.); Baham. (Hitche.).

Exostemma spec.

Saba: locality unknown: n. $107 \mathrm{~L}$.

\section{Mussaenda Linn.}

Mussaenda frondosa Linn. Spec. (1753) 177.

Cultivated. [India orient.] (Ind. Kew.).

\section{Gonzalagunia Ruiz. et Pav.}

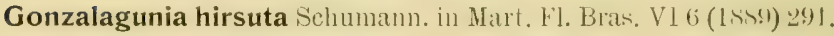
Gonzalea spicata DC. Prodr. IV (1830) 437; Gris. Fl. 321; Duss 332.

Saba: between Bottom and Marypoint 350 M.: n. 1493 B., 1552 B.; Mountain near Hellsgate: n. 2ost B.; Mountain: n. 171, 172; Devilshand: n. 173.

Antill., Guiana, Bahia. (Gris. E'l.).

\section{Randia Linn.}

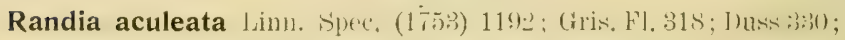
Millspaugh 531. Fishing rod, Black cherry.

St. Eustatius: Slope of the lower part of Signalhill: 11. $1100 \mathrm{~B}$ : top of the Quill 400 M.: n. 280 B; slope of the lower part of the (yuill near Bengalen: 11. T12 B.; hetween oranjestad and Fort de Wind on the top of the Clift: 11 . $7: 10$ B.: slepe of sisnalhill: 11. 12.5. 14: Quill: n. 1S1: White Wall: n. 125; Little Hountain: 11, 126: (ijlloohill: 11, 124; locality unknown: n. 176 (i., 195 $\mathrm{H}$. 
I also saw Randia aculeata: on the slope of the Quill on the side of White Wall; Tumbledowndickbay-Venusbiy.

Saba: between Bottom and Marypoint 100-200 M.: 1. 1519 B.; Saddle: n. 165S B; Hellsgate: 1). 120; locality unknown: n. 123.

St. Martin: from Filipsburg to Guanabay: n. 2416 B.; Prince (puarter (coll. Georgo): n. 2474 B; near Fort Willem: 11. 2798 B.; top of the hill on the northern side of Cul de Sac 200-300 M.: n. $2991 \mathrm{~B}$; Mildrumhill $300-400$ M.: 1. 3140 B.; Vineyardhill : 11. 121,122 .

I also saw Randia aculeata: Marigotthill-Marigot; top of Nakedboylitl; near Bellevue; hill between Mont des Accords and Centryhill; along roak neat Grunde Cite; Mount Paradis 300-400 M.; Lowlands.

St. Croix ex Millspaugh.

Bermud., Florida, Antill. (Hemsley 1884).

\section{Guettarda Linn.}

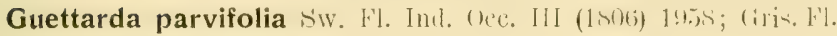
333; Duss 337 ; Millspaugh 531. Wild cherry.

St. Eustatius: from Little Mountain to Venusbay: 11. 961 B.; slope of the Quill on the side of White Wall 200 M.: n. 1181 B.

Saba: between Bottom and Marypoint 100-200 M.: n. 146+ B.; Springloaygut: 11. 2079 B.

St. Martin: from Filipsburg to Belvedere: n. 2571 B.; Nakedboyhill 280 M.: n. 2737 B.; mountain between Nont des Accolus and Centryhill 200-300 M.: 1). 2887 B.; lower part of the mountains between Mount Vernon and Mont Chambord: n. 2450 B.; Milldrumbill 200-300 M. : n. 3122 B.; hills on the eastern side of Cul de Sac: n. 3438 B.

St. Croix ex Millspaugh.

Antill., Venezuela. (Gris. $\mathrm{H}^{3} \mathrm{l}$.).

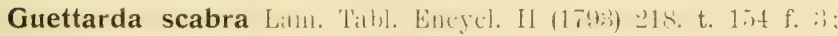
Gris. Fl. 332; Duss 337; Millspaugh 531. Candle wood, Wild guave.

St. Eustatius: Top of the Quill 380 M. : 11, 255 B., 319 B., 50\% B.; from Little Nountain to Venusbay: n. 998 B.; Signalhill: n. 76 ; Quill: n. 78 .

I also saw Guettarda scabra on the slope of the Quill on the side of White Wall.

Saba: between Bottom and Marypoint 100-200 M. : n. 1463 B.; Greathill: $1.75 \mathrm{~A}:$; Hellsgate: $\mathrm{n}$. $75 ;$ locality unknown: $\mathrm{n} .7$. 
I also sar Guettarda seabra: in the gut between Hellsgate and Masticgut; Crespeenhill.

St. Martin: Mount Paradis $300-400$ M.: n. 3224a B.

Anguilla Boldingh.

St. Croix ex Millspaugh.

Baham., Antill, Yucatan, Brazil. (Gris, Fl.).

\section{Erithalis Linn.}

Erithalis fruticosa Limn. Syst. ed. X (1759) 9:30; (rris. Fl. :336; Duss 338; Millspaugh 530. Flambeau.

St. Eustatius: from Little Mountain to Venuslay: n. 97:3 B.;

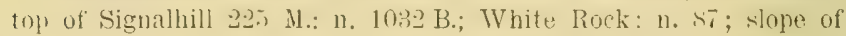
the Quill near White Wall: n. S6; White Wall: n. 83, 151; Sugarloaf: n. 152; Boven: n. 84; Oranjestad n. 85.

St. Martin: (olehay (coll. (ienrge): n. 24 (3) Hi; near Mulletpond: 11. 2752 B.; from Belvedere to Oysterpond: n. 3047 B.; T'intamare: n. $3390 \mathrm{~B}$.

Anguilla Boldingh.

St. Croix ex Millspangh.

Antill. (Herb. Krug et Urban); Key West (Melvill.); Baham. (Hitchc.)

\section{Chiococca Linn.}

Chiococca racemosa Jilcy. Sielect. $\lambda \mathrm{m}$. (1763) (is; (iris. F']. 83ti; Duss 389; Millspaugh 530. Buckroot.

St. Eustatius: Top of the Quill 400 M.: n. 199 B., 336 B., 4ho B.: slope of the Quill near Bengalen 200 M.: 1), liss B.; from Little Mountain to Venusluay: n. $972 \mathrm{~B}$; slope of the Quill on the side of White Wall 200 M.: n. 1170 B; top of the Quill: n. 141, 142, 143, 152; Quill: n. 156; locality unknown: n. 157, $187 \mathrm{G} ., 271 \mathrm{H}$.

Saba: along roads between Bottom and Windwardside 2100 M.: n. 1355 B.; Greathill 400 M.: n. 1385 B.; between Bottom and Marypoint 350 M.: n. 1497 B.; Boobyhill 300 M.: n. 1574 B.; Hellsgate-Marypoint: 11. 2038 B.; , Fringhaygut: 11. 20\$1 B., 2082 B.; Paris: n. 154, 155, 159; Rendez-vous: n. 158; Middle Island: n. 153; Wellgut: n. 151; locality unknown: 11. $137 \mathrm{~L} .909 \mathrm{~L}$.

St. Martin: Marimothill-Marigot 0-200 M.: 11. 2(13:37 B.; moun. tain betwern Mont des Aceorls and Centryhill 200 - 300 M.: $\mathrm{n}$. 25 - 9 B.; top of the hills on the northern side of Cul de Sie $200-$ 
300 M.: 11. 3009 B.: from Belvedere to oysterpond: N. $306 ; 0$ B.; Milletrumbill $200-300$ M : 11. 31:33 13.; $3100-400$ M.: 11. 315:3 13.; Mount Paradis $300-400$ M.: n. 3225 B.; 100-200 M.: 11. 3298 B.; gut from Bethlehem to Cul de Sac 200 M.: n. 3429 B.

St. Croix ex Millspaugh.

Antill., Mexico, Amer. austr. (E. a. P.).

\section{Coffea Linn.}

Coffea arabica Limn. Spec, 1175:3) 172; firis. Fl. 33s; Duss :341. Coffee.

St. Eustatius: Bottom of the Quill 300 M.: n. 900 B.; inner slope of the Quill 350 H.: 11. 1220 B., 101 II., 215 G.

Saba: Windwardside: n. 19:35 B.; Gainbygut: 11. 98; Hellsgategut: 11.99 .

Indig. in Abyssinia, Angola and Mosambique. (F. a. P.).

\section{S384. Ixora Jimn.}

Ixora coccinea Linn. Spec. (1753) 110.

Cultivated. [Asia trop.] (Ind. Kew.).

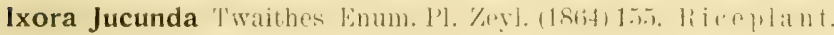
Cultivated. [Zeylan.] (Ind. Kew.).

\section{Strumpfia Jacq.}

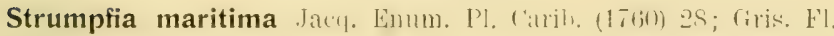
336; Duss 338.

St. Eustatius: White Wall: n. 813 B., 1264 B., 138.

St. Martin: Colebay (coll. George): 11. 2519 B.

Anguilla Boldingh.

Baham., Antill., Aruba, Bonaire, Curaralo. (ITerh. Kirug et Trisan).

\section{Psychotria Linn.}

Psychotria horizontalis iw. Prod. Veg. Int. ()e. (17sis 14t: Gris. Fl. 344; Duss 344; Millspaugh 531.

St. Eustatius: Slope of the Quill near Bengalen 5) - 22(x) M.: n. $609 a$ B., 657 B.; slope of the Quill above White Wall : n. $1191 a$ B.; locality unknown: 13. $218 \mathrm{G}$.

Saba: near the Sulphurmines: 11. 1701 $a$ B; near Devilshand: n. 2075 B., 2076 B.; Springbaygut: 11. 2123 B.

St. Martin: from Filipsure to Belvedere: n, 2575 B., 2.ivi B.:

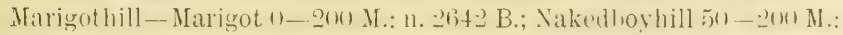

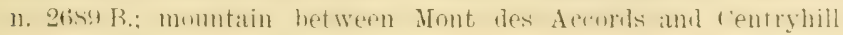


200 -300 M.: 11, 2483 B.; along roads in Cul de sic: n. $2968 \mathrm{~B}$; from Belvedere to Oysterpond: n. 3063 B; lower part of Mill. drumhill: $3161 \mathrm{~B}$; along roarls trom Colebay to Simsonsbay: n.

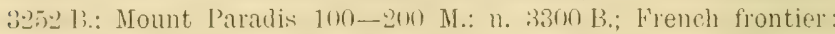
ก. 149 .

St. Croix ex Millspaugh.

Antill., Guiana. (Gris. Fl.).

Psychotria pendula Urh. Symb. Ant. I (1900) 445 .

Psychotria parasitica Sw. Prodr. (1788) 44; Gris. F'. 344 (p. p.); Duss 344.

Mapouria parasitica K. Sch. in Engl. u. Prantl. Nat. Pll. Eam. IV. 4. (1891) 112.

Psychotric erassa Gris. Flor. W. I. (1861) 344 (p. p.); Duss 344. (Symb.).

Saba: Mountain 800 M.: n. 148, 153.

Cuba, St. Kitts, Montserrat. Fuadelnupe, Ihminicil, Martini,une, St. Vincent, Grenada. (Symb.).

Psychotria rufescens H. B. ct $k$. Nor. (ien. At S. III $11 \mathrm{~S} 1 \mathrm{~s}$ 364; Gris. Fl. 342. Jastard canckerberry.

St. Eustatius: Top of the Quill to0 M.: n. $174 \mathrm{~B} ., 349 \mathrm{~B}$. 403 B., 500 B., 117; locality unknown: n. 116, 175 G., $213 \mathrm{H}$.

Saba: along rouds letween lintom and Windwatsisle $200 \mathrm{M}$. 11. 1353 B.; Bottom: n. 1456 B.; Crispeen 400 M.: n. 1762 B.; Marypoint: n. 118; Jenkinsbay: n. 114.

St. Martin: Centryhill: 11.115.

Antill, Cuba and Nexico to Nova Granatil. (Gris. Fl.).

Psychotria uliginosa Sir. Pror. Tet. Int. Oer. (1-is) ti?; Gris. Fl. 340: Duss 342.

St. Eustatius: Bottom of the Quill 300 H.: n. 899 B.

Saba: alunes rouds between linttom and Wimewardside $200 \mathrm{M}$.: n. 1306 \& B., 1365 B.; Bottom: 11. 1448 B.; Mountain : 11. 11!) Gainlygut: n. 150.

Antill., Amer. centr., Guiana. (Gris. Fl.).

8401. Palicourea Aubl.

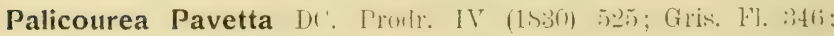
Duss 345; Millspaugh 531.

St. Eustatius: inner slope of the Quill 350 M.: 11. $1216 \mathrm{~B}$., 122+ B.; Quill: n. 146, 147. 


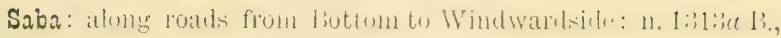
$136 \pm$ B.; Mountain: n. 1755 a B.

St. Croix ex Millspaugh.

Cuba, Jamaica, St. Thomas, Guadeloupe. (ITerb. Krug et Urian).

\section{6, Faramea Aubl.}

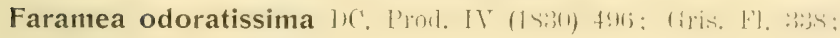
Duss 341 ; Millspaugh 531.

Saba: from Marypoint to Bottom 300 M.: 11. $2071 \Leftrightarrow$ B. Antill., Mexico. (E. a. P.).

\section{Ernodea Swartz.}

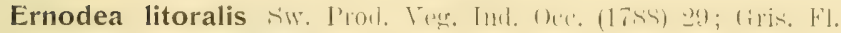
347; Duss 347; Millspaugh 530.

St. Eustatius: White Wall: 11. 806 B., 1263 B.; White rock: 11. 96; locality unknown: n. 97.

Saba: Hellsgate-Marypoint: n. 2037 B.

St. Martin: near Mulletpond: n. 2759 B. ; Lowlands: n. 3279 B.; near Orientbay: n. 3357 B.

Anguilla Boldingh.

St. Croix ex Millspaugh.

Baham., Antill. (Gris. Fl.).

\section{Diodia Linn.}

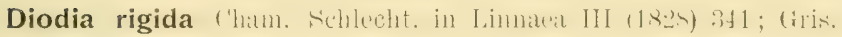
Hil. 348 .

St. Eustatius: 'Top of the ('litf leetween (iallowshall and Whit" Wall: $11,765 \mathrm{~B}$.

Amer: austr. (Ind, Kew.).

\section{Hemidiodia K. Schum.}

Hemidiodia ocimifolia K. Shlum. in Mart. Fl. Bras. IV. 4 (1888) 29.

St. Eustatius: Flope of the (huill near Bengalen 5n-on M.: 11. 650 B.; slope of the Quill 200 M.: n. 876 B., 72, 73.

Saba: ahong rodo hetween Bottom and Windwardside zon H.: n. 1337 B.; Hellsgate: n. 1691 B.; Hountain 800 M.: n. 1806 B.; Mountain near Windwardside 600 M.: n. 2174 B., 2183 B.; Ladder: n. $74 a$; Gainbygut: n. 74 ; locality unknown: n. 138 L., $205 \mathrm{~L}$.

St. Martin: Marigothill-Marigot 0-209 M.: n. 2648 B.; hill on the northern side of Cul de Sac: n. 2982 B.

Antill., Mexico. (E. a. P.). 
8473. Borreria G. F. Mey.

Borreria ocymoides DC. Prodr. IV (1830) 54t.

Saba: Ilellsgategut: $n .196$.

Amer., Africa, Asia. (li. a. P.)

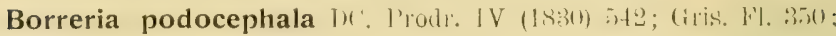
Duss 349 .

St. Eustatius; White Wall: 11. S1, B.. slsb B.; near signallill: n. $943 \alpha$ B., 1039 J3. ; seashore near English Quarter: n. 1009 B.

Cuba, Pinos, Dosirarle, Antigua, Guadeloupe. (Herb. King et Urban).

\section{\$475. Spermacoce Crïltn.}

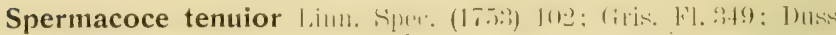
347: Millspaugh 532.

St. Eustatius: 'Top of the (yuill $4100 \mathrm{M}$ : 11. 2:30 13.: Bengilen: n. 625 B.; in a garden in Oranjestad: 11. 1084 B.; along roads near Glassbottle: n. 1115 B; slope of the Quill: n. 133 A.; locality unknown: n. $301 \mathrm{G}$.

Saba: lower part of the Bottom Mountain 300 M.: n. 1428 B.; between Bottom and Marypoint 100-200 M.: 11. 1521 B.; along the Forthgut $150-200$ N.: n. 1630 B.

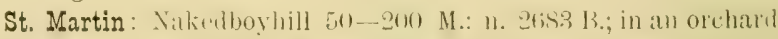

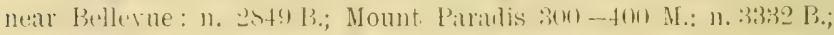
Colebayhill: n. 133.

Anguilla Boldingh.

St. Croix ex Millspaugh.

Antill., Amer. centr. (E. a. P.).

\section{Mitracarpus Zucc.}

Mitracarpus polycladus UH. in Symbolate Antillande III (190:3) :SS?.

Saba: along roads from Juttom to Windwarisile n. $1322 \mathrm{~B}$.: Paris: n. 1877 B.; Ladelergut: n. 1966a B., 175; locality unknown: 11. $86 \mathrm{I}$.

Portorico. (Symh).).

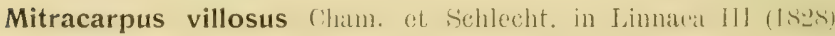
36is; (tris. Fl. 350.

Afitracermes hirtus DC. Prod. IV (1830) 572; Duss 349).

St. Eustatius: Slope of the Quill: 11. 164; roar in Oranjest xd: n. 173 .

Antill., Cuba and Mexico to Equarlor and Veneznela. (Ciris. Fl.). 


\section{Caprifoliaceae.}

8515. Sambucus Linn.

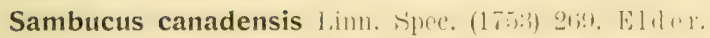

Cultivated. [Amer. sept.] (E. a. P.).

\section{Lonicera Linn.}

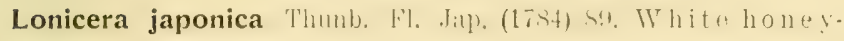
suckle.

Cultivated. [Himalaya]. (Ind. Kew.).

\section{Cucurbitaceae.}

\section{5̃62. Melothria Linu.}

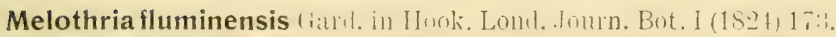

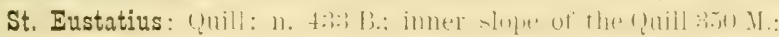
n. 418 B.

Saba: Windwardside: n. 1933 B.; Gainbygut: 11. 35.

Antill, Amer. cent. and austral. (Cogniaus 1881).

\section{S5๊ 1. Momordica Linn.}

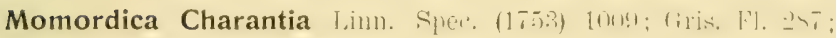
Duss 307; Millspaugh 583. Ma id enaple le.

St. Eustatius: on the slope of the Cliff near Oranjestad: 11. $58 \mathrm{~B}$; along roads in Oranjestad: n. $562 \mathrm{~B} ., 35 \mathrm{H} .4,42 \mathrm{G}$.

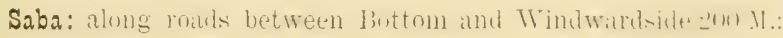
n. 1825 B., 1706 ; locality unknown: n. $163 \mathrm{~L}$.

St. Croix ex Millspaugh.

In all trop. and subtrop. countries. (Cogniaux 1881).

\section{Cucumis Linn.}

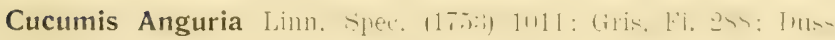
309; Millspaugh 582. Pu mpin.

St. Eustatius: Locality unknown: 11. $156 \mathrm{G}$.

Saba: along roads: n. 1707 B, locality unknown: n. $276 \mathrm{I}$.

St. Croix ex Mfillspaugh.

Florida austr, Antill., Texas, Amer. centr. and austr. sept. (Cogniaux 1S81). 


\section{Lagenaria Ser.}

Lagenaria vulgaris Sir. in Mem. Sox. I'lyys. (itmil. III. 1 (1S:5) 25; Gris. Fl. 288; Duss. 308 ; Sw e et gourd.

Saba: Mountain 800 M.: n. 2230 B.; locality unknown: n. 275 L., 308 L.

St. Croix ex Millspaugh.

Indig. in Afr. trop. and India orient. (Cogniaux 1881). Also cultivated.

\section{Cucurbita Linn.}

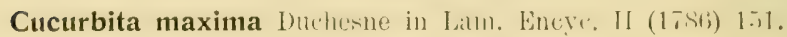

Cultivated. [Asia merid.] (Cogniaux 1881).

\section{Sechium Juss.}

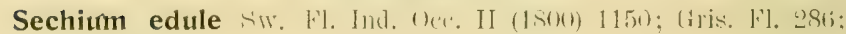
Duss 306.

Saba: Boobyhill: n. 1567 B.; Windwardside: 11, 1595 B.; Mountain 800 M.: n. $2198 a$ B.

Antill., Mexico, Amer. centr., Panama., Nova Granata, Venezuela, Brasilia. (Cogniaux 1881). Also cultivated.

\section{Goodeniaceae 8716. Scaevola Linn.}

Scaevola Plumieri Vahl Syml. II (1791) itt; 1iris. Fl. 3isis; Duss 378; Millspaugh 533.

Sccuevola Lobelia Murr. Syst. ed. XIII (1774) 178.

St. Martin: Colebily (coll. George): n. 2503 a 13.; nual ,imsonsbaylagoon: $11,30356 \mathrm{~B}$.

Bermud., Baham., Antill. (Her). Krug et Urban).

\section{Compositae.}

8751. Vernonia Schreb.

Vernonia punctata Sw. ex Wikstr. in Vet. Acul. Handl. Stuclih. (1827) 72 ; Gris. I1. 353; Duss 351.

Vernonia longifolia Pers. Syn. II (1807) 404, Wild tobacco.

St. Eustatius: ,ignalhill 30 M.: n. 1:38 B.; top of the Quill 40) M.: n. 206 B., 30! B., 328 B.; slope of the quill near Bengalen 50-200) MI: 11. 658 I3., 669 B.; along roads near linglish Quarter: n. $1006 \mathrm{~B}$; along roads near Bengalen: 11. 1197 B.; slope of the Quill: n. 55,57 ; slope of Signalhill: n. 56; locality unknown: 1 . $198 \mathrm{H}, 272 \mathrm{i}$. 
Saba: along roads hetween Bottom and Windwardsito 200 M.: 11. 1280 B.; Crespeen 400 M.: n. 1719 B.; Ladciergut 0-200 M.: n. 1968 B.; Ladder: n. 54.

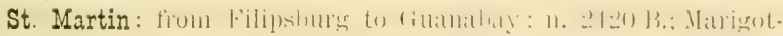
hill-Marigot 0-200 M.: n. $26 \pm 1$ B.; neal Mulletpond: 12. 2755 B.; hill on the northern side of Cul de Sac: n. 2975 B.: from Belvedere to Oysterpond: n. 3059 B.; Mount Paradis 150-400 M.: n. 3194 B., 3348 B.; Centryhill: n. 319.

Antill., Mexico, Honduras, (Gris, Fl.).

\section{Elephantopus Linn.}

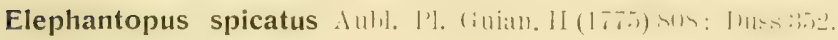
Distreptus spicatus Cilss. in Bull. Soc. 1'hilom. (1817) 66; Gris. F'l. 35.5; Millspaugh 534.

St. Eustatius: Slope of the Quill near Bengalen: 11. $711 \mathrm{a} \mathrm{B.}$

Saba: Boolyyhill: n. $1590 a$ B.; Crespeen-Rendoz-vous: 11, 199; Rendez-vous-Mlountain 4-600 M.: n. 198.

St. Croix ex Millspaugh.

Antill., Cuba and Mexico to Venezucla and Peru. (Gris. Fl.).

\section{Ageratum Limn.}

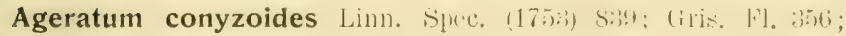
Duss 353; Millspiugh 533. Wh ite cap).

St. Eustatius: in a garden near Oranjestad: 11 . $77 \mathrm{~B}$; atlong roads in Oranjestad: n. 545 B.; slope of the Quill: 13. 290, 291, 292 ; locality unknown: n. $6 \mathrm{G} ., 6 \mathrm{H}, 230 \mathrm{H}$.

Saba: along roads between Bottom and Windwardside -201 M.:

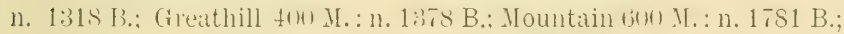
Laddergut 0-200 M.: n. 2022 B.; Crespeen-Rendez-vous: n. 289; Gainbygut: n. 288 ; locality unknown: n. 20 L., 135 L.

St. Martin: Mountain hetween Mont de's Aceorts and Centry.

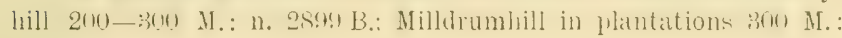
11. $3130 \mathrm{~B}$.

St. Croix ex Millspaugh.

In all warmer countries of both hemisph. (L.a. P.).

8816. Eupatorium Linn.

Eupatorium canescens Tahl, in Wrest Bill. St. Croix (lian) 302; Gris. Fl. 360; Duss 355.

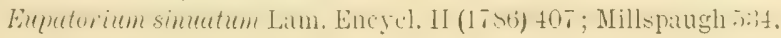


St. Eustatius: Gut near Fort do Wind: 11. 841 a B.; White Wall: 11. $814 a$ B.

St. Croix ex Millspaugh.

C'ula, Inomingo, I'ortorico, Antigua, (iuadelumes. (Herh. Krug et Urban).

Eupatorium conyzoides Valıl. Symb. Bot. 111 (1Mit) 46; (iris. [1. 358.

St. Eustatius: T'op of the Quill 400 M.: 11. $18 t$ B., 258 B., jul B.: along roads near Bengalen: n. 114; B.; slope of the quill: n. 25, 26, 288; road to Roundhill: n. 24 .

Saba: Crispeen 400 M. : n. 1717 B.; Laddergut: n. 1999 B.; liatpoint: 11. 2121 B. ; Peperpot: 11. 233; Mountain: n. 990; Gainhy. grut: 1. 289.

St. Martin: lower part of the Milldrumhill: n. 3162 B..

Bermuil., Florida austr., Autill., Mexico to Brazil. (Humsley 188t).

Eupatorium iresinoides H. B. at K. Nov. (iell. t.t tifue. IV (1820) 105. t. 340; Gris. Fl. 360; Duss 355.

Saba: Bottom: 200 M.: 11. $1+46$ B.; from Buttom tu Millypoint 100-2(1) M.: n. 1515) B.; Hellsgate: 11. 169() B.; Crispeen to0 M. : 1. 1795 B.; Peperpot: 12. 29; Crispeen Rendez-vous: 400 M.: n. 28. Antill., Panama to Venezuela. (Gris. H1.).

Eupatorium macranthum Siv. Fl. Ind. Uce. IIl 1 181ti) 1315.

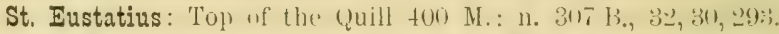

Saba: Mountain near Windwardsisle to0 MI.: 11. 21-4 B., 217s 13.: Crespeen-Rendez-vous 400 M.: 11. 31, 293.

St. Kitts. (Herb. Krug et Urban).

Eupatorium macrophyllum Limn. Apec. (176;:3) 1175: Millspaugh j:34.

Hebeclininm macroplugllum DC. Prodr. V (1836) 136; Gris. Fl. 356 ; Duss 353 .

Saba: between Bottom and Marypoint 300 M.: 1. 1487 B.; 100-200 M.: 11. 1511 B.; Laddergut 0-200 M.: n. 1965 B.; Gainbygut: n. 33 .

St. Martin: Mount Paradis 300-400 M.: n. 3349 B.

St. Croix ex Millspaugh.

Bermud, throughout the Antill. and southward to Brazil. (Hemsley 1884).

Eupatorium paniculatum Schrakl. Ind. S(1n. Hort. (iotting. (18:3:) 2 ; Gris. Fl. 362 ; Duss 356.

Eupatorium guadalupense Spreng. Syst. III (1826) 414. 
Saba: 'Tup of the Mountilin s00 M.: 1., 1805 B.; II cllsgate: 11. 291; Marypointgut: 11. 27.

Antill., Blonduras, Peru, Venezucla, Brazil. (tiris. El.).

\section{Erigeron Limm.}

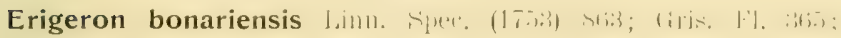
Duss 360.

Saba: Molntain l;-800 M.: 11. 11.

Antill, Amer: centr. to Buenos Ayres. ((tris. li.).

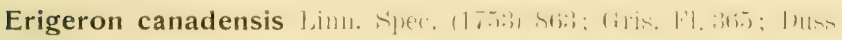
360. Foxtail.

St. Eustatius: Slope of the Quill: n. 13; Cultuurvlakte: 11. 12; locality unknown: 1], $145 \mathrm{G}, 173 \mathrm{H}$.

Saba: Hellsgate-Maryloint: n. 2033 B.; Rendez-vous 400 M.: 11. 296; locality unknown: n. $248 \mathrm{~L}$., $259 \mathrm{~L}$.

St. Martin: near Mullutpond: n. 2760 B.; lower part of Milldrumhill: 11. 3169 B.

Bermud., Canada to Brazil, and naturaliserl in many other countries of hoth hemisph. (Hemsley 1884).

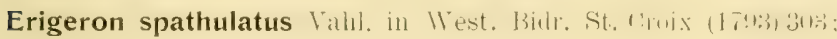
Gris. Fl. 365; Duss 359; Millspaugh 534.

Saba: between Botton and Marypoint 100_-200 M.: 11. 1502 B. 1558 B.; Latddergut 0-200 M.: 11. 1958 B., 286; P'eperpot: 11. 20; Crespeen-Rendez-vous: 11. 19; Gainbygut: 11. 287.

St. Martin: Milldrunhill in plantitions 300 M : 11, 3128 B.

St. Croix ex Millspaughl.

Hispaniola, Portorico, Martiniyuo, Guadeloupe, 'lrinidad. (Herb. krug et Urban).

\section{Gundlachia A. Gray.}

Gundlachia domingensis A. (ilaty But. Cuntr. in l'rue. Amet. Acild. XVI (1880) 100.

Baceharis dioica Gris. Kill. (1857) 85; Gris. L'l. 366; Duss 361.

Saba: near the Sulphurmines: n. 1700 B.

Baham., Antill. (Symb.).

\section{Baccharis Linn.}

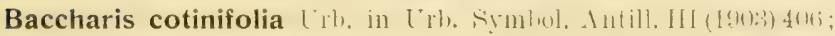
Gris. Fl. 366; Duss 860.

Saba: Top of the Mountain: 11. 274.

Antill., Costarica. (Symb.). 


\section{Pluchea Cass.}

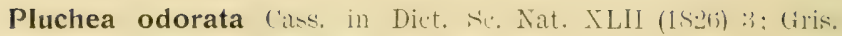
Fl. 366; Duss 361; Millspaugh 535. Wild tobacco.

St. Eustatius: Flope of the Clift near (rallowsbaly: n. (6.2 B.; seashore: n. 30 ; locality unknown: n. $61 \mathrm{H}$., $116 \mathrm{G}$.

Saba: between Bottom and Maryloint 100-200 M.: 11. 1.226 B.; Crispeen--Rendez-vous 400 MI.: n. 303; locality unknown: n. 228 L., 234 L.

St. Martin: Milldrumhill in plantations 300 M.: n. 3127 B.

St. Croix ex Millspaugh.

Antill., Guiana., Amer. centr. (Hemsley 1884).

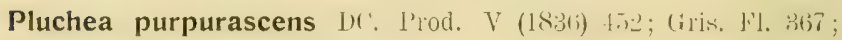
Duss 362; Millspaugh 535.

St. Martin: Cul de Sac-Marigothill 0-200 M.: n. 2621 B.

Anguilla Boldingh.

St. Croix ex Millspaugh.

Bermud., Florida and Mexico, Antill, to Nova Granata. (Hems. ley 1884).

\section{Gnaphalium Linn.}

Gnaphalium purpureum Linn. Spee. (1-5.3) h.j. vel. affin.

Gnaphatium americanum Mill. Gard. Dict. ed. VIII (1768) n. 17; Duss 373.

Saba: Paris 400 M.: n. 1375 B.; between Bottom and Marypoint 100-200 M.: n, $1502 \alpha$ B.; Greathill: n. 37; Jenkeygut: n. 36 .

Amer. and warmer parts of Africa, Asia and Australia. (li. a. P.).

\section{Lagascea Cav.}

Lagascea mollis Cav. in Anal. (Genc. Nat. VI (1S0:3) :3:3 t. 44.

Saba: along roads between Bottom and Windwardside $200 \mathrm{M}$.: n. 1310 B.; along roads 200-300 M.: 11, 1708 B.; Windwardside: n. 2091 B.. 167.

St. Martin: from Filipshury te (iuanabay: 12.2403 B., 24t5) B.; Cul de Sac-Marigothill 0-200 M.: n. 2631 B.; hill on the northern side of Cul de Sac: n. 2972 B.; French frontier: n. 68; Marigotroad: n. 69, 299.

Anguilla Boldingh.

Indig, in Amer, centr. (E. a. P.). 


\section{Clibadium Linn.}

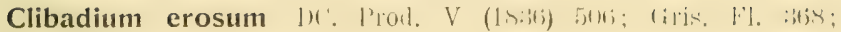
Duss 362.

Saba: Mountain ti-800 M.: 11. 179.

Portorico, Dominica, Hartinique, St. Vincent. (IFer). Krug et Urban).

\section{Acanthospermum Schrank.}

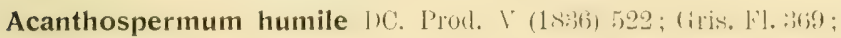
Millspaugh 533 .

St. Martin: Cul de Sac-Marigot 0-200 M.: 11. 2657 B.; along roads from Filipshurg to Marigot: 11. $3109 \mathrm{~B}$.

St. Croix ex Millspaugh.

Jamaica, Cuba, Haiti, I'anama. (Gris. F'l.).

\section{Parthenium Linn.}

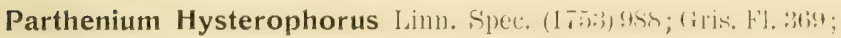
Duss 365; Millspaugh 535.

St. Eustatius: in a girden in (1):anjestad: 11. 7!l B., 10; alung roads in Oranjestad: n. 520 B., $3 \mathrm{G} ., 3 \mathrm{H}$.

Saba: Laddergut: n. $195 \pm$ B., 11; Mountain near Windwardside 600 M.: n. 2165 B.; locality unknown: n. 56 L.9 95 L.

St. Martin: from kilipsburg to Guanabay: n. 2398 B.

Anguilla Boldingh.

St. Croix ex Millspaugh.

Antill., Amer. sept. and centr., and in other parts of both hemisph. (E. a. P.).

\section{Ambrosia Linn.}

Ambrosia hispida P'urel. F'. Am. Sipt. suppl. (1>1t) it:3. Ambrosia crithmifolia DC. Prod. V (1836) 525.

St. Eustatius: in a garden in Oranjestad: $12.157 \mathrm{G} ., 1102 \mathrm{~B}$. Saba: along roark between Bottom and Windwarlside zon 11. 11. 1303 B.; Windwardside: n. 1949 B.

St. Martin: from Filipsburg to Belvedere: n. 2561 B.

Florida. (Ind. Kerv.). Also cultivated.

\section{Xanthium Linn.}

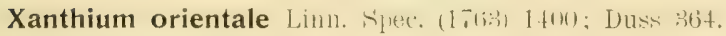

Saba: Bottom: n. 62 .

In almost all warmer countries of both hemisph. (E. a. P.). 


\section{5ั. Zinnia Lim!.}

Zinnia elegans Jacer. Coll. 111 152.

Cultivated. [Patria ignotio.].

\section{Eclipta Linn.}

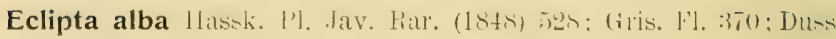
366; Nillspaugh 534.

Liclipte erecte Linn. Hant. II (1771) 2S6.

St. Martin: lower part of Mount Paradis: 11. 3187 B.

St. Croix ex Millspangh.

In the warmer countries of loth hemisph. (k. a. P.).

\section{Borrichia Adlans.}

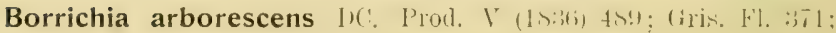
Duss 366; Millspaugh 533.

Saba: Peperpot: n, 278.

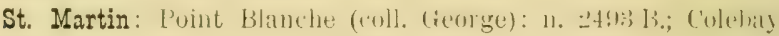
(coll. George): n. 2506 B.; near Mulletpond: 11. 2750 B.; near Simsonshayvillage: n. 3030 B.; 'l'intamare: n. 3373 B.; Oysterpond: 11. 30t; Guanabay: 11. 30t, 305.

St. Croix ex Millspaugh.

Antill., Bermud., Key of F'lorida, seashore of the Carib. Scia to Poru. (Hemsley 1884 ; E. a. P.).

\section{9:. Wedelia Jacr.}

Wedelia buphthalmoides (iris. in (inett. Ab). IH (1,45) 2:35); Gris. Fl. 372; Duss 367; Millspaugh 536. Y ellow sage.

St. Eustatius: Tor of the Quill to0 M.: n. 27+ B., 2int B.; shpet of the Quill near Bengalen 150 M.: n. 681 B., 687 B.; gut near Fort de Wind 50 M,: $\mathrm{n}, 836 \mathrm{~B}$; along roads near Glassbottle: 11. siti B.; from Little Momtain to Vellusway: 11. !196 B.; slupe of the quill on the side of White Wall 200 M.: n. 1171 B.; along roats neal Bengalen: 13. 119s B.; (iilhoohill: n. 58; Sugarloaf: n. 317 ; locality unkown: 1n. $165 \mathrm{G} ., 233 \mathrm{H}$.

Saba: along roads between Botton and Windwandside 20n M.: 11. 12st B., 12ss B.; between Bottom and Marypoint zon M.: 1). 15:3) B.; Helsgate: n. 169t B.; Laddergut 0-200 M.: 11. 1960 B.; gut hetween Ifellsgate and Mastiegut $300-4110$ M.: 11. 20.s B.; Windwardside: n. 2315 B.; Peperpot: 11. 318: Paris 2-410 M.: n. 61: Crespeen-Rendez-vous: n. 60; locality unknown: n. 311;, 2! L.

St. Martin: from Filipsburg to (iuanabay: n. 2410 B.; Nilked- 
boyhill 50-200 M.: 11. 2703 B., 200-260 M.: 11. 2719 B.; Mountain

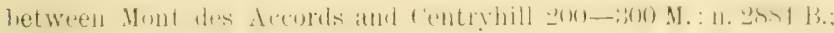
hills on the northern sile of Cul de Sac: 11.2984 B.; lower part of

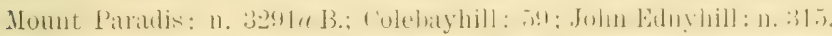

I also saw Wedelia buphthalmoides: on the top of Mount Paradis $400 \mathrm{M}$. hills on the eistem side of Cul de sac.

Anguilla Boldingh.

St. Croix ex Millspaugh.

Baham. (Hitchc.); Antill. (Herb, Krug et Ulban).

9196. Tithonia Dest.

Tithonia tagetiflora Desf. in Antr. Mus. Pizl. I (IStiz) fli. t. t. Cultivated. [Mexico]. (E. a. P.).

\section{Spilanthes Jacy.}

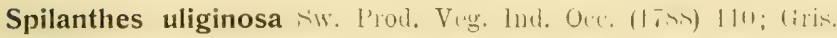
11. 376; Duss 370.

St. Eustatius: near English Quarter: n. 1018 B.

Warmer countries of both hemisph. (E. a. P'.).

\section{Synedrella Gïrtn.}

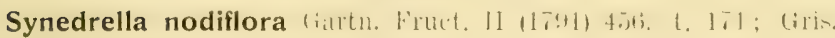
H. 377; Duss 371.

Ucacoua nodiflora Hitch. in Missouri. Bot. Gard, Rep, IV (189:3) 100 ; Millspaugh 535.

St. Eustatius: Slope of the Quill: 1,47 ; locality unknown: 11. $2+2 \mathrm{H}$.

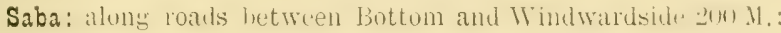
1332 B.; lower part of the Bottom Mountain 250 M.: n. 1397 B.; along the Forthgut 150-200 M.: n. 1629 B.; Mountain 600 M.: 11. 1754 B.

St. Martin: Nilkethoyhill .00-100 M.: 1. 2-T6 B.: mountain

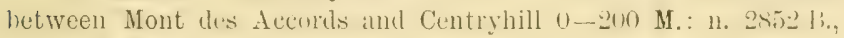
200-300 M.: 11. 2892 B.

St. Croiz ex Millspaugh.

Indig. in Amer, trop.; dispersed in the Old world. (E. a. P.).

\section{Bidens Linn.}

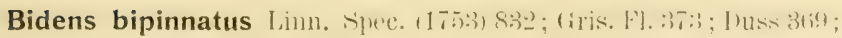
Millspaugh 533. 


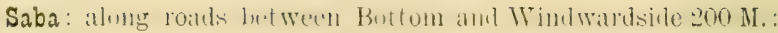
11. 1333 B., 1366 B.; Rendez-vous: n. 275 ; Rendez-vous-Crespeen: 11. 276 ; locality unknown: n. $255 \mathrm{~L}$.

St. Martin: Hill on the northern side of Cul de Sac $0-100$ M.: 11. :3003 B.; along roarls from Grimde Case to Marigot: 11. 3099 B.; French frontier: n. 295.

St. Croix ex Millspaugh.

Indig, in Amer, dispersed in the warmer countries of the Old world. (F., a. P.).

Bidens leucantha Willd. Sp. I’l. III (Ist()) 17l9; (iris. Fl. 3̈7: ; Duss 368; Millspaugh 533.

Bidens pilosa Linn. Spec. (1753) $\$ 32$.

St. Eustatius: Slope of the Quill: n. 302.

Saba: along mads between Bottom and Windwarlside n. $1282 \mathrm{~B}$; Windwardside-Hellsgate: n. $1672 \mathrm{~B}$; seashore near Fiat Point: 17. 2139 B.; Crespeen-Rendez-vous n. 301 ; Gainbygut: 11. 303 .

St. Martin: Hill on the northern side of Cul de Sac $0-100$ M.: 11. 2978 B.; Marigotroad: n. 279; locality unknown: n. 3602 B.

St. Croix ex Millspaugh.

Indig. in Amer, dispersed in the warmer countries of the Old world. (L. a. P.).

\section{Cosmos Cav.}

Cosmos sulfureus Cav. Ic. I (1791) 56 t. 79. Poppy.

Cultivated. from Cuba and Mexico to Brazil.] (Gris. Fl.).

9306. Gaillardia Fouger.

Gaillardia aristata Purch. 1\%. Am. Supt. II (1<14) $57: 3$. Cultivated. [Amer, bor.] (Ind. Kew.).

\section{Porophyllum Adans.}

Porophyllum ellipticum Cass. I)ict. XIIII (15:36) 56.

Porophyllam muderale Gris. Flor. W. I. (1861) 379; Duss 372. (p. p.). (Symb.).

St. Eustatius: along roads in (Minjestad: 11. $1111 \mathrm{~B}$.; slope of the Quill: n. 36, 37, 38; locality unknown: n, $277 \mathrm{H} ., 28+\mathrm{G}$.

Saba: lower part of the Bottom Mountain 300 M.: n. 1435 B.; hetween Bottom and Maryoint 100-200 M.: n. 1555) B.; locality unknown: n. $269 \mathrm{~L}$. 
St. Martin: Locality unknown: n. 3603 B.

Baham., Antill, Amer. cont. trop. (Symb.).

\section{Pectis Tinn.}

Pectis febrifuga van Hall. in Amn. Hort, of But, ou F'lome Jart. des Pays-Bas vol. IV (1861) 333 cum tab.

P'ectis linifolia Less. in Limmaer VI (1831) 709. (excl, syn.); (Symb.).

Pectis Swartziena Borg et Pauls. Veget. Dansk-vestind. Oer (1898) 110 - non Less; (Symb.).

Saba: Seashore near Forthloay: n. 1641 B.

Antill., Bonaire, Curaça, Aruba, Columbia. (Symb.).

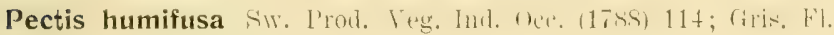
378; Duss 372; Millspaugh 535.

St. Eustatius: Whito IVall: 11. 824 B.: Gilboohill 150 M.: n. 1122 B.; near Boven 200 M.: n. 1249 B.; near White Wall: n. 1258 B.; Teuuslay: n. 20 ; locality unknown: n. 3586 B.

Saba: along the lower part of korthgut: n. 1642 B.; St. Giles: 11. $1869 \mathrm{~B}$.

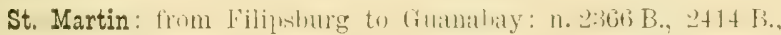
19); near Fort Willem: 11. 2801 B, near Fort Amsterdam: n. 18 . Anguilla Boldiugh.

St. Croix ex Millspaugh.

St. Domingo, Portorico, St. Thomas, St. Jan, St. Barthélemy, St. Kitts, Antigua, (rudeloupe, Desirale, Narie-(ialante, Dominica, Martinique, St. Lucia, St. Vincent, Mustique, Barhados. (Symb.).

\section{Neurolaena R. Br.}

Neurolaena lobata R. Br. in Trams. Limn, soc. XT (1S17) 1:2): Gris. Fl. 381; Duss 373.

St. Eustatius: Slope of the Quill ahore White Will: 13. 118.5 l3. Saba: Top of the Hountain 800 M.: n. 1836 B.; locality unknown: n. $210 \mathrm{~L}$.

Antill., Mexico, Columbia. (E. a. P.).

\section{Erechthites Raf.}

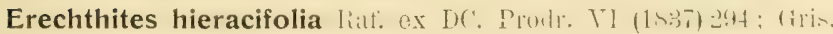
Fl. 381; Juss 374; Millspaugh 534.

Erechthiles praealta Raf. Fl. Ludov. (1817) 65. 
St. Eustatius: Slope of the Quill near Bengalen: $n .9$.

Saba: between Bottom and Marypoint 300 M.: n. 1485 B.; Gainbygut: n. 10 .

St. Croix ex Millspaugh.

In all warmer parts of Amer, also in the Old world. (E. a. P.).

Erechthites valerianaefolia DC. Prorl. VI (15:37) 29.

Saba: between Bottom and Marypoint 350 M.: n. 1491 B.;

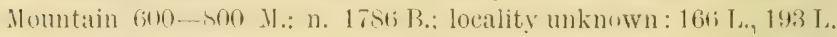

Amer. centr. and sept., Java. (Herh. Berlin).

\section{Senecio Linn.}

Senecio sonchifolius Mornch. Mrth. Suppl. (1.802) 231.

Eimilia sonchifolia DC. Prod. VI (1837) 302; Gris. Fl. 381; Duss 374 ; Millspaugh 534.

St. Eustatius: near Boven 200 M.: n. 1234 B.; locality unknown n. $126 \mathrm{G}$.

Saba: along matds betwern Bottom and Windwarikide $200 \mathrm{M}$.: 11. $1285 \mathrm{~B}$., $1348 \mathrm{~B}$; Windwardsile: n. $2309 \mathrm{~B}$.

I also saw Senecio sonchifolius in the Laddergut.

St. Martin: Naliedboyhill 5)_-200 M.: n. 270. B.; Motuntain between Mont des Accorls and ('entryhill 20(1) - 306) M.: 11. 2890 B.; Milldrumhill in plantations 300 M.: n. 3129 B.

I alsn saw Senicio sonehifolins: Momnt Paratis $300-400$ M.

St. Croix ex Millspaugh.

Indig. in the trop. of the old workl. (K, i. P.).

\section{Chaptalia Vent.}

Chaptalia nutans Hemsl. Biol. Centl. Am. Bot. II 25is; Mill. spaugh 533.

Leria mutans DC. Ann. Mus. Par. XIX (1812) 68; Gris. Fi. 383: Duss 375.

St. Eustatius: Slope of the Quill: 1n. 310.

Saba: along roals between Bottom and $1 \mathrm{~T}$ indwarisile $200 \mathrm{M}$. n. 1815 B.; Mountain 400 M.: n. 1769 B., 1774 B., :308; CrespeenRendez-vous: n. 307, 309.

St. Martin: Milldrumlill 300 M: n. 3179 B.; Mount l'aradis 300-400 M.: n. 3339 B.

St. Croix ex Millspaugh.

fn all warmer countrios of $A$ mer. (E. a. $\mathrm{l}^{3}$.). 
9595. Sonchus Limn.

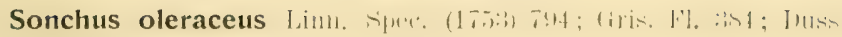
375 ; Millspaugh 535. W ild salad.

St. Eustatius: Glassgut at the seashore near Oranjestal: n. $48 \mathrm{~B}$; Bengalen: n. $606 \mathrm{~B}$; along roads near Glassbottle: n. S.s B B; slope of the Quill: n. 41 ; Roundhill: n. 302.

Saba: lower part of the Bottom Mountain 300 Mr: n. 1430 B.: Mountin 600 M.: n. 17s0 B.; Bottom: n. 40; locaity unknown: 11. $40 \mathrm{~L}$.

St. Martin: Milldrumhill in plantations 300 M.: n. 3133 B.

St. Croiz ex Millspaugh.

Widely dispersed in both homisph. (k. a. P.).

\section{Lactuca Limn.}

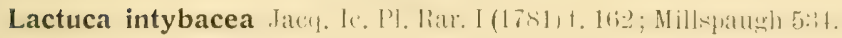
Brachlyteamplens intybaceus DC. Prod. VII (1888) 177; Gris. Fl. 384: Duss 376.

St. Eustatius: Cultuurvlakte: 11. 57: seashore: 11.307.

St. Croix ex Millspaugh.

Antill., Nicaragua, last Indies, Africa trop. (Gris. Fl.). 



\section{SECOND PART}

\section{HISTORICAL}





\section{HISTORICAL}

\section{CHAPTER I \\ HISTORICAL SKETCH.}

The following persons have given informations about the Flora of the Dutch West Indian Islands. Liter. Urban Symbolae Antillanae Vol. I and Vol. III.

\section{St. Eustatius.}

J. B. Labat, between 1694 and 1705.

I. B. Labat in his: Nouveau voyize aux isles de l'Amérique.... Editio 1724. La Haye, does not give any compendium of the vegetation of the Dutch Antilles.

N. J. Jacquin between 1755 and 1757.

N. J. . Tacquin in his: Selectarum stirpium americanum historia, in

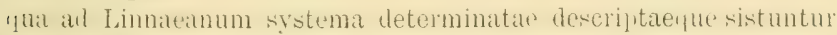
flantae sllae, yuas in instulis Martinica, Jamaica, Dontingo alimpue et in vicinae continente parte olwervavit rariores; adjectis iconibus in solo natali delineastis. Vinclobonae 1763, mentions only 6 plants found in St. Eustatius, of which I point out only those quoted by Grisebach in his: Systematische Untersuchungen über die Tegetation der Karaben, insbesomdere der Jnsel tiuadeloupe: Justicia sessilis.

Justitia eustachyana. (Beloperone custachiana Benth.).

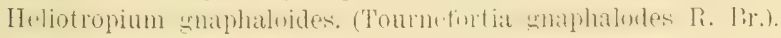

Coccoloba nivea.

Cactus triangularis. (Cereus triangularis Haw.).

(Heri). Banks. Brit. Mus. London; Herb. Willd. Berlin).

F. Masson 1780.

I only saw Masson mentioned by 0 . Swartz in his: Flora Indiae occidentalis, concerning Microtea debilis.

(Brit. Mus. London). 
S. Fahlberg between 1785 and 1834 .

I nnly saw Fullherg mentioned hy o. Sicatz in his Floril Indiate occidentalis with reference to a few plants from St. Barthélémy.

(Herb. Swartz. Stockholm; herb. Thunberg Upsala).

Placide Duchassaing de Fontbressis about $18 \pm 8$.

P. D. de Fonturessis collected only at few plants in St. Eustatins; I saw the following mentioned in Gris buch: Systematische Untersuchungen über the Vegetation der hiraibu, inshesondere der Insel Guadeloupe:

no. 16. Lepidium virginicum Linn.

no. 22. Polanisia viscosi DC.

no. 24. Capparis cynophallophora Linn. $\gamma$ attenuata.

no. 162. Sida spinosa Linn.

no. 2st. Tribulus maximus Jimm. (Kallstromia maxima Wight et Arn.).

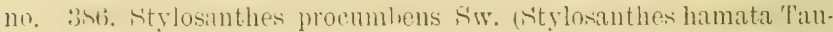
bert).

no. 94.2. Cyphiacanthus babalensis Ns. (Ruellia tuberosi Limn.). no. 1047. Jeliophytum indicum Dr: (Heliotropium mdicum Linn.). no. 1074. Lantana camara Linn.

no. 1076. Lantana involucrata Linn.

(Herb. Göttingen).

B. A. Euphrasen 1788.

B. A. Eupherasen in his: Beskrifning ofver srenska vestindiska an St. Barthélimi samt öarne st. Eustache och St. Christopher. Stockholn 179s, makes alin mention of a ferw plants from st. Eustatius. I did not think it necessary to quote them here as, according to the statement of Urban (Symbolae Antillanae 1 1. 4(1) it is impossible to control the determination of Euphrasen.

\section{F. L. l'Herminier 1815.}

M. D. Feenstra about 1836 .

The few botanical data I could tike from his: De Nellerlandsche West-indische eilanten in dery lver tegenwordigen toestand. Am. sterdam. 1836-1837 are of 110 use to the Systematic Part. In clescribing the regetation in the Thim laut I shall say something about this paper of Feenstra. 
IV. F. R. Suringar 1885.

$W . F$. R. Suringar made a botanical investigation on the Dutch West Indian Islands in 1885.

Further details about this expedition will he linum in ('hapter II of the Historical Part of this Flora.

(Herb. Leiden, Herb. Krug et Urban Berlin).

J. J. Walch about 1889 .

In the Report of the 60 meeting of the Br. Ass. f. Adv. Sc. Leeds 1890 p. 448 the following is said about the collection Walch: A small collection of plants numbering 143 specimens, was receiver from $M r . J . J$. Walch, $R$. $N$. This collections includes plants from Dominia, St. Martin, it. Eustatius, St. Kitts, st. Lueia and (irenildil. Most of the plants consisted of common West Indian spe. cies, presumably such as would be met with in the more accossible spots in the various places visited.

(Herbar. Kew.).

F. A. F. C. Went 1902

F. A. F. C. Went is his: Rapport omtrent den toestand van landen tuinhumw op de Iederlantsche Antillen, bijlage $V$ van het koloniaal verslag van Curaçao van 1902, gives some observations on the vingetation of tit. Eustatius; in so fill as thesenbservation will be interesting to us I shall deal with them in the Third Part of this Flora.

J. van Grol-Meyers 1904-1906.

Two collections of plants gathered by Mrs. van Grol-Meyers will he treated more fully in chapter. II of th. Historital Part of this Elora.

(Herb. Utrecht; Herb. Koloniaal Museum Haarlem).

\section{Boldingh 1906.}

The botanical investigations I made in 1906 will more largely be dwelt on in Chapter II of the Historical Part of this Flora.

Besides the abovo mentioned data I found in Grisebach: Flora of the British W. I. Islands that the following plants were also collected in St. Eustatius:

p. 66. Lithophila muscoides Siv.

p. 75. Sida ulmifolia Cilv.

And in Grischech: Systematishe [ntersuchungen äher die Vegetation der Killaben, inshesomere der Insel Guadeloupe I lound: 147.2. Aspidium macrophyllum Sw. (Aspidium martiniconso Spr.). 


\section{Saba.}

J. H. Labat between 1694 and 1705 .

Conformable to what I mentioned under St. Eustatius.

I. E. Forsstrüm 1805-1812.

I saw Forsström mentioned only in reference to a few plants in Urban, Symbolae Antillanae.

(Herb. Swartz and Herb. Stockholm).

M. D. Fieenstra about 1836.

Conformable to what I mentioner under St. Eustatius.

Placide Duchassaing de Fontbressis about 1848.

According to (ris burh: Systematische. [ntersuchungen über dic Vegetation der Karaiben, insbesondere der Insel Cuadelonle, P. D. de Fontbressis collected in Saba: 12. 187. Guazuma tomentosa Kth.

\section{O. Kuntze 1874.}

Besides at Caesalpinia ciliata Urb. I did not see O. Kunlze mentioned at the label of any plant in the Herbarium of Krug and Urban in Berlin. In his: Um die Erde. Reisebericht eines Naturlinschers, Leipzin 1881, Kienar mentions only Melucactus communis Link et Otto.

(Herb. Krug et Urban Berlin).

IV. F. R. Suringar 1885.

F. A. F. C. Went 1902.

I. Boldingh 1906.

For these three persons I refer to what was said under St. Eustatius.

A. C. W. Lionarons 1907.

'The plants gathered by Liunuruts will fully be treated in Chapter' II of the Historical Part of this Flora.

\section{St. Martin.}

J. B. chu I'ertre about $1650-1656$.

.T. 13. Labat between 1694 and 1705 .

Conformable to what I mentioned under St. Eustatius.

N. J. Jacquin between 1755 and 1757.

N. J. Jacquin in his: Select. Stirp. Amer., does not mention but 8 pitnts found in St. Martin. I will quote only the plants enu. 


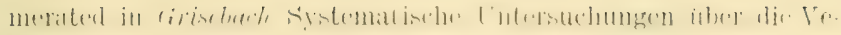
getation der Karaben, insbesondere der Insel Guadeloupe:

Myginda uragogil cf. Symb. Antill. V. p. 80. (Rhacoma crossopetalum Linn.).

Rhamnus colubrinus (Colubrina ferruginosa Brong.).

Malpighia linearis (Mralpighia angustifolia Limn.).

Corchorus hirsutus Linn.

Lobelia Plumieri (Scaevola Plumieri Vahl).

A. Plée 1821.

After a discussion I had with Prof. I. Urban, I think it superfluous to quote the few plants that are collected by Mr: Plie. (Herb. Mus. Paris).

MI. D. Feenstra about 1836.

W. F. R. Suring(t) 1885.

J. J. Wulch about 1889.

F. A. F. C. Went 1902.

I. Boldingh 1906.

For these 5 persons I refer to what was said under St. Eustatius.

Accorling to Grisebuch: Systematische Untersuchungen über die Vegetation der Karaiben, insbesondere der Insel Guadelonpe, also the following plants woro collected in St. Martin:

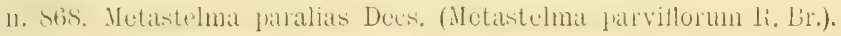

n. 1009. Cuscuta americana Limn.

According to the notes I made about the persons and collections afore said 1 did not think it desirable to treat any of the smaller collections. The difficulties in gretting all these pliants would not be in proportion to the small amount of greater completeness of the plants then mentioned in this Flora.

So I have treated in the Systematic l'art only the collcetions male by Suringar, van Grol-Mleyers, Boldingh and Lionarons.

It will appear from these collections that I have about 5000 numbers of plants at my disposal, a lig number when we take into consideration that the surface of the 3 Islands does not sur. pass $140 \square \mathrm{km}$. 


\section{CHAPTER II}

\section{NOTES ON THE LARGER COLLECTIONS.}

\section{Collection W. F. R. Suringar.}

Dr. J. Valckenier Suringar was so kind as to allow me to make use of the diary written by his father Prof. W. F. R. Suringar during his hotanical investigations of the Dutch West Indian Islands in 1885 .

From these notes together with the datal drawn from the lalush attacher to the collected plants I was enalued to make the fol. lowing list of the localities visited by suringu and the numbers of plants collected there.

St. Eustatius: April 8th-18th and May 7th-18th.

April 8th. Concordiabay, Turtlebay.

Ma 15̆th. Fairplay, English Quarter.
May 7th. Bengalen.

April 9th. Signalhill and environment.

"11th. Little Mountain, Tumbledowndickbay.

17th. Boven, Little Mountain.

coll. 50 nos.

May sth. Fort Amsterdam.

" 9th. Gilboohill.

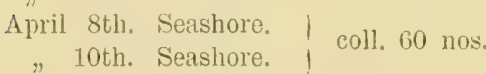

May 11th. Sugarloaf.

April 13 th.

$"$ 14th.

,16th. Quill. coll. 170 nos.

May 18th. |

On lis 16 excursions through St. Eustatius Suringar has ovidently collected 385 nos.

All the lnealities visited hy simingar were also investigated by me. 
In proportion to the other two islands st. Eustatius was inves. tigated by Suringar best of all, thanks to a longel stay there.

Saba: April 21-28th.

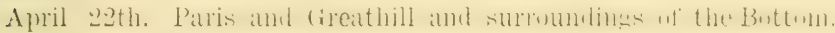
coll. 50 nos.

22th. Peperpot near Springbaygut. coll. $3 \check{\text { nos. }}$

23th. Top of the Mountain. coll. 100 nos.

24 th.

26th. Surroundings of the Bottom. coll. 50 nos.

28th. I

25th. Gainbygut. coll. 30 nos.

27th. Round the Island. coll. 90 nos.

27th. Laddergut, coll. 40 nos.

Suringar has evidently collected during these ? excursions 395 nos.

I myself saw all the localities visited by Suringar.

St. Martin: April 30th-May 5th.

April 30th. Saltpond and Fort Amsterdam. coll. 40 nos.

May 1st. Centryhill and Surroundings. coll. 45 nos.

2nd. from Filipsburg to Simsonsbay. coll. 50 nos.

3rd. Nakedboyhill, Princequarter. coll. 25 nos.

" 4th. from Filipsburg to Cul de Sac and French frontier. coll. 60 nos.

5th. from Filipsburg to Guanabay. coll. 20 nos.

Suringar has collected during these 6 excursions 240 nos.

'The short time Suringar could spend on St. Martin is the reason why this island was explored but on a very small scale; the French part of St. Martin has not been studied; all the localities named by Suringar were also visited by me.

The collection suringar is preserved is "'s Rijks-herbarium" at Loiden; duplicates are to be found in the collection Herl. Krug et ("rhan in Berlin (h. Bot. (iart. unn Museun , Steglit\%. Dahlem). The wreater jart of the suringir plants were leterminatrel hy I. Urban before I took up the study of the WVest Indian Flora and another part was determined by him afterwards.

\section{Collections Mrs. J. van Grol-Meyers.}

The two collections of dried plants given by Mrrs. .I. van

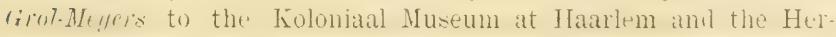


barium of the Botanical Laboratory of the Universily of Utrecht consist both of about 350 numbers of well driel plants with ample notes on the colour of flowers and on the way the inhabitants of St. Eustatius make use of these plants.

In my paper: Lijst van Planten, die door de Bervoners van de Wrie Nederlandsche Antillen st. Eustatius, Maba en st. Martin als weneeskrachtig worlen beschouwl. Bull. Kolon. Mus. Hatarlem 11. 38 (1907) p. 93-112, these notes have been of great use to me.

As might be expected the greater part of the plants in these collections are common tropical plints growing in the more accessible parts of the Island; there is moreover a fine collection of plants from the Quill. The eximination these plant- hefore I entered upon my voyage, has rendered it easy for me to recognise them in their natural state.

The cultivated plants sent in by Mrs. van Grol fucilitated my paying attention also to these plants, and this was the more agreable for me as I could thus the better comply with the wishes of the "Commissie van het F. W. van Eeden-fonds." Thanks to the great number of flowers and fruits occurring in the collection van Grol-Meyer's it was not too difficult for me, though beginning with the study of the West Indian Flora to find out the names.

'The following list contains the names of the plants collected by Mrs. J. van Grol-1eyers.

\section{Collection J. van Grol-Meyers of the Koloniaal Museum at Haarlem.}

1 Asclepias nivea.

2 Commelina virginical.

3 Parthenium Hysterophorus.

4 Cassia occidentalis.

5 Chenopodium ambrosioides.

6 Ageratum conyzoides.

7 Heliotropium parviflorum.

8 Euphorbia pilulifera.

9 Chenopodium murale.

10 Scoparia dulcis.

11 Capraria biflora.

12 Datura Metel.

13 Datura Stramonium.

14 Heliotropium indicum.
15 Sida carpinifolia.

16 Amarantus tristis.

17 Argemone mexicana.

18 Antigonon leptopus.

19 Ipomoea pentaphylla.

20 Triphasia trifoliata.

21 Passiflora quadrangularis.

22 Cassia bicapsularis.

23 Ricinus communis.

24 Capsicum dulce.

26 Abrus precatorius.

28 Polanisia viscosa.

29 Gynandropsis pentaphylla.

30 Poinciana regia. 
:il Plumbago seandens.

32 Euphorbia heterophylla.

33 Salvia serotina.

3t Phyllanthus niruri.

85) Momordica Charantia.

iti Acacia macracantha.

3it Leucaeni glauca.

is Terminalia Catappa.

34 Acacia macracantha.

40) T'amarindus indica.

41 Psidium guajava.

t. Zephyranthes carinati.

4:) Calotropis procera.

4 Melia azedarach.

4.) Anacardium occidentale.

46 Capparis cynophallophora.

47 Solanum racemosum igneum.

the Plumiera rubra.

4 4) Plumiera alba.

5) Oncidium spec.

.1 Brassavola rigida.

5: T'ecoma leucoxylon.

33 Picrasma antillana.

54 Croton flavens.

5) Eugenia axillaris.

ix Lantana involucrata.

if Mangifera indica.

58 Punica Granatum.

59 Achras sapota.

60 Lochnera rosea.

(j1 Pluchea odorata.

(i:) MIalpighia punicifolia.

66 Vigna repens.

67 Caesalpinia pulcherrima.

is Caesalpinia pulcherrima.

69 Caesalpinia ciliata.

70 Mammea americana.

il Spondias lutea.

72 Spondias purpurea.

73 Chrysobalanus icaco.

7 Justicia sessilis.

75 Hymenaea Courbaril.
76 Eugenia unitlora.

77 Linociera compacta.

is Piper reticulatum.

7!) Passitlora laurifolia.

se) Byrsonima spicata.

\$1 Cassia glandulosi.

ר) Byrsonima spicata.

s.) Ruellia tuberosa.

s.) Opuntia Ficus indica.

sti Melicocca bijuga.

si Citrus spec.

is Anona squamosa.

s.) Anona muricata.

(4) Phyllantus distichus.

!1 Mloringa oleifera.

(13 foeniculum vulgare.

4.) Carica Papaya.

!ki Solanum lycopersicum.

97 Solanum lycopersicum.

4s Solanum esculentum.

(4) Cuscuta americana.

(111) Clusia alba.

101 Coftea arabica.

10.) Theobroma Cacao.

1113 Eugenia Jambos.

10. Centrosema virginianum.

10.) Cecropia peltata.

106 Bursera simaruba.

107 Citharexylum quadrangulare.

108 Artocarpus incisa.

109 Eugenia ligustrina.

110) Lantana Camara.

111 Comocladia ilicifolia.

11.- Persea americana.

$11+$ Myrcia paniculata.

11ti Nectandra coriacen.

117 Heliconia Bihai.

118 Tillandsia utriculata.

11) Canavalia obtusifolia.

120 Coccoloba urifera.

121 Costus speciosus. 
1.:3 Aristolochia odoratissima.

124 Verbena chamaedrifolia.

12.) Larvsonia inermis.

126 Larwsonia inermis.

127 Brunfelsia americana.

125 Amarantus paniculatus.

$1: 9$ Cissus sicyoides.

1:30 Urera caracasana.

131 Codiaeum variegatum.

13: Phaseolus lunatus.

133 Phaseolus vulgaris.

1:3.7 Antigonon cinerascens.

$1: 7$ Aegiphila martinicensis.

135 Althaea officinalis.

1:3! Duranta repens.

1 to Bixa Orellana.

141 Brassica integrifolia.

14: Hura crepitans.

14:3 Jasminum spec.

141 Stigmatophyllum periplocifolium.

14.) Cordia Sehestena.

146) Fourcroya gigantea.

147 Clerodendron aculeatum.

149 Plumbago capensis.

1.5) Parkinsonia aculeata.

1.). Quisqualis indica.

1.). Ipomoea pes caprate.

1.::) Mirabilis Jalapa.

15t Mirabilis Jalapa.

15:) Chrysobalanus icaco.

1.if Picrasma antillana.

157 Indigofera suffruticosa.

1.is Coleus scutellarioides.

159 Cosmos sulphureus.

1 tio Sambucus canadensis.

161 Hibicus Rosa-sinensis.

14: Nopalea coccinellifera.

11i:; Opuntia 'T'una.

16. Gomphrena globosa.

165 Codiaeum variegatum.

167 Codiaeum variegatum.
168 Jatropha curcas.

169 Hibiscus radiatus.

170 Nymphaea spec.

171 Jatropha multifida.

172 Leonotis nepetaefolia.

173 Erigeron canadensis.

174 Stylosanthes hamata.

175 Hymenaea Courbaril.

$175 a$ Boerhaavea hirsuta.

176 Achyranthes obtusifolia.

177 Croton lobatus.

178 Stenolobium stans.

179 Stylosanthes hamati.

181 Waltheria americana.

182 Sida cordifolia.

183 Thunbergia fragans.

184 Urechites suberecta.

185 Triumfetta rhomboidea.

188 Passiflora suluerosa.

189 Neurogramme calomelanos.

190 Solanum aculeatissimum.

191 Lonicera japonica.

192 Clerodenitron fallax.

193 Sesbania granditlora.

194 Leonurus sibiricus.

195 liandia aculeata.

196 Jatropha gossypifolia.

197 Jatropha gossypifolia.

198 Vernonia punctata.

200 Krameria ixina.

201 Abutilon indicum.

203 Rauwolfia Lamarckiana.

$20 t$ Galactia rulsa.

210 Cajanus indicus.

211 Rivina humilis.

212 Aegiphila martinicensis.

213 Psychotria rufescens.

214 Andropogon Schoenanthus.

215 Aristida americana.

216 Mimosa pudica.

217 Tillandsia recurvata.

218 Cissampelos Pareira. 
220 Ipomoea fastigiata.

221 Porana paniculata.

222 Jacquemontia pentantha.

223 Boerhanvea erecta.

224 Operculina ventricosa.

225 Ilratia maritima.

2.7 Solanum lycopersicum.

a.d Bouchea Ehrenbergii.

ㄴ.s!) Euphorbia thymifolia.

230 Ambrosia hispida.

2:)1 Russelia juncea.

2:i- Helochia tomentosa.

23:3 Wedelia buphthalmoides.

2ist Panicum livaricatum.

23i) Clitoria ternatea.

2:37 Fleusine indica.

238 Petiveria allincea.

23: Allophylus accidentalis.

zt) Tephrosia cathartica.

$\because+1$ Hyptis pectinati.

4.) Synedrella nodiflora.

24:3 Nedicago sativa.

244 Gossypium barbadense.

245 Dioscorea alata.

2ti Beloperone oustachyana.

24!) Desmodium triftorum.

2.00 Alysicarpus nummularifo. lius.

251 Hibiscus esculentus.

252 Hibiscus esculentus.

25.3 Canna indica.

y.t Canna indica.

עos Crescentia Cujete.

siti Crotalaria incana.

257 Polypodium phyllitidis.

25. Dryopteris parasitica.

Lis! Bignonia ungius-cati.

260) Oplismenus setarius.

-61 Polypodium lycopodioides.

2ri.) Mariscus hrizaeus.

26i: Smilax coriacea.

-lit Xanthosoma spec.
265 Cenchrus tribuloides.

266 Cyperus elegans.

267 Tournefor tia gnaphalodes.

268 Epidendrum ciliare.

269 Scleria lithosperma.

271 Chiococer racemosi.

272 Allium ascalonicum.

2.:-) Pilea semidentati.

$2 i t$ Anona montana.

275 Sida glomerata.

2.7 Porophyllum ellipticum.

278 Allamanda cathartica.

.sul Calonyction speciosum.

$\therefore 1$ Ipomoea pentaphylla.

2x-3 Maranta arundinacea.

2s:; Cueurbita maxima.

$29+$ Pithecolobium ungius-cati.

2. Shi Crotalaria rerrucosa.

isti Crotalaria retusa.

¿st Bryophyllum pinnatum.

בny Ricinus communis.

2.S! Ricinus communis.

ע9) Manihot utilissima.

-2!1 Haematoxylon campechi. anum.

2!+ Salvia occidentalis.

293 Stachytarpheta jamaicensis.

294 Cardiospernum halicacaloum.

295 Aloe vera.

296 Ocimum micranthum.

297 Bryophyllum pinnatum.

29s Hymenocallis caribaea.

29) Eucharis grandiflora.

300 Sanseviera guineensis.

301 Pilea microphylla.

302 Pilea microphylla.

304 Blechum Brownei.

305 Jasminum pubescens.

306 Panax fruticosum.

307 Panax fruticosum.

308 Codiaeum variegatum.

309 Codiacum variegatum. 
310 Codiaeum variegatum.

311 Codiaeum variegatum.

312 Gaillardia aristata.

$: 31+$ Piscidia piscipula Sarg.

:31i Angelonia angustifolia.

317 Hibiscus schizopetalus.

$31 \mathrm{~s}$ Bromelia pinguin.

$3: 1$ Ceiba pentandra.

3.2. Pisonia subcordata.

32: Villamilla octandra.
$32+$ Hippomane mancinella.

325 Musa paradisiaca.

326 Verbena chamaedrifolia.

329 Desmodium supinum.

380 Lochnera rosea.

331 Panax fruticosum.

:3i2 Comocladia ilicifolia.

334 Musa paradisiaca.

336 Nerium Oleander.

339 Portulaca halimoides.

\section{Collection J. van Grol-Meyers of the Herbarium of the Botanical Institute of the University in Utrecht.}

1 Vigna repens.

$\because$ Commelina virginica.

:) Parthenium Hysterophorus.

\& Cassia occidentalis.

5) Chenopodium ambrosioides.

is Ageratum conyzoides.

- Heliotropium parviflorum.

is Euphorbia piluliferi.

() Chenopodium murale.

10) Scoparia dulcis.

11 Capraria biflora.

1.- Datura Stramonium.

1i) Datura Metel.

14 Datura Stramonium.

15) Heliotropium indicum.

11: Sida carpinifolia.

17 Amarantus tristis.

is Amarantus spinosus.

19) Amarantus polygonoides.

20 Argemone mexicana.

-1 Antigonon leptopus.

¿2 Lochnera rosea.

23 Capsicum dulce.

24 Passiflora quadrangularis.

25 Cassia bicapsularis.

2t; Ricinus communis.

.27 Ricinus communis.

¿- Portulaca oleracea. g!! Portulaca oleracea.

30) Kallstroemia maxima.

:i Jasminum azoricum.

3i Polanisia viscosa.

33 Gynindropsis pentaphylla.

3it Poinciana regia.

35) Plumbago scandens.

iti Euphorbia heterophylla.

3 Salvia serotina.

3is Priva echinata.

:3! Melochia tomentosi.

to Murraya exotica.

+1 Phyllanthus niruri.

4.- Momordica Charantia.

$4: 3$ Microtea debilis.

4 Leucaena glauca.

45. Terminalia Catappa.

46. Solanum racemosum igneum.

47 Tamarindus indica.

ts Psidium Guajava.

f!) Allamanda cathartica.

(x) Acacia macracantha.

il Capsicum frutescens.

.- Jatropha curcas.

.3. Cordia speciosa.

ift Cilotropis procern.

y) Cajanus indicus.

sti Clerodendron fillax. 
57 Brunfelsia americana.

is Ixora Jucunda.

ก) Solanum torvum.

61 Lagerstroemia indiea.

15. Canna indica.

(6.) 'Talinum triangulare.

if Plumbago capensis.

(i.) Quisqualis indica.

(iti Jatropha multifidi.

it Clusia alba.

isi Plumiern rubra.

(i)! Peperomia petiolaris.

7) Peireskia aculeata.

71 Eleutherine plicata.

i: Verbena chamaedrifolia.

i: Parkinsonia aculeata.

it Caesalpinia pulcherrima.

7) Caesalpinia pulcherrima.

it Ixora coccinen.

77 Punica Granatum.

is Malpighia punicifolia.

i!) Sambucus canadensis.

i1 Panax fruticosum.

S. Panax fruticosum.

(i) Panax fruticosum.

int Citrus spec.

8.) Tabernaemontana coronaria.

st) Boerhanvea erecta.

Si Boerhaavea hirsuta.

sis Lantana Camara.

S? Lantana involucrata.

$\$ 10$ Trianthema portulacastrum.

(1) Anona squamosi.

12 Croton lobatus.

(1) Achyranthes obtusifolia.

94 Lonicera japonica.

95 Clerodendron aculeatum.

96 Bauhinia Kapplerj.

97 Jasminum undulatum.

98 Gaillardia aristata.

99 Cassia glanclulosa.

100 Stenolobium stans.
101 Fourcroya gigantea.

102 Aloe vera.

103 Bromelia pinguin.

104 Phoenix dactylifera.

105 Amarantus paniculatus.

106 Achras sapota.

107 Codiacum variegatum.

108 Nerium Oleander.

109 Tecoma leucoxylon.

110 Artocarpus incisa.

112 Russelia juncea.

113 Hibiscus Rosa-sinensis.

114 Hippeastrum puniceum.

115 Mariseus brizaeus.

116 Plucher odorata.

117 Coleus scutellarioides.

119 Angelonia angustifolia.

120 Croton flavens.

121 Althaea officinalis.

122 Foeniculum vulgare.

123 Begonia spec.

121 Codineum variegatum.

125 Codiaeum variegatum.

126 Senecio sonchifolius.

127 Cassia fistula.

128 Citharexylum quadrangulare.

12 !) Ruellia tuberosa.

180 Mangifera indica.

131 Ocimum micranthum.

132 Jatropha gossypifolia.

133 Jatropha gossypifola.

134 Asclepias nivea.

135 Fleurya aestuans.

136 Leonurus sibiricus.

137 Stigmatophyllon periplocifolium.

138 Leonotis nepetaefolia.

139 Nelia azetlarach.

140 Neurogramme calomelanos.

141 Justicia sessilis.

142 Nicotiana 'Tahacum.

143 Solanum lycopersicum. 
144 Passiflora Iaurifolia.

145 Erigeron canadensis.

146 Persea americana.

147 Hura crepitans.

148 Phyllanthus distichus.

149 Crescentia Cujete.

150 Pilea microphylla.

151 Opuntia Ficus indica.

152 Opuntia T'una.

154 Cardiospermum halicacabum.

155 Cissus sicyoides.

156 Cucumis Anguria.

157 Ambrosia hispida.

158 Tephrosia cathartica.

159 Cyperus rotundus.

160 Caesalpinia crista.

161 Rivina humilus.

162 Desmantlius virgatus.

163 Medicago sativa.

$16 \pm$ Ipomoea dissecta.

165 Trerdelia buphthalmoides.

166 Beloperone eustachyana.

167 Maranta arundinacea.

168 Nectandra coriacea.

169 Linociera compacta.

170 Cassia glandulosa.

171 Croton astroites.

17:- Indigoferal suffruticusil.

173 Anona muricata.

174 Piper dilatatum.

175 Psychotria rufescens.

176 Randia aculeata.

177 Myrcia splendens.

178 Bignonia ungius-cati.

179 Polypodium lycopodioides.

180 Pisonia fragans.

181 Pisonia subcordata.

182 Sida cordifolia.

183 Polypodium phyllitidis.

184 Mammea americana.

185 Clusia alba.
186 Cuseuta americana.

187 Chiococca racemosa.

188 Smilax coriacea.

189 Capparis frondosa.

190 Capparis cynophallophora.

191 Capparis cynophallophora.

192 Pilea semidentata.

193 Peperomia glabella.

194 Panicum divaricatum.

195 Anthurium cordatum.

196 Casearia parvifolia.

198 Coccoloba uvifera.

199 Carica Papaya.

200 Bursera simarubí.

201 Mfussaenda frondosa.

202 Eucharis grandiflora.

203 Codiaeum variegatum.

204 Codiaeum variegatum.

205 Carica Papaya.

206 Allium ascalonicum.

207 Anacardium occidentale.

209 Phoradendron trinervimm.

210aPiscidia piscipula.

213 Myrcia splendens.

214 Theolroma Cacao.

215 Caesalpinia coriaria.

216 Hymenaea Courbaril.

217 Quararibea turbinata.

21s Taberniemontana citrifolia.

$218 a$ Psychotria horizontalis.

219 Indigofera suffruticosa.

220 Philodendron dispar.

221 Theobroma Cacio.

222 Urera caracasana.

223 Boerhaavea erecta.

$223 a$ Heliconia Bihai.

224 Chrysobalanus icaco.

225 Pharus glaber.

226 Triphasia trifoliata.

227 Moringa oleifera.

228 Gomphrena globosa.

229 Hibiscus radiatus. 
2:30 Solanum lycopersicum.

2:31 Bixa Orellana.

2:32 Boussingaultia baselloides.

23:3 Ocimum micranthum.

:-34 Lawsonia inermis.

2:i.) Abrus precatorius.

3:) Centrosema virginianum.

:3: Rauwollia Lamarckiana.

2:3!) Mirabilis Jalapa.

4t) Mirabilis Jalapa.

$\because+1$ Codiaeum variegatum.

4:? Cosmos sulphureus.

I4 Canaralia spec.

¿45 Caesalpinia coriacea.

¿tti Chrysobalanus icaco.

$2+7$ Mirabilis Jaliapa.

24 Antigonon cinerascens.

$\because 4 !$ Cenchus tribuloides.

2.51 Gossypium barbadense.

2.)- Stylosanthes hamata.

25 Operculina ventricosa.

2.)t Melicocer bijuga.

25.) Euphorbia thymifolia.

25 . Malvastrum spicatum.

25. Ricinus communis.

259 Cassia alata.

260 Petiveria alliacea.

"til Ocimum Basilicum.

$\because 63$ Solanum aculeatissimum.

$26+$ Eleusine indica.

26.) Ipomoen fastigiata.

$21 ; 6$ Ipomoea fastigiata.

-67 Cucurbita maxima.

¿tis Achyranthes obtusifolı.

(2)9) Corchorus olitorius.
970 Ipomoea fastigiata.

271 Passiflora suberosa.

272 Vernonia punctata.

274 Oplismenus setarius.

276 Dryopteris parasitica.

277 Ibatia maritima.

278 Setaria setosa.

279 Oplismenus setarius.

280 atpidendrum papilionaceum.

281 Epidendrum ciliare.

282 Galactia rubra.

283 Capsicum dulco.

281 Porophyllum ellipticum.

285 Crotalaria retusa.

286 Citrus spec.

287 Andropogon zizanioides.

289 Andropogon Schoenanthus.

290 Sesbania grandiflora.

291 Sesbania grandiflora.

292 Brassica integrifolia.

294 Lawsonia inermis.

295 Tillandsia usneoicles.

296 Celosia argentea.

297 Polana paniculata.

298 aHymenocallis caribaea.

299 Bouchea Ehrenbergii.

300 Stylosanthes hamati.

301 Spermacoce tenuior.

302 Desmanthus virgatus.

303 Crotalaria verrucosa.

304 Acacia macracantha.

305 Stachytarpheta jamaicensis.

306 Musa paradisiaca.

307 Phaseolus lunatus.

308 Epidendrum ciliare.

\section{Collection Lionarons.}

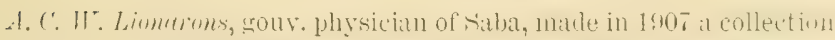

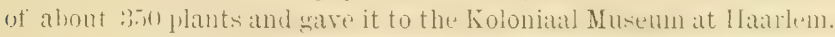
Dr. M. Greshioff, Director of this Museum was so kind as to give me an apportanity to study these plants. 
The greater part of the plants are cultivated and another part has evidently been collected on the top of the Mountain.

There are some interesting plants, which I myself saw but once during my rambles through Saba.

'I'he following list gives the names of the plants collected by

Lionarons.

1 Anona muricata.

$1 a$ Melicocca bijuga.

2 Passiflora laurifolia.

4 Jasminum azoricum.

7 Cansicum frutescens.

8 Capsicum frutescens.

9) Capsicum ceratocarpum.

12 Swietenia Mahagoni.

15 Commelina virginica.

16 Cajanus indicus.

17 Thunbergia fragans.

18 Chrysobalanus icaco.

21 Tillandsia fasciculata.

22 Carica Papaya.

23 Eleusine indica.

24 Beloperone eustachyana.

25 Desmodium supinum.

26 Brassica integrifolia.

28 Cassia bicapsularis.

29 IVedelia buphthalmoides.

30 Ipomoea pentaphylla.

31 Boerhaavea paniculata.

32 Rivina humilis.

33 Amarantus tristis.

34 Pharus glaber.

35 Salvia occidentals.

36 Thunbergia alata.

37 Fryngium foetidum.

39 Peperomia petiolaris.

40 Sonchus oleraceus.

42 Zebrina pendula.

44 Foeniculum vulgare.

46 Antigonon leptopus.

47 Gossypium barbadense.

48 Capparis jamaicensis.
49 Polypodium lycopodioides.

50 Oxalis corniculati.

53 Oplismenus setarius.

55) Canua indica.

56 Parthenium Hysterophorus.

57 Sambucus canadensis.

61 Polypodium crassifolium.

63 Carica Papaya.

64 Ricinus communis.

65 Crescentia Cujete.

(i6 Crotalaria retusi.

67 Manilot utilissima.

68 Ipomoea fastigiata.

69 Centrosema virginianum.

72 Petiveria alliacea.

74 Lochnera rosea.

75 Abutilon indicum.

76 Ardisia coriacen.

78 Allium ascalonicum.

79 Allium ascalonicum.

80 Portulaca oleracea.

S2 Operculina ventricosa.

85 Plumbago capensis.

87 Indigofera suftruticosa.

88 Aleurites moluccana.

90 Lochnera rosea.

91 Tephrosia cathartica.

92 Zinnia elegans.

93 Jasminum azoricum.

95 Parthenium Hysterophorus.

96 Nicotiana Tabacum.

97 Triphasia trifoliata.

99 Punica Granatum.

101 Coccoloba nivea.

103 Peperomia acuminata. 
104 Cissus sicyoides.

106 Cuscuta americana.

109 Eugenia Jambos.

110 Quisqualis indica.

111 Hibiscus Rosa-sinensis.

112 Mirabilis Jalapa.

113 Calotropis procera.

114 Mammea americana.

117 Tamarindus indica.

118 Manihot utilissima.

119 Psidium guajava.

128 Lepidium virginicum.

124 'lalinum paniculatum.

125 Melia azedarach.

127 Hillia longiflora.

128 Euphorbia hypericifolia.

129 Leucaena glauca.

132 Scleria pterota.

133 Setaria setosa.

134 Stenolobium stans.

135 Ageratum conyzoides.

136 Leucaena glauca.

137 Chiococea racemosi.

140 Myrcia paniculata.

142 Pilea microphylla.

148 Hiliscus schizopetalus.

145 Solanum Iycopersicum.

146 Iresine paniculata.

147 Panicum brevifolium.

147 bPanicum fuscum.

148 Pisonia aculeata.

149 Persea americana.

151 'lecoma capensis.

153 Gynandropsis pentaphylla.

154 Fleurya aestuans.

155 Argemone mexicana.

156 Mangifera indica.

1 o. Maranta arundinacea.

159 Piper reticulatum.

$159 a$ Piper marginatum.

160 Asclepias nivea.

161 Euphorbia hypericifolia.
162 Cassia occidentalis.

163 Momordica Charantia.

164 f'oeniculum vulgare.

165 Epidendrum ciliare.

168 Belamcanda punctata.

169 Phytolacca icosandra.

170 Passiflora laurifolia.

171 Peperomia acuminata.

172 Nephrolepis exaltata.

174 Peperomia hernandifolia.

175 Phytolacca icosandra.

176 Physurus plantagineus.

177 Casuarina equisetifolia.

178 Plantago major.

181 Stenochlaena sorbifolia.

182 Citharexylum quadrangulare.

183 Ardisia coriacea.

184 Scleria pterota.

186 Leucaena glauca.

187 Charianthus crinitus.

188 Hemitelia horrida.

189 Hemitelia horrida.

192 Cecropia peltata.

194 Urera caracasana.

196 Neurogramme calomelanos.

196๘ Nangifera indica.

197 Charianthus crinitus.

201 Desmodium axillare.

202 Polybotrya cervina.

203 Charanthus crinitus.

204 Polybotrya cervina.

206 Ichnanthus pallens.

208 Cladium restioides.

209 Phytolacca icosandra.

210 Neurolaena lohata.

211 Asplenium serratum.

212 Aspidium Plumierii.

214. Selaginella flabellata.

216 Miconia laevigata.

218 Ipomoea fistigiata.

220 Dryopteris opposita.

221 Colocasia esculenta. 
222 Nerium Oleander.

223 Caesalpinia pulcherrima.

224 Codiaeum variegatum.

225 Codiaeum variegatum.

227 Anacardium occidentale.

229 Quistqualis indica.

230 Russelia juncea.

231 Nicotiana Talacum.

232 Gossypium barbaciense.

233 l'hespesia populnea.

234 Plucher odorata.

236 Villamilla octandra.

237 Cassia occidentillis.

238 Phyllanthus distichus.

239 Euphorbia pilulifera.

240 Panicum maximum.

241 Jatropha gossypifolia.

245 Bixa Orellana.

247 Leonotis nepetaefolia.

251 Solanum nigrum nodiflorum. 251.aIndigofera suffruticosa.

25. Amatiatus spinosus.

25:3 Lantant camara.

254 Achyranthes obtusifoliz.

256 'Talinum triangulare.

257 Jatropha multifida.

260 Datura fastuosa.

262 Desmodium spirale.

265 Lawsonia inermis.

269 Mussaenda frondosa.

$269 a$ Porophyllum ellipticum.

270 Rauwolfia Lamarckii.

273 Panicum sanguinale.

276 Cucumis Anguria.

278 Quispualis indica.

279 Galphimia gracilis.

280 Brunfelsia americana.

281 Murraya exotica.

282 Sanchezia nobilis.

284 Tecoma leucoxylon.

285 Rhoeo discolor.

286 Colens amboinicus.
287 Anthurium cordatum.

288 Bryophyllum pinnatum.

290 Bursera simaruba.

291 Solanum tuherosum.

29.2 Bixa Orellana.

293 Wittmackia lingulata.

294 Panicum insulare.

297 Ruellia tuberosa.

298 'Thrinax spec.

299 Antigonon leptopus.

301 Cuesalpinit coriaria.

302 Mangifera indica.

303 Panicum divaricatum. $303 a$ Colocasia esculenta.

306 Miconia laevigata.

309 Chiococea racemosi.

311 Callisia repens.

312 Abrus precatorius.

313 Jatropha gossypifolia.

315 Croton flavens.

316 Blechum Brownei.

320 Salvia serotina.

321 kugenia Jambos.

322 Colocasia esenlenta.

324 Colocasia esculenta.

325 Colocasia esculenta.

328 Petiveria alliacea.

330 Codiaeum variegatum.

330 aLochnera rosea.

335 Chrysobalanus icaco.

336 Citharexylum quadrangulare.

337 Casearia parvifolia.

338 Aleurites moluccana.

:3:39 Codiaeum variegatum.

340 Coccoloba diversifolia.

343 Panicum divaricatum.

345 Codiacum variegatum.

346 Stachytarpheta jamaiconsis.

348 Hymenaea Courbaril.

354 Caesalpinia crista.

356 Poinciana regia. 
357 Clusia alba.

359 Albizzit leblek.

360 Coccoloba uviferi.
361 Canna indica.

363 Ipomoea pentiphylla.

\section{Collection I. Boldingh.}

I do not think it necessary to give more details about my own oxcursions; the different dati, which are found in the Systematic Part are all taken from my notes and collections made during my rambles.

The following list may give an ilea of the localities visitul hy me. In the 'Third Part Section C. I give a detailed description of the vegetation also taken from my personal observations.

\section{St. Eustatius: May 25th-July 5th.}

Miıy 25th. Signalhill, T'umbledowndickbay.

"26th. round the Quill.

"27th. Quill.

"28th. Seashore near Oranjestad.

. 29uh. Seashore near Oranjestad, Gallowshay.

, 30th. 'Tumbledowndickbay.

"31st-June 5th. Quill.

June 6th-8th. Quill.

"9th-10th. Cultuurvlakte.

"12th. Bengalen-seashore.

"13th-14th, Quill near Bengalen.

" 15th-16th. White Wall and seashore.

"17th. Quill.

" 19th. Cultuurvlakte.

"21st. Schotsenhoek, Concordia, Venusbay.

" 22nd. English Quarter and seashore.

" 23th. Signalhill.

"25th. Cultuurvlakte.

"26th. Gilboohill, Venusbiy.

"27th. Schotsenhoek, Maryglory, Bergje, Jenkinsbay.

"28th. Quill.

"30th. Jenkinsbay, Bergje.

July 2nd. White Wall.

"3rth. Zeelandia, Concordiabay.

"5th. Bengalen. 


\section{Saba: July 6th-29th.}

July 6th. St. John's.

"7th. Windwardside, Paris, Great hill.

" Sth. Bottom.

. 9th. Bottom to Marypoint.

. 10th. Boobyhill.

" 12th. Bottom, Forthbaygut, Saddle.

" 13th. Hellsgate, Sulphurmines.

. 14th. Mountain, Crespeenhill.

"16th. Mountain.

" 17th. St. John's, Giles gut.

" 18th. Windwardside.

" 19th. Laddergut.

"20th. Bottom, Windwardside, IIellsgate, Marypoint.

. 21st. Springbaygut.

. 23th. Sulphurmines, Green Island, Flat Point.

" 24 th. Mountain, Windwardside.

. 25th. Mountain.

. 26ith. Bottom.

. 27th. Mountain, Hellsgate.

, 29th. Windwardside, Bottom.

St. Martin : August 14th-September 14th.

August 4th. Filipsburg, Marigot.

6th. Filipsburg, Guanabaly.

Sth. Filipsburg, Belvedere, Mont Chambord, Grande Case, Marigot, Filipsburg.

17th. Fort Willem.

. 18th. Prince quarter.

" 19th. Filipsburg, Cul de Sac, Marigothill, Marigot, Colebay.

. 20th. Bethlehem.

. 21st. Nakedboyhill, l'ort Willem.

.. 22nd. Simsonsbay, Cherry cap, Anse des Bourgaux.

" 23th. Fort Willem.

.. 24th. Bellevue,

. 25th. Hill between Centryhill and Marigothill.

27th. Mont Chambord, Orientbay.

"28th. Hills on the north s.de of Cul de Sac.

29th. Filipsburg, Simsonsbay.

30th. Oysterpond. 
September 4th. Filipshurg, Grande Caso, Milldrumbill. 5th-7th. Anguilla.

" Sth. Grando Case, Anso des l’ères, Marigot, Filipsburg.

" 10th. District Colombier, Hount Paradis.

" 11th. Lowlands, Simsonsbaylagoon.

" 12th. Bethlehem, Mount Paradis.

" 13th. Green Key, Tintamarre.

" 14 th. Point Blanche, Bethlehem, Cul de Sac. 



\section{THIRD PART}

\section{PHYTOGEOGRAPHICAL}

Section A. Orological, Geological

and Meteorological 



\section{PHYTOGEOGRAPHICAL}

\section{A. Orological, Geological and Meteorological \\ CHAPTER I}

\section{GEOLOGICAL AND OROLOGICAL NOTES.}

\section{Introduction.}

In making noter on the dieolugy and orulogy of the athe mentioned Islands I have made use of the following treatises:

P. 1. Cleve. On the Geology of the Northeastern West Indian Islands, Kongl. Vetensk. Akad. Handl. 1870. Bd. 9, no. 12. p. 19 a. f.

A. (7. Högbum. '/ur l'etrographie der Ḱleinen Antillen. Bull, tieol. Institution of Upsala. VI. 1905. p. 228-229.

E. O. Hovey. Volcanoes of Martinique, Guadeloupe, and Saba. Report ith Internat. (ieogr. Congress. 19k1. London, 1'. 4t7-t5l. E. O. Horey. Volcamoes of 'st. Vincent, st. Kitts, and statial. ihid., p. $452-454$.

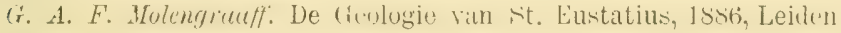
[Dissertat. Utrecht].

G. A. F. Holongrauff. Het dieologisch verband tusschen de WestIndische ellinden, Handelingen vim het ecrste Nederlanilsche Natuur- en Geneeskundig Congres 1888, p. 288.

G. A. F. Molengraaff. Uber vulkanischen Schwefel aus WestIndien. Zeitschr. f. Krystallogr. XIV. 1888, p. 43-48.

J. W. W. Spencer. On the Geological and Physical Development of Anguilla, st. Martin, st. Bartholomew, and Sombrero. Quarterly Journal of the (ieological Society of London. 1901, vol. is. p. 520 a. 1 .

J. II. Hr. Spenert, ()n the (ieological and physical Development uf the st. C'hristopher ("hain and Salna Banks. Guarterly Journal of the Geological Society of London, 1901. vol. 57. p. 534. a. f. K. Suprer. Ein Besuch von St. Eustatius und Silla. ('entralblatt für Mineralogie etc. 1903, p. 314-318. 
K. Samper. In den Vulkangebieten Mittel Amerika's und WestIndien. Stuttgart 1905.

It will appear that our genlogical and orological information about St. Eustatius is sufficient, whereas Siba is all but ab. solutely unknown in literature, so that almost all that I can communicate rests on my personal observation with the addition of some information borrowed from maps.

St. Martin is somewhat better kwown than Saba lut a good description has not yet appeared. The maps from which I borrowed different data ar the following:

St. Eustatius: MLolengraaff 1886 l. c. pl. I.

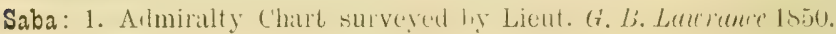
London 1864 .

2. West Indies. den Harg, od. Nov. 1903 by the Navy-Department.

3. A. map of Siba borrowed from one of the inhabitants, evidently made by G. B. Lavrance afore said.

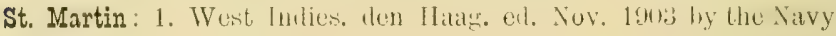
Department.

2. A map of St. Martin horrowed from MIr. A. J. C. Brounor, Administrator of St. Martin.

3. Molengraaff 1888 1. c. p. 288.

4. C. J. Hudig, the Saltwork's in the Isle of St. Martin. de Ingenieur May 24. 1905.

From these maps it appeared to me that there is reason for calling to mind the words spoken by $K a n^{1}{ }^{1}$ ) in 1888: It can "hardly be called superfluous to refer to the value of a good "map.... both for science and practice....

"In time of peace such a map might of course render invaluable ,ssrices in agricultural and inlustrial enter prises, in making

1) C. M. Kan. Onze aardrijkskundige kennis van de Molukken. Handelingen van het Eerste Ned. Nat. en Geneesk. Congres. Haarlem 1888. p. 285:

"Het kan schier overbodig geacht worden... op de waarde eener goede kaart... te wijzen. In vredestijd kan zulk een kaart natuurlijk onschatbare diensten bewijzen: bij cultures en industrieele ondernemingen, bij den aanleg van wegen, mijnontginningen, regeling van grondbezit en belastingen, aanleg van openbare en waterstaatwerken, enz, enz.

Doch evengroot is het nut van zulk een kaart voor verschillende beoefenaars der wetenschap, dus niet enkel voor den geograaf. Hebben niet de II.H. Martin en Suringar op meer dan een der W. I. eilanden het gemis van goede kaarten gevoeld?" 
"roads in working mines, in settling landed property and taxes, "and in public works.

"Still not only for the geographer but also for those who apply "themselves to any science, such maps are of great use.

"Iave not Messrs Martin and Suringar in more than one of the "West Indian Islands felt the want of good maps"?

In my rambles I also greatly felt the want of really good maps. By means of panorama photos I tried to see my way in difierent poinits of the islands. Probably these photos may not be of any use in making a topographic map, still I dire say they have had for me such lesults as to hold myself entitled to deriate sometimes from the maps consulted hy me. In this way I perhaps contributer a little to obtain a somewhat clearer rescription of Saba and St. Martin.

\section{St. Eustatius.}

The Isle of St. Eustatius extends from $17^{\circ} 28^{\prime} 12^{\prime \prime}$ to $17^{\circ} 31^{\prime} 35^{\prime \prime} \mathrm{N}$. ancl from $62^{\circ} 56^{\prime} 25^{\prime \prime}$ to $62^{\circ} 59^{\prime} 48^{\prime \prime} \mathrm{W}$.

'The greatest length runs from N.W. to S.F. whereas the brealth from S. to W. except a small narrowing in the middle gradually decreases: the length of St. E'ustatius amounts to $7.8 \mathrm{~km}$. whereas the breadth in the southern part amounts to $3.7 \mathrm{~km}$. in the middle decreases to $2.6 \mathrm{~km}$. and in the northern part it does not exceed $2.7 \mathrm{~km}$. 'The Surface is $28 \square \mathrm{km}$.

'The structure of st. Eustatius is ocrasioned ly rocks belonging to one and the same geological period.

Still from their relative age we can sharply trace out two parts viz. the northern hils on one side and the principal vulcamo with its eruptions on the other side. 'These two groups of mountains are clearly separated from each other by a wide plain. The hilly soil taling up the whole northern part of the islinds con. sists of remnants ot old vulcanoes.

'I'he' southern part chicfly consists of a single very regularly built mountain which has the form of a truncated cone. The highest point of this mountain, named Quill is $581 \mathrm{~m}$. above the level of the sea. On the top of it is found a circular valley shut in on all sides and of which the bottom is $297 \mathrm{~m}$. above the surface of the sea.

The inner slopes of the crater are very steep and in most parts inaccessible on account of numerous perpenticular clifls; the 
slope has here a declivity of $34^{\circ}$ to $36^{\circ}$ the average slope in other places being $67^{\circ}$ to $78^{\circ}$.

The bottom of the crater is in general tolerably flat but uneren on account of the numerous pieces of rock that have fallen down from the sides. The whole surface is covered with a bed of humus.

The fragments of rock that have served in the structure of the crater are for the greater part Augite-Audesite only here and there occurs Hornblende-Augite-Andesite.

The quill is hordered on all sides hy very ster], not seldom prerpendicular rocks. The brown of these rocks form a ridge which has a width of only a few $\mathrm{m}$. and the height of which varies betweon 391 and 581. On the outside the slope of the lill is at first, in general. very steep, but it is gradually declining to shelve away at last in a plain in a northwestem direction 11. to be suddenly cut off ly the steep sea coast. (1n the southern side only inaccessible rocks called the White Wall, which with a declivity of $45^{\circ}$ rising from the sea disturb the regularity of the profile of the mountains.

The second group of mountilins taking up the N.W. part of the islanil consists of a great number of hills of which the highest called "Boven" has a height of $295 \mathrm{~m}$.

'The very steep coast that begins neall Heiligenbatai hordering the further northern side of the islimi, is formed ly a gigatutic, perpendicular wall of lava (Augite-Anidesite.)

By a leep) ravine Boren is separated from Gilhoohill. 'This hill lit $\mathrm{m}$ high, is formed partly from ashes and partly from solid volcanic matter. The other hilly ground consists out of a number of more or less sharp tops which are connected by a ridge. This ridge runs close to the seashore from Boven to 'I'umbledowndickbay and extends from there in eastern direction as far as Mary Glory, the highest top of the range. Going eastward from there we perceive that the ground is consiclerably sloping.

To the northern hills of St. Eustatius belongs also : crater; the exat form of a horse shoe lyings south of the Tumhledownlickbay aml fircing the town. The highest astern top of this crater is Sirnallhill $(220 ; \mathrm{m}$.) the northwestern one is called l'ilothill.

As to petrography the nolthern part of st. Eustitius offers but little rariety; the mineral most occurring is Augite-Amlesite. Almost the whole northern part of the island is covered with it kind of good humus, moducen from voleanic ashes mostly mixed 
with lapilli and pumice. 'The slopes of these hills especially on the seaside are generally very steep, as a rule even inaccessible. Of the plains that are to he found in St. Eustatius only the large one that connects the two mountainous parts is of importance. If we include in the plain that part only of which the general declivity does not surpass 4 degrees, the extent of it may be estimated at $5 \square \mathrm{km}$. A small plain at $0.5 \square \mathrm{km}$. may moreover be found in the Quill whereas there are some level parts in the Concordiavalley and the Venusvalley.

The formation of dells talies place on a small suale. In the northrun part of the island are of importance the two large valleys that open upon the Venusbay, the Concordiabay and Tumbledowndiclibay.

These valleys are bordered by rocks suddenly rising from the plain. The average declivity of these rocks is seldem less than $30^{\circ}$ and often exceeds $50^{\circ}$.

In the southern part of the island few dells are to be found. The outside of the principal volcano is furrowed by numerous, narrow and deep ravines, the so called "guts" which show a beginning of dell formation by erosion.

Rumning water is found mowhere in st. Fustatius hut during the heavy showers that will sometimes fall in the rainy season all aforesaid valleys and ravines are turned into beds of strong currents which by their havy fall and temporary considerable masses of water possess a great force in carrying off.

The coast of St. Eustatius is partly a steep coast.partly a flat one bordering the precipices.

A steep coast occurs in the whole northern part except in places where valleys face the sea. In the southern part a steep coast is found on the eastern border of the White Wall as far as Gallowsbay and moreover in some places east of the White Wall. A flat coakt hortering the precipices meurs near oranje Bay and on the other side of the island near concorliabay, Turtlehay and further in most places on the south-eastern and southern side. An easily practicable sandy beach, nowhere broader than $50 \mathrm{~m}$. is to he found only near Oranjebay the Concordialray and Turtlebay. The beach on the south-eastern sicle is no more than 10 to $20 \mathrm{~m}$. wide and is covered by pieces of rock wom loy the seawater.

\section{Saba.}

The isle of Sala extends from $17^{\circ} 37^{\prime} 10^{\prime \prime}$ to $17^{\circ} 39^{\prime} 18^{\prime \prime} \mathrm{N}$. and from $63^{\circ} 12^{\prime} 25^{\prime \prime}$ to $63^{\circ} 15^{\prime} 5^{\prime \prime} \mathrm{N}$. 
Round the island may be drawn a parallelogram of which the longer diagonal going from Ladderpoint to Flat Point has a length of $5.25 \mathrm{~km}$.; the other from Torrents Bay to SouthEast Point $4.5 \mathrm{~km}$. The surfaces is $16 \square \mathrm{km}$.

In oriter to get a better insight in the geological condition I col. lected a number of minerals during my rambles on this island. I am greatly indebted to Prof. Dr. C. E. A. Wichmann at Utrecht for his determination of these minerals. It proved to me that I brought only Andesites except some stones containing sulphur. which, especially with a view to the geological condition of St. Eustatius, was howerer to be expected.

As far as may rexult from my ohservations the rocks rising stowly from the sea consist of Andesites.

The conical mountain (of which Mountain Peak is $580 \mathrm{~m}$. allove the sea-level), rising steeply from the sat, perfectly-ommands the form of the island. Its top lies in the intersection of the mentioned diagonals an? ands in an almost sharp summit that, viewed from St. Barthelemy seems somewhat level. To the North this mountain is sloping almost down to the seashore, higher up at an angle of $30^{\circ}$ lower it is less steep; only near. Flat point there is a tolerably large plain where the sea may be reached.

Also on the other sides of the island the rocks rise from the sea at a steep angle but do not go up to the Mountain top. I'hey rise as far as 300,400 or $500 \mathrm{~m}$. and end in different cones which seem to rest on the central part of the island at $200-300$ $m$. alove the level of the sea.

Southwestrard of Mountain P'eak there is the small plain where the chief village. 'The Bottom, is to be found. I estimate its surface at $0.5 \square \mathrm{km}$.

South East of Mountain Peik lies another plain of still smaller surface where the little village IVindwardsitce is situated alout $300 \mathrm{~m}$. ahove the level of the sea.

On one of the least steep parts of the conical mountain Marypoint, a knot of houses is built X.W. of the P'eak at alout $400 \mathrm{~m}$. above the sea level whereas a group of houses, Jellsgate is huilt. on a gentle slope N. E. of the Mountain Peak.

So the Botton is surrounded on all sides by mountain tops anm the situation is such as might easily be taken for the bottom of the crater:

In the sulphurmines that extend in the rivinity of Itellagate near Flat Point, occurs Andesite, changed into Opal. 
Here as well ats in Sit. Eustallus there is but little doll formation;

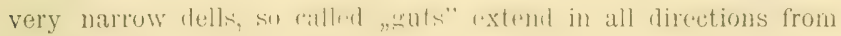
the mountain top towards the sea. So there are two issuing

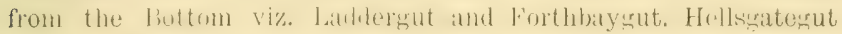
is onte of the most chatretryistic and especially at the seatshore: wider than the others; all ravines abound in big stones.

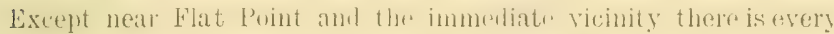
where a steep coast in front of which allo fomm howlder stones to a height of $2 \mathrm{~m}$.

The Mountain Paak and neighhouring tols are covered with a thick bed of humus; foing lownward it is getting graslually thinner and on the proper arable land on the steep slopes it must be

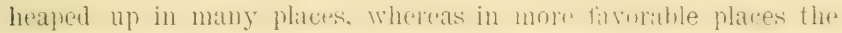
immense suantity of stones must he limeved. In the neighbumhood of the sulphurmines the soil consists of very fine tuff.

\section{St. Martin.}

The isle of st. Martin extends from $14^{\circ}$ to $18^{\circ} 7^{\prime} 20^{\prime \prime} \mathrm{N}$. amb from $63^{\circ} 1^{\prime} 20^{\prime \prime}$ to $63^{\circ} \mathrm{W}$. The greatest distance in the direction $\mathrm{N}$. to S. is $13.5 \mathrm{~km}$. and from W. to E. $15 \mathrm{~km}$. The surface (the large lagoons not included) is about $90 \square \mathrm{km}$.

Te form of St. Martin is chiefly commanded by a number of ridges that extend in the direction from $\mathrm{N}$. to $\mathrm{S}$.; the eastern ridge is ruming pretty near the shore fium (osterpome to Point Blanche (Nackedboyhill $280 \mathrm{~m}$.).

A valley $2.5 \mathrm{~km}$. wide separates this ridge from the other mountainous land of the Island. 'This mass of hills reaches in Mount. Paradis ( $112 \mathrm{~m}$.) its highent print and in all directions extend ridges of which Mont ('hamburl, Mnnt Ternon and Mill. drumhill (3S0 m.) (nd very near the seatshore in the nortwestern part of the Island; separated from it by a cross valley there is somewhat lower hilly country in the murthern part of st. Martin. Two ridges extend from Mount Palaldis to the District Colombier, whereas the two largest rallges repariate Cul de Sac from the large plain and Colehay District. The rirge of hills between Cul de Sac and the pliain ends in Fort Willem and Fort Amsterdam: the highest tops are $250 \mathrm{~m}$. above the sea level. The largest ridge of hills cast of Cul de Sac rises in Centryhill to $408 \mathrm{~m}$. of great importance in the structure of sit. Martin is a sedimen- tary formation, visible to the naked eye (Pointe Blanche for- 
mation) consisting alternately out of Breccia, Sandstone and Limestone with a large proportion of Flint.

In general the Pointe Blanche formation covers the northern slope of the large range of hills and the south eastern slope of the small range. These older selimentary layers are probally of Cretaceous origin. Later sediments have but a short extension on St. Martin. On the promontary near Simsonsbay a hard limestone is found which perhaps is Miocene.

The Lorvlands separated by simsonsbaylagoon from the other part of the Island is almost flat with some hills of which the highest rises to about $90 \mathrm{~m}$. and consist for the greater part of a horizontal layer of soft marly limestone: it is of Miocene age and quite resembles the Miocene formation of Anguilla.

The minerals I hrought from this island were also determined by Prof. Wichmann and proved to contain nothing particular for this island; I only mention gypsum, which I found on Green Key. Plains of some importance are found between the eastern and western ridges of hills, in the southern part (Prince quarter) however hordered by a few isolated conical hills. By a gentle slope the plain gradually passes into the western ridge of hills. Cul de Sac as an important plain is bounded on the eastnorthern- and western sirle by rather steep hills; especially Centryhill and surrounding is much more steep than the other part of the hilly country of St. Martin.

The principal rocks of the Island are composed of Quartz-diorite, Mica-diorite or Phonalite; the part that is exposed to view is generally found unchanged only on the seacoasts. In the large plain it is broken up $1 \mathrm{~m}$. deep into a coarse-grained sand, containing a large proportion of mica. It was not however regularly crumbled down for there are a great many conical parts that resisted the breaking up. 'These continued to stand out when the wastel part wils carried down by the water. So the plain contains for the greater part an abundance of fragments of rocks of considerable dimension. They form a real "Felsenmeer" as is frequently met with on granitic soil.

Further there are some pieces of flat land between the ends of the different ridges of the hilly country between Grande Case and Marigot.

In some places from Eethlehom to Mount Paradis deep valleys are found whereals the villey in the District Colomisier is one of the few spots in st. Martin where a typical tropical vegretation is met with. Especially here in the District Columbier and also near 
Mont Chambord are some sinill eurents when the weather is not too dry.

The larger valleys open on a bay and there the coast is flat whereas almost everywhere clse a rather sterp coast is to be found.

The size of these hays is in direct proportion to the plains that end in them. The larger bays are separated from the sea by shoals and the inner seas thus formed are made use of as Saltponds.

The largest bays are found near the transversal villey Gramir. Case, near Orientbay and at Filipsburg, which is built on a sandbank.

\section{General notes.}

Whereas the map alled indicates the distances broween the islands, the following may serve to inform.

Clece ${ }^{1}$ ) says: "The islands north of (inadelnupe from two parillel "chains from North-west to South-east. The western chatin com"mences with saha and consists of St. Eustatius, St. Kitts, Nevis, "liedonda and Montserrat. All of those islands are volcanoes and "if the line were extended farther to the north it would reach "the island of Amegada, of postplinciune date and all the volca. "noes sem to be of the sime or nearly the same geolosical time. "East of the volcanic range is another conpletely different range "of islands. They are not volcanic and commence with Sombrero "comprising Ansuilla, St. Martin, St. Bartbolomew, Barbuda and "Antigua. All of these islands are of the tertiary age, eocene, mio"cene and pliocene."

Althoush I do not agree with cleve here. who wants to separate the group of st. Hartin from the Virgin Islands and denies as such, that a connection might have existed between the great Antilles and the group St. Martin I want to state that we see clearly mentioned here how wide a difference thre "xists between the two grouns the St. Martin plateau and the Christoffer chain.

About the connection between Anguilla and St. Martin Spencer-2) says:

"The same limestone (we find in st. Martin) also occurs a short

1) Cleve: On the Geology of the Northerstern West Indian Islands p. 19.

2) Spencer: On the Geological and Physical Development of Anguilla, St. Martin, St. Bartholomes and Sombrero p. 526. 
"distance arvay in the island of Anguilla, which was once evi"dently a part of the coastal plain extending from the mountains „of St. Martin. T'intumarre, an arljacent outlier of St. Martin, re"presents a fragment of the dissected coastal plain. It is underlain "by the same white limestones."

According to spencer the various heights and lepths of the diffe. rent groups of St. Hartin plateau and st. ('hristoffer chain seem to agree. As to the connection lietween the Carihean isles he says ${ }^{1}$ ): $n \ldots$ if this inference be true, then the modern islands of the "West Indies formed an elevated plateau bridge between the two "Americas during the two epochs of elevation, namely in the "Pliocäne and Pleistocäne period."

1) Spencer: Recorstruction of the Antillean Continent p. 132. 


\section{CHAPTER II}

\section{METEOROLOGICAL NOTES.}

There is not much known about the elimate of the Dutch West Indian Islands.

As they form part of the Carihean Islands it will be sufficient for me to give all I found about the climate of these Islands in Hamn, Handbuch der Metrorolosie', Je Aufl. Stuttgart 1897. The direction of the winds does not change very much and throughout the year there is generally the N. E. tradewind.

The amount of rain is mostly about $1200-2000 \mathrm{~mm}$. a year; the maxima of rainfall are in May and october; the dry months are February and March. The mean temperature varies from $24-26^{\circ}$ in January and $26-28^{\circ}$ in July.

The following list shows the quantity of rain that will fall on the Dutch West Indian Islands and the neighlouring Islands St. Croix and St. Kitts. 

Rainfall in $\mathrm{mm}$. of the West Indian Islands St. Croix,
St. Kitts, St. Eustatius, Saba and St. Martin.

\begin{tabular}{|c|c|c|c|c|c|c|c|c|c|c|}
\hline & & & & Averas & ge qua & antity & & & $\left.906 .{ }^{4}\right)$ & \\
\hline & & & $\begin{array}{l}\frac{x}{0} \\
\dot{0}= \\
\dot{0}\end{array}$ & 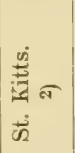 & 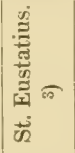 & $\frac{\pi^{\pi}}{\tilde{\pi}}$ & 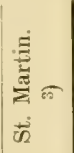 & 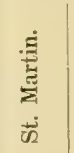 & 劳 & 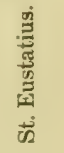 \\
\hline January . & . & . . & 72 & 103 & 63 & 61 & 60 & 56 & 100 & 31 \\
\hline February & . & . . & 61 & 48 & 43 & 28 & 41 & 16 & 71 & 46 \\
\hline March. & . & . & 24 & 55 & 40 & 21 & 33 & 55 & 67 & 31 \\
\hline April . . & . & . . & 77 & 90 & 60 & 32 & 70 & 17 & 38 & 36 \\
\hline May . & .. & . . & 79 & 98 & 106 & 89 & 118 & 23 & 18 & 27 \\
\hline June ... & . & . . & 126 & 92 & 96 & 78 & 80 & 123 & 53 & 124 \\
\hline July, ... & - & . . & 191 & 105 & 118 & 121 & 85 & 70 & 65 & 66 \\
\hline August . . & - . & . . & 112 & 144 & 126 & 143 & 116 & 83 & 30 & 61 \\
\hline September . & - . & . & 123 & $15 t$ & 139 & 189 & 145 & 368 & 465 & 419 \\
\hline October. . & 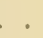 & . . & 156 & 173 & 121 & 123 & 128 & 48 & 66 & 66 \\
\hline November . & $\cdot$ & . . & 181 & 134 & 131 & 162 & 139 & 137 & 88 & 98 \\
\hline December. & $0^{\circ}$ & . . & 82 & 96 & 82 & 72 & 69 & 214 & 223 & 148 \\
\hline Total & ${ }^{\circ}$ & . & 1284 & 1294 & 1125 & 1119 & 1084 & 1213 & 1282 & 1159 \\
\hline
\end{tabular}

1) and 2) from Hann 1. c.

3) from Went Rapport 1. c.

4) from In pectie tan den Landhum in IVest-Indu. Meteorologriche Waarnemingen gedaan op de Meteorologische stations in de koloniën Suriname en Curaçao in het jaar 1906.

As appears from the preceding list there is a great harmony about the data concerning the rainfull on the different Islands and especially when we take in consideration that the first data about the rainfall in the Dutch West Indian Islands are most probably not s) accurate as the last, there is almost the same amount of rain on each of them.

Moreover I am sur: (and here I yuite agree with what Went says in his lieport) that the quantity of rail that falls on the higher parts of the Mountain in st. Eustatius and Saba is much more than elsewhere on these Islands. 


\title{
THIRD PART
}

Section B. Distribution of the Wild Plants

\author{
enumerated in the First Part
}





\title{
B. Distribution of the Wild Plants enume- rated in the First Part
}

\author{
CHAPTER I \\ DISTRUBUTION OF ALL THE WILD PLANTS \\ ENUMERATED IN THE FIRST PART.
}

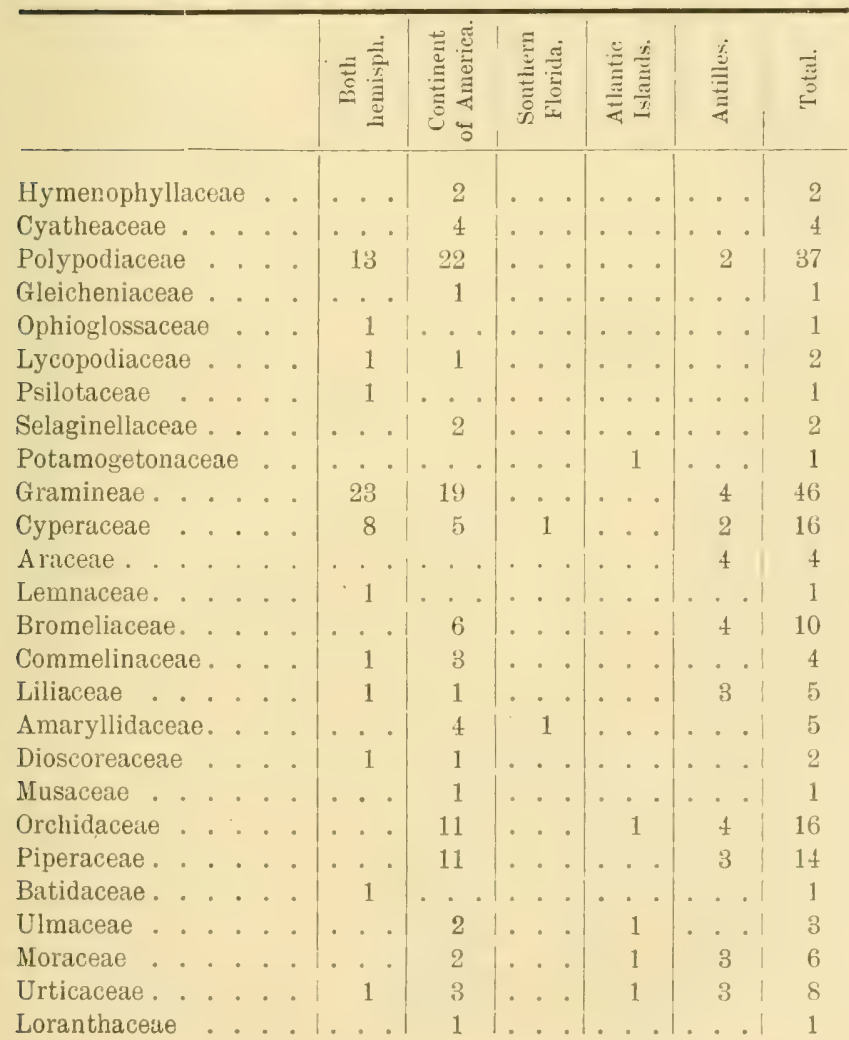




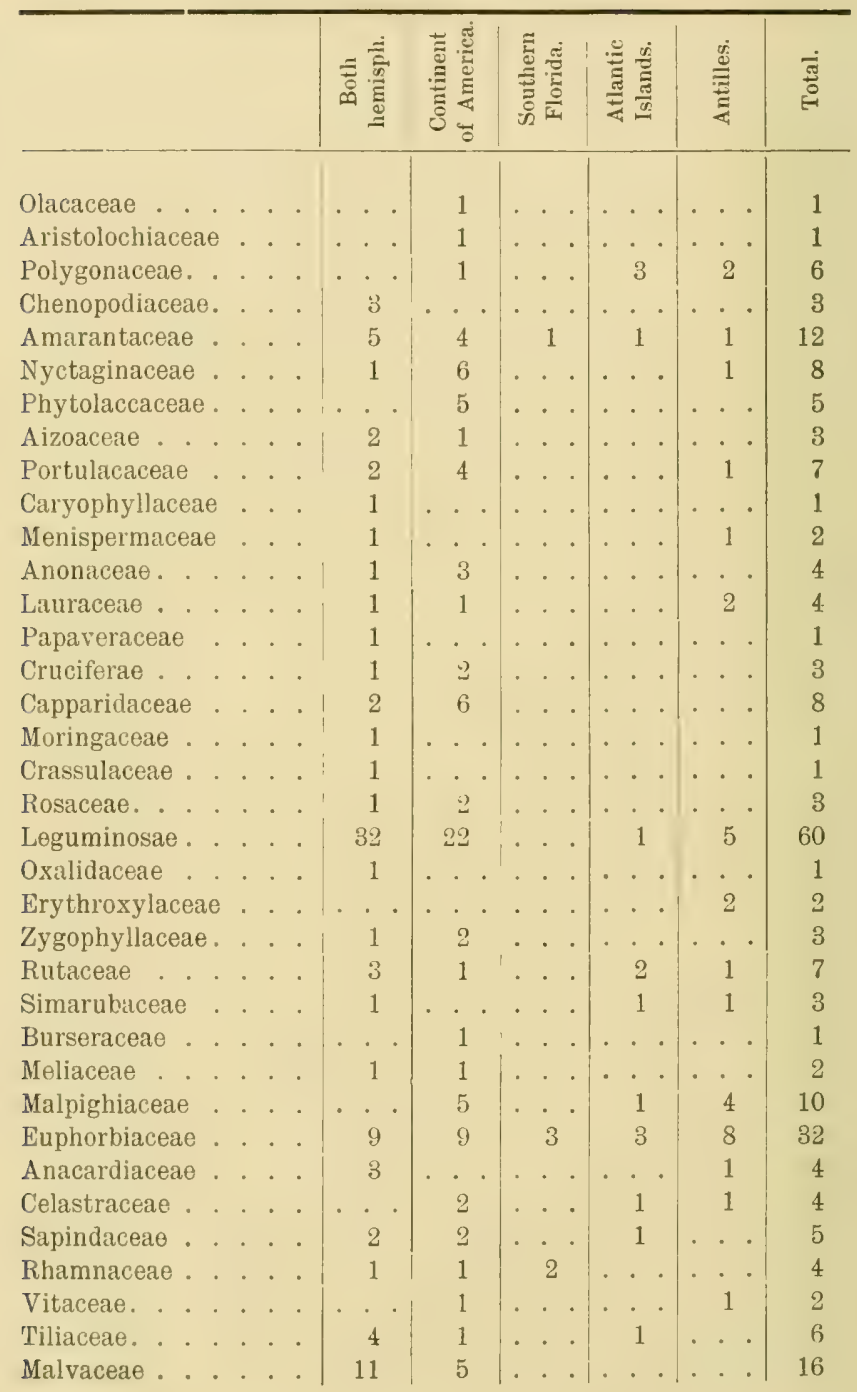




\begin{tabular}{|c|c|c|c|c|c|c|c|c|c|}
\hline & & & & 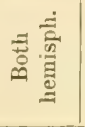 & 苛 & 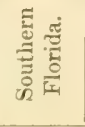 & 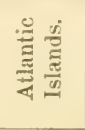 & 兽 & हं \\
\hline Bombacaceae. & & & & 1 & 1 & . . & . . & . . & 2 \\
\hline Sterculiaceae. & . & . & & 2 & 5 & . . . & . . . & . . & 7 \\
\hline Ochnaceae. . & . & . & & 1 & . & . . . & . . & . . . & 1 \\
\hline Maregraviaceae & . & . & & . . . & 1 & . . . & . . . &.. & 1 \\
\hline Theaceae . . & . & . & & . . . & 1 & . . . & . . . & 1 & 2 \\
\hline Guttiferae . . & . & . & & . . . & 2 & . . . & . . . & 2 & 4 \\
\hline Bixaceae . . . & . & . & & . . . & 1 & . . . & . . . &.. & 1 \\
\hline Canellaceae . . & . & . & &.. & . . . & 1 & .. & . . . & 1 \\
\hline Flacourtiaceae . & . & . & & . . & 2 & . . & 1 & 1 & 4 \\
\hline T'urneraceae . . & . & . & & ... & 2 & . . & .. . & . . . & 2 \\
\hline Passifloraceae. & . & . & & . . . & 5 & ... & . . . & . . . & 5 \\
\hline Begoniacene . . & . & . & . & . . & . . . & . . & . . & 1 & 1 \\
\hline Cactaceae. . . & . & . & & 1 & 3 & . . . & 1 & 2 & 7 \\
\hline Thymelaeacea $\theta$. & . & . & & . . . & . . & . . & . . & 1 & 1 \\
\hline Rhizophoraceae. & . & . & & . . . & 1 & . . . & . . . & .. & 1 \\
\hline Combretaceae . & . & . & & 2 & ... & . . . & . . & .. & 2 \\
\hline Myrtacea. . . & . & . & . & 1 & 5 & 4 & 1 & 4 & 15 \\
\hline Melastomataceae & . & . & & . . . & 4 & . . . & . . . & 1 & 5 \\
\hline Umbelliferae. . & . & . & & . . . & 1 & . . & . . . & . . . & 1 \\
\hline Theophrastaceae & . & . & & . . & . . & . . . & 1 & 1 & 2 \\
\hline Myrsinaceae . & . & . & & . . . & 1 & . . . & . . . & 1 & 2 \\
\hline Plumbaginaceae & . & . & &.. & 1 & . . . & . . & .. & 1 \\
\hline Sapotaceae . . & . & . & &.. & 2 & 1 & . . . & 3 & 6 \\
\hline Symplocaceae. & . & . & & . . & . & . . . & . . . & 1 & 1 \\
\hline Oleaceae ... & . & 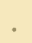 & &. .1 & 1 & . . . & . . . & 1 & 2 \\
\hline Loganiaceae . . & . & . & & . . . 1 & 1 & . . . & . . & . . & 1 \\
\hline Gentianaceae. . & & . & & . . . & 1 & . . . & . . & . . . & 1 \\
\hline Apocynaceae & . & . & & 1 & 2 & . & . . & 2 & 5 \\
\hline Asclepiadaceae. & . & . & & 2 & . . . & . . & . & 2 & 4 \\
\hline Convolvulaceae & & . & & 4 & 9 & . . & . . & 3 & 16 \\
\hline Hydrophyllaceae & . & . & & . . . & 1 & . . & . . & . . . & 1 \\
\hline Borraginaceae . & & . & & 2 & 4 & 1 & 2 & 6 & 15 \\
\hline Verbenacoae. . & & . & & 4 & 7 & . . & . . & . . & 11 \\
\hline Labiatae . . & & & & 5 & 2 & 1 & . . & . . . & 8 \\
\hline Solanaceae . . & & ${ }^{\circ}$ & & 9 & 4 & . . & . . & 3 & 16 \\
\hline Scrophulariaceae & & & & 1 & 2 & & & . . . 1 & 3 \\
\hline
\end{tabular}




\begin{tabular}{|c|c|c|c|c|c|c|c|c|}
\hline & & & $=\frac{\dot{a}}{\overline{\vec{a}}}$ & 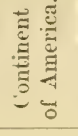 & 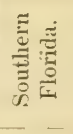 & 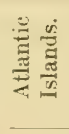 & 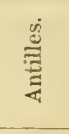 & $\frac{\vec{\pi}}{0}$ \\
\hline Bignoniaceae. . & . & - & . . . & 4 & . . & . & . & 4 \\
\hline Gesneriaceae. . & . & . . & . . . & . . & . . . & . & 2 & 2 \\
\hline Lentibulariaceae & . & .. & . . . & 1 & . . . & . . & . . . & 1 \\
\hline Acanthaceae. . & . & . . & 2 & 2 & . . . & 1 & 2 & 7 \\
\hline Plantaginaceae. & . & . . & 1 & . . . & . . . & . . & - . & 1 \\
\hline Rubiaceae . . . & . & . . & 3 & 13 & 1 & 4 & 4 & 25 \\
\hline Cucurbitaceae . & . & . . & 2 & 3 & . . & . . & . & 5 \\
\hline Goodeniaceae . & . & . & . . . & . . . & . . . & 1 & . . & 1 \\
\hline Compositae . . & . & - . & 17 & 14 & 1 & 2 & 6 & 40 \\
\hline & Tot & & 207 & 301 & 18 & 34 & 114 & 674 \\
\hline & & & & & & & & \\
\hline
\end{tabular}

I have enumerated in the Systenatic Part Sot species;. 132 of these are cultivaterl, and as will be seen from the list before given there are 674 wild plants, 166 of these are West Indian plants, of which 114 ouly are known on the Antilles. Another 34 are distributed in the Antilles and the other Atlantic Islands, whereas 18 are to be found both in the Antilles, the other Atlintic Islands and Southern klorida. From the 508 plants that are not restricted to the West Indies, there are 301 that occur on the Continent of America, whereas another 207 are to be found as well in the Continent of America as in the old world.

From the plints enumerated in the Systematic Part there are 550 namer in Grisebach: Flora of British West Indian Islands. I could make out by what I found in Grisebuch: Die geographische Verhreitung der Pflanzen West Indiens, that from these 550 plants there are alout 180 indigenous in the Antilles and the Bahamas.

When the results given hy Grisebach in 1865 had not been changed by more recent investigations on the Flora of the West Indian Islands than the number of indigenous plants I 


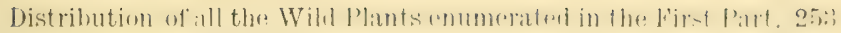

enumerate is this Flora ought to be between 180 and $304=$ $(180+124)$. As it appears that my number is but 148 , it is evident that the data we had in 1865 , abouth the West Indian plants have changed by the newer collections we have got from the West Indies and the neighbouring countries.

Whereas I draw this conclusion from our knowledge about the Dutch West Indian Islands so I believe that a similar study of the other Antilles would show us that there would be a still greater difference between what we know now and what Grisebach stated as the result of his studies on the West Indies in 1865 .

In order to show that there is probilily not a very large number of plants of the. J)utch sutilles St. kiustatius, Siala and St. Martin that might have heen overlooked by suringur and myself I sive the next list where I comprare the numbers of the species from the larger families of the Island of St. Croix (Egyers: Floral of St. Croix and the Tirgin Islands, 1 s 79 ) and the Duteh West Indian Islands above named.

Leguminosae

Graminea

Compositae

Polypodiaceae

Euphorbiaceae

Rubiaceae

Moraceae

Ulmaceae

Urticaceae

Convolvulaceae

Cyperaceate

Malvaceae

Solanaceae

Borraginaceae

Myrtaceae

Caryophyllaceae |

Portulaceae

Amarantaceae
Dutch West Indian Islands.

60

46

40

37

32

25

17

16

16

16

16

15

15

12

12
St. Croix.

57

39

36

34

33

26

14

27

18

25

18

20

22

10

13 
The following list may give any idea about the percentage of the species of the larger families in the Flora of the Islands St. Eustatius, Saba and St. Martin.

$\begin{array}{ll}\text { Leguminosae } & 9 \% \\ \text { Gramineae } & 6.5 \% \\ \text { Compositae } & 6 \% \\ \text { Polypodiaceae } & 5.5 \% \\ \text { Euphorbiaceae } & 4.5 \% \\ \text { Rubiaceae } & 3.5 \% \\ \text { Borraginaceae } & 2.5 \% \\ \text { Convolvulaceae } & 2.5 \% \\ \text { Cyperaceae } & 2.5 \% \\ \text { Malvaceae } & 2.5 \% \\ \text { Myrtaceae } & 2.5 \% \\ \text { Orchidaceae } & 2.5 \% \\ \text { Piperaceae } & 2.5 \% \\ \text { Solanaceae } & 2.5 \% \\ & \end{array}$

As may be seen from the following list there is no great difference between the numbers of plants known from each of the Islands St. Eustatius, Saba and St. Martin.

\begin{tabular}{|c|c|c|c|}
\hline St. Eustatius & Saba & St. Martin & 249 \\
\hline St. Eustatius & Saba & & \\
\hline St. Eustatius & & St. Martin & \\
\hline St. Eustatius & Saba & St. Martin & \\
\hline & Saba & & 100 \\
\hline & & St. Martin & \\
\hline 464 & 456 & 424 & \\
\hline
\end{tabular}




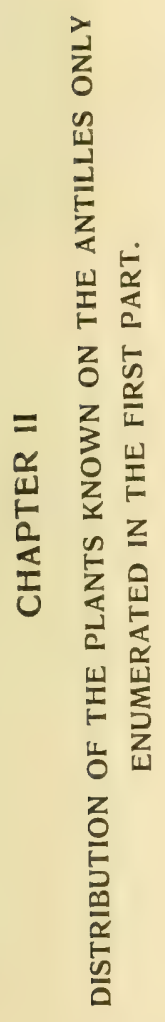

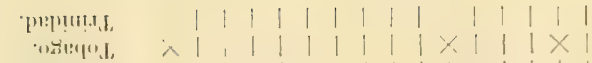

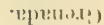

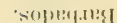

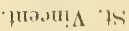

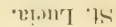

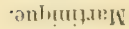

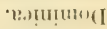

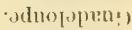

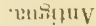

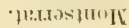

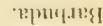

shey is

4แ?

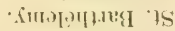

(5)

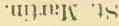

'?

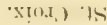

. TII: is

- का:แ11

$0.01 .107 .141 \mathrm{~d}$

ifle?

- 1:.)

' $:[1]$,

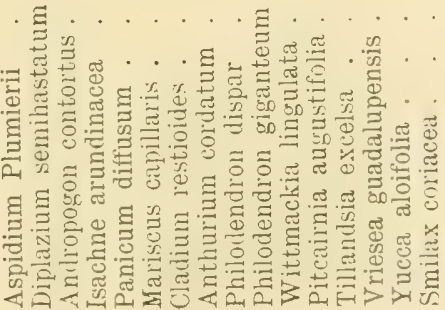



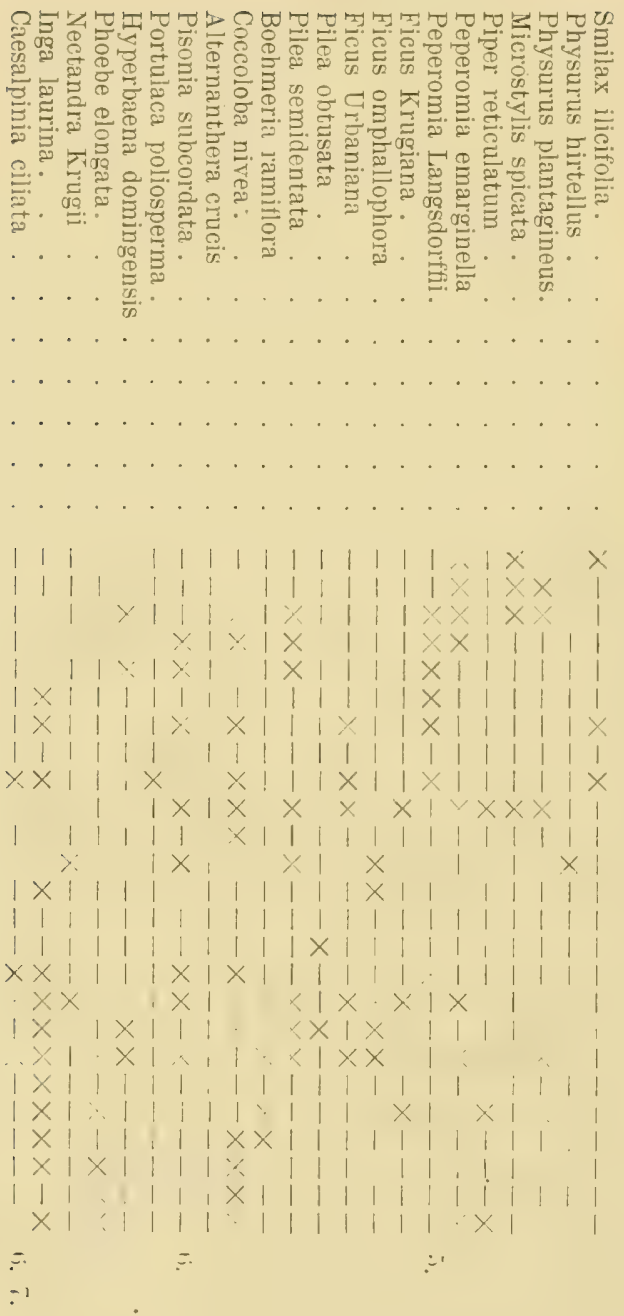

Cuba.

Jamaica

Haïti.

P'ortorîco.

St. Thomas.

St. Jan.

St. Croix.

Inguilla.

st. Martin.

Salua.

St. Barthélemy.

St. Eustatius,

St. Kitts.

Barbudit.

Montserrat.

Antigua.

Guadeloupe.

Dominica.

Martinique.

St. Lucia.

Sit. Vincent.

Barbatos.

Grenadiı.

Tobngo.

'Triniolas. 


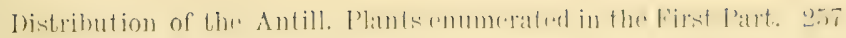

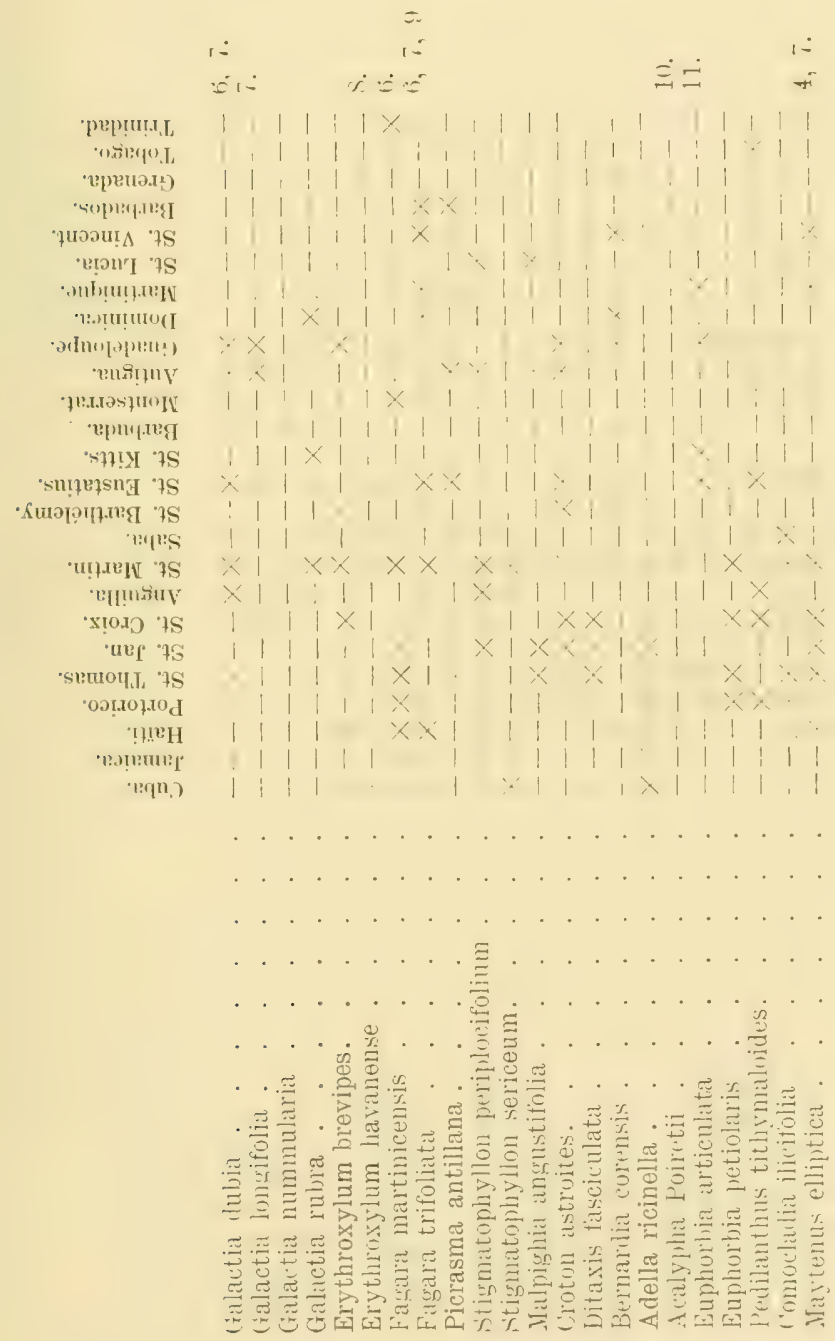




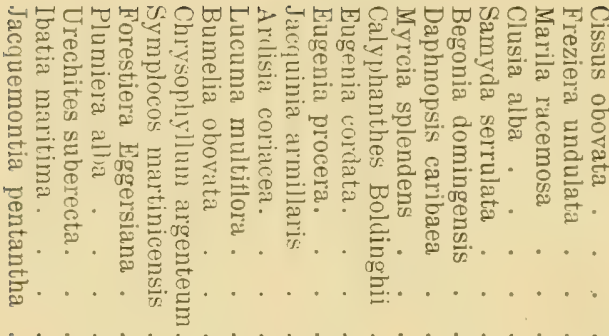

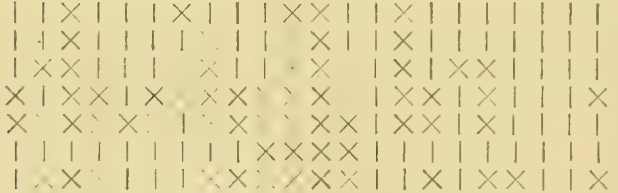
$\begin{array}{llllllllllllllllllll} & 1 \times x & 1 & 1 & 1 & 1 & 1 & 1 & 1 & 1 & 1 & 1 & 1 & 1 & 1 & 1 & 1 & 1 & 1 & 1\end{array}$ $x \times x|1 \times 1| x|\cdots x \times 1 \times 1| x \mid x$ $x: x \therefore 1 \times \therefore \times x \times 1 \times 1 \times x+1 \times x \mid x \times 1$ $|1| 1|1| 1|1| 1|1| x|1| 1$ $x \times x \quad 1 \quad 1 \quad 1 \times 1 \times 1 \times x \times x \times 11$

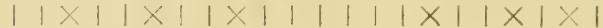
$\begin{array}{llllllllllllllllllll}x & 1 & 1 & 1 & 1 & 1 & 1 & 1 & 1 & 1 & 1 & 1 & 1 & 1 & 1 & 1 & 1 & 1 & 1\end{array}$

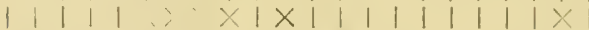

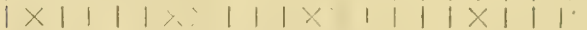
$x \quad 1 ; 1, \cdots x \times x|1,1 \times x| 1 \times x \times$

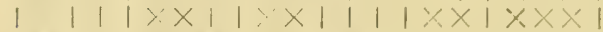
$x \quad 1 \times 1 \because \cdots x \mid 1 \times 1 \times 1 \times 1 \times 1 \times 1$ $1|1 \times 1: 1: x, 1| 1 \times 1,1 \times 1 \mid 1 \times 1$ $x \times 1|1 ; x+x| x|x| x|1| 1 \mid x \times 1$

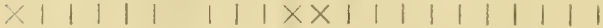

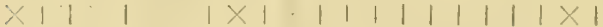

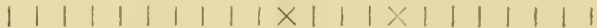

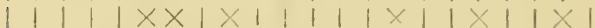
$+$

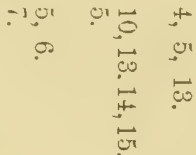

Culua.

Jimaica.

Haiti.

Portorico.

St, Thomas.

St. Jan.

St. Croix.

Anguilla.

St. Martin.

Silba.

St. Barthélemy.

sit. Eustatius.

St. Kitts.

Barlouda.

Montserrat.

Antigua.

Guadeloupe.

Dominiea.

Martinique.

st. Lueia.

St. Vincent.

Barbados.

Grenada.

Tobago.

Trinidad. 
Distribution of the Antill. Plants enumerated in the First Part. 259

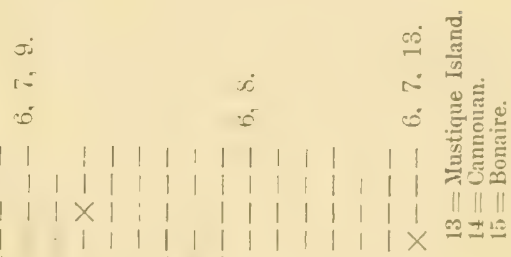

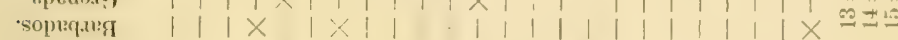

.

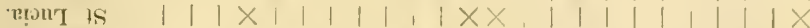

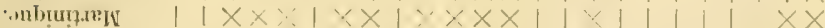

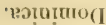

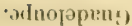

'еกริเฺแบ

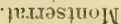

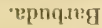

S 7719.78

-sm!n?⿻肀一𠃋 7s

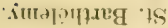

"zopes

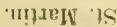

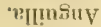

$\left.x \underline{0} \cdot x^{\prime}\right)$ is

шер. is

S1:11001 75

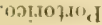

I)!n:!1

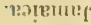

e(in:)

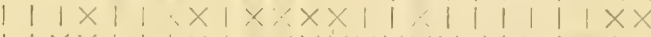

$1 \times x+1 \quad 1 \times 1 \times x \times x \times x \times x \times 1 \% 1 \div 1 \times$

$|1| x|||||x||x \times 1|<|x|, \mid x$

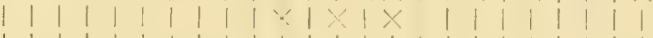

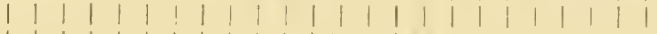

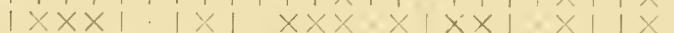

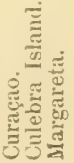

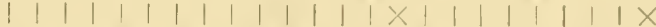

$|1 \times x=1 \times 1 \times x \times x \times x| x \mid x \times x$

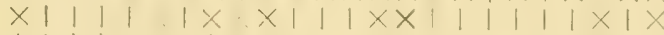

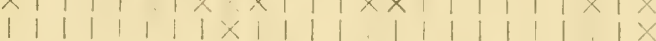

$x|1 \times 1| x|x, 1,1, x| 1 \times 1 \times 1 \times$

\section{$1|1|$}

$x \mid+1 \times 1,1,1 \times 1$

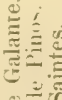

$x|1 \times| \times|x|$

$\Xi$

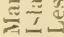

II II

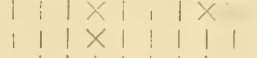

$\begin{array}{llllll}1 & 1 & 1 & 1 & 1\end{array}$

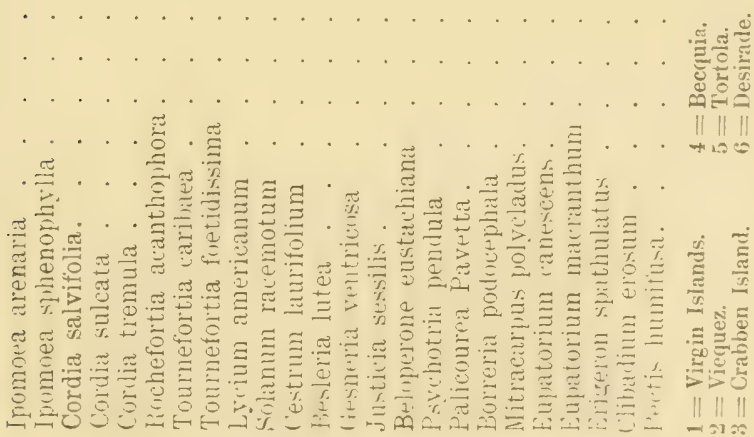


The list given in this chapter neals no further explanition.

As may be seen from the great amount of plants occurring in such a small number of different Islands it will be clear that our knowledge of the Flora of the West Indian Islands is far from being complete.

That is the reason why I will not try to draw any conclusion about the distribution of the different species except a single one and this with all kinds of reserve.

I draw the attention to the data that may be gathered from the following lists.

On the lisands only to the north of the Dutch West Indian Islands together with these Islands are collected:

\begin{tabular}{|c|c|c|c|c|c|c|c|}
\hline & & & & & $\begin{array}{c}\text { St. } \\
\text { Martin. }\end{array}$ & Saba. & $\begin{array}{c}\text { St. } \\
\text { Eustatius. }\end{array}$ \\
\hline Diplazium semihastatum & . & . & . & . & - & - & X \\
\hline Mariscus capillaris . . & . & . & . & - & $x$ & $x$ & - \\
\hline Tillandsia excelsa . . & . & . & . & . & - & $x$ & - \\
\hline Yucca aloifolia . . . & . & . & . & . & $x$ & - & - \\
\hline Smilax coriacea . . . & . & . & . & . & - & - & $x$ \\
\hline Smilax ilicifolia. . & . & . & . & . & $x$ & - & - \\
\hline Alternanthera crucis . & . & . & . & . & $x$ & 一 & - \\
\hline Portulaca poliosperma . & . & - & . & . & $x$ & - & - \\
\hline Erythroxylum havanense & . & . & . & . & $\times$ & $x$ & $x$ \\
\hline Stigmatophyllon sericeum & . & . & . & . & $x$ & - & - \\
\hline Ditaxis faxiculata . & . & . & - & - & $x$ & - & 一 \\
\hline Adelia ricinella . . . & . & . & . & . & $x$ & - & - \\
\hline Acalypha Poiretii & . & . & . & . & $x$ & - & - \\
\hline Cissus obovata . . . & . & . & - & . & $x$ & - & - \\
\hline Forestiera Eggersiana . & . & . & . & . & $x$ & - & - \\
\hline Ipomoea arenaria . & - & . & . & . & $x$ & - & - \\
\hline Rochefortia acanthophora & - & - & - & - & $x$ & 一 & $x$ \\
\hline Lycium americanum . & . & . & . & . & $x$ & - & - \\
\hline Mitracarpus polycladus. & . & . & . & . & - & $x$ & - \\
\hline
\end{tabular}


Distribution of the Antill. l'lants anmmerated in the First l'art. 2601

On the Islands only to the south of the Inteh West Indian Islands together with these Islands are collected:

\begin{tabular}{|c|c|c|c|c|c|c|c|c|}
\hline & & & & & & $\begin{array}{c}\text { St. } \\
\text { Martin. }\end{array}$ & Saba. & $\begin{array}{c}\text { St. } \\
\text { Eustatius. }\end{array}$ \\
\hline Philodendron dispar & - & . & . & . & . & - & $x$ & $x$ \\
\hline Vriesea guadalupensis & . & . & - & . & - & - & $\times$ & $\times$ \\
\hline Piper reticulatum . & . & - & . & - & - & - & $x$ & $x$ \\
\hline Ficus Krugiana . . & - & - & - & - & - & - & $x$ & $x$ \\
\hline Ficus omphallophora & - & . & - & - & . & - & $x$ & $x$ \\
\hline Boehmeria ramiflora & . & - & . & - & - & - & $x$ & x \\
\hline Caesalpinia ciliata . & - & - & & . & . & $x$ & x & $x$ \\
\hline Galactia rubra . . & . & & & . & . & $x$ & $x$ & $x$ \\
\hline Freziera undulata . & . & & & - & . & - & x & - \\
\hline Marila racemosa. . & - & & & . & - & - & $x$ & 一 \\
\hline Cordia salvifolia. & . & & & . & . & - & $x$ & $x$ \\
\hline Cordia tremula . . & . & & - & - & - & - & $x$ & - \\
\hline Tournefortia caribaea & $\cdot$ & & & - & - & - & $x$ & - \\
\hline Beloperone eustachiana & & . & . & - & - & $x$ & $x$ & $x$ \\
\hline Eupatorium macranthus & & - & - & - & - & 一 & $\times$ & $x$ \\
\hline
\end{tabular}

As may be seen from these two lists the grater number of the plants found on the northern Islands only are but to be met with on St. Martin, the greater number of the plants found on the southern Islands only are but to be met with on Sabd and St. Eustatius.

In conelusion, I believe the different indigenous plants of the Antilles make it sufficiently clear that the vegetation of St. Martun has been influenced ly some other circumstances than the vegetation of Saba and St. Martin.

Siecies only known from the Dutch West Indians Islands are:

Galactia nummularia

Calyptranthes Boldinghii

Ipomoea sphenophylla
St. Martin.

St. Martin.

St. Eustatius. 



\section{THIRD PAR'T}

Section C. The vegetation of St. Eustatius, Saba and St. Martin 



\section{The vegetation of St. Eustatius, Saba and St. Martin}

\section{CHAPTER I}

\section{NOTES ON THE ASPECT OF THE VEGETATION OF THE THREE DUTCH WEST INDIAN ISLANDS. \\ Introduction.}

The vegetation of St. Eustatius, Saba and St. Martin is that of a tropical zone, without any pronounced dry season. As may be seen from the meteorological data the quantity of rain is almost sufficient to malie possible the existence of at tropical rainforest vegetation.

The N. E. tradewinds however are of great influence upon the vegetation; where these winds are not hroken by mountains we may meet with the typical vegetation of trees blown in a certain direction as well as with the Cactacene and thormplants, characteristics of a dry climate.

From the eliaphic factors that exert influence upon the charikter of the different Plantformations I will in this chapter only draw the attention to the amount of humus that covers the rocks. It is one of the principal factors, as there are a great number of localities where but a small quantity of humus is to be found. Only there were no strong wind is blowing, where the atmosphere is humid and where the yuantity of humus has surpassert a minimum the tropicil vegetation shows itselt in its wellknown typical features.

But the ahove named fitctors have not exclusively determined the plantcovering of the Islands; one of the greatest fiactors hats heen the influence of man, who hils been destroying the wools ant introducing many plants in culivating the soil. So there are a great number of plants introduced that have superseder the primitive vegetation. 
When taking the vegetation in general and without entering into Ietails we may consider it to be identical on the three Islands. From the short notes however I give in this chapter on the aspect of the different Islands it will be obvious that on each of the three Islands a particular Formation gives a predominant chatracter to each of them.

\section{St. Eustatius.}

The monotony of the level part of St. Eustatius is relieved by some plantations surrounden by trees and by ruats also bordered by trees and shrubs.

The formation that predominates in the "cultuurvlakte" is a dry shrublyy vegetation of a greyish aspect that I will call the Crotun vegetation, following the nomenclature of Eggers in his Flora of St. Croix and the Virgin Islands.

The sreater part of the hills near Signalhill and Concordia are also covered with the same vegetation.

Only the higher parts of these hills as well as the Quill are covered with a greenish wood that I will call the Eriodendron vegetation also in analogy with Eggers 1. c.

These two parts of the vegetation are not wholly separated and both of them are intermixed with those plants which form the vegetation of the cultivated region.

'l'he impracticahle parts of the rocky clift's covered only with few plants form a typical vegetation with thicker or more coriacenus leaves in close relation with the vegetation of the seashore; the latter is not very well developed in St. Eustatius as there is but a small beach and the Cultuurvlakte is about $40 \mathrm{~m}$. above the sealevel.

Without entering into details we can mactically divide the vegetation of St. Eustatius in:

1. Vegetation of the higher parts of the mountains.

2. Vegetation of the Cultuurvlakte and the lower parts of the mountains.

3. Vegetation of the seashore and the rocky localities of the Island.

4. Vegetation of the cultivated region.

Whereas a survey of the vegetation of St. Eustatius based on these principles is given in the following lists it may he men. tioned here that an almost synomical list could be given, viz: 
1. Eriodendron vegetation.

2. Croton vogetation.

3. Littoral vegetation.

4. Eriodendron- and Croton vegotation intermixed with and partly replaced by plants of the cultivatod region.

We will see in Chapter If of this sertion that these two series of names do not quite agree and this is the reatson why I use the first mentioned numes when in the following lists I givo a survey of the distrihution of the plints on the diflerent puts of St. Eustatius. In these lists as well as in the other ones I give in this Chap. ter the West Indian plants are printed in italics whereas the plants that are cultivated on the Dutch IVest Indian Islanils ant: printed in small type.

\section{Vegetation of the Higher Parts of the Mountains of St. Eustatius.}

Acacia marracantha, Acacial tortuosa, Aceriphilat martinicensis, Allophylus oceidentalis, Amomis caryophyllata, Anacardium orci. dentale, Andira jamaicensis, Anona muricata, Anona reticulata. Anthurium cordatum, Aspidium Plumieri, Asplenium cristatum,

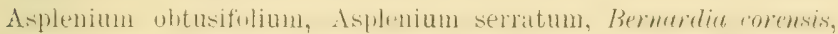
Besleviu huter, Bignonia ungius cati, Blechnum ocrilentale, Bumelin obmeter, Bursera simaruha. Byrsonima spicita, calotropis procera, Capparis Brevnia, (apparis cynophallophora, (apparis fromkona, Calpparis jamaicensis, Casearlia parvifolial. Cassial glandulosil, Catopsis nutans, (eeropia peltata, Ceilna pentandra, Celt is Iguanacea, Centrosema virginianum, Chiococera racemosia, ('hrysolsalanus ieaco,

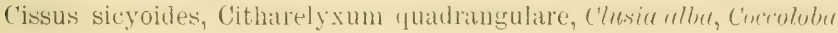
diversifolia, Coccoloba nivea, Coccoloba uvifera, Coffea arabica, Corchorus hirsutus, Cordia salvifolia, Crotalaria retusa, Croton astroites, Cyathea serra, Ihphnmpsis creribara, Desminthus depressus, Desmodium smpinum, Dipholis salicifolia, Dodonaeal visiosid, Elapho. slossum contorme, klaphoglussum petiolatum, Ejpirtentrum anceps,

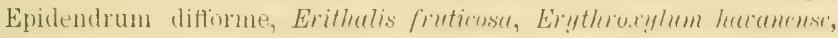

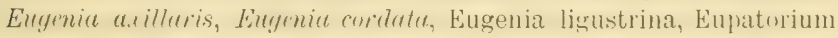

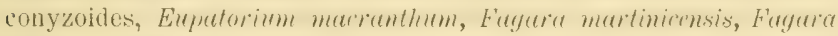

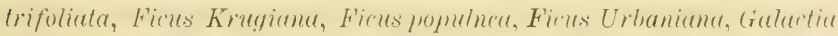

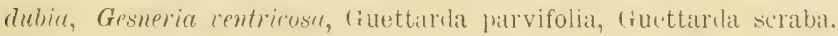
Gymindal latifolia, cilmmunthes luride, Heliennia Bihai, Heteropteris lanceolata, Heteropteryx furpurea, Hillia longifolia, Hyperbuena

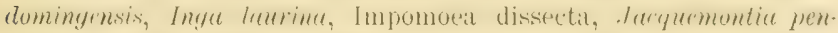
fenther, Krugiodendron forrum, Liuntana cilluila, Lantana involu- 
crata, Leiphaimos aphylla, Linociera compacta, Lonchocarpus violaceus, Lycopodium taxifulium, Mammea americana, Laytenus ellip. licus, Melothria fluminensis, Miconia impetiolaris, Miconia laevigata, Mimosa pudica, Morisonia ampricana, Myrcia paniculatit, Myrcia splendens, Nectandra coriacea, Nectundra Krugii, Neurogramme caIomelanos, ()plismenus setarius, Pulicoure Pevetta, Panicum divaricatum, Panicum insulare, Paspalum hemisphatericum. Passiflora laurifolia, Pilsiflora suberosil, Pectis humifusu, Peperomia acuminata, Peperomiat stabella, Peperomiu Langstorffi, P'eperomia obtusifolia, Peperomia seandens, Persea anericana, Pharus glaber, Philodendron dispor, Philutendron oxyeardium, Phorbe elongutu, Physurus plentugineres, Picrasma Antillana, Pilsa semidentulu, l'jper dilatatum, l'iper medeun, Pijer reticulutum, Pirifueta villosa, Pisonia aculeata, P'isonia fragans, Pisonia subrordute, Pitcaimia andystifolia, Polypodium brasiliense, Polypulium lycopodivides, Polypodium phyllitikles, Polypodium plumuli, Polystachya luteola, Prescotria stachyoides, Prunus myrtifolius, Psychotria horizontalis, Psychotria rufescens, Psychotria uliginosi, Remdiu aculeata, Rauwoltia Lamarckii, Rhynchosia reticulata, Samyda serrulata, Schaefferii frutescens, Sichoepfia Sicheberi, scleria lithosperma, Smilax coracea, Smilix guianensis, Solanum lanceaefolium, Sulamem racemosum, Solinum racemosum ig. neum, Spermacoce tenuior, Stenochlaena sorbifolia, Stenolobium stans, Stenorrhynchus orchioides, Stigmatoplu!llon periplocifolizm, Swictonia mahagoni, symplocos martinicensis, Taliernaemontana citrifolia, Tecoma leucoxylon, T'ernstroemia peducularis, Theobroma Cacao, Toumefortic foetidissima, Tragia volubilis, Trema Lamarckiconem, Trema micrunthum, Triumfetta rhomboidea, Lrera Calacasani, Vernonia punctata, Vittaria lineata, IViesurenudulupensis, Waltheria americana, Wedelia buphthalmoides, Iylosma buxifolium.

\section{Vegetation of the Cultuurvlakte and the Lower Parts of the Mountains of St. Eustatius.}

Acacia macracautha, Acaciil tortuosil, Amarantus polygonoides, Andira jamaicensis, Andropogon contortus, Aristida americana, Ayena pusilla, Beloperone enstuchyana, Bernardia corensis, Borreria podocephale, Brassavolit cueullatid, Camavalia olstusitulia, Capparis cynophallophora, Calplaris frondoria, Capparis jamaicensis, Cassia bicapsularis, Cassia glandulosi, Catopsis nutans, Celtis Iguanacea, Cenchrus tribuloides, Centrosema virginianum, Citharexylum quadrangulare, Coccoloha uviferi, Commelina virginica, Comocladia ilicifolia, Corchorus hirsutus, Cordia salvifolia, Cracca caribaea, 


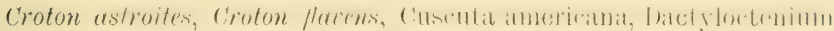

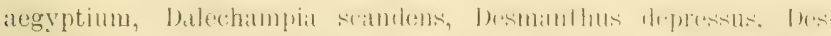

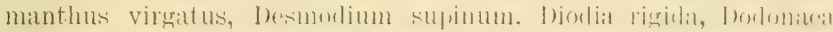

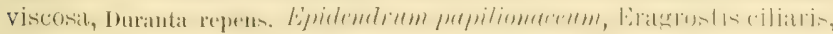
Erithalis fruticosa, Ermodec litoralis, Engenia cordata Sintensii,

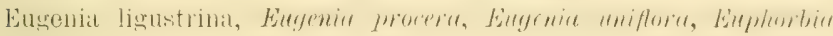
articulata, Evolvulus sericous, Fourcroya gigantea, Galactia dubia,

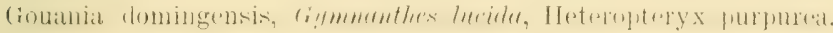

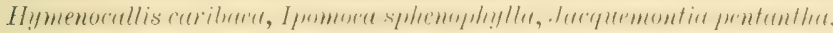

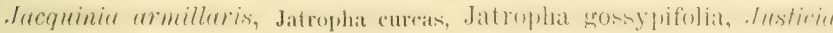
sessitis, Krameria Ixina, Lantana camara, Lantana involucrata, Malpighia junicifolia. Malvastrum spicatum, Monstems ellipliera,

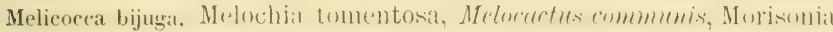
americana, (p)untia Ficus indicat. Opuntiat trianantha, ()puntia T'una, Panicum insulate, Pappophorum alopecuroilemm, Pectis humifuna, Phorillendron trinervium, Phyllanthus distichus, Pilea microphylla, Pisonia fragans, Pisonice subrondata, Piteaimnio angustifolia, Pithe. colobium ungus cati, Polypodium lycopoliobles, Portulara oleraces,

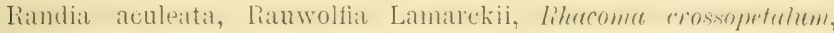

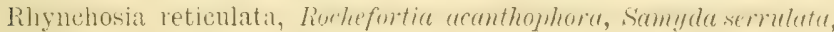
Schatfieria frutescens, Setaria setosa, Sida ciliaris, Sicla suphini, Solanum racemosum, Solanum racemosum igneum, Sporobolus virginicus, Steuolobium stans, Stigmatophlyllon priplucifolium, Strumpice marifimu, stylosauthes hamata, Talinum triangulare, T'amarindus indica, Tecoma lencoxylun, Tephrosia cinerea, Tommefortiat volubilus, Tragus racemosus, Turnera ulnifolia, Lreblites suberectu, Vernonia punctati, Waltheria americana.

\section{Vegetation of the Seashore and Rocky Parts of St. Eustatius.}

Amarantus polygunuides, Andropogom rontortus, Argemone mexicana, Aristida anericana, Borrerin frolocrphale, Carsalpinia crista, Calonyetion tulra, Camavalia uhtusifolia, Cassia bieapsubaris, Como-

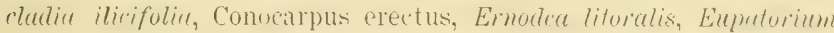
comescons, Euphorbiu artioulutu, Euphorlia buxifulia, Evolvulus sericeus, Heliotropinm curassavicum, Hibircus tiliacens, Hipno mane: mancinella. I ponoea pesalprate, Jacquiniu amilluris, Lither. phila muscoles, Malpighia punicifolia, Melorertus commmenis, (1)untia

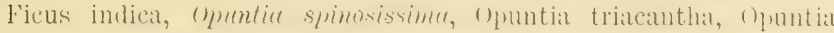
T'una, Punirum aliffusum, L'appophorum alopecuroideum, harromu

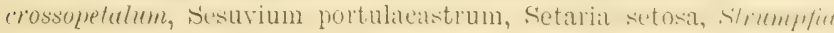


maritima, 'Tephrosia cinerea, Thespesia populnea, Tournfortiagnaphalodes.

\section{Vegetation of the Cultivated Region of St. Eustatius.}

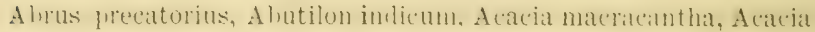

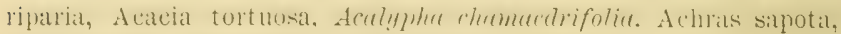
Achyranthes ubtusifulia, Ageratum comyoides, Altrmanthera repens, Amarantus spinosus, Antrantus tristis, Ambrosia hisplidu, Anoma montana, Anona muricata, Anthephora hemaphrorlitica, Antignon leptopus, Bofrhatea hirsuta, Boerhatrea paniculata, Bryo. phyllum pinnatum, ('alotropis procera, ('apparis Brevola, Capparis cymophallophora, Capraria biflorat, f'ardiosfumum halicacalum, Cassia oceidentalis, Cenchrus tribuloides: Centrosema virginiana, cheilanthes microphylla, Chenopotium ambrosioides, chemplodium murile, Cissampelus Partira, Clerodendron ateuleatum, Coccolobil uviferal, Coleus amhoinicus, Commelinat virgini.a, corchorus acentangulus, corchores hirsutus, Cratcea caribatea, Crotalaria rerrucosa. crotum astroites, ciutom flacens, croton lohitus. (Ymoulon dactylon, Cyperus rotundus, llatura Metel, Datura Stramonium, Desman. thus deprusins, Desmanthus virgatus, Dusmodium -uninum, Elensine intica, Eragrostis cilinris, Eupatorium engyoiles, Euphorbia heteruhyla, Euphorbia hypercofolia, Euphorbia pilulifera, Festuca Myurus, Flemya aestuans, (iymandropsis fentaphylla, Hatematoxylon campechiamum, Heliotropium indicum, Holintropium parviforum, Hura crepitans, Hyptis pectinata, lluth martimu, Indignferal suffiutiensa, Iuequmentia pentunthe. Jatrophat go sypifolia, Kallstroemia maxima, Lantana imvolnerata, Lenuntis nepetaefolia, Ltommos sibirieus, Letuaenal whluea. Inchnera roxea, Malvastrum spricatum, Meli. aneat bijuga, Meluewtus inmmomis, Melochial promidati, Melochia tomentosa, Microten dehilis, Mimosa furlica, Mirabilis Jalapa, Mollugo verticillata, Momordira Chanantia, Hỵlrolea jamateensis, Nexurogramme calomelanos, () cimum micranthum, Opuntia Ficus indica, 1)umtia triacantha. (1)untia 'T'unal, l'anicum insulal', l'anicum maximum, Panicum sillsuinale, Palthenium Hysterophorus, Pax. palum fimbriatum, Paspalum bemisphatericum, Passillem laurifolia, Prablumthus tith!muluidrs, Peperomia petiolaris, l'haseolus lathyJobles, Phrllanthus niruri, I'ilea microphylla, Pirigueta rillosil, P'umbagn scamdens, Pulanisia viscosa, Pulypodium aturemm, Poros.

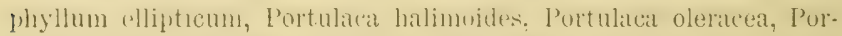

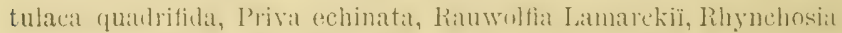

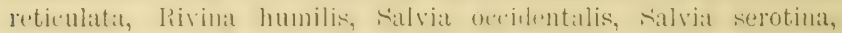
Setaria glatuea, setarja setosa, sirla carpinifolia, Sida cordifolia, 


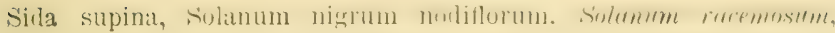

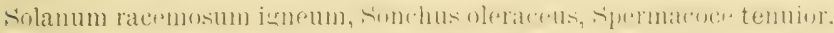

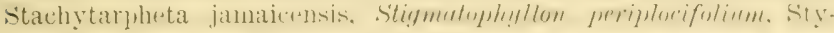

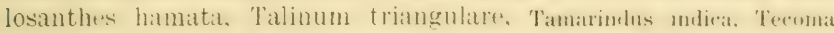

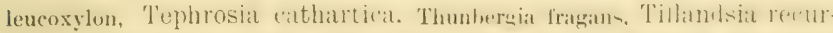

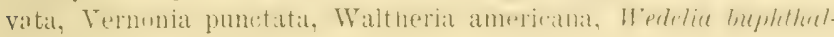
moides, Zornia diphylla.

\section{Saba.}

The Island of Siba makes a vory ditlirent impresion as St. Eustatius does, especially hy the total alsence of al levil country like the Cultuurvlakte on the latter Island.

Owing to the conical form of satua there are only stexp, slopess of hills and these hills are covered by the same rewetation as those in St. Eustatius, viz.: the Eriodendron veretation. Murrover there is a good quantity of almost naked rock where a very poor flora is to be found.

According to the fact that almost in all the localities that art apt for cultivation the soil is occupieal by the inhalitants. there is a well developed regetation of the cultivatud remion inter. mixed with the Eriodendronvegetation.

The Croton vesetation is only to be seen in a rery ferr Incalitis: and mowhere on Saha it is so premlominant as in st. Eustatius.

That part of the Mountain higher than $500 \mathrm{~m}$. above the sealevel, though also greatly influenced by ruderal plants has a very well developed tropical rainforest which, withrut lusing the typical character of the st. Eustatius forests is charactrised by its great number of Ferntrees.

The vegetation of the seashore is very lool and has no influmer on the general aspect of the Sabavegetation.

Whereas it is not so easy to makt a sharp diffirence lis tween the. four l'antformations still I think it hett+e alsis to thace out here the four following typical groups:

1. Vegetation of the Mountain top and the greater part of all the slopes of the Island.

2. Vegetation of some arid hills.

3. Vegetation of the seashore and rocky Iocalities.

4. Vegetation of the cultivated region.

Referring to page 267 we might take take the same series of names I gave there fir the tirst three divisons wheleas the 
fourth divisinn could be replateel by: Eriodendronvegetition intermixed with and partly replaced by ruderalplants.

\section{Vegetation of the Mountaintop and the Greater Part of the Slopes of Saba.}

Ageratum conyzoides, Alsophila phalerata, Amomis caryophyllata, Andropogon glomeratus, Anona muricata, Anthurium rordutum, Aspistium Plumierii, Aspirtium trifoliatum, Asplenium eristatum, Baceharis cotinifolia, Begonia domingensis, Bester ia lutea, Beureria succuleata, Blechmum ocrilentale, Blechum Brownei, Boels. mrice ramiflora, Bromelia pinguin, I3ryophyllum pinnatum, Burserat simaruba, Callisia repens, (apparis Breynia, ('apparis cynophallophora, Catstariat parvifolia. Cecropia preltata, Celtis Ignanicea, Cestrum leurifolium, Chaptalia nutaus, Choococea racemosil, Chloris radiatia, Chrysobalanus jeaco, (Hrysophyllum argenteum, Cissus

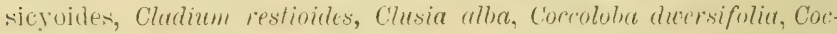
rolobe niven, Commelina virginica, Comoclude ilicifolia, (ondia salvifoliu, Crotalaria retusil, Cyathea arhorea, Cyathea sema, Cyperus distans, Cyperus rotumlus, Laphonosis raribaea, Desmodium axillare, Desmodium supinum, Dichromena rulicans, Dioscorea alata, Dioseoreil trifida, Diplazium striatum, Dryopteris opposital, Dryopteris reticulatil, Duranta repens, Eliphoglosisum conforme, Elaphoglossum latifollum, Epidendrum anceps, Epidentirum ciliare, Epidendrum ulohosum, Eragrostis ciliaris, Eragrostis pilosa, Erechthites valerianafolia, Eryngium foetidum, Erythroxylom haranense, Eugeniu arilluris, Eugeniu proceru, Eupatorium iresinoiles, Eunatorium macrantum, Eupatorium paniculatum, Fagaru martinicensis, Ficus omphallophora, Ficus Urbaniana, Fipziera vendulata, Gissueria venfricose, Gleichenia bifida, Gonzalagunia hirsuti, Guettarda parvi. folia, Heliconia Bihai, Heliotropium indicum, Heliotropium parviflorum, Ifemidiodia ocimifolia, ITemitelia horrida, Hillia longifolia, Hirtella triandra, Hypprtaena domingensis, Hypoxis decumbens, Ichnanthes pallens, Indigofera sufruticosi, Ipomoea fastigiata, Isuchne arundinaceu, Isachne rigens, Lagascea mollis, Lagenaria vulgaris, Leiphaimos aphylli, Lepidium virginieum, Leptochloa virgata, Loch. nera roseil, Lycopolum taxifolium, Marcgravia umbellata, Murismes cuprllaris, Mariseus flavus, Melia Azedarach, Melochia tomentosi,

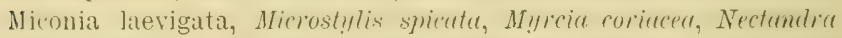
Krugii, Nephrolepis exaltatin, Neurogramme calomelanos, Neuroliena Lohata, oplismenus setarius, ()puntia triacanthi, Ornithidiun cocci-

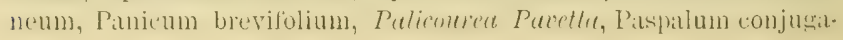




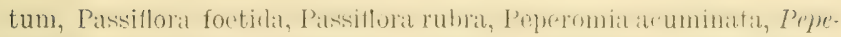

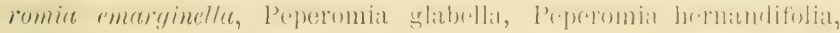

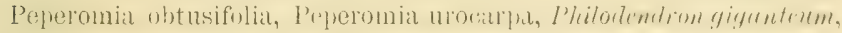

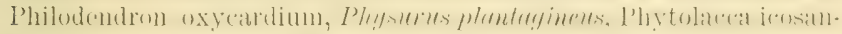

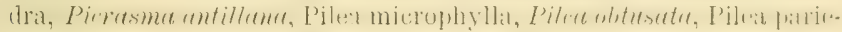

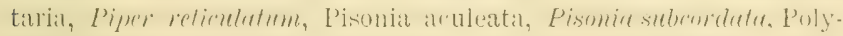

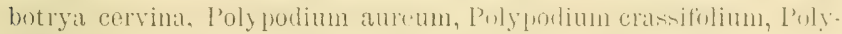

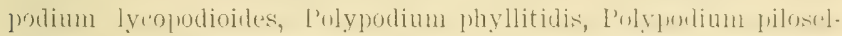

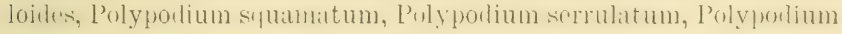
trichomanoides, l'silotum nulum, P'scehotria hurizuntalis, Psyoho-

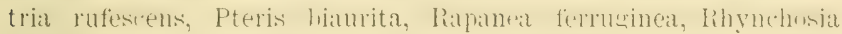

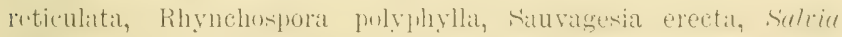

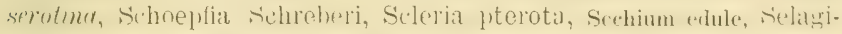
mella flabellata, Selaginella substipitata, Sitaria glancil, Sretaria setosil, Solanum lanceatefolium, suliumum nigrum nodiflorum, Solte-

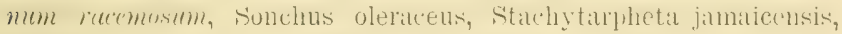

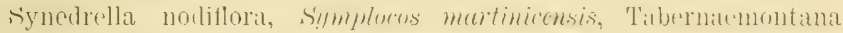
ritrifolia, 'Talinum triangulare, Teenna lenroxylen, T'ibou hina strignal, Tillandsia ereelsa. Torulinium ronfertum, Tourn fortier forctictissimu. Trawia volubilis, Trithommes alatom, Triumfetta semitriloba, Ureni,

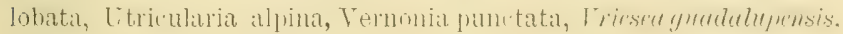
Wedelia buphthalmoides.

\section{Vegetation of Dry Hills of Saba.}

Acacia Farnesiana, Anona muricata, Capparis cynophallophora,

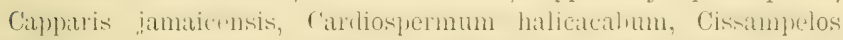

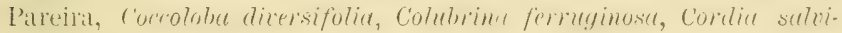
folia, Croton flavens, Ernodea litoralis, Gundlachia domingensis,

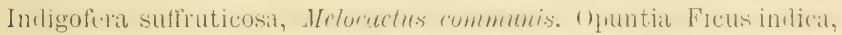
(1)puntia triaccinthat, Opuntia Tuna, Pertis humifusa, P'ismniu suluendeffe, Polyporlium filosellodes, I'sychotrial horizontalis, sisurium portulacastrum, Talinum triangulare, Tecoma leucoxylon, Tragus racemosus.

\section{Vegetation of the Seashore and Rocky Parts of Saba.}

Aristida americana, Bursera simaruba, Calotropis procera, Canavalia olutusifolia, Capraria bithora, Cheilanthe's mierophylia, 'heno.

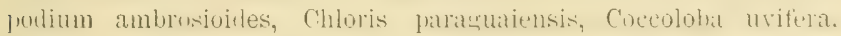

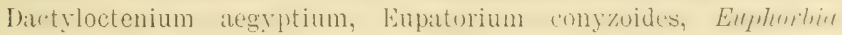

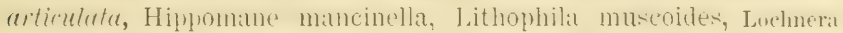

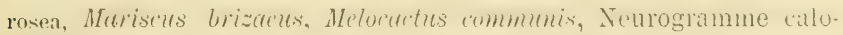
melanos, Opuntia Ficus inslica, Opuntia triacantha, (1)untia Tuna. 
Panicum insulare, Pappophorum alopecuroideum, Pectis febrifuga, Pisonia aculeati, Pisonia subcordutu, L'ortulaca oleracea, Sesuvium portulacastrum, Sida rhomhifolia, Solanum racemosum, Stylosanthes hamata, Tecoma leucoxylon, Turnera ulmifolia.

\section{Vegetation of the Cultivated Region of Saba.}

Abrus precatorius, Acacia Farnesianil, Acalypha chamaedrifolia, Acnistus arborescens, Achyranthes obtusifolia, Adiantum tenerum, Ageratum conyzoides, Alternanthera repens, Alysicarpus nummulirrius, Amilantus paniculatus, Amarantus spinosus, Amarantus tristus, Ambrosic hispicla, Anacardium occidentale, Anona montana, Anona muricata, Anona reticulata, Anona squanosa, Anthephora hermaphruditica, Antigonon leptopus, Argemone mexicuna, Aristida americana, Asclepias nivea, Asplenium pumilum, Beloperone erestachiana, Besleria lutea, Beureriu succulenta, Bidens bippinatus, Bidens leucantha, Bignonia ungius-eati, Bixa Orellana, Blechnum occidentale, Blechum Brownei, Boerhaavea hirsuta, Boerhatvea paniculata, Borreria podocephala, Bouchea Ehrenbergii, Boussingatultia laselloides, Brassicil integrifolia, Bromelia pinguin, Bryophyllum pinnatum, Bumelia obovata, Bursera simaruba, Byr'sonima spicata, Catesalpinia crista, Cajanus indicus, Callisia repens, Capparris Breynia, Capparis cynophallophora, Capparis trondosa, Capparis jamaicensis, Capraria biflora, Cardiospermum halicacalum, Casearia parvifolia, Cassia licapsularis, Cassia glandulosa, Cassia occidentalis, Catopsis nutans, Celtis Iguanacen, Cenchrus tribuloides, Centrosema virginianum, Cestrum laurifolium, Chaptalia nutans, Cheilanthes microphylla, Chenopodium ambrosioides, Chiococea racenosa, Chloris paraguaiensis, Chrysobalanus icaco, Cissus sicyoides, Citharexylum quadrangulare, Clerodendron aculeatum, Chusia alba, Coccoloba diversifolia, Coccoloba nivea, Coccoloba obtusifolia, Coccolulia uvifera, Coleus amboinicus, Commelina virginica, Comoclatia ilicifolia, Cordia salvifolia, Cordia sulcute, Cordia tremula, Crescentia Cujete, Crinum americanum, Crotalaria incana, Crotalitria retusa, Croton flavens, Croton lobatus, Cucumis Anguria, Cuscuta americana, Cyperus rotundus, Dactyloctenium aegyptium, Daphnopsis caribara, Desmanthus virgatus, Dusmodium axillare, Desmodium spirale, Desmodium supinum, Desmodium uncinatum, Dichromena radicans, Drymaria cordata, Dryopteris opposita, Duranta repens, Eleusine indica, Epidendrum anceps, Epidendrum ciliare, Epidendrum papilionaceum, Eragrostis ciliaris, Eragrostis pilosa, Erechthites hieracifolia, Erechthites valerianaefolia, Erigeron canadensis, Erigeron spathulatus, Eryngium 


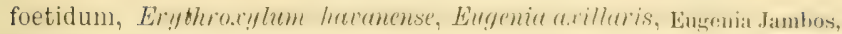
Eugenia moersa, Eugenier mniflora, Eupratorimn conyzuides, Eupattorium iresinoirles, Eupatorium macrophyllum, Euphorbia hy. pericifolia, Euphorlia jilulifera, Euphorbia pulcherrima, Fongure mat-

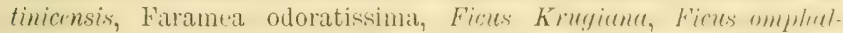
lophora, Ficus populnea, Ficus Erbaniuna, Fleuryal asstuans, Galactia rubra, Gonzalagmnia hirsuta, Gouania domingensis, ciuet. tarla parvifolia, cruettarda seahral, cyymondropsis pentaphylla, Heliotropium parviflorum, Hemidlodia ocimifolia, Iymenaea cumbaril, Hymenorallis caribaer, Hyptis lectinata, Ingar lanerine, Ipomoexa fustigiata, Ipomoeil Nil, Indigofera suffuticosa, Iresine panieulatil. Jacquemontia pentuntha, Jatrophit curas, Jatropha gossypifulia, insticiu sessilis, Kallstroemia maxima, Lilgiscea mollis, Lantillal Cimmara, Lantana involucrata, Leonotis nepetaefolia, I.enurus sibj. ricus, Lepirlium virginicum, Leptochlna rirgita, Linociera com. pacta, Lochnera rosea, Malpighia punicifolia, Malvastrum spicatum, Mammea aumerima, Mariscus brizucus, Murisens capillaris, Melothria fluminensis, Miconia laevigitil, Mitrarmpnes pol!rlundus, Momordinal charantia, Morisonia americana, Mrreia coriacea, Murcin sylentens, Nertandrit coriacea, Nectandra hrugii, Neurogramme calomelanos, ()cimun micranthum, Oncilium slec., Oplismenus setarius, ()xalis coniculata, Palirourea Pacetta, Panicum hrovifoliun, Panicum divaricatum, Panicum insulare, Panicum maximum, Panicum molle, Panicum sanguinale, Palprphorum alopecuroideum, Parthenium Hysterophorus, Paspalum eonjugatum, Paspalum fimliatum, Paspalum hemisphaericum, Passiflora laurifolia, Passiflora rubra, Passiflora suberosa, Paronia spinifex, Pectis humifusa, Pedilunthus tithymaloides, Peperomia glahella, Peperomia petiolaris, Petiveria alliacea, Pharus glaber, Philodendron dispar, Philodendron oxycardium, Phoradendron trinervium, Phyllanthus niruri, Physalis pubescens, Picrasma antillana, I'ilea microphyllat. Pilca semidentata, l'iper dilatatum, Piper reticulatum, Pisonia aculeata, Pisoma subcorduta, Pitcainia angustifolia, Pithecolohium ungius-cati, Plantago major, Pluchea purpurascens, Plumbago standens, Plumier allu, Polanisia viscosa, Polypodium aureum, Polypodium lrasiliense, Polypodium lycopodioides, Polypodium phyllitidis, Polypodıum piloselloides, Polypodium squamatum, Porophyllum ellipticum, Portulaca oleraces. Portulaca pilosa, Priva echinata, Psidium Guajava, Psychotria rufescens, Psychotria uliginosa, Pteris biamita, Rumliu aculcatu, Rauwolfia Lamarckii, Rhacoma crossopetulum, Rhynchosia reti. culati, Rivina humilis, Ruellia tulerosa, Salvia oceidentalis, Sulriu serotina, Situvagesia erecta, Schattieria frutescens. Schoepfia 
Schreberi, Scleria pterota, Scoparia dulcis, Sechium edule, Senecio sonchifolius, Setaria glauca, Setaria setosa, Sida carpinifolia, Sida cordifolia, Sida supina, Sideroxylon foetidissimum, Smilax guianensis, Solanum aculeatissimum, Solanum lanceaefolium, Solanum nigrum nodiflorum, Solanum racemosum igneum, Solanum torvum, Sonchus oleraceus, Spermacoce tenuior, Spigelia Anthelmia, Sporobolus indicus, Stachytarpheta jamaicensis, Stenolobium stins, Sty. losanthes hamata, Synedrella nodiflora, Tabernaemontana citrifolial, Talinum paniculatum, Talinum triangulare, Tamarimdus indica, Tecoma lencoxylon, Tephrosia cathartica, Tetrazygia discolor, Thespesia populnea, Thunbergia alata, Tillandsia fasciculata, Tillandsia recurvata, Tillandsia usneoides, Tillandsia utriculata, Tounefortia foetidissima, Tragia volubilis, Trema Lamarckiamem, Triumfetta semitriloha, Urechites suberecta, Urena lobata, Vernonia punctata, Villamilla octandri, Waltheriil amerianil, Iredelia buphthalmuides, Writhmeliat lingulata, Zebrina pendula.

\section{St. Martin.}

Widely different from the former two Islands the vegetation of St. Martin is chiefly composed of a Crotonvegetation much more developed than on St. Eustatius, whereas the tropical woorls that are to be found on St. Eustatius and Saba are not to be met with on St. Martin.

The greater part of the Island heing cultivated the ruderalfliants are dispersed in almost the whole Island, along the roads and on the plantations and meadows, except in the typical Crotonvegetation which on the outer parts of St. Martin is gradually changing into a well developed Littoralflora.

'This Littoralflora together with the Crotonvegetation commands the whole aspect of St. Martin.

Only where the hills are a little higher the Eriodendronvegetation like that one of the northern part of St. Eustatius is to be found.

The vegetation in the neighbourhood of the Saltponds with species that are not to he seen on the other Islands has also a typical aspect.

According to the results I got in studying the vegetation of st. Martin more minutely I lare say that we can also trace out here the following formations. 
1. Vegetation of the tops of the hills.

2. Vegetation of the level parts of the Island and of tho lower slopes of the hills.

3. Vegetation of the seashore and the rocky parts.

4. Vegetation of the cultivated region.

Referring to page 2157 we could substitute for the first three divisions the sime names I give there, whereals the fourth division is almost synonym with: Crotonvegretition and in some cases also kriodendronvegetation intermixed with, and partly replaces hy ruderal plants.

\section{Vegetation of the Tops of the Hills of St. Martin.}

Abrus precatorius, Acacia macracantha, Acacia riparia, Actinostemon earibatum, Adelie ricinclle, Ageratum conyzoides, Amarantus sinosus, Amomis Ciryophyllata, Anonil muricata, Anona slutamusa, inthurium cordatum, Argithamnie rendicans, Aristolochial trilohata, Asclepias nivea, Asplenium cristatum, Asplenium rumilun, Betuperone cersturhiunu, Bernardia corensis, Bignonia unguis-(ati, Blechnum orcidentale, Brassarolia cucullati, Bryophyllum jinnatum, Bunchosia nitiła, Bursera simaruha, Callisia repens, Canellu alba, Capparis Breynia, Capparis coccolubifolia, Capparis cynophallophora, Capparis jamaicensis, Capraria biflora, Capsium frutescens, Cardiospermum halicacabun, Casearia parvifolia, Casearia syl vestris, Cassia hicipsularis, Catopsis nutans, ('enchrus tribuloides, Chipptalia nutans, Chiococa racemosa, Chloris ciliata, Cissampelos Pareira, Cissus obovutu, Cissus sicyoides, Clusia rosea, Coccoloba nirea. Commelina virginica, Comocludia ilirifolia, Corchures hirsutus, Corchorus silipuosus, Crotalaria incana, Croton betulinus, Cyperus elegans, Dilechampia scandens, Daphnopsis cariban, Desmodium supinum, I) smoulium triflorum, Dorstenia contrajervil, Epidendrum ancels, Epidendrum viliare, Erigh ron spathelutus. Eryngium foetidum, Er?! throxylum hurunense, Ëugeniu frugrane, Eugenia Jambos, Eugeniu monticola, Eisgentu juocera, Eupatorium macrophyllum, Euphorbia Intioluris, Exostemma ranibaem, Fions popmelnea, Ficus Uibeniana, Fleurya aestuans, Guettarda parvifolia, Guettarda seabra, Hemionitis palmata, Heteropteris lanceolata, Hymenocullis caribueu, Inga luerina, Krugiodendron ferrem, Kyllinga brevitolia, Lantana Camara, Lantana involucrata, Leonurus sibirica, Lepirdium virginicum, Leucatnal glatuca, Lonicieral compactia, Mammea imerieana, Mangi. fera indica, Moeftems ellipticus, Miconia lievigatal, Mrreia paniculata, Ifyrciu splendens, Neurogranme calomelamos, Inciclium spec, Oplis- 
menus setarius, Oxalis corniculatus, Ponicum diffusum, Panicum divaricatum, Panicum insulare, Panicum maximum, Panicum prostribtum, Panictum simguinale, P’aspalum conjugatum, Passiflora laurifolia, Passiflora suberosa, Pedilanthus tith!/mulvides, Peperomia acuminata, Peperomia glabelli, Peperomia ol,usifolia, Pharus glaber, l'horitdendron trinervium, Phyllanthus nolilis, Phytolacea icosandra, Picramnia pentandia, Pirrasma antillana, Pilea microphylla, Piper dilatatum, Pisonia aculeati, Pisonia fragrans, Pisonia subcordate, Pithecolobium ungius-cati, I'luchea furpurascens, Polypodium aureum, Polypodium brasiliense, Polypodium lycopodioides, Poly. podium phyllitidis, Polypodium piloselloides, Polypodium stpatmattum, Psidium Guajava, Psychotria horizontalis, Rencliut aruleutu, Lihymchosia reticulata, Rivina humilis, Rochefortia uranthophora, Ruellia tuherosa, Samydu servulatu, Schaefferia frutescens, Senecio sonchifolius, Sida carpinifolia, Sida rhombifulid, Smilax ilicifuliu, Solanum lanceaefolium, Solunum rucemosum, Sonchus oleraceus, Spermacoce tenuior, Spigelia Anthelmia, spiranthes elata, Sporoholus virginicus, Stenolobium stans, Synedrella noditlora, Tabernaemontana citrifolia, Teenmi leuexylon, Tillandsia fasciculata, T'illandsici recurvata, Tillandsia ntriculati, Tournefortia fortidissima, Tour. uefortia hirsutissime, Tralescantia geniculata, Tragia volubilis, Triumfetta rhomboiriea, Vernonia punctata, Wrelik buphlhulmoides, Wittmackia lingulata.

\section{Vegetation of the Level Parts and of the Lower Parts of the Hills of St. Martin.}

Abrus precatorius, Abutilon indicum, Amarantus polygonoides, Amarintus tristis, Ammris elemiforu, Anacitrdium oecidentale, Anonil muricata, Anona squamosa, Aryithemnice candiecens, Brloperone eusterchianc, Beureria succulenta, Boerhatitea paniculati, Bunchosia nitida, Bursera simaruba, Calotropis procera, Calyptranthes Buldinghii, Canella alba, Capparis eynophallophora, Capparis frondosa, Capparis janaicensis, Cardiospermum halicucahum, ('assia bicapsularis, Cassia occidentalis, Cenchrus tribuloides, Centrosema virginianum, Chloris paraguaiensis, Chrysolalamus icaco, Cissus sicyoides, Clusia rosea, Cuccoloba uvifera, Commelina virginica, Comorludiu ilicifoliu, Conocillpus erectus, Crotalaria retusa, Croton astroites, Cioton betulinus, Croton flecens, Cuseuta americitna, Cyperus elegans, (yperus rotun. dus, Desmanthus depressus, Jitaxis fusriculatu, Dodontaea riscusil, Eleusine indicit, Equdendrum pepilionucem, Eragrostis ciliaris, Eratgrostis pilosa, Eragrostis tephrosanthos, Erigeron canadensis, 


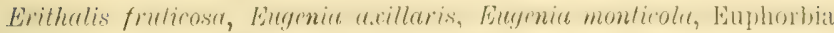
buxifulia, Emphorbin petiolaris, Euphorbia thymifulia, Evolvulus glaber, Evolvulus sericens, Erostemma cariberem, Fagara spinifex,

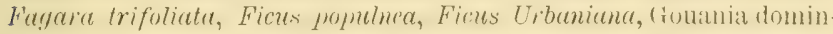
gensis, (ruajacum officinale, Gueltardar parvitolia, Gyminda lati. folia, Heliotropium inlicum, Holiotropium microphyllum, Hymeno. callis coribuea, I pomoed fastigiata, Jacruinia armillaris, Krugioden. dron ferreum, Lantana Camara, I antana involucrata, Lemma paucicostata, Leucaena glanca, Iochnera rosea, Malprighice unemotifoliu, Malpighia punicifolia, Mangifera indica, Melochia nodiflora, Melochia tomentosa, Myreia paniculata, Ponicum diffusum, Panicum nositra. tum, Panicum sanguinale, Paspalum hemisphatericum, Passiflora suberosa, Peetis humifusa, Pedilanthas tithymuloides, Picrommia pentandra, Piscielia piscipula, P'isonia aculeata, Pisonia fragrans, Pisonia subcordatu, Pithecolobium unguis-cati, Plumiera alba, Portulaca halimoides, Portulaca oleracea, Porfulace poliospermu, Portulaca lpuadrifida. I'sidium Guayava, Rendiu aculeate, Rauwolfia Lamarkii, Rharoma crossopetalum, Rhynchosia reticulata, Rohefortia aranthophora, Ruellia tulerosa, Salvia serotima, Samodu sermata, Schatferia frutescuns, sidit carpinitolia, Sida ciliaris, Sida cordifolia, Sida supina, Smilax ilicifolia, Solanm racemorm, Solanum racemosum igneum, Solanum torrum, Śporobolus virginjeus, Stachytarpheta jamaicensis, Stiymatophyllon periplocifolium, Stiymatophyllon serierm, Stylosan. thes hamata, Tamarinlus indica, Tecoma leucoxylon, Ternstroemia peduncularis, Thllandsia recurvata, 'lournefortia rolubilis, Tragus racemosus, Tribulus cistoides, Vernonia punctata, Waltheria americana.

\section{Vegetation of the Seashore and Rocky Parts of St. Martin.}

Acacia Firnesiana, Alternanthera crucis, Anthephnra hermat phroditiea, Aristida americana, Avicennia nitida, Batis naritima, Borrichia arborescens, Bumeliel obocele, Byrsonima crassifolia, Caesalpinice rilutu, C'esalpinire rriste, Canavalia obtnsifolia, Cassia obuvata, Cassiu polyalem, Clerorlendron aculeat un, Coctoloba Krugii, Coccoloba uvitera, Conocarpus erectus, Ernoden littoralis, Euphorbia prostrata, Fimbristylis spathacea, Galartia dubia, Heleocharis capitata, Heliotropium eurassavicum Hippomane maneinella, H!lpelate

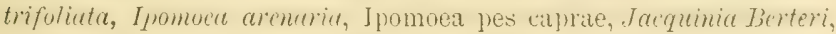
Jumetlus litevigatus. Laguncularia ratemosil, Lithophila muscoiles,

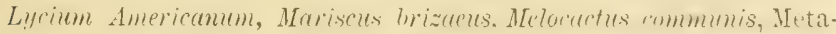
stelma parviflorum, (1)untia Ficus indicil, opuntia triacantha, ()puntia Tuna, Pappophorum ilnpecuroideum, Phullanthes epriphy!- 
lenthes, P'lumbilgo scandens, Rhizophora Ningle, Silicurnia herba-

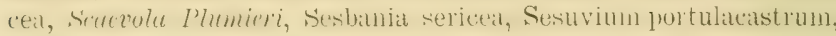
Surianil maritimit, Tephrosia cinereal, Tournefortine gnophulodes, Turnera ulmifolia, Urechites suberecta, Yucca aloifolia.

\section{Vegetation of the Cultivated Region of St. Martin.}

Abrus precatorius, Abutilnn indicum, Acacia macracantha, Acacia riparia, Aculyplea chanuedrifolia, Acalyplue Poiretii, Acanthosprermum humile, Achyranthes asperi, Adelin icinelle, Adiantum tenerum, Alternanthera repens, Alysicapos nummularifolius, Amu-

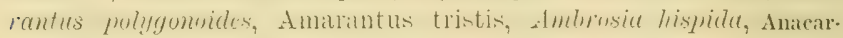
dium occidentale, Anona muricata, Anona squamosa, Antigonon leptopus,

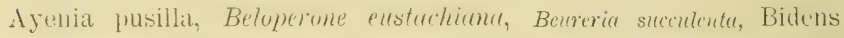
bipinnatus, Blechnum occidentale, Blechum Brownei, Bochlatvea erectal, Boerhatasea hirsuta, Boerhatrea janiculata, Boerhatvea seandens, Bratsiavolat encullata, Bromelia pinguin, Bryophyllum jinnatum, Bursera simaruba, Caesalpinia cortaria, Citesilpinia puleherrima, Callisia repens, Calotrupis proceral, Cappraris Breynia, Capparis coccolobifolia, Cappatis cynophallophorih, Capparis frombosa, Capparis jamaicensis, Capmaria hiflora, Capsieun frutencens, (ardiospermum halicacabum, Casearia parvifolia, C'assia bicapsularis, Cassia ghandulosi, Cassia occidentalis, Catopsis nutans, Ceiba pentandra. Celosia nitidi, Celtis Igumacea, Cinchrus trihuloides, Centrosema virginianum, Chenoporlium ambrosioides, Chenopolium murale, Chio. cocca racemosil, Chloris paralguaiensis, ('hrysobalanus icaco, Cissus sicyoides, Citharexylum qualdrangulare, Corcoloba niren, Coccolola urifera, Coleds amboinicas, Commedinat virginica, Comochedice ileri. folie, Corchores hirsutes, Cracca caribatea, Crotalaria incima, Crotalitria retusa, Crotalinlia relruesosi, Croton ustroites. Croton betulimus, Ciolon flerens, Croton lohatus, Cuscuta americana, Cyperus elegans, ('yperus rotundus, Dactyloctenium aterptium, Datura firtuosia, Daturat Metel, Desmanthus depressus, Desmanthus virgatus, Desmodium spirale, Iesmodium supinum, Dituris fusciculute, Durantat repens, Eleusine indica, Eragrestis aliaris, Eragrostis tephresanthos,

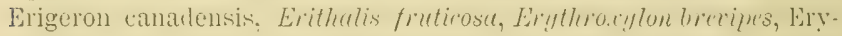
thrina corallodendron, Eugenie axilluris, Eutgenia monticole, Eugeniu procera, Eupatorium conyzoides, Euphorbia huxifolia, Euphorbia heterohylla, Euphorhia hypericifolia, Euphorbia putioluris, Euphorhia pilulifera, Evolvulus glaber, Evolvulus scricens, Erostrmmu curibuenn, Fagara martinicensis, Fagana spinifex, Fugara trifoliatu, Finus popelnee, Galactia rubri, Goumia domingensis, Guettarda parvi- 
folia, (iymindal latifolia, Gymambopsis pentaphylla, lfelinlropium

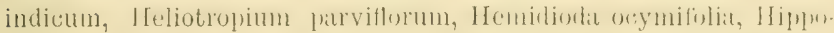

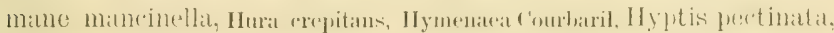

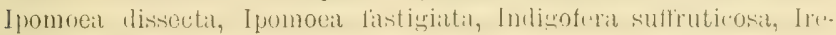

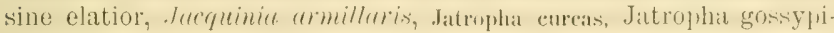

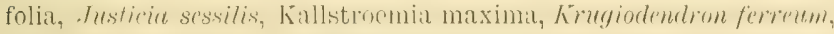
Kyllingia brovifolial, Latarkeca mollis, Lantana ranara, Lantana jnvolucrata, Leonotis nepetiefnlia. Lemants sibiricus, Letpidiun vir. srinicum, Leucaena slaura, Letucas matinicensis, Lippia reptans,

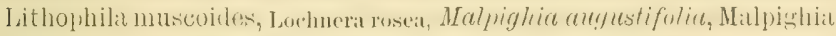
punicifolia, Malvastrum spieatum, Mangifera imliea, Mariscus copille. ris, Melia azedaracl. Melienera hijuga, Melochia tomentosi, Microted de bilis, Moringa oleifera, Horisonia annerieana, Murraya exotica, Neptunia

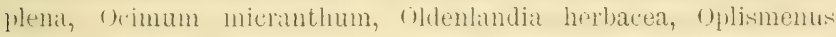
setarius, I'anicum colonmm, P'anicum diffesum, P’anicum geminatum, Panicum insulare, Panicum prostratum, P'anicum sanguinale, Parkinconia aculeata, Parthenium Hysterophorus, l'aspalum hemisphate. ricum, l'avonial spinifex. Peetis humifusu, Pedilunthes tithlymalnides, Peperomia Lambsolffi, Persea americana. Petiveria alliacea, l'hilo. xerus vermirularis, Phylanthus niruri, P’bysalis angulata, Prorm. nia pontuntra, P'iscidia piscipula, P'isonia aculeata, Pisonia fragalls, Pisonia subcordata, P'ithecolobium unguis cati, Plumicra alla, I'lumiena rubra, Polanisia viscosa, Portulaca halimoides, Portulica oleracea, Priva echinata, Psidium fuajava, Psychotria horizontalis, Randir

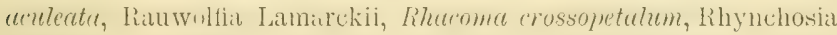
reticulata, Rivina humlis, Ruellia tuberosa, Sulviu serotima, Sam!du sermuluta, schaefferia frutescens, Setaria setosi, , Setaria viridis, Sida carpinifolia, réida ciliaris, Sida cordifolia, Sida strpina, Solanum nigrum nodiflorum, Solanum racemosum igneum, Solanum torvum, spermacoce tenuior, Sporobolus indicus, sporololus virginieus, Stachytarphetal janaicensis, Strnumbum rupestre, Stenolobiumstam, Stifmetophepllon periphlorifolium, Stylosinthes himmata, T'alinum triangulare, Tamarindus indiea, Tecoma lencoxylon, Terammus dabliatis, Thespesia populnea, Thumbergia fragans, Tillandsia recurrata, Tunne. fortia forticissime, Tournetortia volubilis, Tranus racemosus, 'Tri.

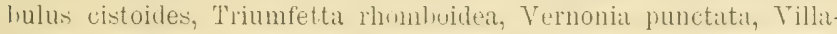
milla octandra, IValtheria americana, W'olelice buththulmoiles. Zizyphus Jujuba. 


\section{CHAPTER II}

\section{DESCRIPTION OF THE DIFFERENT TYPES OF THE VEGETATION.}

\section{Introduction.}

In chanter I of the Third Part I divided the regetation of the Dutch West Indian Islands into three regions: 1. the higher parts, 2. the level plains and the lower part of the slope of the hills, 3. the slope of the hills joining the seashore together with the seashore. Whereas I refer for further letails to the Geographical Part I draw the attention to the fact that on St. Eustatius the three regions are well leveloped. In Silla, however there is almost no level plain to be seen, whereas in St. Martin there is but a very small part of the Island that belongs to the first region.

There is a close connection between the above given data and tho development of the three typical forms of the vegutation.

\section{ERIODENDRONVEGETATION. \\ 2. CROTONVEGETATION. \\ 3. LITTORALVEGETATION.}

The first two names are taken from Egyer's Flora of St. Croix and the Virgin Islands.

\section{ERIODENDRONVEGETATION.}

This type of vegetation is hest characterised by the following trees: Capparis cynophallophora, Capparis frondosa, Capparis jamaicensis, Ardisia coriacea, Rauwoltia Lamarkiana, Linociera compacta, l'isonia fragans, Daphnopsis caribaea, Coccoloba diversifolia, Clusia alba, Ternstroemia peduncularis, Nectandra coriacea, Inga laurina, Picrasma antillana, Ficus populnea, Ficus Urbaniana, Ficus Krugiana, Allophylus occidentalis, whereas Eugenia axillaris, Eugenia cordata, Eugenia ligustrina, Myreia splendens, Palirourea Pavetta, Psychotria horizontalis, Psychotria rufescens, Chiococca 
racemosil, Samyda serrulata, Casearia parvifolia, Byrsonima spicatar, birythroxylon havanense, Vextrmal punetati, are the most predomi. nant shrubs. In very humid localities the soil ss covered by I'e. peromia glathella, Peperomia obtusifolia ambl l'ileat semidentatal.

\section{St. Eustatius.}

The Eriodendrunvegetation is well developer on the higher parti of the Quill and on those parts of the northern hilly country that are shut in between the higher tops, viz the ravines.

Quill. The higher partis of the Quill are coverol ty a forest of ever. green plants and intersperserl heween these evergreens there arr some species with leciduous leaves; all the plants named on pag. gris and 253 as typical for the Erivelendronvegetation atle to be seen here.

The lower parts of this region show some plants that occur also in the Crotonvegetation viz. Acacia, Croton, Lantana, śolanum. Shrubs and smaller trees: Chrysubilanus icaco, Symplocos mattinicomsis, Rauwolfia Lamarckiana, T'ahelnaemontana critrifolia, Lonchoranpus violacea, Casearia parvifolia, are lorming is dense mixture and gradually pass into the forust of the higher tols; there we see a real tropical rainforest with its typical features. The trees that dominate on this top are: Linociera compacta, Nectandra coriacea, Inga laurina, Myrcia paniculata, Myreia splendens, I'sychotria rufescens, Eugenia ligustrina, Micoma laevigati, Byr. sonima spicata, Clusia allu, Ceiba pentandra, Pisnnia fragans, Coccololia diversifolia, Ardisial guadalupensis, Daphnopsis carabaea, Bursera simaruba, Ficus Krugianal, Cestrun laurifolum, Mlophylus occidentalis, Tecona leucoxylon, Celtis Iguanicea, Casearia parvifolia, Persea gratissimil, Ternstroemia pedunculiris, Tabernaemontana citrifolia, Guettarda scalra, Ficus laevigati. On humid localitixs we see the following plants: Peperomia glabella, Pilea remidentata, Anthurium oxycardium. Especially the eastern part of the Tol) of the Quill is rery humid and there Clusial alba, Linociera compacti and Myrtaceae are covered with 'Tillanisia recurvata. The inner slope of the Quill has the same plants, which occur on the outer slope, hut moreover the following plants are to be seen here: Ceiba pentandri, Piper ruticulatum and Piper dilatatum, Ureril Caracasana, Pharus glaber, Panicum divaricatum, Oplismenus setarius, Smilax coriacea, Stenochlaena sorbifolia, cyathea serra, Begonia domingesis, Phoebe elongata, Hyperkaena domin. gensis, Pisonia aculeata, Psychotria uligimosa, Palicourea Pavetta, 
Heliconia Bihai, Asplenium ohtusifolum, Asplenium cristatum, Anlenium serratum, Stenochlaena solbifulia, l'olypodium Phyllitidis, Polypodium Jytopodioides, Dryopteris parasitica, whereas Inehneria raniflora, l'epuromia scantens together with Aractac cover humid rocks. There occur in the Quill also Coffea arabica, Theobroma Cacao, Spondius lutea and Mammea americana.

Northern hilly country. In the northern hilly country the Erio. windronvegetation is to be found in the ravines and other parts which are not exposed to the heavy winds and where a certain amount of humus is covering the rocks.

It may easily be recosnisen here hy the great amount of smaller trees and shrulis mentioned on lage 282 and 283 , whereas the higher trees that are to be seen in the Quill are almost absent. Moreover there is a great quantity of trees, which certainly are not wild here. intermixed with the lower shrubs riz: T'amarindus indica, Nelicueca lijuga, Noringa oleifera, spondias purpurea, Persea americana.

The species that fredominate here in the northern Eriodendron. vegetatiun are: Rliacoma crossopetalum, lugenia ligustrina, Eugenia procera, Samyda serrulata, Eugenia axillaris, Trema Lamallekiana. l'ithecolobum unguis-catis, Celtis Iguanacea, Guettarda parvifolia, Trehaefferia frutescens, Krugiolendron ferreum, Chiococea racemosa, liandia aculeata, Erythroxylon hrevipes, Erythroxylon havanense, l'isonia aculeata, Guettarda seabria, Bumelia ohorata, Anona muri. cata, Anona squamosa, Rauwolfia Lamarckiana.

Here in the northern hilly country no sharp difference can be traced between the Eriodendronvesctation and the Crutonvegetation, nor between the latter and the Vegetation of the rocky slopes of the hills.

Where this Crotonvegetation begins to change into the Lriodendronregetation we see the following plints: Lantanal Camara, Lantana involucrata, Croton flavens, Cruton astroites, Bernarilia corensis, Wedelia buphthalmoides, Melochia tomentosa, lourcroya gigantea, Vernonia punctata, Stylosinthes hamita, Portulaca oleracea, Pani. cum divaricatum, Melocactus communis, Tragus ricemosus, Ruelliat tuherosa, Pectis humifusa, Desmanthus virgatus, sida ciliaris, Justicia sessilis, Commelina virginica, Setaria setosa, Jatropha gossypifolia, Opuntia triacantha, Cordia sulcata, Gouania domingensis, Cissus sicyoides, Rhynchosia reticulata, Stigmatophyllon periplo. cifolium, Tillandsia utriculata, Phoradendron trinervium.

The are very ferw herlaceous plants; only in humid localities some species of Peperomia are to be seen. 


\section{Saba.}

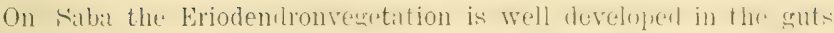
and those parts of the lower slopes of the hills which are not used for cultivation. The plants typical for this vegetation are Enerenia axillaris, Pisonia obtusata, Anoma muricata, stemolobiun Stans, Iantana Camara, T'ournefortia foetidissima, 'hiocncea racesmosid, 'lrema Lamarkiana, Pithecolobium unguiscati, Allophylus occidentalis, Nectandra Krusii. Schoeptia Schreberi, (apharis cynophallophora, Camearia parvifolia, Hyperbacma domingensis, P'sonia aculeati, Celtis Ignanaceal, symplocos martinicencis, (incrolobiat diversifolia, Myreia coriacea, Tibernaemontana citritolia, (occoloba nivea, Capparis frondosa, Chrysobalanus icaco, Inga laurina, Lrythroxylon havimense, Ficrasma antillatil, Daphnopsis caribatal, Amomis caryophyllata, Clusia allat, Miconia laevigata, Ardisia coriacea, Citharexylum quadrangulare, Cestrum Iaurifolum, Tecoma leucoxylon, Psychotria rufescens.

It must however be understood that there are but few parts of Saba where the Eriodendronvegetation has the silme feature as that of the northem hilly country of st. Eustatius. Whereas the shrubs of the latter contain some Myrtaceae, these plants do not occur in Saba in a great quantity; the trees that give a typical aspect to the Quill vegetation viz. Linociera comprati, Nectandrit coriacea, do not occur on siabil except sporadically.

There is however another part of the Eriodendronvegetation in Saba covering the higher tops of the Mointain, where the typical plants of a real tropical rainforest occur.

The greater part of the plants we meet in this part of Silua are not known from sit. Eustatius and st. Martin and chiefly occur in Guadaloupe and other Antilles with high mountains. The feature of this rainforest is given to it by the Treeterns: Hemitelia horrilia, Alsophila phaleratat, (yathea serra, Cyathea arlorea togethe? with kuterpe.

On the most humid localities occur: Psychotria pendula, Hillia parasitica, Maregravia umbellata, Heliconia Bihai, Fiagara martinicensis, Nectandra Krugii, Psychotria rutescens, Besleria luteil. Gesneria ventricosa, Charianthus crinitus, P'eperonia emareinellit, Pepteromia urourya, P'eperomia hernandifolia, Hymenphyllum hirtellum, Trichomanes alatum, Polypulium trichomanoiles, Ltri-

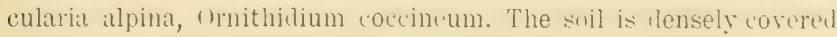
by: Dryolteris palasitical, Dryopteris reticulata, Aspidium I'lumierii, Aspidium trifoliatun, Polybotrya cervina, Neptrolepis exal- 
tata, Diplazium striatum, Blechum occidentale, Stenochlaena sorbifolia, Neurogramme calomclanos, Lonchitis hirsuta, Polypodium brasiliense, Polypodium crassifolium, Polypodium loriceum, Polypodium piloselloides, Elilphoglossum conforme, Elaphoglossum lati. folium, Gleichenia bifida.

\section{St. Martin.}

The well developed Eriodendronvegetation of Saba and St. Eustatius with its typicill plants is not so pronounced in St. Martin. small remnants of an in former times well developed Eriodendron vegetation are to be found on the tops of the Milldrumhili, Mount Paradis, Nakedboyhill, Centryhill and on the higher parts of the road from Filipsburg to Marigot. The following plants make it sure that these parts are covered by the Eriodendronvegetation: Punicum divaricatun, Peperomia obtusifolia, Peperomia glabella, Polypodium aureum, Daphnopsis caribaea, Miconil laevigata, Tabernaemontana citrifolia, Samyda serrulata, Inga laurina.

Moreover there are to be seen the following plants: Guettarda scalira, Myrcia coriacea, Casearia parvifolia, Pisonia fragrans, Peperomia Langsdorffii. Especiallly typical for St. Martin are the many individuals that may be found of Samyda serrulata, Coccoloha nivea, Pisonia fragrans, Comocladia ilicifolia, Schaefferia frutescens and of the following ones unknown from St. Eustatius and Saba: Picramnia pentandra, Bunchosia nitja, Casearia sylvestris, Eugenia monticola, Tournefortia hirsutissima.

Coccoloha diversifolia, Allophylus occidentalis, Ardisia coriacea, Linociera compacta, Cestrum laurifolium do not occur on St. Martin. Where the Crotonvegetation comes in connection with the Eriorlenironvegetation, especially in the northern parts of St. Martin we find another number of plants not known on the other Dutch West Indian Islands viz. Malpighia augustifolia, Bunchosia nitida, whereas there occur also in great quantity, Samyla serrulata, Pisonia subcordata, Capparis frondosa and Capparis janaicensis.

\section{CROTONVEGETATION.}

The Crotonvegetation is characterised by Croton, Acacia, Litntana, Solinum racemosum, Melochia tomentosa, Opuntial triacanthi, Opuntia Tuna, Opuntia Ficus indica and Melocactus.

\section{St. Eustatius.}

The level country and the lower parts of the slopes of the hills 
which atre mot used hy man for ilgricultural purposes, are (1)vered with the Crotonvegutation. Moreover this regetation is to bes seen also in the northern hilly country its well on the slupes of the hills as on the higher localities viz. Boven.

kispecially in the hilly country there is not a great difference hetweren in porly developed Crotonvegetition and that of the rocky slopes. The Crotonvergetation on st. Eustatius has not the same as inet over the whole Island.

Where Croton thavens is predominant and but a few trees like Pisonia subcordata are growing among the lower shrubs, the Crotonvegetation hats a very monotonous aspect and it is not very difticult to walk through it; we find this form of the Crotonvegetation near Boven.

In the lower parts of St. Eustatius the Croton is much more intermixed with other plants such as Acacia tortuosa, Solanum ricemosum and igneum, Pisonia subcordata, Tecuna leucoxylon. Crescentia Cujete, Ceiba pentamira, Lencaena glauca, Plumbago scandens, liandia aculeata, Jatropha gossypifolia, Lochneri rusea, Cissia hicapsularis and other ruderalylants. From the ractafeae there are to he seen (Ipuntia Tuma, Opuntia Ficus Indica, ()puntia triacantha and Melocactus communis.

Moreuver there is a great puantily of Lantana Camara, Lantana involucrata, Waltherial americana, Nelochia tomentusa, Nigmatophylion periplocifolium and T'uurnefortia voluhilis. T'his type op the Crotonvegetation. is to he seen especially near Bengalen and between Oranjestad and Fort de Wind.

still another feature of the crotonvestation is to be mentioner? where Krameria ixina is fredominant; this typical crotonvegetil. tion is only to be seen near Fort de Wind; here the plants are much smaller and the soil is covered bij ruderalplants.

\section{Saba.}

The Crotonvegetation of Salna is not well developed; from the plints typical for this vegetation on st. Eustatius Acacia Firne. siana is not to he seen in Sala, whereas the other plants although they oceur in Saba are nowhere in such quantities as to predo. minate and to form a real Crotonvegetation.

\section{St. Martin.}

The level part of St. Martin as far as it is not usen for agricul. tural purposes and the slopes of the surrounding hills are corered with the Crotonvegetation. 
'l'his Crotonvegetation has another aspect as the one of St. Eustatius, owing to different other plants that are intermixed with the St. Lustatiusplants viz. Croton hetulinus, Euphorbia petiolaris, Plumera allia, Figara sppinifex, Fagara trifoliata, Ditaxis filsciculata, Argithamnia candicans, Arlelia ricinella, Comocladia ilicifolia, Melochia tomentosa, Phyllanthus epyphyllanthus, Coccoloha Krugii, Jacquinia Berteri.

Here also the Crotonvegetation shows some likeness with the Birionlemlronvegetation and especially in the Lowlands and near mrienthay we see the Crotonvegetation gradually intermixed with or replaced by the Eriodendronvegetation; there we find the following plants: Engenia axillaris, (appraris frondosa, Chiococca racemosa, Picrumnia pentandra, Capparis cynophallophora, Pithecolobium ungiuseati, Comocladia ilicifolia, Casearia parvifolia, Samyda serrulatil, Croton flivens, Meluchia tomentosa, Plumiera alla, Acacia Farnesia, Lantani Camari, Lantana involuerata, Argithania candicans, Rauwoltia Lamarkiana, Clerolentron aculeatum, como. cladia ilicifolia, Capparis jumaicensis. Byrsonima lucida, Eugenia axillaris, Myrcia corikea, Calyptranthes Boldinghii, 'I'erntroemia peduncularis, Chrysobalanus leaco, Eugeniil ligustrina, Ficus laevigrata. Clusia alba, Psidium Guajava, Randia aculeata, Pisonia subcorlata, Bursera simaruha, Euphorbia petiolaris, Cassia polyaldena, Paspalum paniculatum, Stigmatophyllon sericeum, Croton hetulinus, Ruellia tuberosa. Cassia occidentalis, Stuhytarpheta jamaicensis, Melocactus communis.

\section{LITTORAL VEGETATION.}

T'he Littoralvegetation may easely be divided into:

\section{Vegetation of the beach.}

2. Vegetation of the rocky slopes near the seashore.

3. Vegetation of the lagoons and the saltponds.

\section{Vegetation of the Beach.}

This vegetation is characterised by:

IIippomane mancinelli, Erithalis fruticosil, Exostemma carilaeum, Suriama maritima, Cimella alha, T'ournefortia gntiaphalodes, realevolil Plumieri, Coccoloba uvifera, Ipomocal feccaprae, Heliotropium curassavicum, Sporobolus virginicus. 


\section{St. Eustatius.}

This vegetation is only to be seen at the different Bays.

The trees which mostly oceur are Iippomane mancinella and Coccoloba uvifera. The smaller plants are Tournefortia snaphalodes, Suriana maritima and Clerodendron aculeatum, Ipomoea pescaprae, Canavalia obtusifolia, Sporobolus viryinicus, IIeliotropium curassavicum and Lithophila muscoides.

On localities where the ruderal plants have also invaded into the seashore we see the following plants: ('aesalpinia ciliata, Jatropha gossypifola, Lantanil Camara, Acacia macracantha, Plumbago scandens and Solanum racemosum igneum. TWhere the seashore does not consist of sand but only of stones the regetation is still less developed and we only find Portulica halimoi. des, Euphorbia buxifolia, Tephrosia cinerea and Borreria podocephala.

\section{Saba.}

The seashore and accorlingly also the Seashorevegetation is almost not developed on siaba; only at Flatpoint there is a small beach and the plants to be found there are: Lithophila muscoi. des, Sesuvium portulacastrum, Stylosanthes hamata and Turnera ulmifolia.

\section{St. Martin.}

Here the beach is very well developed along the southern part of the Island near Grande Case and near Oricntbay; the typical plants to be met with are: Erithalis fruticosa, Exostcrmma caribaoum, Canella alha, Borrichia argentea, Jaçuinia armillaris, Suriana maritima, Chrysobalanus icaco, Conocarpus erectus, Strumpia maritima, Jaçuinia Berteri, Phyllanthus epiphyllanthus, Caesalpinia ciliata, Caesalpinia crista, Tournefortia gnaphaludes, Scatevoli Plumierii, Bumelia obovata, Coccoloba uvifera, Emodea litoralis, Ipomoea pescaprae, Heliotropium curassavicum, Sporoholus virginicus.

\section{Vegetation of the Rocky Slopes near the Seashore.}

I refer to the Geographical and Orological Section where may les seen where these rocks are to be found.

The plants typical for this vegetation are: Coccoloha uvifera, Jacquinia armillaris, Tecoma leucoxylon, Rhacoma crossopetalum, Conocarpus erectus. Erythalis fruticosa. Mrolocactus communis, ['s. 
dilanthus tithymaloides, Dictyloctenium aegyptium, Aristida americana.

\section{St. Eustatius.}

This vegetation is formed here by the following plants: Fedilanthus tithymaloides, Foureroya gigantea, Opuntia spinosissima, Lochnera rosea, Croton flavens, Jacruinia armillaris, Erythalis fruticosa, Melocactus communis, Opuntia triacanthi, Opuntia Tuna, Opuntia Ficus indica, Jacipuinia armillaris, Conocarpus erecti, Acacia macracantha, Tecona leucoxylon, Dactyloctenium aegyptium, Pappophorum alopecuroideum, Euphorhia articulata, Eragrostis ciliaris, Aristida americana, Pectis humusa. Emodea littoralis and Strumpfia maritima are to be seen on the White Wall only.

\section{Saba and St. Martin.}

Whereas the plants occurring on the rocky parts of these Islancls do not differ from those on sit. Eustatius this vegetation needs no further description.

I will only mention that Jacquinia armillaris has not heen seen on Saba. The following summary of the plants found on the small Islands Green Island, Green Key and Tintamarre may give an idea of the vegetation there.

On Green Island I collected the following plants:

Euphorbia articulata, Coccoloba uvifera, Tecoma leucoxylon, Mariscus brizaeus, Opuntia Funa.

On Green Key I collected the following plants: Euphorhia huxifolia, Portulaca halimoides, Lantana involucrata, Croton flavens, Acacia Farnesiana, Beloperone eustachiana, Lithophila muscoides, Tournefortia gnaphalodes, Batis maritima, Hippomane mancinella, Opuntia Ficus indica, Melocactus communis, Coccoloba uvifera. The vegetation is a mixture of Croton- and Littoralvegetation.

The Island of Tintamarre that partly is used for cultivation of Cotton and consists also out of limestone I saw the following plants: Mariscus brizacus, Guajacum officinale, Erolvulus argyraeus, Helio. tropium microphyllum, Borrichia arborescens, Jacquemontia pentantha, Desmanthus depressus, Fagara flava, Panicum prostratum, Beureria succulenta, Tournefortia volulilis, Plumiera alba, Pedilanthes tithymaloides, Bignonia ungius-cati, Hippomane mancinella, liauwolfia Lamarckii, Melochia tomentosa, Croton flavens, Croton betulinus, Amarintus polygonoides, Cenchrus tribuloides, Pappophorum alopecuroideum, Sesuvium portulacastrum, Erithalis fruti- 
cosil, Myreia splendens, lihatoma crossofutalum, stigmatophyllon periplocifolium, Argithamnia candicans, 'L'urnera ulmifolia.

()) this Island ats mat be sexp from the above mentioned species there is a typicial Littoral- and ('rotonversetation which wholly resembles the one on St. Martin.

\section{Vegetation of the Lagoons and the Saltponds.}

This Vegetation is to be seen on St. Martin only; the typical plants are Rhizophora Mangle, Laguncularia racenosa, Aricennia nitida. Accordingly we find here a Mangrovevegetation whereas in the neighbourhood of the Saltponds are to bu seen Batis maritima, Pycreus flavescens, Eleocharis capitata together with other small plants of the beach.

\section{VEGETATION OF THE CULTIVATED REGION.}

This vegetation neels no further explanation as it is of no phy. togengraphical interest; for further details I refer to page 270,274 , 275,280 and 281.

From the data given by Feenstra in his: De Nederlindsche WestIndische eilanden ete. as well as from the data gathered by myself when collecting the plants on the different parts of the is. lands it is evident that in former time a much reater part of St. Eustatius was in cultivation; I refer also to the Ralport of Went; not only the northern hilly country but also the inner part of the Quill shows some plants that certainly were intruduced there by man.

The greater part of the localities of Siba that are apt for culti. vation is used for that purpose whereas on St. Martin the greater part of the level plains and the lower slopes of the hills are in cultivation; the top of Mount Paradis certainly was used in former times for the same purposes.

The well developed mearlows in St. Martin take up a great part of this Island. 


\section{LITERATURE.}

Boldingh $=I$ Boldingh: A Contribution to the Knowledge of the Flora of Anguilla (B. W. I) Rec. d. Trav. Botan. Néerlandais, vol. VI (1909) p. 1-36.

I. Boldingh: Lijst van Planten, die loor de Bewoners van de drie Nederlandsche Antillen St. Eustatius, Saba en St. Martin als geneeskrachtig worden heschouwa. Bull. Kolon. Mus. Haarlem n. 38 (1907) p. 93-112.

Brand $1901=$ A. Brand: Symplocaceae in Engler: Regnum vegetabile IV, 242, 1901.

Christensen $1906=$ C. Christensen: Index Filicum 1906.

cogniaux $1881=A$. Cognianx: Cucurbitaceae in De Candolle Monographiae Phanerogamarum, vol. III 1881 p. 325-954.

Cogniaux $1891=A$. Cogniaux: Melastomaceae in De C'andolle Monographiae Phanerogamarum, vol. VII, 1891.

Duss $=R$. P. Duss: Flore phanérogamique des Antilles françaises (Guadeloupe tet Martinique). Avec annotations du professeur Dr. Edouard Heckel sur l'emploi de ces plantes. [Heck. Ann. de l'inst. colon. de Marseille t'ème année 1896, vol. III]. Macon 1897.

H. F. A. Eggers: Flora of St. Croix and the Virgin Islands, West Indies. (Bull. of the United States National Museum no. 13. Washington 1870,133 p. $8^{0}$ ).

E. a. P. $=1$. Engler und $K$. Prontl: Die naturlichen Pflanzenfamilien, up to April 1909.

Engler $1883=A$. Engler: Burseraceae et Anacardiaceite in the Candolle Monographiae Phanerogamarum, vol. IV, 1883.

Engler $1898=A$. Engler: Araceae in De Candolle Monographiae Phanerogamarum, vol. II, 1889.

B. A. Euphrasen: Beskrifning öfver Svenska restindiska ör St. Birthélémi samt üarne St. Eustache och St. Christopher. Stock. holm 1759.

M. D. Feenstra: De Nederlandsche West-indische eilanden in derzelver tegenwoordigen toestand, Amsterdam 1836-1837.

A. H. R. Grisebach: Systenatische Untersuchungen üher die Vegetation der Karaiben, insbesondere der Insel Guadeloupe. [Ahh. Kgl. Gesellseh. Wiss. Güttingen. VII (1857) p. 151-286]. Gris. Fl. = A. H. R. Grisebach: Flora of the British West Indian 
Islands. London. 1, 1-195: $18 \div 9 ; 1,19: 3-315: 1860 ; \mathrm{p}, 315-506$ : 1861; p. 507-789, Tit. et Index: 1864.

A. H. R. Grisebach: Die geographische Verbreitung der P'llanzen Westindiens, [Abh. Kgl. (resellsch. Wiss, (ivittingen, vol. XII (1865) p. 3-80] - Gesamm. A hhandl. Leipzig 1sso. $8^{\circ}, 1$ ). 2.2. - 285. Hackel $1889=E$. Hackel: Androprogoneae in the Candolle Mfonographiae Phanerogamarum, vol. VI, 1889.

Ifemsley $185 t=W$. Botting Hemslry: Report on the Botany of the Bermudas and various other Islands of the Atlantic and Southern Oceans. The Bermulis. | Rep. (s) the seient. results of the Voy of H. M. S. Challenger. London, $4^{\circ}$. Botany vol. I part I (1884) p. 1-135, tab. I-XIII et introduction (1885) p. 48-49. Hitchc. = Albert S. Hitchoock: List of plants collected in the Bahamas, Jamaica and Grand Cayman. [Fourth annual rep. of the Missouri Bot. Gard. vol. IV (Mart. 1893) p. 47-179, tab. XI-XIV. $8^{\circ}$.

Ind. Kew. = Index Kewensis Plantirum Phanerogamarum by B. D. Jackson 1898-1905.

Nicoluze Joseph. Fucquin: Selectarum stirpium anericanarum historia, in yua all Linnaeanum systenia determinatae descriptaeque sistuntur plantite illae, yuas in insulis Martinica, Jimaica, Domingo alisque et in vicinae Continentis parte ohservavit rariores, ar. jectis iconibus in solo natali delineatis. Vindobonae 1763.

Koelne $1903=E$. Koelme: Lythraceae in Engler: Regnum vegetabile IV, 216, 1903.

Otto Kuntze: Um die Erde. Reiseberichte eines Naturforschers. Leipzig 1881.

Melvill $=J$. Cosmo Melvill: List of the phanerogams of huy West, South Floridi, mostly observed there in March 1872. Memoirs of the Manchester Liter, and I'hilus. Society III ser. vol. VIII p. 138-154, Londen 1884.

Mez $1896=$ C. Mez: Bromeliaceae in the Candolle Monographiae Phanerogamarum, vol. IX, 1896.

Millspaush = Charles Frederick Millspaugh: Flora of the Island of St. Croix. [Field Columbian Museum. Chicago. $8^{\circ}$. Publication 68. Botanical Series. Vol. I. Nr. 7 (Nov. 1902) p. $441-546$ with map of St. Croix.].

Niedenzu $1899=F$. Niedenzu: De genere Malpighia in Ind. lect. in lyceo Hosiano aest. instit. Brunsbergae 1899.

Planchon $1883=J$. E. Planchon: Ampelidae in De Candolle Monographiae Phanerogamarum, vol. V. 1883-1887.

Pulle $=A$. Pulle: An Enumeration of the Vascular Plants known 
from Surinam, together with their Distribution and synonymy. 1906.

Report of the 60 meeting of the Br. Ass. f. Adv. Sc. Leuds 1890 p. 448.

Schumann $1903=K$. Schumann: (resamtbeschreibung ler Kakteen (Monographia Cactacearum) Neudamm 1903.

olof Swartz: Flora Indiae occidentalis aucta atque illustrata sive descriptiones plantarum in Prodromo recensitarum. Erlangae. 1797-1806. 3 vol. $8^{\circ}$ : vol. I. 1797. VIII et 640 p., tab. 1-15; vol. II. 1800 p. $641-1230$, tab. 16-24; vol. III. 1806. p. $1231-$ 2018, ind. X p., tab. 25-29.

Ir. F. R. Suringar: Melocacti novi ex insulis archipelagi indicioucidentalis neerlandicis Curiçao, Arula et Bonaire (Bijdrage tot de kennis der Melocacti. ' $V$ in Versl. d. Natuurk. Afd. Kon. Akad. Wetensch. Amsterdam. Deel VI (1897) p. 178.

symb. $=$ I. Urban: Symbolae Antillanae seu Fundamenta Florie Indiae Occidentalis. Berolini, Parisiïs, Londini $8^{\circ}$.

Vol. I. fasc. I 1898, p. 1-192, fase. I 1899, p. 384-471.

Vol. II. fase. I 1900, p. 1-160, fasc. II 1900, p. 161-336, 1901, p. 344-388, fasc. III 1901. p. 337-508.

Vol. III. fasc. I. 1902. p. 1-160, fasc. II. 1902. p. 161-352, fasc. III. 1903. p. 352-546.

Vol. IV. fasc. I. 1903. p. 1-192, fasc. II. 1905. p. 193-352.

Vol. V. fasc. I. 1904. p. $1-176$, fasc. II. 1907. p. 177-352, fasc. III 1908. p. 353-552.

Vol. VI. fasc. I. 1909.

Urban $1893=\mathrm{I}$. Urbun: Additimenta al cognitionem florae Indiae occidentalis in Engler's Botan. Jahrb. I. in vol. XV (1892) p. $286-361$.

Urban $1895=I$. Urban: Alditamenta ad cognitionem florae Indiae occidentalis in Engler's Botan. Jahrb. II. in vol. XIX (189.4-95) p. $562-681$.

Urban $1896=I$. Urban: Additamenta ad cognitionem florae Indiae occidentalis in Englel's Botan. Jahrb. III. in vol. XXI (1896). p. $514-638$.

Vesque $1893=I$. Vesque. Guttiferae in De Candolle Monographiae Phanerogamarum, vol. VIII. 1893.

F. A. F. C. Went: Rapprort omtrent den toestand van land. en tuinhouw op de Nelderlandsche Antillen, bijlage $V$ vau het Koloniaal verslag van Curaçao van 1902. 


\section{VERNACULAR NAMES.}

Adam's needle $=$ Yucca aloifolia Linn. 36.

Adonis $==$ Duranta repens Linn. 170.

Adonisabbot $=$ Leonotis nepetaefolia R. Br. 172.

Almond tree $=$ 'lerminalia Catappa Linv. 143.

Aloe $=$ Aloo vera Linn. 36 .

Antigua balsam $=$ Rauwolfia Lamarkii DC. 157.

Arrowroot $=$ Maranta arundinacea Linn. 40.

Avocado $=$ Persea americana Mill. 70 .

Bachelor's button $=$ Gomphrena globosa Linn. 58.

Bahama grass $=$ Cynodon dactylon Pers. 22.

Balsam tree = Bursera simaruba Sarg. 103.

Banso = Samyda serrulata Linn. 136.

Basil = . Ocimum basilicum Linn. 175 .

Basket hibisc $=$ Hibiscus schizopetalus Hook. f. 130 .

Basket hoop $=$ Smilax guianensis Vitman. 37.

Bastard black berry $=$ Eugenia procera Poir. 148 .

Bastard bough = Picramnia pentandra Sw. 103.

Bastard cancker berry = Psychotria rufescens H. B. et K. 192 .

Bastard catclauw $=$ Polypodium lycopodioides Linn. 8.

Bastard cherry $=$ Malpighia augustifolia Linn. 105.

Bastard cinnamom $=$ Ardisia coriacea Sw. 151.

Bastard nettle = Acalypha chamaedrifolia Müll-Arg. 110.

Bastard white root $=$ Aegiphyla martinicensis Jacq. 171.

Bayberry = Amomis caryophyllata Kr. et Urban. 144 .

Bellapple = Passiflora laurifolia Linn. 138.

Big yellow popbush $=$ Crotalaria retusa Linn. 87.

Billyache $=$ Rauwolfia Lamarkii DC. 157.

Bitterroot $=$ Cassia occidentalis Linn. 84 .

Birdsberry $=$ Eugenia monticola DC. 147.

= Myrcia paniculata Kr. et Urban. 145 .

Bitterpot $=$ Urechites suberecta Müll.Arg. 157.

Blackberry $=$ Eugenia ligustrina Willd. 147 . 
Blackcherry = Eugenia ligustrina Willd. 147. = Randia aculeata Linn. 188.

Blaci lobloly tree $=$ Pisonia fragrans Dumont. 61 . Black mangeniel $=$ Euphorbia petiolaris Curt. 116. Black sage $=$ Croton betulinus Vahl. 109. Black streetwood = Nectandra Krugii Mez. 71. Black toarch = Melochia tomentosa Linn. 132. Blisterbush $=$ Plumbago scandens Linn. 152. Birdpepper $=$ Capsicum dulce Hort. 176. Blueberry = Symplocos martinicensis Jacq. 153. Blydog = Cassia bicapsularis Linn. 83 . Boulanger $=$ Solanum Melongena Linn. 177.

Boxwood = Murraya exotica Linn. 101. $=$ Schaefferia frutescens Jacq. 121.

Bracelet = Erythroxylum havanense Jaç. 99.

Breadfruit tree $=$ Artocarpus incisa Linn. f. 48 . Bridgo tree = Linociera compacta R. Br. 154 . Bruckroot $=$ Chiococca racemosa Jacq. 190.

Bugal plant $=$ Russelia juncea Zuccar. 180 . Bullgrass = Mariscus brizaeus C. B. Clarke. 27. Burrgrass $=$ Cenchrus tribuloides Linn. 20.

Button wood = Conocarpus erectus Linn. 143.

Calabash $=$ Crescentia Cujete Linn. 183.

Calaloe $=$ Amarantus tristis Linn. 57.

Canckerberry $=$ Solanum racemosum Jacq. 177.

Candle wood = Guettarda scabra Lam. 189.

Cane grass $=$ Panicum divaricatum linn. 16 .

Cannon = Canna indica Linn. 40.

Casha $=$ Acacia Farnesiana Willd. 79.

Cashew = Anacardium occidentale Linn. 118.

Cassave $=$ Manihot utilissima Pohl. 114.

Castoroil plant $=$ Ricinus communis Linn. 112.

Casuarine $=$ Casuarina equisetifolia Linn. 44.

Cat claw = Bignonia unguis-cati Linn. 181.

Cat nip = Salvia serotina Linn. 173.

Centepeeplant $=$ Comocladia ilicifolia Sw. 119.

Charlotten $=$ Allium ascalonicum Linn. 36.

Cherry = Anacardium occidentale Linn. 118.

= Malpighia punicifolia Linn. 106.

Choaky berry = Eugenia axillaris Willd. 146.

Chickenweed = Euphorbia pilulifera Linn. 116.

Children chickenweed $=$ Euphorbia thymifolia Linn. 117. 
Churchblossom $=$ Capparis frondosa Jacq. 75.

Churchyardblossom = Lochnera rosea Reichb. 156.

Cinnamon tree $=$ Amomis caryophyllata $\mathrm{Kr}$. et Urban. 144.

Cockspur $=$ Pisonia aculeata Linn. 61 . = Xylosma buxifolium A. Gray 136 .

Cocoa = Theobroma Cacao Linn. 133.

Cocoaplum $=$ Chrysobalanus icaco Linn. 77.

Coffee $=$ Coffea arabica Linn. 191.

Coralita $=$ Antigonon leptopus Hook. et Arn. 55 .

Coralplant $=$ Jatropha multifida Linn, 113.

Crabgrass $=$ Pappophorum alopecuroideum Vahl. 24.

$=$ Paspalum conjugatum Bergius. 14 .

Crabwood $=$ Pithecolobium unguis-cati Benth. 78 .

Crack open $=$ Casearia parvifolia Willd. 137.

Creole casha $=$ Acacia macracantha Humb. et Bonpl. 79 .

Creole corn $=$ Andropogon sorghum Brot. 13.

Creole cotton $=$ Gossypium barbadense Linn. 130.

Croton $=$ Codiaeum variegatum Bl. 114 .

Curaçao prickle $=$ Nopalea coccinellifera Salm-Dyck. 142 .

Custard apple $=$ Anona reticulata Linn. 69.

Devil's bit $=$ Ruellia tuberosa Linn. 185 .

Different coloured jessamine = Quisqualis indica Linn. 143.

Divi divi $=$ Caesalpinia coriaria Willd. 86.

Dogwood = Piscidia piscipula Sarg. 92.

Dutch casha $=$ Acacia tortuosa Willd. 80 .

Dutch grass $=$ Eleusine indica Gärtn. 23.

Elder $=$ Sambucus canadensis Linn. 195.

Eucharis lily = Eucharis grandiflora Planch. et Linden. 38.

Eye bright $=$ Heliotropium indicum Linn. 166.

$=$ Heliotropium parviflorum Linn. 167.

Fat pork $=$ Chrysobalanus icaco Linn. 77 .

Fire weed $=$ Datura fastuosa Linn. 179.

= Datura Metel Linn. 179.

= Stramonium Linn. 179.

Fishing rod $=$ Randia aculeata Linn. 183.

Flambeau $=$ Erithalis fruticosa Linn. 190.

Flamboyant $=$ Poinciana regia Boy. 85 .

Four o' clock $=$ Mirabilis Jalapa Linn. 60.

Fox tail = Erigeron canadensis Linn. 199.

French casha $=$ Acacia macracantha Humb. et Bonpl. 79 .

Gingerbush $=$ Pavonia spinifex Cav. 129.

Goatbush $=$ Stigmatophyllon periplocifolium A. Juss. 105. 
Gooseberry $=$ Phyllanthus nobilis Müll-Arg. 108.

Granadilla = Passiflora quadrangularis Linn. 139.

Grave physicnut = Jatropha curcas Linn. 113.

Guaveberry = Eugenia floribunda West. 146.

Guave tree = Psidium Guayava Linn, 144.

Gum tree = Bursera simaruba Sarg. 103.

Guineacorn = Andropogon sorghum Brot. 13.

Guineagrass $=$ Panicum maximum Jacq. 17.

Guinea tamarind $=$ Adansonia digitata Linn. 130 .

Haguebush $=$ Clerodendron aculeatum Gris. 171.

Haryplum = Hirtella triandra Sw. 78 .

Hawthorn $=$ Hibiscus radiatus Cav. 129.

Haygrass $=$ Panicum sanguinale Linn. 18.

Hibisc $=$ Hibiscus Rosa-sinensis Linn. 129.

Hog meat $=$ Boerhaavea hirsuta Willd. 60 .

Hollyhock $=$ Byrsonima spicata L. Cl. Rich. 107 .

Hollystalk $=$ Hyptis pectinata Poit. 174 .

Honeyberry $=$ Eugenia uniflora Linn. 148.

Hoofdpijnblad $=$ Operculina ventricosa Peter. 161.

Indian shot = Canna indica Linn, 40.

Ipecacuanha $=$ Asclepias nivea Linn. 158.

Ironberry = Krugiodendron ferreum Urb. 123.

Jeruzalem thorn $=$ Parkinsonia aculeata Linn. 85 .

Joseph's coat = Euphorbia heterophylla Linn. 115.

July tree $=$ Poinciana regia Boj. 85 .

Jumbybeans $=$ Abrus precatorius 93.

Jumby pepper = Rivina humilis Linn. 63 .

Karata = Fourcroya gigantea Vent. 38.

Kinnup tree $=$ Melicocca bijuga Linn. 122.

Klapper = Pitcairnia angustifolia Redouté 32.

Lace plant $=$ Pilea microphylla Liebm. 50 .

Lady of the night $=$ Brunfelsia americana Linn. 180 .

Leaf of live $=$ Bryophyllum pinnatum S. Kurz. 77.

Lemongrass $=$ Andropogon Schoenanthus Linn. 13.

Liberty tree $=$ Calotropis procera $\mathrm{R} . \mathrm{Br} .158$.

Lilac = Melia azedarach Linn. 104.

Lilac bell $=$ Calonyction speciosum Choisy 163 .

Lilac bush $=$ Croton lobatus Linn. 110.

Lilac verbena $=$ Verhena chamaedrifolia Juss. 167.

Lime tree = Bursera simaruba Sarg. 103.

Liquorice plant $=$ Abrus precatorius Linn. 93 .

$=$ Cassia fistula Linn. 84. 
Lizardsgrass $=$ Sporobolus indicus R. Br. 22.

Locust $=$ Hymenaea Courbaril Linn. 82.

Logwood $=$ Haematoxylon campechianum Linn. 85.

Longgras = Panicum insulare G. F. W. Mey 17.

Love wine $=$ Cuscuta americana Linn. 159.

Mahagony $=$ Swietenia mahagoni Jacq. 104.

Maho $=$ Daphnopsis caribaea Gris. 142.

Maidenapple $=$ Momordica Charantia Linn. 195.

Naidenhair $=$ Adiantum tenerum Sw. 7.

Mammie = Mammea americana Linn. 134.

Man better man = Achyranthes obtusifolia Lam. 57.

Man of war bush = Capparis cynophallophora Linn. 74.

Mangeniel tree $=$ Hippomane mancinella Linn. 114.

Mango tree = Mangifera indica Linn. 118.

Man jack $=$ Cordia sulcata DC. 164 .

Mappoo = Pisonia subcordata Sw. 62.

Maria pompoen = Ibatia maritima Dene. 159.

Marrown $=$ Croton flavens Linn. 109.

Mash mellow = Sida cordifolía Linn. 128.

= Waltheria americana Linn. 132.

Massamby = Gynandropsis pentaphylla DC. 73 .

Milky tree $=$ Tabernaemontana critifolia Jacq. 156.

Mastic tree $=$ Sideroxylon foetidissimum Jacı. 152.

Millerwood = Quararibea turbinata Poir. 131.

Miminet $=$ Lawsonia inermis Linn. 142.

Mimosa = Leucana glauca Benth. 80 .

Mispel = Achras sapota Linn. 152.

Monkeytail $=$ Anthurium cordatum G. Don. 30.

Moringo = Moringa oleifera Lam. 76.

Mosquito basil $=$ Ocimum micranthum Willd. 175 .

Mountaingruss $=$ Scleria lithosperma Sw. 29.

Mulegrass = Aristida americana Linn. 21.

Mustard = Brassica integrifolia O. E. Schulz 72 .

Mustard tree $=$ Capparis cynophallophora Linn. 74 .

Myrtle lemon $=$ Triphasia trifoliata P. DC. 101.

Noyeaux $=$ Ipomoea pentaphylla Jacq. 162.

Nutgrass $=$ Cyperus rotundus Linn. 27.

old man's beard = Tillandsia recurvata Linn. 33.

$$
=\text { Tillandsia usneoides. Linn. } 33 .
$$

Oleander $=$ Nerium Oleander Linn. 158.

Orchids purslane $=$ Peperomia glabella A. Dietz. 45 .

Orebian jessamine $=$ Jasminum undulatum Ker. Gawl. $15 t$. 
Papao = Carica Papaya Linn. 139.

Paragrass $=$ Panicum molle Sw. 18 .

Parakiet $=$ Pedilanthus tithymaloides Port. 117.

Pea nut $=$ Arachis hypogaea Linn. 90.

Pea withe $=$ Rhynchosia reticulata P. DC. 97.

Pear tree $=$ Persea americana Mill. 70 .

Pepper cinnamon = Canella alba Murr. 135.

Physicnut $=$ Jatropha gossypifolia Linn. 113.

Picrous bark = Jacquinia armillaris Jacq. 150.

Pigeonberry = Eugenia axillaris Willd. 146.

Pigeonwood = Plumiera alba Linn. 155.

Pingwing $=$ Bromelia pinguin Linn. 31 .

Pita $=$ Fourcroya gigantea Vent. 38.

Plum rose $=$ Eugenia Jambos Linn. 147.

Pomegranate tree $=$ Punica Granatum Linn. 143

Popehead $=$ Melocactus communis Link et Otto 140 .

Poppy $=$ Cosmos sulfureus Cav. 204.

Potatoes = Solanum tuberosum Linn. 179.

Potta potta $=$ Boerhavea hirsuta Willd 60 .

Prickly balsam = Justicia sessilis Jacq. 186.

Pride of Barbados $=$ Caesalpinia pulcherrima Sw. 86.

Prince plum $=$ Amarantus paniculatus Linn. 56.

Pudding withe $=$ Cissus sicyoides Linn. 124.

Pumpkin = Cucumis Anguria Linn. 195.

Purple popbush $=$ Crotalaria verrucosa Linn. 87.

Purple wormhush = Stachytarpheta jamaicensis Vahl. 169.

Quaker blossom = Plumbago capensis Thunb. 151.

Queen of Cribflower = Lagerstroemia indica Linn. 142.

Rabbitmeat $=$ Salvia occidentalis Sw. 173.

Red frangepane = Plumiera rubra Linn. 156.

Red $\operatorname{man}=$ Comocladia ilicifolia Sw. 119.

Red plum $=$ Spondias purpurea Linn. 119.

Red wood $=$ Coccoloba diversifolia Jacq. 53.

= Inga laurina Willd. 78 .

= Myrcia paniculata Kr. et Urban 145 .

Rhamni $=$ Sanseviera guineensis Willd. 36.

Rice plant = Ixora Jucunda Twaithes 191 .

Roucou = Bixa Orellana Linn. 135 .

Runninggrass $=$ Panicum prostratum Lam. 18.

Rumning mountaingrass = Oplismenus setarius R. et Sch. 19. Sage $=$ Lantana Camara Linn. 167. = Lantana involucrata Linn. 168. 
Saltpond tree = Avicennia nitida Jacq. 172.

Sandbox tree = Hura crepitans Linn. 115.

Sapodille $=$ Achras sapota Linn. 152.

Scarlet accordia $=$ Cordia Sebestena Linn. 164.

Sea grape $=$ Coccoloba uvifera Jacq. 54 .

Sea lavendel $=$ Heliotropium curassavicum Linn. 166.

Sea peas = Canavalia obtusifolia P. DC. 96 .

Sea purslane $=$ Tournefortia gnaphalodes R. Br. 165.

Sea wine = Ipomoea pescaprae Roth. 162.

Senna $=$ Tephrosia cathartica Urb. 88.

$=$ Tephrosia cinerea Pers. 88 .

Shame lady shame = Mimosa pudica Linn. 81 .

Shellplant $=$ Costus speciosus Sw. 40.

Silk cotton purslane $=$ Portulaca halimoides Linn. 66 .

Silk cotton tree $=$ Ceiba pentandra Gärtn. 131 .

Sistern pea nut $=$ Alysicarpus nummularifolius P. DC. 92.

Sistern weed $=$ Desmodium triflorum P. DC. 91.

Slingercactus $=$ Cereus triangularis Haworth 140 .

Small yellow pophush $=$ Crotalaria incana Linn. 87 .

Snakeberry tree = Rauwolfia Lamarckii DC. 157.

Snaky $=$ Celtis Iguanacea Sarg. 47.

Snap dragon $=$ Angelonia angustifolia Benth 180 .

Snijboontjes = Phaseolus vulgaris Linn. 97.

Soapbush $=$ Sida carpinifolia Linn. f. 127.

Sour prickle = Opuntia Tuna Mill. 141.

Sour sap = Anona muricata Linn. 68 .

Spanish casha = Acacia macracantha Humb) ot Bonpl. 79.

Spanish dagger $=$ Yucca aloifolia Linn. 36.

Spanish lady $=$ Opuntia triacantha Haworth. 141.

Sprainbush $=$ Chenopodium murale Linn. 55.

Sprainbush wine $=$ Cardiospermum halicacabum. Linn 1:1.

Sprain leaf $=$ Calotropis procera $\mathrm{R} . \mathrm{Br}, 158$.

Star of Bethlehem = Euphorbia pulcherrima Willd. 117.

Stinging nettle $=$ Fleurya aestuans Gaudich. 50 .

Stinging thyme $=$ Coleus amboinicus Lour. 174 .

Stinkwood = Piscidia piscipula Sarg. 92 .

Strawberry $=$ Cereus triangularis Haworth. 140 .

Sugarygrape $=$ Coccoloba nivea Jacq. 53.

Surinaam bitter $=$ Phyllanthus niruri Linn. 108.

Surinaam cherries $=$ Eugenia uniflora Linn. 148.

= Myrcia splendens DC. 145.

Susanna berry $=$ Citharexylum (quadrangulare Jacı. 170. 
Sweet broom = Scoparia dulcis Linn, 181. Sweet gourd = Lagenaria vulgaris Ser. 196 . Sweet potatoes = Ipomoea batatas Poir. 161. Sweet pricklo $=$ Opuntia Ficus indica Mill. 141. Sweetwood $=$ Nectandra coriacea Griseb. 70 . Tamarind $=$ Tamarindus indica Linn. 82.

T'antan = Leucaena glauca Benth. 80 .

'Thistle = Argemone mexicana Linn. 71.

Tomate $=$ Solanum lycopersicum Linn. 177.

Tiger tongue $=$ Sesbania grandiflora Pers. 89. Vervine $=$ Bouchea Ehrenbergii Cham. 169.

Wall purslane = Peperomia petiolaris C. DC. 47 .

Waterthistle $=$ Neptunia plena Benth. 82 .

Watergrass $=$ Commelina virginica Linn. 34 .

White beans $=$ Phaseolus lunatus Linn. 97 .

White cap $=$ Ageratum conyzoides Linn. 197.

White ceder = Tecoma leucoxylon Mart. 182.

White coralita = Porana paniculata Roxb. 160 .

White frangepane $=$ Plumiera alba Linn. 155.

White honeysuckle $=$ Lonicera japonica Thunb. 195.

White hoop $=$ Villamilla octandra Hook. f. 63 .

White lavendel = T'ournefortia gnaphalodes $\mathrm{R} . \mathrm{Br} .165$.

White polly $=$ Amarantus polygonoides Linn. f. 56 .

White root $=$ Gouania domingensis Linn, 123.

White chank $=$ Beureria succulenta Jacq. 164.

White snow $=$ Drymaria cordata Willd. 67 .

White snowdrop = Zephyrantes carinata Herb. 37.

White snowplant $=$ Iresine elatior L. Cl. Rich. 58.

White withe $=$ Smilax coriacea Spr. 37 .

Wild banana $=$ Batis maritima Linn. 40 .

$=$ Heliconia Bihai Linn. 40 .

Wild cane $=$ Panicum divaricatum Linn, 16.

$=$ Piper reticulatum Linn. 45.

Wild cherry $=$ Guettarda parvifolia Sw. 189.

Wild clover $=$ Stylosanthes hamata Taubert. 89.

Wild coffee = Picramnia pentandra Sw. 103.

Wild date $=$ Aleurites moluccana Willd. 113.

Wild guave = Guettarda scabra Lam. 189.

= Samyda serrulata Linn. 136.

Wild indigo $=$ Indigofera suffruticosa Mill. 88.

Wild maho $=$ Triumfetta rhomboidea Jacq. 125.

Wild mammie $=$ Clusia alba Jacq. 135. 
Wild marrow $=$ Croton astroites Dryand. 108.

Wild massamby $=$ Polanisia viscosa DC. 73.

Wild misple $=$ Morisonia americana Linn. 76.

Wild papaw $=$ Cecropia peltata Linn. 50 .

Wild peas $=$ Cassia glandulosa Linn. 84 .

$=$ Desmodium supinum P. DC. 91.

Wild pepper $=$ Solanum nigrum nodiflorum Linn. 177.

Wild pine $=$ Tillandsia utriculata Linn. 33 .

Wild potatowine $=$ Ipomoea fastigiata Sweet 161 .

Wild salad $=$ Sonchus oleraceus Linn. 207.

Wild senna $=$ Krameria ixina Linn. 85.

Wild tantan $=$ Desmanthus virgatus Willd. 81 .

Wild tea $=$ Capraria biflora Linn. 180.

Wild tobacco $=$ Vernonia punctata Sw. 196.

Wonder tree $=$ Parkinsonia aculeata Linn. 85.

Wormbush $=$ Chenopodium ambrosioides Linn. 55.

Yellow balsem $=$ Croton flavens Linn. 109.

Yellow bell $=$ Allamanda cathartica Linn. 155.

Yellow blossom = Stenolobium stans Seem. 182.

Yellow plum $=$ Spondias lutea Linn. 119.

Yellow prickle $=$ Fagara martinicensis P. DC. 100.

Yellow sage $=$ Wedelia buphthalmoides Gris. 202.

Yellow saunder $=$ Fagara flava $\mathrm{Kr}$. et Urb. 100. 


\section{INDEX TO THE FIRST PART.}

*Abelmoschus moschatus Médik. 130.

Abrus precatorius Linn. 93.

Abutilon crispum Sweet. 126. Abutilon indicum Sweet. 126. Acacia Farnesiana Willd. 79. Acacia macracantha Humb. et Bonpl. 79.

Acacia riparia H. B. K. 80. Acacia sarmentosa Gris. 80. Acacia tortuosa Willd. 80. Acalypha chamaedrifolia MüllArg. 111.

Acalypha Poiretii Spreng. 111. Acalypha reptans Siv. 111.

*Acalypha Wilkesiana Müll-Arg. 112.

Acanthaceae 184.

Acanthospermum humile DC. 201.

Acnistus arborescens Schlecht. 175.

*Achras sapota Linn. 152.

Achyranthes aspera var, obtusifolia Gris. 57.

Achyranthes obtusifolia Lam. 57.

Acrostichum conforme Sio. 10. Acrostichum latifolium Sw. 10. Acrostichum viscosum Vent. 10. Actinostemon caribaeum Gris.

114.
*Adansonia digitata Linn. 130. Adelia pedunculosa A. Rich. 111. Adelia ricinella Linn. 111.

"Adenanthera pavonina Linn. 82. Adiantum tenerum Sw. 7. Adicea microphylla 0. Ktze. 51. Aechmea lingulata Baker. 32. Aegiphila martinicensis Jacq. 171.

Ageratum conyzoides Linn. 197. Aizoaceae 64.

*Albizzia lebbek Benth. 79.

*Aleurites moluccana Benth. 113.

-Allamanda cathartioa Linn. 155. *Allium ascalonicum Linn. 36. Allophylus occidentalis Radek. 121.

Aloe vera Linn. 36. Aloe vulgaris Lam. 36. Alternanthera achyrantica $\mathrm{R} . \mathrm{Br}$. 58.

Alternanthera crucis. Comb. nov. 58.

Alternanthera muscoides Bent. et Hook. 59.

Alternanthera repens 0. Ktze. 58. Alsophila phalerata Mart. 2. *Althaea officinalis Linn. 126. Alysicarpus nummularifolius $\mathrm{P}$. DC. 92. Alysicarpus vaginalis P.DC.92. Amarantaceae 56. 
Amarantus caudatus Linn. 56. Amarantus gangeticus Linn. 57. Amarantus gracilis Desf. 56.

*Amarantus paniculatus Linn. 56. Amarantus polygonoides Linn. 56 .

Amarantus spinosus Linn. 56. Amarantus tristis Linn. 57.

Amaryllidaceae 37.

Amaryllis carinata Spreng. 37. Amaryllis equestris Ait. 38. Amblogyne polygonoides Raf. 56. Ambrosia crithmifolia DC. 201. *Ambrosia hispida Purch 201. Amomis caryophyllata $\mathrm{Kr}$. et Urb. 144.

Amyris elemifera Linn. 101. Anacardiaceae 118.

*Anacardium occidentale Linn. 118.

Anamonis fragrans Gris. 146. Anamonis punctata Gris. 146.

"Ananas sativus Schult. 32. Anatherum bicorne Beauv. 13. Anatherum macrouram Gris. 13 Ancliva inermis H. B. K. 93. Andira jamaicensis Urb. 93. Andropogon bicornis Linn. 13. Andropogon contortus Linn. 13. Andropogon glomeratus Britton. 13.

Andropogon macrouros Michx. 13. "Andropogon Schoenanthus Linn. 13.

Andropogon secundus Willd. 13. *Andropogon sorghum Brot. 13. Andropogon squarrosus Linn. $f$. 13.

Andropogon zizanoides Urb. 13. *Angelonia angustifolia Benth. 180.

Anonacoar 68 .
Anona inontana Macf. 68. *Anona muricata Linn. 68.

*Anona reticulata Linn. 69.

*Anona squamosa Linn. 69. Anthephora elegans Schreb. 13. Anthephora hermaphroditica $\mathrm{O}$.

Ktze. 13.

Anthurium cordatum G. Don. 30. *Antigonon cinerascens Mart, et Gal. 55.

*Antigonon leptopus Hook. et Arn. 55.

Apocynaceae 155.

Araceae 30.

*Araclis hypogaea Linn. 90. Ardisia coriacea Sw. 151.

Ardisia guadalupensis Duchass. 151.

Árgemone mexicana Linn. 71. Argithamnia candicans Sw. 110. Aristida americana Linn. 21.

\section{Aristolochiaceae 53.}

*Aristolochia odoratissima Linn. 53.

Aristolochia trilobata Linn. $5: 3$. Artanthe Bredemeyeri Gris. 44 . Artocarpus communis Forst. $t$. Artocarpus incisa Linn. fil. 4 s. Asclepiadaceae 158.

Asclepias Curassavica Limn. Asclepias nivea Linn. 158. Aspidium conterminum Willd. 2. Aspidium exaltatum Sto. 3. Aspidium molle Sio. 2. Aspidium Plumierii Presl. 3. Aspidium trifoliatum Sw. 3. Asplenium auritum Sw. 4. Asplenium bipartitum Spr. 4. Asplenium cristatum Lam. 4. Asplenium monanthes Linn. 4. Asplenium obtusifolium Linn. 4. Asplenium pumilum Sw. 5. 
Asplenium rhizophorim Linn. cicutarium Sw. 4.

Asplenium serratum Linn. 5. Asplenium striatum Presl. 4. Avicennia nitida Jacq. 17\%. Ayenia pusilla Linn. 132. Baccharis cotinifolia Urb. 199. Baccharis dioica Gris. 199.

\section{Balsaminaceae 128.}

Basellaceae 67.

Batidaceae 47.

Batis maritima Linn. 47.

"Bauhinia Kappleri Sagot. 83.

\section{Begoniaceae 139.}

Begonia domingensis DC. 139.

*Belamcanda punctata Moench.39. Beloperone eustachiana Benth. 186.

Benthamantha caribaea Benth. 89. Bernardia corensis Müll-Arg.111. Besleria lutea Linn. 183.

Beureria succulenta Jacq. 164. Bidens bipinnatus Linn. 203. Bidens leucantha Willd. 204. Bidens pilosa Linn. 204.

Bignoniaceae 181.

"Bignonia Unguis-cati Linn. 181. Bignonia spec. 182.

\section{Bixaceae 135.}

*Bixa Orellana Linn. 135. Blechnum occidertale Linn. 5. Blechum Blechum Millsp. 185. Blechum Brownei Juss. 185. Boehmeria ramiflora Jacq. 52. Boerhavea erecta Linn. 60. Boerhaavea hirsuta Willd. 60. Boerhaavea paniculata L. Cl. Rich. 60.

Boerhaavea repens Linn. 60. Boerhavea scandens Linn. 61. Bombacacear 130.

"Bontia daphnoides Linn. 187.
Borraginaceae 164.

Borreria ocymoides DC. 194. Borreria podocephala DC. 194. Borrichia arborescens DC. 202. Bouchea Ehrenbergii Cham. 169. *Boussingaultia baselloides H. B. K. 67.

Bouteloua juncifolia Lag. 21. Bouteloua litigosa Lag. 21. Brachyrrhamphus intybaceus DC. 207.

Bradburya virginianum 0. Ktze. 94.

Brassavola cucullata R. Br. 43 . Brassavola rigida comb. nov. 43. Brassica integrifolia O. E. Schulz. 72.

\section{Bromeliaceae 31.}

*Bromelia pinguin Linn. 31.

* Brunfelsia americana Linn. 180. Bryophyllum calycinum Salisb. 77.

Bryophyllum pinnatum S. Kurz. 77.

Bumelia cuneata Siv. 153.

Bumelia obovata A. DC. 153.

Bunchosia jamaicensis Urb. et Niedenz. 106.

Bunchosia nitida L. Cl. Rich. 106. Burseraceae 103.

Bursera gummifora Jacq. 103. Bursera simaruba Sarg. 103. Byrsonima crassifolia H. B. K. 107.

Byrsonima lucida L. Cl. Rich. 107.

Byrsonima martinicensis $K r$. et Urb. 107.

Byrsonima spicata L. Cl. Rich. 107.

Cactaceae 140.

Caesalpinia ciliata Urb. 86. 
Caesalpinia coriaria Willd. 86. Caesalpinia crista Linn. 86.

"Caesalpinia pulcherrima Sw. 86. Cajames cajan Millsp. 96.

*Cajanus indicus Spreng. 96.

Cakile aequalis L'Herit. 72.

Cakile lanceolata O. E. Schulz. 72.

Callisia repens Linn. 35.

Calotropis procera R. Br, 158.

Calonyction speciosum Choisy. 163.

Calonyction tuba Colla. 163.

Calyptranthes Boldinghii Urb. 146.

Canavalia obtusifolia P. DC. 96. Canavalia spec. 96.

Canellaceae 135.

Canella alba Murr. 135.

Cannaceae 40.

Canna indica Linn. 40.

Capparidaceae 73.

Capparis amygdalina Lam. 73. Capparis Breynia Jacq. 73.

Capparis coccolobifolia Mart. 74. Capparis cynophallophora Linn. 74.

Capparis cynophallophora var. latifolia Gris. 74.

Capparis frondosa Jacq. 75.

Capparis jamaicensis Jacq. 75. Capraria biflora Linn. 180.

Caprifoliaceae 195.

*Capsicum ceratocarpum Fing. 176.

*Capsicum dulce Hort. 176.

"Capsicum frutescens Linn. 176. Cardiospermum halicacabum Linn. 121.

Caricaceae 139.

*Carica Papaya Linn. 139.

Caryophyllaceae 67.

Casearia parvifolia Willd. 137.
Casearia sylvestris Sw. 137.

"Cassia alata Linn. 83.

Cassia bicapsularis Linn. 83.

"Cassia fistula Linn. 84.

Cassia glandulosa Linn. 84.

Cassia obovata Collad. 84.

Cassia occidentalis Linn. 84.

Cassia polyadena DC. 85.

Casuarinaceae 44.

${ }^{*}$ Casuarina equisetifolia Linn. 44.

Catopsis nutans Griseb. 34.

Cecropia peltata Linn. 50.

${ }^{*}$ Ceiba pentandra Gärtn. 131.

Celastraceae 119.

*Celosia argentea Linn. 56.

Celosia nitida Vahl. 56.

Celtis aculeata Sw. 47.

Celtis Iguanacea Sarg. 47.

Cenchrus tribuloides Linn. 20.

Centrosema virginianum Benth. 94.

Cereus compressus Mill. 140.

Cereus grandiflorus Mill. 140.

Cereus Peruvianus Mill. 140.

Cereus triangularis Haw. 140.

Cereus spec. 140.

Cestrum laurifolium L'Hér. 179.

Chaetochloa glauca Linn. 19.

Chaetochloa setosa Sw. 20.

Chaetogastra chironioides Gris. 148.

Chaetogastra strigasa Cogn. 148.

Chamaecrista glandulosa Linn. 84.

Chaptalia nutans Hemsl. 206.

Charianthus crinitus Naud. 148.

Charianthus nodosus crinitus

Naud. 149.

Cheilanthes microphylla Sw. 6.

Chenopodiaceae 55.

Chenopodium ambrosioides Linn. 55. 
Chenopodium murale Linn. 55. Chiococca racemosa Jace. 190. Chloris ciliata Sw. 23.

Chloris paraguaiensis Steud. 23. Chloris radiata Sw. 23.

Chrysobalanus icaco Linn. 77.

Chrysophyllum argenteum Jacq. 153.

Chrysophyllum glabrum Jacq. 153

Cicca antillana A. Juss. 108.

Cicca disticha Linn. 107.

Cissampelos Pareira Linn. 68.

Cissus obovata Vahl. 124.

Cissus sicyoides Linn. 124.

Citharexylum quadrangulare Jacq. 170.

"Citrus limonum Risso. 102.

Cladium restioides Benth. 29.

Cleome graveolens Rafin. 73.

Cleome pentaphylla Linn. 73.

Cleome viscosa Linn. 73.

Clerodendron aculeatum Gris. 171.

Clerodendron fallax Lindl. 171. Clerodendron fragans Willd.171. Clerodendron Thomsonae Balf. 171.

Clibadium erosum DC. 201.

*Clitoria ternatea Linn. 93.

Clusia alba Jacq. 135.

Clusia rosea Jacq. 135.

Clusia spec. 135.

Coccoloba barbadensis Jacq. 53. Coccoloba diversifolia Gris. 53. Coccoloba diversifolia Jacq. 53. Coccoloba excoriata Linn. 53. Coccoloba Krugii Lindau. 53. Coccoloba nivea Jacq. 53. Coccoloba obtusifolia Jacq. 54. Coccoloba punctata Gris. 53, 54 . Coccoloba punctata var. microstachya Gris. 54.
Coccoloba uvifera Jacq. 54. Cocculus domingensis DC. 68. ${ }^{*}$ Cocos nucifora Linn. 39.

${ }^{*}$ Codiaeum variegatum Bl. 114. Coffea arabica Linn. 191.

Coleus amboinicus Lour. 174.

Coleus aromaticus Benth. 174. *Coleus scutellarioides Benth. 174.

*Colocasia esculenta Schott. 31. Colubrina ferruginosa Brong. 123.

Combretaceae 143. Commelinaceae 34 .

Commelina elegans H. B. K. 34 . Commelina virginica Linn. 34. Comocladia ilicifolia Sw. 119.

Compositae 196.

Condalia ferrea Gris. 123.

Conocarpus erectus Linn. 143.

Convolvulaceae 159.

Convolvulus pentanthus Jacq. 161.

Corchorus acutangulus Lam. 125.

Corchorus hirsutus Linn. 125.

Corchorus olitorius Linn. 125.

Corchorus siliquosus Linn. 125.

Cordia salvifolia Juss. 164.

*Cordia Sebestena Linn. 164.

Cordia sulcata DC. 164.

Cordia tremula Gris. 164.

*Cosmos sulphureus Cav. 204.

${ }^{*}$ Costus speciosus Sm. 40.

Cracca caribaea Benth. 89.

Crassulaceae 77.

Crescentia Cujete Linn. 183.

*Crinum americanum Linn. 37.

Crotalaria incana Linn. 87.

Crotalaria retusa Linn. 87.

Crotalaria verrucosa Linn. 87.

Croton astroites Dryand. 108.

Croton balsamifer Jacq. 109.

Croton betulinus Vahl, 109.

Croton flavens Linn. 109. 
Croton lobatus Linn. 110.

Cruciferae 72.

*Cryptostegia grandiflora R. Br. 158.

Cucumis Anguria Linn. 195.

Cucurbitaceae 195.

*Cucurbita maxima Duchesne. 196.

Cuscuta americana Linn. 159. Cuscuta umbellata H. B. et K. 160.

\section{Cyatheaceae 1.}

Cyathea arborea J. E. Sm. 1. Cyathea serra Willd. 1.

Cymodocea manatorum Aschers. 12.

Cynodon dactylon Pers. 22.

\section{Cyperaceae 26.}

Cyperus bruneus Sw. 27.

Cyperus capillaris Sw. 27.

Cyperus confertus Gris. 26.

Cyperus distans Linn. fil. 26.

Cyperus elegans Linn. 26.

Cyperus ferax L. C. Rich. 28.

Cyperus flavus Nees. 27.

Cipperus flavomariscus Gris. 28.

Cyperus mucronatus Rottb. 26.

Cyperus purpureo-variegatus Boeck. 27.

Cyperus rotundus Linn. 27.

Cyperus viscosus Sio. 26.

Dactyloctenium aegyptium Willd. 24.

Dalechampia scandens Linn. 112. Daphnopsis caribaea Gris. 142. Datura fastuosa Linn. 179.

Datura Metel Linn. 179.

Datura Stramonium Linn. 179. Desmanthus depressus Humb. et Bonpl. 81.

Desmanthus virgatus Willd. 81 . Desmodium axillare P. DC. 90.
Desmodium incanum P. DC. 91 . Desmodium scorpiurus Desv. 90. Desmodium spirale P. DC. 91. Desmodium supinum P. DC. 91. Desmodium tortuosum P. DC.91. Desmodium triflorum P. DC. 91. Desmodium uncinatum P. DC. 92. Dianthere sessilis Gris. 186.

Dichromena radicans Schlecht. 28.

Dicotyledoneae 44.

Digitaria marginata $L k .18$.

Digitaria setigera Roth. 18.

Diodia rigida Cham. 193.

Dioscoreaceae 39.

*Dioscorea alata Linn. 39.

Dioscorea trifida Linn. 39.

Dipholis salicifolia A. DC, 153.

Diplazium semihastatum C. Christensen. 4.

Diplazium striatum Presl.

Distreptus spicatus Cass. 197.

Ditaxis fasciculata Vahl. 110.

Dodonae viscosa Linn. 122.

Dolicholus minimus Medic. 96.

Dolicholus reticulutus Millsp. 97.

Dolicholus lablab Linn. 98.

Dorstemia contrajerva Linn. 48. Drymaria cordata Willd. 67.

Dryopteris opposita Urb. 2.

Dryopteris parasitica 0 . Ktze. 2. Dryopteris reticulta Urb. 3. Duranta Plumieri Jacq. 170. Duranta repens Linn. 170. Echites suberecta Jacq. 157. Eclipta alba Hassk. 202. Eclipta erecta Linn. 202. Elretia Bourreria Linn. 164. Elaphoglossum conforme Schott. 10.

Elaphoglossum latifolium J. Sm. 10. 
Elaphoglossum petiolatum Urb. 10.

Elephantopus spicatus Aubl. 197.

Elersine aegyptiaca Desf. "24.

Eleusine indica Gärtn. 23.

*Eleutherine plicata Herb. 39. Emilia sonchifolia DC. 206. Enckea Sieberi Gris. 44.

Enckea smilacifolium C. DC. 45. *Enterolobium Timbouwa Mart. 78.

Epidendrum anceps Jacq. 41. Epidendrum ciliare Linn. 42. Epidendrum difforme Jacq. 42. Epidendrum fuscatum Sm. 41. Epidendrum globosum Jacq. 42. Epidendrum papilionaceum Vahl 42.

Epidendrum strobuliferum Reichb. f. 43.

Epidendrum umbellatum Sio.42. Eragrostis ciliaris Lk, 25. Eragrostis pilosa Beauv. 25. Eragrostis poaeoides Gris. 25.

Eragrostis tephrosanthos Schult. 25.

Erechthites hieracifolia Raf. 205. Erechthites praealta Raf. 205. Erechthites valerianaefolia DC. 206.

Erigeron bonariensis Linn. 199. Erigeron canadensis Linn. 199. Erigeron spathulatus Vahl 199. Eriodendron anfractuosum $D C$. 131.

Erithalis fruticosa Linn. 190. Ernodea litoralis Sw. 193.

Eryngium foetidum Linn. 150. Erythrina corallodendron Linn. 94.

Erythroxylaceae 99.
Erythroxylum brevipes P. DC. 99.

Erythroxylum havanonse Jaç. 99.

Erythroxylum obtusum DC. 99. * Eucharis granditlora Planch. 38. Eugenia axillaris Willd. 146. Eugenia buxifolia Gris. 147. Eugenia cordata var Sintensii Kr. et Urb. 146.

Eugenia floribunda West. 146. Eugenia fragrans Willd 146. Eugenia Jambos Linn. 147. Eugenia ligustrina Willd. 147. Eugenia monticola DC. 147. Eugenia obtusata Willd. 147. Eugenia pallens Gris. 147. Eugenia Poiretii Berg. 147. Eugenia procera Poir. 148. Eugenia rhombea Kr. et Urb. 148. Eugenia sessiliflora DC. 146. Eugenia uniflora Linn. 148. Eupatorium canescens Vahl 197. Eupatorium conyzoides Vahl 198. Eupatorium guadalupense

Spreng. 198.

Eupatorium iresinoides H. B. et K. 198.

Eupatorium macranthum Sw. 198.

Eupatorium macrophyllum Linn. 198.

Ėupatorium paniculatum Schrad. 198.

Eupatorium sinuatum Lam. 197. Euphorbiaceae 107.

Euphorbia articulata Burm. 115. Euphorbia buxifolia Lam. 115. Euphorbia cyathophora Murr. 115.

Euphorbia heterophylla Linn. 116. 
Euphorbia hypericifolia Linn. 116.

Euphorbia linearis Retz. 115.

Euphorbia petiolaris Curt. 116. Euphorbia pilulifera Linn. 116. Luphorbia prostrata Ait. 117. Euphorbia pulcherrima Willd. 117.

Euphorbia thymifolia Linn. 117. Euphorbia verticillata Poir. 116. Euterpe spec. 30.

Euxolus caudatus Moq. 56.

Evolvulus argyraeus Choisy 160.

Evolvulus glaber Spreng. 160.

Evolvulus incanis Pers. 160.

Evolvulus mucronatus Sio. 160.

Evolvuius sericeus Sw. 160.

Fxcoccaria caribaea Gris. 114.

Excoecaria hucida Sw. 114.

Exostemma caribaeum Roem.

et Schult. 188.

Exostemma sp. 188.

Fagara flava Kr. et Urb. 100.

Fagara martinicensis Lam. 100.

Fagara microphylla Dess. 100.

Fagara spinifex Jacq. 100.

Fagara trifoliata Sw, 101.

Faramea odoratissima DC. 193.

Festuca Myurus Linn. 26.

Ficus Krugiana Warb. 49.

Ficus laurifolia Duss. 49.

Ficus laevigata Vahl. 49.

Ficus omphallophora Warb. 49.

Ficus pallida Gris. 49.

Ficus pertusa Gris. 49.

Ficus populnea Willd. 49.

Ficus Urbaniana Warh. 49.

Fimbristylis spathacea Roth. 28.

Flacourtiaceae 136.

Fleurya aestuans Gaudich. 50.

*Foeniculum vulgare Mill. 150.
Forestiera Eggersiana $\mathrm{Kr}$. et Urh. 154.

Fourcroya gigantea Vont. 38. Freziera undulata Willd. 124. *Gaillardia aristata Purch. 204. Galactic angustifolia Griseb. 95. Galactia dubia P. DC. 95. Galactia filiformis Griseb. 95. Gulactia longiflora Griseb. 95. Galactia longifolia Benth. 95. Galactia nummularia Urb. 95. Galactia rubra Urb. 95.

Galactia tenuiflora Wight. et Arn. 95.

*Galphimia gracilis Bartl. 105.

Gentianaceae 155.

Gesneriaceae 183.

Gesneria ventricosa Sw. 184.

Gleicheriaceae 11.

Gleichenia bifida Spreng. 11.

Gnaphalium americanum Mill. 200.

Gnaphalium purpureum Linn. 200.

*Gomphrena globosa Linn. 58.

Gonzalagunia hirsuta Schumann 188.

Gonzalea spicata DC. 188.

Goodeniaceae 196.

*Gossypium barbadense Linn. 130. Gouania domingensis Linn. 123.

Gramineae 12.

*Graptophyllum pictum Griff. 186. Guaiacum officinale Linn. 99. Gundlachia domingensis A. Gray 199.

Guettarda parvifolia Sw. 189. Guettarda scabra Lam. 189. Guillandina Bonduc Gris. 86. Guillandina glabra Gris. 86. Guillandina Grisebachiana $\mathrm{Kr}$. et Urb. 86. 
Guttiferae 134.

Gyminda latifolia Urb, 120.

Gymnanthes lucida Sw. 114.

Gymnogramme calomelanos

Kaulf. 6.

\section{Gymnospermae 12.}

Gynandropsis pentaphylla DC.75. Haematoxylon campechianum

Linn. 85.

Hebeclinum macrophyllum DC. 198.

Heckeria peltata Kunth. 45.

Heleocharis capitata Boeck. 28.

Heliconia Bihai Linn. 40.

Helicteres Isora Linn. 133.

Helicteres jamaicensis 133.

Heliotropium curassavicum Linn. 166.

Heliotropium indicum Linn. 166. Heliotropium microphyllum Sw. 167.

Heliotropium parviflorum Linn. 167.

*Heliotropium peruvianum Linn. 167.

Hemidiodia ocimifolia K. Schum. 193.

Hemionitis palmati Linn. 5. Hemitelia horrida R. Br. 2. Heteropteris lanceolate F'ee. 7. Heteropteryx purpurea H. B. K. 104.

*Hibiscus esculentus Linn. 129.

*Hibiscus radiatus Cav. 129.

"Hibiscus Rosa-sinensis Linn. 129.

*Hibiscus Sabdariffa Linn. 129.

"Hibiscus schizopetalus Hook. 130.

Hibiscus tiliaceus Linn. 130. Hillia longiflora Sw. 187. Hillia parasitica Jacq. 187.
Hippeastrum equestre Herb. 38. *Hippeastrum puniceum Urb. 38. Hippomane mancinella Limn.114. Hirtella triandra Sw. 78. *Hoya carnosa R. Br. 159. Hura crepitans Linn. 115. Hydrolea jamaicensis Raeusch. 163.

\section{Hydrophyllaceae 163.}

Hymenaea Courbaril Linn. 82. Hymenocallis caribaea Herb. 38.

\section{Hymenophyllaceae 1.}

Hymenophyllum hirtellum Sw. 1.

Hypelate trifoliata Sw. 122.

Hyperbaena domingensis Benth. 68.

Hypoxis decumbens Linn. 39. Hyptis pectinata Poit. 174. Ibatia maritima Dcne 159. Ibatia muricata Gris. 159. Ichnanthus pallens MIunro. 19. Ichthiomethia piscipula Hitchc. 92. *Impatiens Balsamina Linn. 123. Indigofera anil Linn. 88. Indigofera suffruticosa Mill. 88. Inga laurina Willd. 78. Ipomoea arenaria Steud. 161. *Ipomoea batatas Poir. 161. Ipomoea biloba Forsh. 162. Ipomoea bona nox Linn. 163. Ipomoea dissecta Pers. 161. Ipomoea fastigiata Sweet. 161. Ipomoea grandiflora Lam. 163. Ipomoea hederacea Jacq. 162. Ipomoea Nil Roth. 162. Ipomoea pentaphylla Jacq. 162. Ipomoea pes caprae Roth. 162. Ipomoea sinuata Orteg. 161. Ipomoea sphenophylla Urb. 163. Ipomoea tuba G. Don. 163. Ipomoea violacea Linn. 163. 
Iresine celosioides Linn. 58. Iresine elatior L. Cl. Rich. 58. Iresine paniculata 0. Ktze 58. Iridaceae 39.

Isachne arundinacea Gris. 15. Isachne rigens Trin. 15.

Isoloma hirsuta Reg. 184.

*Ixora coccinea Linn. 191.

*Ixora Jucunda Thwaites 191.

Jacquemontia pentantha G. Don. 161.

Jacquemontia violacea Choisy. 161.

Jacquinia armillaris Jacq. 150. Jacquinia Berteri Spreng. 151. Jambosa Jambos Millsp. 147. Jambosa vulgaris DC. 147.

*Jasminum azoricum Linn. 154.

*Jasminum pubescens Willd. 154. "Jasminum undulatum Ker. Gawl. 154.

Jatropha curcas Linn. 113. Jatropha gossypifolia Linn. 113. *Jatropha multifida Linn. 113. Juncellus laevigatus C. B. Clarke 26.

*Juniperus bermudiana Linn. 12. *Juniperus virginica Linn. 12. Justicix eustachiana Jacq. 186. Justicia sessilis Jacq. 186.

Kallstroemia maxima Wight et Arn. 99.

*Kohleria hirsuta Reg. 183.

Krameria ixinia Linn. 85.

Krugiodendron ferreum Urb. 123.

Kyllinga brevifolia Rottb. 28. Kyllinga monocephala Thunb.28.

Iabiatae 172.

Lablab vulgaris Savi 98.

Lachnostoma maritimum Nichols. 159.
Lactuca intylacea Jaç. 207. Lagascea mollis Cav. 200. Lagenaria vulgaris Ser. 196. *Lagerstroemia indica Linn. 142. Laguncularia racemosa Gärtn. 144.

Lantana Camara Linn. 167.

Lantana involucrata Linn. 168. Lappago alienc Spreng. 14.

Lauraceae 70 .

Lawsonia inermis Linn. 142. Lebidibia coriaria Schlecht. 86.

Ieguminosae 78.

Leiphaimos aphylla Gilg. 155.

Lemnaceae 31.

Lemna minor Griseb. 31.

Lemna paucicostata Hegelm. 31.

Ientibulariaceae 184.

Leonotis nepetaefolia $\mathrm{R}$. Br. 172. Leonurus sibiricus Linn. 172. Lepicystis piloselloides Diels 9. Lepicystis squamata J. Sm. 10. Lepidium virginicum Linn, 72. Leptochloa filiformis Beauv. 24. Leptochloa mucronata Kth. 24. Leptochloa virgata Beauv. 24. Leria nutans DC. 206.

Leucaena glauca Benth. 80.

Leucas martinicensis R. Br. 172. Liliaceae 36 .

Linociera compacta $R, B r .154$. Lippia reptans H. B. et K. 169. Lithophila muscoides Sw. 59.

Lithophila vermiculata Uline. 59. *Lochnera rosea Reichb. 156.

\section{Loganiacea 154.}

Lonchitis hirsuta Linn. 7.

Lonchocarpus violaceus H. B. et K. 92 .

*Lonicera japonica Thunb. 195.

Loranthaceae 52.

Lucuma multiflora A. DC. 152. 
Lycium americanum Jacq. 175. Iycopodiaceae 11.

Lycopodium taxifolium Sw. 11. Lycopodium verticillatum Linn. fil. 11.

Iythraceae 142.

Machaerina restioides Valll. 29. Malpighiaceae 104.

Malpighia angustifolia Linn. 105. Malpighia punicifolia Linn. 106. Malvaceae 126.

Malvastrum coromandelianum Garcke 127.

Malvastrum spicatum A. Gray 126.

Nalvastrum tricuspidatum A. Gray 127.

"Nammea americana Linn. 134. * Mangifera indica Linn. 118.

*Manihot utilissima Pohl. 114. Mapouria parasitica K. Sch. 192. Marantaceae 40.

*Maranta arundinacea Linn. 40. Marcgraviacea 133.

Marcgravia umbellata Linn. 133. Marila racemosa Sw. 134.

Mariscus brizaeus C. B. Clarke 27.

Mariscus capillaris Vahl. 27.

Mariscus flavus Vahl. 27.

Maurandia semperflorens Jaeq̣. 180.

Mayepea caribaea O. Ktze. 154. Maytenus elliptica Kr. et Urb. 119.

*Medicago sativa Linn. 88. Meibomia axillaris 0 . Ktze 90. Meibomia incana O. Ktze 91. Meibomia scorpiurus O. Ktze 90. Meibomia spiralis O. Ktze 91. Meibomia triflora 0. Ktze 91.

Molastomataceae 148.
Meliaceae 104.

*Melia aredarach Linn. 104. Melia sempervirens Sio. 104. *Melicocca bijuga Linn. 122. Melocactus communis Link, et Otto 140.

Melochia nodiflora Sw, 131. Melochia pyramidata Linn. 131. Melochia tomentosa Linn. 132. Melothria fluminensis Gard. 195. Meniscium reticulatum $S w .3$.

Menispermaceae 68.

Mertensia furcata Gris. 11. Mesosphaerum pectinatum 0 . Ktze 174.

Metastelma Paralias Dcne 159. Metastelma parviflora R. Br. 159. Metastelma Schlechtendalii Gris. 159.

Miconia impetiolaris D. Don. 149. Miconia laevigata DC. 149. Microstylis spicata Lindl. 41. Microtert debilis Sw. 64 . Mimosa pudica Linn. 81. *Mirabilis Jalapa Linn. 60. Mitracarmes hirtus DC. 194. Mitracarpus polycladus Urb. 194. Mitracarpus villosus Cham. et Schlecht. 194. Mollugo verticillata Linn. 64. Momordica Charantia Linn. 195.

Monocotyledoneae 12.

Moraceae 48.

Moringaceae 76.

Morin:7a moringa Millsp. 76.

*Moringa oleifera Lam. 76. Moringa pterygosperma Gärtn. 76.

Morisonia americana Linn. 76. *Mucuna pruriens P. DC. 95. * Murraya exotica Linn. 101. Musaceae 39. 
Musa sapientium Linn. 39.

"Musa paradisiaca Linn. 39.

*Mussaenda frondosa Linn. 188. Myginda latifolia Sw. 120.

Myginda pallens Sarg. 120.

Myginda pallens Smith 120.

Myginda rhacoma Swo. 120.

Myoporaceae 187.

Myrcia coriacea DC. 145.

Myrcia paniculata Kr. et Urb.145.

Myrcia splendens DC. 145.

MIyrodia turbinata Sio. 130.

Myroxylon buxifolizm $K r$. et Urb. 136.

Myrsinaceae 151.

Myrsine laeta Gris. 151.

Myrtaceae 144.

Nama jamaicensis Linn. 163.

Nazia aliena Millsp. 14.

Nectandra coriacea Gris. 70.

Nectandra discolor Gris. 71.

Nectandra Krugii Mez. 71.

Nectandra Wildenoviana Nees. 70.

Nephrodium oppositum Diels. 2.

Nephrolepis exaltata Schott. 3.

Neptunia plena Benth. 82.

*Nerium Oleander Linn. 158.

Neurogramme calomelanos

Diels. 6.

Neurolaena lobata R. Br. 205.

*Nicotiana Tabacum Linn. 180.

*Nopalea coccinellifera Salm. Dyck 142.

Nyctaginaceae 60 .

Nymphaeaceae 67.

Nymphaea spec. 67.

Ochnaceae 133.

*Ocimum basilicum Linn, 175.

*Ocimum gratissimum Linn. 175. Ocimum micranthum Willd. 175.

Olacaceae 52.

Oldenlandia herbacea DC. 187.
Oleaceae 154.

Olfersia cervina $0, K l \approx e .3$.

Oncidium silvestre Lindl. 43 .

Oncidium tetrapetalum W. 43.

Oncidium variegatum Sw. 43 .

*Operculina ventricosa Peter. 1 ti1.

Ophioglossaceae 11.

Ophioglossum reticulatum Linn. 11.

Oplismenus setarius R. et Sch. 19. Opuntia Ficus indica Mill. 141. Opuntia spinosissima Mill. 141. Opuntia triacantha Haworth. 141.

Opuntia Tuna Mill. 141.

Orchidaceae 40.

*Oreodoxa caribaea Damm. et Urb. 30.

Ornithidium coccineum Salisb. 43.

Orthopogon setarius Spreng. 19. Oxalidaceae 98.

Oxalis corniculata Linn. 98.

* Oxalis Martiana Zucc. 98.

Pachygone domingensis Eichl. 68.

Palicourea Pavetta DC. 192.

Palmae 29.

Paltonium lanceolatum Pr. 7.

*Panax fruticosum Linn. 150.

Pancratium caribaeum Limn. 38.

Panicum barbinode Trin. 18.

Panicum brevifolium Linn. 15.

Panicum colonum Linn. 15.

Panicum diffusum Sw. 15.

Panicum divaricatum Linn. 16.

Panicum fuscum Sw. 16.

Panicum geminatum Forsk. 16. Panicum insulare G. F. W. Mey. 17.

Panicum lanatum Rottb. 17.

Panicum leucophaeum H. B. K.17.

Panicum maximum Jacq. 17. 
Panicum molle Sw. 18.

Panicum pallens Sio. 19.

Panicum paspaloides Pers. 16.

Panicum prostratum Lam. 18.

Panicum sanguinale Linn. 18.

Papaveraceae 71.

Pappophorum alopecuroideum Vahl. 24.

Pappophorum laguroideum

Schrad. 25.

Paritium tiliaceum St. Hill. 130.

*Parkinsonia aculenta Linn. 85.

Parthenium Hysterophorus

Linn. 201.

Paspalum conjugatum Bergius. 14.

Paspalum fimbriatum H. B. K. 14.

Paspalum hemisphaericum Poir. 14.

Paspalum paniculatum Willd. 14.

Passifloraceae 138.

Passiflora foetida Linn. 138.

*Passiflora laurifolia Linn. 138.

Passiflora maliformis Linn. 138.

"Passiflora quadrangularis Linn. 139.

Passiflora rubra Linn. 139.

Passiflora suberosa Linn. 139.

Pavonia spinifex Cav. 129.

Pectis febrifuga van Hall. 205.

Pectis humifusa Sw. 205.

Pectis linifolia Less. 205.

Pectis Swartziana Borg et Pauls. 205.

Pedilanthus tithymaloides Poit. 117.

*Peireskia aculeata Mill. 142.

*Peireskia bleo DC. 142.

Pennisetum setosum Rich. 21.

Pentarrhaphia longiflora Lindl. 184.
Peperomia acuminata C. DC. 45. Peperomia emarginella C. DC. 45. Peperomia exilis Griseb. 45.

Peperomia glabella A. Dietr. 45. Peperomia glabella Griseb. 45.

Peperomia glabella var. nigro. punctata Duss. 45.

Peperomia hernandifolia A.Dietr. 46.

Peperomia Langsdorffiï Miq. 46. Peperomia magnoliaefolia $A$. Dietr. 46.

Peperomia obtusifolia A. Dietr. 46.

Peperomia petiolaris C. DC. 47. Peperomia repens. Kth. 47.

Peperomia scandens $R$. et Pav. 47.

Peperomia urocarpa Fisch. et Mey. 47.

*Persea americana Mill. 70. Persea gratissima Gärtn. 70 . Persea Persea Cock. 70. Petiveria alliacea Linn. 64. *Petunia violacea Lindl. 180. Pharbitis Nil Choisy. 162.

Pharus glaber H. B. K. 21. Phaseolus lathyroides Linn. 97. *Phaseolus lunatus Linn. 97.

Phaseolus semierectus Linn. 97.

*Phaseolus vulgaris Linn. 97.

Philodendron dispar Schott. 30. Philodendron giganteum Schott. 31.

Philodendron oxycardium Schott. 31.

Philoxerus vermicularis $R$. Br. 59.

Phoebe elongata Nees. 70.

*Phoenix dactylifera Linn. 29.

Phoradendron trinervium Gris. 52. 
Phyllanthus distichus Müll.Arg. 107.

Phyllanthus epiphyllanthus Linn. 107.

Phyllanthus falcatus Sio. 107.

Phyllanthus niruri Linn. 108.

Phyllanthus nobilis Mull.-Arg. 108.

Physalis angulata Linn. 175.

Physalis pubescens Linn. 176.

Physurus hirtellus Lindl. 41.

Physurus plantagineus Lindl. 41.

Phytolacoaceae 68.

Phytolacca icosandra Linn. 64.

Picraena excelsu Gris. 102.

Picramnia pentandra Sw. 103.

Picrasma antillana Urb. 102.

Pilea ciliaris Wedd. 51.

Pilea elegans Wedd. 51.

Pilea microphylla Liebm. 50.

Pilea muscosa Lindl. 51.

Pilea obtusata Liebm. 51.

Pilea parietaria Bl. 51.

Pilea pubescens Gris. 51.

Pilea semidentata Wedd. 51.

Pimenta acris Kostel. 144.

Pinaceae 12.

Piperaceae 45.

Piper Bredemeyeri Jacq. 44.

Piper ceanothifolium Kunth. 44.

Piper dilatatum L. Cl. Rich. 44.

Piper marginatum Jacq. 44.

Piper medium Jacq. 44.

Piper peltatum Linn. 45.

Piper reticulatum Linn. 45.

Piper smilacifolium P. DC. 45.

Piriqueta cistoides G. F. W. Mey 137.

Piriqueta villosa Aubl. 137.

Piscidia erythrina Linn. 92.

Piscidia piscipula Sarg. 92.

Pisonia aculeata Linn. 61.
P'isonia fragans Dumont 61.

Pisonia inermis Gris. 61.

Pisonia obtusata Sio. 61.

Pisonia subcordata Sw. 62.

Pitcairnia angustifolia Redouté 39.

Pithecolobium ungius-cati Benth. 78.

Plantaginaceae 187.

Plantago major Linn. 187.

Pluchea odorata Cass. 200.

Pluchea purpurascens DC. 200.

Plumbaginacaea 151.

*Plumbago capensis Thunb. 151. Plumbago scandens Linn. 152.

Plumiera alba Linn. 155.

*Plumiera rubra Linn. 156.

*Poinciana regia Boj. 85.

Polanisia icosandra Wight et Arn. 73.

Polanisia viscosa DC. 73.

Polyboea corensis Klotsch. 111.

Polybotrya cervina Klfs. 3.

Polygonaceae 53.

Polypodiaceae 2.

Polypodium aureum Linn. 8.

Polypodium brasiliense Poir. 8 . Polypodium crassifolium Linn. 8. Polypodium loriceum Linn. 8.

Polypodium lycopodioides Linn. 8.

Polypodium neriffolium

Schkuhr. 8.

Polypodium phyllitidis Linn. 9.

Polypodium piloselloides Linn.9.

Polypodium plumula $\mathrm{H}$. B.

IVild. 9.

Polypodium serrulatum Mett. 10.

Polypodium squamatum

Linn. 10.

Polypodium trichomanoides

Sw. 10. 
Polystachya luteola Hook. 41. *Porana paniculata Roxb. 160.

Porophyll um ellipticum Cilss. 204 Porophyllum ruderale Gris. 204.

Portulacaceae 65.

Portulaca halimoides Linn. 66.

*Portulaca oleracea Linn. 66.

Portulaca pilosa Linn. 66.

Portulaca poliosperma Lirb. 67. Portulaca quadrifida Linn. 67.

\section{Potamogetonaceae 12.}

Pothomorphe peltata AIiq. 45.

Prescottia stachyoides Lindl. 41.

Priva echinata Juss. 170.

Priva lappulacea Pers. 170.

*Prockia Crucis Linn. 136.

Prunus myrtifolia Urb. 77.

Prunus sphaerocarpa Sw. 77.

Psidium Guajava Linn. 144.

Psilotaceae 11.

Psilotum nudum Gris. 11.

Psilotum triquetrum Sw. 11.

Psychotria crassa Gris. 192.

Psychotria horizontalis Sw. 191.

Psychotria parasitica Sw. 192.

Psychotria pendula Urb. 192.

Psychotria rufescens $\mathrm{H}$. B. et K. 192 .

Psychotria uliginosa Sw. 192.

\section{Pteridophyta 1.}

Pteris biaurita Linn. 7 .

Pteris laciniata Willd. 7.

Punicaceae 143.

*Punica Granatum Linn. 143.

*Quamoclit vulgaris Choisy 163. Quararibea turbinata Poir. 181.

*Quisqualis indica Linn. 143. Randia aculeata Linn. 188.

Rapanea ferruginea Mez. 151. Rauwolfia Lamarckii DC. 157. Rhacoma crossopetalum Linn. 120.
Rhacoma latifolia Loesener 120.

Ramnaceae 123.

Rhizophoraceae 143.

Rhizophora Mangle Linn. 143.

*Rhoeo discolor Hance 35.

Rhynchosia minima P. DC. 96. Rhynchosia reticulata P. DC. 97.

Rhynchospora Humboldtiana

Gris. 29.

Rhynchospora Persooniana

Gris. 29.

Rhynchospora polyphylla Vahl. 29.

*Ricinus communis Linn. 112.

Rivina humilis Linn. 63.

Rivina laevis Linn. 63.

Rivina octandra Linn. 63.

Rochefortia acanthophora Gris. 165.

\section{Rosaceae 77.}

Rousselia humilis Urb. 52.

Rousselia lappulacea Gaudich. 52.

Rubiaceae 187.

Ruellia tuberosa Linn. 185.

*Russelia juncea Zuccar. 180.

Rutaceae 100.

*Saccharum officinarum Linn. 12. Salicornia herbacea Linn. 55. Salvia occidentalis Sw. 173. Salvia serotina Linn. 173.

*Sambucus canadensis Linn. 195. Samyda serrulata Linn. 136. *Sanchezia nobilis Hook. 185.

*Sanseviera guineensis Willd. 36.

Sapindaceae 121.

Sapotaoeae 152.

Sapota Achras Mill 152.

Sauvagesia erecta Linn. 133.

Scaevola Lobelia Murr. 196.

Scaevola Plumieri Vahl. 196.

Schaefferia frutescens Jacq. 121. 
Schmidelia occidentalis Sw. 121. Schoepfia Schrebori J. F'. Gimel. 52.

Scirpus capitatus Linn. 28. Scirpus obtusifolius Gris. 28. Scleria filiformis Sro. 29. Scleria lithosperma Sw. 29. Scleria pratensis Nees. 29. Scleria pterota Pres]. 29. Scoparia dulcis Linn. 181.

Scrophulariaceae 180.

Sechium edule Sw. 196.

Selaginellaceae 11.

Selaginella flabellata Spring. 11. Selaginella substipitata Spring. 12.

Senecio sonchifolius Moench. 206.

* Sesbania grandiflora Pers. 89.

Sesbania sericea P. DC. 89.

Sesuvium portulacastrum Linn. 64.

Setaria glauca Beauv. 19.

Setaria setosa Beauv. 20.

Setaria viridis Beauv. 20.

Sida acuta Burm. 127.

Sida carpinifolia Linn. 127.

Sida ciliaris Linn. 127.

Sida cordifolia Linn. 128.

Sida glomerata Cav, 128.

Siđa rhombifolia Linn. 128.

Sida spinosa Linn. 128.

Sida supina l'Herit. 128.

Sideroxylon foetidissimum Jacq. 152.

Sideroxylon mastichodendron Jacq. 152.

Simarubaceae 102.

Sinapis brassicata Gris. 72.

Sinapis juncea Eichl. 72.

Smilax coriacea Spr. 37.

Smilax guianensis Vitmann 37.
Smilax havanensis Gris. :37.

Smilax jlicifolia Kth. 37.

Smilax macrophylla Willd. 37.

Solanaceae 175.

Solanum aculeatissimum Jaç.

176 .

Solanum igneum Linn. 178.

Solanum lanceaefolium Jacq. 176.

*Solanum lycopersicum Linn. 177.

*Solanum Melongena Linn. 177.

Solanum nigrum nodiflorum $A$. Gray 177.

Solanum nigrum nodiflorum Linn. 17\%.

Solanum nodiflorum Dunal 177. Solanum racemosum Jacq. 177. Solanum racemosum Jacq. igneum (Linn.) O. E. Schulz. 178. Solanum torvum Str. 178.

"Solanum tuberosum Linn. 179. Sonchus oleraceus Linn. 207. Spermacoce tenuior Linn. 194. Spigelia Anthelmia Linn. 154. Spilanthus uliginosa Sw. 203. Spiranthes elata L. Cl. Rich. 40. *Spondias lutea Linn. 119.

*Spondias purpurea Linn. 119. Sponia Lamarckiana Dcne 48. Sponia micrantha Done 48.

Sporobolus indicus R. Br. 22. Sporobolus virginicus Kth. 22. Stachytarpheta indica Vahl 169. Stachytarpheta jamaicensis Vahl 169.

Stenandrium rupestre Nees 186. Stenochlaena sorbifolia J. Sm. 5. *Stenolobium stans Seem. 182.

Stenorrlunnchus lanceolatus $\mathrm{L} . \mathrm{Cl}$. Rich. 40.

Stenorrhynchus orchioides L. Cl. Rich. 40.

Sterculiaceae 131. 
Stigmatophyllon ciliatum A. Juss. 105.

Stigmatophyllon periplocifolium

A. Juss. 105.

Stigmatophyllon sericeum

Wright 105.

Stigmatophyllum diversifolium

A. Juss. 105.

Strumpfia maritima Jacq. 191. Stylosanthes hamata Taubert 89. Stylosanthes procumbers Sio.89. Suriana maritima Linn. 102. *Swietenia mahagoni Jacq. 104.

Symplocaceas 153.

Symplocos martinicensis Jacq. 153.

Synedrella nodiflora Gärtn. 203. Syntherisma insularis Millsp. 17. Syntherisma sanguinale Dulac. 18.

Tabernaemontana citrifolia Jacq. 156.

*Tabernaemontana coronaria Willd. 156.

* Tabernaemontana Cumingiana A. DC. 156.

Taenitis lanceolata $R, B r .7$. T‘alinum paniculatum Gärtn. 65. Talinum patens Willd. 65. Talinum triangulare Willd. 65. *Tamarindus indica Linn. 82. *Tecoma capensis Lindl. 182. T'ecoma stans Juss. 182.

*Tecoma leucoxylon Mart. 182. Telanthera crucis Moq. 58. Tephrosia cathartica Urb. 88. Tephrosia cinerea Pers. 88. Teramnus labialis Spreng. 94.

*Terminalia Catappa Linn. 143. T'ernstroemia elliptica Siv. 134. Ternstroemia peduncularis DC. 134.
Tetramicra rigida Lindl. 43. Tetrazygia discolor DC. 149. Theaceae 134.

Theobroma Cacao Linn. 133.

Theophrastaceae 150.

Thespesia populnea Soland. 130.

*Thevetia neriifolia Juss. 157.

Thrinax sp. 30.

*Thunbergia affinis pulvinatus Moore 184.

*Thunbergia alata Boj. 184.

*Thunbergia fragans Roxb. 184.

Thymelaeaceae 142 .

Tibouchina ornata Baill. 149. Tibouchina strigosa Cogn. 148. Tiliaceae 125 .

'T'illandsia excelsa Gris. 33. Tillandsia fasciculata Sw. 33. Tillandsia recurvata Linn. 33. Tillandsia usneoides Linn. 33. Tillandsia utriculata Linn. 33. *Tithonia tagetiflora Desf. 203. Tobinia punctata Gris. 101. Tobinia ternata Desv. 101. Torulinium confertum Desv. 28. Tournefortia bicolor Siv. 165. Tournefortia caribaea Gris. 165. Tournefortia foetidissima Linn. 165.

Tournefortia gnaphalodes $R$. Br. 165.

'Tournefortia hirsutissima Linn. 166.

Tournefortia volubilis Linn. 166. Tradescantia geniculata Jacq. 35. Tragia volubilis Linn, 112. Tragus racemosus Haller 14. 'Trema Lamarckianum Bl. 48. Trema micranthum Bl. 48. Trianthema monogynum Linn. 65. 'Trianthema portulacastrum Linn. 65. 
T'ribulus cistoides Linn. 99. Tribulus maximus Linn. 99.

Tricholaene insularis Gris. 17. Trichomanes alatum Sw. 1.

Triphasia aurantiola Lour. 102. *1'riphasia trifoliata P. DC. 101. 'Triumfetta rhomboidea Jacq. 125.

T'riumfetta semitriloba Jacq. 125.

Turneraceae 137.

I'urnera ulmifolia Linn. 138.

Ucacona nodiflora Hitchc. 203.

Ulmaceae 47.

Umbelliferae 150 .

Urechites suberecta Müll.Arg. 157.

Urena americana Limn. 129.

Urena lobata Linn. 129.

Urera alceaefolia Gaudich. 50.

Urera caracasana Gris. 50.

Urticacene 50.

Utrieularia alpina Jaeq. 184.

Utricularia montana Jacq. 184.

Valerianoides jamaicensis Meclic. 169.

Verbenaceae 167.

*Verbena chamaedrifolia Juss. 167.

Vernonia longifolia Pers. 196.

Vernonia punctata Sw. 196.

Vetiveria amendinacen Gris. 13.

Vigna glabra Savi 89.

Vigna luteold Benth. 97.

Vigna repens O. Ktze 97.
Villamilla octandra Hook. 63. Vincr rosea Linn. 156.

Vitaceae 124.

Vitis sicyoides Miq. 124.

Vittaria lineata Sw. 7.

Voyria aphylla Pers. 155.

Voyria unitlora Pers. 155.

Vriesea guadalupensis Mez. 34.

Waltheria americana Linn. 132.

Waltheria indica Linn. 182.

Wedelia buphthalmoides Gris. 202.

Wittmackia lingulata Mez, 32.

Xanthium orientale Linn. 201.

*Xanthosoma spec. 31.

Tanthoxylum clava Herculis

Sw. 100.

Xanthoxylum flavum Vahl.100.

Xanthoxylum martinicense $P$. DC. 100.

Xanthoxilum spinifex $P . D C$. 100.

Xanthoxylum ternatum Sio. 101.

Xiphopteris serrulata Kaulf. 10.

Xylosma buxifolium A. Gray 136.

Yucea aloifolia Linn. 36.

*Zea Mays Linn. 12.

Zebrina pendula Schnizl. 36.

*Zephyranthes carinata Herb. 37.

Zingiberaceae 40 .

*Zinnia elegans Jacq. 202.

Zizyphus Jujuba Lam. 123.

Zornia diphylla Pers. 90.

Zygophyllaceae 99. 



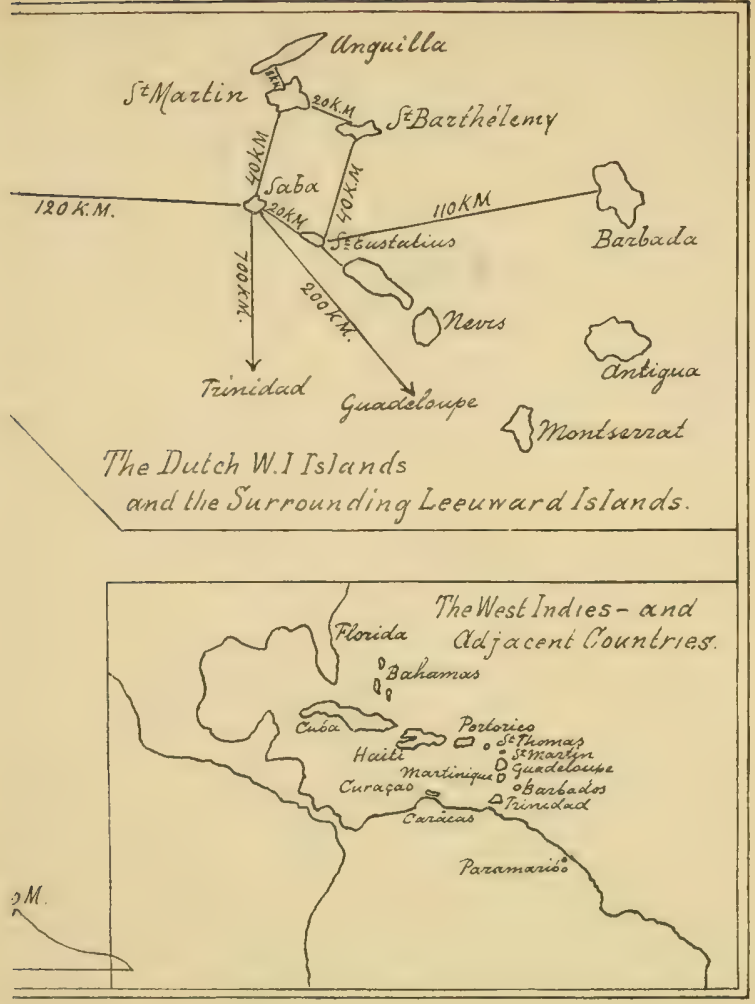




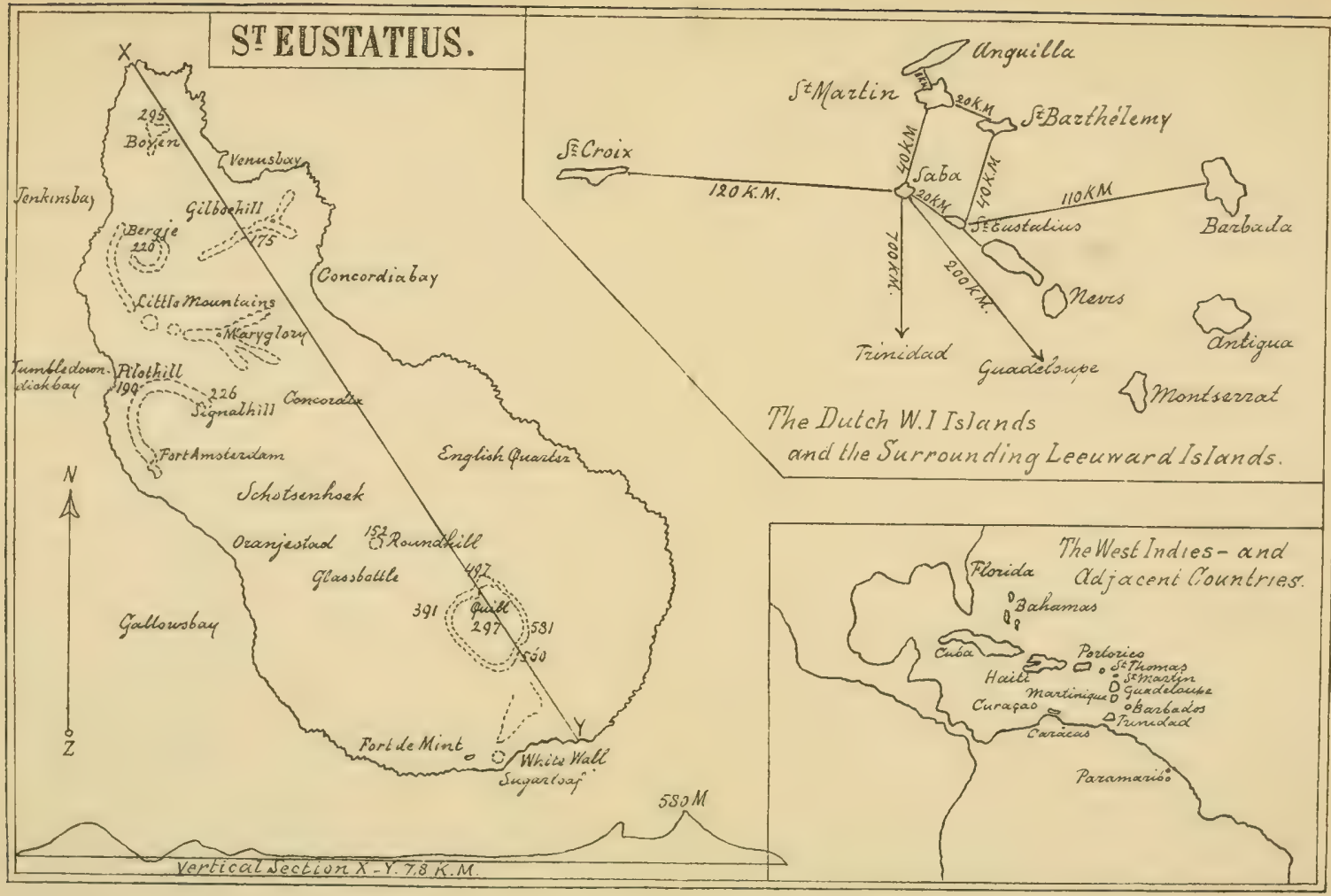

Boldingh. - Flora Dutch, Wost 1nd., Islanđ's. 


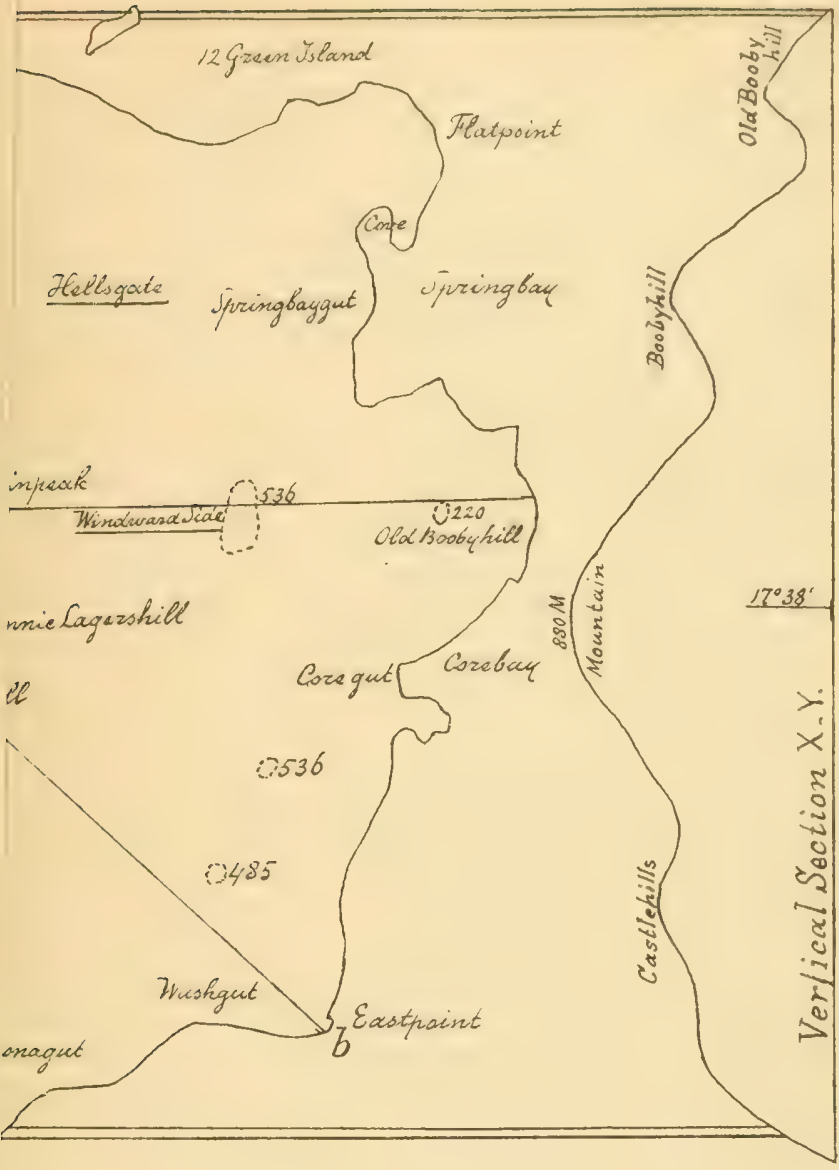




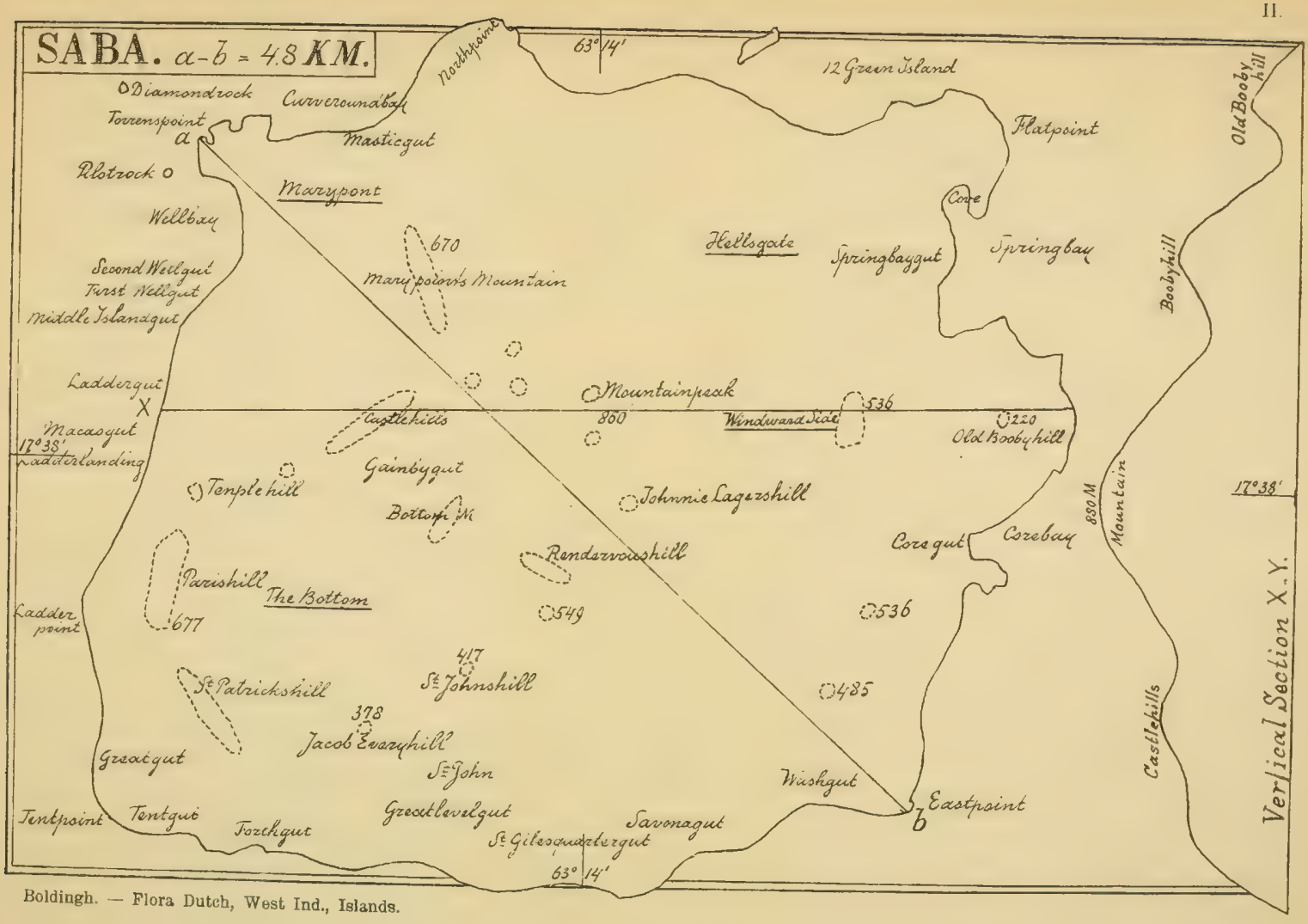




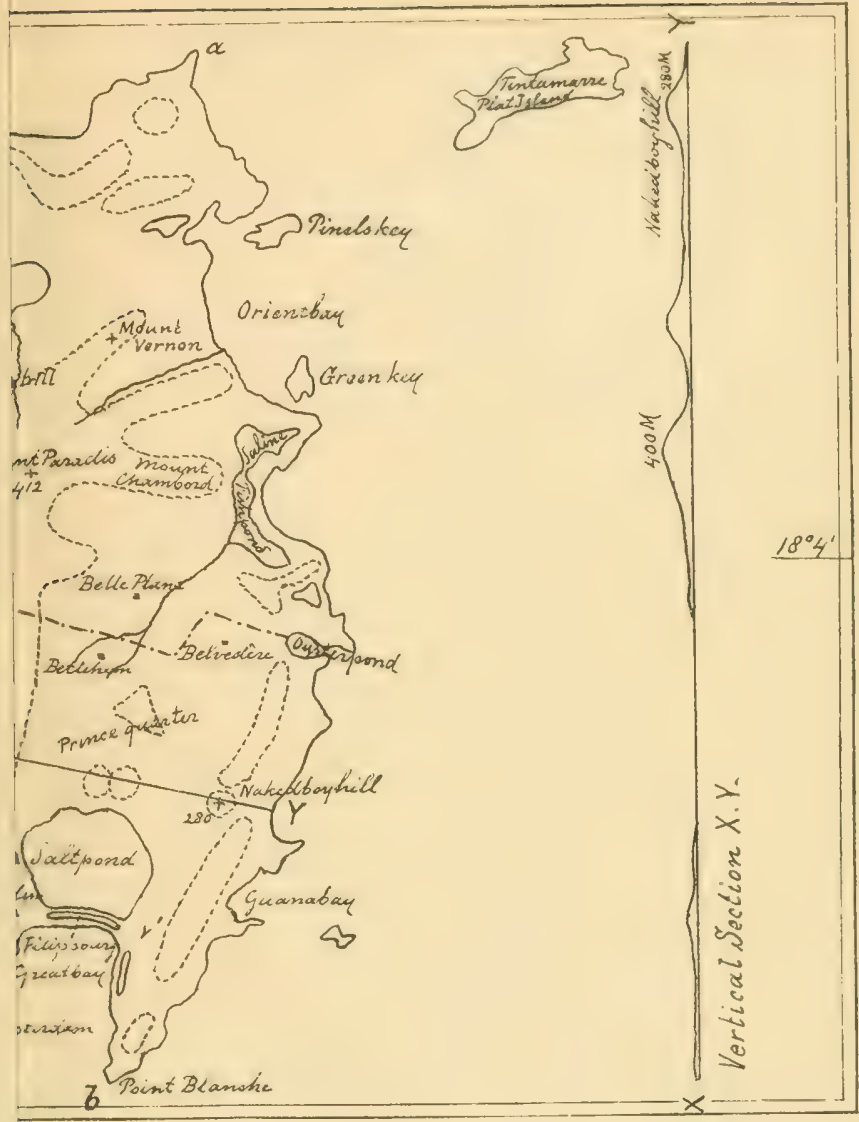




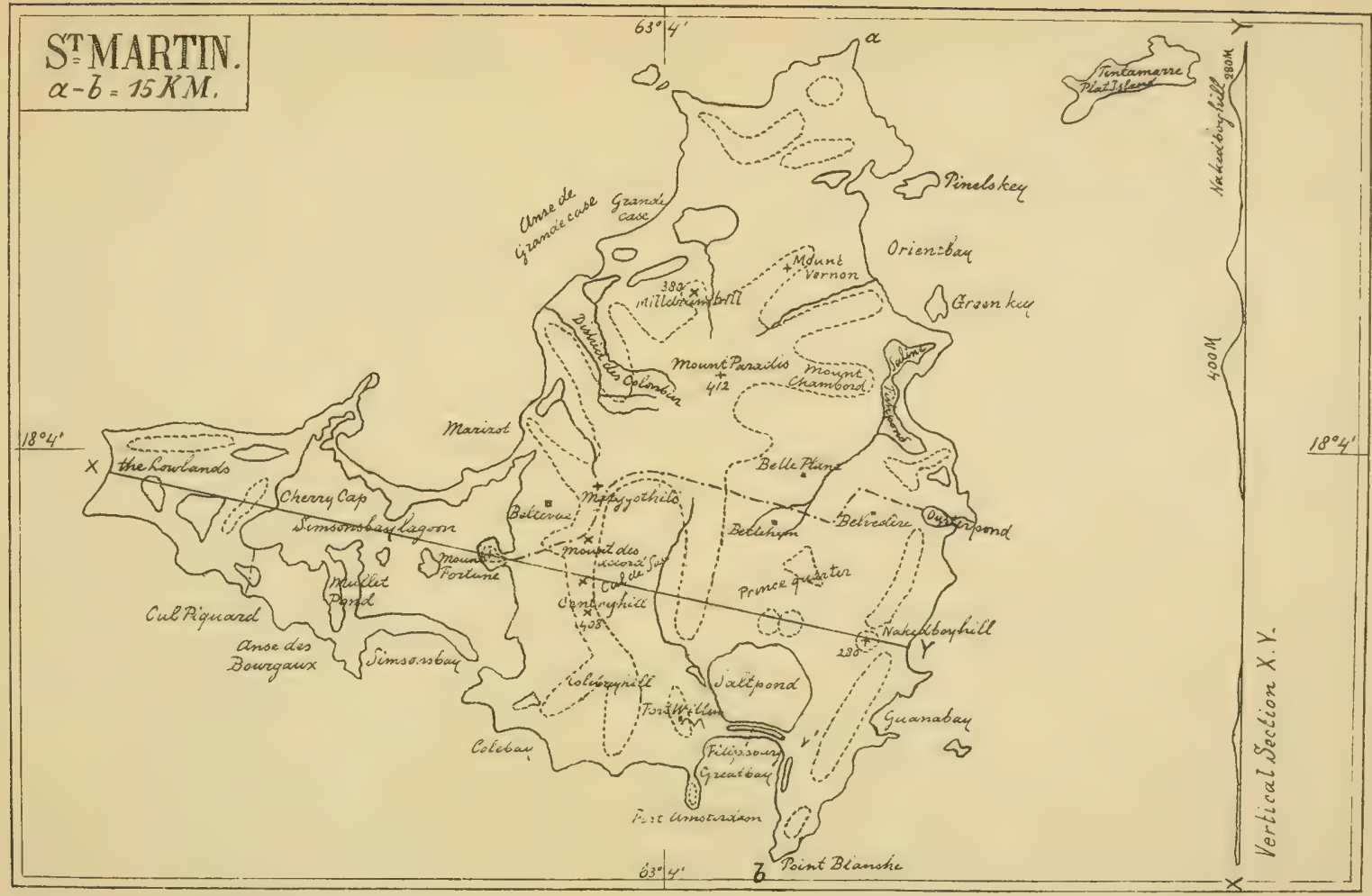









QK 238. Bow

Bolding. B6 238 .

Iingh Isaac/The flora of the Dutch $w$

|| || || || || || || || | | | || |||

${ }_{35185}^{\|||}$

\begin{tabular}{l}
5185001014065 \\
\hline
\end{tabular} 
